\title{
QUALITY OF LIFE RESEARCH
}

Official Journal of the International Society for Quality of Life Research

\author{
Volume $10 \quad$ Number 32001
}

This journal issue contains abstracts that have been accepted for presentation at the

\author{
8th Annual Conference of the
}

\section{International Society for Quality of Life Research (ISOQOL)}

7 - 10 November 2001, Amsterdam, The Netherlands

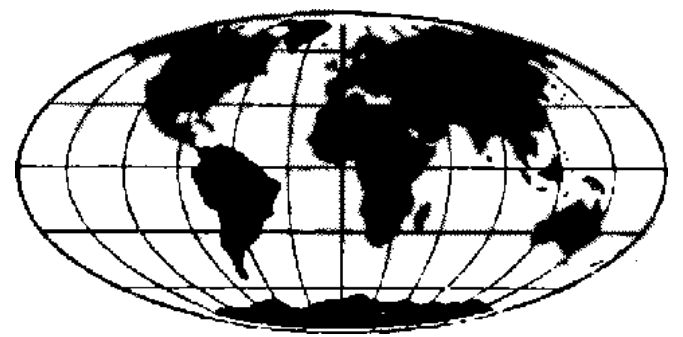

The formatting of the abstracts within this issue was done in cooperation with Degnon Associates, ISOQOL's management team.

ISOQOL Executive Office 6728 Old McLean Village Drive McLean VA 22101-3906 USA

Phone: (703) 556-9222

Fax: (703) 556-8729

E-mail: info@isoqol.org Internet: www.ISOQOL.org 



\section{International Society for Quality of Life Research (ISOQOL)}

\section{Mission Statement}

The scientific study of Quality of Life Relevant to health and healthcare is the mission of the International Society for Quality of Life Research (ISOQOL). The Society promotes the rigorous investigation of health-related quality of life measurement from conceptualization to application and practice. ISOQOL fosters the worldwide exchange of information through:

- Scientific Publications

- Educational Outreach
- International Conferences

- Collaborative Support for HRQL Initiatives

\section{Membership Benefits}

- Reduced subscription rate for the Quality of Life Research Journal

- Reduced registration rates at ISOQOL's Annual Meeting and workshops which are held all over the world throughout the year

- Opportunity to present and hear cutting edge research presentations and posters at the Annual Meeting

- Membership Directory

- Access to the Members Only section of the website

- Complimentary receipt of quarterly newsletters

- Opportunity to have special interest group meetings at the Annual Meeting

- Exposure to and participation with other professionals involved in quality of life research activities

The 2001 ISOQOL Annual Meeting will take place 7-10 November in Amsterdam, The Netherlands

Chairs: Neil Aaronson, PhD and Mirjam Sprangers, $\mathrm{PhD}$

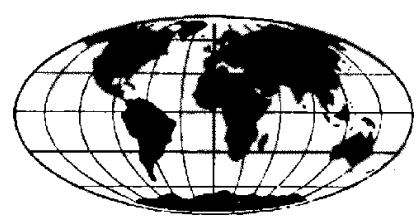

International Society for Quality of Life Research 6728 Old McLean Village Drive McLean, VA 22101-3906 USA

Phone: 703-556-9222; Fax: 703-556-8729

Email: info@isoqol.org; WWW.ISOQOL.ORG 

1/ COMPARING FIVE METHODS OF UTILITY ASSESSMENT ACROSS DIFFERENT DISEASE STATES

Patrick W. Sullivan, Michael B. Nichol, Denise R. Globe, Pharmaceutical Economics and Policy, University of Southern California, Los Angeles, CA

The objective of this study was to (1) compare five methods of utility assessment across different disease states and (2) to analyze the construct validity of each method. The study population included 6,921 members of Southern California Kaiser Permanente. The HUI Mark II, SF-36 and visual analogue scale (VAS) were directly administered The Nichol/Sengupta/Globe (NSG) algorithm and the Brazier method (both VAS and standard gamble [SG]) were applied to SF-36 scores to calculate summary utility scores. Dummy variables for 23 different comorbidities were created from AHFS codes. OLS was used to regress utility scores on the 23 comorbidities controlling for sociodemographic variables for all five methods. Generic mean utility scores were also compared. The majority of the dummy variables representing different comorbidities were statistically significant $(0.05$ level) for each of the five methods. The particular comorbidities that were statistically significant varied by method. For example, for the larger diseases $(n>300)$, the Brazier SG method captured the marginal effect of 13 different diseases, but did not capture the remaining 2 (thyroid replacement and epilepsy). The differences in the parameter estimates and the standard errors between the five methods varied depending on the disease. As an example, the parameter estimates (standard errors) of the marginal impact of Depression on utility for all five methods are as follows: HUI2 -0.08 (.007); NSG -0.08 (.005); Brazier SG -0.04 (.003); Brazier VAS -0.09 (.007) and VAS 0.06 (.007). Generic mean utility scores were: HUI2 (.80), NSG (.80), Brazier SG (.90), Brazier VAS (.65), and VAS (.68). All five methods appear to be valid measures of disease-related morbidity for a variety of comorbidities. The five methods differ in their ability to capture morbidity associated with certain diseases. The Brazier SG method and the HUI2 were substantially different in their estimates of generic utility under uncertainty.

\section{2/ COMPARISON OF THREE MEASURES OF UTILITY WITHIN DI- VERSE ETHNIC GROUPS \\ Denise R. Globe, Joanne Wu, Pharmaceutical Economics and Policy,}

University of Southern California, Los Angeles, CA

The purpose of this study was to compare health utility scores generated from three different methods. The data set consisted of sociodemographic, pharmacy, and survey data collected from 6,924 members of a large managed care organization. Subjects who completed both the SF-36 and the HUI were included in this study. HUI scores were calculated using the standard algorithm. Two utility score estimates were calculated from the SF-36; (1) a previously published Ordinary Least Squares (OLS) algorithm and (2) the SF-6D. Mean subgroup scores by ethnicity were compared using one-way ANOVA to assess the utility scores between race subgroups. The cohort included Caucasian (59\%), African-American (21\%), Latino (12\%), and Asian $(6.8 \%)$ subgroups. The HUI was not significantly different across ethnic groups (Caucasians 0.79, African-Americans 0.80, Latinos 0.81, Asians 0.82). Asians had significantly higher unadjusted estimated utility scores from both the OLS model (mean score 0.83 ) and the SF-6D (mean score 0.92). After adjusting for gender, income, smoking, employment status, and the presence of chronic disease, Caucasians rated utility significantly lower compared to the other ethnic subgroups (mean HUI score range 0.79-0.81). In the two estimated utility scores (mean OLS range $0.80-0.82$, mean SF-6D range 0.90 $0.91)$ Caucasian scores were lower compared to the other three ethnic subgroups $(P=0.0001)$. HUI and OLS scores were significantly lower (mean difference 0.10) than SF-6D scores for both the total cohort and within ethnic subgroups. The results of this study suggest that among diverse, English speaking ethnic groups, Caucasians rate health utility lower, regardless of the method used to calculate utilities. Furthermore, mean utility scores differ between the three different methods, irrespective of ethnic group. These findings suggest the need to account for differences in utility scores by ethnicity and utility measurement method when incorporating these values for clinical and policy decisions.
3/ CAN UTILITY-WEIGHTED HRQOL ESTIMATES CAPTURE HEALTH EFFECTS OF QI FOR DEPRESSION?

Cathy Sherbourne, Health Program, RAND, Santa Monica, CA

Utility methods that are responsive to changes in desirable outcomes are needed for cost-effectiveness (CE) analyses and to help in decisions about resource allocation. We evaluate the responsiveness of several different methods that assign utility weights to subsets of SF36 items to improvements in health resulting from two quality improvement (QI) interventions for depression. The data for these analyses are from a group level, randomized, control trial conducted in 46 primary care clinics in 6 managed care organizations. Clinics were randomized to 1 of $2 \mathrm{QI}$ interventions or usual care. The sample includes 1136 patients with current depressive symptoms and either 12-month, lifetime, or no depressive disorder identified through screening 27,332 consecutive patients. We estimated utility values using 7 different methods (developed by ourselves and other investigators) to convert items from the 12- or 36-item Short Form Health Surveys to utility weights. Utilities elicited directly from patients were also evaluated We conducted intent-to-treat analyses for each utility measure, specifying linear time trends over the 2 years of follow-up. Several utilityweighted measures showed stronger increases in utility values for patients in one of the interventions, relative to usual care, that were similar in pattern to the improved health effects seen for measures of probable depression and emotional well-being. However, gains in QALYs were small. Directly elicited utility values showed a paradoxical result of lower utility during the first year of the study for intervention patients relative to controls. The results raise cautions about the use of utility measures as outcomes in effectiveness studies for depression. Choice of measure may lead to different conclusions about the benefit and CE of treatment. Utility measures which capture the mental health and non-health outcomes associated with treatment for depression are needed.

\section{4/ CONVERGENT VALIDITY OF SF-36 UTILITIES IN PATIENTS} WITH INTERMITTENT CLAUDICATION

Johanna L. Bosch, Decision Analysis and Technology Assessment Group, Massachusetts General Hospital, Harvard Medical School, Boston, MA; Molly T. Beinfeld, Elkan F. Halpern, Scott G. Gazelle, DATA Group, MGH, HMS, Boston, MA

To use SF-36 data for economic evaluation in health care, Brazier et al developed an algorithm to assess preference-based utilities. In this study, we assessed the convergent validity of the SF-36 utilities. The SF-36, Health Utilities Index mark 2 (HUI2) and mark 3 (HUI3), EuroQol EQ-5D, standard gamble, and rating scale were assessed in 87 patients with intermittent claudication before percutaneous revascularization. Societal preference-based SF-36 utilities were calculated based on the algorithm of Brazier et al (which they developed using standard-gamble utilities assessed in the general population of the UK). The utilities and scores were analyzed using paired t-tests and uni-variate linear regression analyses. The mean SF-36 and HUI3 utilities were the same $(0.66$ vs. $0.66, \mathrm{P}=0.92)$. The mean $\mathrm{HUI} 2$ utility, standard-gamble utility, and rating-scale score were significantly higher than the mean SF-36 utility $(0.70,0.91,0.72, P<0.05)$; the EQ-5D score was significantly lower $(0.57, \mathrm{P}<0.05)$. Uni-variate linear regression analyses demonstrated that the best relationship between the sf-36 utilities and outcomes of the other measures was found between the sf-36 and HUI3 (B-coefficient 0.25, Adjusted $\mathrm{R}^{2} 0.30$ ) and the poorest relationship between the sf-36 and standard gamble (B-coefficient 0.10 , Adjusted $R^{2} 0.02$ ). The relationship between the sf-36 and HUI2, EQ-5D, and rating scale were moderate to good (Bcoefficient $0.25,0.21,0.27$, Adjusted $R^{2} 0.30,0.33,0.26$, respectively). In conclusion, SF-36 utilities were very similar to the HUI3 utilities in patients with intermittent claudication. Face validity was demonstrated between the SF-36 and the other measures. These results suggest that the SF-36 utilities estimated with the preference-based algorithm of Brazier et al can be used interchangeably with HUI3 utilities in economic evaluation of new interventions in health care. 
5/ ESTIMATION AND VALIDATION OF DERIVED UTILITY SCORES FROM THE SF-36 IN LUNG TRANSPLANT PATIENTS

Francis S. Lobo, Cynthia R. Gross, Barbara Mathees, University of Minnesota, Minneapolis, MN

The purpose of this study is to predict and validate utility scores from the SF-36 in a sample of lung transplant patients and to examine the extent to which the resulting scores correspond to self-reported health. Data including the SF-36, Center for Epidemiologic Studies Depression (CES-D) Scale, Symptom Distress Scale (SDS), Illness Intrusion Scale (IIS), Internal Health Locus of Control (IHLOC) and Health Status (VAS) were collected from 99 lung transplant patients via a mail survey. Methods from two studies (Fryback et. al. and Brazier et. al.) were used to estimate utilities. Correlations and group differences methods were used to validate predicted scores. The Fryback scores ranged from 0.43 to 0.83 (Mean $=0.643, S D=0.086$ ); Brazier scores ranged from 0.12 to 0.92 (Mean $=0.596, S D=0.188$ ). High positive correlations were observed for both scores with the SF-36 PCS and VAS that ranged from 0.61 to 0.83 . The SF-36 MCS was highly correlated with the Brazier scores $(r=0.65)$ and moderately correlated with Fryback scores $(r=0.32)$. Moderate positive correlations were observed for both scores with the IHLOC ( $r=0.47$ to 0.51$)$. Moderate to high negative correlations were observed for both scores with the SDS and the IIS $(-0.36$ to -0.79$)$. On both scores significant differences were found among patients grouped by quartiles of CES-D, SDS, IIS IHLOC, and VAS and at five levels of general health. The CES-D was highly negatively correlated with the Brazier scores $(r=-0.71)$ as opposed to moderate correlations with the Fryback scores $(r=-0.41)$. The results support evidence of validity for these two derived scores as representative measures of Health Related Quality of Life. The Brazier scores appear to capture mental health aspects like depression and illness intrusion to a larger extent. On the other hand the Fryback scores correlate well with physical health measures like the VAS and general health status. However, there is further need to demonstrate the validity of the use of the derived scores in cost-utility analyses.

\section{6/ CANCER PATIENTS' PREFERENCES FOR COMMUNICATING} QUALITY OF LIFE INFORMATION: A QUALITATIVE STUDY

Michael D. Brundage, Radiation Oncology Research Unit, Queen's University, Kingston, ON, Canada; Anne Leis, Psychology, University of Saskatchewan, Saskatoon, Saskatchewan, Canada; Andrea Bezjak, Radiation Oncology, University of Toronto, Toronto, ON, Canada; Deb Feldman-Stewart, Radiation Oncology Research Unit, Queen's University, Kingston, ON, Canada; Lesley Degner, Nursing, University of Manitoba, Winnipeg, Manitoba, Canada; Karima Velji Radiation Oncology, University of Toronto, Toronto, ON, Canada; Dongsheng Tu, Joseph Pater, NCIC Clinical Trials Group, Queen's University, Kingston, ON, Canada; Paul Ritvo, Family Physicians Research Section, SCRN, University of Toronto, Toronto, ON, Canada

Quality of life (QOL) is a prevalent outcome measure in clinical trials but it is not known how to best communicate QOL results to new patients with cancer. Our purpose was to explore cancer patients' attitudes toward ten visual and written formats for communicating QOL information. Using standardised qualitative methods, six focus groups were held (two groups in each of three cancer Centres) with patients who had completed treatment for cancer 6-24 months earlier. Groups were stratified according to patients' education. To ensure consistency across Centres, group moderators used the same detailed guide training video, and props. Three investigators independently coded the resulting (audiotaped) transcripts. Identified themes were then compared between coders, and subsequently between educational strata and between Centres. Participants also rated each of the 10 presentation formats (Likert scale) as to their perceived usefulness. Fourteen men and 19 women with a variety of cancer diagnoses participated; $13(39 \%)$ in the three lower-education groups and $20(61 \%)$ in the three higher-education groups. Three preliminary themes were identified: First, simple formats (simple graphs or written text) were generally preferred to more complex graphical information. Second, patients did not wish to receive QOL information out of context or without explanation. Third, individual patients varied substantially as to which visual format they most preferred. The same format, however, was rated favourably by the highest proportion of participants $(85 \%)$ in both the high- and low-education groups. Additional themes identified by the groups, and variation in findings between groups, will be presented. Cancer patients desire QOL information, but vary in their preferences for its communication. Simple formats are generally preferred. Further quantitative studies of patients' preferences are planned.
7/ DOCTOR-PATIENT COMMUNICATION ABOUT HRQL ISSUES IN DAILY CLINICAL ONCOLOGY PRACTICE

Symone B. Detmar, Child Health Division, TNO Prevention and Health, Leiden, The Netherlands; Neil K. Aaronson, Psychosocial Research and Epidemiology, Netherlands Cancer Institute, Amsterdam, The Netherlands

Although improving health-related quality of life (HRQL) is an important goal of palliative cancer treatment, very little is known about actual doctor-patient communication regarding HRQL topics during the treatment period. The aim of this study was to investigate the content of communication regarding $\mathrm{HRQL}$ issues between oncologists and their patients, and to identify patient-, physician- and visit-specific factors associated significantly with the discussion of such issues. Discussions between 240 patients and 10 physicians were audiotaped and content-analyzed using the Roter Interactional Analysis System and a substantive content checklist. In addition, patients and physicians were asked to complete a number of questionnaires. Physicians devoted approximately two-third of their conversation to medical/technical issues and one-quarter to HRQL issues. Patients' communication behavior was divided more equally between medical/technical issues $(41 \%)$ and $H R Q L$ topics $(48 \%)$. Of the independent variables investigated, patients' self-reported HRQL was the most powerful predictor of discussing HRQL issues. Nevertheless, in between $20 \%$ and $54 \%$ of the consultations in which patients were experiencing serious HRQL problems, no time was devoted to the discussion of those problems. In particular, patients' emotional functioning and fatigue were often left unaddressed. The discussion of HRQL issues was not found to be related significantly to patients' preferences for discussing these topics. In addition, the evaluation of tumor progression was not related to the discussion of HRQL topics. The main conclusion to be drawn from this study was that, despite increasing recognition of the importance of maintaining patients' HRQL as a goal of palliative treatment, the amount of doctor-patient communication devoted to such issues remains limited, and does not appear to contribute, at least in an explicit sense, to the evaluation of treatment efficacy in daily clinical practice.

\section{8/ PHYSICIAN'S COMMUNICATION STYLE, CONSULTATION} TIME AND PATIENT'S PARTICIPATION IN ONCOLOGY SETTINGS Tomoko Takayama, Yoshihiko Yamazaki, Health Sociology, The University of Tokyo, Bunkyo-ku, Tokyo, Japan; Atsushi Fukuuchi, Tsunehiro Nishi, Mitsui Memorial Hospital, Chiyoda-ku, Tokyo, Japan; Masakazu Toi, Katsumasa Kuroi, Kazuo Hayashi, Tokyo Metropolitan Komagome Hospital, Bunkyo-ku, Tokyo, Japan

Patient's participation has been recognized as important to the patient's quality of care and quality of life. In this study, we examined the effects of the physician's communication style and visit-specific factors on the patient's participation during the consultation. 86 breast cancer consultations were audiotaped and analyzed using the Roter Interaction Analysis System. We employed dependent variables as follows: three observational measures of patient's communication behavior (patient's biomedical information-giving, psychosocial information-giving, question-asking) and patient's perception of the participation. Independent variables: three observational measures of physician's communication behavior (information-giving, attentive listening, question-asking), physician's collaborative communication style perceived by patient, and the visit-specific factors (consultation length, patient's perceived time pressure). The limited duration of the encounter suppressed the patient's communication behavior, psychosocial information-giving $(p<0.05)$ and especially question-asking $(p<0.0001)$. Patient's biomedical information-giving and perceived participation increased when the physician's attentive listening or question asking was accompanied by the patient's perception of physician's collaborative style. When the patient felt the consultation was time pressured, on one hand patient's psychosocial informationgiving and question-asking were suppressed, on the contrary, patient's perceived participation was enhanced. This study suggests that the effects of the physician's communication style and the consultation length on the particular patient's communication behavior. Also, the patient's perceived time pressure not only influences patient's communication behavior but also distorts the patient's sense of participation. 
9/ MEASURING QUALITY OF LIFE IN CLINICAL PRACTICE IMPROVED PATIENT SATISFACTION

Fabienne Empereur, Epidemiologie Evaluation Cliniques, Chu de Nancy, Nancy, France; Emmanuel Desandes, Recherche Clinique, Centre Alexis Vautrin, Vandoeuvre-les-Nancy, France; Francis Guillemin, Isabelle Leonard, Sylvie Klein, Serge Briancon, Epidemiologie Evaluation Cliniques, Chu de Nancy, Nancy, France

Quality of life measurements use is widespread in the field of clinical and epidemiological research, especially for the assessment of therapy. The value of these questionnaires for individual medical decision-making is yet a matter of debate. The aim was to investigate whether the systematic measurement of QoL at admission, and whether scores being returned or not to the care staff modify patients QoL, care management and satisfaction with care. The CliniQualVie randomized controlled trial was designed as a $2^{*} 2$ factorial design and a control group : having not QoL measures at admission (control group), having Qol measures at admission and returning results to physicians (Bp group), and returning results to nurses (Bn group), and returning results to both (Bpn group), and no returning results (B0 group) Fifteen days after discharge, all included inpatients received and returned the same type QoL questionnaires and the $\mathrm{Pa}$ tient Judgments of Hospital Quality questionnaire (PJHQ). Two thousand seven hundred and four inpatients have been included since April 1997 to April 2000 at the Nancy University Hospital Center (France) (1/5 per group). B0 group patients have better satisfaction scores (0-100 scale) than control group patients, for several dimensions: "admission" (71.2 Vs 67.2, p<0.01)," hospital environment and ancillary staff" (67.2 Vs 64.9, $p<0.05)$, "recommendations/intentions" (82.6 Vs $79.4 ; p<0.05)$. In all inpatients who completed QoL questionnaires at admission, there was no significant differences in al satisfaction dimensions between $\mathrm{B} 0, \mathrm{Bp}, \mathrm{Bn}$ and $\mathrm{Bpn}$ groups. Complete a QoL questionnaire seems to improve satisfaction level in few dimensions, but the provision of feedback on patient's quality of life to health professionals seems to have no effect on satisfaction level. Work supported by a PHRC 1996 grant.

\section{0/ THE IMPACT OF SHARED TREATMENT DECISION-MAKING} ON QUALITY OF LIFE IN WOMEN WITH BREAST CANCER

Ann F. Chou, Health Services and Policy Analysis, Joan R. Bloom, School of Public Health, University of California at Berkeley, Berkeley, CA

Biomedical advances have offered women with breast cancer a variety of treatment options that are comparable in health outcomes. Moreover, there is a recent shift toward a view of shared decisionmaking, which encourages the individual to be an informed patient and the physician to be a respectful provider. This process may be determined by individual beliefs towards participation, the amount of information received and processed, relationship with the physician, the environment in which the decision process occurs, or knowledge of treatment choices. Nevertheless, literature has been limited on this type of decision-making model and its impact. The objective of this study is to understand the impact of the decision-making process on survival and patient's quality of life. The study sample included 722 women diagnosed with breast cancer between 1994-1997. Descriptive statistics show that women in this cohort were mostly married $(80 \%)$, White $(70 \%)$, had some college education $(80 \%)$, and diagnosed at age 50 or younger. The distribution of disease stage was $14 \%$ in-situ cancer, $46 \%$ local, $37 \%$ regional, and $2 \%$ remote. Excluding the in-situ cases, $47 \%$ of the women who received surgical treatment had a lumpectomy and $53 \%$ had a mastectomy. Fifty-four percent of the women reported having undergone radiation therapy, $66 \%$ had chemotherapy, and $29 \%$ used Tamoxifen. The Cox proportional hazards model was used to examine the impact of decisionmaking involvement on survival and the multiple regression analysis is used to determine the impact on quality of life. Overall, $20 \%$ of women left the decision to their physicians, $29 \%$ shared the decisionmaking, $43 \%$ made the decision with physician input, and $8 \%$ reported making the decision on their own. The mortality rate for this cohort was $8 \%$. Those who reported having a good understanding of cancer and treatment had significantly better mental heath outcomes. Controlling for demographic factors and disease stage, preliminary results suggest that participation in shared decision-making is correlated with better mental health but poor physical health. On the other hand, decision-making independent of physician input is correlated with poor mental health but better physical health. Overall, different levels of patient participation in decision-making had a varying effect on quality of life. Understanding the effects of participation in medical treatment decision-making is important for designing educational in- terventions for patients and providers, and updating hospital/clinic policies to include patients in medical treatment decisions.

\section{1/ EVALUATING THE LATENT DISTRIBUTION IN IRT MODELS OF HRQOL DATA \\ Jakob B. Bjorner, Department of Work Psychology and Sociology,} National Institute of Occupational Health, Copenhagen, Denmark

IRT analyses of questionnaire data concerning HRQOL must frequently deal with the problem of skewed data distributions. This may reflect floor and ceiling problems of the items or scales and skewness in the latent distribution. In case of a non-normal distribution of the latent variable, item parameters can still be estimated using conditional maximum likelihood estimation (Rasch type models) or discrete empirical approximation of the latent distribution and marginal maximum likelihood estimation (other IRT models). Floor and ceiling problems in the data can create problems for statistical analyses like regression analyses or analyses of variance even if IRT-based scores (i.e estimates of the latent variable) are used as the outcome variable. One solution is to use models that combine the IRT model with the regression model or the analysis of variance model. In such a latent regression analysis the dependent variable is the latent variable itself and not an estimate hereof. However, since such techniques assume a conditional normal distribution of the latent variable we still need to evaluate the latent distribution. We show how latent regression analysis can be applied in research on HRQOL and we compare two approaches for examining the latent distribution: (1) examining the empirical (estimated) latent density as implemented in Parscale/Bilog, and (2) comparing the observed and expected distribution on a simple sum scale of the items (building on work by Thissen et al 1995). We use data from HRQOL research and simulated data. We show that in case of floor or ceiling problems, the estimated latent density can be non-normal even when the true latent density is normal. Comparison of the observed and expected sum scale distribution appears promising for testing assumptions about the latent distribution.

12/ CAN THE CAMCOG MEASURE OF COGNITIVE STATUS BE MODELLED USING ITEM RESPONSE THEORY?

Rob J. de Haan, Rebecca Holman, Robert Lindeboom, B. Schmand, Clinical Epidemiology and Biostatistics, Academic Medical Center Amsterdam, The Netherlands

When considering the quality of life of those suffering from dementia, it is important to examine their cognitive status. This can be measured using one of many multi-item instruments. One of the best known is the CAMCOG. It has been used as a screening instrument and in numerous scientific investigations, having been validated in many populations. In spite of its widespread use, in-depth psychometric consideration of the CAMCOG has not often been carried out. This paper will examine the patterns of responses to the items in the CAMCOG. Firstly, a brief classical psychometric (sum score) analysis will be presented. We will then consider whether more information about the psychometric properties of the CAMCOG can be obtained using some simple graphical techniques. These will lead onto the examination of how the responses to the items can be placed on a single, hierarchical scale with linear measurement properties using the powerful techniques of item response theory. The classical psychometric analysis shows an instrument with a high internal consistency. However, when the response patterns on individual items were compared to the sum scores it became apparent that a large number of the items in the CAMCOG demonstrated an obvious ceiling effect. These items provide very little information on the cognitive ability of patients. The item response theory analysis highlighted a number of items with strange, often random, behaviour in relation to the disease stage of patients. These items can give misleading results when used to measure the cognitive ability of patients. It has been possible to place approximately one half of the 60 items in the CAMCOG on a unidimensional linear scale using the Rasch model. It is hoped that the results in this paper will encourage researchers to explore the use of item response theory in the analysis of data resulting from multi-item instruments. 
13/ COMPARISON OF THREE DIFFERENT STATISTICAL ANALYSIS STRATEGIES FOR ANALYZING SEATTLE ANGINA QUESTIONNAIRE QUALITY OF LIFE DATA

Colleen Norris, APPROACH Project, Edmonton, AB, Canada; W. Ghali, University of Calgary, Calgary, AB, Canada; D. Saunders, University of Alberta, Edmonton, AB, Canada; R. Brant, University of Calgary, Calgary, AB, Canada; D. Galbraith, Foothills Hospital, Calgary, $A B$, Canada; P. Faris, University of Calgary, Calgary, $A B$, Canada; M. Knudtson, Foothills Hospital, Calgary, AB, Canada

The Seattle Angina Questionnaire (SAQ) is being used with increasing frequency in clinical research to address the quality of life (QOL) outcomes of patients with coronary artery disease (CAD). Results of a review that systematically identified all published studies analyzing SAQ scores, demonstrated that inappropriate analysis methods are commonly used to analyze SAQ scores. The purpose of this paper was to compare three different statistical analysis strategies for analyzing skewed SAQ QOL data. Using the Physical limitation scale of the SAQ, three regression models were constructed. Two logistic models were fitted using binary outcomes and one ordinal regression model was fitted using an ordinal scale as the outcome variable. Comparison of the 3 models demonstrated that the ordinal regression model is more sensitive to the characteristics, specifically the ordinality, of the SAQ data. The ordinal regression model generated estimates of effect that consistently yielded the narrowest $95 \%$ confidence intervals. More importantly, the results (summary odds ratios) are generalizable to the entire patient population under study, as the estimated proportional odds ratio is not based on a particular dichotomization of the outcome variable. Although this paper focuses on the $S A Q$, the issue of the most appropriate statistical analysis for disease specific $Q O L$ scales may not be unique to this particular scale. Ordinal scales (particularly common in QOL instruments) even when transformed, risk generating skewed results. The 'average' scores for patients with chronic diseases may be concentrated at the top of the scale (ceiling effect) or the bottom of the scale (floor effect) severely limiting the range of scores possible. This makes it difficult using parametric methods to describe differences in QOL post-treatment or changes in QOL over time. The results of this study suggest that analysis of the SAQ should be done using ordinal regression modeling.

14/ THE AMSTERDAM LINEAR DISABILITY SCORE PROJECT (ALDS): DEVELOPMENT AND CALIBRATION RESULTS OF AN ADL-ITEMBANK

Robert Lindeboom, Rebecca Holman, M. Vermeulen, Rob de Haan, Clinical Epidemiology and Biostatistics. Academic Medical Centre, KEB, Amsterdam, The Netherlands

The functional status or QoL of patients is frequently expressed as a total score obtained by summing the (weighted) individual items in a scale. The disadvantage of this approach is that all items have to be presented to patients regardless of their functional level, cross-instrument comparability is not assured and the scores obtained are ordinal rather than linear. Also, because of the correlational methods used in their development, the items in many functional status or QoL scales cluster in the mid-range of the construct of interest. Hence, the extremes of the scales tend to be poorly defined and are obscured by floor and ceiling effects. The goal of the ALDS-project is to calibrate an Activities of Daily Living (ADL) item bank using IRT statistical models that claim to correct for these problems. The item bank consists of 190 items selected from a pool of more than 110 existing ADL scales and covers a large range of the construct. We will discuss the theoretical framework used in the item selection and the specific methods to put all 190 items on a common difficulty scale. The calibration estimates of the more challenging half of the item bank containing the more difficult ADL item tasks will be presented. The estimates were obtained by applying Rasch's IRT model to the responses of about 1000 outpatients with a range of chronic conditions.
15/ THE NOTTINGHAM HEALTH PROFILE IN POPULATION RESEARCH: FLOOR EFFECTS PERSIST IN GROUPS WITH IMPAIRED HEALTH

Sonja Boehmer, Thomas Kohlmann, Institute for Social Medicine, Medical University of Luebeck, Luebeck, Germany

The Nottingham Health Profile (NHP) is known to produce strong floor effects (i.e. percentage of "zero scorers") when used in surveys of the general population. Recently, in a representative German population sample up to 83 percent of the respondents showed zero scale scores on the NHP. The purpose of this study was to investigate if floor effects decrease substantially in population subgroups when only persons with at least moderate health problems are included. Mail survey data from a random sample consisting of $\mathrm{N}=600$ residents of Luebeck, North Germany, aged between 50 and 69 was used for this analysis (51 percent male, response rate 61 percent). Floor effects were calculated for the total sample and for respondents reporting (A) fair or poor general health (26 pct), (B) moderate, severe or very severe pain (33 pct) or (C) depressed mood (CES-D score >23; 5 pct). In the total population sample score distributions of the NHP subscales were highly skewed in the direction of positive well-being with the following floor effects: Energy 72 pct, Pain 59 pct, Emotional Reaction 69 pct, Sleep 51 pct, Social Isolation 86 pct and Physical Mobility 55 pct. In all three subgroups (A-C) these floor effects decreased. In group $A$ and $B$, smallest floor effects were observed for Physical Mobility and Pain, respectively (A: 14 and 20 pct; B: 19 and $17 \mathrm{pct}$ ). In group $\mathrm{C}$, floor effects were markedly reduced in three subscales: Sleep (0 pct), Emotional Reaction (3 pct) and Energy ( 3 pct). Like in the total population sample Social Isolation produced the highest rate of zero scorers in each of the three subgroups (A: 70 pct; B: 76 pct; C: 25 pct). The study showed that floor effects of the NHP decrease in population subgroups when only persons with at least moderate health problems are included. Yet, this reduction has not been substantial in all scales. According to these results the NHP seems to be suitable as a survey tool only in population subgroups with relevant health problems.

16/ USING ITEM RESPONSE THEORY TO SHORTEN THE LENGTH OF SCALES IN A QUESTIONNAIRE FOR PALLIATIVE CARE. AN EORTC QUALITY OF LIFE GROUP STUDY

Mogens Groenvold, Morten A. Petersen, Department of Palliative Medicine, Bispebjerg Hospital, Copenhagen NV, Denmark; Jakob B. Bjorner, National Institute of Occupational Health, Copenhagen OE, Denmark

When evaluating physical and psychological symptoms experienced by patients in palliative care, the questionnaire should be short but still satisfy standard requirements regarding content validity and psychometric properties. The EORTC QLQ-C30 was judged to satisfy such standard requirements and has the advantage of being widely used. However, ideally, a questionnaire for palliative care should be shorter than 30 items. This project will reduce the QLQ-C30 from 30 to approximately 20 items relevant for patients in palliative care. $\mathrm{Re}$ sults obtained with the shortened version should be directly comparable with scores on the (full-length) QLQ-C30. Two approaches are used: (1) Shortening multi-item scales using Item Response Theory (IRT). (2) Interviews with patients and professionals across Europe to identify items or scales that can be eliminated. We here present results from the first approach. A database containing QLQ-C30 data from 10,815 European cancer patients was created. We developed an IRT model, used it to select items for shortened scales and to predict scores on the original scales from the shortened scales, examined agreement between the shortened scale and the original scale, and compared their measurement abilities. We have analysed the 4item Emotional Function (EF) scale and the Fatigue scale. The EF scale was shortened from 4 to 2 items. There was very high agreement between EF scores obtained with the original 4-item scale and scores predicted from the shortened 2-item EF scale: the mean deviation was 1.00 on a 100 point scale, the correlation was 0.94 . Judged from 'known group comparisons' in 24 data sets, there was virtually no loss of statistical power despite the use of only 2 items. The Fatigue scale was shortened from 3 to 2 items which resulted in a small loss of statistical power for this scale. The surprisingly good preservation of measurement power in shortened scales may be caused by (1) the use of a prediction system (predicting the original scale scores) rather than just estimating a 2-item sum score from the shorter scale, and (2) the omission - following the IRT analyses - of a relatively uninformative item from the EF scale. 
17/ A COMPARISON OF ANALYSIS METHODS FOR HEALTH-RELATED QUALITY-OF-LIFE (HRQL) OUTCOMES OF CLINICAL TRIALS

David Osoba, QOL Consulting, West Vancouver, BC, Canada; Grazyna Lieberman, Biostatistcs, Genentech, Inc., South San Francisco, CA

The purpose of this study was to determine differences between three methods of longitudinal analysis of HRQL data: means of scores, means of changes in scores from baseline, and proportions of patients with improvement or deterioration (success/failure rate). These methods were assessed in a randomized trial of trastuzumab (Herceptin) $(\mathrm{H})$ and chemotherapy $(\mathrm{C})$ vs $\mathrm{C}$ alone in 469 women with HER2-overexpressing, metastatic breast cancer (Slamon DJ et al. NEJM 2001;344:783). HRQL was assessed in 400 women using the EORTC Quality of Life Questionnaire (QLQ-C30, version 1.0) at baseline, 8, 20, 32, 44 and 56 weeks of treatment. The data for 6 preselected domains (global QL, physical, role, social and emotional functioning, and fatigue) were analyzed. Mean scores (for women still on study compared to the mean baseline score for the entire group) and mean change scores from baseline (the difference at each time point for women remaining on study minus their own baseline scores) were determined by repeated measures ANOVA. Success/failure rates were assessed by calculating the proportions of women whose scores either improved or deteriorated by $=/>10$ points for at least 12 weeks (Osoba D et al. JCO 1998;16:139). Mean scores, as compared to baseline, improved over time to a similar extent in all 6 domains (e.g., global $\mathrm{QL}$ in the $\mathrm{H}+\mathrm{C}$ group $=66.8$ at week 44 vs 59.3 at baseline; in the $C$ group $=62.9$ at week 44 vs 58.4 at baseline). However, the means of changes in scores from baseline showed statistically significant improvement $(P<0.05)$ only in the global $Q L$, physical functioning and fatigue domains in the $\mathrm{H}+\mathrm{C}$ group but not in the $\mathrm{C}$ group. The success rate was statistically significantly higher $(P<0.05)$ for global $Q L$ and fatigue in the $\mathrm{HC}$ group than in the $C$ group. The failure rate was similar for all domains in both treatment groups. In conclusion, calculation of the mean scores over time is biased by a "survivor effect" in patients remaining on study, regardless of the treatment they receive. Mean change scores and success/failure rates present a more accurate picture of HRQL outcomes. (Supported by Genentech, Inc.)

\section{8/ THE GOAL INTERFERENCE SCALE: EVALUATION OF A NEW} INDIVIDUALIZED HEALTH OUTCOME MEASURE

Amy Peterman, David Cella, Marianne Brady, Northwestern University, Evanston, IL

Despite the proliferation of health-related quality of life measures, most do not provide information about individually meaningful problems that are amenable to intervention. One reason for this may be that most do not focus upon personally relevant goals which vary across people and which are differentially affected by illness/treatment. Existing literature and patient input were utilized to create the Goal Interference Scale (GIS), a questionnaire that incorporates both idiographic and nomothetic information. Thus, it provides a method for capturing the highly individual impact of illness/treatment while still allowing for comparisons of scores across groups of patients. Thirty-three cancer patients who were currently receiving chemotherapy completed the GIS, the FACT-G (Cella, et al.,1993) and the FACIT-Fatigue Scale (Yellen, et al.,1997)at an initial assessment (T1) and $28(85 \%)$ subjects also completed a 2 month follow-up (T2). The sample was $75 \%$ female: median age was 61 years. Descriptive statistics demonstrated a wide range of scores (T1: 8-77; T2: 10.5-79), with a possible range of $0-100$. Subjects experienced a moderate degree of interference at both $\mathrm{T} 1$ (mean $=49.8 ; \mathrm{SD}=17.2$ ) and T2 (mean=47.1; $S D=18.3$ ). The scale demonstrated good internal consistency (Cronbach's alpha $=.87$ at T1 and .83 at T2). Concurrent validity was demonstrated by significant correlations between the GIS and the total FACT-G, functional well-being, fatigue and global QOL (r's from -.46 to $-.78, p$ 's<.001). In addition, subjects indicated that the GIS is easy to complete and asks about meaningful and personally relevant issues. Initial testing of the GIS indicates promising psychometric properties and the likelihood that goal interference is a meaningful concept for cancer patients. Further evaluation of the scale's validity and clinical relevance is ongoing
19/ COMPARISON OF THE HEALTH UTILITY INDEX MARK III (HUIIII) AND THE SELF-ASSESSED HEALTH STATUS IN THE CANADIAN POPULATION

Julie Bernier, Jean-Marie Berthelot, Health Analysis and Modeling Group, Statistics Canada, Ottawa, ON, Canada; Ivan Barofsky, The Johns Hopkins University, Baltimore, MD; Pennifer Erickson, Pensylvania State University, State College, PA

The objective of this presentation is to compare a population based multiple domain measure of health, the HUI-III, with the single item self-assessed health status measure (SAHS). The household component ( $n=17,626$ individuals) of the 1994 National Population health Survey (NPHS) was designed to be representative of the Canadian household population. Both the HUI-III and the SAHS have been measured with a variety of socio-demographic (e.g., sex, age, language) and health-related (e.g., diabetes, high blood pressure, restriction of activities) variables. In order to have both the HUI-III and SAHS evaluated on a 0 to 1 scale, the categorical responses to the SAHS question have been transformed into the values provided by Stewart and Ware. Using transformed data, the difference between the HUI-III and SAHS was calculated and studied within socio-demographic groups and for individual having specific health conditions. In order to account for the strong relationship between the socio-demographic and health variables, a multiple regression model was estimated to provide an overall picture of the sources of the observed differences between HUI-III and SAHS. Comparing HUI-III and SAHS in the total sample revealed a mean difference of only 0.10 . However, this difference varies greatly with age and level of SAHS. The mean difference can be as large as 0.44 for people aged 25 to 34 who self-report fair health status. In general, the difference is higher for people reporting fair and poor health, aged between 55 and 74 or reporting diabetes, high blood pressure or asthma. The two measures have been shown to be different at the individual level and analysis of these differences reveals information about how individuals respond to different types of items within a questionnaire. In addition, the magnitude of these differences varies as a function of personal characteristics.

20/ HEMOPHILIA: CONSTRUCT VALIDITY OF THE HEALTH UTILITIES INDEX

William J. Furlong, Department of Clinical Epidemiology and Biostatistic Ronald D Barr, Department of Pediatrics, McMaster University, Hamilton, ON, Canada; Mahassen Saleh, Department of Pediatrics, Hamilton Health Sciences Corporation, Hamilton, ON, Canada; John Horsman, Department of Clinical Epidemiology and Biostatistic, McMaster University, Hamilton, ON, Canada; Julia Sek, Mohan Pai, Department of Pediatrics, Hamilton Health Sciences Corporation, Hamilton, ON, Canada; Irwin Walker, McMaster University Medical Centre, Hamilton Health Sciences Corporation, Hamilton, ON, Canada

This population-based study assessed the comprehensive health status and health-related quality of life (HRQL) experienced by males age $>13$ years, with a diagnosis of hemophilia $A$ or $B$, and active in a geographically-defined health care program. Diagnostic variables included virologic status and disease severity. A mail survey, using a standard Health Utilities Index Mark 2 (HUI2) and Mark 3 (HUI3) selfcomplete questionnaire, collected information from patients about their health status during the previous 4 weeks. The HUI2 and HUI 3 are generic systems for measuring health status and HRQL. A priori hypotheses included reduced health status being associated with increasing severity of disease, hepatitis $B$ and $C(B / C)$ sero-conversion, and human immunodeficiency virus (HIV) infection. The survey response rate was 101 of $115(88 \%)$. Non-response was due to refusal $(n=10)$, terminally ill from AIDS $(n=2)$, inability to communicate in English $(n=1)$, unable to contact $(n=1)$. Patients' HRQL mean HUI2 score was 0.81 (range 0.20 to 1.00 ) and $\mathrm{HUI} 3$ was 0.71 (range -0.23 to 1.00$)$. There were statistically significant $(p<0.05)$ and important size ( $>0.10$ units) differences in mean overall HRQL scores within the following pairs of patient groups: Pair I - mild disease $(n=41)$ versus severe disease \& HIV+ $(n=23)$; Pair II - moderate disease $(n=17)$ versus severe disease \& HIV+ $(n=23)$; Pair III - severe disease \& HIV$(n=20)$ versus severe disease \& HIV+ $(n=23)$; Pair IV - moderate disease \& HIV- \& B/C- $(n=7)$ versus moderate disease \& HIV- \& B/C+ $(n=8)$. Within Pairs I and II, there were significant differences in mean single-attribute utility scores for HUI2 mobility, HUI2 pain, HUI3 ambulation and HUI3 pain. Within Pair III there was a significant difference in mean single-attribute scores for HUI2 pain. The significant differences between clinically important groups were in the expected directions and provide evidence of construct validity for HUI. 
21/ ONE YEAR OUTCOMES OF HEMODIALYSIS AND PERITONEAL DIALYSIS IN AN INCIDENT COHORT OF PATIENTS WITH END STAGE RENAL DISEASE (ESRD)

Albert W. Wu, Health Policy and Management, Jane Marsh, Nancy E. Fink, Department of Epidemiology, Kraig Kinchen, Neil R. Powe, Department of Medicine, Johns Hopkins University, Baltimore, MD

After nearly three decades of Medicare coverage of dialysis care, the relative outcomes of hemodialysis (HD) and peritoneal dialysis (PD) are not known. A few cross-sectional studies have suggested better health related quality of life (HRQOL) on PD. We examined HRQOL and survival in an incident cohort of patients with ESRD. Patients from over 80 dialysis clinics across the US were enrolled in Choices for Healthy Outcomes in ESRD (CHOICE) between 1995 and 1998. Patients completed the CHOICE Health Evaluation Questionnaire (CHEQ) which includes the SF-36 and 14 ESRD specific domains (Cronbachs alph > .67), at baseline and 12 months later. Dependent variables included HRQOL, survival and renal transplant. Independent variables included age, gender, race, education and comorbidity score. Analyses examined unadjusted and adjusted differences in changes in HRQOL scores, and change (worse, same, better) in overall health status in which death or increase in comorbidity = worse, transplant or decrease in comorbidity =better, and HRQOL was categorized based on +2 SEM. Of 928 patients who completed the baseline CHEQ, 585 also completed the 12 month CHEQ; 101 had died, and 55 had renal transplant. PD patients were slightly younger, more likely to be Caucasian, well-educated, employed and had less comorbidity. Unadjusted baseline scores showed better HRQOL for PD patients (physical function, pain, role emotional, travel, diet, and dialysis access $(P<.05))$. A 1 year SF-36 scores improved, while ESRD specific domains were mixed. There were relative improvements for HD in SF-36 (physical, general) and mixed results for ESRD domains (PD better for finances, HD better for sleep, unadjusted and adjusted). No differences between $\mathrm{HD}$ and $\mathrm{PD}$ in overall health status were found. In conclusion, in one of the first longitudinal studies to compare HD and PD patients' HRQOL, we found substantially similar outcomes for the two treatments. General health status improved more for HD patients. However, for ESRD specific domains, there appeared to be trade-offs, with PD better in some, and HD better in other domains. In advising patients about which modality to choose, clinicians should discuss the individual's preferences for specific aspects of HRQOL.

22/ USE OF ERYTHROPOIETIN IN EARLY ANAEMIA IMPROVES HRQOL IN PREDIALYSIS CHRONIC RENAL FAILURE PATIENTS M. S. Salek, Centre for Socioeconomic Research, Cardiff University Cardiff, UK; N. Pratheepawanit, Faculty of Pharmaceutical Sciences, Khon Kaen University, Khon Kaen, Thailand

Predialysis patients are usually allowed to become anaemic $(\mathrm{Hb}$ greater than or equal $9.0 \mathrm{~g} / \mathrm{dl}$ ) before the commencement of erythropoietin therapy. Several studies have demonstrated the physiological benefits of correction of anaemia in predialysis patients. However its HRQOL benefits have not been established. An open label, prospective randomised, multicentre study was carried out to assess the impact of maintaining a haemoglobin level of $11.0+/-1.0 \mathrm{~g} / \mathrm{dl}$ on HRQOL in predialysis chronic renal failure patients. Patients who met the inclusion/exclusion criteria were randomised into two groups. In group A, patients commenced erythropoietin therapy at an early stage in the development of their anemia in order to maintain a target hemoglobin level of $11.0+/-1.0 \mathrm{~g} / \mathrm{dl}$. Hemoglobin levels in group B patients were allowed to fall below $9.0 \mathrm{~g} / \mathrm{dl}$ prior to receiving erythropoietin therapy. The HRQOL was measured at baseline and a year later using the Renal Quality of Life Profile (RQLP). This is a 34-item questionnaire consisting of 4 domains: eating and drinking; physical activity; leisure activity; and daily life. A total of 65 patients completed the study; 26 $(40 \%)$ patients were in group $A$ and the rest $(n=39)$ in group $B$ with similar demographic characteristics. Most patients were male (group $A$ $=81 \%$, group $B=68 \%$ ) with mean age of 57 (group $A=60$, group $B=$ $55)$, ranging from 28 to 78 years, and the majority stated that, at baseline, they were still working and led a normal life (group $A=73 \%$, group $B=$ $78 \%$ ). There were no significant differences in baseline RQLP scores between group $A$ and $B$. Results at one year showed a deterioration of patients' quality of life in both groups. However, slightly more impaired quality of life was found in group $B$ than group $A$, except physical activities where group A showed an improvement. In comparison, the difference of quality of life scores in physical activities was statistically significant between both groups $(p=0.03)$. The findings suggest that maintaining hemoglobin level of $11.0+/-1.0 \mathrm{~g} / \mathrm{dl}$ can improve patients physical activities and delay the deterioration of patients' overall HRQOL.
23/ ORODENTAL HEALTH, ACUTE AND LATE MORBIDITIES, AND QUALITY OF LIFE IN IRRADIATED INDIAN HEAD AND NECK CANCER PATIENTS

Manishi Bansal, Bidhu Mohanti, Naseem Shah, Rama Chaudhry, Sudhir Bahadur, Nootan Shukla Institute Rotary Cancer Hospital, AlIMS, Ansari Nagar New Delhi, India

Approximately $50 \%-70 \%$ of head and neck cancer $(\mathrm{H} \& \mathrm{~N} \mathrm{Ca})$ patients receive radiation therapy in India. Assessment of orodental status, radiation-related morbidities and impact of irradiation on quality of life is not consistently carried out and this study has evaluated the above issues in the Indian patients prospectively. Radiation therapy (RT) was delivered to $45 \mathrm{H} \& \mathrm{~N}$ Ca patients (70 Gy, Group A) and 20 patients of cancer other than $\mathrm{H} \& \mathrm{~N} \mathrm{Ca}$ (46-60Gy, Group B). Orodental health, dental care and oral flora culture, acute and late radiation morbidites (RTOG and LENT/SOMA systems), and quality of life scales (EORTC QLQC30) were recorded in both groups. Temporal assessments were carried out in 5 phases-Pre-RT (phase I), during 4th week of RT course (phase II), at end of radiation (phase III), after 1 month of RT (phase IV) and after 4 months of RT (phase V). The caries incidence increased from $0.37 \%$ in phase II to $2.8 \%$ at phase $V(p<0.0001)$. Periodontal disease recorded in $51 \%$ patients before RT increased to $86.6 \%$ at end of therapy $(p<0.001)$. Candidiasis was detected in $24(53.3 \%) \mathrm{H}$ \& $\mathrm{N}$ Ca patients in phase II. Acute radiation morbidites of head and neck cancer patients were prominent in phase III, affecting skin, mucosa and salivary gland in $15.5 \%, 66.6 \%$ and $97 \%$ of patients respectively. Late radiation effects were observed in salivary gland $(90.6 \%)$ and teeth $(58.3 \%)$ at 4 months after RT course. QOL assessment showed decline in scores for $\mathrm{H} \& \mathrm{~N}$ Ca patients in physical and social function $(p<0.0001)$, and emotional function $(p<0.001)$ scales, for phase I vs IV. The pre-RT global health status was higher in $\mathrm{H} \& \mathrm{~N} \mathrm{Ca}$ compared to others (82.31 vs 62.05), but showed dramatic decline in phase II (30.66 vs $50.95, p<0.0001)$ and in phase IV (58.53vs 68.70, $p<0.021)$. Single item symptoms showed significant declines related to fatigue, appetite loss, and pain in H\&N Ca patients $(p<0.0001)$ during RT course. Poor orodental hygiene in Indian head and neck cancer patients increases the incidence of post-irradiation periodontal disease and caries. Radiation to $\mathrm{H} \& \mathrm{~N}$ region severely affects physical and social functions and global health status, significantly in mid-course and immediately after therapy.

\section{4/ QUALITY OF LIFE AFTER ESOPHAGEAL RESECTION FOR} CANCER AND BARRETT'S

Claude Deschamps, Francis C. Nichols, Mark S. Allen, Peter C Pairolero, Daniel L. Miller, Victor F. Trastek, Division of General Thoracic Surgery, Mayo Clinic, Rochester, MN

Early detection and resection of esophageal carcinoma provide the best chance for cure. We reviewed and analyzed quality of life in 64 patients who survived more than five years after resection of esophageal carcinoma. We used the Medical Outcomes Study 36-Item ShortForm Health Survey (MOS SF-36). Physical function scores were decreased significantly $(p<0.01)$ compared to the national norm. Ability to work, social interaction, daily activities, emotional dysfunction scores, and perception of health were similar to the national norm. Level of energy was decreased compared to the national norm but the significance was borderline $(p=0.05)$. Our patients had higher scores in the area of mental health $(p<0.05)$. The occurrence of a postoperative anastomotic leak adversely affected the physical functioning and the health perception scores in our population $(p<0.05)$. Also, the need for postoperative dilatation adversely affected the social functioning score $(p<$ 0.01 ). Age, sex, time interval since the surgery, location of lesion, histology, type of surgery, and adjuvant therapy did not significantly affect any of the 8 conceptual areas. More recently, 44 patients who had esophagectomy for Barretts with high-grade dysplasia (HGD) responded to the same written survey. Patients with HGD only (i.e. no cancer) find themselves to be better than the norm in role-physical and role-emotional $(p<0.03)$. The occurrence of an anastomotic leak adversely affected the social functioning scores $(p=0.02)$. Age was significantly correlated with physical functioning $(r=-0.49, p=0.0007)$ and role-physical $(r=-0.33, p=0.03)$. Time from surgery is significantly correlated with social functioning $(r=0.34, p=0.02)$. While functional outcome after esophagectomy is acceptable but less than ideal, the operation had no consistent and measurable negative impact on these patient's quality of life, as measured by the MOS SF-36. 
25/ DIABETES AND CHANGE IN PSYCHOSOCIAL WELL-BEING Siri Naess, John Eriksen, NOVA - Norwegian Social Research, Oslo, Norway

Previous research has documented that people with diabetes report lower quality of life than do people with no reported disease. In recent years new treatment regimes for diabetes have been introduced, including improved insulin, simpler blood sugar tests and transfer of responsibility from doctor to patient. Have these improved methods for controlling diabetes resulted in improved psychosocial well-being? In the this paper we analyse changes in psychosocial well-being between 1984-86 and 1995-97. On these two occasions the entire adult population of one county in Norway was called in for health screenings (the Nord-Trondelag Health Surveys, HUNT1 and HUNT2). The questionnaires included questions on several known diseases and impairments, self-assessed health and psychological well-being. 77,224 and 62,784 persons respectively responded to the questionnaires (response rates were 88.5 per cent in 1984-84 and 69.3 per cent in 1995-97). In a second questionnaire both in HUNT12 and HUNT2 more questions on psychosocial well-being were asked (with lower response rates, 72.6 and 58.0 per cent respectively). Preliminary analyses indicate that people with diabetes reported lower well-being than people with no reported diabetes, in HUNT1 as well as in HUNT2. Further, data indicate that the relationship between diabetes and well-being was weaker in HUNT2 than in HUNT1. More specifically, self-assessed health, the feeling of being strong and fit, the use of tranquillisers, and life satisfaction had improved during the 11 years between the two surveys, for people with diabetes compared to people with no reported diabetes. Reduced well-being was related to additional diseases (late complications), such as macrovascular disease. Controlled for reports on these complications, the differences between people with and without diabetes were slight. In the paper these changes in psychosocial well-being from 1984-86 to 1995-97 will be pursued in more detail by multivariate analysis techniques (like ANOVA and "sandwich"(Huber/White).

26/ THE REVISED KINDL-R: FINAL RESULTS ON RELIABILITY, VALIDITY AND RESPONSIVENESS OF A MODULAR HRQOL INSTRUMENT FOR CHILDREN AND ADOLESCENTS

Ulrike Ravens-Sieberer, Child and Adolescent Health, Robert Koch Institute, Berlin, Germany; Monika Bullinger, Department of Medical Psychology, University of Hamburg, Hamburg, Germany

Children's self report of HrQoL has become an important outcome in pediatrics and public health. This paper reports the validation project of the 24 item generic self-report QoL questionnaire for healthy and ill children: the revised Kindl-R, intended to be used as a discriminant, predictive and evaluative instrument in community and clinical pediatric populations. In developing the Kindl- $R$, the goal was to design a modular approach to measure HrQoL in healthy and ill children with a generic core and disease specific modules as outlined by the WHO The measure can be completed by children and parents as paperpencil ond/or computer assisted version and is available for different age groups (4-7, 8-12, 13-16). Items were derived from focus groups with children. The measure (languages: German, English, French, Italian, Spanish, Greek, Swedish) can be augmented by disease-specific modules for obesity, bronchial asthma, atopic dermatitis, cancer and diabetes. 5781 healthy and chronically ill children and adolescents (4 to 17 years) and 3200 parents completed the Kindl-R in a number of longitudinal studies together with clinical and psychosocial predictors over a period of up to 2 years. Six dimension scores (physical, psychological well-being, family, friends, self-esteem, school) were computed as well as an index. Item response theory was used to determine optimal item and scale characteristics as well as multimethod-multitrait analysis and regression analysis. Content and construct validity was confirmed, factor validity was shown. Small to medium scale intercorrelation supported multidimensionality. The KINDL-R is reliable in terms of internal consistency (alpha $>0.80$ ) and test-retest results $(r=0.69)$. Convergent validity was satisfactory. The acceptance of the measure was high $(<15 \mathrm{~min})$. The questionnaire has been able to distinguish between different acute and chronic health conditions $(p<.05)$ and different health behavior(known groups). It has been proven to be sensitive to change over time in patients under treatment with moderate to high effect size estimates (Kazis) related to treatment $(d>0.6$ in modules). The results support the KINDL-R as a flexible, modular, psychometrically sound measure for use in public health research and clinical applications.
E. Andresen, T. Catlin, K. Wyrwich, Saint Louis University, St. Louis, $\mathrm{MO}$; J. Jackson-Thompson, Missouri Department of Health, Jefferson City, MO

Measures of health-related quality of life (HRQoL) are increasingly common to clinical research, but uncommonly used in surveillance systems. For surveillance use, HRQoL measures need to be brief, generic, and broadly applicable to public health questions. HRQoL has been proposed as a method to monitor health in the American public health plan, "Healthy People 2010." We investigated the reliability and validity of the $4 \mathrm{HRQ}$ oL surveillance questions from the Behavioral Risk Factor Surveillance System - an ongoing nation-wide randomdigit dialed telephone survey of Americans (aged ${ }^{3} 18$ ). In 1999, randomly sampled adults from a midwestern state were re-interviewed for a retest of the HRQoL questions; 868 adults completed both interviews. The HRQoL questions include Self-Reported Health and 3 "days" questions measuring poor physical health, poor mental health, and activity limitation in the past 30 days. Poor mental and physical health days combine for a positive summary measure of Healthy Days. Mental and physical health days were also dichotomized using a cutoff of 14 days. Reliability is reported as kappa statistics for categorical questions and intraclass correlation coefficients for continuous questions. Validity of continuous "days" questions was examined by linear trend analysis across Self-Reported Health categories. Retest reliability was excellent $(0.75$ or higher) for Self-Reported Health and Healthy Days measures, and moderate $(0.58$ to 0.71$)$ for other measures. Reliability was lower for older adults. Other demographic subgroups (e.g., gender, race/ethnic group) showed no regular pattern of differing reliability. Validity of the "days" questions showed strong linear trends across categories of Self-Reported Health, especially for physical health days. Retest reliability of the HRQoL surveillance measures is moderate to excellent, and internal validity is strong. Scaling options will require future attention, as will research into appropriate metrics for what constitutes important population differences.

28/ THE SEXUAL RELATIONS SCALE: PSYCHOMETRIC CHARACTERISTICS IN PATIENTS WITH HIV DISEASE, GERD, UTERINE FIBROIDS, AN PERIPHERAL ARTERIAL DISEASE

Dennis A. Revicki, Karin Coyne, Carol Koro, Center for Health Outcomes Research, MEDTAP International, Bethesda, MD; Albert W. Wu, Health Services Research and Development Center, Johns Hopkins University, Baltimore, MD; Anne Rentz, Center for Health Outcomes Research, MEDTAP International, Bethesda, MD

Many chronic diseases and medical treatments impact on patient sexual functioning and behavior. Impairments in sexual functioning may decrease health-related quality of life, especially social and intimate partner relationships. Brief, psychometrically sound instrument are needed to evaluate sexual functioning and relations for clinical trials and clinical monitoring. The objective of this study was to evaluate the reliability and validity of the Sexual Relations Scale (SRS) in patients with HIV disease $(n=163)$, GERD $(n=138)$, uterine fibroids $(n=139)$, or peripheral arterial disease $(P A D)(n=60)$. All patients were recruited from clinical centers and the sample was $57 \%$ women and $50 \%$ non-white, with average ages of 31 to 63 years, depending on the sample. The SRS consists of 6 questions evaluating intimate relationships: health interference with sexual relations or ability, satisfaction with sexual relations, decrease in sexual activity, and intimate relations with partner. For all four samples, the SF-36 Health Survey and disease-specific HRQL measures were included; for the HIV disease and uterine fibroid samples, the MOS Sexual Problems Scale was included; and for the HIV sample, the CESD was also included. Internal consistency reliability (Cronbach's alpha) ranged from 0.84 to 0.92 across patient samples. The SRS was significantly correlated with the MOS Sexual Problems Scale in men $(r=0.82, p<.05)$ and women $(r=.73, p<.05)$ with HIV disease and in uterine fibroid patients $(r=71, p<.05)$. Patients with AIDS or symptomatic HIV disease reported more impairments on SRS scores compared with those who had asymptomatic HIV disease $(p<.05)$. SRS scores were most strongly correlated with SF-36 social function, general health perceptions, vitality and mental health scores. In the HIV sample, SRS scores varied significantly by depression status $(p<.0001)$. Uterine fibroid patients reported mean SRS scores that were significantly more impaired than controls $(p<.05)$, and SRS scores were significantly correlated with a disease-specific $H R Q L$ measure $(r=.34, p<.01)$. Based on this study, the SRS had acceptable reliability and evidence of construct validity across several chronic disease populations. Future research needs to evaluate the responsiveness of the SRS and determine guidelines for interpreting clinical meaningfulness of SRS scores. 
29/ VALIDATION OF THE UFS-QOL, A DISEASE-SPECIFIC SYMPTOM AND HEALTH-RELATED QUALITY OF LIFE QUESTIONNAIRE FOR UTERINE FIBROIDS

Karin Coyne, Center for Health Outcomes Research, MEDTAP International, Bethesda, MD; James Spies, Department of Inter-Radiology, Georgetown University, Washington, DC; Deneane Boyle, Center for Health Outcomes Research, MEDTAP International, Bethesda MD; Kerry Skyrnarz-Murphy, Sheila Gonzalves, Department of Inter Radiology, Georgetown University, Washington, DC

Uterine fibroids (UF) cause significant symptoms and health-related quality of life (HRQL) impairment in women who experience them. The purpose of this research was to validate a newly created instrument to assess symptom severity and HRQL in women with UF. The UFS-QOL was generated from focus groups, literature reviews and clinical opinion. Participants (pts) for the validation study were recruited from 5 area gynecologist offices, an interventional radiology department, and a University campus. Instruments used for validation were the SF-36, Ruta's Menorrhagia questionnaire, the RevickiWu sexual functioning scale, and physician and patient assessments of severity. Item and exploratory factor analysis were performed to assess the subscale structure of the questionnaire. Psychometric evaluation was conducted to assess reliability and validity. Test-retest was performed on a random subset of the study group within 2 weeks of the initial visit. 110 patients with confirmed fibroids and 30 normal subjects participated in the validation. Using defined decision rules for item reduction, the questionnaire was reduced to 8 symptom questions and $29 \mathrm{HRQL}$ questions with 6 subscales. Subscale cronbach's alphas ranged from 0.81 to 0.94 . Small to moderate correlations were present with the SF-36 subscales, Menorrhagia questionnaire and the Revicki-Wu sexual functioning scale. The UFS-QOL showed excellent discrimination among UF patients with varying degrees of symptom severity and from normals. Subscale intra-class correlations for two week retest reliablilty ranged from $0.76-0.92$. The UFS-QOL appears to be a useful new tool for detecting differences in symptom severity and HRQL among patients with uterine leiomyomata. Further investigation regarding the responsiveness of the UFSQOL to therapies for UF is underway.

30/ PSYCHOMETRIC EVALUATION OF TWO OBESITY AND WEIGHT LOSS QUALITY OF LIFE INSTRUMENTS: THE OWLQOL AND WRSM

Donald L. Patrick, Department of Health Services, University of Washington, Seattle, WA; Donald M. Bushnell, Health Research Associates, Seattle, WA; Laura A. Glauda, Dennis D. Gagnon, Margare Rothman, The R. W. Johnson Pharmaceutical Research Institute, Raritan, NJ

To report U.S. validation of two measures specific to obesity and weigh loss: the Obesity and Weight-Loss Quality of Life Instrument (OWLQOL) and Weight-Related Symptom Measure (WRSM), including measurement model, psychometric properties and burden. Two groups were recruited: 160 obese enrollees in diet, exercise, and health programs, and 180 obese non-enrollees from the general public. Respondents had a Body Mass Index (BMI) of 27 through 50 and were between the ages of 18-75. All completed the OWLQOL, the WRSM, the SF-36, the CES-D (depression), the Perceived QOL scale, and standard demographic items at baseline, 1 week (for test/retest of OWLQOL and WRSM), and again at 12 weeks (for responsiveness based on weight loss and global rating of change). Psychometric testing followed standardized procedures. 340 patients completed baseline assessments: $60.0 \%$ female, $77.9 \%$ white, and 50.3 married. The overall OWLQOL score was internally consistent (alpha=0.96) and reproducible $(I C C=0.90)$. As predicted, OWLQOL scores had stronger associations with the SF-36 vitality subscale $(0.54)$, the more generic quality of life scale (QCOL, 0.54$)$, weightrelated symptom bothersomeness (0.53), and depressive symptomotology (CES-D, 0.49) than the SF-36 physical function subscale $(0.40)$. The overall WRSM was also internally consistent $(0.87)$ and reproducible $(0.83)$. OWLQOL scores were able to discriminate between levels of BMI, levels of symptoms, levels of depressive symptomotology (CES-D), and gender. The total sample (irregardless of being in a program) was evaluated for responsiveness at 12 weeks. Effect size for $2.5 \%$ or greater decrease in the patients' weight, were 0.76 for the OWLQOL, 0.54 for the WRSM, and ranged from $0.20-0.60$ for the 8 subscales of the SF-36. In this stand alone study, the OWLQOL and WRSM proved valid, reproducible, and responsive to weight-loss and global rating of change.
31/ COPING STYLES PREDICT QUALITY OF LIFE IN ADOLESCENTS WITH INFLAMMATORY BOWEL DISEASE

Hester J. Loonen, Bert H. Derkx, Pediatrics, Martha A. Grootenhuis, Bob F. Last, Psychosocial Department, Emma Children's Hospital, Academic Medical Centre, Amsterdam, The Netherlands

In clinical practice, more attention is being paid to ways to ameliorate patients' QoL. Inflammatory bowel disease (IBD) is a chronic relapsing disorder with heavy treatment protocols that challenges patients' coping abilities considerably. The aim of this study was to analyse which variables (including sex, disease activity and reliance on certain coping strategies) best predict HRQoL. We used a cognitive control strategy scale (CCSS), which measures four coping styles: predictive coping (having positive expectations about the future), vicarious coping (attributing power to medical caregivers and treatment), interpretative (searching for meaning and information), and illusory control (wishful thinking), and we used a disease-specific HRQoL questionnaire (Impact-II (NL)), measuring six domains of QoL (bowel and systemic symptoms, emotional and social functioning, body image and treatment related concerns). Adolescents with IBD (12 to 18 years old) were asked to fill out the CCSS and the Impact-II (NL). To investigate which variables predict adolescents $\mathrm{HRQ}$ oL, regressions analyses in steps were performed with the Impact domains as the dependent variable, and sex, diseease activity and predictive and vicarious control as predictor variables. More reliance upon predictive (optimism about course of disease) and vicarious coping (attributing power to medical care givers) predicts better QoL. Gender and disease activity also attributed to the model, although less than the coping styles. Coping is an important predictor of HRQoL, even more than the widely accepted gender and disease activity variables. Our findings demonstrate that interventions with adolescents with IBD could focus on enhancing the efforts to appraise their disease more positively, and on strengthening the reliance upon the doctor, besides traditional therapies aimed at decreasing disease symptoms.

32/ "I THINK MY LIFE IS BETTER THAN MOST KIDS" LIFE SATISFACTION IN HEALTHY AND CHRONICALLY ILL CHILDREN

Kirsten Moenkemoeller, Kinderkrankenhaus, Universität Witten/ Herdecke, Köln, Germany; Anne Wrede, IFAS, Universität zu Köln, Köln, Germany; Guido Buerk, Vestische Kinderklinik, Universität Witten/Herdecke, Datteln, Germany; Ulrike Ravens-Sieberer, Forschungsgruppe, Robert-Koch-Institut, Berlin, Germany

Life satisfaction (LS) as the cognitive component of well being refers to a "judgemental process, in which individuals assess the quality of their lives on the basis of their own unique citeria" (Shin, D.C., Johnson, D.M.1978). Do chronically ill children judge their quality of life any different than healthy children when applying their own criteria? The "Student's Life Satisfaction Scale" for children aged 7-14 (Huebner, E. 1991) was translated into German and adapted for children aged 8-12. Additionally the satisfaction in five domains (school, family, friends, living environment, and self) was registered applying an adapted version of the "MSLSS" (Huebner, E. 1994). The questionnaires were administered to a group of 347 children aged 8-12. 130 children with Asthma $(n=39)$, Diabetes $(n=45)$ and Rheumatic Diseases $(n=46)$ were interviewed using the revised version. Teachers of healthy children and parents of the chronically ill were interviewed as external raters. Both scales showed acceptable psychometric properties. Convergent validity could be shown with teachers and parents ratings in expected directions. T-Test showed a significant difference between the global LS of the two groups $(p=.001)$. Applying Linear Regression, the satisfaction in the five domains explained $47 \%$ of global LS for healthy children but only $27 \%$ for the chronically ill. Including all variables in a Backwards Linear Regression, sickness parameters explained $9 \%$ with function and severity rated by the children being the strongest predictors. Logistic Regression showed that $86 \%$ of the extremely satisfied/unsatisfied children (SD>1) can be classified by the MSLSS and $87 \%$ applying all variables. Evaluating specific living domains as well as disease specific concepts might not grasp the criteria the children apply to judge their quality of life. Complementing HRQL interviews by global LS ratings can enhance the potential to classify healthy or chronically ill children who need special support. 
33/ QOL AFTER PEDIATRIC STEM CELL TRANSPLANTATION: A CASE FOR RESPONSE SHIFT?

Susan K. Parsons, Christopher J. Recklitis, Dana-Farber Cancer Institute, Boston, MA

Hematopoietic stem cell transplantation (HSCT) raises central quality of life (QOL) issues and significant 'adaptational' challenges for survivors. Treatment is intense, including risk of sequelae and fear of recurrence. In pediatric HSCT, QOL evaluation is complicated by reliance on parents' proxy report. The transplant affects parents and their adaptation has a major impact on the child. To understand children's QOL after HSCT both the children and parents' ratings and the interaction between the ratings over time must be considered We performed a cross-sectional analysis using the Child Health Ratings Inventory and the Disease-Specific Impairment Inventory-HSCT of 150 children (5-12 yrs.) and adolescents (13-21 yrs.) after HSCT. For children surveyed soon after transplant (i.e., $<6$ mo.), parent reports were universally lower in all dimensions ( 1-2 s.d.; $p<0.001)$. Later post transplant (i.e., > 12 mo.), a dramatic shift occurred with parent scores equaling or exceeding all child scores. These parentchild ratings were not correlated in either time period. Child selfreports,however, were more strongly correlated with disease severity than parent reports. The pattern in the adolescent sample is slightly different. Early on, parents scored significantly lower than adolescents in only two of the seven dimensions. Later on, no significant differences were detected between raters, and correlations within parent-adolescent pairs were robust $(r=0.36-0.97)$. In contrast to the younger sample, adolescent self-report was generally not associated with disease severity, whereas their parents'ratings were. These results suggest that response shifts may differ as a function of age and cognitive development. Further, over time the parent and the child derive different meaning from the same external reality. Parental scores may reflect an internal recalibration, influenced by the child's survival and resumption of normative activities. The data will be discussed within the context of a response shift model.

34/ PEDIATRIC SPINE DEFORMITY: ASSESSING PATIENT OUTCOMES AND QUALITY OF LIFE

Michael G. Vitale, Pediatric Orthopaedics, Children's Hospital of New York, New York, NY; Julie C. Choe, Douglas E. Levy, Pediatric Orthopaedics, Annetine C. Gelijns, Alan J. Moskowitz, Surgery, InCHOIR, New York, NY; Joshua E. Hyman, Francis Y. Lee, David P. Roye, Pediatric Orthopaedics, Children's Hospital of New York, New York, NY

Several new outcome measures have been recently published to assess quality of life (QOL) outcomes in pediatric orthopaedics and pediatrics, in general. Although these measures have shown promise, none have been validated for use in children with scoliosis. Parents of 279 children with scoliosis completed the Child Health Questionnaire (CHQ) and the American Academy of Orthopaedic Surgeons (AAOS) Pediatric Outcomes Instrument between 1997 and 2000; the Scoliosis Research Society (SRS) instrument was completed directly by the affected children. The treating physician completed a subjective rating of health and documented pertinent socioclinical data for each child. Scores were compared with one another and to the clinical parameters. The effect of surgery on QOL (responsiveness) was assessed for 40 patients who had scoliosis surgery. Ceiling effects were noted for each instrument, especially in the physical function and self esteem domains. However, patients with scoliosis scored significantly lower than published values for "healthy" children in several CHQ domains (t-test): physical function $(p<0.005)$, bodily pain $(p=0.01)$, parental time impact $(p=0.02)$, parental emotional impact $(p<0.005)$, behavioral scale $(p<0.005)$. Moreover, patients with larger curves exhibited lower QOL scores, as measured by the $\mathrm{CHQ}$, supporting the face validity of this measure. In general, the $C H Q$ showed superior psychometric characteristics over the AAOS instrument in this population. Four SRS domains were correlated with degree of curvature: physical function $(r=-0.17, p=0.008)$, bodily pain $(r=-0.32$, $p<0.005)$, self image $(r=-0.24, p<0.005)$, and satisfaction $(r=-0.29$ $p=0.02$ ). Preliminary analysis of longitudinal data on patients at 1 year after surgery showed no significant changes. The CHQ, AAOS, and SRS instruments all suggest that scoliosis has a negative effect on the physical and psychosocial health status of affected children.
35/ HEALTH RELATED QUALITY OF LIFE OF CHILDREN WITH CHRONIC DISEASES

Martha A. Grootenhuis, Bob F. Last, Psychosocial Department, Emma Children's Hospital, Academic Medical Centre, Amsterdam, The Netherlands

Advances in pediatrics and pediatric surgery, have led to the need to evaluate the physical, psychological and social consequences of medical treatment in children. In several patient populations Health Related Quality of Life (HRQOL) has been measured with the TACQOL-CF, which measures the domains: physical, motor, cognitive and social functioning, autonomy, and positive and negative emotions. Chronically ill children aged 8-18 years with different diagnosis: inflammatory bowel disease $(n=82)$, congenital heart disease $(n=73)$, familial hypercholesterolemia $(n=69)$, end stage renal failure $(n=18)$ and cancer $(n=17)$ participated in different ongoing studies. Aim of this study was to compare the HRQOL of children with a chronic illness with healthy controls and to investigate within-group differences. Children with a chronic disease were compared with a random sample of 400 healthy children aged 8-15 years using t-tests. Analysis of variance were used to investigate differences between illness-groups. Children with a chronic illness (complete sample $n=259$ ) differed from healthy children on the domains of motor functioning and autonomy with lower HRQOL. Analysis of variance showed differences between illness groups on the domains of motor and cognitive functioning and on autonomy. Major findings are: Children with a congenital heart disease reported lowest HRQOL on motor functioning (difficulties with walking, running and power of endurance). Children with cancer report lower quality of life in autonomy (doing things on their own or independently) whereas children with end stage renal failure report affected HRQOL on cognitive functioning (concentration, difficulties with schooltasks). Although all specific groups have their own issues, the findings show some of the disease-specific problems of children with a chronic illness. Intervention programs should take these problems into account.

\section{6/ THE RELATIONSHIP BETWEEN RESOURCE CONSUMPTION AND ASTHMA SEVERITY AMONG ASTHMATIC OUTPATIENTS} IN THREE EUROPEAN COUNTRIES

David U. Himmelberger, Health Outcomes Group, Palo Alto, CA; Leonardo Antonicelli, Hospital Umberto I, Ancona, Italy

This study was designed to evaluate the relationship between asthma severity as measured by the GINA Guidelines (intermittent, mild, moderate and severe persistent) and resource consumption among patients with asthma. A total of 1,128 patients from Spain, Italy and France participated in the study. Data were collected from patients based upon their self reported recall using a questionnaire designed to measure the relevant components of resource consumption and asthma severity. Additional data about the patients' asthma status and severity was collected from their physicians. The data of this study confirm the hypothesis that more resources are consumed as the severity of asthma increases. The total resource consumption in monetary units varied from 6-14.5 times greater for patients with severe persistent asthma than for patients with intermittent asthma $(\$ 2,720$ vs. $\$ 428$ for Spain and $\$ 19,670$ vs. $\$ 1,350$ for Italy). As severity increased from intermittent to severe persistent, a 2-3 fold increase in limitation in daily activities was observed. Significant increases in time lost from paid and non-paid work were also observed. Peak flow was below the predicted values for all four GINA classifications of severity, with the magnitude of the differential varying greatly across the three mildest GINA classes (8-29\%) and was slightly greater for the patients with severe persistent asthma (32-45\%). Similar to the results with limitation of daily activities and asthma symptoms, consumption of individual resources increased two to three fold in terms of natural health units in all areas measured as the severity increased from intermittent to severe persistent asthma. The data from this study suggests that resource consumption is more strongly related to asthma severity, as measured by the GINA guidelines, than it is to pulmonary function, as measured by peak flow. 
37/ THE EFFECT OF ASTHMA SEVERITY ON HEALTH-RELATED QUALITY OF LIFE AND WORK PERFORMANCE: DOES THE DEFINITION OF SEVERITY MAKE A DIFFERENCE?

Steven R. Erickson, Duane M. Kirking, College of Pharmacy, University of Michigan, Ann Arbor, MI; Nancy Kline Leidy, Center for Health Outcomes Research, MEDTAP International, Bethesda, MD

This study compares HQL and WP scores across five methods of determining asthma severity: three subjective methods: patient perceived severity (PPS); patient-reported symptom-severity-overall (OSS); symptom-severity nocturnal (NSS); and two objective methods using retrospective claims analyses: oral steroid use (OSU) and multi-drug use (B2, inhaled and oral steroids). Three levels of severity were examined: mild, moderate and severe. Secondary analysis of data from a cross-sectional mail survey of 603 adults treated for asthma in a U.S. managed care organization linked to pharmaceuti$\mathrm{cal} /$ medical claims data. Patients completed the SF-36, Asthma Quality of Life Questionnaire (AQLQ) and a WP scale. Analysis of variance (ANOVA) procedures were used to compare scores across severity level within method and across method within severity level. Within each method, significant severity effects were found for PCS, AQLQ and WPS (except OSU). Significant effects for MCS were also found in the OSS and NSS models. In post-hoc analyses, mild-moderate and mild-severe comparisons were consistently significant $(p<0.05)$. In the cross-method analyses, significant method effects were found at all levels of severity $(p<0.05)$ for most scale scores with ranges as follows: Mild-PCS 47.7 to 50.0; MCS 48.7 to 49.8 ; AQLQ 5.3 to 5.8 ; WPS 89.5 to 93.3 . Moderate-PCS 41.7 to 47.5 ; MCS 46.4 to 48.7 AQLQ 4.0 to 4.8; WPS 81.9 to 88.7. Severe-PCS 38.3 to 43.6; MCS 44.1 to 49.2 ; AQLQ 3.8 to 4.7 ; WPS: 75.4 to 85.7 . Within-method results were consistent with previous studies demonstrating a significant relationship between severity, $\mathrm{HRQL}$ and WP. HRQL and WP scale scores varied by the method used to define severity, however. This variation should be considered when performing cross-study comparisons of burden of illness and treatment effects.

\section{8/ QUALITY OF LIFE AFTER INTENSIVE CARE FOR ACUTE LUNG INJURY}

Cynthia R. Gross, Academic Health Center, University of Minnesota (UMN), Minneapolis, MN: Craig R. Weinert, Medical School, Minneapolis, MN; Carol Albright, Caron L. Bury, William A. Marinelli, UMN, Minneapolis, MN

Progress has been made in reducing in-hospital mortality from Acute Respiratory Distress Syndrome (ARDS), but there is still relatively little information on the health status of survivors. A sample of 51 patients (men=29, women=22, aged 19-78) who recovered from ARDS or acute lung injury completed a battery of mailed, self-report questionnaires at six months or longer after discharge from intensive care (median 8 mos). Their health-related quality of life (HRQL), as measured by the SF-36, showed large and statistically significant decrements compared to norms across all domains. Clinically relevant levels of depression symptoms were found in $46 \%$ of the sample based on the Center for Epidemiologic Studies Depression Scale (CES-D). A subsample $(\mathrm{N}=18)$ completed the Post-Traumatic Stress Disorders Checklist (PCL), and while only one patient scored above the leve considered indicative of the diagnosis, $61 \%(11 / 18)$ reported these symptoms interfered with their normal social activities. In our sample, the SF-36 mental component score is strongly correlated with the PCL $(r=-.80, N=18, p<.01)$ and with the CES-D $(r=-.83, n=49, p<.01)$. Depression and PCL scores were positively correlated $(r=.64, p<.01)$. These findings highlight the need to include HRQL outcomes in ICUbased clinical trials. Currently, our group is investigating the relationships among factors such as medications (sedatives and neuromuscular blocking agents) or therapies (ventilation strategies, new medications) and these HRQL outcomes. Additional research should establish whether clinicians are aware of these long-term decrements in HRQOL or how families and patients are prepared for post-ICU sequelae. Funding: SCOR Clinical Core P50 HL50152.
39/ EFFECTS OF DISEASE MANAGEMENT ON QUALITY OF LIFE IN PATIENTS WITH ASTHMA

Wolfgang Greiner, Matthias J. Graf v.d. Schulenburg, Institut für Versicherungsbetriebslehre, University of Hannover, Hannover, Germany

In the last years, Disease Management has been recognized as a significant element in improving the care situation of chronically ill people in particular, both from a medical and an economic point of view. This study deals with an economic evaluation of a Disease Management Programme for asthma with the help of an electronic control instrument for Home-Monitoring of the patient. Research was made on the improvement in the quality of life as well as the saved costs. 112 adult patients with asthma of varying severities were divided randomly into two groups. The patients are all members of a large German health insurance group in the Bundesland RheinlandPfalz. The first group received an extensive Disease Management Programme with electronic Home-Monitoring, while the control group was treated further by their GP. The patients were observed over a period of 12 months. Lung function tests and investigations on the quality of life were carried out in intervals of six months (at the beginning, after six months and after 12 months; visits one, two and three). The quality of life was measured using the questionnaires Quality of Life Asthma (FLA), a validated disease-specific questionnaire and the EQ-5D (Euro-Qol). In addition, the treatment costs during the study time period were documented and evaluated. During the study, the quality of life of the control group revealed no changes statistically, while the patients in the Disease Management group achieved significantly higher values than those at the beginning of the study. The improvements are related particularly to the "Physical and Psychic Attributes" of the FLA, although they can also be seen in the (less sensitive) generic EQ-5D. Thus, the average EQ-5D value of 62 increased to 72, while in the control group it dropped from 65 to 60 . The study data additionally shows that considerable savings are possible with Disease Management for asthma, especially concerning hospital services. These savings are partly counteracted with higher costs in other area.

40/ COPING AND QUALITY OF LIFE IN ADULTS WITH ASTHMA Edgar A. Correia, Instituto Superior de Psicologia Aplicada, Lisboa, Portugal; Jose L. Pais-Ribeiro, Faculdade de Psicologia, Universidade do Porto, Porto, Portugal; Nuno B. Neuparth, Pedro L. Mata, Fisiopatologia, Faculdade de Ciencias Medicas, Universidade Nova, Lisboa, Portugal

The objective of this cross-sectional study is to explore the relationships between coping strategies and health related quality of life (HRQOL) in patients with asthma. The portuguese versions of the Coping with Health Injuries and Problems Scale (CHIPS) and the Asthma Quality of Life Questionnaire (AQLQ) were applied to 60 adults, from both sexes (52\% females), with asthma diagnostic. $R e$ sults showed some significant correlations between self-reported coping strategies and HRQOL in asthma. Patients who scored higher in Palliative Coping reported significant worst $\mathrm{HRQOL}$ in all domains of the AQLQ (varying from $r(60)=-.27, p<.05$ in the Emotional Function domain, to $r(59)=-.39, p<.01$ in the Exposure to Environmental Stimuli domain), as well as in the Overall Quality of Life, $r(58)=-.35, p<.01$. Similar pattern appeared when correlation's between Emotional Preoccupation coping and AQLQ were maid: effectively, asthmatic patients that reported more use of Emotion-Oriented strategies to deal with their asthma showed significant lower HRQOL $(p<.01$, in the overall $A Q L Q$, as well as in all its domains). Otherwise, patients that referred more resource of Instrumental or Distraction coping had only negative correlations with Environmental Exposure related QOL ( $\mathrm{r}$ $(59)=-.32, p<.05 ; r(59)=-.34, p<.01$, respectively). Results suggests, having in account that some ways of coping have clearly negative implications in $\mathrm{HRQOL}$ in patients with asthma, that it can have advantage in creating self-management education programs that attend to psychological factors, namely coping strategies. 
41/ USING ITEM RESPONSE THEORY TO IMPROVE QOL MEASURES FOR CHILDREN - FIRST RESULTS FROM THE CHILD DYNAMIC HEALTH ASSESSMENT PROJECT

Ute Ellert, Christiane Thomas, Ulrike Ravens-Sieberer, Department for Child and Adolescent Health, Robert Koch Institute, Berlin, Germany; Mark Kosinski, Quality Metric Inc., Lincoln, RI; Jakob B. Bjorner National Institute of Occupational Health, Copenhagen, Denmark, Jim Dewey, John E. Ware, Quality Metric Inc., Lincoln, RI

The aim of the project was to apply Item Response Theory Models to equate and score 3 widely used health related quality of life questionnaires for measuring the emotional well-being of children. Data from a rehabilitation study $(\mathrm{N}=881)$ and a survey with healthy children $(\mathrm{N}=185)$ was analyzed. The children and adolescents (8-16 years) completed three different HrQoL instruments (KINDL-R, CHQ, SF36) pre-treatment and directly, 3 months, and 12 months post-treatment. The total pool of items measuring mental health was 28 . Factor analytic methods were used to test the assumptions of IRT, unidimensionality and local independence if items. IRT methods were used to evaluate the ordinal consistency of response choices and the distinctiveness of items representing a "mental health" dimension for all instruments and to score an overall child mental health concept (theta). Measurement precision using IRT based scoring was evaluated against the developer's scoring for each instrument and all were evaluated in relation to theta. Eight items were dropped since they did not meet the IRT assumptions of unidimensionality or local independence. Response choices to more than one third of the mental health items showed ordinal inconsistencies. Model fit was improved by re-scoring the response choices of these items. For the IRT-based scale scores statistically significant gains in measurement precision were observed compared to the scores based on the developer's method. The pool of items from all instruments combined provided better coverage of the range of subjective mental health measured (theta) in comparison with mental health domains covered by each instrument. The item pool was included in a ChildDynHA demo version running on the Internet and its feasibility was tested on 60 school-aged children indicating good acceptance and a minimum of time and effort for the children. In conclusion, IRT methods are likely to be useful in improving measurement precision and comparing results across different instruments that measure the same health concepts in children. Computer adaptive administration of items from a pool of items from all instruments is likely to yield more precise measures of child health and reduce respondent burden.

\section{2/ ITEM BANKING TO IMPROVE, SHORTEN AND COMPUTER-} IZE SELF-REPORTED FATIGUE

Jin-shei Lai, David Cella, Chih-Hung Chang, Evanston Northwestern Healthcare (ENH), Northwestern University (NU), Evanston, IL; Rita K. Bode, Rehabilitation Institute of Chicago (RIC), Allen W. Heinemann, RIC, NU, Chicago, IL

Fatigue is a common symptom among cancer patients and the general population. Modern computerized adaptive testing (CAT) can enable precise assessment of fatigue across diverse groups using a small number of items from a fatigue item bank. This article illustrates 4 steps to prepare such an item bank, using 13 items from the Functional Assessment of Chronic Illness Therapy-Fatigue Subscale (FACIT-F) as the basis. Samples included 1,022 cancer patients and 1,010 people from the United States general population. Steps included: (1) select core items, (2) partition the fatigue continuum into probabilistic segments using a pre-selected criterion item, (3) position item response categories of the selected core item onto the fatigue continuum, and (4) create an item bank matrix for CAT application. Using an Item Response Theory-based measurement model, Rasch measurement model, we selected 9 items based on their fit to the underlying model and their lack of differential item functioning The items were then positioned on the fatigue continuum. The fatigue level for $66.8 \%$ of the general population and $82.6 \%$ of the cancer patients was well-captured by these 9 items. A precise fatigue score was obtainable using as few as 4 of the 10 banked items (including 1 criterion item from the FACIT-general). This study demonstrates that 10 banked items can precisely measure fatigue, but also that a more extensive item bank covering the entire fatigue continuum will further enhance measurement precision. Four steps to create the item bank and derive the CAT algorithm will be presented.
43/ ONE-VERSUS TWO-PARAMETER ITEM RESPONSE THEORY (IRT) MEASUREMENT MODELS APPLIED TO MOS PF-10 SCORES: HOW MUCH DOES IT REALLY MATTER?

Chih-Hung Chang, David Cella, Center on Outcomes, Research and Education, Evanston Northwestern Healthcare, Evanston, IL

Item response theory (IRT)-based models have increasingly been applied in health status questionnaire development and assessment. There is debate as to which of these models, often classified as 1versus 2-parameter $(P)$ models, is most appropriate. This study examined the comparability of original and IRT scoring methods for the MOS 10-item Physical Functioning (PF-10) scale. The PF-10 data were collected from 5 sites as part of a larger project to equate five commonly used quality of life instruments in a total of 1,714 cancer and HIVIAIDS patients. Physical functioning scores were obtained using algorithms in Ware et al (1994) and Hays et al (1998), as well as two 1-P models: Masters' partial credit model (PCM); Andrich's rating scale model (RSM), and two 2-P models: Samejima's graded response model (GRM) and Muraki's generalized partial credit model (GPCM). Scores obtained by Hays, PCM, and RSM methods were all highly correlated with the original PF-10 scoring as in the SF-36 manual (all $r>.975, p<.0001$ ), while scores based on GRM and GPCM correlated only moderately with the original scoring $(r=.60, p$ $=.02$ ). The two possible misfitting items (PF01 and PF10) identified by RSM and PCM also had the lowest slope parameters obtained from the GRM and GPCM, evidenced by the high degree of association between fit statistics in the RSM and PCM versus slope parameters in the GRM and GPCM ( $r=-.76$ to -.77). Item difficulty (location) hierarchies obtained from the four IRT models were almost identical (correlation range $=.998$ to .999 ), indicating these models yield almost identical ordering of patients in measuring physical functioning. We conclude that these approaches yield comparable results and have good potential for providing complementary rather than conflicting data.

44/ DO MEASURES OF FUNCTIONAL ABILITY FULFIL THE ASSUMPTIONS ON DIMENSIONALITY REQUIRED BY ITEM RESPONSE THEORY?

Rebecca Holman, Robert Lindeboom, M. Vermeulen, Rob de Haan, Clinical Edpidemiology and Biostatistics, Academic Medical Center (AMC), Amsterdam, The Netherlands

One of the most important determinants of the level of health related quality of life enjoyed by patients is their functional health status. This is often defined in terms of their ability to perform activities of daily life and measured using multi-item instruments. The dimensionality of these instruments has often been well considered using classical psychometric methods, such as factor analysis. However, recently there has been a surge in interest in the application of item response theory to the analysis of such multi-item instruments. Unidimensional item response theory models are now relatively well known, and their utility in modelling and analysing the responses to multi-item instruments has been proven, in many clinical fields, including the measurement of $\mathrm{HRQOL}$. However, interest is beginning to move towards models, which can accommodate a number of measurement dimensions. In this article we will examine a multidimensional item response theory model and how it can be used to shed light on the dimensionality of the instruments developed to measure the functional status. The item bank developed during the Amsterdam Linear Disability Score project to measure the functional status of chronically ill, but stable patients, will be considered in depth. The item bank consists of approximately 200 items and was constructed using item response theory techniques with the aim of placing all items on a single measurement scale. We will present the results of both multi- and unidimensional analyses of the data and consider whether the results are worth the extra input required to estimate a multidimensional item response theory model. We hope that the results obtained in this paper will provide some important guidelines for considering the dimensionality of multi-item instruments for the measurement of functional status when using item response theory techniques. 
45/ FACTORS ASSOCIATED WITH STABILITY IN QUALITY OF LIFE RATINGS AMONG PEOPLE LIVING WITH HIVIAIDS: RESPONSE SHIFT'S "SMOKING GUN"?

Bruce D. Rapkin, Rosy Chhabra, Carolyn Springer, Psychiatry and Behavioral Sciences, Memorial Sloan-Kettering Cancer Center, New York, NY; Bruce Agins, Clemens M. Steinbock, AIDS Institute, New York State Department of Health, New York, NY; Mark Sharp, Ira Feldman, AIDS Institute, New York State Department of Health, Albany, NY

During intervals when global QOL ratings remain constant, significant changes may nevertheless occur in component quality of life scales. Such underlying changes necessarily indicate response shifts in the appraisal of overall QOL. In this paper, we will examine this phenomenon in order to better to understand how people living with HIVIAIDS maintain their overall QOL. Data are based on 1307 Medicaid recipients interviewed at two or more points in time. Measures included the MOS-HIV, health status, service use, life events, and demographics. Initial analyses compared change from first to last interview, average of 3 years (range 6 to 48 months). Of 1307 observations, $460(35.2 \%)$ provided the same answer to the overall health rating at first and last interviews. However, these patients displayed variance and covariance among MOS subscales similar to the full sample. Cluster analysis based on the stable subsample revealed six patterns of change in MOS subscales. $43.2 \%$ demonstrated stability on all MOS subscales. $56.8 \%$ showed change on one or more subscales despite stability in overall health. Discriminant analysis suggested three major patterns of sub-scale change: (1) Increases in emotional well being and energy offsetting losses in cognitive, socia and role functioning; (2) General improvement in social, cognitive and role functioning; and (3) Improved emotional well-being and cognitive functioning offsetting decline in physical functioning and increased pain. Changes were associated with longer intervals between interviews, role changes, substance use and declining health. Findings demonstrate processes involved as individuals adapt to changing circumstances while maintaining their sense of well-being. Implications of these results for understanding how people modify their perspectives to maintain QOL will be discussed.

46/ RESPONSE SHIFT EFFECTS OF AN ADVANCE CARE PLANNING INTERVENTION WITH AMBULATORY GERIATRIC PATIENTS

Carolyn E. Schwartz, Department of Family Medicine \& Community Health, University of Massachusetts Medical School, Worcester, MA Noreen Basque, VNA Care Network, Worcester, MA; George Reed, Yungsheng Ma, Medicine, $\mathrm{H}$. Brownell Wheeler, Surgery, University of Massachusetts Medical School, Worcester, MA

There is a large discrepancy between the wishes of dying patients and their actual end-of-life (EOL) care, with a negative impact on patients and survivors alike. It is postulated, however, that early intervention to facilitate advance care planning (ACP) can reduce this discrepancy and may lead to response shifts in quality of life (QOL). This randomized trial $(n=61)$ evaluated the impact of the LaCrosse early intervention in planning EOL care for ambulatory geriatric patients, and investigated how patient's values, conceptualizations, and internal standards of QOL change as they engage in ACP. Patients were randomized either to a non-directive control condition or to an intervention in which an ACP-trained nurse facilitated a discussion between the patient and his/her health care proxy. They discussed the benefits and burdens of life-sustaining treatments, and the patient's goals and preferences for life-sustaining treatments. Two-month follow-up revealed that the intervention achieved higher congruence between patients and proxies in reporting patients' EOL care preferences with $81 \%$ in complete agreement vs $57 \%$ [Effect Size (ES) of difference in overall congruence index $=0.54$ ] and a larger increase in knowledge about ACP ( $E S=0.22)$. Intervention patients also experienced response shifts in values and conceptualizations of $Q O L$, becoming less willing to undergo life-sustaining treatments for a new serious medical problem $(E S=0.46)$ or for an incurable progressive disease $(E S=0.24)$, and less willing to tolerate poor health states $(E S=0.79)$. There was no detectable shift in internal standards. We conclude that an intervention of facilitated discussion may improve the congruence of patient wishes and EOL care, and may help define patient values and conceptualizations of QOL at EOL. Consequently, ACP should be an iterative process that is revisited as life experiences and one's understanding of the benefits and burdens of lifesustaining treatments change over time.
47/ USING ITEM RESPONSE THEORY (IRT) AND STRUCTURAL EQUATION MODELING (SEM) TO INVESTIGATE RESPONSE SHIFTS

Frans J. Oort, Pythia T. Nieuwkerk, Mirjam A. Sprangers, Medical Psychology, Academic Medical Centre, University of Amsterdam, Amsterdam, The Netherlands

When assessing change in quality of life (QOL), we must account for response shifts such as reconceptualization, reprioritization, and recalibration. To detect response shifts we use the IRT\&SEM approach, i.e., (1) find appropriate measurement model, using generalized linear IRT, (2) investigate changes in model parameters, using SEM, (3) interpret findings, using a change typology. We illustrate this approach with QOL data. HIV infected patients (216) completed the MOS-HIV Health Survey at the start of antiretroviral therapy and 36 weeks later (T2). The survey measures patient's health status (PHS) with 11 scales. We used 7 scales: general health $(\mathrm{GH})$, social function (SF), energy/fatigue (EF), quality of life (QL), cognitive function (CF), mental health (MH), and health distress (HD). We applied the IRT\&SEM approach with (1) a linear model with 2 common factors, named overall $\mathrm{GH}(\mathrm{OvGH})$ and overall $\mathrm{MH}(\mathrm{OvMH}),(2)$ likelihood-ratio tests to test for invariance of model parameters (e.g., factor loadings and means, variances, and correlations of common and specific factors), and (3) the response shift typology to interpret parameter changes. We found no significant differences between factor loading patterns or values, so neither reconceptualization of OvGH or OvMH, nor reprioritization of the 7 underlying scales occurred. We did find a higher T2 specific factor mean of the HD scale indicating recalibration. At T2 we also found: lower specific factor variances indicating that the patient group became more homogeneous in HD, $\mathrm{CF}$, and SF; a higher correlation between OvGH and OvMH indicating reconceptualization of $\mathrm{PHS}$; and higher common factor means indicating general improvement of OvGH and OvMH. Application of the IRT\&SEM approach is useful because it reveals different types of change that would remain hidden otherwise. Underlying assumptions of the IRT\&SEM approach, and caveats, are subject of discussion.

48/ RESPONSE SHIFT IN THE ASSESSMENT OF HEALTH RELATED QUALITY OF LIFE (HRQL) POST-STROKE

Sara Ahmed, Nancy E. Mayo, Sharon Wood-Dauphinee, Jim Hanley, Epidemiology, McGill University, Montreal, QC, Canada

The validity of assessing changes in HRQL over time is threatened if people experience a response shift. This study assessed whether the recovery process following stroke altered individuals' perceptions of past health status and the impact that change in internal standards had on rating of HRQL over time. Individuals were recruited through a randomized trial of acute post-stroke care. Health status was evaluated at baseline and 6 and 24 weeks later using the thermometer scale of the EQ-5D. At 6 and 24 weeks, subjects were asked to retrospectively re-evaluate their health status for the preceding evaluation using the then test technique. Retrospective rating of baseline health status was $10 \%$ lower than the prospective rating $(p<0.0001)$ and at 6 weeks, $13 \%$ lower $(p<0.0001)$. Measured prospectively, mean health status significantly improved from 67 (/100; SD: 19) at baseline to 76 (SD: 17$)$ at 6 weeks. When baseline health status was retrospectively measured, it was re-evaluated at 60 (SD:20) and correspondingly there was an increase in the magnitude of change. Prospectively, between 6 and 24 weeks, there was no measured change in health status. When the retrospective re-evaluation of the 6 -week rating was incorporated, there was a significant improvement between 6 and 24 weeks. Memory had an impact on response shift; subjects with "poor" memory had greater response shift and variability. The results suggest that there was a change in internal standards, and that measures of improvement in health status are different based on prospective versus retrospective ratings. Further understanding of the impact of memory on the assessment of response shift using the then test will help to validate the use of this technique. 
49/ RESPONSE SHIFT IN PATIENTS' LIVING WITH ADVANCED CANCER

Nessa Coyle, Richard Payne, Neurology, Memorial Sloan-Kettering Cancer Center (MSKCC), New York, NY

Clinical work with patients living with advanced cancer involving palliative care and psychological and social support has led to the observation that at least in some individuals, a response shift occurs in what they consider to be an acceptable QOL. The purpose of this research was to identify, through listening to a series of personal narratives, evidence of this response shift and influencing factors. This is a qualitative exploratory study on the experience of 7 patients living with advanced cancer. Adult English speaking patients with advanced disease, who were followed by the Pain and Palliative Care Service at MSKCC, had expressed attitudes towards life and death to their physician or nurse, and were not known to the principle investigator from her clinical practice were eligible for the study. A series of from 2-6 open-ended audio taped interviews were conducted with each participant either in the hospital, clinic, terminal care facility or their home. Time between each interview varied from 24 hours to a week. Five patients died within one month and two within one week of their last interview. Thematic analysis of their narratives was used. We are developing an indepth sytem to track processes and changes. An illustrative case of response shift was seen in a 60 -year old man who was interviewed 6 times over one-month. He spoke of how he came to view care from his wife and sons not as an overwhelming burden that had initially led him to desire a hastened death, but as a positive aspect of their relationship and as a "gift." He also changed the way he saw his progressive illness from one of fighting to one of acceptance. Initial analysis of other participants' narratives illustrates the impact of the environment, relationships and forms of communication on shifting responses to progressive illness. This study highlights the potential for rapid and potentially radical swings in the way terminally ill patients appraise QOL, indicating the need to take response shifts into account in any study with this population.

50/ SYMPTOM EXPERIENCE AND HEALTH RELATED QUALITY OF LIFE AFTER IMPLANTATION OF A CARDIOVERTER DEFIBRILLATOR

Susan F. Marden, Clinical Center Nursing Department, National Institutes of Health, Bethesda, MD

The efficacy of implantable defibrillator (ICD) therapy is well established, yet the impact of recipients' appraisals of side effects associated with disease and treatment is uncertain. This study examined whether symptom experience (SyE) alters Health related Quality of Life (HRQL) in ICD recipients ( $n=117)$. The Important Concerns and Distress Questionnaire (ICDQ) and Short Form-36 (SF-36) were completed by mail survey. SyE is a combination of perceived frequency and associated distress of 29 concerns on the ICDQ. Recipients ( $81 \%$ male; 62 years; 3.9 years post ) had experienced at least 1 shock $(57 \%)$ and cardiac arrest (50\%). Most $(73 \%)$ reported $>10$ concerns with women reporting more than men $(t=1.79 ; p<.05)$. Frequent concerns were also most distressing: tiredness, less sexual activity, trouble sleeping, ICD awareness, shortness of breath, forgetfulness, frustration, weakness, future anxiety, dependency, shock worry. Factorial ANOVA ( SyE-high/low; age $<65$ or $>65$; years post $<2,2-5,>5$ ) indicated a main effect for SyE on mental and physical component summary scores of the SF-36 (MCS: $F=35.9$, p< 05; PCS: $F=15.7$, $p<05)$. High SyE levels were associated with a significant, independent reduction in both mental and physical HRQL. Interventions to ameliorate symptom experiences of ICD recipients are warranted and may improve HRQL.
51/ PREDICTING UTILIZATION AND COSTS IN A ONE-YEAR FOLLOW-UP OF HEART FAILURE PATIENTS: THE CONTRIBUTIONS OF GENERIC AND DISEASE SPECIFIC HRQOL ASSESSMENTS Deborah M. Miller, Mellen Center, Neurology, Cleveland Clinic Foundation, Cleveland, OH; D. Nelson, M. Karafa, Biostatistics and Epidemiology, J. Michaels, Center for Clinical Outcomes, J. B. Young, Cardiology, $\mathrm{CCF}$, Cleveland, $\mathrm{OH}$

The purpose of this study was to determine the factors, including generic and disease-specific HRQoL measures, that contribute to unscheduled health care utilization anywhere and treatment costs at a tertiary care center for cardiac patients who were followed for a maximum of 14 months. Patients who gave informed consent completed two HRQoL measures, the MOS SF-36 and the disease specific Minnesota Living with Heart Failure (LwHF) and reported unscheduled health care utilization (HCU) for the preceding 6 months. They agreed to provide phone follow-up at 6-month intervals for the next year. Objective measures of disease severity, including New York Heart Association classification, type of cardiac disease and medications were retrieved at baseline only. 794 patients provided baseline date. Using a professional survey organization, 181 of these patients were successfully contacted and provided phone follow-up once (either between 6 to 8 months or between 12 to 14 months) and 60 provided follow-up at both assessment phases. When combining all available follow-ups, among individuals who had not had previously reported $\mathrm{HCU}$, either recent LwHF or change in LwHF was significantly higher for those with recent HCU. However, LwHF scores did not differ between those with and without HCU in the subgroup with a history of $\mathrm{HCU}$. Logistic regression was used to compare the relationship of LwHF and the SF-36 with recent HCU. For each of the components of $\mathrm{HCU}$, previous $\mathrm{HCU}$ was most strongly associated with recent utilization. Among the survey instruments, LwHF had the strongest association with recent hospitalization, and SF-36 scores had the strongest associations with ED visits and unscheduled medical visits. Average cost of care during one-year study period was $\$ 25,449$. Of baseline data, SF-36 PCS was most highly correlated with this cost $(r=-0.17, p<0.001)$.

\section{2/ LONG TERM QUALITY OF LIFE AFTER OPEN HEART SUR- GERY}

Djordje Jakovljevic, Zoran R. Potic, Bogoljub Mihajlovic, Marijela M. Potic, Ninoslav D. Radovanovic, Cardiovascular Surgery, Institute of CVD, Sremska Kamenica, Vojvodina, Yugoslavia

To estimate quality of life as an outcome of open heart surgery in long term follow-up (five years). For assessment of quality of life, we used diseases and treatment specific health related quality of life questionnaire and derived integral numeric QOL index QOLi-NS (zero value represents complete inability for any functioning and value 100 means perfect health). The prospective study involved 793 patients who were interviewed preoperatively. Until now, 165 of them completed all of six postoperative controls $(6,12,24,36,48$ and 60 months after the operation). Average QOLi-NS before the operation was 44.1. Six months after the QOLi-NS was 83.8, after one year 87.2, after 2 years 85.6 , after three years 84.4 , after four years 77.9 and 77.8 after five years. Statistically (and clinically) significant difference $(p<0.001)$ in QOL was observed comparing QOL before and after open heart surgery. The highest value of QOLi-NS was one year after the surgery. Quality of life of cardiac surgery patients (average QOLi-NS) increase significantly after the operation and remains very stable till fourth year. At that time it starts to decline, but still is significantly much better than before the treatment. 
53/ DETERMINANTS OF THE QUALITY OF LIFE OF PATIENTS WITH CONGENITAL HEART DISEASE

M. Rose, K. Köhler, Psychosomatic, F. Köhler, Cardiology, Charité Humboldt University, Berlin, Germany

What factors determine the health-related quality of life of patients with congenital heart defects? 111 patients (aged $33 \pm 12$ years) with different degrees of cardiac dysfunction were examined (NYHA 0: 2 I: 56, II: 38, III: 13 , IV: 2). All patients underwent a treadmill ergometry in order to determine their level of cardiopulmonary functioning (peak oxygen consumption: VO2max) and answered established questionnaires concerning their quality of life (WHOQOL-Bref), their cardiac complaints (Giessener Complaint Questionnaire GBB), their personality traits (Giessen Test GTS), and their social support (Social Support Questionnaire SOZU-k22). The data was analysed by means of a linear structural equations model. The mode proposed was valid, showing good indices of fit $\left(\mathrm{chi}^{2}=1.18 \mathrm{p}=.55, \mathrm{GFI}=.996\right)$. It could be shown that the level of cardiopulmonary functioning was most significant for the reporting of specific cardiac complaints $(b=-28)$ and for the physical component of the general, health-related quality of life $(b=32)$ although the former was also influenced by a depressed disposition $(b=$ .20) and the extent of social support experienced $(b=18)$. The objective findings, however, had virtually no individual significance for the psychological $(b=.09)$ and social domains $(b=-.02)$ of the HRQL. These HRQL domains are primarily influenced by depressive personality traits $(b=-26 /-$ $.16)$ and the social support experienced $(b=.51 / .51)$. All three predictors have individual significance for the global assessment of the quality of life $(b=.12 / .-15 / .28)$. In general, there is a small bond between subjective complaints and objective findings, suggesting that it is necessary to look for factors which influence the expression of specific complaints and other components of the quality of life construct. In the sample studied here, we found that a patient's depressed disposition and his/her reported experience of social support had added significance for the understanding of the subjective well-being of the patient.

\section{4/ PSYCHOMETRIC PROPERTIES OF THE MACNEW HEART DIS- EASE QUESTIONNAIRE IN PATIENTS AFTER MYOCARDIAL INFARC-} TION

Stefan Höfer, Medical Psychology and Psychotherapy, University of Innsbruck, Innsbruck, Austria, Werner Benzer, Working Group on CR \& SP, Austrian Society of Cardiology, Feldkirch, Austria; Dieter Brandt, Working Group on CR \& SP, Austrian Society of Cardiology, St. Radegund, Austria; Herbert Laimer, Working Group on CR \& SP, Austrian Society of Cardiology, Bad Tatzmannsdorf, Austria; Peter Schmidt, Working Group on CR \& SP, Austrian Society of Cardiology, Bad Schallerbach, Austria; Arthur Bernardo, Clinic of Cardiac and Psychosomatic Rehabilitation, Gais, Switzerland

Health-related quality of Life (HRQL) represents an important aspect within the assessment of medical treatment. Myocardial infarction (MI) is a major life event and impairs HRQL. After Ml important restrictions can be found in physical, emotional and social aspects. For the evaluation of the results of treatment and rehabilitation, appropriate instruments are required. The purpose of this study was the psychometric testing of the translated and linguistic-validated MacNew Heart Disease Questionnaire (MacNew) in MIpatients. 199 subjects (161 male, 38 female) with documented MI received the self-administered MacNew Heart Disease Questionnaire and the SF36 as the "gold standard" at the beginning of the rehabilitation period. The MacNew identifies disease specific physical, emotional, social and global HRQL. Test-retest reliability, Cronbachs alpha, convergent and discriminate validity coefficients were calculated. Item response rates were $>92 \%$ for the MacNew. Test-retest reliability [ 4 weeks] ranged between $r=0.72$. 0.87 . Cronbachs alpha between $0.89-0.93$. Convergent validity "with the SF-36 scales ranged for the questionnaire from $r=0.36-0.75(p<.001)$ Discriminate validity established by different grades of severity was high for all scales (ANOVA p<.01). Our results show, that the German MacNew is a reliable and valid instrument to measure $H R Q L$ in patients with $\mathrm{MI}$. It enables to discriminate between different grades of severity. Therefore it can also be considered to be sensitive. The high item response rate shows the acceptance of this questionnaire by the patients. The German MacNew can be recommended for evaluative purposes in patients with MI.

55/ THE FACTOR STRUCTURE OF THE SF-36 PHYSICAL AND MENTAL HEALTH SUMMARY MEASURES BY RACE/ETHNICITY AND LANGUAGE IN A US PATIENT POPULATION

Leo S. Morales, Department of Medicine, Steven P. Reise, Department of Psychology, Ron D. Hays, Department of Medicine, University of California, Los Angeles, CA

The growing diversity of the US population was made evident by the 2000 census. Until recently, few researchers have given attention to the equivalence of self-report measures among subgroups within the US population. In this study, we examine the factor structure of the SF-36, in four racial/ethnic subgroups: Whites, Blacks, Asians and Latinos. Subjects: A random sample of patients receiving medical care from a medical group association in United States was studied. A total of 7,093 questionnaires were returned for an overall response rate of $59 \%$. Approximately $80 \%$ of patients completing surveys selfidentified as White $(n=5,508), 10 \%$ self-identified as Latino $(n=713)$, $4 \%$ as Asian $(n=298)$, and $3 \%$ as Black $(n=210)$. Latinos were further divided among who those responded using a Spanish questionnaire $(n=181)$ and English questionnaire $(n=532)$. SF-36 Health Survey: The SF-36 is a generic HRQOL measure that consists of 36 items, yielding 8 scales (physical functioning, bodily pain, role limitations due to physical health problems, role limitations due to emotional problems, emotional well-being, social functioning, energy/fatigue, and general health perceptions) as well as summary physical and mental health measures. The 8 SF-36 scales were factor analyzed using a full information factor analysis program designed to handle ordinal data. Based on several number of factor criteria, two factors were extracted and rotated using the Promax rotation method. The general health scale score loaded primarily on the physical health factor among Whites, Blacks, and Latinos-English and primarily on the mental health composite among Asians and Latinos-Spanish. The role-emotional scale loaded primarily on the mental health factor among Whites, Asians and Latino-Spanish, and loaded approximately equally on both factors among Blacks and Latino-English. The energy/fatigue scale loaded primarily on mental health among Whites, Asians, Latinos-English and Latinos-Spanish and loaded approximately equally on the two factors among Blacks. Finally, the social functioning scale loaded primarily on physical health among Asians, Blacks, Latino-Spanish and primarily on the mental health factor among Latino-Spanish. The social functioning loaded approximately equally on the two factors among Whites. In general, the hypothesized 2 -factor model fit the responses to the SF-36 items very well for all racial/ethnic and language subgroups. However, the pattern of factor loadings varied significantly among the groups. These results have implications for scoring of summary scores from the SF-36. It is possible that distinct scoring protocols are needed for racial/ethnic and language subgroups represented within the United States.

\section{6/ VALIDATION OF THE WHOQOL-BREF IN A NORWEGIAN GEN-}

Berit R. Hanestad, Department of Public Health and Primary Health Care, University of Bergen, Bergen, Norway; Astrid K. Wahl, Faculty of Health, Buskerud College, Drammen, Nonway; Tone Rustoen, Faculty of Nursing, Oslo University College, Oslo, Norway; Anners Lerdal, Øistein Knudsen, Faculty of Health, Buskerud College, Drammen, Norway

WHOQOL-BREF is a quality of life questionnaire developed for cross-cultural adaptation. The purpose of this study was to validate the questionnaire in a general Norwegian population. 4000 randomly assigned people were sent the questionnaire by mail and 1867 returned it after one reminder. $48 \%$ were female and mean age was 45 years(SD 15.9 years). WHOQOL-BREF contains a total of 26 items in relation to the physical health (7items, alpha.83), psychological (6items,alpha.82), social relationships(3items,alpha.60) and environmental domain (8items,alpha.79) and two overall items measuring overall quality of life and general health. Domain score range from 4-20. Higher scores indicate better quality of life. Mean domain scores were 15.8 (SD 2.8), 15.2 (SD 2.4), 15.0 (SD 2.7) and 15.2 (SD 2.4) for the physical health, psychological, social relationships and environmental domain respectively. The results showed that women scored significantly lower within the social relationships domain $(p=.005)$ and higher within the psychological domain $(p<.000)$. Higher age was negatively associated with quality of life within the physical health domain $(p<.000)$ and positively associated within the environmental domain $(p<.000)$. Higher educational level was associated with increased quality of life within the physical health, psychological and environmental domain $(p<.000)$. The questionnaire discriminated in all domains between those who were reporting having a chronic illness compared to those who did not $(p<.000)$ and those living alone compared to those living together with either a spouse or children $(p<.000)$. Correlation analysis between overall quality of life and the different domains ranged from .52 to $.65(p<.000)$ for the social relationships and psychological domain respectively and between overall health and the different domains the correlation ranged from .34 to $.69(p<.000)$ for the social relationships and physical health domain respectively. Principal component factor analysis with varimax rotation resulted in a four factor solution explaining $53 \%$ of the variance. The rotated matrix partly confirmed the domain structure. In conclusion the questionnaire seemed to be a valid instrument discriminating different subgroups as expected. 
57/ HOW WELL DO HEALTH STATE VALUATION TECHNIQUES CROSS CULTURES? IMPLICATIONS OF CONCEPTIONS OF DEATH AMONGST THE KAMBA OF KENYA

Charles Nzioka, Sociology, University of Nairobi, Nairobi, Kenya; Julia A. Fox-Rushby, Public Health and Policy, London School of Hygiene and Tropical Medicine, London, UK; Patrick I. Muia, Anthropology, University of Nairobi, Nairobi, Kenya

Valuation techniques developed in the USA and Europe are being used around the world either to revalue an existing descriptive system or to weight newly developed questionnaires. Whilst the crosscultural applicability of HRQL measures is being questioned, there is little debate concerning valuation techniques other than whether values differ. It is important that valuation methods are acceptable and meaningful in a new context. As 'dead' is used in all valuation techniques to facilitate calculation of quality adjusted life years, we focussed on conceptions of death amongst the Kamba of Kenya - with a view to considering whether or not to adopt existing valuation techniques to value the KENQOL instrument. Our methods included: examining the ideas embedded within existing valuation tools; reviewing literature on the Kamba; participant observation during funeral and burial activities; and other qualitative methods including key informant interviews. Local literature highlighted a range of ceremonies associated with death, dying and the after life but also that talking about death was taboo. However, the literature was often old and descriptive - we gained little insight into why death was difficult to talk about in public. Early fieldwork results suggest that, once dead, a person is considered to remain living (and active in the lives of physically visible people) for around six generations before moving to the spirit world. We also found that one of the reasons for being unwilling to talk about death in public was because a major cause of death is believed to be witchcraft and that simply thinking about death can cause it to happen - which is divinable by witchdoctors. Alongside a range of other findings we consider the implications for using and interpreting health state valuations, for the methods currently used to evaluate the cross-cultural equivalence of health state valuation techniques and what other alternatives for valuation might include.

\section{8/ TRANSLATION AND DEVELOPMENT OF A DUTCH PROS-} TATE CANCER QUALITY OF LIFE QUESTIONNAIRE

Ida J. Korfage, Marie-Louise Essink-Bot, Joanna M. Madalinska, Department of Public Health, Wim J. Kirkels, Erasmus Medical Centre Rotterdam (EMCR), Rotterdam, The Netherlands; Mark S. Litwin, Department of Urology, UCLA, Los Angeles, CA; Harry J. de Koning, Department of Public Health, EMCR, Rotterdam, The Netherlands

We developed a Dutch adaptation of Litwin's UCLA Prostate Cancer Index (PCI) on urinary function (UF, 5 items), bowel function (BF, 4 items), and urinary and bowel bother (UB and BB, 1 item each). Independent forward and backward translation procedures were applied The $\mathrm{PCl}$ sexual function and bother scales were replaced by an existing Dutch questionnaire on sexual activities (SAM), as PCl could not differentiate among causes of sexual inactivity. After qualitative testing 369 prostate cancer patients (age 50-82) completed 1 baseline questionnaire and 2 questionnaires after primary treatment. Psychometric properties (feasibility, reliability, construct validity and responsiveness) of the new instrument were analyzed. Response rates were high (88 to $93 \%$ ). Ranges for missing values were $0.9-5.4 \%$ (UF), $1.1-7.2 \%$ (UB), $0.7-1.5 \%(B F), 0.4-2.5 \%(B B)$ and $1.6-3.2 \%$ (SAM) Cronbach's alphas were .79 for UF and .75 for BF. UF and UB, and $\mathrm{BF}$ and $\mathrm{BB}$ correlated significantly $(r=.58 ; P<.001$ and $r=.73 ; P<.001$ respectively). As expected SF-36 scales showed non-significant correlations with urinary and bowel modules. Correlations of Dutch UF and BF with SF-36 scales were lower than those found by Litwin. Effect sizes for pre-/post-prostatectomy UF and UB scores were 1.09 and .72 respectively. Effect sizes for pre-/post-radiotherapy BF and BB scores were .56 and .59 respectively. Differentiation among various causes for sexual inactivity requires further adjustment. The instrument is comprehensible to and well accepted by respondents, and proved to be a reliable and valid measure. Most respondents agreed to participate in the follow-up study. We conclude, that the Dutch version of the urinary and bowel modules is suitable for further use. We will adapt the sexual activities module by e.g. comparison with Rosen's International Index of Erectile Function.
59/ QUALITY OF LIFE ASSESSMENT IN HIV: MODIFYING INSTRUMENTS FOR A CHANGING DISEASE?

Waldo H. Belloso, Infectious Diseases Section, Hospital Italiano de Buenos Aires, Buenos Aires, Capital Federal, Argentina; Ezequiel Consiglio, CVAr Coordinating Center, Buenos Aires, Capital Federal, Argentina

Quality of life (QoL) assessment is an important tool for the evaluation of progression and intervention in HIV disease. Most QoL questionnaires have been developed prior to the widespread use of Highly Active Antiretroviral Therapy (HAART), and none of them has included south-american people in its generation process. The objective of this study was to identify most relevant QoL items in an argentine HIV population during the HAART era. Patients were evaluated for demographics and for medical, work, educational and HIV-related histories. QoL domain identification was performed by a semi-structured, self-assessment questionnaire, allowing for spontaneous responses and categorization (by 6 level Likert-scale) of $33 \mathrm{QoL}$ aspects obtained from other questionnaires and from ad-hoc medical focus groups. HIV relation of each answer was assessed by a paired question for each domain. Analysis of 198 patient profiles is presented. Male/Female ratio was 2,4:1, median current CD4+ cell count was 479,5 cells $/ \mathrm{mm} 3$, and $22 \%$ of the patients had history of AIDS. Traditional QoL domains such as Pain, Rest, Food and Activities of Daily Living were overall not considered as priorities. New and less usual domains as Medication, Quality of Health Care and Social Services, Fear to Discrimination, and Fear to Health Deterioration were identified as most relevant. Slight differences were seen by gender and risk factor. By Factor Analysis, most of these new areas were grouped together in a different principal component than the rest of the domains. Changes in HIV disease were reflected in the identification of new significant QoL issues not generally assessed by current available instruments. Factor analysis showed that this information was not redundant with the usually obtained in this setting. Updating content validity appears as an important mechanism in order to maintain the accuracy of QoL instruments in the context of the changing clinical scenario of HIV disease.

60/ NEGATIVE UTILITY SCORES: THEORETICAL AND PRACTICAL DIFFICULTIES WITH AN ESSENTIAL COMPONENT OF UTILITY INSTRUMENTS

Graeme Hawthorne, Centre for Health Program Evaluation, The University of Melbourne, West Heidelberg, Victoria, Australia; Jeffrey Richardson, Centre for Health Program Evaluation, Monash University, West Heidelberg, Victoria, Australia

Multi-attribute utility (MAU) instruments permit negative utilities; ie health states worse than death. The literature fails to adequately describe the theory, practical meaning and utility implications of these. This paper shows the assumptions are implausible and may lead to utility computation errors. There are two problems: (a) the treatment of negative utility values for poor health states, and (b) difficulties mapping MAU utility scores on the 'life-death scale'. For (a) there is no lower limit to negative utilities implied by time trade-off. A person not accepting any time in a health state worse than death implies a utility score of minus infinity. This and other large negative scores have no meaning and must be transformed. The arbitrary transformation rules used are unsatisfactory and threaten the validity of instrument scores. For (b) the disutility values of MAU models vary from 0.00 to 1.00 . These 'model utilities' must be rescaled to represent 'life-death' utilities where 1.00 is full health and 0.00 death. Since full health has a common value on both scales the transformation only requires correspondence between one other point on the two scales. The simplest point is the 'all worst' (the health state when each item is at its worst level). However, scaling this requires evaluation of a complex and unusual state. For example, the AQoL 'all worst' is a health state with 12 dimensions. However, the raison d'être of the MAU approach is avoidance of the need to carry out such complex tasks. The likelihood of error is increased when the all worst health state is used to establish the nexus, if that health state is close to death and respondents have never experienced a health state so bad that death was equally (un)desirable. Procedures to overcome these two problems are presented, along with illustrated examples. It is concluded both these problems are important and have received too little discussion in the literature. 
61/ MEASURING THE QUALITY OF LIFE AND QUALITY-ADJUSTED LIFE SPAN OF THE U.S. POPULATION, 1984-1994 Pennifer Erickson, Biobehavioral Health and Health Evaluation Sciences, Penn State University, State College, PA

This paper presents trends in the health of the US population from 1984-1994 using the Health and Activity Limitation Index (HALex) and the Healthy People 2000 Years of Healthy Life (HP2000YHL) measure, a type of quality adjusted life year. Data are from the U.S National Health Interview Survey and the Vital Health Statistics System. Trends in HALex scores, scores for its two domains, and years of healthy life for the total population, as well as by gender and racial background, are examined across the 11 year period. Years of healthy life rose from 63.4 in 1984 to 64.5 years in 1990 and declined to 64.0 in 1994. Over the 1984-1994 interval, life expectancy at birth rose steadily from 74.7 to 75.7 years. Years of healthy life are lower for males than for females, a difference that can be attributed to the less favorable mortality experience, as reflected in life expectancy at birth, of males. While years of healthy life generally increased for the White population, those for the African-American population remained approximately constant over the 1984-1994 interval, suggesting that this population experienced no gain in either mortality or quality of life. HALex scores as well as those for the domains of perceived health and activity limitation followed similar patterns over the interval, with some unexpected differences between the majority and minority population. This analysis indicates that population measures of quality of life can be sensitive to change over time, a finding that has implications for their use as normative data.

62/ WHOSE VALUES COUNT? A CRITIQUE OF DISABILITY ADJUSTED LIFE YEARS (DALYS)

Julia A. Fox-Rushby, Public Health and Policy, London School of Hygiene and Tropical Medicine, London, England

Disability-adjusted life years were launched in the World Development Report 1993 to estimate the burden of disease, and as an outcome measure for use in cost-effectiveness analysis. Combined with the health sector priorities review in low and middle-income countries, it has become an influential tool in the hands of international and national decision-makers. This paper examines the beliefs and preferences embedded within the DALY to understand whose values count and how. The critique is based on a systematic search of the literature that included: a search of eleven electronic data bases and the world-wide web, as well as contact of researchers known to be working in the field and includes the construction, use and critiques of DALYs in the published literature. Results highlight the narrow perspective taken in judging the impact of disease. Its' orientation to biomedical concepts of disease and disability have a major impact on the types of interventions considered to alleviate the impact of disease. Restriction of valuation exercises to health professionals and refusal to consider context in the experience of illness suggest the interests of biomedicine and the medical profession are served best. It would appear that a measure designed to serve global public health, has sidelined the global public in the identification, measurement and valuation of the impact of disease on life to date. The paper concludes by considering the impact embedded values have on the choice of interventions designed to improve 'health.'
63/ COMPARING COMMUNITY-PREFERENCE BASED AND DIRECT STANDARD GAMBLE UTILITY SCORES: EVIDENCE FROM ELECTIVE HIP ARTHROPLASTY

David Feeny, Chris Blanchard, Institute of Health Economics, Edmonton, AB, Canada; Jeffrey Mahon, Department of Epidemiology and Biostatistics, Robert Bourne, Cecil Rorabeck, Department of Surgery, Larry Stitt, Susan Webster-Bogaert, Department of Epidemiology and Biostatistics, University of Western Ontario, London, ON, Canada

The purpose is to assess agreement between standard gamble (SG) utility scores for current health and utility scores from the Health Utilities Index Mark 2 (HUI2) and Mark 3 (HUI3)systems for the same individuals based on self assessments made at the same points in time. HUI scores are based on community preferences. SG scores reflect the individual's assessment and valuation of current health. SG scores for three hypothetical marker states and the patient's current health state were obtained at multiple points in time. Patients were assessed serially throughout the process of waiting to see a surgeon, waiting for surgery, and recovery after total hip arthroplasty (THA). Patients also completed specific and generic health-related quality of life instruments, including HUI2 and HUI3. Descriptive statistics will be presented. Agreement is assessed using the intra-class correlation coefficient (ICC). The mean SG, HUI2, and HUI3 (SD) scores at assessment \#1(A1) are $0.62(0.31), 0.62(0.19)$, and 0.52 $(0.21) ; n=103$. At $A 2$ the means are $0.67(0.30), 0.68(0.30)$, and $0.58(0.22) ; n=84$. At $A 3$ results are $0.65(0.28), 0.70(0.19)$, and $0.57(0.24) ; n=35$. At $A 4$ results are $0.75(0.31), 0.70(0.19)$, and $0.61(0.20) ; n=12$. ICCs at the group level range from 0.11 to 0.87 . At the mean level for the group, HUI2 and SG scores match closely. At the individual level, however, agreement is poor. Multi-attribute utility scores such as HUI appear to be reasonable for group level analyses but are a poor substitute for directly measured utility scores at the individual level.

64/ PATIENT PREFERENCE AND COMPREHENSION OF UTILITY INSTRUMENTS

Deborah G. Dobrez, Elizabeth Calhoun, Institute for Health Services Research \& Policy Studies, Northwestern University, Chicago, IL

There is no consensus regarding the best utility measurement method. However, it appears that instruments that conform more closely to expected utility theory are also more difficult to understand. Our study compares subject comprehension and preference for 3 utility instruments. Sixty-one cancer patients were interviewed using standard gamble (SG), time trade-off (TTO) and visual analog scale (VAS) instruments. Subjects were queried about question difficulty and relevance, and whether the questions were too personal. Subjects were randomized to 1 of 2 comprehension tests: an assessment of two hypothetical health states and a quiz. For the first test, the health state "being blind," was expected to have a lower quality of life than the health state "requiring glasses." Reverse rankings were considered logically inconsistent and suggestive of comprehension difficulties. For the second test, a quiz described responses to the SG and TTO for 2 hypothetical respondents. Subjects determined which response indicated a higher quality of life. Incorrect answers were suggestive of comprehension difficulties. Nearly all subjects rated each questionnaire as easy to complete (59-SG, 56-TTO and 56-VAS). Most felt that the questions were at least somewhat important to their quality of life (54-SG, 49-TTO, and 51-VAS), and were not too personal (59-SG, 60-TTO, and 58-VAS). Thirty-one subjects completed the SG and TTO for 2 hypothetical health states. Requiring glasses was usually assigned a score equal to 1 (65\%-SG and $81 \%$-TTO), and was always rated to be at least as good as being blind. Four of 30 subjects completing the quiz gave incorrect answers (2 each for the SG and TTO). Our study demonstrates the acceptability of utility questions to patients. Comprehension is a concern; although most subjects understand the questions, a minority should be expected to have difficulty. The use of comprehension checks may improve the reliability of utility data by identifying subjects who may have misunderstood the utility questions. 
65/ THE COMPLEX RELATIONSHIP BETWEEN COGNITIVE IMPAIRMENT AND HEALTH-RELATED QUALITY OF LIFE AFTER CLOSED HEAD INJURY

Bernd-Otto Hütter, Joachim-Michael Gilsbach, Department of Neurosurgery, Medical Faculty of the RWTH Aachen, Aachen, Germany

The assessment of health-related quality of life (HRQOL) in braindamaged patients becomes increasingly important in clinical research and practice. However, not much is known about the relationship between measures of HRQOL and the performance in neuropsychological tests. A series of 87 patients with an age mean of 37,8 years (25 females and 62 males) were studied one to two years after $\mathrm{CH}$ by means of the Aachen Life Quality Inventory (ALQI), a modified German version of the SIP and cognitive tests including attention, figural and verbal short- and long-term memory, aphasia, concentration and spatial cognition. $13 \%$ of the patients had sustained a severe, $28 \%$ a moderate and $59 \%$ a mild $\mathrm{CHI}$. Proxy ratings could be obtained of 71 life partners. In the case of self-rated HRQOL, the statistically significant correlations were modest, ranging between $r=0,26(p<0,01)$ and $r=0,46(p<0,001)$. Functions of attention and memory exhibited the closest relationship to subjective HRQOL. The associations between the cognitive functional level and HRQOL as rated by the proxies were consistently weaker. Analyses of variance showed significant ( $p<0,05$, respectively) effects of the degree of neuropsychological impairment. Multivariate analyses revealed figural short- and long-term memory and the simple reaction time as the most important predictors of overall HRQOL sharing $27 \%$ of common variance while the psychosocial aspect of $\mathrm{HRQOL}$ was explained by the capacity of figural short- and long-term memory and finally the simple reaction time sharing $24 \%$ of common variance. The associations between HRQOL and neuropsychological test performance are at best modest. The reasons for this finding remain unclear and should be investigated further. The results underline that cognitive testing alone is not sufficient to judge the degree of impairment in daily life and to evaluate the efficacy of rehabilitation in patients with brain damage.

\section{6/ VALIDATION OF THE EUROQOL STROKE UTILITY MEASURE (EQ-SUM)}

Lise Poissant, Nancy E Mayo, Sharon Wood-Dauphinee, Ann Clarke, Division of Clinical Epidemiology, McGill University, Montreal, QC, Canada

The EQ-SUM is the first stroke utility measure to describe health status post-stroke. The purpose of this study was to assess the validity of the EQ-SUM in a stroke population. Our sample of subjects came from a RCT looking at the effectiveness of teaming a patient's personal physician with a stroke case manager. Subjects were interviewed at their home at 6 weeks and 6 months on various measures of activity and HRQoL. As no utility function is yet available for the EQ-SUM, an individual's stroke health status was determined according to their classification on each of the 10 domains, ordered according to their preference weights. Speech was identified as the first domain on the EQ-SUM health state and memory as the second. The ranking of stroke health state showed moderate correlations, 6 weeks poststroke, with 6 of the 8 SF-36 sub-scales (Pearson's $r$ between -0.452 to $-0.754, p<0.0001$ ). Subjects were then grouped on the basis of the ranking of their stroke health status (top-middle-bottom tertile). Subjects in the top tertile had significantly higher scores on all measured variables. Our results provide evidence of convergent validity of the EQ-SUM with the SF-36. The EQ-SUM was particularly useful for identifying people with good stroke health status reducing the problem of ceiling effects observed with other stroke instruments. Information on the optimal time to evaluate stroke health status was also gathered because of changes occurring between 6 weeks and 6 months.

\section{7/ CONTRIBUTIONS OF HEALTH-RELATED QUALITY OF LIFE (HRQL) TO UNDERSTANDING HEALTH CARE COSTS POST STROKE}

Sharon L. Wood-Dauphinee, School of Physical and Occupational Therapy, McGill University, Montreal, QC, Canada, Josephine Teng, Nancy E. Mayo, Clinical Epidemiology, McGill University Health Center, Montreal, QC, Canada

The value of HRQL as an endpoint is that it encompasses physical, psychological, social, and role domains as well as a general sense of well-being. Yet, $\mathrm{HRQL}$ is rarely selected as the primary outcome in studies evaluating the impact of treatments or prognostic factors on the consequences of diseases. The basis for choosing HRQL as an important endpoint would be strengthened in the eyes of researchers and policy makers by empirical data linking HRQL domains to health care costs. This link would be especially relevant for chronic diseases where costs and consequences continue far beyond the onset of care. Costs are generated for a variety of reasons arising from both planned and unplanned health care encounters. The purpose of this study was to examine the link between HRQL domains and costs in persons with stroke, taking into consideration age, sex and stroke severity. The underlying hypothesis is that better HRQL, and its various domains, would be associated over time, with lower health care costs related primarily to unplanned to physician visits and hospitalizations. The data for this study came from a randomized trial of the effectiveness of home care and rehabilitation in the sub-acute phase of stroke. A total of 114 persons participated in the study. Measures of severity of stroke and function were made at baseline ( 9 days) and 1 and 3 months later; measures of community reintegration and HRQL components were made at the latter time intervals. Costs associated with in-patient care were obtained from provincial sources and direct costs for fee-for-service acts were generated using administrative data. The only component of HRQL associated with combined costs from physician and emergency room visits and re-admissions was mental health. A 10 point decrease in the mental health index of the SF-36 was associated with a $21 \%(95 \% \mathrm{Cl}: 11 \%-29 \%)$ increase in total costs Higher costs from emergency room visits and re-admissions were also predicted by poorer daily functioning and social reintegration: a one standard deviation change was associated with a $40 \%$ change in costs. The results of this study indicate that components of HRQL are indeed cost-drivers.

\section{8/ THE MS SYMPTOM AND IMPACT DIARY: A NEW INSTRU-} MENT TO MEASURE THE DAY TO DAY IMPACT OF MS

Joanne Greenhalgh, Nuffield Institute for Health, University of Leeds, Leeds, West Yorkshire, UK; Helen Ford, Neurology, St. James's University Hospital, Leeds, West Yorkshire, UK; Amanda Georgiou, Keith Hurst, Nuffield Institute for Health, University of Leeds, Leeds, West Yorkshire, UK; Ann Jacoby, Department of Primary Care, Whelan Building, Liverpool, Merseyside, UK; Andrew F. Long, Health Care Practice R\&D Unit, University of Salford, Salford, Greater Manchester, UK; Carolyn A. Young, Walton Centre for Neurology and Neurosurgery, Lower Lane, Liverpool, Merseyside, UK

For many people with MS, different symptoms and their impact vary from one day to the next. Currently available, single point health status instruments are not designed to capture the fluctuating impact of these various symptoms. This study aimed to further develop the MS Symptom and Impact Diary (MS SID) developed by Parkin et al (1998) and assess its psychometric properties. The study was conducted in three phases. Phase 1: two focus groups $(n=16)$ and in-depth interviews $(n=10)$ were used to establish the impact of MS from the patient's perspective and to further develop the original MS SID. Phase 2: the adapted MS SID was pretested using cognitive interviews ( $n=$ 9 ), and a 2 week pilot study ( $n=14)$ assessed the new instrument's potential feasibility and personal utility. Phase 3 : a three month longitudinal study $(n=77)$ in two centres assessed the psychometric properties of the MS SID in comparison with currently available single point measures of health status (MSQol-54), quality of life (Leeds MSQoL), disability (Barthel Index) and handicap (London Handicap Scale and Reintegration to Normal Living Index). Phase 1 revealed that MS prevented or limited people from doing valued activities and that this impact often varied from day to day, confirming that a diary would be an appropriate method of assessing the impact of MS. Phase 2 showed that the MS SID was moderately easy to complete and $70 \%$ felt the diary was personally useful or would be useful to others. Initial psychometric analyses from Phase 3 suggest that the MS SID is able to distinguish between people with progressive and relapsing remitting MS in terms of the duration of symptoms concerned with mobility, the severity of their impact on daily activities and the degree to which MS had prevented people from doing what they wanted to do on a daily basis. Future analyses will further assess the construct validity of the diary through the relationships between symptom and impact patterns over time and (1) clinical and demographic characteristics of participants and (2) single point measures of disability, handicap, health status and quality of life. 
69/ PDQ-39SI, PDQ-8SI: TOWARDS A FRENCH VERSION OF THESE TWO SUMMARY INDEXES IN PARKINSON'S DISEASE Christophe Sapin, Public Health Department, Medical School Marseille, France; Marc Ziegler, Neurology Department, Bellan Hospital, Paris, France; Mohammed El Khammar, Public Health Department, Medical School, Marseille, France; Jean-Luc Thiault, Lilly France Laboratories, St. Cloud, France; Pascal Auquier, Public Health Department, Medical School, Marseille, France

The PDQ-39 is a self-administered HRQL instrument which explores 8 dimensions. Its cultural adaptation in French has been established, but its summary indexes, the PDQ-39SI and the PDQ-8SI, have not been validated yet. The PDQ-39SI was defined by the mean of the 8 dimensions explored by the 39 items. The PDQ-8SI was defined by the mean of the 8 items the most correlated with their own dimension. These scores ranged from 0 (best HRQL) to 100 (worst HRQL). Using the data of the validation of the French version, we decided to explore the psychometric properties of the French PDQ-39SI and PDQ-8SI. The second-order factor analysis confirmed the relevance of such summary indexes in French. They showed a good external validity, like the original version: high levels of correlations with the SF-36 dimensions scores (range: $[-0.36 ;-0.63]$ ) and the Unified Parkinson's Disease Rating Scale (UPDRS) scores (range: $[0.31 ; 0.44])$. These indexes were statistically linked to the Hoehn \& Yahr classification: the worst the patients were, the greater PDQ-39SI and PDQ-8SI were. The reliability of the French PDQ-39SI is better than the original one: the internal consistency assessed by Cronbach's alpha is good ( 0.94 versus 0.89 for the English version), and the results of the stability over time (test-retest correlations) led us to conclude that the French PDQ-39SI and PDQ-8SI are reliable measures. The sensitivity was clearly demonstrated: the PDQ-39SI scores of patients whose health status was improved decreased from $35.1 \pm 12.3$ (mean $\pm S D$ ) to $23.2 \pm 12.7$; the score of patients whose health status was stable or impaired decreased from $38.5 \pm 15.2$ to $36.3 \pm 14.2$. Even if this work needs further investigations, the French versions of the PDQ-39 SI and PDQ-8SI seem to be valid, reliable and sensitive. These easy-to-use tools can help the neurologists' community to add the HRQL evaluation in the medical decision process, in terms of treatments' compliance or observance.

70/ DEFINING SYMPTOM AND FUNCTIONAL HEALTH "RESPONSE" IN CLINICAL TRIAL-BASED HEALTH-RELATED QUALITY OF LIFE (HRQL) ASSESSMENT IN ONCOLOGY

Martin J. Muller, Psychosocial Research and Epidemiology, The Netherlands Cancer Institute, Amsterdam, The Netherlands; Johannes A. Langendijk, Radiation Oncology, Vrije University Hospital, Amsterdam, The Netherlands; Neil K. Aaronson, Psychosocial Research and Epidemiology, The Netherlands Cancer Institute, Amsterdam, The Netherlands

While mean scores are often sufficient for demonstrating betweengroup differences and within-group changes over time in HRQL, they can sometimes mask heterogeneity in the patterns of responses to serial HRQL assessments. In this paper, we present a complementary approach that allows one to define a HRQL "response," analogous to the clinical response criteria typically employed in cancer clinical trials.Drawing on previous work by Stephens et al. (Br J Cancer 1999;79:538-544) and Osoba et al. (J Clin Oncol 1998;16:139-144), we constructed a classification system for defining both symptom response and functional health response based on serial administrations of the EORTC QLQ-C30. Symptom response (or palliation) was defined as: (1) patients with a moderate or severe symptom at baseline for whom that symptom was mild or absent on 2 successive follow-up assessments (improvement); (2) patients with a mild symptom at baseline for whom that symptom was absent on 2 successive followup assessments (improvement); (3) those with a mild symptom at baseline who remained stable over 2 successive follow-up assessments (control); and (4) patients without a given symptom at baseline who did not develop that symptom during 2 successive follow-up assessments (prevention). A similar algorithm was employed for defining an HRQL response based on the functional scales of the QLQC30. Patients who dropped-out before study completion due to death or severe illness were also incorporated in the evaluation. Data from a prospective, randomized trial comparing external radiation with external radiation plus endobronchial brachytherapy in non-operable non-small cell lung cancer are used to illustrate the advantages and limitations of this approach to analysing and interpreting serial HRQL data.
71/ AGREEMENT BETWEEN PROXY ASSESSMENT AND PATIENT SELF-ASSESSMENT IN STROKE: A COMPARISON OF GENERIC HEALTH STATUS MEASURES

Simon Pickard, Jeffrey Johnson, David Feeny, KC Carriere, Institute of Health Economics (IHE), Edmonton, AB, Canada; Ashfaq Shuaib, University of Alberta, Edmonton, AB, Canada

The use of proxies to substitute for missing patient self-assessment of health status is an alternative to more rigorous statistical methods for incomplete data. The purpose of this investigation was to examine the extent of agreement between patient self-assessment and proxy assessment for cross-sectional and change scores of selected generic health status measures in stroke. Patient-proxy pairs $(n=97)$ were administered the SF-36, the EQ-5D, and the Health Utilities Index Mark 2 and $3(\mathrm{HUI} / 3)$ at baseline (within 2 weeks of stroke), 1 month, 3 months and 6 months following stroke. Agreement was evaluated using the intraclass correlation coefficient (ICC) on the summary scores of each measure: the HUI2/3 multi-attribute utility scores (HUI2/ 3 MAUS), EQ-5D visual analog scale (EQ-VAS), EQ-5D index score, and the SF-36 mental and physical composite summary scores (MCS36, PCS-36)). Cross-sectional agreement at baseline was strongest for the HUI2 MAUS (ICC=0.74) and poorest for the $E Q-V A S$ $(I C C=0.40)$ and MCS-36 $(I C C=0.44)$; agreement was generally good $($ ICC >0.70) after baseline except for the MCS-36 and EQ-VAS (ICCs 0.61 to 0.69 ). Change score agreement was strongest between baseline and 1 month (ICCs ranged from 0.32 to 0.70 ) then declined, with weak agreement between patient and proxy change scores for months 3 to 6 (ICCs ranged from 0.08 to 0.43 ). The findings indicate that among summary scores studied, proxy respondents may reliably substitute for patient self-assessment at the group level using the HUI2/3 MAUS, EQ-5D index-based scores, and the PCS-36 at a point in time, especially when more than month has elapsed since the stroke. However, in considering the substitutibility of proxy for patient selfassessments in clinical trials, the use of proxy-based change scores may be limited to time periods when the magnitude of change is expected to be relatively large (as an effect size), such as between baseline and month 1 in stroke patients.

\section{2/ THE IMPORTANCE OF CONSIDERING MISSING DATA IN CO- HORT STUDIES OF STROKE OUTCOME}

Nancy E. Mayo, Sharon L. Wood-Dauphinee, Clinical Epidemiology, McGill University Health Center, Montreal, QC, Canada

Traditionally, studies that follow individuals over time tend to focus the analysis on those who remain in the study to the end. More recently, there has been an emphasis on dealing with those persons who drop out early and create the problem of data that are "not missing at random". The objective of this study is to examine similarities and differences among subjects who remain in a study for the duration or drop out for a variety of reasons, and to demonstrate the impact of dropouts on estimates of longitudinal change. The data for this study came from a two-year outcome study of persons with acute stroke who survived to return to the community. A total of 604 persons had at least one follow-up interview but only 318 (53\%) completed the study as designed: $44(7 \%)$ moved away from the study area; $125(21 \%)$ refused to continue in the study; $51(8 \%)$ were admitted to long-term care; and $66(11 \%)$ died during the follow-up period. In general, those who left the study did so within one year. Right from entry into the cohort, those who completed the study scored higher on all measures of function, re-integration and quality of life and consistently showed improvement over time. To estimate longitudinal change, the data were stratified according to completion status and analyzed using mixed effects, repeated measures models. When the data were considered without stratification, the estimated slope was positive; with stratification the lower starting point of the dropouts was observed. Grouping all subjects together served to bring the left tail down, creating the artificial positive slope. With stratification the slopes were flat showing neither improvement nor deterioration in either group. These data have ramifications for the design of prospective studies. Extra effort to maintain participation should be made for persons with "low" baseline scores and for those who show deterioration of mental health over time, as these groups were more likely to drop out. In addition, preliminary analysis demonstrating the magnitude of the difference between those who leave and those who complete should be undertaken before finalizing the analytic strategy. 
73/ INCORPORATING DEATH INTO THE STATISTICAL ANALYSIS OF LONGITUDINAL HEALTH STATUS DATA

Laura L. Johnson, Department of Biostatistics, University of Washington, Seattle, WA

Information on health status is collected in many clinical trials and observational studies. This information usually is longitudinal, and in studies involving the elderly or chronic diseases a number of study participants may die while the study is still collecting information. These data are not 'missing' in the usual sense, because of compliance problems, and to analyze it in such a manner often will not make sense. It may produce biased results to exclude patients who die from the analysis. What is needed is a method where health status is the outcome which includes patients until the time of their deaths, and allows the deaths to affect the statistical analysis. This methodological issue has been discussed in the statistical literature using several different ways of incorporating death into the analysis of longitudinal data. We compare the methods for analysis of longitudinal data that include death currently found in the literature and modify a generalized estimating equations method to include death. Examples using SF-36 data from an observational trial and a randomized clinical trial format will be used to illustrate the differences in statistical accuracy and ease of interpretation between the methods. We believe this work has the potential to provide important information to investigators on ways to analyze trial data and in turn to provide better information on changes in health status to clinicians and patients.

74/ IMPACT OF MISSING HRQOL DATA. IS IT REALLY MISSING? Diane L. Fairclough, Center for Research Methodology and Biometry, AMC Cancer Research Center, Denver, CO

Missing data presents one of the most significant challenges to the interpretations of the health-related quality of life (HRQOL) of patients on a clinical trial. From the analysts viewpoint, the definition of what data is actually 'missing', depends on the research objectives and the population relevant to the intended inferences. For example, assessments that are not obtained after a subject discontinues therapy are informative (missing) if the objective is pragmatic and we are interested in the patient's HRQOL regardless of whether they responded to treatment, switched therapy, etc. Alternatively, the same assessments may not be informative (not missing) if the objective is exploratory and we are interested in the expected HRQOL of patients remaining on therapy. The choice among various analytic methods will be determined by the research objectives and will affect the interpretation of the results. The impact of design and analysis on the interpretation of $\mathrm{HRQOL}$ outcomes is illustrated in a clinical trial of cancer patients. Limiting analyses to patients with complete data limits inference to the small proportion of subjects who respond and remain on therapy without side effects. Repeated univariate analyses describe the HRQOL in different populations of patients over time. Maximum likelihood estimation of all available data assumes that patient discontinuing will follow a similar trajectory as continuing patients with the same history. Selection and pattern mixture models will require restrictive assumptions that may reflect more realistic changes in HRQOL in subjects with significant morbidity. Examination of graphical displays of estimates across patterns of dropout will facilitate the communication of the se concepts and the interpretation of overall results.

75/ HRQOL OUTCOMES AMONG MEN MANAGED WITHOUT SURGERY OR RADIOTHERAPY FOR LOCALIZED PROSTATE CANCER: RESULTS FROM THE PROSTATE CANCER OUTCOMES STUDY

Arnold L. Potosky, Bryce B. Reeve, Applied Research Program, Limin $X$. Clegg, Surveillance Research Program, National Cancer Institute, Bethesda, MD; Richard M. Hoffman, Medical Service, Veterans Affairs Medical Center, Albuquerque, NM; Robert A. Stephenson, Division of Urology, University of Utah, Salt Lake City, UT; Peter C. Albertsen, Division of Urology, University of Connecticut Health Center, Farmington, CT; Frank D. Gilliland, Preventive Medicine, USC Keck School of Medicine, Los Angeles, CA; Janet L. Stanford, Division of Public Health Sciences, Fred Hutchinson Cancer Research Center, Seattle, WA

The purpose of this study was to assess practice patterns and healthrelated quality of life outcomes among a US population-based cohor of men who received neither surgery nor radiotherapy for diagnosed clinically localized prostate cancer. Subjects included men $(n=661)$ newly diagnosed with clinically localized prostate cancer from 6 geographic regions in 1994-95 who participated in the Prostate Cancer Outcomes Study (PCOS) and who received neither surgery nor radiotherapy with the intent to cure within 12 months of initial diagnosis. Treatment and outcome comparisons were statistically adjusted for patient sociodemographic factors, clinical characteristics, comorbidity, and baseline sexual function. Approximately one-third of the study patients received systemic androgen deprivation $(A D)$ therapy and the remaining patients received no therapy. Two-thirds of the patients receiving $A D$ therapy had poor prognostic risk factors, such as baseline Gleason grade greater than 6 or PSA values over $20 \mathrm{ng} / \mathrm{Ml}$. Among men who were potent before diagnosis, $80 \%$ of the AD therapy patients compared with $30 \%$ of the untreated patients reported being impotent one year later. Similar declines were observed with respect to sexual libido and frequency of sexual activity. $A D$ therapy patients reported significantly more physical discomfort due to prostate cancer one year after diagnosis, but were more likely to be satisfied with their decision about treatment than the untreated men. AD therapy patients also experienced a greater decline in vitality and physical function, but these differences did not remain statistically significant after adjustment for potentially confounding factors. Primary AD therapy among men with newly diagnosed clinically localized prostate cancer is very common, occuring in nearly 20,000 men in the US each year despite limited evidence concerning its efficacy. Men with prostate cancer considering primary $A D$ therapy as an alternative to other treatment strategies should be informed that sexual function and overall physical function and vitality are likely to be affected by this treatment.

76/ IS MORE BETTER? QUALITY OF LIFE (QOL) RESULTS OF A CANADIAN PALLIATIVE RADIOTHERAPY TRIAL IN PATIENTS WITH UNRESECTABLE LUNG CANCER (NCIC CTG SC.15)

Andrea Bezjak, DRO, Princess Margaret Hospital, Toronto, ON, Canada; Michael Brundage, Department of Radiation Oncology, Kingston Regional Cancer Center, Kingston, ON, Canada; Peter F. Dixon, Department of Radiation Oncology, Kingston Regional Cancer Center, Kingston, ON, Canada; Michael Palmer, Joseph Pater, Dongsheng Tu, Central Office, NCIC Clinical Trials Group, Kingston, ON, Canada; Clive Grafton, Department of Radiation Oncology, BC Cancer Agency, Vancouver, BC, Canada; Paul Blood, Department of Radiation Oncology, Vancouver Island Cancer Centre, Vancouver BC, Canada; Carson Leong, Department of Radiation Oncology, Surrey Memorial Hospital, Surrey, BC, Canada; Liam A. Mulroy, Department of Radiation Oncology, Nova Scotia Cancer Centre, Halifax NS, Canada; Jim Wright, Department of Radiation Oncology, Hamilton Regional Cancer Center, Hamilton, ON, Canada

Palliative radiation (RT) is a frequently employed treatment for patients (pts) with lung cancer (NSCLC). Previous studies have demonstrated palliative efficacy of single or few fractions of thoracic RT. We wished to compare single fraction 10Gy RT (F1) to the most frequently employed schedule in Canada, 20Gy/5fractions (F5). Pts with thoracic symptoms due to NSCLC, deemed unsuitable for radical treatment, were randomized to F1 or F5. Endpoints were: symptom palliation (with a patient-completed daily diary card), QOL (with the EORTC QLQ-C30 and the Lung Cancer Symptom Scale, LCSS), treatment toxicity and overall survival. 230 pts were randomized in 13 cancer centers across Canada. The treatment arms were balanced with respect to age (median 70$)$, gender $(63 \%$ male), performance status (most ECOG 1 or 2 ), degree of weight loss ( $>10 \%$ in $38 \%$ ), extent of disease (24\% had known metastases) and index symptom (the symptom considered as the main indication for palliative RT). At 1 month following RT, there was no difference in reported symptom control (the primary endpoint) between arms. EORTC QLQ-C30 change scores comparing 5 weeks post RT to baseline showed that F5 pts had greater improvement in dyspnea $(-4.5$ in F5 vs +7.14 in F1 $p=0.027)$, and pain $(-7.8$ vs $+3.7, p=0.07)$. Changes in $Q O L$ domain scores tended to favour the fractionated arm. Similarly, change scores on LCSS revealed greater improvement in symptoms related to lung cancer $(p=0.037)$, pain $(p=0.017)$ and the ability to carry out normal activities $(p=0.047)$ on the F5 arm. F5 pts survived significantly longer (median survival $6.0 \mathrm{mo}, 95 \% \mathrm{Cl} 5.2-7.9 \mathrm{mo}$ ) than those given a single fr (median $4.2 \mathrm{mo}, 95 \% \mathrm{Cl} 3.7-5.2 \mathrm{mo}$ ); $\mathrm{p} 0.014$. Treatmentrelated toxicity in the two arms was comparable. All endpoints favoured the fractioned RT arm. QOL analysis was particularly helpful in comparing the two RT schedules. 
77/ SYMPTOMATOLOGY OF CANCER PATIENTS IN PALLIATIVE CARE: CONTENT VALIDATION OF SELF-ASSESSMENT QUESTIONNAIRES AGAINST MEDICAL RECORDS

Annette S. Stromgren, Mogens Groenvold, Lise Pedersen, Alf K. Olsen, Department of Palliative Medicine, Per Sjogren, Department of Palliative Medicine, Bispebjerg Hospital, Copenhagen, Denmark

The aim of the study was to elucidate which symptoms or problems to measure in the evaluation of palliative care. We assessed the content validity of selected patient self-assessment questionnaires used to evaluate palliative care: the 3rd version of the EORTC Quality of Life Questionnaire (EORTC QLQ-C30) and the 1991 version of Edmonton Symptom Assessment System (ESAS). The content of these questionnaires was compared against the symptoms and problems noted in the medical records of 171 consecutive cancer patients on their first admission to a department of palliative medicine. Of 63 different symptoms listed in the medical records, only 24 were covered in the questionnaires. However, the most prevalent symptoms/problems were covered by the questionnaires; the EORTC QLQ-C30 covered 10 whereas the ESAS covered 5 of the 10 most frequent problems. Researchers selecting instruments for evaluating palliative care may use the present study and other reviews to examine to what degree a given selection of instruments cover the important symptoms.

Table/ Prevalence of Palliative Needs in 171 Cancer Patients
\begin{tabular}{|l|l|l|l|l|}
\hline Symptom/problem & $n$ & ESAS & EORTC QLQ-C 30 \\
\hline Pain & 157 & $\times$ & $\times$ \\
\hline Fatigue & 74 & & $\times$ \\
\hline Loss of appetite & 62 & $\times$ & $\times$ \\
\hline Nausea & 60 & $\times$ & $\times$ \\
\hline Depression/sadness & 55 & $\times$ & $\times$ \\
\hline Poor physical function & 51 & & $\times$ \\
\hline Concentration or memory & 49 & & $\times$ \\
\hline Constipation & 41 & & $\times$ \\
\hline Breathlessness & 32 & $\times$ & $\times$ \\
\hline Vomiting & 28 & & $\times$ \\
\hline
\end{tabular}

78/ BRIEF ASSESSMENT OF PRIORITY SYMPTOMS IN ADVANCED CANCER

S. Yount, Evanston Northwestern Healthcare (ENH), Northwestern University (NU), Evanston, IL; D. Cella, ENH and NU, Evanston, IL; D. Paul, R. Winn, National Comprehensive Cancer Network (NCCN), Rockledge, PA; C.H. Chang, ENH and NU, Evanston, IL, J. Weeks, Dana-Farber Cancer Institute, Boston, MA; D. Banik, ENH, Evanston, IL; N. Heffernan, Memorial Sloan-Kettering Cancer Center, New York, NY; K. Webster, ENH, Evanston, IL; R. Van Gool, Janssen Research Foundation, Beerse, Belgium; L. Odom, ENH, Evanston, IL

Increasing interest in health-related quality of life (HRQL) assessment among clinicians has led to a need to develop a bridge between disease symptoms that are primary targets of therapy and HRQL. Clinicians treating advanced cancer have most familiarity with the symptom component of HRQL assessment, resulting in interest in a more symptom-focused approach to assessment, whereby disease symptoms measured by multidimensional questionnaires are aggregated in a clinically relevant and psychometrically acceptable manner. In collaboration with the National Comprehensive Cancer Network (NCCN) and using symptoms from the Functional Assessment of Cancer Therapy (FACT), we developed brief "NCCN/FACT symptom indices" for advanced bladder, brain, breast, colorectal, head \& neck, hepatobiliary/pancreas, lung, ovarian, and prostate cancers. Surveys of disease-related symptoms were presented to 455 experts at 17 NCCN institutions. Experts narrowed each list to the 5 most important to attend to when treating a specific advanced cancer. Symptoms endorsed at a frequency greater than chance probability were retained for each index. In a parallel study, an advanced hepatobiliary cancer symptom index was constructed with input from an international sample of 95 experts and validated using data from 51 patients with advanced hepatobiliary disease. Experts from the 2 projects reached consensus on priority symptoms for advanced hepatobiliary cancer (pain, fatigue, nausea, weight loss, jaundice). The 8-item patient-validated index showed good internal consistency (.79), test-retest reliability $(.86)$, high association with mood (-.56), ability to differentiate patients by performance status rating $(p<.0001)$ and treatment status $(p=.057)$, and performed comparably to conventional QOL endpoints. Symptom scaling in advanced cancer is feasible and can produce efficient, rapid assessment of the symptom component of QOL to evaluate treatment effectiveness in clinical trials.

79/ DEVELOPMENT OF THE SHORT FORM MCGILL QUALITY OF LIFE QUESTIONNAIRE
N. Pratheepawanit, Faculty of Pharmaceutical Sciences, Khon Kaen University, Khon Kaen, Thailand; M. S. Salek, P. L. Lua, Centre for Socioeconomic Research, Cardiff University, Cardiff, UK; I. G. Finlay, Velindre NHS Trust \& University of Wales College of Medicine, Cardiff, UK

There is an increasing demand for compact and quick-to-administer quality of life (QoL) questionnaires in routine practice, particularly in areas such as advanced cancer where patients are very ill. Therefore, the aim of the study was to develop a short form version of the McGill Quality of Life questionnaire (MQOL-SF). The QoL data was obtained from results of routine MQOL administration during a two-year study $(4 / 98-4 / 00)$ at Velindre Hospital, Cardiff, UK. Items were selected if they had repeatedly received affirmative responses by the patients, those indicated by the doctors as being useful in their clinical decisionmaking, and those constituting the conceptual framework of the measure by demonstrating close correlation with the global QoL score. A total of 910 palliative care consultations yielded $601(66 \%)$ completed Revised MQOL of which only $582(64 \%)$ were evaluable. This represented 219 patients (female $=149 ;$ mean age $=59$, age range $=26$ to 84). There was a low level of missing data (1.4 to $5.2 \%)$ except for physical symptoms which showed $10-30 \%$ noncompliance. The openended question asking patients to report factors affecting their QoL was completed in 366 forms (63\%). Only 128 (22\%) completed questionnaires had written comments from the doctors stating the 'usefulness' of QoL information (36-65\%), mainly physical symptoms, physical well-being, psychological (item 5 - 7) and existential (item 14) domains. The revised MQOL had a high internal consistency reliability (alpha $=0.75-0.92$ ) and most domains correlated well ( $r s=0.44-$ 0.70 ) with the overall QoL. Items strongly correlating with both overall QoL and their respective domain were those from physical well-being, psychological (item 5,7) and existential (item 9, 10,12,14) domains. Finally, the items considered for the short form included the overall QoL, physical symptoms (item $1-3$ ), psychological (item 5, 7), existential domains (item 12,14) and the open-ended question. Factor analysis revealed two factors with a total variance of $63.9 \%$ which closely resemble the structure in the Revised MQOL. Further studies to evaluate psychometric properties of the MQOL-SF are underway.

80/ DEVELOPMENT AND VALIDATION OF QUALITY OF LIFE MEASUREMENT IN SCHIZOPHRENIA: SCHIZO-QOL

Christophe Lancon, Psychiatry Department, Pascal Auquier, Public Health Department, Marie-Claude Simeoni, Public Health Department, Medical School, Jean-Michel Azorin, Psychiatry Department, Marseille, France; Jean Dallery, Psychiatry Department, Lyon, France; Alain Leplege, Inserm U292, Le Kremlin Bicêtre, France; Pierre-Michel Llorca, Psychiatry Department, Clermont-Ferrand, France; Michel Goudeman, Psychiatry Department, Lille, France; Julien-Daniel Guelfi, Marie-France Poirier, Psychiatry Department, Paris, France; Gilles Reine, Psychiatry Department, Toulon, France; Jean-Claude Samuelian, Psychiatry Department, Marseille, France; Hélène Verdoux, Psychiatry Department, Bordeaux, France

Four schizophrenic patients 16 major QoL instruments are described in the literature. Those instruments reflect a wide variability concerning their content and only three are specific measures for schizophrenic patients. None were developed both: - from the exclusive patients' viewpoint; - to be sensitive to change. Then, we report the development and the validation of the first schizophrenia-specific instrument in French: the SCHIZO-QoL questionnaire. We have conducted more than 20 videotaped interviews with schizophrenic patients (in- and outpatients, different levels of severity, different subgroups of DSM-IV, acute and chronic illness). Content analysis of the interviews (classical and computerised process) isolated 97 candidate items. Face validity was explored among 40 patients. 53 items were selected after the item reduction step, conducted on 160 patients. After validation study (more than 300 in- and out-patients), the final self-administered questionnaire included less than 40 items. The major dimensions (70\% of total variance) were mental health, social relationships with friends, with family, leisure, self-esteem... Cronbach's alpha ranged from 0.7 to 0.9 . Reproducibility was confirmed (one-month follow-up). Correlations between the dimensions of SCHIZO-QoL and SF-36, QoLI, EuroQoL were reported. Known group comparisons were studied according to the different forms of schizophrenia (DSM-IV), socio-demographic criteria, course of disease. Criterion validity (PANSS, Calgary Scale, GCl, ESRS) was shown. The percentage of missing responses was low (fewer than $10 \%$ for all the questions), completion time was always under 20 minutes, therefore in schizophrenia, it seems possible to assess QoL with this only specific self-administered questionnaire constructed on the basis of patient's viewpoint: SCHIZO-QoL. 
81/ THE PATIENT'S PERSPECTIVE IN SCHIZOPHRENIA: IS IT VALID, RELIABLE, AND RESPONSIVE?

Benoit Arnould, Mapi Values, Lyon, France; Anthony F. Lehman, University of Maryland School of Medicine, Baltimore, MD; Céline Laforêt Mapi Values, Lyon, France; Mike Ingham, Inge Duchesne, Janssen Research Foundation, Beerse, Belgium

Despite an increasing use of Quality of Life (QoL) assessment in schizophrenic patients (SCHI), two issues remain discussed: $1 /$ the validity and reliability of the patient's perspective; 2 / the lack of published information about the responsiveness of the questionnaires used. We discuss these issues in the light of the results from a clinical trial conducted in 397 stable schizophrenic patients in the US. The QoL was assessed every 6 month using the QOLI-89. The QOLI Subjective Scales (SS) assess 8 domains: General Life Satisfaction (GLS), Living Situation (LIS), Daily Activities (DA), Family (FAM), Social Relations (SR), Finances (FIN), Legal and Safety issues (SAF), Health (HLT); the Objective Scales (OS) assess 5 domains: DA, FAM, SR, FIN, SAF. The construct was analysed by Principal Components Analysis (PCA), item-discriminant and convergent validity, scale-scale correlations; the internal consistency reliability was measured by Cronbach's alpha (alpha), the clinical validity was tested according to PANSS and CGI, the responsiveness was assessed by Effect Sizes (ES) calculated in PANSS worsening and improving patients. The construct validity was excellent for all SS and for 3 out of $5 \mathrm{OS}$. All SS, but only the FIN OS, had alpha $>0.7$. SS correlated significantly ( $p$-values $<0.0002$ ) with the related OS. At baseline SS scores GLS DA, FAM, SR and HLT correlated significantly ( $p$-values $<0.0006$ ) to the PANSS scores; DA was significantly related to CGI; no relation was found between the OS scores and PANSS neither CGI. 3 SS (DA, FAM and HLT) had moderate responsiveness (ES between 0.2 and 0.5 for both improved and impaired patients). The patient's perception, measured by SS is consistent with the facts they report in $\mathrm{OS}$. The SS do not clearly match the CGI, but most of them are validated by the PANSS, and some are responsive to PANSS change. The patient's perception of HRQL assessed by the QOLI SS is valid reliable, and may be responsive to clinical change in stable $\mathrm{SCHI}$.

82/ SOAP-51: A SELF-ADMINISTERED, HEALTH-RELATED QUALITY OF LIFE INSTRUMENT FOR INDIVIDUALS WITH SCHIZOPHRENIA LIVING IN THE COMMUNITY

Judith T. Barr, Gerald E. Schumacher, School of Pharmacy, Emanual Mason, Counseling Psychology, Susan Ohman, NERCOA, Northeastern University, Boston, MA

To assess the perceptions of individuals with schizophrenia about aspects of their health-related quality of life, we have developed and validated a 15-minute, self-administered, 51-item measure to assess 8 factors in their life: satisfaction, self-concept, work/role, mental health, interpersonal, medication effects, activities of daily living, and physical function. Based on 4 administrations to a cohort of 1500 consumers (age 43.6 years, $60 \%$ males) with schizophrenia in 5 ethnically and geographically diverse states (AZ, MA, SC, WA, WI), Cronbach alpha of the 51 -item survey was $0.711-0.881$ and test-retest reliability was $0.781-0.994$. Each item had a stronger correlation with its factor score (minus the item) than with other factors. For each factor, consumer factor scores progressively lowered as consumers reported that their condition had more negative impact on their lives $(p<0.001)$. For all factors but medication effects, consumer factor scores improved with improving staff assessments of consumer functionality $(p<0.001)$. Overall consumer scores from the lowest to highest staff-assigned quartile of function also progressively increased: $42.7 \%, 49.3 \%, 54.7 \%$, and $60.0 \%$ respectively. $92 \%$ of consumers considered the survey useful and $92 \%$ of staff considered the survey valuable for monitoring consumer progress. Based on input from 193 consumers in a followup item importance weighting study, there were no differences among the importance weights of the 8 factors nor the 51 items $(p>0.05)$. More than 2100 consumers have been involved in various stages of SOAP-51 development: item identification, item reduction, reliability and validity studies, and item importance weighting. In conclusion, we established reliability and concurrent and discriminatory validity for the SOAP-51, a consumer-centered instrument, which can be used as a self-administered outcome measure in patient monitoring and management and in health policy decision making.
83/ETHNIC DIFFERENCES IN VALUES FOR HEALTH OUTCOMES IN SCHIZOPHRENIA

Leslie Lenert, Health Services Research, VA San Diego Healthcare System, San Diego, CA; Ann Sturly, San Diego Veterans Medical Research Foundation, San Diego, CA; Mark Rapaport, Shannon Chavez, Department of Psychiatry, University of California, San Diego, La Jolla, CA; Marcia Rupnow, Amy Grogg, Janssen Pharmaceuticals, Titusville, NJ

If ethnic groups perceive and value mental illnesses and mental health outcomes differently, then allocation of health resources based on the preferences of the majority could result in ineffective and inefficient healthcare delivery systems. To study, in a cost-effective way, whether there are differences in values for outcomes in schizophrenia across ethnic groups in the United States, we created streamedvideo materials portraying health states with different combinations of schizophrenia symptoms, and linked them to a web-based survey that measured visual analog scale and standard gamble (SG) ratings. We sent announcements to 30,100 randomly selected members of a large Internet survey panel, deliberately over-sampling minority ethnic groups. This procedure resulted in a convenience sample of 640 participants, $54 \%$ of whom were Caucasians (CAUC), $15 \%$ Asians (ASIAN), 14\% African-Americans (AA), 12\% Latinos (LAT), $3 \%$ "other" and $3 \%$ no information). Preference measurements showed good internal consistency ( $7 \%$ error rate) and reliability ( $r=0.74$ to 0.90 for SG ratings.) We used repeated measures ANOVA, simultaneously examining ratings of mild, moderate, severe and extreme states to look for associations between demographic factors and preferences. In univariate analyses, subjects' age, ethnicity and gender were associated with their preferences $(p=0.001)$; however, in multivariate analyses, only the ethnicity effects remained significant $(p=0.004)$. Minority groups rated the severe and extreme states 0.06 to 0.08 higher than CAUC, resulting in differences of $30 \%$ or more in the gain in utility with complete remission of certain syndromes. Moreover, while $64 \%$ of the CAUC thought controlling severe symptoms at the cost of inducing an adverse drug effect was a favorable trade-off, only $42 \%$ of AA, $55 \%$ of ASIAN and $49 \%$ of LAT agreed $(p<0.001)$. The results suggest potentially important differences in perceptions of quality of life in schizophrenia in persons of different ethnicity.

\section{4/ WHAT ROLE DO SYMPTOMS PLAY IN HEALTH-RELATED QUALITY OF LIFE?}

Nancy Kline Leidy, Deborah L. Jonas, MEDTAP International, Inc., Bethesda, MD; Cheryl C. Silberman, AstraZeneca, Wilmington, DE Mary Kay Margolis, MEDTAP International, Inc., Bethesda, MD; Anne Heyes, AstraZeneca, Cheshire, UK

Health-related quality of life (HRQL) has been defined as a person's subjective perception of the impact of health status on physical, psychological, and social functioning and well-being. Intuitively, physical symptoms play a role in $\mathrm{HRQL}$ - but what role do they really play? Are they part of HRQL? A contributor to HRQL?, Or, as some have proposed, are symptoms and HRQL synonymous? This paper will describe a theoretical framework in which a distinction is made between symptom occurrence (characterized by the following attributes: number, frequency, intensity, duration), symptom distress (discomfort attributed to symptom occurrence), and HRQL. The logical outcome of this distinction is the ability to model and test the extent to which each attribute of a given symptom or symptom cluster is distressing and which dimensions of HRQL are affected. Thresholds of symptom tolerance can be defined by examining the symptom-HRQL relationship cross-sectionally and over time. This framework also enables the investigator to test whether a given intervention has an effect on any or all of these outcomes and whether symptom occurrence moderates or mediates the relationship between treatment and HRQL. Evaluating outcomes of new treatments for chronic conditions such as lung cancer and advanced chronic pulmonary disease offers an example of the utility of this approach. The primary goal of treatment for these conditions is to contain the symptoms of illness such that activity limitations and role compromise are minimized, that is, to reduce or minimize symptom occurrence, relieve distress, and improve or optimize HRQL. Thus, prospective studies and clinical trials should include a comprehensive assessment of these outcomes. The paper will draw from the empirical literature in oncology and chronic respiratory disease to illustrate the utility of this framework for critiquing the literature, designing prospective studies and clinical trials, and interpreting study results. 
85/ SUBJECTIVE VERSUS OBJECTIVE QUALITY OF LIFE IN WOMEN WITH POSTMENOPAUSAL OSTEOPOROSIS

Maren Gesina Glueer, Bundesanstalt fuer Milchforschung, Hamburg Germany; Helmut W. Minne, Institut fuer klinsche Osteologie, Bad Pyrmont, Germany; Ana D. Lazarescu, Klinik Der Fuerstenhof, Bad Pyrmont, Germany; Michael Pfeifer, Bettina Begerow, Institut fuer klinsche Osteologie, Bad Pyrmont, Germany

Vertebral osteoporotic fractures affect patients' Health Related Quality of Life (HRQoL) and may result e.g. in enhanced functional limitations and chronic back pain. Still frequently patients who are suffering from these impairments nevertheless report an excellent HRQoL while some patients with very few impairments report a poorer HRQoL. Thus, when investigating HRQoL aspects in osteoporosis the individual perception (i.e. subjective HRQoL aspects) should be taken into account to improve treatment strategies which should not solely be directed at objective HRQoL aspects (e.g. cure back pain). Aiming to differentiate subjective and objective aspects of HRQoL, we investigated 283 women with diagnosed postmenopausal osteoporosis (age: $63.47 \pm 7.92$ ) with regard to various $H R Q o L$ aspects, e.g. wellbeing (BBS Hobi 1985), pain (Hoppe 1990, Miltner 1988), limitations in daily living (Leidig 1991), load of disease (Badura 1987), life satisfaction (Bullinger 1990). Using factor analysis on a dataset of 90 questions, each variable could be distinctly assigned to one of two main factors. The first reflects more 'objective' aspects like pain and limitations while the second reflects more 'subjective' aspects like wellbeing, load of disease, satisfaction with health, quality of life, and social support. Together the two factors explained $29 \%$ of the overall variance. Current HRQoL research in osteoporosis mainly focuses on the improvement of the more objective HRQoL aspects like pain reduction and improvement of functional limitations. Our data challenges the view that objective HRQoL aspects also reflect the subjective perception of the patient. Assessment of these subjective aspects will allow to verify whether current treatment strategies also help to improve subjective HRQoL. This is needed to comprehensively improve HRQoL in patients with chronic disease, like osteoporosis.

86/ HOW IMPORTANT IS HEALTH FOR OVERALL QUALITY OF LIFE?

Thomas V. Perneger, Philippe Garnerin, Quality of Care Unit, Geneva University Hospitals, Geneva, Switzerland

How much health contributes to overall quality of life remains poorly understood. To explore the relative contribution of various aspects of life, including health, to perceptions of overall quality of life, we surveyed 216 volunteers, members of the general public. Participants rated their satisfaction with 13 aspects of their lives (between "none" and "very strong"), as well as the perceived importance of these domains for their overall quality of life (also between "none" and "very strong"). The outcome variable was a rating of overall quality of life (between "poor" and "excellent"). Twenty-six respondents (12\%) rated their quality of life as excellent, $86(40 \%)$ as very good, $91(42 \%)$ as good, $7(3 \%)$ as fair, and $6(3 \%)$ did not respond. Satisfaction scores for six aspects of life (living environment, money, physical health, mental health, relationships, and opportunity for personal development) were independently associated with quality of life; the others were not independent predictors (basic needs, work, leisure activities, autonomy, love life, role in society, and ability to influence things). Satisfaction ratings were positively associated with importance ratings for the same domain, but importance ratings were not associated with quality of life. A comparison of stated importance and revealed importance (i.e., adjusted regression coefficient reflecting association with overall quality of life) for the 13 dimensions indicated that people tend to overstate the importance of health, love life and basic needs, and underestimate the importance of money. These results indicate that (a) aspects of people's lives other than health bear strongly on perceptions of quality of life, and (b) perception by individuals of what matters for their quality of life may differ from what is revealed by a group-level analysis.
87/ WHAT IS SUBJECTIVE QUALITY OF LIFE? A PHILOSOPHICAL PERSPECTIVE

Bengt Brülde, Department of Philosophy, Practical Philosophy, Helge Malmgren, Department of Philosophy, Theoretical Philosophy, Göteborg University, Göteborg, Sweden

The recent discussion about the relative advantages of "subjective" and "objective" measures of quality of life (QoL) is marred by several confusions. It is not always clear what the term "subjective" means in this context, or to what the "subjectivity" is attributed. We first have to distinguish between subjectivist conceptions of what QoL is (valuetheoretical subjectivism), and subjective methods of QoL measurement (methodological subjectivism). There are two main kinds of subjectivist value theories of QoL. Some theories (e.g., hedonism) are subjectivist in the weak sense that the things to which they attribute final value are certain mental states (like happiness). This sense is better expressed by using the term "internalism". However, a theory can also (like preferentialism) be subjectivist in the sense that it defines a person's QoL in terms of that person's own valuing attitudes. If, for example, a person sincerely declares that physical excellence is what she values most in the world, then subjectivism of this brand claims that physical excellence is good for that person. When we talk about a measurement method being subjective, we normally mean that it is essentially based on the judgments of only one person. Subjectivist conceptions of QoL tend to give rise to subjective measurement, since it is assumed that the subject is an expert on how good she feels or what she wants. There is no necessary connection between value-theoretical subjectivism and methodological subjectivism, however. Just as objective variables like work can be measured subjectively, subjective variables can be measured objectively. For example, the severity of a person's depressive feelings can be independently estimated by different psychiatrists. In the present paper, we argue that the concept of QoL which is most relevant for therapy evaluation is subjective $\mathrm{QoL}$ in the preferentialist sense. The main reason for this is that the role of QoL measurements is to help decide whether a treatment is good for the patient, and this evaluative question can hardly be answered without referring to the person's own values.

88/ QUALITY OF LIFE IN SERIOUSLY ILL ADULTS AFTER HOSPITALIZATION: WHAT IS IT LIKE AND CAN WE PREDICT IT? Kitty S. Chan, RAND Corporation, Santa Monica, CA; Kenneth Covinsky, Medicine, University of California, San Francisco, San Francisco, CA; Joel Tsevat, Medicine, University of Cincinnati, Cincinnati, $\mathrm{OH}$; Albert W. Wu, Health Policy and Management, Johns Hopkins University, Baltimore, MD

This study describes the global quality of life (QOL) of seriously ill hospitalized patients and evaluates the feasibility of predicting future QOL in these patients. The sample was subjects from the Study to Understand Prognoses and Preferences for Outcomes and Risks of Treatment (SUPPORT) who had an estimated probability of 0.5 for surviving six months. Subjects included those who survived and responded to a global QOL item at the month-two interview. Model development used 1378 patients from the observational phase of SUPPORT; model validation used 1665 comparable patients from the intervention phase. QOL at study entry and two months later were described. The ability of baseline QOL, chart data and interview variables to predict QOL (excellent/very good/good vs. fair/poor) 2 months after hospitalization was explored through bivariate analyses and logistic regression. The proportion of subjects reporting at least a good QOL increased from $39 \%$ at baseline to $61 \%$ after two months. Of the 1378 subjects, the QOL of $48.4 \%$ improved, $20.4 \%$ worsened and $31.2 \%$ remained stable. Dependence in activities of daily living at baseline did not reduce the likelihood of a good future QOL. As expected, baseline $Q O L$ predicted future $Q O L(C=0.65)$. Adding chartdata improved prediction only modestly $(C=0.69)$. Including both chart and interview data produced a model that predicts future QOL with good accuracy $(C=0.73 ; 0.72$ in validation sample). QOL prospects were positive for many patients after hospitalization, even some who were functionally dependent at baseline. The prediction of QOL was more difficult than that for mortality or functional status, but our model was sufficiently discriminative to be useful. As QOL is an important outcome for these patients, clinicians should consider prognosis for QOL alongside survival and functioning to provide their patients with a comprehensive picture of what life would be like after hospitalization. 
89/ ELECTRONIC VERSUS PAPER QUESTIONNAIRES: A FURTHER COMPARISON IN PEOPLE WITH ASTHMA

Donald M. Bushnell, Mona L. Martin, HRA, Inc, Seattle, WA; Bhash Parasuraman, Health Economics and Outcomes Research, AstraZeneca, Wayne, PA

The use of electronic data capture (EDC) to assess health related quality of life (HRQoL) using validated questionnaires is increasing however it must be determined how data collected electronically correlates with the original mode of administration used in validation. Our objective was to compare paper and electronic administration of the standardized Asthma Quality of Life Questionnaire (AQLQ(S)), Pediatric Asthma Quality of Life Questionnaire (PAQLQ(S)), and Pediatric Asthma Caregiver's Quality of Life Questionnaire (PACQLQ). Using a cross-over design, adults and children with asthma and caregivers of children with asthma were recruited from clinics. Subjects were asked to complete both forms of the appropriate HRQoL measures at enrollment and 24-48 hours later. 30 subjects from each group repeated the EDC version 1 week after their initial electronic survey. Psychometric properties were assessed for each of the EDC versions. Intraclass correlation coefficients (ICC) and Pearson correlations were calculated to compare EDC and paper versions. 51 adults (mean age: $37,73 \%$ females), 52 children (mean age: $13,38 \%$ females), and 51 caregivers (mean age: $43,92 \%$ females) were evaluated. Internal consistency (Cronbach's alpha) for the overall score of each questionnaire was: 0.96 for the $A Q L Q(S)$ and the PAQLQ(S), and 0.92 for the PACQLQ. Overall ICCs comparing paper to EDC were: 0.96 for the $A Q L Q(S), 0.91$ for the PAQLQ(S), and 0.82 for the PACQLQ. Pearson's correlations were identical. One-week reproducibility (ICC) of the EDC versions was: 0.88 for the AQLQ(S), 0.78 for the PAQLQ(S), and 0.85 for the PACQLQ. When asked which method subjects preferred, the electronic version was chosen by $69 \%$ of adults, $77 \%$ of children, and $73 \%$ of caregivers. Additionally $14 \%$ of adults $14 \%$ of children, and $18 \%$ of caregivers reported no difference in preference. As in previous studies comparing electronic to paper questionnaires, this study revealed statistical evidence to support the use of EDC of the AQLQ(S), PAQLQ(S) and PACQLQ for populations with asthma.

\section{0/ ADMINISTRATION OF SEIQOL-DW: THREATS TO RELIABIL-} ITY

Marjan J. Westerman, EMGO/Social Medicine, Vrije Universiteit, Amsterdam, The Netherlands; Tony Hak, Methodology, Faculteit Bedrijfskunde / Erasmus Universiteit, Rotterdam, The Netherlands

In our study of "Response shift in Quality of Life in palliative treatment of small cell lung cancer patients", a qualitative exploratory longitudinal multiple case-study with 30 patients (cases), each patient is interviewed five times at equivalent "assessment points" in their illness trajectory, resulting into a data base of 150 interviews. Part of this study is an exploration of the validity and reliability of SEIQoL-DW as an instrument for measuring response shift. SEIQoL-DW allows respondents to "define" QoL by nominating the five most important aspects of their lives at that moment ("elicited cues") and by indicating the relative contribution ("weight") of each cue to their overall QoL. At different points in time, patients might nominate different "cues" and the same "cues" might get a different weight. As Schwartz \& Sprangers have indicated, both types of change could be considered indications of response shift. From the transcripts of interviews in which SEIQoLDW was administered, it appeared that "elicited cues" were in most cases not just the result of a process of elicitation and subsequent recording of the respondents' cues. Rather, these cues were the result of an interactional process of "working up" initial remarks made by the respondent into categories deemed relevant by the interviewer. We conclude that SEIQoL-DW data as produced in these interviews are artefacts resulting from interviewer intervention rather than representations of independent judgments made by respondents. This implies that observed changes of SEIQoL-DW cues and weights between two assessment points cannot be taken as reliable measurements of response shift. This affects the reliability of SEIQWoL-DW scores in QoL measurements as well. Inspection of the SEIQoL-DW manual and of instructions for interviewers (as made explicit in training practices) shows that this problem is hardly acknowledged and that remedies (e.g., advice aimed at standardizing interviewer behavior) are not yet developed. This makes SEIQoL-DW data vulnerable to unaccounted interviewer bias.
91/ TOWARDS BRIEFER QOL INSTRUMENTS: A POPULATION APPROACH

T. G. Ganiats, K. M. David, E. J. Groessl, W. J. Sieber, R. M. Kaplan, UCSD Health Outcomes Assessment Program, University of California San Diego, La Jolla, CA

Respondent burden is an obstacle in QOL assessment. This study evaluates an estimation method for assessing population health status. The method administers part of a quality of life instrument to each individual and combines these parts to estimate the score for the whole instrument. Data was taken from a registry of 2,264 self-administered Quality of Well-being (QWB-SA) scale QOL assessments from a wide range of clinical trials and clinic patients. The QWB-SA score is a function of 4 subscales (Symptom Complex (CPX), Mobility (MOB), Physical Activity (PAC), and Social Activity (SAC)). The QWB-SA was split into 5 pre-determined parts, each with about 14 questions, creating a pool of $5 \times 2,264$ elements for use in the simulation. These elements were randomly selected, and then assembled and scored. A second simulation method, keeping the symptom list as a single part, was also constructed, run, and scored. The mean score for the actual sample was $4 \%$ higher than that of the first simulation method ( 0.641 vs. $0.613, p<0.001$ ), with essentially all of the difference resulting the estimate of the CPX score: CPX (Actual $=-0.322$, Simulation $=-0.351)$, MOB $(-0.002,-0.002)$, PAC $(-0.025,-0.025)$, SAC $(-$ $0.010,-0.010)$. This pattern was consistent across several runs of the simulation. The lack of agreement in the CPX score is not surprising in part because the length of the symptom list requires that the CPX comprise 4 of the 5 parts. Agreement in the other components may also have resulted from less variance in the weights assigned to items within the MOB, PAC, and SAC scores. Allowing the CPX section to remain intact improved the performance of the second simulation method (mean of 0.639 vs. 0.641). Investigators wishing to decrease respondent burden and who are not interested in following the scores of individual subjects can consider splitting the QWB-SA into smaller parts that are combined later. Keeping the CPX section intact improves the accuracy of the estimate.

92/ DIFFERENCES IN PERCEPTION OF QUALITY OF LIFE AND GOALS FOR IMPROVEMENT WITH TREATMENT AMONG PATIENTS, FAMILY MEMBERS AND PROVIDERS

Marion A. Becker, David Thornton, Department of Mental Health Law and Policy, Louis de la Parte Florida Mental Health Institute, Tampa, FL; Ronald J. Diamond, Department of Psychiatry, University of Wisconsin-Madison, Madison, WI

The objectives of this research were to determine whether there are important differences in how patients, family members and mental health providers' value treatment outcomes and to assess the nature and degree to which such differences, if they exist, could adversely affect perception of quality of life. While Quality of Life (QoL) data in persons with severe and persistent mental illness are accumulating, little is known about the agreement between patient and proxy responses to a QoL instrument, and the association between patientproxy agreement on goals for improvement with treatment and the patients' QoL outcomes. The study used a convenience sample of 454 adult outpatients with severe and persistent mental illness receiving treatment in the public mental health system, their treatment providers and family/significant others. Subjects were administered the Wisconsin Quality of Life Index (W-QLI), a multidimensional instrument for measuring QoL along major domains including life satisfaction, occupational activities, psychological well-being, physical health, social relations, economics, activities of daily living, and symptoms. The W-QLI also captures information about the patient's most important goals for improvement with mental health treatment. The W-QLI has separate forms for multiple responders and takes into account the different perspectives of the patient, family/significant other, and the clinician, both concurrently and over time. There are consistent systematic differences in goals for improvement with mental health services and perceptions of QoL outcomes among patients, providers and family/significant others. Family/significant others had goals and QoL evaluations that were more similar to those of patients than were those of mental health providers. The results emphasize the importance of participation by patients (or family/significant other proxies) in clinical decision-making and outcomes evaluation. 
93/ THE CHILD HEALTH QUESTIONNAIRE (CHQ): COMPARISON OF THE QUALITY OF LIFE (QOL) SCORES OBTAINED BY THE PARENT REPORT (PF50) AND THE SELF REPORT (CF87)

Chantal Rodary, Biostatistics, Bellon Nicolas, Institut Gustave Roussy, Villejuif, France; Jeanne Landgraf, Health Act, Boston, MA; Chanta Kalifa, Institut Gustave Roussy, Villejuif, France; André Baruchel, hôpital Saint Louis, Paris, France; Erica Quintana, Institut Curie, Paris, France

To show whether the degree of agreement on overall scale scores of quality of life (QOL), provided by parent report and self report, was sufficiently good to allow use of the parent-form only when the child is unable to complete the questionnaire. The French CHQ-CF87 (modified version: 81 items, a single scale "role/social limitations due to health" instead of 3 scales on role), and CHQ-PF50. They have seven dimensions in common : Physical Functioning (PF), Self Esteem (SE), General Health Perception (GH), Bodily Pain (BP), General Behavior $(\mathrm{BE})$, Family Activities (FA), and Mental Health (MH), each providing, an overall score from 0 (worst QOL) to 100 (best QOL). Children (C) aged 9 to 18 years, diagnosed with cancer were included in the study. Both the parent $(P)$ and the child completed their specific questionnaire once at the follow-up visit, a minimum of 6 months after completion of treatment. Ten centers participated. The degree of agreement was measured by Intra Class Correlation-coefficients (ICC). 235 P/C pairs were obtained by November 2000 . The median age was 14 years, and sex ratio 1.3. Compliance was high, about $99 \%$ for both $C$ and $P$. Psychometric properties were verified in both. The ICC were rather low, ranging from $0.12(\mathrm{BE})$ to $0.45(\mathrm{BP})$. The relative mean difference $(d=(C-P) / C)$ between overall scores, indicated lower results from $\mathrm{P}$, in all dimensions with the exception of FA. Parent scores were significantly lower $(p=0.001)$ for $\mathrm{BE}(20 \%)$, MH $(7 \%)$, SE $(6 \%)$ $\mathrm{GH}(6 \%)$. The low ICC for the different dimensional and overall scale scores may be due to the fact that the items in the 2 questionnaires were not totally identical (number and wording). Nevertheless, it is noteworthy that the scores assigned by parents are worse than children's, in the psychosocial and GH dimensions, whereas they are very similar for physical dimensions.

\section{4/ ITEM REDUCTION: DO DIFFERENT METHODS PRODUCE DIF-} FERENT SCALES?

Jeremy C. Hobart, Afsane Riazi, Neurological Outcome Measures Unit, Institute of Neurology, London, UK; Donna L. Lamping, Health Services Research Unit, London School of Hygiene \& Tropical Medicine, London, UK; Ray Fitzpatrick, Health Services Research Unit Oxford University, Oxford, UK; Alan J. Thompson, Neurological Outcome Measures Unit, Institute of Neurology, London, UK

Most multi-item measures are developed by the statistical reduction of a large item pool. Although different methods of item reduction are used, few studies have examined the extent to which they may generate different instruments. We compared the item content and psychometric equivalence of scales resulting from four methods of item reduction. Patient interviews, expert opinion, and a literature review generated a pool of 32 items concerning activity limitations in multiple sclerosis (MS). These were administered to 1530 people randomly selected from the UK MS Society database. Test-retest reproducibility was studied in a subsample of 400 . Four short-form measures were generated by selecting items on the basis of the item-item correlations, corrected item-total correlations, equidiscriminatory itemtotal correlations, and a stepwise regression. Resulting scales were transformed to have a 0 to 100 range, and examined for their descriptive statistics, reliability, convergent and discriminant validity, agreement with each other, and agreement with scores generated by all 32-items. 766 completed questionnaires were returned (response rate $64 \%$ ). Factor and Rasch analysis supported the unidimensionality of the 32 items. Each item reduction method generated a different scale. The maximum item overlap was two. All scales demonstrated good score variability, small floor and ceiling effects, high alpha coefficients $(>0.85)$, high test-retest reliability (ICC $>0.94)$, and had near identical convergent and discriminant validity. Agreement between scores generated by each scale and the 32 -items was high (ICC > $0.94)$. Agreement among scores generated by the four scales was high (ICC 0.89 to 0.97 ). These different methods of item reduction generated different scales that are psychometrically equivalent.
95/ PSYCHOMETRIC PROPERTIES OF FACT-AN NORMATIVE DATA: RESULTS FROM A WEB-BASED SURVEY

D. Gagnon, RWJ Pharmaceutical Research Institute, Raritan, NJ; Christina Vandoros, Janssen-Cilag Pharmaceutical SACI, Pefki, Athens, Greece; M. Zagari, B. Shilling, RWJ Pharmaceutical Research Institute, Raritan, NJ; D. Cella, Northwestern University, Evanston, IL

We investigated the psychometric properties of the Functional Assessment of Cancer Therapy-Anemia (FACT-An), as administered using a web-TV survey based upon a random sample $(\mathrm{N}=1,078)$ drawn from a representative US panel. The FACT-An is composed of the following: Physical Well-Being (PWB, 7 items); Social/Family WellBeing (SFWB, 7 items); Emotional Well-Being (EWB, 6 items); Functional Well-Being (FWB, 7 items); FACT-G (PWB + SFWB + EWB + FWB); Fatigue (FAT, 13 items); Non-Fatigue (NFAT, 7 items); Anemia (FAT + NFAT); and FACT-An (FACT-G + Anemia). Six items from the FACT-G could not be collected in this venue because of references to treatment and/or illness. We investigated the scaling and psychometric properties of the FACT-An given missing values for these 6 items. The six missing items are in the PWB (1), SFWB (2), EWB (2), and FWB (1). Scores were derived for these 4 scales if there were $<50 \%$ missing items in that scale. FACT-G scores are usually derived if $<20 \%$ of its items are missing, but here they were derived if none of the 4 sub-scales were missing. Convergent and discriminant validity, internal consistency, and Likert scaling assumptions were assessed using item-scale correlations and by inspecting item-level variances within scales. Finally, FACT-G and FAT were tested for discrimination between cancer and anemia versus no chronic illness, respectively, using independent sample t-tests. All scales except NFAT showed excellent convergent and discriminant validity, internal consistency, Likert scaling properties and reliability. The FACT$\mathrm{G}$ discriminated between cancer and no chronic illness (13.88 points, $p<0.001)$ and FAT discriminated between anemia and no chronic illness ( 9.96 points, $p<0.001)$. Thus, FACT-An data collected by a webTV-based survey, given six items were not asked, appear to have good scaling and psychometric properties. Reference data from this representative US sample can now be use to render clinical trial data more interpretable.

96/ PRECISION AND BREVITY: VITALITY ASSESSMENTS USING COMPUTERIZED ADAPTIVE TESTING METHODS

Julie H. Zhao, Quality Metric, Inc., Lincoln, RI; Jakob B. Bjorner, The National Institute of Occupatioinal Health, Copenhagen, Denmark; Mark Kosinski, Kimberly A. Laliberte, John E. Ware Jr., Quallity Metric, Inc., Lincoln, RI

Fatigue is a common reason for seeking treatment. Accordingly vitality (energy/fatigue) is one of the most frequently measured HR-QoL concepts. This study examined the precision and validity of vitality scores estimated from very brief computerized adaptive test (CAT) administrations based on Item Response Theory (IRT) models. Adults were randomly sampled through the Internet $(\mathrm{N}=900)$. Questionnaire items were selected from 12 widely used vitality scales (e.g. Copenhagen Burnout Inventory, Multidimensional Fatigue Inventory, SF-36). A pool of 33 items that met the assumptions of a Generalized Partial Credit IRT model were calibrated. CAT administrations of items based on these calibrations were simulated to investigate measurement precision, validity, and reduction in respondent burden, in comparison with scores estimated using all items. The Pearson P-M correlation between CAT-based vitality scores and scores based on all items was 0.95 . The latter showed better measurement precision at all levels, as expected. The standard error of measurement (SEM) ranged from 0.13 to 0.46 for estimates based on the total item pool, in comparison with SEM's of 0.25 to 0.48 for CAT-based estimates. Only 7 or fewer items were needed to achieve the latter's levels of precision, resulting in a $79 \%$ reduction in respondent burden. In tests of discriminant validity (e.g., comparing people with or without a chronic disease), the CAT-based scores were $94 \%$ as valid as scores based on all items. CAT-based assessments of vitality are likely to produce precise score estimates with substantial reductions in respondent burden. The generalizability of these results based on simulations is currently being tested using actual CAT-based assessments on the Internet. 
97/ PSYCHOMETRIC PROPERTIES OF THE GLOBAL INDEX OF SAFETY (GIS): A NEW INSTRUMENT TO ASSESS DRUG SAFETY Luis Prieto, José A. Sacristán, Juan C. Gómez, Clinical Research Department, Lilly SA, Alcobendas, Spain

The Global Index of Safety (GIS) is an Adverse Event (AE) based instrument designed to evaluate with a single, weighted score the safety profiles of drugs. This paper presents the evaluation of the inter-rater reliability, unidimensionality and construct validity of a 94item GIS for anti-psychotics (AP). AE severity weights were obtained through a rating scale procedure: a total of 194 psychiatrists participating in an outpatient pharmacoepidemiologic study of Olanzapine in schizophrenia (EFESO study) were asked to rate the severity that each $A E$ would have on a 5-point scale. Reliability was determined through a paired comparison design involving the new independent ratings of 101 different psychiatrists participating in another study of Olanzapine, but this time in the treatment of schizophrenia at acute inpatient units (EUROPA study). Spearman's, Pearson's and twoway mixed parametric Intraclass (ICC) correlation coefficients were used to estimate the inter-rater reliability of the AE weights. A graphical comparison approach was also used to test the null hypothesis that the differences between the weights obtained by the two subsamples (EFESO vs EUROPA) were attributable to measurement error. For this purpose, numerical calculations were summarized in a scatter plot of $A E$ weights and $95 \%$ confidence bands. Unidimensionality and construct validity were analyzed through the Rasch rating scale model by examining the distributions of residuals for $A E$ weights and raters. Reliability coefficient estimates were excellent (Spearman=0.99, Pearson=0.99, ICC=0.98). The regression fit line fell evenly between the prediction intervals defined by the confidence bands. Of the $94 \mathrm{AE}$ and the 295 raters of the overall study, $89 \%$ and $79 \%$ respectively demonstrated excellent goodness-of-fit on their weights and ratings (Infit Mean Square<1.3). The results of this study support the inter-rater reliability, unidimensionality, and construct validity for the GIS as a tool to be used in analytic studies to evaluate drug safety.

98/ A COMPARISON OF LIKERT SCALE AND VISUAL ANALOGUE SCALES IN CHILDREN: RESPONSE DIFFERENCES AND PREFERENCES

Henriette van Laerhoven, Bert H. Derkx, Hester J. Loonen, Paediatrics, Emma Children's Hospital, Academic Medical Centre, Amsterdam, The Netherlands

To measure QoL in children it is important to choose the most reliable methodology. Since little research has been dedicated to instrument development in children the purpose of the study was to examine which response options children prefer and how they correlate. A consecutive group of 120 children coming to a general paediatric outpatient clinic were studied. A seven-item questionnaire was developed that contained two affective questions (dreams, current mood) three questions asking about the child's opinion (school, sports, height), two frequency questions (watching tv, going by bus). Children were given one page with the seven questions and the Likert scale as answering mode, one page with the same questions but the numeric VAS scale, and a page with the simple VAS scale. The pages were combined in random order. All children were asked to give a preference for each answering option on a scale from zero to ten. On average, the numeric VAS correlated with both the Likert scale and simple VAS with a coefficient of 0.85 . The simple VAS and the Likert scale correlated with a coefficient of 0.81 . The mean preference scores children gave for the different answering options were: 8.5 for the Likert scale, 7.5 for the numeric VAS, and 6.3 for the simple VAS There was no difference between younger and older children. Overall, the simple VAS, numeric VAS and Likert scale can be considered comparable. However, most children prefer the Likert scale. The simple VAS, which we expected to be popular amongst children, scored very low.

99/ FATIGUE AND QUALITY OF LIFE IN PATIENTS WITH RHEUMATOID ARTHRITIS

Ines Rupp, Department of Social Medicine, Academic Medical Centre/University of Amsterdam, Amsterdam, The Netherlands; Hendriek C. Boshuizen, Division of Public Health Research, National Institute of Public Health and Environment, Bilthoven, The Netherlands; Catharina E. Jacobi, Department of Social Medicine, Academic Medical Centre/University of Amsterdam, Amsterdam, The Netherlands:
Huibert J. Dinant, Jan van Breemen Institute, Amsterdam, The Netherlands; Geertrudis A. van den Bos, Department for Health Services Research, National Institute of Public Health and Environment, Bilthoven, The Netherlands

Rheumatoid arthritis (RA) is a chronic, inflammatory disease with longterm consequences for daily functioning and health related quality of life (HRQoL). Fatigue is a common symptom in RA. We evaluated the impact of fatigue on quality of life. Our study was conducted in 1999 within a longitudinal survey among patients with RA. Fatigue was measured with the Multidimensional Fatigue Inventory (MFI-20), which covers 5 different dimensions of fatigue: general, physical and mental fatigue, reduced motivation and reduced activity. Scores range from 4 to 20, with higher scores indicating higher levels of fatigue. HRQoL was assessed with the RAND-36. The impact of the subscales of fatigue on different dimensions of HRQoL was evaluated with multivariate regression analyses after adjusting for sociodemographic and health characteristics. A total of 683 patients participated (mean age 61.5 years (SD 13.8), $71 \%$ female). The mean disease duration was 10.7 years (SD 9.3). Patients with both quiet and active RA (range mDAS: $0.8-8$ ) as well as good and bad functional status (range $\mathrm{HAQ}$ : $0-2.9)$ were included. 190 patients $(29 \%)$ reported symptoms of possible clinical depression (CES-D score of 16 and more). The mean scores of the MFI-20 were 13.6 (SD 5.0) for general fatigue, 12.7 (SD 4.7) for physical fatigue, 8.2 (SD 4.1) for mental fatigue, 10.2 (SD 4.4) for reduced motivation and 11.5 (SD 4.9) for reduced activity. Within the different dimensions of HRQoL, the impact of the subscales of fatigue varied. Physical functioning was associated with physical fatigue only. Mental health was associated with mental fatigue, but also with general and physical fatigue. Social functioning was affected by general fatigue and reduced activity, but not by the more mental aspects of fatigue (mental fatigue and reduced motivation). The impact of fatigue in RA on HRQoL depends on both the dimension of fatigue and the dimension of quality of life.

100/ QUALITY OF LIFE IN SYSTEMIC LUPUS ERYTHEMATOSUS Ayhan A. Lash, School of Nursing, Northern Illinois University, DeKalb, IL

Systemic lupus erythematosus (SLE) is a chronic, multisystem disorder that primarily affects women in their reproductive years. The disease has a lifelong trajectory characterized by day-to-day fatigue, sudden flare-ups, life threatening complications such as kidney failure and stroke. The purpose of the study was to investigate the impact of SLE on the quality of life (QOL) of individuals with SLE in terms of physical, psychological, and social well-being, and to identify the domains of QOL most affected by SLE. The study also investigated the reliability and validity of two instruments: the Sickness Impact Profile (SIP) (Bergner, 1988) and Arthritis Impact Measurement Scale 2 (AIMS2) (Meenan, Mason, Kaziz \& Guccione, 1990). The target population was all persons diagnosed with SLE who were members of the Lupus Foundation of America, Illinois Chapter. Only those with SLE diagnosis confirmed by their physicians, which was consistent with the American Rheumatism Associations revised criteria for diagnosing SLE (1982), were accepted into the study. Using survey methodology, two QOL instruments, one demographic questionnaire and a cover letter were mailed to 39 subjects. Of the 39 subjects, $37(94 \%)$ returned the questionnaires. All were usable. Of the 37 subjects, 34 were women, with a mean age of 36 . The analysis of SIP and AIMS2 scores showed that SLE affected all areas that are considered essential for QOL such as alertness behavior, recreation/pastime, sleep, rest, emotional balance, home management, social interaction, mobility and ability to work. Analysis of SIP dimensions showed that alertness behavior was the area most affected by SLE. The alertness behavior had a significant correlation with other SIP dimensions and also with the physical dimension of AIMS2. ANOVA and Scheffe tests for all statistically significant variables showed that age and marital status impacted on the QOL in SLE: older individuals and those divorced, widowed, or unmarried had poorer QOL due to physical impairment (age) and poor social support (marital status). An analysis of the QOL in SLE using the AIMS2 scale showed similar results. For example the correlation between the AIMS2 mood dimensions and the SIP's emotional balance was $r=.77(p=.000)$ corroborating the presence of a poor emotional state in SLE. The two areas measured by AIMS2 that are absent from SIP are health perception and the global impact of disease. The normalized scores (means) of these areas showed that SLE patients perceived their status to be poor and that the disease was having a major impact in their lives. 
101/ THE OUTCOME OF HIP AND KNEE REPLACEMENT FOR OSTEOARTHRITIS: LONG-TERM FOLLOW-UP

Francois A. Bethoux, Deborah M. Miller, The Mellen Center / U10, Mary LoGrasso, George M. Muschler, Department of Orthopaedic Surgery / A41, The Cleveland Clinic Foundation, Cleveland, $\mathrm{OH}$ JoAnna B. Younts, Younts Consulting, Raleigh, NC

Prior to implementing an outcomes registry for joint replacement in a large orthopaedic surgery center, a pilot study was conducted on patients undergoing total hip or knee replacement (THR or TKR) for osteoarthritis $(\mathrm{OA})$. Consecutive patients scheduled for primary unilateral THR or TKR with a diagnosis of OA were assessed before and up to 15 months after surgery, using the American Academy of Orthopaedic Surgeons MODEMS questionnaire, which includes the SF36 and disease-specific questions assessing symptom severity, functional status, expectations and satisfaction with treatment. Sixty-one patients completed both pre-surgical and long-term ( $>=6$ months) post-surgical assessments ( $61 \%$ THR). Mean delay between surgery and follow-up was $342+/-60$ days. Mean age was $65.8+/-12.3$ years $56 \%$ of the patients were women, and $64 \%$ reported a caregiver at home. The proportion of patients walking without aid increased from $58 \%$ to $87 \%$ between baseline and follow-up. Statistically significant improvement of scores was observed for SF-36 physical (PCS, $p=0.0001$ ) and mental (MCS, $p=0.021$ ) components, as well as 6 of the 8 SF-36 subscales (except Mental Health and General Health). Disease-specific item scores also improved significantly $(p=0.0001)$. Most disease-specific items, both at baseline and follow-up, were correlated with concurrent PCS scores. PCS change scores were significantly higher after THR than after TKR. PCS change scores were correlated with most items evaluating symptom severity and disability at baseline, and with patient satisfaction at follow-up. Change in MCS scores correlated with age and ambulation status at baseline, and with satisfaction with symptom relief at follow-up. In conclusion, long-term improvement of health status after THR or TKR was detected by both generic and disease-specific measures. The SF-36 (particularly SF36-PCS) appears as a valid measure of subjective treatment outcomes in this population.

\section{2/ THE RELIABILITY AND VALIDITY OF THE QUALITY OF LIFE} INSTRUMENT FOR THE JAPANESE PATIENTS WITH RHEUMATOID ARTHRITIS

Takako Morita, Department of Preventative Medicine, Kyushu University, Fukuoka, Japan; Jun Nagano, Institute of Health Science, Kyushu University, Kasuga, Japan; Suminori Kono, Department of Preventative Medicine, Kyushu University, Fukuoka, Japan; Yasurou Nishibayashi, Mikisanyo Hospital, Miki, Japan

The goal of this study was to develop a well validated questionnaire which is sensitive to assess the quality of life (QOL) of the Japanese patients with Rheumatoid Arthritis (RA) in clinical trials. It is a selfadministered instrument that consists of the three scales such as ADL, RA-specific QOL and generic QOL. In this report, the reliability and validity of the instrument were examined. A sample of 270 out- or hospitalized patients with RA who were diagnosed according to the 1992 version of the American Rheumatism Association criteria was used for analysis. The Japanese version of the Modified Health Assessment Questionnaire (MHAQ) and WHO/QOL-26 were used for assessment of the concurrent validity. The clinical data of the patients such as the C-reactive protein (CRP) level, the disease duration, the disease severity by stage and functional class were also collected for assessment of the construct validity. The questionnaire was re-administered after two to four weeks to examine the reproducibility, and the Chronbach's alpha coefficient was estimated to test the internal consistency. $91.2 \%$ of the patients answered the questions within $20 \mathrm{mts}$. The obtained Chronbach's alpha coefficient was $0.95,0.93$ and 0.92 for each ADL, RA-specific QOL and generic QOL. The correlation coefficient between the first and second administration was ranged from 0.70 to 0.90 . As a whole, the ADL summary score was most strongly correlated with the various clinical data among the three sections. For the ADL, RA-specific QOL and generic QOL section, the correlation coefficient with the score of MHAQ was 0.92 , 0.80 and 0.49 , and with the score of WHO/QOL-26 was $0.39,0.57$ and 0.74 respectively. The results of the psychometric examinations proved that our original instrument has high reliability and validity. It is suitable instrument to assess QOL of the Japanese patients with RA in clinical trials.
103/ PERSPECTIVES ON PEDIATRIC OUTCOMES: A COMPARISON OF PARENTS' AND PATIENTS' RATING OF QUALITY OF LIFE FOLLOWING ORTHOPAEDIC INTERVENTION

Julie C. Choe, Soo J. Im, Pediatric Orthopaedics, InCHOIR, New York, NY; Michael G. Vitale, Pediatric Orthopaedics, Children's Hospital of New York, New York, NY; Mark E. Spellmann, School of Social Work, New York University, New York, NY; David P. Roye, Pediatric Orthopaedics, Children's Hospital of New York, New York, NY

Orthopaedic interventions are often aimed at increasing quality of life (QOL), rather than extending life expectancy. Therefore, a more rigorous approach to assessing QOL is necessary, especially in children who are unable to communicate. The purpose of this study was to compare the parents' and children's perceptions of QOL in order to determine whether parents are a good proxy for assessing QOL of their children. A prospective study comparing parents' and children's (ages 11-18 years) responses to QOL questions was conducted on 78 parent-child pairs presenting to our institution from 1999 to 2000 . QOL was assessed using two instruments - Child Health Questionnaire $(\mathrm{CHQ})$ and the American Academy of Orthopaedic Surgeons Outcomes Instrument (AAOS), both of which consist of parent and child versions. Univariate analysis and Bonferroni multiple comparisons test were used to determine whether there were any significant differences in QOL domain scores between parents and children. Parent and child responses to the AAOS questionnaire did not differ significantly in any QOL domains, but there were several differences in $\mathrm{CHQ}$ domains: mental health $(\mathrm{p}=0.045)$, role/social physical limitations (limitations in school-related activities) $(p=0.042)$, global health item $(p<0.0001)$, and general health $(p=0.02)$. Children tended to perceive that they were in a better general health status and had fewer limitations in school-related activities than what their parents believed. Parents were more likely to perceive that their child had a better global health and mental health status than what their child perceived. There were more differences in parent vs. child QOL responses in the $\mathrm{CHQ}$ than in the AAOS instrument. This study suggests that if a child's QOL cannot be assessed directly, it is possible to gather such information indirectly from the parent. This is especially true in the case of the AAOS questionnaire.

104/ SATISFACTION AND DISSATISFACTION: ARE THEY MERELY OPPOSITES?

Elizabeth A. Hahn, David Cella, Center on Outcomes, Research and Education, Evanston Northwestern Healthcare, Evanston, IL

Patient satisfaction has become a key outcome of healthcare and is especially important in treatment of chronic illnesses. Hundreds of instruments are now available to measure self-reported satisfaction with healthcare. The use of both favorably and unfavorably worded items on the same questionnaire has often been recommended to control for certain types of response bias, and it has been assumed that positive and negative items reflect opposing approaches to measuring the same thing. Little attention has been given to evaluating the effects of both types of items on the measurement of patient satisfaction, and to exploring the relationship between satisfaction and dissatisfaction. A psychosocial intervention study with 213 cancer patients used a satisfaction questionnaire with a balanced number of positively and negatively worded items; each set covered similar item content. Responses to the two sets of items were analyzed using Andrich's extension of the Rasch measurement model for rating scale data. Principal components factor analysis of the standardized itemresponse residuals identified two underlying dimensions related to the direction of phrasing - not the content - of the items. Within patients the association between the two measures was moderate $(r=0.53)$ and measures based on positively worded items tended to be higher than measures based on negatively worded items. Person fit statistics detected a high proportion of misfitting responses, some of which seem attributable to the interspersing of positive and negative items. The fit statistics also detected most instances of acquiescent response bias. This approach demonstrates several interrelated applications of item response theory measurement to the challenges of measuring satisfaction. First, measurement of satisfaction differs from measurement of dissatisfaction. Second, one can reduce the problem of ceiling effects by introducing more negatively worded stems. Third, if both positive and negative items are to be used in a questionnaire, it is recommended that they be separated to avoid patient confusion and to better elicit sensitive estimates of attitudes. 
105/ RELATIONSHIPS BETWEEN SATISFACTION WITH LIFE DOMAINS AND SATISFACTION WITH LIFE AS A WHOLE IN PEOPLE WITH PHYSICAL DISABILITIES

Marcel W. Post, Luc P. de Witte, Research, iRv, Institute for Rehabilitation Research, Hoensbroek, The Netherlands

Subjective well-being has been conceptualised as consisting of two major components: an emotional or affective component (happiness) and a judgmental or cognitive component (life satisfaction). However, it is unknown whether both satisfaction with life as a whole and satisfaction with specific life domains belong to this cognitive component and whether satisfaction with life as a whole can be inferred from satisfaction with life domains. For this study, data of people with Spinal Cord Injury ( $\mathrm{SCl} ; \mathrm{N}=318$ ) or Spinal Muscular Atrophy (SMA; $\mathrm{N}=99$ ) were re-analysed. Fugl-Meyer's Life Satisfaction Questionnaire was used, containing one question about satisfaction with life as a whole and eight questions about satisfaction with life domains. All item scores range from 1 (very dissatisfying) to 6 (very satisfying). In both patient groups, people were satisfied with life as a whole (SMA group 4.9; $\mathrm{SCl}$ group 4.4). In the SMA group, satisfaction with life domains ranged from 2.9 (self care) to 5.1 (relationships with friends and acquaintances). In the $\mathrm{SCl}$ group, this was between 3.1 (sexual life) and 4.9 (relationships with friends and acquaintances). Spearman correlations between satisfaction with life as a whole and life domains were between 0.17 and 0.48 in both groups. Satisfaction with life domains predicted only a minor part of satisfaction with life as a whole (SMA $27 \%$ and SCI $35 \%$ of variance explained). In the SMA group, significant beta values were seen for relationships with friends and acquaintances (0.26), leisure time (0.26) and self care (0.21). In the SCl group satisfaction with leisure time was the best predictor $(0.38)$, followed by sexual life $(0.15)$ and self care $(0.15)$. In conclusion, in both patient groups, at best moderate relationships between satisfaction with life as a whole and satisfaction with life domains were seen. Satisfaction with social functioning appeared more important for satisfaction with life as a whole than satisfaction with self-care abilities (best reflecting the seriousness of the injury).

106/ QUALITY OF LIFE OF CHINESE CANCER PATIENTS: OPTIMISM AS MEDIATOR

Clara L. Yu, R. Fielding, Com. Med. C. L. Chan, Social Work and Soc. Administration, J. S. Sham, Clinical Oncology, Hong Kong University, Hong Kong, China

Nasopharyngeal carcinoma (NPC) is prevalent in southern China. In non-welfare countries, eating difficulties after radiotherapy may hinder NPC patients' short-term quality of life (QoL) especially in work and finance. This study explored the positive mediating role of optimism on the relationship between eating ability and QoL of Chinese NPC patients who received radiotherapy in Hong Kong. A sample of 211 newly refered patients were recruited and followed-up face-to-face or by telephone at four (post-radiation 1) (FU 1) and eight (post-radiation 2) (FU 2) months from Baseline. QoL was measured by the FACT$\mathrm{G}(\mathrm{Ch})$. Optimism and eating ability were measured by two elevenpoint self-rated items. Mediating effects were tested with a series of multiple regressions. Adjusting for pre-radiation QoL, socio-economic status and cancer stage, results indicated that optimism significantly mediated the relationship between eating ability at FU 1 and overall QoL at FU 2, in particular physical and social/family well-being. This study is the first to underpin the mediating role of optimism on QoL of NPC patients. Faced with aversive side-effects of radiotherapy, facilitating Chinese NPC patients to adopt positive thinking may accelerate their short-term post-treatment QoL.
107/ MAJOR DEPRESSIVE DISORDER (MDD) IN CHRONIC PAIN PATIENTS: CORRELATION OF THE SF-36 WITH PHYSICIAN DETERMINED DIAGNOSIS USING DSM-IV CRITERIA

Thomas E. Elliott, Division of Education and Research, Colleen M. Renier, Donna K. Anderson, Jeanette A. Palcher, St. Mary's/Duluth Clinic Health System, Duluth, MN

Major depressive disorder (MDD) is common among chronic pain patients resulting in great disease burden and significantly reduced quality of life. The diagnosis of MDD is dependent upon physician use of the DSM-IV criteria. The SF-36 may be a useful tool for detecting MDD, assessing patient-reported quality of life, and measuring patient outcomes. The purposes of the study were to (1) determine the correlation of the SF-36 Mental Composite Score (MCS) with the diagnosis of MDD made by a physician using DSM-IV criteria, and (2) document any difference in SF-36 MCS between chronic pain patients with and without MDD. A convenience sample of 114 consecutive patients with chronic non-cancer pain completed the SF-36 and was assessed by the same physician at entry into a pain management program. Pearson's correlations evaluated the correlation between physician diagnosed MDD and SF-36 scores. Student's ttests were used to compare the SF-36 scores of those patients with and without MDD. The study sample was $61 \%$ female, mean age of 45.4 (range 19 to 83 ), of whom $41 \%$ met DSM-IV criteria for MDD. Many patients had multiple pain diagnoses, including back pain $(56 \%)$, fibromyalgia $(36 \%)$, neuropathic pain $(41 \%)$, headache $(22 \%)$, and arthritis (9\%). The SF-36 MCS was significantly correlated with the physician diagnosis of MDD using DSM-IV criteria $(P=.000)$. MDD was significantly correlated with 6 of the 8 domains in the SF-36. Patients with MDD had significantly lower scores than patients without MDD on the following SF-36 items: MCS, PF, VT, MH, RE, SF, and $\mathrm{GH}$ scores $(\mathrm{P}=.000)$. The SF-36 MCS was highly correlated with the physician diagnosis of MDD using DSM-IV criteria in chronic noncancer pain patients. In addition, chronic pain patients with MDD had significantly lower MCS and lower scores on 6 domains of the SF-36. The SF-36 in this patient population can be a useful clinical tool to detect MDD, assess their quality of life, and potentially measure patient outcomes.

108/ PREDICTING PSYCHOSOCIAL OUTCOME OF PATIENTS UNDERGOING LUMBAR DISC SURGERY

Max Leibetseder, Social Medical Service, Government of Salzburg, Salzburg, Salzburg, Austria; Karoline V. Greimel, Institute of Clinical Psychology, General Hospital, Salzburg, Salzburg, Austria; Wolfgang P. Piotrowski, Gabriele Kohlboeck, Neurosurgery, Christian Doppler Medical Center, Salzburg, Salzburg, Austria; Regine Klinger, Institute of Psychology III, University of Hamburg, Hamburg, Germany

The aim of the study was to predict the psychosocial outcome of patients undergoing lumbar disc surgery based on a complex, multidimensional criterion. Forty-eight consecutive patients were included in this study (50\% male, $50 \%$ female). Medical, psychological and social predictor variables were assessed 1 week before surgery. The medical predictors were lasegue-sign (straight leg raising test), duration of pain, and radicular distribution of pain. The following psychological predictors were used: pain intensity, depression, pain disability, sensory and emotional perception of pain, and working distress. In addition we measured educational and social status, occupational characteristics, and the duration of inability to work. The psychosocial outcome variables were measured 6 months after lumbar disc surgery, regarding pain intensity, pain locations, functional status, return to previous job and health-related quality of life. The hierarchical cluster analysis showed three groups of outcome of surgery. Good results were observed in $29 \%$ patients with no pain, high functional status and high quality of life, while $27 \%$ of patients achieved only a moderate success rate. $44 \%$ of all patients reported a poor operation outcome with severe pain, low functional status and quality of life. Discriminatory analysis showed that $92 \%$ of the three outcome groups could be correctly classified by the combination of clinical, psychological and social predictors. The results indicate that psychological aspects are important predictors in long-term outcomes of surgery. Consequently, they should be included in clinical decision-making of patients with low back pain. 
109/ ASSOCIATION OF GENERIC AND SPECIFIC HEALTH RELATED QUALITY OF LIFE WITH MORTALITY IN COPD PATIENTS A. Domingo-Salvany, HSR Unit (HSRU), R. Lamarca, M. Ferrer, HSRU, J. Garcia-Aymerich, Respiratory and Environmental Research Unit (RERU), J M. Antó, RERU, G Vilagut, HSRU, IMIM, Barcelona Spain, M Félez, Hospital del Mar, Barcelona, Spain, A Khalaf, Hospital de la Magdalena, Castelló, Spain, E Monsó, Hospital Germans Tries i Pujol, Badalona, Spain; R. Marrades, Hospital Clínic, Barcelona, Spain, J. Serra-Batlle, Hospital General, Vic, Spain, J. Alonso, HSRU, IMIM, Barcelona, Spain

Generic health related quality of life (HRQL) has been found associated with all cause mortality in COPD patients. However it is unclear whether this association was specific both for $\mathrm{HRQL}$ and cause of death. Very rarely the association between HRQL and mortality has been studied in specific disease populations. To assess whether generic and specific $\mathrm{HRQL}$ predicts total and respiratory mortality in COPD patients, independently of clinical predictors, a cohort of 321 male COPD patients were followed from April 1993 to November 1999 (mean follow-up time 4.8 years, SD 1.8). Mean age at entry was 64.9 years, SD 9.6. HRQL was assessed through the Spanish SF-36 and Saint George's Respiratory Questionnaire (SGRQ). Among other variables, age, smoking status, body mass index (BMI), FEV1, bronchial responsiveness, self reported chronic conditions, social class and education were recorded. Vital status at November 30th, 1999 was obtained through reinterviews and the Mortality Register in 303 $(94.4 \%)$ patients, 197 of whom were alive $(65 \%)$. Comparing baseline data of those individuals alive with those dead at the end of follow-up, they were younger ( 62.5 vs 69.8 years), a higher proportion were current smokers ( $43.1 \%$ vs $25.3 \%$ ), had a higher BMI ( 27.1 vs 25.4 ) a higher \%FEV1 (49.2 vs 36.2 ) and had higher physical and mental summary scores for SF-36 (40.6 and 51.0 vs 37.1 and 49.1 ) and a lower total score of SGRQ (39.9 vs 49.9). No differences were found in bronchial responsiveness, chronic conditions, education and social class. In two independent Cox models including age, FEV1, and BMI, both SF-36 physical summary and SGRQ total scores showed a significant association with total mortality (standardised hazard ratio 1.32 and 1.30, respectively, per impaired SD units). Both generic and specific $\mathrm{HRQL}$ measures equally predict mortality in COPD patients, independently of clinical severity. (supported by FIS grant 99/ 0690.)

\section{0/ PREDICTORS OF MORTALITY IN PATIENTS WITH CHRONIC} RESPIRATORY FAILURE

Mauro Carone, Respiratory Disease, Fondazione Salvatore Maugeri, Veruno, (NO), Italy; Sabina A. Antoniu, Clinic of Pulmonary Disease, GR. T. PoPa University of Medicine and Pharmacy, lasi, lasi, Romania; Paul W. Jones, Division of Physiological Medicine, St. George's Hospital Medical School, London, UK; Claudio F. Donner, Division of Pulmonary Disease, Fondazione Salvatore Maugeri, Veruno, (NO), Italy

The Quality of Life Evaluation and Survival Study (QuESS, ERJ 1998 $12: 296 \mathrm{~s}$ ) is a multinational project (nine countries) which aims to evaluate over a three-year follow up period the natural history, prognostic factors of mortality and health care utilisation in chronic respiratory failure. Four-hundred forty-two patients on LTOT and/or overnight mechanical ventilation are presently enrolled (males 307 ); 173 have been followed up for one year (interim data). Baseline FEV1 $=37 \pm 16 \%$. Fiftytwo patients died in the first year ( $23 \%$ mortality). We stratified baseline results splitting patients died vs. survivors at the first year. Baseline FVC\%, FEV1\%, RV\%, TLC\%, desaturation during the Shuttle Test and blood gases were not significantly different between groups. Shuttle distance was slightly less in the non-survivors group $(-43 \mathrm{~m}$; $p<0.03$ ). Conversely, all the beseline measures of health status were statistically and clinically more impaired in the subgroup of of patients who died, with the exception of the mental component (MCS) of the SF36 and the activity component of the SGRQ (Table). The interim QuESS data show that Health Status (Quality of Life) is a better predictor of mortality than functional measures in patients with chronic respiratory failure.

\begin{tabular}{|l|l|l|l|}
\hline Parameter & Difference (p-value) & Parameter & Difference (p-value) \\
\hline SGRQ, Symptoms & $10.4(0.005)$ & MRF28, Invalidity & $14.7(0.003)$ \\
\hline SGRQ, Activity & $6.2(\mathrm{NS})$ & MRF28, Activity & $18.8(<0.0001)$ \\
\hline SGRQ, Impacts & $11.8(0.0001)$ & MRF28, Cognitive & $17.8(0.001)$ \\
\hline SGRQ, Total & $10.9(<0.0001)$ & MRF28, Total & $16.4(<0.0001)$ \\
\hline
\end{tabular}

111/IS IT POSSIBLE TO PREDICT PATIENT'S QOL AFTER OPEN HEART SURGERY?

Zoran R. Potic, Djordje Jakovljevic, Marijela M. Potic, Miroslava Popov, Ninoslav D. Radovanovic, Cardiovascular Surgery, Institute of CVD, Sremska Kamenica, Vojvodina, Yugoslavia

Using QOLi-NS index, an integral numerical value for measuring disease specific health related Quality of life in cardiovascular patients undergoing open heart surgery, we tried to develop a simple (additive) model for predicting improvement of QOL after surgery. Target variable was improvement of QOL six months after open heart surgery calculated as difference between QOLi-NS scores before and after surgery. For factors influencing changes in QOLi-NS we chose widely available preoperative clinical data. We collected relevant data for 294 consecutive patients (both risk factors and QOLi-NS scores before and six months after operation). After applying multivariate stepwise linear regression using QOLi-NS improvement as dependent variable and set of preoperative risk factors as independent ones, a simple linear (additive) model with 18 risk factors was created. Correlation between predicted QOL improvement and observed was very strong $(r=0.78 p<0.001)$. Patients form training set were stratified into four groups according to the predicted QOLi-NS improvement. The developed method was applied to another group of 465 patients and also has shown strong capabilities of prediction. Obtained method may be used for prediction of patient's outcome after open heart surgery in order to help patients to weight risks and benefits of an operation and as quality monitoring tool for healthcare professionals.

Table/ Predicted and Observed Improvement of QOLi-NS Score

\begin{tabular}{|l|l|l|l|l|l|}
\hline QOLi-NS improvement & $<30$ & $30-45$ & $45-60$ & $>60$ & Overall \\
\hline Predicted QOLi-NS & 11.4 & 39.3 & 51.8 & 64.9 & 40.7 \\
\hline Observed QOLi-NS & 11.0 & 40.5 & 51.2 & 65.1 & 40.7 \\
\hline
\end{tabular}

112/ RELATIONSHIP BETWEEN COMORBIDITIES AND HEALTHRELATED QUALITY OF LIFE IN CHRONIC RESPIRATORY FAILURE: A QUESS GROUP STUDY

Sabina A. Antoniu, European Respiratory Fellow at Divisione di Pneumolo, Mauro Carone, Claudio F. Donner, Divisione di Pneumologia, Salvatore Maugeri Foundation, Veruno, NO, Italy; Paul W. Jones, Division of Physiological Medicine, St. George's Hospital Medical School, London, UK

The Quality of Life and Survival Study (QuESS) is a multicentric, multinational, observational study, designed to assess health related quality of life and survival predictors in patients with chronic respiratory failure (Monaldi Arch Chest Dis 2001). 449 patients have been initially enrolled ( 311 males). Mean baseline FEV1 was $37 \pm 16 \%$. All patients are on LTOT and/or overnight mechanical ventilation. At baseline $240(53 \%)$ patients with comorbidities were detected, cardiovascular diseases being the most prevalent, $n=92(20 \%)$. We analysed health-related quality of life (SGRQ, MRF28, MRC dyspnoea scale, anxiety, depression) in patients with versus patients without comorbidities (Table). Baseline data show that comorbidities do not influence health status as measured by QoL questionnaires. In particular, when considering SGRQ and MRF28, this means that both questionnaires are well constructed in that they appear really specific to the underlying disease (chronic respiratory failure) and are not confounded by comorbidities.

\begin{tabular}{|l|l|l|l|}
\hline & Comorbidities $\mathbf{Y}$ & Comorbidities N & p-value \\
\hline SGRQ & $52 \pm 18$ & $53 \pm 16$ & NS \\
\hline MRF28 & $47 \pm 25$ & $43 \pm 23$ & NS \\
\hline MRC dyspnoea & $3.1 \pm 1.4$ & $2.9 \pm 1.2$ & NS \\
\hline HADS anxiety & $6.5 \pm 4.6$ & $6.2 \pm 4.3$ & NS \\
\hline HADS depression & $6.8 \pm 4.3$ & $6.7 \pm 4.1$ & NS \\
\hline
\end{tabular}


113/ SURVIVAL, QUALITY OF LIFE AND THE SEVERITY OF SYMPTOMS OF CANCER PATIENTS

Tito R. Mendoza, Shelley Wang, Cielito Reyes-Gibby, Charles S. Cleeland, Pain Research Group, The University of Texas M.D. Anderson Cancer Center, Houston, TX

The purpose of our study was to evaluate the relationship between quality of life, severity of symptoms and survival in cancer patients. One hundred thirteen patients were administered the M.D. Anderson Cancer Symptom Inventory (MDASI), the SF36 and three VAS items assessing health-related quality of life in a cross-sectional survey. Survival and vital status data were matched with the survey data Results showed that patients who subsequently died reported significantly poorer quality of life as measured by the physical $(p<.003, r=.28)$ and mental $(p<.032, r=.21)$ subscales of the SF36 compared with those who were still alive at the time of last contact. The groups did not significantly differ in their health-related quality of life using the three health-related quality of life VAS items. Only the physical subscale of the SF36 is significantly related with survival $(p<.02, r=.22)$. The study also showed that the number of severe symptoms, those rated seven or greater on a 0 to 10 scale, is significantly related with reported physical $(p<.001, r=.67)$ and mental $(p<.001, r=.64)$ subscales of the SF 36 but not with survival. The data suggest that reported healthrelated quality of life is significantly associated with survival. However, patients' symptom ratings were not predictive of survival in this study.

114/ DEFINING RECOVERY: THE APPLICATION OF CLUSTER ANALYSIS TO LONGITUDINAL HEALTH STATUS DATA IN PHYSIOTHERAPY PATIENTS

Dorcas E. Beaton, Neuromusculoskeletal Program, St Michael's Hospital, Toronto, ON, Canada; Carol Kennedy, Michael Manno, Deirdre McKenzie, Sheilah Hogg-Johnson, Institute for Work \& Health, Toronto, ON, Canada; Laurie Hurley, Marla Nayer, College of Physiotherapy of Ontario, Toronto, ON, Canada

Traditionally final health status or change in health status score are used to define "recovery". However longitudinal patterns of health status data can combine initial and final state with speed of change to offer a more informative description of health over time. The purpose of this study was to apply cluster analytic methods to longitudinal health status data in order to identify any 'typical' patterns of recovery. 362 patients undergoing physiotherapy for shoulder tendinitis completed the DASH (Disabilities of the Arm Shoulder and Hand) on admission, four week and at discharge (variable time up to 12 weeks) visits. K-means cluster analysis identified groups of patients with similarity in four variables: the three DASH scores plus time to discharge. The model (number of clusters) was determined by a break in the distortion plot, a high pseudo-F statistic and the clinical sensibility of the clusters. Four clusters were identified (Pseudo-F=204). Cluster A $(n=53)$ had high disability at intake and little change over a longer time. Cluster $B(n=53)$ high initial disability but a rapid change in health state. Cluster $C(n=129)$ had midrange disability and changed slowly, Cluster $D(n=124)$ had very low level of disability and had a very quick change in DASH. Cluster A had a longer duration of symptoms. Cluster $B$ and $D$ had earlier discharge than the others and achieved their treatment goals at the time of discharge. Conclusions: cluster analysis was a useful way to identify four unique patterns of recovery in these patients. Descriptions of patients in the clusters demonstrate the differences. Patterns of recovery may prove more clinically useful than absolute change or final health status scores alone.
115/ ROC ANALYSIS OF WEIGHT LOSS TO ACHIEVE CLINICALLY MEANINGFUL IMPROVEMENTS IN HEALTH-RELATED QUALITY OF LIFE

Ross D. Crosby, Biomedical Statistics, Neuropsychiatric Research Institute, Fargo, ND; Ronette L. Kolotkin, Psychiatry, Duke University Medical Center, Durham, NC; G. R. Williams, Knoll Pharmaceuticals, Abbott Laboratories, Mount Olive, NJ; Guilford G. Hartley, Department of Medicine, Hennepin County Medical Center, Minneapolis, $\mathrm{MN}$

Health-related quality of life (HRQOL) has consistently shown improvement after weight reduction. One question that has not been addressed to date is the amount of weight loss necessary to produce clinically meaningful improvements in HRQOL. The purposes of the present research project were to determine (1) the amount of weight loss necessary to produce clinically meaningful improvements in HRQOL in obese patients, and (2) whether different subgroups of patients (e.g., male vs. female, initial $\mathrm{BMI}$ ) require different amounts of weight loss to achieve clinically meaningful improvements in $\mathrm{HRQOL}$. Baseline and 6-month outcome data were compiled from over 1000 obese subjects enrolled in 1 of 6 weight loss treatment studies. HRQOL was measured at baseline and 6 months using the Impact of Weight on the Quality of Life-Lite (IWQOL-Lite, Kolotkin \& Crosby, 2001). Clinically meaningful improvements in HRQOL were defined as a minimum reduction of 1.96 standard error of measurements (SEMs) in IWQOL-Lite scores. Receiver operating characteristic (ROC) analyses were performed to determine the amount of weight loss necessary to produce clinically meaningful improvements in HRQOL. ROC curves were determined for various patient subgroups (e.g., male vs. females, BMI at baseline) and areas under the curve (AUC) were compared. Results indicated that (1) weight loss was associated with clinically significant improvements in IWQOL-Lite total score (AUC = .697) and all scales (AUCs = .592-.681) except Work, (2) weight loss which maximized sensitivity and specificity for clinically meaningful improvements ranged from 5.4\% (Total) to $6.7 \%$ (Public Distress), (3) weight loss was more strongly associated with clinically significant improvement in IWQOL-Lite Total score for males (AUC = .728) than females (AUC $=.671$ ), and for those with higher initial BMls, (4) males required less weight loss than females to achieve clinically meaningful improvement.

116/ RESPONSIVENESS OF THE QUALITY OF LIFE IN EPILEPSY INVENTORY (QOLIE-89) IN AN ANTIEPILEPTIC DRUG TRIAL Sehyun Kim, Preventive Medicine, College of Medicine, Pochon CHA University, Sungnam, Kyonggi-do, Korea; Ron D. Hays, General Internal Medicine, UCLA, Los Angeles, CA; Gretchen L. Birbeck, Neurology \& Epidemiology, Michigan State University, East Lansing, Ml Barbara G. Vickrey, Neurology, UCLA, Los Angeles, CA

This study examined relationships among responsiveness indices for health-related quality of life (HRQOL) measures administered to adults with epilepsy enrolled in an antiepileptic drug trial. The Quality of Life in Epilepsy (QOLIE)-89 was completed at baseline and at a 28-week follow-up. Six responsiveness indices (effect size, standardized response mean, responsiveness statistic, paired t-test, area under receiver operating characteristic curve or ROC, F-statistic) were calculated for each of the 16 QOLIE-89 scales, using two different external criteria for clinically significant change: (1) attainment of complete seizure freedom, and (2) a 2-category improvement between baseline and follow-up in a self-rating of the subject's overall condition. Spearman correlations among the 6 responsiveness indices for the 16 QOLIE-89 scales tended to be moderate to large (Spearman's rho $=0.53$ to $1.00 ; p$ 's $<0.05$ for 29 out of the 30 correlations). Rankings of the 16 scales across the two external criteria for change were similar for the responsiveness statistic (Spearman's rho $=0.62 ; p<0.05$ ), but dissimilar for the other responsiveness indices (all $p>0.05$ ). Both effect size and standardized response mean were well predicted by the other indices, except for ROC, using regression modeling. In conclusion, results using different responsiveness indices are comparable for a given external criterion. However, only the responsiveness statistic yielded robust results across 2 different external criteria. Responsiveness of this HRQOL measure can be reported in terms of previously established benchmarks for effect sizes, which can be predicted from other indices. 
117/ WHAT IS A CLINICALLY MEANINGFUL CHANGE IN THE MEASUREMENT OF FATIGUE?

David Cella, David T. Eton, Jin-Shei Lai, Amy H. Peterman, Evanston Northwestern Healthcare (ENH), Northwestern University (NU), Evanston, IL

The objective of this analysis was to determine clinically meaningful changes (CMCs) on the Functional Assessment of Cancer Therapy Fatigue (FACT-F) and Functional Assessment of Cancer Therapy Anemia (FACT-An). Anchor-based and distribution-based methods were used to provide ranges of $\mathrm{CMC}$ for five targeted scale scores of the FACT-F and FACT-An. Three samples of cancer patients were used: Sample 1 was 50 patients participating in a validation study of the FACT-An; Sample 2 included 131 patients participating in a longitudinal study of chemotherapy-induced fatigue; Sample 3 included 2,402 patients enrolled in a community-based clinical trial evaluating the effectiveness and safety of a treatment for anemia. Three clinical indicators (hemoglobin level; performance status; response to treatment) were used to determine anchor-based differences. One-third and $1 / 2$ of the standard deviation and 1 standard error of measurement were used as distribution-based criteria. Analyses supported the following whole number estimates of a CMC for these five targeted scores: Fatigue Scale $=3.0 ;$ FACT $-G$ total score $=4.0 ;$ FACT An total score $=7.0$; Trial Outcome Index - Fatigue $=5.0$; and Trial Outcome Index - Anemia $=6.0$. These estimates provide a basis for sample size estimation when planning for a clinical trial or other longitudinal study, when the purpose is to ensure detection of meaningful change over time, or to estimate the proportion of people in a given cohort who improve or decline significantly. They can also be used in conjunction with more traditional clinical markers to assis investigators in determining treatment efficacy for a population of patients. Caution is recommended when interpreting the magnitude of change in a given individual or group over time.

\section{8/ MULTISAMPLE MEAN AND COVARIANCE STRUCTURE} MODELS FOR ASSESSING RESPONSIVENESS

Thomas Kohlmann, Institute for Social Medicine, Medical University of Luebeck, Luebeck, Germany; Joachim Leonard, Boehringer Ingelheim Pharma KG, Ingelheim am Rhein, Germany; Heiner Raspe, Institute for Social Medicine, Medical University of Luebeck, Luebeck, Germany; Henning Zeidler, Hannover Medical School, Hannover, Germany

Measurement of quality of life is often based on psychometric models involving latent variables such as factor analysis or latent trait models. Yet, responsiveness of these measures is usually determined referring to observed indicators (items, composite scores) only. The objective of this paper is to explore the applicability of structural equations models including mean structures for assessing responsiveness on the observed and on the latent level. As part of a drug monitoring study (Meloxicam) $\mathrm{N}=2,377$ patients with rheumatoid arthritis (RA) and $\mathrm{N}=6,211$ patients with osteoarthritis $(\mathrm{OA})$ completed a 21 item questionnaire comprising items from three condition-specific (OARA-, and back pain-specific) versions of the Hannover Functional Ability Questionnaire (HFAQ). Multisample mean structure models were fitted to the data using the structural equations program EQS Overall model fit was evaluated by normed fit indexes and standardized residuals. Each set of condition-specific items was analyzed in separate models. Differential responsiveness was found for RA- and $O A$-specific items in observed and latent variables. RA-specific $(O A-$ specific) items were more responsive in RA (OA) patients. Tests of invariance constraints imposed across groups indicated that itemspecific differences in change over time exist. Analysis of latent variables provided more clear-cut and comprehensive figures of differential responsiveness and demonstrated that relevant violations of some basic assumptions underlying the standard factor analysis mode (e.g. zero correlation of error terms) occurred. Multisample mean and covariance structure models represent a versatile method for studying responsiveness of quality of life measures within a latent variable framework. Results provide additional information not usually available when only observed variables are analyzed. However, multisample mean structure models appear to be rather complex and difficult to apply in particular data sets and may therefore not be adequate in all situations.

119/ A COMPARISON OF THE DISCRIMINATIVE AND EVALUATIVE PROPERTIES OF SF-36 SCALES AND SUMMARY MEASURES AND THE SF-6D INDEX
John Brazier, Scharr, The University of Sheffield, Sheffield, UK: Samuel J. Sinclair, Barbara Gandek, John E. Ware, Jr., Health Assessment Laboratory, Boston, MA

A concern in moving from a profile of health scores to a single index is a potential loss in sensitivity to group differences and change over time. A significant loss would result in the need for larger samples in clinical studies to detect differences and risk a false acceptance of the null hypothesis due to the loss of power. The recent development of a preference-based index for the SF-36 (the SF-6D) provides an opportunity to examine this issue for a common descriptive system. We compared the sensitivity of the eight SF-36 scales, the physical (PCS) and mental (MCS) health summary scores, and the SF-6D index for patients with COPD $(n=91)$, osteoarthritis (medical treatment $[n=102]$ and surgery [ $n=95])$, leg ulcer $(n=231)$, depression $(n=412)$, and seniors who were in an exercise program $(n=6722)$. Patients were classified into severity groups at baseline (e.g., degree of breathlessness, clinician rating) and by responsiveness to treatment over time or self-evaluated change in health. As reported below comparisons between measures were made in terms of effect sizes. The SF-6D index was found to compare favorably to the scales and summary measures in tests in which no one outcome dominated. In tests in which one outcome was predominant (e.g., mental health in depression), the SF-6D was stronger than some measures but not as strong as the dominant outcome. Overall, however, these findings suggest that the use of the SF-6D index in analysis does not significantly reduce the power of a study compared to scale scores and summary measures.

Table/ Comparison of Effect Sizes

\begin{tabular}{|l|l|l|l|l|l|l|l|l|}
\hline & $\begin{array}{l}\text { X-Sectio- } \\
\text { nal }\end{array}$ & & & & Longitudinal & & & \\
\hline & Scales & PCS & MCS & SF-6D & Scales & PCS & MCS & SF-6D \\
\hline COPD & $0.12-1.27$ & 0.70 & 0.68 & 0.94 & $0.03-0.66$ & 0.47 & 0.15 & 0.25 \\
\hline OA Medical & $0.26-0.73$ & 0.49 & 0.56 & 0.72 & $0.01-0.52$ & 0.31 & 0.27 & 0.68 \\
\hline OA Surgical & $0.23-1.00$ & 0.67 & 0.51 & 1.08 & $0.13-0.69$ & 0.49 & 0.02 & 0.38 \\
\hline Leg UIcer & $0.08-0.37$ & 0.22 & 0.06 & 0.22 & $0.19-0.57$ & 0.52 & 0.24 & 0.53 \\
\hline Depression & $0.03-1.55$ & 0.18 & 1.64 & 0.92 & $0.01-0.85$ & 0.18 & 1.02 & 0.33 \\
\hline Elderly & $0.50-0.78$ & 0.65 & 0.55 & 0.72 & $0.01-0.08$ & 0.05 & 0.06 & 0.07 \\
\hline
\end{tabular}

120/ USING STRUCTURAL EQUATION MODELS (SEM) TO INTERPRET TREATMENT SATISFACTION AND QUALITY OF LIFE DATA: THE CASE FOR INHALED INSULIN

Ralph R. Turner, PHASE V Technologies, Inc., Wellesley Hills, MA, Marcia A. Testa, Biostatistics, Harvard School of Public Health, Boston, MA

Intensive insulin therapy reduces symptoms and long-term complications of both type 1 and type 2 diabetes. Glycemic levels remain high due to treatment-related barries. Better glycemic control improves QOL and decreases health care costs, making understanding barriers to use important for treatment planning. Insulin delivery systems now include inhaled doses. We employed SEM to analyze self-administered QOL and treatment satisfaction (SAT) change data in a sample (164 inhaled, 156 injected) of type 1 patients randomly receiving pre-meal inhaled insulin plus single injection or 2-3 injections daily for 24 weeks. The structural model comprised 2 QOL latent variables (LV) (Health Status and Subjective Filter, e.g. perceived health) and 2 SAT LVs (Positive, e.g. general satisfaction, and Hinderence, e.g. burden). The measurement model comprised 5 Health Status, 4 Subjective Filter, 4 Positive, and 6 Hinderence scales. Factor loadings within the measurement model, and covariances among the structural LVs were assessed. Significant factor loadings confirmed the measurement model for both treatment groups. The results revealed a good fit $\left(X^{2}=13.9, p>.05, C F I=.94\right.$, RMSEA $\left.=.04\right)$ ). The $Q O L$ (Health Status, Subjective Filter) and SAT LVs (Positive, Hinderence) were significantly correlated. Significant correlations between the QOL LVs and Hinderence were found for the injected group. Significant LV mean differences favored the inhaled group for Health Status, Positive, and Hinderence but not Subjective Filter. Patients with similar levels of glycemic control, achieved via two different delivery methods, reported different QOL impact and treatment satisfaction. The inhaled group had larger Health Status changes coupled with more positive and less negative satisfaction changes. Significant assocations between health status and subjective filter changes and injection-related barriers provide important guidance regarding the adopting alternative treatment approaches to achieve glycemic control. 
121/ VALUE SHIFT AFTER DISABILITY ONSET: A MINOR ISSUE? Marcel P. Dijkers, Rehabilitation Medicine, Mount Sinai School of Medicine, New York, NY

It has been claimed that significant life events such as disability result in shifts in values that affect quality of life (QOL) judgments. Through reconceptualization, reprioritization, and recalibration individuals change what they value and how they value it, maintaining high overall QOL assessments. While longitudinal designs are optimal to investigate the phenomenon, comparison of values between groups characterized by a disability/ chronic illness and "normals" has also been used The purpose of this study was to evaluate to what degree this phenomenon occurs, and how much difference it actually makes for QOL assessment. The Flanagan QOL questionnaire (FQOLQ) was completed by samples of persons with a spinal cord injury $(\mathrm{N}=100)$, traumatic brain injury $(\mathrm{N}=400)$; other type of brain disorder $(\mathrm{N}=125)$; HIVIAIDS $(\mathrm{N}=230)$; liver transplant $(\mathrm{N}=100)$ and non-disabled (ND) persons $(N=280)$. The FQOLQ consists of ratings of the importance of 15 broad domains of life (health and personal safety; work; material concerns; etc.) to QOL, followed by 15 ratings of the degree to which one's needs in the area in question are met. Overall QOL for each subject in the clinical samples was calculated in three ways: weighted sums based on (1) one's own importance ratings; (2) one's own disability group's average importance ratings; and (3) the ND group's average importance ratings. There were statistically significant differences between individual disability samples and the ND group in importance ratings. However, the profiles of both mean ratings and percent of cases selecting the "very important" response were very similar for all groups. The FQOLQ total scores calculated in different ways correlated strongly with one another: ICC3.1 between methods (1) and (2) and between methods (1) and (3) was at least 0.80 and up to 0.86 for all samples and for the samples combined. These results suggest that importance ratings of $Q O L$ domains are of limited consequence, and that investigators using the FQOLQ or similar measures may do better focusing on reliable assessment of needs satisfaction in the various life domains.

122/ COMPARISON OF UNWEIGHTED SATISFACTION SCORES AND SCORES WEIGHTED BY RELATIVE IMPORTANCE IN MEASURING QUALITY OF LIFE FOR ADULTS WITH CHRONIC HEALTH PROBLEMS

Janet E. Jeffrey, School of Nursing, York University, Toronto, ON, Canada

The purpose of this presentation is to compare scores for the Qualify of Life Index - QOLI (Ferrans \& Powers, 1985) that have been weighted (as designed) by subjects' ratings of importance of items to unweighted scores that measure satisfaction with life domains. Weighted scores are reflect values assigned by subjects to measure perceived QOL. Weighting is accomplished by multiplying the ratings of satisfaction with each life domain by the rating of importance with the same life domain. Data included in this comparison were provided by adults with chronic illnesses: rheumatoid arthritis (samples of 96 and 141) or fibromyalgia syndrome (142) who were primarily women, and coronary heart disease (103), primarily men. Subjects ranged in age from 21 to 70 , with mean 47 to 59 years. Most were married and had completed high (secondary) school or some post-secondary (college/university) education. Overall quality of life was significantly lower for weighted scores for all groups than for unweighted scores, using univariate statistics (paired t-test) with $p<.001$, although the numerical difference in means is small and may be of little obvious clinical significance. This pattern held for scored of four QOL subscales: physical function, socioeconomic, psychological/spiritual, and family. However, differences in the family subscale were not different in all samples. Most of the differences between weighted and unweighted scroes disappeared when the effect of age was controlled, but not when sex was controlled (likely from very uneven groups of men and women). The findings of this secondary analysis pose interesting questions given the QOL measure used is one of the few that condisers the values and perspective of the subjects rather than focusing on satisfaction alone. It appears that such weighting may not make any real difference, but suggests that the effect of known covariates be considered. Other questions will also be posed with further data analysis.
123/ ASSESSMENT OF QUALITY OF LIFE IN CHRONIC BRONCHITIS AND ITS ACUTE EXACERBATIONS: COMPARISON OF A GENERIC AND A CONDITION-SPECIFIC MEASURE

Helen A. Doll, Oxford Outcomes, Oxford, Oxon, UK; Isabelle DupratLomon, Health Economics and Outcomes Research, Bayer Pharma, Paris, France; Erika D. Ammerman, Oxford Outcomes, Oxford, Oxon, UK; Pierre-Philippe Sagnier, Health Economics and Outcomes Research, Bayer plc, Slough, Berkshire, UK

Chronic bronchitis (CB) and its acute exacerbations (AECB) are associated with marked effects on QoL. This study reports on a comparison of the psychometric properties of the generic Nottingham Health Profile (NHP) and the condition-specific St. George's Respiratory Questionnaire (SGRQ) in assessing the QoL of CB patients during an $A E C B$ and later at a stable phase. The data for this comparison come from a study in which data on a wide range of sociodemographic, risk and clinical factors were collected on 320 subjects during an AECB and around 6 months later at a stable phase. While the two instruments are significantly but moderately associated with each other at the stable phase (Spearman correlation coefficients 0.4-0.6, $p<0.001$ ), the SGRQ has stronger associations than the NHP with other demographic, risk and clinical factors, thus providing at non-AECB a more sensitive measure of QOL. For example, only the NHP energy and physical mobility domains are significantly associated with physician-assessed CB severity, while all SGRQ domain scores show trends for increasing score with increasing $C B$ severity. The NHP is also not as sensitive to the deterioration in QoL associated with the presence of air pollution and increasing co-morbidity. A AECB, however, the SGRQ does not provide such a sensitive QoL measure, with the Symptom domain (which records symptoms over the previous 12 months) showing similar scores at both assessments. This is further illustrated by poorer correlation between the NHP and SGRQ at AECB (Spearman correlations $0.3-0.5$, all $p<0.06$ ). The SGRQ, and particularly its Symptom domain, is thus less likely to provide a responsive measure when measuring QoL change from $A E C B$ to a stable phase (mean improvement in SGRQ symptom score in those with mild, moderate, or severe $C B$ at non-AECB $=2.1,5.3$, 1.7, NS; NHP Energy improvement $=34.6,11.6,0.6, p<0.05)$. The implications of this apparent limitation of the SGRQ will be discussed. 
124/ AN INDIVIDUALIZED APPROACH IN THE MEASUREMENT OF QUALITY OF LIFE IN LONG-TERM SURVIVORS OF HODGKIN'S DISEASE

Lena Wettergren, Magnus Björkholm, Department of Medicine and Division of Hematology, Karolinska Institutet and Hospital, Stockholm, Sweden; Ann Bowling, Department of Primary Care and Population Sciences, Royal Free and University College Medical School, London, UK; Ulla Axdorph, Division of Hematology, Karolinska Hospital, Stockholm, Sweden; Ann Langius-Eklöf, Department of Nursing, Karolinska Institutet, Stockholm, Sweden

The aim of this study was to delineate what areas in life that are important for long-term survivors of HD and how life is influenced of the disease, using an individual approach in addition to standardized questionnaires measuring health-related quality of life. Furthermore, the findings were to be compared with a sample from the general population. Long-term survivors from $\mathrm{HD}(\mathrm{n}=121)$ were compared with a randomly selected sample of the general population in Stockholm $(n=236)$. They were approached with one semi-structured interview, The Schedule for the Evaluation of The Individual Quality of Life Direct Weighting (SEIQoL-DW) which explores important areas in life crucial for quality of life and rates the current status of the nominated areas. In addition three self-administrated questionnaires were used: 1. The Hospital Anxiety and Depression (HAD) scale. 2. Short Form 12 health survey (SF-12) and 3. The Sense of Coherence (SOC) scale. The results of the SEIQoL-DW method showed that the most commonly nominated areas, by more then fifty percent of the survivors as well as by the controls, were the family, own health, work and relations to other people. Generally the problems following HD were few and the reported limitations showed great individual variation. No differences regarding the mean scores were found between the HD survivors and the controls with the exception of the SF-12 $(p<0.01)$ the patients considered themselves to be in poorer physical health than the controls. It is suggested that future studies focusing on quality of life in HD patients should include the SEIQoL-DW as a complement to existing standardized instruments for measuring quality of life at different points during and after treatment, including clinical trials.

125/ QUALITY OF LIFE IN LONG-TERM PROSTATE CANCER SURVIVORS AND THEIR SPOUSES

Carolyn C. Gotay, Miles Y. Muraoka, Cancer Research Center of Hawaii, University of Hawaii, Honolulu, $\mathrm{HI}$

This report examines quality of life (QOL) in 181 long-term prostate cancer survivors and their spouses. Participants were identified by a population-based tumor registry, based on prostate cancer diagnosis five $(n=86)$, eight $(n=76)$, or $11(n=19)$ years ago. Survivors received no therapy ("watchful waiting") $(n=17)$, surgery $(n=84)$, or radiation $(n=80)$. Data were collected through mailed questionnaires including standard measures of depression (CES-D), functioning (MOS), sexual functioning, and treatment satisfaction. Mean age was $77 \mathrm{yrs}$ for survivors and 72 yrs for spouses. $75 \%$ of patients and wives were of Asian/Pacific Islander (API) descent. Overall, survivors and spouses were doing well, with mean scores of 5.6 or higher on a 7-point global QOL scale for all groups, and $14 \%$ reporting depression levels above CES-D cutoffs. Survivors who received radiation therapy had significantly impaired physical and bowel functioning and role limitations due to physical health and emotional problems, compared to surgery patients. Group differences were maintained for physical and bowel functioning using analyses of covariance (ANCOVA) with age as the covariate. Men who received surgery reported significantly more sexual problems than survivors who received watchful waiting or radiation. These differences persisted when patient age was included in an ANCOVA. The groups did not differ in urinary functioning satisfaction. Treatment satisfaction ratings showed that wives in surgical and radiation groups were more pleased with their husbands' treatment than the survivors were. Survivors who received watchful waiting and their wives were significantly more likely to report that they would choose the same treatment again, compared to surgery-treated survivors and their wives. This study indicates that prostate cancer treatment is associated with related morbidities even many years after treatment cessation. Treatment satisfaction appears to derive from factors other than our measures of QOL and symptom experience.
126/ QUALITY OF LIFE OF MALIGNANT BRAIN TUMOR PATIENTS UNDERGOING GAMMA KNIFE RADIOSURGERY

Peggy A. Ward-Smith, School of Nursing, The University of MissouriKansas City, Kansas City, MO

Brain tumors are second only to stroke as the leading neurological disease in the United States. Despite medical advances in the treatment of brain tumors, the disease remains uniformly fatal. Gamma knife radiosurgery (GKR), a palliative treatment, is a method of delivering a single focused beam of radiation therapy directly to the tumor. Quality of life (QOL), as a treatment outcome, has become an important attribute of health care, especially for those who treatment is not curative. A review of the current literature finds no research related to GKR's potential impact on QOL, as evaluated by the patient. The purpose of this multiple-method descriptive study was to evaluate QOL before and after GKR. Quantitatively, a previously developed instrument (FACT-Br), was administered before and after treatment. Qualitatively, a semi-structured interview was done after treatment to elicit information not covered by the instrument. Demographic information was also obtained to describe the sample population. A total of 65 participants, all who underwent GKR at the same Midwestern treatment center participated in the study. T-tests were utilized to compare before and after treatment FACT-Br (version 3) scores. The FACT-Br scores, as well as three of the subscale scores (functional well-being, emotional well-being and additional concerns) were statistically different. ANOVA was utilized to compare genders, and different tumor types. Neither were statistically different. The qualitative data uncovered six themes. The most prominent theme was that GKR had no effect on the participant's QOL. The results of this study should be carefully applied to the clinical area, for its results are conflicting. Additional research needs to be done prior to applying these findings in a clinical situation. The instrument utilized for this study appears to have sound psychometric properties when utilized in a before and after treatment situation.

127/ EFFECTS OF PSYCHOSOCIAL INTERVENTION ON QUALITY OF LIFE IN PATIENTS WITH HEAD AND NECK CANCER

Karin M. Petruson, Ewa Silander, Eva Hammerlid, Department of Otolaryngology, Head and Neck Surgery, Sahlgrenska University Hospital, Göteborg, Sweden

The purpose of this study was to evaluate if health-related quality of life $(H R Q L)$ in Head and Neck (H\&N) cancer patients was improved by psychosocial intervention. All patients with newly diagnosed $\mathrm{H} \& \mathrm{~N}$ cancer were asked to participate in a HRQL study. Patients living close to the hospital (within 30 min drive) were also offered weekly/ monthly counselling by a dietitian and a nurse, during the first year after diagnosis. Otherwise both groups were treated according to our depatment standards. Patients HRQL was evaluated according to the European Organization for Research and Treatment of Cancer Quality of Life Core Questionnaire (EORTC QLQ-C30), the EORTC QLQ-Head and Neck Cancer module (QLQ-H\&N35), and the Hospital Anxiety and Depression Scale (HADS). HRQL was measured at diagosis and 1,2,3,6,12 and 36 months after the start of treatment. In total 144 patients with $\mathrm{H} \& \mathrm{~N}$ cancer were included in the study. Fifty-two of the patients (mean age $60,81 \%$ male) were offered counselling. Visits ranged from 2-50 (mean 20). The remaining 92 patients (mean age $61,73 \%$ male) served as a control group. The two groups were clinically statistically alike at diagnosis, and evaluated their HRQL similarily, with a few exceptions. After 3 years $71 \%$ of the patients were alive in the control group, wereas $77 \%$ were alive in the study group. When changes over time for the two groups were compared, rather few significant differences were found. Both groups had worse HRQL the weeks after the treatment had finished. After one year, the control group had a better globalQL $(p=0.04)$, but this difference was not found after three years. The control group had a better role function $(p=0.017)$ and better sleep $(p=0.03)$ after three years The results will be further analysed, but so far no major HRQL differences have been found in favour for counselling in $\mathrm{H} \& \mathrm{~N}$ cancer patients. 
128/ PSYCHOSOCIAL CONSEQUENCES OF PREVENTIVE HEALTH OPTIONS AMONG WOMEN AT INCREASED RISK OF DEVELOPING OVARIAN CANCER: A PILOT STUDY

Judith Hollenstein, Division of Psychosocial Research and Epidemiology, The Netherlands Cancer Institute, Amsterdam, The Netherlands; Eveline M. Bleiker, Division of Psychosocial Research and Epidemiology, Marc van Beurden, Department of Gynecology, The Netherlands Cancer Institute, Amsterdam, The Netherlands; Heiddis Valdimarsdottir, Department of Cancer Prevention and Control, Mount Sinai Hospital, New York, NY; Neil A. Aaronson, Division of Psychsocial Research and Epidemiology, Netherlands Cancer Institute, Amsterdam, The Netherlands

Women at increased familial or genetic risk of (breast and) ovarian cancer may choose to undergo a prophylactic oophorectomy or annual gynaecological screening. This pilot-study was undertaken to investigate: (1) the psychosocial consequences of these two preventive health strategies; and (2) the sociodemographic, clinical and psychosocial factors related significantly to the choice between these strategies. The study sample included 36 women who attended the Department of Gynecology of the Antoni van Leeuwenhoek Hospital between 1995 and 1999 because of familial or hereditary (breast-) ovarian cancer. The sample was stratified according to preventive health strategy and personal history of breast cancer. In total, 29 women $(81 \%)$ completed a self-administered questionnaire by mail, 16 of whom had undergone a prophylactic oophorectomy and 13 of whom had opted for periodic screening. Women who had undergone an oophorectomy reported, on average, significantly less cancer-related worries, less generalized distress (MHI-5), fewer intrusive thoughts about cancer (IES), and perceived their risk of developing (extra-) ovarian cancer as lower than did women who opted for screening. Women who had undergone preventive surgery were also significantly more satisfied with their choice of preventive health action as compared to those who opted for screening. In a logistic regression model, only two variables were found to be significantly associated with the choice of prophylactic oophorectomy: mutation status (DNA carriership) and an active coping style characterized by information-seeking behavior. This pilot investigation is currently being replicated in a larger, multicenter study.

129/ GENDER DIFFERENCES IN FACTORS PREDICTING QUALITY OF LIFE AFTER SURGERY FOR COLORECTAL CANCER PATIENTS

M. Berend, J. Siebmann, P. Boll, T. Küchler, D. Henne-Bruns, B. Kremer, Reference Centre Quality of Life, Christian-Albrechts-University, Kiel, Germany

In 1998 the model "Operative Oncology" was established by the Department of Surgery of the University of Kiel. A main focus of the pilot project is to promote a high standard of treatment for cancer patients in order to improve their survival rates as well as their Quality of Life $(Q \circ L)$. A routine assessment of $Q o L$ in 7 diagnostic subgroups starting before the operation up to two years follow-up is part of the quality management. Diagnosis specific modules that complete the EORTC QLQ-C30 (core questionnaire) were developed prior to the study, so that detailed symptoms and problems of the different groups can be analysed. Besides answering clinical research questions we were interested which factors predict QoL over the course of the disease. In the presented analysis data from the preoperative time point to 6 month postoperatively (4 time points) were included. Furthermore since clinical experience indicates differences in profiles of QoL of different diagnostic subgroups, only patients $(N=100)$ with colorectal cancer were included. Exploring different models of prediction we found substantial gender differences: while postoperative QoL in men is mostly predicted by hope and confidence and medical factors for female patients somatic symptoms are predictive. Further analysis including more patients and a longer follow-up will assert if a) this model is stable and b) this model is applicable to other diagnostic subgroups of patients with gastrointestinal cancer.
130/ COLORECTAL CANCER SCREENING: COMPLIANCE OF FAMILES AT HIGH RISK

Eveline M. Bleiker, Department of Psychosocial Research and Epidemiology, Netherlands Cancer Institute, Amsterdam, The Netherlands; Fred H. Menko, Department of Clinical Genetics, University Hospital Vrije Universiteit, Amsterdam, The Netherlands; Lidwina D. Wever, Miranda A. Gerritsma, Department of Psychosocial Research and Epidemiology, Netherlands Cancer Institute, Amsterdam, The Netherlands; Irma Kluijt, Department of Clinical Genetics, Academic Medical Center, Amsterdam, The Netherlands; Babs G. Taal, Department of Gastroenterology, Neil K. Aaronson, Department of Psychosocial Research and Epidemiology, Netherlands Cancer Institute, Amsterdam, The Netherlands

For individuals with familial/ hereditary colorectal cancer, periodic screening of the colorectum is advised. The aims of this study were to investigate: 1) the extent to which high-risk individuals adhere to screening recommendations, 2) the self-reported reasons for noncompliance, and 3) factors related to compliance. In this cross-sectional study, questionnaires were sent to individuals counseled for hereditary (HNPCC) or familial colorectal cancer during 1986-1996 at three Family Cancer Clinics in Amsterdam. Of the 178 eligible individuals, $149(84 \%)$ participated in the study. Of the respondents $15 \%$ $(n=23)$ indicated having undergone fewer colon controls than advised on a self-report questionnaire, and $25 \%$ based on data obtained from medical files. The most important reasons for self-reported non-compliance were the unpleasant, painful and embarrassing nature of the screening and 'having no physical complaints'. All gene-mutation carriers reported being compliant versus $79 \%$ of those for whom the DNA-tests were non-conclusive. Psychosocial factors, including risk perception, levels of distress, cancer worries, and knowledge about colon cancer and its prevention, were not found to be significantly related to colon screening adherence in these high risk families. The results based on both self-report and medical records data suggest that compliance rates may be increased when colon screening becomes less unpleasant, embarrassing and painful.

131/ GENETIC COUNSELING FOR COLORECTAL CANCER: DISTRESS 1-11 YEARS LATER

Eveline M. Bleiker, Lidwina D. Wever, Miranda A Gerritsma, Department of Psychosocial Research and Epidemiology, Netherlands Cancer Institute, Amsterdam, The Netherlands; Fred H. Menko, Department of Clinical Genetics, University Hospital Vrije Universiteit, Amsterdam, The Netherlands; Babs G. Taal, Department of Gastroenterology, Netherlands Cancer Institute, Amsterdam, The Netherlands; Irma Kluijt, Department of Clinical Genetics, Academic Medical Center, Amsterdam, The Netherlands; Neil K. Aaronson, Department of Psychosocial Research and Epidemiology, Netherlands Cancer Institute, Amsterdam, The Netherlands

Although genetic counseling for colorectal cancer has been available for many years, its impact on psychological well-being is unclear. This study examined (1) the long-term impact of genetic counseling for colorectal cancer on levels of cancer-specific distress and well-being, and (2) the association between sociodemographic, clinical and psychosocial factors and heightened levels of distress. Of the 214 individuals who were counseled for the familial occurrence of colorectal cancer during 1986-1998, 178 (83\%) participated in the study by returning our questionnaire. In this cross-sectional study, the respondents had a mean follow-up of 4 years after their last counseling session. Five percent of the respondents had clinically significant levels of cancer-specific distress. The best predictor of present levels of distress and current well-being is having had contact with a professional psychosocial worker for cancer-risk in the past 10 years. At univariate level, strong personal involvement in the disease-process of a relative, and high perceived risk (but not actual risk) were found to be associated with higher levels of cancer-specific distress. Genetic counseling for colorectal cancer appears to have a clinically meaningful, negative effect on distress for only a small minority of counsellees and is not related to actual risk of cancer. 
132/ QUALITY OF LIFE ASSESSMENT IN RANDOMIZED PHASE III TRIAL IN STAGE IV NON SMALL CELL LUNG CANCER (NSCLC) PATIENTS WITH THE QOL-ANTICANCER DRUGS (QOL-ACD) QUESTIONNAIRE

Taketoshi Matsumoto, Division of Thoracic Oncology, National Cancer Center Hospital East, Kashiwa, Chiba, Japan; Yasuo Ohashi, Department of Biostatistics, University of Tokyo, Bunkyo-ku, Tokyo, Japan; Hideo Kunitoh Department of Internal Medicine \& Thoracic Oncology, National Cancer Center Hospital Central, Chuo-ku, Tokyo, Japan; Kazumasa Noda, Department of Thoracic Oncology, Kanagawa Cancer Center, Yokohama, Kanagawa, Japan; Koshiro Watanabe, Division of Respiratory Medicine, Yokohama Municipal Citizen's Hospital, Yokohama, Kanagawa, Japan

We prospectively compared the relationship between chemothrapy and QOL in patients (pts) with untreated stage IV NSCLC. A primary endpoint of this study was survival, secondary endpoints were response rate (RR), tolerability and QOL. The QOL-ACD consists of functional ( 6 items), physical (5 items), mental (5 items), psychosocial (5 items) and a global face scale. Questionnaires were collected before each treatment cycles(Cy), and every 3 months (mts) after finishing the treatment. From APR/98 to MAR/00, 302 pts were received either Docetaxel+CDDP (Arm-A) or Vindesine+CDDP (ArmB) and the $\%$ of questionnaire collection was $81 \%$ before treatment and $67 \%$ for $3 \mathrm{mts}$ after finishing the treatment. Pts characteristics were: median age 64 (30-74), median PS 1(1-3), M/F 200/102. ArmA showed a significantly higher RR $(37 \%$ vs. $21 \% p<0.01)$. We validated the structure of the QOL-ACD. The result of factor analysis with promax rotation confirmed factorial validity. According to the twoway analysis of variance, female aged less than 65 years showed a significantly lower social QOL than the other pts population. The leastsquares mean (LSM) from the pre-treatment indicated that Arm- $A$ was relatively superior to $A r m-B$ in each $Q O L$ domain, however, there was no significant difference between the arms (See Table: the values shown as; before $2 \mathrm{nd} \mathrm{Cy/3} \mathrm{mts} \mathrm{after} \mathrm{end} \mathrm{of} \mathrm{treatment.} \mathrm{Positive}$ values mean the improvement in QOL). The results of correlation analysis with RR and adverse events, and comparison with other randomized studies will be presented at the meeting.

LSM from the Pre-treatment (2nd Cy/3mts after end of treatment)

\begin{tabular}{|l|l|l|l|l|l|l|}
\hline & Arm & Functional & Physical & Mental & Psychosocial & $\begin{array}{l}\text { Face } \\
\text { Scale }\end{array}$ \\
\hline & A & $-11.1 /-12.6$ & $-4.5 /-7.7$ & $-0.6 /-4.2$ & $5.4 / 3.0$ & $2.0 /-1.8$ \\
\hline COPD & B & $-15.5 /-23.1$ & $-12.2 /-13.6$ & $-5.7 /-6.9$ & $1.6 / 0.7$ & $-0.1 /-2.0$ \\
\hline
\end{tabular}

133/ HOW POTENT IS POTENT? EVALUATION OF SEXUAL FUNCTION AND BOTHER IN MEN WHO REPORT POTENCY: DATA FROM CAPSURE

Deborah P. Lubeck, Gary Grossfeld, Peter Carroll, Urology, University of California San Francisco, San Francisco, CA; James M. Henning, Outcomes Research, TAP Pharmaceutical Products Inc., Lake Forest, IL

Impotence may be a side effect of prostate cancer treatment. An accurate assessment of potency is important in assessing the effects of treatment. This study evaluates sexual function and bother for men who indicate they have erections or intercourse after treatment for prostate cancer. CaPSURE is a national database of men who have prostate cancer. Men are recruited from communicty, academic and $\mathrm{VAH}$ urology practices. Questionnaires covering sexual function and bother are completed every six months by patients in the study using the UCLA Prostate Cancer Index. Scales are scored from 0-100, with $100=$ optimal function. 898 men $(26 \%$ reported potency. Sexual function and bother correlated with age. Median and first and third quartile scores are reported in the table by age group. Sexual function and bother vary considerably in men who are reported to be potent. Therefore, clinicians should use more quantatitive instruments to measure the degree of function both before and after treatment rather than just describing a patient as potent or impotent.
134/ EVALUATION OF QUALITY OF LIFE AFTER LIVER TRANSPLANTATION IN JAPAN

Hirohisa Imai, Public Health, Miyazaki Medical College, Miyazaki, Miyazaki, Japan; Katsuhiko Oga, Radiological Technology, College of Medical Technology Hokkaido, Sapporo, Hokkaido, Japan

Liver transplantation has been undergone since 1989 in Japan. However, health-related quality of life of the patient who undergone liver transplantations have hardly been evaluated using the measuring tools. There is little quantitative analysis concerning how liver transplantation can improve the health-related quality of life in the recipients. Since quality of life after transplantation may reflect how successful the operation is undergone, measuring quality of life could contribute to the improvement of the liver transplantation technology. The aim of this study is to examine and measure the health-related quality of life for the recipients of the liver transplantation from a donor in brain death and the partial liver transplant. The patients measured health-related quality of life were the patients who underwent liver transplantation in Hokkaido University hospital. Three tools for the measuring the quality of life were adopted: Originally in English, the measuring tools, Short-Form 36 (SF-36), Health Utility Index (HUI), and EuroQol-5D (EQ-5D) were translated into Japanese language. The measuring of quality of life of the patients was prospectively performed and described both preoperatively, as well as three, six, twelve and twenty-four months after transplantation. The recipient coordinator was in charge of delivering and collecting these questionnaire tools for the patients. This study was investigated based on the informed consent written by the patient. The analysis showed some findings. First, the data showed that there was little difference among the tools. Second, the recipient's quality of life scores were significantly improved at six months after liver transplantation compared to preoperative scoring. Third, there is a little difference between HUI and $E Q-5 D$ in Visual analogue scale (VAS). In conclusion, liver transplantation not only prolongs life but also increases health-related quality of life including an enhanced capacity to work and a diminished need of medical care.

135/ A PROSPECTIVE COMPARISON OF QUALITY OF LIFE INSTRUMENTS IN PATIENTS WITH OESOPHAGEAL CANCER

V. Kavadas, J. M. Blazeby, Division of Surgery, Bristol Royal Infirmary, Bristol, UK; C. W. Vickery, Department of Surgery, R. G. Berrisford, Department of Thoracic Surgery, Royal Devon and Exeter Hospital, Exeter, Devon, UK; C. P. Barham, D. Alderson, Division of Surgery, Bristol Royal Infirmary, Bristol, UK

Accurate assessment of quality of life (QL) is important in oesophageal cancer. The aim of this study was to compare two valid QL instruments and corresponding oesophageal modules: EORTC QLQC30/EORTC QLQ-OES24 (the generic questionnaire and oesophageal cancer specific module) with FACT-E, the general measure combined with an oesophageal cancer scale. Sixty-one patients, who had undergone oesophagectomy, were posted the QL instruments, a socio-demographic questionnaire and a debriefing form. Missing data were recorded and imputed following published guidelines. Questionnaire data were analysed using Spearman rank correlation coefficients between subscales. Fifty-five patients (36 men, mean age 68 ) completed and returned all forms (response rate $90 \%$ ). Data were missing in $0.05 \%$ (14 items) of EORTC QLQ-C30/EORTC QLQOES24 questions and $0.1 \%$ (26 items) of FACT-E questions ( $p=$ $0.01)$. Correlations between corresponding scales of the EORTC QLQ$\mathrm{C} 30$ and the FACT-E ranged from $r=0.04$ for the social domain (very poor), to $r=0.67$ (good) for the physical domain with $r$ values for the other domains more than 0.4 . The oesophageal cancer scale from FACT-E correlated poorly with all domains in the EORTC QLQ-C30. Its highest correlation was with the swallowing scale of EORTC QLQOES24 $(r=0.43)$. The swallowing, eating and emotional scales of the EORTC QLQ-OES-24 showed good correlations with the total FACT-E score and the FACT-G score. These data suggest that the physical, emotional and functional scales in the EORTC QLQ-C30 and FACT-E overlap, but that the social scale of the FACT-E focuses on different aspects of QL. The EORTC QLQ-OES24 appears to provide a more comprehensive view regarding the multidimensional nature of QL than the FACT-E oesophageal cancer scale. 
136/ A PROSPECTIVE RANDOMIZED STUDY ON THE IMPACT OF PSYCHOONCOLOGICAL INTERVENTION IN CANCER PATIENTS INCLUDING QUALITY OF LIFE AND PSYCHOENDOCRINO-IMMUNOLOGICAL PARAMETERS

Mechthild M. Determann, Holger Kalthoff, Department of General Surgery, Volker E. Kollenbaum, Cancer Centre, Nicholas Zavazava Institute of Immunology, Bernd Kremer, Doris Henne-Bruns, Department of General Surgery, University Hospital, Kiel, Germany

The objective of this study was to evaluate psychooncological support for inpatients with colorectal cancer undergoing surgery. The design was a randomized controlled trial with repeated measurements. Patients in the experimental group received individualized psychotherapeutic support during the stay; those in the control group were provided with a daily program of classical music. All patients were assessed 1 day before surgery and 10 days and 3 months after surgery using the EORTC-QLQ-C30 for Quality of Life, and the STAI-X1 for State Anxiety (SA). Measurements of the cortisol level (IMMULITE) activity of Natural Killer (NK) cells (LDH) and counts of NK and T cells (FACS) were made. Of 203 patients screened for the study, 106 met the inclusion criteria, 53 in each group. Results indicated that the groups were comparable at baseline on socio-demographic and clinical characteristics, SA and all scales of the QLQ. At baseline, there were significant correlations between SA and Global Health Status (QL) $(r=0.41)$ as well as with five other scales of the QLQ. Preliminary results using 57 of 106 patients showed significant correlations between counts of NK cells and SA $(r=-0.30)$ and $Q L(r=0.44)$ at baseline General Linear Models showed, that the number of treatment sessions in combination with the group allocation had significant effects on Emotional Functioning (EF), Cognitive Functioning, Fatigue and Pain. For example, EF demonstrated a significant within subject effect over 3 months $(F(d f=2)=6.23)$ with significant between subject effects for the type of group (exp./control) $(F(d f=1)=4.71)$ and the number of treatment sessions $(\mathrm{F}(\mathrm{df}=1)=4.43)$. The number of treatment sessions were dependent on postoperative length of stay, suggesting that the amount of intervention may be important in determining patient outcomes. The study was sponsored by German Cancer Help.

\section{7/ QUALITY OF LIFE IMPACT ON SATISFACTION WITH CARE} IN CANCER INPATIENTS

Emmanuel Desandes, Thierry Conroy, Recherche Clinique, Centre Alexis Vautrin, Vandoeuvre-les-Nancy, France; Serge Briancon Francis Guillemin, Fabienne Empereur, Epidemiologie et Evaluation Cliniques, Chu de Nancy, Nancy, France; Francois Guillemin Chirurgie, Isabelle Leonard, Recherche Clinique, Centre Alexis Vautrin, Van doeuvre-les-Nancy, Nancy, France; Anne Bredart, Unite de Psycho-oncologie, Institut Curie, Paris, France; Pierre Bey, Radiotherapie, Centre Alexis Bautrin, Vandoeuvre-les-Nancy, France

There is an increasing interest to incorporate patient views into a comprehensive assessment of quality of care. The aims were to investigate satisfaction with care and its possible relationships to quality of life (QoL) at admission and QoL changes after discharge among cancer inpatients. Three hundred and fifty-one patients completed three QoL questionnaires (SF-36, EuroQoL, EORTC QLQ-C30) at admission and received the Patient Judgments of Hospital Quality (PJHQ) questionnaire and the same QoL questionnaires fifteen days after discharge. Construct validity of PJHQ French version was checked Multiple linear regression was used to determine relationships between QoL levels of satisfaction with care ratings. Responses were obtained from $75.5 \%$ of patients. Overall scores of 265 respondents on the PJHQ questionnaire (0-100 scale) were high (means ranging from $72.5 \pm 16.1$ for "Hospital environment and ancillary staff" to 87.7 \pm 15.2 for "Recommendations and Intentions"). High levels of internal consistency, items convergent and discriminant validity were achieved except for the two-items dimensions "Recommendations and Intentions" and "Overall quality of care and services". We identified several QoL determinants of highest postdischarges patients ratings of satisfaction with care: decrease of physical functioning, low initial pain ratings, high initial social functioning and fatigue levels. Satisfaction with care is correlated to QoL level at admission and QoL changes fifteen days after discharge. Adjustment for $\mathrm{QoL}$ is essential to evaluate satisfaction with care. QoL measurement is of high interest in interpreting results of satisfaction questionnaires. Work supported by a PHRC 1996 grant.
138/ IMPACT OF COPING, FUNCTIONALITY, AND SYMPTOMS ON THE QUALITY OF LIFE OF HAEMATOLOGICAL CANCER PATIENTS

Jose L. Pais-Ribeiro, Faculdade de Psicologia, Universidade do Porto, Porto, Portugal; Eunice Sa, ISPA, ISPA, Lisboa, Portugal

The aims of the present study is to identify the contribution of coping variables, health status variables, and demographic variables, to the prediction of Quality of Life of haematological Cancer Patients. The sample includes 122 haematological cancer patients ( $52.5 \%$ females), aged between 18 and 74 years $(M=45.95) ; 63.9 \%$ are married; school level $M=9.7$ years. We use the EORTC QLQ-C30 (v.3), a 30-item scale that includes five functional scales, three symptom scales, six single symptom items, and a global heath status/QOL scale. We use also one coping scale based on the Mental Adjustment to Cancer Scale with four dimensions: Fighting Spirit; Helpless/Hopeless, Anxious Preoccupation, Fatalism. A standard multiple regression analysis was performed between the QOL scale as the dependent variable (DV), and the four scales of coping, and the 14 dimensions of QLQ$\mathrm{C} 30$, as independent variables (IV). Analysis was performed using SPSS regression (Stepwise procedure). The IVs, Fatigue $(p=.0001)$, Dyspnoea $(p=.0001)$, Fatalism $(p=.01)$, Financial Difficulties $p=(.02)$, and Pain $(p=.07)$, stay in the equation, and contributed significantly to the prediction of QOL. All the other variables were excluded from the equation. The four IVs in combination predicted $50.88 \%(48.27 \%$ adjusted) of the variability of QOL. Results suggest that for this group of patients, the specific cancer items (or the symptom items) more the fatalistic scale of the coping scale (accepts the diagnosis, does not seek further information, adopts a fatalistic attitude) where the best predictors of QOL.

139/ AN EMPIRICAL META-ANALYSIS OF FIVE DIFFERENT STUDIES EXPLORING QUALITY OF LIFE AFTER PROSTATE CANCER

J. U. Siebmann, M. Berend, T. Küchler, Reference Centre Quality of Life, Christian-Albrechts-University, Kiel, Germany

Recently the debate on the impact of different treatment modalities for patients with prostate cancer on different aspects of quality of life has increased among urologists. One of the mayor issues of this discussion in Germany is which instrument to use to cover not just general quality of life aspects but as well prostate specific symptoms. Between 1996 and 20005 different groups (Ester, M., Klän, R., Galalae, R., Biermann, C., Kurek, R.) conducted retrospective studies using the EORTC QLQ-C30 and the disease specific module on prostate cancer developed by Biermann and Küchler (1996). We performed an empirical meta-analysis on those five studies with a total $\mathrm{N}$ of 1185 , of which 947 had complete quality of life data. First steps of analysis indicate the overall good psychometric properties of the module which divides into the following nine factors: "urination," "continence," "erection," "sexuality," "relationship," "psychic strain," "pain," "heat," and "diet" in which Cronbach's alpha ranks from 0.70 to 0.86 except for "urination" $(a=0.61)$ and "diet" $(a=0.57)$. Since these retrospective data cover up to 10 years after cancer treatment (for very few patients even more: $\mathrm{N}=23$, max. 14.38 years), we are able to present results that show the development of various (especially prostate specific-) quality of life aspects within this comparatively large timeframe. In short, "physical functioning" and "role functioning" tend to decline over the years while "global health" remains relatively stable on a moderate level between 60 and 70 . We found that erectile dysfunctions (av. mean on symptom scale $>90$ ) and a related decrease in sexual pleasure and activity (av. mean on symptom scale $>80$ ) are main problems after treatment, which seem to stagnate. Furthermore, QLQ-C30 and prostate module both indicate a slow and steady rise of "pain" beginning two years after treatment. 
140/ THE NEED FOR PSYCHOSOCIAL SUPPORT IN PATIENTS WITH MALIGNANT CHOROIDAL MELANOMA

Jens Reimer, Department of Neurology, University of Bochum Bochum, Germany; Anja Fleiss, Gabriele H. Franke, Institute of Medical Psychology, University of Essen, Essen, Germany

The malignant choroidal melanoma (MCM) is the most common ocular tumor in adults. Five year mortality ranges, depending on tumor size, between $16 \%$ and $53 \%$. To evaluate the need for psychosocial support, 117 patients with MCM after radiotherapy were asked to complete the Symptom Checklist 90-R (SCL-90-R) and the Inventory of Interpersonal Problems (IIP). Psychological distress was defined as a T-score of 60 or higher regarding the Global Severity Index of the SCL-90-R. The IIP was used to assess the relation between somatic and interpersonal problems. Patients were aged in average 61.5 yrs (\&\#61617; 11.2), and time from diagnosis was in average 50 mths $(\& \# 61617 ; 42)$. Visual acuity decreased in the sick eye from .48 $(\& \# 61617 ; .3)$ at diagnosis to $.16(\& \# 61617 ; .21)$ at the time of study participation, whilst visual acuity remained stable in the other eye (between .88 and .85 (\&\#61617;23). Relevant psychological distress was detected in 33 patients $(28 \%)$, out of which $9(8 \%)$ were strongly distressed. Among distressed patients, somatic problems were stronger than interpersonal problems in 17 patients, in 16 patients interpersonal problems were stronger than psychological problems. Visual acuity of the other ("healthy") eye correlated negatively with the SCL-90-R subscales "Obsessive-compulsive," "Anxiety," "Phobic anxiety," "Pschoticism" and the global indeces "GSI" and "PSDI" $(p<.05)$. Relevant psychological distress is prevalent in nearly every third longterm survivor of MCM. In the clinical context, special attention should be drawn to patients with low visual acuity of the other ("healthy") eye. Interpersonal problems could serve as a basis in integrating psychosocial support-models for patients with malignant choroidal melanoma.

141/ DETERMINING CLINICALLY SIGNIFICANT CHANGES IN FATIGUE SUBSCALE HEALTH-RELATED QUALITY OF LIFE SCORES FOR CANCER PATIENTS

Heather-Jane Au, Melina Dharma-Wardene, Martin Palmer, Cross Cancer Institute, Edmonton, AB, Canada; David Dupere, Queen Elizabeth II HSC, Halifax, NS, Canada; John Hanson, Cross Cancer Institute, Edmonton, $\mathrm{AB}$, Canada

The primary objective of this study is to determine what is a clinically significant change in cancer patients' Fatigue subscale scores using FACT-F. The second objective is to evaluate the ability of this subscale to discriminate between patients with or without fatigue progression while on chemotherapy. Forty-two consecutive patients with advanced lung cancer undergoing platinum-based chemotherapy at a tertiary care cancer centre in Canada were followed prospectively. FACT-F was self-administered at baseline pre-chemotherapy, following each cycle of chemotherapy, and at the end of treatment. Patients also completed a single item on fatigue progression: "Compared to my last cycle of chemotherapy, I am:", with three possible responses "more tired","as tired", or "less tired". This single item was administered following each cycle, and at the end of treatment. For each category of fatigue progression, corresponding FACT-F Fatigue subscale mean change scores (from prior assessment) were calculated along with standard errors. Analysis of variance was performed to determine whether Fatigue subscale change scores were able to discriminate the three fatigue progression categories (more, same, less). Results revealed mean Fatigue subscale change scores of +4.88 (SE 1.06) for patients who rated themselves as more tired, +1.26 (SE 1.01) for those who rated themselves the same, and -1.52 (SE 0.84) for those who rated themselves less tired. The Fatigue subscale change scores were able to discriminate between these three categories of fatigue progression $(p<0.001)$. In conclusion, we were able to demonstrate that the FACT-F Fatigue subscale is able to discriminate between patients with and without fatigue progression. The amount of change seen in the Fatigue subscale scores was small ( $<5$ units) though patients noted clinically important changes. Therefore, small changes in Fatigue subscale scores are clinically meaningful and should not be ignored.

142/ TESTING THE LUNG CANCER SYMPTOM SCALE (LCSS) MODEL IN PATIENTS WITH MALIGNANT PLEURAL MESOTHELIOMA (MPM)

Patricia J. Hollen, School of Nursing, Northeastern University, Boston, MA; Richard J. Gralla, Division of Medical Oncology, Columbia
University, New York, NY; Astra Liepa, Global Economic Affairs, Xiaochun Li, Department of Statistics, James Rusthoven, ALIMTA team, Eli Lilly and Co., Indianapolis, IN

The LCSS is a site-specific instrument used to assess health-related quality of life (HRQoL) in patients (pts) with lung cancer. The testing of the LCSS conceptual model has been described by Hollen et al (Support Care Cancer, 1994). The 9-item patient-rated scale, using a $100 \mathrm{~mm}$ visual analogue scale, was modified slightly for MPM, as no MPM-specific instrument was available. The modified LCSS was included in 2 multinational trials of pemetrexed disodium (ALIMTA) in pts with MPM. As in the previous report of the model, baseline data $(\mathrm{N}=461)$ were analyzed using stepwise multiple regression to determine the contribution of predictive factors (symptom [Sx] items: anorexia, fatigue, cough, dyspnea, hemoptysis, pain) to the summation items (Sx distress, activity level, global QoL). Fatigue, cough, dyspnea and pain were significant predictors $(p<0.05)$ for Sx distress. Fatigue, dyspnea and Sx distress were significant for activity level. Anorexia, fatigue, dyspnea and activity level were significant for global HRQoL. The LCSS model explained $52 \%$ of the variance for Sx distress, $49 \%$ for activity level and $61 \%$ for global HRQoL experienced by pts with MPM at presentation. Significant factors were as expected (eg, fatigue was a significant predictor for all summation items but hemoptysis was not). The contribution of each factor (using $R^{2}$ ) for 461 MPM pts will be compared to 144 lung cancer pts. We conclude that relationships in the expected direction were found, thus providing support for construct validity. A model focusing on physical and functional dimensions as the best predictors of HRQoL for progressive lung disease, with other dimensions captured in less depth, was again supported. A practical instrument suitable for serial measurement that explains $61 \%$ of the variance in overall HRQoL will be useful in MPM clinical trials. Stability of the model at several timepoints during the trials will be tested to further support construct validity when data are available.

143/ RELATIONSHIP BETWEEN CANCER LOCUS OF CONTROL AND QUALITY OF LIFE

Vitor L. Rodrigues, Pedro L. Ferreira, Helena F. Ribeiro, Centre for Health Studies and Research, University of Coimbra, Coimbra, Portugal

This study aimed at searching the relationship between Cancer Locus of Control and the Quality of Life in women with breast cancer, submitted to oncology treatment at two hospitals in Coimbra: The Oncology Hospital Francisco Gentil (IPOFG) and Coimbra University Hospital (HUC). The Cancer Locus of Control Scale (CLCS) was developed as an area-specific measure for prediction of illness-related behaviour of cancer patients. It includes an assessment of the perception that the causes of specific events will be attributed to personal (internal control) or situational (external control) elements. For the assessment of the impact of the disease and its treatment on the Quality of Life of the patient, we used measures recommended by the European Organization for Research and Treatment of Cancer (EORTC). The QLQ-C30 incorporates nine multi-item scales: five functional scales: physical, role, cognitive, emotional and social; three symptom scales fatigue, pain and nausea and vomiting. Several single-item symptom measures are also included: dyspnoea, sleep disturbance, appetite loss, constipation and diarrhoea, also the financial impact, a global health and quality of life scale. The questionnaire was administered during the oncology treatment at $82(70.1 \%)$ women submitted to radical surgery and $35(29.9 \%)$ women submitted to conservative surgery. Mean age is 57.8 years for the group mastectomy and 55.4 years, for the tumorectomised women. The results showed statistical differences in the function and symptoms scales of the QLQ-C30 of Quality of Life in the groups of women, submitted to chemical treatment. Comparing the mean values in the two samples they suggest that there exists a statistically significant difference, suggesting that Quality of Life in the two samples is different. Statistically significant correlations were found between Locus of Control and QLQ-C30 internal control over course with role function and the Global Quality of Life and between religious control and cognitive function in the women submitted to radical surgery. Statistically significant correlation between the religious control and social function, were found in conservative surgery women. In conclusion, cancer is an important disease in our society. The relation quantity/quality of life is important in the evaluation of disease and/or outcome in health. 
144/ THE RELATIONSHIP BETWEEN INFORMATION SATISFACTION AND QUALITY OF LIFE IN CANCER

Clara L. Yu, R. Fielding, Community Medicine, C. L. Chan, Social Work and Soc. Administration, J. S. Sham, Clinical Oncology, Hong Kong University, Hong Kong, China

Patient satisfaction with doctor consultation and information giving in particular might have significant bearing on QoL in cancer. Nasopharyngeal carcinoma (NPC) is highly prevalent in Southern China. Prominent acute treatment side-effects create problems in daily living and work which can generate considerable financial problems. Better adjustment to NPC associates with improved rate of recovery and better quality of life $(\mathrm{Q} o \mathrm{~L})$ in terms of quicker return to work and normal functioning. This study explore if satisfaction with information giving improves post-treatment QoL of NPC patients in China. Newly referred Hong Kong Chinese NPC patients $(n=211)$ completed interview measures at Baseline before radiotherapy, at four months (immediate post-treatment consultation) (FU 1), and again at eight months (short-term post-radiation period) after Baseline (FU 2). Information satisfaction was measured by five items selected from the cognitive subscale of the MISS. QoL was measured by the FACT-G (Ch). After adjustment for overall patient satisfaction (the PSQ-9), optimism, worry about family, anger, eating ability, subjective health, family income and occupation at FU 1 follow-up, treatment between Baseline and FU 1, and relapse after Baseline, the five-item MISS at FU 1 (Beta=0.21, $P<0.01$ ) significantly predicted $Q o L$ at $F U 2$. Adjustment for Baseline $\mathrm{QoL}$ and cancer stage did not alter this relationship (Beta=0.20, $P<0.01$ ). These results reinforce the relationship between satisfaction of information giving and QoL with Chinese cancer patients.

145/ IMPROVEMENT IN HEMOGLOBIN LEVELS REDUCES FATIGUE AND IMPROVES HEALTH-RELATED QUALITY OF LIFE (HRQOL) OF ANEMIC CANCER PATIENTS

J. D. Kallich, Health Economics and Epidemiology, H. Erder, G. Rossi, E. Poulsen, A. Colowick, J. Isitt, The NESP 980290/980297 Study Groups, Amgen Inc., Thousand Oaks, CA

Anemia often occurs in cancer patients due to disease or therapy, negatively impacting on HRQOL. NESP(darbepoetin alfa) - a novel erythropoiesis stimulating protein - has been developed, with a 2- to 3 -fold greater serum half-life than rHuEPO. In this analysis, data from 2 trials (conducted in Canada, USA, Australia, and Europe) involving solid tumor patients (517) receiving NESP, rHuEPO or placebo for 12 weeks, were used to examine the relationship between $\mathrm{Hgb}$ and HRQOL. Fatigue (FACT-F) improved by 3.9 points at end of treatment when $\mathrm{Hgb}$ increased by 2 or more $\mathrm{g} / \mathrm{dL}$. Physical, functional, and emotional well-being, and anemia symptoms also improved with $\mathrm{Hgb}$ increases. Social/family support results did not show a relationship with $\mathrm{Hgb}$ change. There was a correlation between changes in the fatigue score and changes in $\mathrm{Hgb}(r=0.19, p=0.002)$ and $\mathrm{ECOG}$ performance scales $(r=-0.24, p<0.001)$. Variation in baseline and change from baseline in fatigue scores by age, geographic region, and gender was observed. Hgb improvement had the greatest impact on patient-reported fatigue levels, with moderate impact on physical and functional well-being. HRQOL tends to decline if $\mathrm{Hgb}$ levels are not increased. Magnitude of response to $\mathrm{Hgb}$ changes may vary by demographic characteristics.

\begin{tabular}{|c|c|c|c|c|c|c|c|c|c|}
\hline & & & & & $\mathrm{Hgb}(\mathrm{g} / \mathrm{dL})$ & & & & \\
\hline & & Less than 0 & & & $0-2$ & & & More than 2 & \\
\hline Scale score & Hgb change & $95 \% \mathrm{Cl}$ & $n$ & Hgb change & $95 \% \mathrm{Cl}$ & $n$ & Hgb change & $95 \% \mathrm{Cl}$ & $n$ \\
\hline \begin{tabular}{|l} 
Fatigue \\
\end{tabular} & -1.7 & $(-3.6,0.2)$ & 137 & 1.6 & $(0.2,0.3)$ & 221 & 3.9 & $(1.9,5.8)$ & 153 \\
\hline Physical & -0.4 & $(-1.3,0.5)$ & 137 & 0.9 & $(0.2,1.5)$ & 220 & 1.5 & $(0.6,2.4)$ & 151 \\
\hline Functional & -0.9 & $(-1.7,0.0)$ & 137 & 0.9 & $(0.2,1.5)$ & 220 & 0.9 & $(0.1,1.8)$ & 153 \\
\hline Emotional & 0.4 & $(-0.3,1.2)$ & 135 & 0.9 & $(0.4,1.4)$ & 220 & 1.1 & $(0.5,1.7)$ & 152 \\
\hline Social & -0.3 & $(-1.0,0.4)$ & 135 & -0.4 & $(-0.9,0.1)$ & 217 & -0.8 & $(-1.6,0.1)$ & 151 \\
\hline An symptoms & -0.9 & $(-1.7,-0.1)$ & 137 & 0.7 & $(0.2,1.2)$ & 221 & 0.7 & $(0.1,1.3)$ & 151 \\
\hline
\end{tabular}

146/ PSYCHOLOGICAL DISTRESS AND SEXUAL FUNCTIONING OF OVARIAN CANCER SURVIVORS

Karen Basen-Engquist, Behavioral Science, Diane Bodurka-Bevers, Gynecologic Oncology, Cindy L. Carmack Taylor, Behavioral Science, The University of Texas, M. D. Anderson Cancer Center, Houston, TX

Epithelial ovarian cancer is the second most common gynecologic cancer, but the leading cause of death from gynecologic cancer. Difficult to detect, the disease is often diagnosed in its late stages; fewer than $40 \%$ of women survive longer than five years. This presentation will characterize psychological distress and sexual functioning among women with epithelial ovarian cancer. The sample comprises 246 women with ovarian cancer seen at M. D. Anderson Cancer Center. Women were classified in four survivorship categories: newly diagnosed patients receiving treatment $(n=40)$; patients on treatment for recurrent or persistent disease $(n=59)$; patients who were off treatment and had not had a recurrence $(n=88)$; and patients who were off treatment after experiencing a recurrence $(n=48)$. Women with recurrence were more depressed, regardless of whether they were currently receiving treatment $(p=.011)$; the groups did not differ significantly in anxiety. Women receiving treatment were less likely to be sexually active than those off-treatment $(p=.02)$, regardless of whether they were being treated for newly diagnosed or recurrent disease. Women receiving treatment also rated the importance of sex as lower $(p=.011)$ and were more likely to report being too tired for $\operatorname{sex}(p=.024)$. They rated their level of sexual desire as slightly lower $(p=.093)$. Among sexually active survivors, neither treatment status nor having had a recurrence was related to satisfaction and enjoyment of sex. However, women who had had a recurrence were more likely to report vaginal dryness during sex $(p=.005)$. These results indicate that ovarian cancer survivors with recurrent disease should be evaluated for interventions to address psychological distress and specific sexual functioning problems related to vaginal lubrication/discomfort during sex. Survivors who are currently receiving treatment may require intervention to address decreased interest in sex if it causes distress to them or their partners.

147/ SYMPTOMS PREDICT REDUCED HRQOL DURING RADIOTHERAPY AMONG PATIENTS WITH LUNG, COLORECTAL AND PANCREAS CANCER

Xin Shelley Wang, Pain Research Group, Nora A. Janjan, Ritsuko Komaki, Radiation Oncology, Mary Easley, Tito Mendoza, Gary Mobley, Charles S. Cleeland, Pain Research Group, University of Texas, MD Anderson Cancer Center, Houston, TX

The objective of this study was to assess the symptom severity and its impact on HRQL among lung and GI cancer patients undergoing radiotherapy. A longitudinal symptom study conducted in the Division of Radiation Oncology, UT M.D. Anderson Cancer Center in 2000. The assessment included the M.D. Anderson Symptom Inventory (MDASI) and SF-36. Patient completed surveys before and after radiotherapy. The MDASI is a newly validated multi symptoms assessment tool. It assesses the severity and interference of cancer related symptoms. Among patients $(\mathrm{N}=47)$ with NSCLC, colorectal and pancreatic cancer, fatigue, pain, distress, sleep disturbances, drowsy, and sadness were most common symptoms reported before radiation. Post therapy, nausea, poor appetite and dry month were added to the above symptoms, while shortness of breath, cough got better in NSCLC group. There is not significant difference of physical or mental health component score among three cancer groups either pre or post therapy (all ANOVA $p>.05$ ). The pre-therapy regression model for the physical health component score of SF-36 (PHC) included pain, fatigue and distress $(\mathrm{R} 2=740, \mathrm{p}<.001)$, while pain and distress $(R 2=.708, p<.001)$ for the mental health component score of SF-36 (MHC). For the post-therapy regression PHC model, fatigue was the only predictor $(R 2=540, p<.001)$, pain and sadness were the predictors of $\mathrm{MHC}(\mathrm{R} 2=.615, \mathrm{p}<.001)$. 1) Significant numbers of patients develops severe fatigue during radiation. It directly impacts on the patient's physical and mental functional status. This finding supports the need for better management of cancer-related fatigue. 2) Uncontrolled pain, distress, and sadness are also major predictors of reduced HRQoL for patients on XRT. 
148/ EARLY QUALITY OF LIFE IN LOCALIZED PROSTATE CANCER: AN EXAMINATION OF TREATMENT-RELATED, DEMOGRAPHIC, AND PSYCHOSOCIAL FACTORS

David T. Eton, Center on Outcomes, Research, \& Education, Evanston Northwestern Healthcare \& Northwestern University, Evanston, IL; Stephen J. Lepore, Department of Psychology, Brooklyn CollegeCUNY, Brooklyn, NY; Vicki S. Helgeson, Department of Psychology, Carnegie Mellon University, Pittsburgh, PA

Men with localized prostate cancer are faced with important treatment decisions and quality of life information has become a crucial element of decision-making. The first objective of this study was to compare the early, health-related quality of life (HRQoL) of men with localized prostate cancer who were treated with radical prostatectomy, external beam radiotherapy, or brachytherapy. A second objective was to identify demographic and psychosocial variables that predict HRQoL. Two-hundred fifty-six men with localized prostate cancer were interviewed within 7 weeks of treatment initiation. The interview included measures of prostate-specific HRQoL (the UCLA Prostate Cancer Index), general HRQoL (the SF-36), and psychosocial variables. After adjusting for covariates, treatment-group differences were found on both prostate-specific and general HRQoL. Men treated with prostatectomy reported more urinary and sexual problems and more general physical dysfunction than men treated with either form of radiation. Men treated with brachytherapy reported the fewest problems in sexual function and the least general physical dysfunction. Few treatment-group differences were found in mental functioning. Both demographic and psychosocial factors predicted HRQoL. Older men and African-American men reported more physical problems than younger and Caucasian men, respectively. A supportive social environment, high self-efficacy and high self-esteem were predictive of better HRQoL. It appears that shortly after treatment for localized prostate cancer, men treated by radical prostatectomy, older men, and African-American men are at heightened risk for experiencing prostate-specific and general deficits in HRQoL. Having psychosocial resources from which to draw may enhance HRQoL.

149/ MENTAL ADJUSTMENT TO CANCER AND SOCIAL SUPPORT: IMPORTANT FACTORS FOR THE QUALITY OF LIFE OF OVARIAN CANCER SURVIVORS

Georg Kemmler, B. Holzner, V. Meraner, T. Bodner, Department of Psychiatry, G. Zeimet, Department of Internal Medicine, B. SpernerUnterweger, Department of Psychiatry, Innsbruck University Clinics, Innsbruck, Austria

While it is well-known that subjective quality of life (QOL) is more than a combination of the patient's functional status and symptomatology, the additional effect of other factors on subjective QOL has not often been studied systematically. Mental adjustment to cancer as well as social support have been discussed to be of special importance for the well-being of cancer survivors. In this contribution the joint effect of functioning, clinical symptoms, mental adjustment to cancer and social support is investigated in survivors of ovarian cancer. In 82 ovarian cancer survivors (mean age 58 years $+/-12$, median time since diagnosis 48 months, $44 \%$ in stage III/IV) the following aspects were assessed using self-administered questionnaires: quality of life (FACT-G), subjective ratings of symptoms and functioning (subscales of EORTC QLQ-C30), strategies of mental adjustment to cancer (MAC Scale), and social support (FSOZU: Questionnaire for Assessing Social Support). Data were analysed using multiple linear regresssion. The number of independent variables was reduced by secondary factor analyses. Functional aspects and symptomatology alone predicted $45 \%$ of the variation (corrected R-square $=0.45$ ) of the patients' global QOL. Inclusion of coping strategies led to a significant increase of R-square to 0.58 . Both a 'fighting spirit' and 'anxious-depressive' coping strategies independently contributed to this increase. Taking social support into consideration resulted in a further rise in R-square to 0.72 . This final model contains a factor measuring 'social distress' as well as the two above-mentioned coping strategies, a general 'functioning' factor and one symptom, pain. Other symptoms (sleep disorders, fatigue) become redundant when social support/distress is entered into the model. Implications of the findings will be discussed.
150/ THE RELATIONSHIP BETWEEN PAIN SEVERITY, PAIN-RELATED INTERFERENCE AND HEALTH RELATED QUALITY OF LIFE IN SWEDISH CANCER PATIENTS

Tito R. Mendoza, Pain Research Group, University of Texas, M.D. Anderson Cancer Center, Houston, TX; Bengt Bergman, Department of Respiratory Medicine, Sahlgrenska University Hospital, Goteborg, Sweden; Colin Durnin, Knoll Pharmaceuticals, London, UK; Charles S. Cleeland, Pain Research Group, The University of Texas, M.D. Anderson Cancer Center, Houston, TX

In order to judge the overall functional status of patients, it is important to examine the effects of various symptoms on patients' level of functioning. Typically, generic quality of life measures consist of selfreport ratings that take into account the following areas of a person's life: functional status, physical well-being, emotional well-being, social functioning, and spiritual well-being. Chronic pain is typically the most distressing symptom experienced by patients with malignant disease. If a measure of functional health and well-being is to be treated as an outcome variable, the independent effects of pain on a cancer patient's level of functioning need to be understood. One way to address this question is to examine the effects of various symptoms, such as pain, measured by symptom-specific scales, on the more generic measures of health-related function that are increasingly used in medical outcomes research. This study examines the relationship between pain as measured by the Brief Pain Inventory (BPI) and health-related quality of life assessed using the European Organization for Research and Treatment of Cancer Quality of Life Questionnaire (EORTC QOL30) among 111 Swedish cancer patients. Statistical methods used were analysis of variance, common factor analysis and regression analysis. Results showed that role functioning, sleep trouble and pain are the only EORTC QOL30 subscales that are significantly different across varying levels of pain severity with the greatest increase in the severe level as measured by the BPI. Pain interference (but not pain severity) is strongly associated with perceptions of overall quality of life. Finally, global quality of life is significantly related to pain interference even after adjusting for the effect of ECOG performance status.

\section{1/EFFECT OF CAREGIVER PERCEPTION OF PREPAREDNESS ON BURDEN IN AN ONCOLOGY POPULATION}

Mary J. Scherbring, Nursing, Mayo Medical Center, Rochester, MN

The health care environment in the United States is being affected by a movement towards limited hospital care for many patients with cancer, with one outcome being that lay persons function in a caretaking role for these patients. The concept of caregiver burden is being increasingly recognized as a phenomenon affecting a significant number of these individuals. The caregiver's perception of preparedness to assume the caregiving role is one that can be modified by the healthcare team. This study was undertaken to identify the level of caregiver burden and perception of preparedness at hospital discharge, one week, and one month after discharge. Utilizing a descriptive, quantitative, correlational design with repeated measures, data were obtained to measure caregiver burden and caregiver perception of preparedness in a sample of caregivers of recently hospitalized medical oncology patients. The research questions included: 1. What is the level of caregiver burden and caregiver perception of preparedness prior to hospital discharge, one week, and one month, after discharge? 2. What is the change in caregiver burden and caregiver perception of preparedness from pre-dismissal to one week to one month following dismissal? 3 . What is the relationship between the level of caregiver burden and caregiver perception of preparedness, from pre-discharge to one month following discharge? The results indicate that a moderate level of burden existed, and that preparedness was measured at about 3.5 on a 5 point scale. Over time there was not a significant change in either measure. The relationship between preparedness and burden was found to be significant and inverse. Increasing levels of preparation were associated with decreased burden. This relationship did not change over time. This finding mandates that the healthcare team provides appropriate interventions to assure that caregivers are prepared for their responsibilities. Attention to this component of the patient/family's experience provides a means to enhance the quality of life for all involved. 
152/ THE PATIENT AND THEIR DOCTOR: A CONTEXT FOR UNDERSTANDING THE DESIRE TO HASTEN DEATH

Brian Kelly, Frank Varghese, Psychiatry, University of Queensland, Brisbane, Queensland, Australia; Marguerite Robertson, Palliative Care Hospice, Mount Olivet Hospital, Brisbane, Queensland, Australia; Paul Burnett, Research and Graduate Training, Charles Sturt University, Wagga Wagga, NSW, Australia; Dan Pelusi, Shirlene Badger, Psychiatry, University of Queensland, Brisbane, Queensland, Australia

An exploratory study was conducted investigating the factors associated with the desire to hasten death among a sample of terminally ill cancer patients and their referring or nominated doctors. Semi-structured interviews conducted on 72 patients and on a subgroup of doctors $(n=24)$ involved in the patients' treatment were analysed using QSR-NUDIST. The main themes to emerge among patients suggested that patients with a high desire to hasten death had greater concerns with physical symptoms and psychological suffering, perceived themselves to be more of a burden to others and experienced higher levels of demoralisation, while also reporting less confidence in symptom control, fewer social supports, and less satisfaction with life experiences compared to those with moderate or no desire to hasten death. Doctors of patients who endorsed a high desire to hasten death reported high levels of conflict and inadequacy in fulfilling their 'doctor role,' whereas doctors of patients who did not endorse a desire to hasten death felt comfortable to perform the role of doctor due to reported 'team support.' An examination of the doctor-patient relationship will be discussed.

\section{3/ QOLLTI-F: A MEASURE OF THE QOL OF FAMILY} CAREGIVERS OF PALLIATIVE CARE PATIENTS

S. Robin Cohen, Department of Oncology, McGill University, Montreal, QC, Canada; Anne Leis, Community Health \& Epidemiology, University of Saskatchewan, Saskatoon, Saskatchewan, Canada; Patricia Porterfield, David Hsu, Palliative Care, Vancouver Hospital \&Health Sciences Centre, Vancouver, BC, Canada; David Kuhl, Family \& Community Medicine, Providence Health Centre, Vancouver, BC Canada; Cecile Charbonneau, Palliative Care Research Team, Maison Michel Sarrazin, Quebec City, QC, Canada; Jane Poulson, Palliative Medicine \& General Internal Medicine, The Toronto Hospital, Toronto, ON, Canada; Carmelita McNeil, Palliative Care Research, McGill University, Montreal, QC, Canada; Paul Ritvo, Preventive Oncology, Cancer Care Ontario, Toronto, ON, Canada

The goal of this study was to test the acceptability and psychometric properties of a self-report instrument to measure the quality of life (QOL) of family caregivers of palliative care patients. The instrument was designed to have 7 subscales that would each have psychometric properties appropriate for measuring the QOL of groups. A goal of the study was to reduce the number of items in the questionnaire. The Quality of Life in Life-Threatening Illness - Family Caregiver version (QOLLTI-F) content was designed to incorporate the following 7 domains, derived from a qualitative study: Own State; Patient State; Outlook; Relationships; Quality of Care; Environment; Finances. Participants were 243 family caregivers of terminally ill cancer patients followed by 1 of 8 palliative care services in 5 Canadian cities. Fortyone percent were caring for the patient at home while $59 \%$ of the patients were admitted to palliative care units. On 3 occasions participants were asked to complete a single item rating the quality of their day (from very bad to very good), a 2-item global measure (QOLLTI-G), QOLLTI-F, and a single-item numerical scale rating the extent to which completing QOLLTI-F was tiring. On the last two occasions they also completed single-item numerical rating scales directly measuring change in each of the domains included in QOLLTI$\mathrm{F}$ as well as for overall QOL. During data analysis QOLLTI-F was reduced from 24 to 16 items through deletion of redundant items and those that did not fit well in an exploratory principal components analysis, as well as of one item that was difficult to answer when the patient was unable to communicate. Otherwise, QOLLTI-F was found to be acceptable to the participants. The results of the principal components analysis reflect the 7 domains and were used to create subscales. The psychometric properties of the subscale and total scores will be reported.
154/ DECISIVE FACTORS OF QUALITY OF LIFE OF BLADDER CANCER SURVIVORS FIVE YEARS AFTER THE TREATMENTS IN FRANCE

Tomohiro Matsuda, Inserm U558, Toulouse, France; Catherine Exbrayat, Registre des Cancers de l'Isere, Meylan, France; Isabelle Aptel, Pascale Grosclaude, Registre des Cancers du Tarn, Toulouse, France

The objectives of this population-based study were to evaluate quality of life among bladder cancer survivors at least 5 years after diagnosis, to ascertain the long-term effect of treatments and to investigate important pathological and socio-demographic factors influencing the quality of life. Questionnaire, Functional Assessment of Cancer Therapy (FACT-BI vers.4) in French, was sent by mail to patients randomly selected from the database of the Isere and Tarn cancer registry in France. 73 valid answers $(M: F=59: 14$, median age $=73$ yrs [33-90]) were examined with their pathological information. Their health status according to ECOG Performance Status Rating differed significantly in total score of the FACT. Old age (> $80 \mathrm{yrs}$ ) affects their QOL in Physical, Emotional and Functional Well-Being as well. In addition, marital and familial situation had a critical impact on Emotional and Familial/Social Well-Being. Those who live alone had a quite low score. As for the pathological variables, TNM categories, grade or histology of initial tumor had no significant effect on score of the questionnaire. Neither variety in treatments nor time from the most recent major treatment was a factor that modified the FACT score except that an experience of total cystectomy clearly deteriorates their health status and score of the bladder cancer specific subscale. Another finding was that low QOL survivors tended to be dissatisfied with quality and quantity of given information about their disease, treatments and post-treatment life. The present study showed a clear association of overall quality of life of bladder cancer survivors with their actual physical status including age, though scores in some subscales depended on the family situation or on other socio-demographical backgrounds. The sensitivity of the FACT among long-term survivors was validated with French version in this study.

\section{5/ IMPACT OF A SECOND PRIMARY CANCER ON QUALITY} OF LIFE IN BREAST CANCER SURVIVORS

Carolyn C. Gotay, Marya Levintova-Romero, Cancer Research Center of Hawaii, University of Hawaii, Honolulu, $\mathrm{HI}$

As cancer patients survive for increasingly long periods of time after initial diagnosis and treatment, they are at risk of developing additional primary tumors. This study describes the psychological impact of experiencing more than one primary cancer in breast cancer survivors, an area where virtually no information has been reported to date. Participants $(\mathrm{N}=50)$ were identified by a population-based tumor registry. Data were collected through mailed questionnaires. Mean age of participants was $67 \mathrm{yrs}, 76 \%$ were of Asian/Pacific Islander (API) descent, and $86 \%$ received at least a high school education. A second breast cancer was the most prevalent site of second primary by far, reported by $66 \%$ of respondents, followed by skin $(6 \%)$ uterine $(6 \%)$, and other cancers experienced by smaller numbers of individuals. Overall, survivors were doing well, with mean scores of 5.7 on a 7-point global QOL scale. The degree to which survivors felt their QOL was affected by cancer was highly variable: $18 \%$ reported cancer affected their lives "very much" (score of 7 on a 7-point scale), while $16 \%$ reported "no effect at all." Patients believed that they had coped with cancer (both first and second primaries) better than their families had coped. They reported that both they and their families had coped better with the second diagnosis (means of 5.0, and 4.5, respectively, on a 7-point scale), than with the first diagnosis (means of 4.7 , and 4.2). However, these differences were not significant. $34 \%$ reported that dealing with their second cancer was much easier than with the first, although $20 \%$ reported that dealing with the second cancer was much more difficult. This study indicates that the impact of a second primary cancer in breast cancer survivors is highly variable. We are exploring the effects of clinical, sociodemographic, and psychosocial factors in explaining QOL in this group. 
156/ SEXUAL FUNCTION IN WOMEN WITH A HISTORY OF CERVICAL CANCER TREATED BY RADIOTHERAPY

Pernille T. Jensen, Department of Gynecology, Copenhagen University Hospital, Copenhagen, Denmark; Mogens Groenvold, Department of Palliative Medicine, Bispebjerg Hospital, Copenhagen, Denmark; Marianne C. Klee, Department of Oncology, Herlev Hospital, Copenhagen, Denmark; David Machin, Institute of General Practice and Primary Care, Northern General Hospital, Sheffield, UK

The purpose of this prospective study was to evaluate sexual function of women with cervical cancer (CC) treated by radiotherapy. 118 patients with cervical cancer, referred for either radical or adjuvant radiotherapy, were included. The patients completed a questionnaire on health-related quality of life, including EORTC QLQ-C30 and additional ad-hoc-constructed items on sexual, physical and psychosocial function. Questionnaires were completed at the termination of radiotherapy and after $1,3,6,12,18$, and 24 months. All patients completed a sociodemographic questionnaire, in which the patient retrospectively assessed her sexual function before the cancer diagnosis. Comparisons were made with results from an age-matched sample of healthy Danish women. The proportion of women having sexual interest dropped from $70 \%$ pretreatment to $10 \%$ immediately posttreatment. At 12- and 24-months' evaluation the proportions of women with sexual interest were $16 \%$ and $26 \%$, respectively. At 3-months, $62 \%$ indicated that they experienced a decreased sexual interest while most women $(87 \%)$ assessed their partner's sexual interest as unchanged compared to before they were diagnosed with CC. A relatively small proportion ceased to have sexual relations due to their cancer (17\%). However, only $50 \%$ and $57 \%$ of sexually active women were able to complete sexual intercourse at 12 and 24 months', respectively. The proportion of women with vaginal changes (lack of lubrication, shortening of vagina and dyspareunia) was high and remained increased compared to pre-treatment values and those of the general population sample. Also, global satisfaction with sex life was reduced compared to that of the general population. Women treated by radiotherapy for cervical cancer experience long-lasting (probably permanent) sexual morbidity.

157/ QUALITY OF LIFE OF BREAST CANCER SURVIVORS IN THE ACUTE, EXTENDED AND PERMANENT PHASES OF SURVIVORSHIP

Lori J. Andreas, Adult Oncology/ Bone Marrow Transplant, OHSU, Portland, Oregon

Most of the women that are diagnosed with breast cancer will be survivors. There is belief that quality of life gradually returns to normal following treatment for breast cancer.However,there appears to be evidence that there are negative changes that occur long after treatment. The purpose of this study is to describe the quality of breast cancer survivors within the physical, psychological, social and spiritual domains. The study also describes how quality life of breast cancer survivors differs in the three phases of survivorship(acute,extended,permanent). The QOL-Cancer Survivors(QOL-CS) Tool created by Ferrell and HasseyDow(1995)was used to collect information regarding quality of life in the four domains(physical, psychological, social and spiritual). This 41 item instrument rated each item on a scale of $0-10$, with 0 being the worst possible and 10 representing the best possible. Psychometric analysis based on te administration of the QOL-CS included a test - retest reliability $(r=.89)$, internal consistency $(r=.93)$. The demographic tool gathered data to describe the sample.There were 56 responses: 12 in the acute, 32 in the extended and 12 in the permanent phases.Physical changes such as aches and pains and fatigue, as well as sleep changes and weight gain were undesirable outcomes for survivors. Psychologically, worse outcomes for survivors included distress associated with initial diagnosis and treatment and distress on family. The most distressing outcome for breast cancer survivors socially was the concern for their daughters or relatives regarding breast cancer. Spiritually, breast cancer survivors rated uncertainity regarding their future and degree that illness had made positive changes had the worst outcomes. The analysis of how quality of life varies in the three phases (acute, extended, permanent) demonstrated that there is no significant variation in the quality of life since diagnosis. The research findings can assist nurses to what components of $\mathrm{QOL}$ are important to breast cancer survivors.
158/ HOW TO MEASURE THE ATTITUDE OF WOMEN WITH BREAST CANCER TOWARDS TRADEOFFS BETWEEN QUALITY AND QUANTITY OF LIFE

Wendy J. Post, Office for MTA, University Hospital Groningen, Groningen, The Netherlands; Anne M. Stiggelbout, Department of Medical Decision Making, Leiden University Medical Center, Leiden, The Netherlands

In medical research it is common to use measuring instruments to evaluate quality of life. Most of these measuring instruments are based on the cumulative principle: the larger the scores of a certain patient on the questions (items), the larger the patient's value on the latent variable. The total score on all items is then a positive linear function of the scores on the different items.But what to do when this principle does not hold, i.e.the case where opposite formulated questions are asked? In this paper we will consider such a case.In a study about the attitude towards tradeoffs between quality of life and quantity of life,a questionnaire consisting of 8 items was filled out by breast cancer patients who had to endure treatments with severe side effects (quality) which were aimed at improving life expectancy (quantity).Some of the items were formulated positively towards quality of life and some were formulated positively towards quantity of life.One of the aims of that study was to assess whether different patient groups respond differently on this item set or not. One possibility to analyse such data is to recode the negatively formulated items. The recoded set can then be analysed in the cumulative way.Another possibility is factor analysis, which frequently results in two distinct factors representing one latent variable. From a measurement theoretical point of view, both possibilities are not optimal. This paper will introduce an item resonse model, which is more appropriate for this case.In psychometric research this model is frequently used and is known as the probabilistic unfolding model, or as the item response model with unimodal Item Characteristic Curves. This model will be applied to these data.First we will construct a measuring instrument (scale) of these items for all women.It will be shown that the same scale holds for different patient groups. After that we will test whether the attitude towards qualityquantity of life is different or not for several subgroups.

159/ THE CANCER OUTCOMES MEASUREMENT WORKING GROUP (COMWG): AN NCI INITIATIVE TO IMPROVE THE SCIENCE OF OUTCOMES MEASUREMENT IN CANCER

Joseph Lipscomb, Division of Cancer Control and Population Sciences, National Cancer Institute, Bethesda, MD; Carolyn C. Gotay, Cancer Research Center of Hawaii, University of Hawaii, Honolulu, $\mathrm{HI}$; Claire F. Snyder, Division of Cancer Control and Population Sciences, National Cancer Institute, Bethesda, MD

The importance of outcomes assessment is increasingly recognized by multiple agencies involved in cancer research. To address the diverse needs of users, it is critical to assess the state of the science of outcomes measurement and identify priorities for future research and practice. Thus, in 2001, the National Cancer Institute invited 35 researchers primarily from academia, medical centers, and government to participate in the COMWG. Over an 18-month period, COMWG members will provide their individual input on the state of the science and recommendations to improve measurement of key patient outcomes in cancer. The COMWG is part of NCl's Quality of Cancer Care Initiative, although the COMWG is not an advisory committee or decisional group. It will help the $\mathrm{NCl}$ define the best approaches to measuring key endpoints of quality care: HRQOL, patient satisfaction, and economic burden. Also, the COMWG will help the $\mathrm{NCl}$ develop its agenda in outcomes research. The COMWG is addressing 4 cancers (breast/prostate/colorectal/lung), the continuum of care (screening/prevention, treatment, survivorship, end of life), and multiple applications (individual patient/clinician decision-making, clinical trials, observational studies). COMWG members are working individually or in pairs on a range of research questions: current best practices and recommendations for the future in assessing the 3 outcomes in the 4 cancers across the continuum of care; different perspectives on and uses of the resulting data; and statistical, psychometric, and methodological considerations. Members with similar research questions or areas of expertise are in affinity groups that review each other's work and provide a multidiscliplinary perspective. They will report to the $\mathrm{NCl}$ in May 2002, and the results will likely be published in a book or special journal issue. In this presentation, we will discuss this initiative and its implications for HRQOL research. 
160/ HRQOL IN PEOPLE WITH APHASIA: PRELIMINARY FINDINGS FROM A PILOT STUDY

Katerina Hilari, Department of Language and Communication Science, City University, London, UK; Sally Byng, Connect-the-Communication Disability Network, London, UK

Aphasia is a language impairment caused by organic damage to the brain. It is a common sequel of stroke and it can affect all language modalities i.e., understanding of language, speaking, reading and writing. Aphasia can pose a serious challenge in the assessment of HRQoL in stroke survivors. This is often reflected in stroke HRQoL studies by the exclusion of people with aphasia from the study sample. This presentation will describe an ongoing study of the HRQoL of people with long-term aphasia following stroke. We aimed to assess $\mathrm{HRQ}$ oL in a way that can be replicated in clinical practice. We thus followed a questionnaire-based approach. The Stroke Specific Quality of Life Scale (SS-QOL) (Williams et al., 1999) was chosen, as it is a patient derived measure specifically developed for use in clinical trials. The SS-QOL was modified in order to increase its content validity with people with aphasia and to make it communicatively accessible to this group. The modified version was tested for feasibility in a pilot study with 18 people with long-term aphasia (>1 year post onset)(Hilari \& Byng, 2001). The refined questionnaire is currently being tested for test-retest reliability and construct validity (convergent, discriminant and known groups validity). As this is an ongoing project this presentation will concentrate on how we administered all instruments used in order to facilitate people with language impairment give their experience of stroke and on the preliminary data of the pilot study. Due to the small sample size, the pilot data are limited and should be interpreted with caution. Nonetheless they give a preliminary impression of our findings. The pilot data suggested that people with mild to moderate receptive aphasia -as determined by a Frenchay Aphasia Screening Test (Enderby et al., 1987) score of 7 out of 15 in receptive domains- were able to complete the modified SS-QOL. The findings also suggested that this small group of people with long-term aphasia had lower scores in the psychosocial domains of the SS-QOL than in the physical domains and the most severely affected area of HRQoL was social life.

161/ CONSTIPATION AND QUALITY OF LIFE IN THE CANADIAN POPULATION COMPARED TO PRIMARY CARE PATIENTS

S. Ferrazzi, Janssen-Ortho Inc., Toronto, Canada; E. J. Irvine, McMaster University, Hamilton, ON, Canada; P. Pare, L'Hotel Dieu de Quebec, QC, Canada; W. G. Thompson, University of Ottawa, Ottawa, ON, Canada; L. Rance, Janssen-Ortho Inc., Toronto, ON, Canada; T. Faloon, University of Ottawa, Ottawa, ON, Canada

Few reports address the impact of constipation on health related quality of life (HRQOL). We evaluated HRQOL status in Canadians with constipation, from the general population and in Primary Care patients using Rome II criteria and Self-Report. Participating family physicians recruited a sample of patients who either presented with or were diagnosed with constipation. Using a random digit dial technique, general population subjects were recruited for a national survey on health related issues. With consent, both groups were sent a survey package containing the Rome II questionnaire, SF-36 and constipation related questions. An independent research firm was employed to perform all subject contact and data retrieval by phone. The 184 family physicians recruited 311 patients of whom 220 completed the survey questionnaire. Mean age was 55.2 years and females comprised $79.5 \%$. For the random population sample, of 10,613 households contacted, 2,001 subjects were eligible and willing to participate. Of these 1,149 completed the questionnaire and had a mean age of 42.2 years and $50.7 \%$ were females. The SF-36 component scores for Self-Report and Rome lla of either group were significantly lower than Canadian norms. No difference was observed between the two groups in Rome lla scores. The physical component score was lower in Primary Care patients self-reporting compared to the general population ( $<<0.05$ - Bonferroni adjustment for multiple comparisons) HRQOL is significantly impaired in subjects with constipation, with the greatest detrimental effect in patient's self-reporting and seeking care from their family physician. Table: ${ }^{*}$ In the past 3 months.

\begin{tabular}{|l|l|l|l|l|l|}
\hline & General & Population & Primary & Care & \\
\hline & Self-Report * & Rome lia & Self-Report* & Rome lla & CDN Norm \\
\hline & $(\mathrm{n}=307)$ & $(\mathrm{n}=162)$ & $(\mathrm{n}=196)$ & $(\mathrm{n}=82)$ & $(\mathrm{n}=9000+)$ \\
\hline & & & & & \\
\hline Mental Component & 47.8 & 48.8 & 45.3 & 48.4 & 51.7 \\
\hline Physical Component & 46.8 & 47.3 & 43.2 & 46.1 & 50.5 \\
\hline
\end{tabular}

162/ QUALITY OF LIFE IN UNIVERSITY STUDENTS WITH CHRONIC HEALTH PROBLEMS: COPING AND CONTEXT

Fuschia M. Sirois, Michelle L. Melia-Gordon, Timothy A. Pychyl, Psychology, Carleton University, Ottawa, ON, Canada

Although student well-being has received attention, the quality of life of students with chronic health issues is often overlooked. The demands and challenges of first-year university may be particularly stressful for students managing ongoing health issues. From a sample of 377 first-year university students (252 females, 125 males), 107 (age $=20.6,+/-4.5 \mathrm{yrs}$.) reported one or more chronic health conditions: asthma (58), chronic pain (41), arthritis (17), or diabetes (5). This group was compared to a non-chronic control group $(n=108$, age $=20.6,+/-4.4$ yrs.) selected from the remaining subjects. After one academic term, students completed self-report questionnaires on psychological indicators of adjustment - negative emotionality (NEM), daily hassles, self-esteem, and coping styles - and health indicators - health-related quality of life (HRQOL) and frequency of common health problems experienced (e.g., cold/flu). Multivariate analysis revealed significant differences on both psychological and healthrelated variables between students with chronic conditions versus those without. Significant univariate tests indicated that students with chronic health problems reported higher perceived stress and NEM, a greater number of common health problems, lower self-esteem, and lower vitality (HRQOL). Analysis of coping styles revealed that students with chronic health problems were more likely to use avoidant strategies (behavioral disengagement) and were less likely to suppress competing activities. Behavioral disengagement related to NEM $(r=.35, p<.01)$ and perceived stress $(r=.35, p<.01)$ in the chronic group. Results suggest that students with chronic health conditions are vulnerable to the stress of adjusting to university life, and may feel overwhelmed by these demands. Maladaptive coping strategies may compromise well-being further. Results highlight the importance of person-environment interactions in assessing HRQOL of young individuals with ongoing health concerns.

163/ EVALUATING QUALITY OF LIFE IN PATIENTS AFTER TREATMENT OF AN OPEN TIBIAL FRACTURE BY USING THE NOTTINGHAM HEALTH PROFILE

Jörg Klewer, Public Health, Dresden Medical School, Dresden, Germany; Werner Knopp, Department of Orthopedic Surgery, Medical School of Göttingen, Göttingen, Germany; Joachim Kugler, Public Health, Dresden Medical School, Dresden, Germany

Modern surgical techniques and consequential reduced mortality require reliable and practical instruments to evaluate quality of life in trauma patients, because the influence of surgical treatment on patients' daily life is still unknown. The purpose of the multi-center-study was a) to analyze quality of life in 221 patients after treatment of an isolated open tibial fracture by using the Nottingham Health Profile (NHP); b) to check the reliability of the German NHP version (Cronbachs Alpha) and c) to evaluate the relation between the NHP scales and the surgical classification of the initial soft-tissue-trauma by Gustilo. The investigated patients experienced primarily impairments in their personal quality of life due to "pain" and reduced "physical mobility". These subjective impairments increased according to the severity of the open fracture. Reliability testing of the NHP scales presented alpha-values from 0,60 to 0,84 . Patients with an open tibial fracture classified as Gustilo I or II showed less impairments in quality of life than patients classified as Gustilo III. The findings indicate that quality of life in patients after treatment of open tibial fractures is impaired due to pain and consequential reduced mobility. Primarily patients with open fractures type III present reduced quality of life. Additionally, the results show the usefulness of the German NHP version to evaluate quality of life also in trauma patients. 
164/ DETERMINANTS OF QUALITY OF LIFE IN LUNG TRANSPLANT PATIENTS: IMPACT OF PERSONAL FACTORS, DISEASE STAGE AND COMPLEMENTARY THERAPY USE

Barbara J. Matthees, Nursing, Minnesota State University Moorhead, Moorhead, MN; Cynthia R. Gross, Academic Health Center, Mary Jo Kreitzer, Center for Spirituality and Healing, Kay Savik, School of Nursing, Marshall I. Hertz, Academic Health Center, University of Minnesota, Minneapolis, MN

Certain traits and behaviors are posited as mediating factors that influence valuations of quality of life (QL) and enable patients to be resilient after successful therapy. We hypothesized that positive and negative outlook, complementary therapy use (CAM) and internal locus of control would be such factors. A sample of 166 lung transplant patients (candidates for transplant $(\mathrm{N}=67)$, recipients $(\mathrm{N}=99)$, mean age 52 yrs, $45 \%$ male) completed a battery of self-report questionnaires including $\mathrm{QL}$ and health-related quality of life (HRQL) measures and scales for these putative mediators. Disease stage differentiates candidates from recipients. Regression models including disease stage, internal control, positive outlook and illness intrusion accounted for over $50 \%$ of the variation in QL and physical HRQL outcomes, and a substantial but lesser amount of mental HRQL outcomes $(41 \%)$. CAM use was not associated with either HRQL or QL outcomes. Recipients reported significantly more internal control, less illness intrusion and less negative outlook than candidates, and they were more likely to be working. Despite large differences in QL, physical HRQL and illness intrusion, candidates and recipients did not differ on mental HRQL outcomes. These findings support a model of $\mathrm{QL}$ in transplant that identifies positive outlook and internality as mediating factors. However, they also suggest that stress and illness may influence internality level and outlook; concurrent measures cannot be assumed to be accurate estimates of stable underlying traits, and should not be interpreted as predictors of QL or resiliency to future illness.

165/ FACT-AN SHOWS SENSITIVITY TO CHRONIC ILLNESSES: RESULTS FROM A WEB-BASED SURVEY

Christina Vandoros, Janssen-Cilag Pharmaceutical SACI, Pefki, Athens, Greece; M. Zagari, D. Gagnon, B. Shilling, The R.W. Johnson Pharmaceutical Research Institute, Raritan, NJ; D. Cella, Northwestern University, Evanston, IL

The FACT-An (Functional Assessment of Cancer Therapy-Anemia) quality-of-life (QOL) questionnaire was designed to measure $\mathrm{QOL}$ impairment in cancer patients, especially those with anemia and fatigue. Recently, the questionnaire was expanded for use in chronic disease and renamed the Functional Assessment of Chronic Illness Therapy. This study investigated the sensitivity of the FACT-An to chronic illness as administered to a nationally representative sample of 1400 people participating in a US Web-TV survey panel. 1,078 people responded $(77 \%)$ to the survey. Demographics and disease history were available. We report results for two main scales: FACT$\mathrm{G}$ (general items) and Fatigue (fatigue-specific items). Where higher scores indicate better QOL, overall mean scores were: FACT-G, 80.14 (range 0-108); Fatigue subscale, 40.07 (range 0-52). For a subgroup of people reporting no chronic illnesses $(n=304)$, mean scores were: FACT-G, 86.78; and Fatigue, 44.18. FACT-G scores were compared between those with particular chronic illnesses to those with no chronic illness. Of the 20 chronic disease subgroups examined, 16 demonstrated statistically significant differences $(p<0.05)$ compared with the no-illness population (effect sizes ranging from 0.57 to 1.64). In orde of decreasing impact these were: stroke, anxiety, hepatitis, depression, heart disease, anemia, carpel tunnel syndrome, cancer, acid reflux/heartburn, diabetes, asthma, ulcer, hypertension, arthritis, allergies and back pain. Thus, Internet-based collection of FACT-An data confirms: 1) Internet-based survey techniques are useful for normative QOL data; and 2) The FACT-An discriminates between respondents reporting no illness and those reporting common chronic conditions, including cancer and anemia.
166/ QUALITY OF LIFE AND COPING STRATEGIES IN FEMALE ALOPECIA

Silke Schmidt, Department of Medical Psychology, University Hospital Eppendorf, Hamburg, Germany; Tobias Fischer, Peter Elsner, Department of Dermatology, Bernhard Strauss, Department of Medical Psychology, University Hospital Jena, Jena, Germany

Measurements of quality of life (QoL) have recently become an integral part of dermatological studies. The relationship between QoL, coping strategies or other determinants of QoL and psychosomatic aspects is supposed to be very complex in particular skin diseases, e.g. female alopecia. The aim of our previous study was to develop a German version of the Skindex, adapted for hair loss. The first aim of this study was to analyse how quality of life, in patients with female alopecia, can be impacted by strategies of coping behaviour, as well as by personality traits. The second aim was to show whether QoL is sensitive to change in a clinical trial and whether coping determines the responses. Fifty female patients, diagnosed with either diffuse or androgenetic alopecia, were evaluated by use of Hairdex, an instrument developed to measure QoL in patients with hair loss. At the first point of measurement coping forms, social support as well as sociodemographic details were assessed by self- and observer report instruments. For the purpose of the second part of the study, a clinical trial was conducted and QoL was measured at the end of the treatment period. Findings indicated that patients with highly visible hair loss reported a more negative impact on four Hairdex dimensions (emotions, functioning, self-confidence and stigmatisation) than patients whose hair loss was only slightly visible. However, a subgroup of patients, with non-visible symptoms of hair loss, showed signs of psychological disturbance. These disturbed patients displayed either dysmorphophobic or affective disorder tendencies. Second, QoL did improve significantly in the experimental group, but not in the placebo group. However, there were some hints that not only dermatologic treatment, but also coping patterns affected the QoL outcome. Future studies using QoL as an instrument in research on patients with alopecia should consider that, in cases of female alopecia, these measurements may be impacted by psychological disturbances.

\section{7/ EFFECTS OF COPING ON MENTAL OUTCOME IN PERSONS} WITH SPINAL CORD LESION

Magnus L. Elfström, Anna Rydén, Health Care Research Unit, Göteborg University, Göteborg, Sweden; Margareta Kreuter, Department of Physiotherapy, Sahlgrenska University Hospital, Göteborg, Sweden; Lars-Olof Persson, Marianne Sullivan, Health Care Research Unit, Göteborg University, Göteborg, Sweden

The purpose of this study was to investigate effects of coping strategies on mental outcome reactions in traumatically acquired spinal cord lesion (SCL) when controlling for sociodemographical, disability-related and social support variables. The study sample comprised 255 participants with non-remarkable distribution of neurological deficit, gender, and age. Coping and outcome measures were the SCLrelated coping and mental outcome scales (Elfström et al, submitted). Coping factors were: Acceptance (revaluation of life values), Fighting spirit (efforts to minimise the effects of the lesion), Social reliance (a tendency towards dependent behaviour). Outcome factors were: Helplessness (feeling perplexed, out of control, low selfesteem), Intrusion (bitterness, brooding), Personal growth (positive outcomes of life crisis). Neurological status and two social support measures from the Interview Schedule for Social Interaction were also included. Stepwise multiple regression analyses were performed. High levels of Acceptance and social support predicted decreased Helplessness, while high levels of Social reliance, less neurological deficit and being a woman predicted more Helplessness. Together these predictors explained $55 \%$ of the variance. Elevated levels of Acceptance, higher education and longer duration of disability predicted less Intrusion, while more Social reliance increased levels of Intrusion. Total amount explained variance by these predictors were $39 \%$. Acceptance was a strong predictor of Personal growth, while duration of disability was a weak negative predictor. These two predictors explained $43 \%$ of the variance. In conclusion, coping substantially influenced mental outcome in persons with SCL. The coping factor Acceptance was the strongest single predictor of elevated positive morale and decreased psychological distress. 
168/ ASSESSING QUALITY OF LIFE IN OSTEOPOROSIS CLINICAL TRIALS

Stephen P. McKenna, Lynda C. Doward, Abigail L. Dewar, Galen Research, Manchester Science Park, Manchester, UK

Osteoporosis is one of the most widespread and serious health problems in the industrialised nations. Several questionnaires have been developed during the 1990s to assess the impact of the condition on the patient. This paper reviews the suitability of these instruments for use in clinical trials, with particular reference to their theoretical basis, the constructs they assess, content development, practicality, reliability, validity, responsiveness and availability of language versions. Literature searches were conducted using Medline. The initial search covered all language publications from 1985 onwards and additional relevant literature cited in these publications was also reviewed. The search identified 5 osteoporosis-specific instruments that collect information relevant to QoL: the Osteoporosis Quality of Life Questionnaire, the Osteoporosis Functional Disability Questionnaire, the European Foundation for Osteoporosis Quality of Life Questionnaire, the Osteoporosis Targeted Quality of Life Questionnaire (OPTQoL) and the Osteoporosis Assessment Questionnaire. Information on the theoretical basis for the content of the instruments was not available. However, instruments were found to focus primarily on constructs of impairment and disability. Thus, they measure aspects of health status that influence quality of life $(\mathrm{QoL})$ rather than QoL itself. Of the measures reviewed, the OPTQoL appears to be best suited for inclusion in a trial as its content, which was primarily derived from patients, is more relevant to QoL issues and less focused on symptoms. It also has better test-retest reliability than the other measures reviewed. However, the OPTQoL's authors argue that it is not an appropriate measure for use in trials as it was designed as a population survey tool. The remaining measures are considered less suitable for inclusion in clinical trials, due to their length and/or complexity and their relatively low levels of reliability, which reduces their ability to detect changes associated with effective interventions.

169/ EVIDENCE SUPPORTING THE VALIDITY OF THE NOTTINGHAM HEALTH PROFILE IN RHEUMATOID ARTHRITIS J. Michael Woolley, Wing W. Chan, Amgen Inc., Thousand Oaks, CA

To assess the validity of the Nottingham Health Profile (NHP) healthrelated quality of life measure in subjects with rheumatoid arthritis. Data on both the NHP and the Health Assessment Questionnaire (HAQ) were collected as part of a multicenter clinical trial conducted in Europe $(n=473)$. The NHP was administered at baseline and week 24 . The NHP consists of 38 items which assess physical mobility (MOB), pain (PAIN), energy (ENRG), sleep (SLP), emotional reactions (EMO), and social isolation (ISO). For both the NHP and the $H A Q$, lower scores indicate better HRQOL, and negative change scores indicate improvements. Validity was assessed by estimating the correlation between the scales of the NHP and the HAQ in crosssection and longitudinally (change score from baseline to week 24). It was hypothesized that both relationships would be positive, and that stronger relationships would be seen in the more physical HRQOL scales of the NHP (MOB and PAIN). In cross-section (first two rows of the table), all six of the NHP scales were positively and statistically significantly $(p<0.001)$ correlated with the HAQ. Additionally, as shown in the third and fourth rows, the changes in all six of the NHP scales were positively and statistically significantly $(p<0.001)$ correlated with the change in the HAQ. As predicted, the highest correlations were seen in the more physical HRQOL scales, MOB and PAIN. These data support the validity of the Nottingham Health Profile as a measure of health-related quality of life in subjects with rheumatoid arthritis.

Correlation Between NHP Scales and the HAQ $(n=473)$

\begin{tabular}{|l|l|l|l|l|l|l|}
\hline Baseline & MOB & PAIN & ENRG & SLP & EMO & ISO \\
\hline HAQ & .773 & .537 & .494 & .270 & .425 & .325 \\
\hline Change Scores & MOB & PAIN & ENRG & SLP & EMO & ISO \\
\hline HAQ & .562 & .521 & .435 & .268 & .343 & .220 \\
\hline
\end{tabular}

170/ THE PRACTICALITIES OF QUALITY OF LIFE MEASURES IN OSTEOPOROSIS CLINICAL TRIALS

Christel Naujoks, Global Health Economics, Novartis Pharma AG, Basel, Switzerland

The paper gives, (i) an overview of existing disease specific quality of life (QoL) questionnaires in osteoporosis including recent developments, (ii) presents a review of designs and results of clinical trials in which these instruments had been used, and (iii) recommends how to use these measures in clinical trials. Five instruments (OQLQ, OFDQ, OPAQ, Qualeffo, OPTQoL) have been developed. The psychometric properties of the questionnaires have been tested empirically by using methods from the classic test theory domain. A trend to shorten instruments, e.g.,OPAQ-1.0 (73 items) to OPAQ-SV (34) is observed. Especially OPAQ and Qualeffo were applied in clinical trials (OPAQ: tiludronate trial; OPAQ, Qualeffo: MORE raloxifene trial). In the MORE trial prevalent and incident vertebral fractures (fx) were associated with decreased QoL. Incident vertebral fx occuring during 3 years of observation significantly decreased mean OPAQ scores compared to baseline. Results obtained for Qualeffo were quite similar to OPAQ results. Even though considerable evidence on the applicability of these QoL measures is available, there is still a lack of knowledge whether these instruments show significant results in patient groups with different drug intervention. One major problem is the relative low frequency of osteoporotic fx. Thus, the number of patients going to have significant QoL changes during a 3-5 year interval is low or results show only small changes on the avarage comparing the treatment groups. In conclusion, although validated instruments are available it might be uninformative to include them in osteoporosis clinical trials. This conclusion mainly refers to the use of QoL instruments as a specific outcome measure. They may show informative results when used, e.g., as a tool for screening of nonspecific negative or positive side effects. Furthermore, alternative design options with including QoL evaluation only in patients with increased risk of prospective $\mathrm{fx}$ might be capable of showing significant results even with respect to the primary outcome.

171/ QUALITY OF LIFE IN PATIENTS WITH ATOPIC DERMATITIS: USING THE JAPANEASE VERSION OF THE SF-36 HEALTH STATUS QUESTIONNAIRE

Keiko Fukuroku, Satoshi Ogino, School of Allied Health Science, Faculty of Medicine, Suita, Osaka, Japan; Takuzou Nagano, Nagano Dermatology and Allergology Clinic Osaka Toyonaka, Osaka, Japan; Takao Harada, Schering-Plough K.K. Osaka, Osaka, Japan

Atopic dermatitis is a common skin disorder with an age onset mainly from infancy to adolescence. Patients with this disorder usually have a long history of repeated relief and relapse, and recently, there have been @increasing reports of severe adult atopic dermatitis. The pruritus and skin rush that accompany the disease plauge the patient's daily life. We began by quantifiying the relationship between the QOL scores of patients and symtpomatic characteristics (including severity measured by SF-36). We followed this by comparing the QOL score of atopic dermatitis patients with the QOL scores of patients with allergic rhinitis including previously reported pollinosis. 281 patients of atopic dermatitis who had been treated at the Nagano dermatology and allergology clinic, aged 16 to 66 years (average :28), were selected for this study. 138 of the subjects were male and 143 were female. The severity of atopic dermatitis was classified in four stages on the basis of the standard method authorized by the Japan Society of Dermatology: (1) mild (22 patients), (2) moderate (167 patients), (3) severe (70 patients), and (4) most severe (22 patients). We scored the physical component summary (PCS) and the mental component summary (MCS) of the SF-36 and assessed the results, concluding that mental components were more influenced than physical ones by atopic dermatitis. 
172/ HELIOTHERAPY AT THE DEAD SEA IMPROVES QUALITY OF LIFE IN PSORIATIC PATIENTS : A PROSPECTIVE STUDY Marianne Amir, Behavioral Sciences and Social Work, Daniel Vardy, Department of Dermatology, Tal Narkiss, Gideon Becker, Department of Behavioral Sciences, Dan Buskila, Department of Internal Medicine, Ben-Gurion University of the Negev, Beer-Sheva, Israel

The objective of the present study was to evaluate the quality of life and psychological well being of psoriatic patients that received heliotherapy treatment at the Dead Sea. Eighty-seven psoriatic patients between the ages 21- $86(M=51.18, S d=14.36), 44$ males $(M=$ $50.27, \mathrm{Sd}=13.92)$ and 43 females $(\mathrm{M}=53.14, \mathrm{Sd}=11.89)$ were administered the following questionnaires before arriving at the Dead Sea (time I) and again at the last day of their stay (time II) : 1.The World Health Organization Quality of Life (WHOQOL-Bref) Assessment Instrument; 2 . The Subjective Well Being Scale (SWLS); 3. The SF-36; 4. Psoriasis Disability Index (PDI); 5. Dermatology Life Quality Index (DLQI). In addition, the patients were evaluated by a physician using the PASI before and after heliotherapy. The patients spent two weeks at the Dead Sea. A matched control group from Wolfson Medical Center are currently being treated with phototherapy for a month duration, the approximate equivalent of a two weeks treatment period with heliotherapy at the Dead Sea. Results showed that the patients at the Dead Sea significantly improved on the WHOQOLBref physical and environment domains, on the SWLS, on the Bodily Pain and Vitality subscales of the SF-36 and on the DLQI. It was concluded that the heliotherapy at the Dead Sea improves quality of life in psoriatic patients.

173/ A METHOD FOR ESTIMATING THE CONTRIBUTION OF DISEASE TO HRQL SCORES: A STUDY OF PERSONS WITH PSORIASIS

Stephen Rapp, Psychiatry and Behavioral Medicine, Wake Forest University School of Medicine, Winston-Salem, NC; Catherine Cottrell, Psychology, Arizona Sate University, Tempe, AZ; Mark Leary, Psychology, Wake Forest University, Winston-Salem, NC

The negative correlation between psoriasis and HRQL is well-documented. To isolate the unique contribution psoriasis makes to HRQL we calculated discrepancy scores between current $\mathrm{HRQL}$ ratings and similar ratings by patients of life without the disease. The construct validity of the HRQL discrepancy scores was evaluated and they were correlated with coping strategies used to manage the social impact of the disease. 318, male $(n=137)$ and female $(n=181)$, community-residing adults (18-91 yr.) with psoriasis responded to a postal survey that measured HRQL, disease severity, coping and other variables. Respondents rated 8 dimensions of current $\mathrm{HRQL}$ and then to rate the same 8 dimensions as if they did not have psoriasis. The differences between the scores estmates the perceived contribution of psoriasis to current HRQL. The construct validity of the HRQL ratings and the discrepancy scores are supported. There are significant, negative correlations between the disease severity and each of the eight $\mathrm{HRQL}$ discrepancy scores (median $=-.40$ range $=-.27$ to -.44 , all ps $<.0001$ ). In addition, the number of non-psoriasis comorbidities correlated significantly with all 6 of 8 dimensions of current HRQL, but with only 3 of 8 ratings of life without psoriasis and 4 of 8 discrepancy scores. Moreover, the magnitude of the correlations between the number of comorbidities and current HRQL ratings was stronger (median $=-0.19$ range $=-0.10$ to -0.40 ) than the correlations between comorbidities and both the psoriasis-free $\mathrm{HRQL}$ ratings (median = 0.09 range $=-0.04$ to -0.30 ) and the HRQL discrepancy scores (median $=-0.11$ range $=-0.08$ to -0.23 ). Discrepancies scores were significantly corrleated with the coping. The present results demonstrate one method for refining estimates of the specific impact of disease on HRQL. Also, greater discrepancy scores are associated with poorer coping.
174/ AN ASSESSMENT OF HEALTH-RELATED QUALITY-OF-LIFE OF PATIENTS WITH MIGRAINE

Bala S. Nadesan, Pharmaceutical Sciences, Texas Tech University, HSC, School of Pharmacy, Amarillo, TX; Paul Cady, Vaughn Culbertson, Pharm. Pract. \& Administration Sciences, Idaho State University, Pocatello, ID

Headache is one of the ten most common complaints to physicians. About $85 \%$ of headaches were migraine headaches. When comparing migraine to other chronic diseases, it is noted that migraine headaches are chronic in nature but have relatively acute physical presentation. Migraineurs may consider their health to be excellent between attacks. However the fear and anticipation of another attack may negatively affect their perception of health. The purpose of the study was to assess the Quality-of-Life of migraine patients. The intent was to gain a better understanding of patients and to give health professionals an insight on patients' perception of health. Migraine patients in the Idaho Medicaid population were randomly selected and sent a mail version of the SF-36 questionnaire. Only patients in the system for a period of one year prior to initializing this project were contacted. The Mental Health status and the health profile of the migraine patients were analyzed. The return rate was $31 \%$. The mean age was 35.3 and approximately $86 \%$ were female. Migraine patients had a mean of 5.3 different prescriptions written and filled by a mean of 2.37 different pharmacies, a mean of $\$ 2,131$ was spent on prescriptions and patients had a mean of 1.99 physician emergency visits. These results when compared to congestive heart failure and hypertensive patients, showed higher means on the above three criteria. The results may be attributed to the unpredictable nature of migraines and resulting trauma to patients. Analysis of mental health status showed migraine patients to have significantly lower mental health status than congestive heart failure and hypertensive patients. The implication is that migraine suffers may need counseling to cope with the fear of impending attacks and may need prophylactic treatment to improve their Quality-of-Life.

175/ HOW CONSISTENT IS SELF-REPORTED HEALTH-RELATED QUALITY OF LIFE IN THE FACE OF COGNITIVE IMPAIRMENT IN MS PATIENTS?

Monika L. Baier, Center for Research Methodology and Biometrics, AMC Cancer Research Center, Lakewood, CO; Deborah M. Miller, Department of Neurology, Mellen Center for MS Treatm, Cleveland Clinic Foundation, Cleveland, $\mathrm{OH}$

This study evaluated the consistency of self-reported health-related quality of life (HRQoL) over the long-term in an MS population characterized by various cognitive impairments. Studies have shown that MS impacts the physical dimensions of quality of life. As $50 \%$ of patients with MS are estimated to have cognitive impairment, the reliability of self-report in this population is suspect. This paper examines the accuracy and reliability of self-reported HRQoL with regards to physical functioning at various levels of cognitive functioning. Relapsing-remitting MS patients who participated in the phase III study of interferon b-1a (Avonex) were re-examined 8 years after their baseline visit. Patients were evaluated to determine the predictive value of the Multiple Sclerosis Functional Composite on MRI, clinical, and HRQoL parameters. These clinical outcome data will serve to demonstrate the accuracy of HRQoL measures, represented by the physical dimension subscale of the Sickness Impact Profile (SIP) across various levels of cognitive functioning. Cognitive functioning was assessed with the Paced Auditory Serial Addition Test (PASAT). Of the 172 patients eligible for follow-up, 153 had cognitive, EDSS, and SIP evaluations at each assessment time. The PASAT scores were divided into quartiles. Spearman rank correlation coefficients were computed between EDSS and the physical dimension subscale, stratified by PASAT quartiles, at baseline, 2 years, and follow-up. The correlation coefficients were not significantly different across the PASAT quartiles for any of the time points. Regressing residuals, obtained from regressing physical subscale on EDSS, on the PASAT scores at all time points also gave non-significant results. Residuals obtained from regressing physical subscale on EDSS were not different across PASAT quartiles. This is a good indication that even cognitively impaired individuals can accurately and reliably assess the impact the disease has on their physical functioning. The lowest correlations were observed at baseline, as would be expected due to the restricted range of EDSS at entry; the highest correlations were obtained at follow-up across all quartiles and time points. 
176/ THE NHP INDEX OF DISTRESS IN PARKINSON'S DISEASE Peter Hagell, Section of Restorative Neurology, Birgitta Sandlund, Jan Reimer, Martin Grabowski, Division of Neurology, Olle Lindvall, Section of Restorative Neurology, Department of Clinical Neuroscience, Lund, Sweden

Measures related to patients' perceptions of disease are ascribed increasing importance. The dichotomous 24-item Nottingham Health Profile-(NHP)-index-of-distress (NHPD) was developed from the NHP, a widely used generic health status questionnaire. Although sparsely evaluated, recent studies have pointed toward the NHPD as a useful measure in Parkinson's disease (PD). In this pilot study, we evaluated the validity and internal consistency (Cronbach's alpha) of the NHPD (Swedish version) in a cross-sectional sample of 68 PD patients (age: $69.1 \pm 10.2$ years; PD duration: $10.4 \pm 9.4$ years). The NHPD measures distress related to ill health, which, in chronic disorders such as PD, probably is individual and influenced by, e.g., coping and adaptation abilities. Therefore, only moderate correlations between the NHPD and indices of PD severity were expected. The mean $\pm S D$ NHPD-score was $6.42 \pm 5.71$ ( $\max =24$ ), ranging between 0 and 21 . No ceiling-, but a 19\% floor-effect was observed. Cronbach's alpha was 0.90 and the average item-to-total correlation was 0.50 Spearman correlations between NHPD scores and PD duration, perceived $P D$ severity, and daily medication ranged between 0.40 and $0.61(p<0.001)$. NHPD scores increased by increasing perceived PD severity (Kruskal-Wallis ANOVA, $p<0.0001$ ). Previous studies using the NHPD in PD have provided evidence of responsiveness (Swedish and Spanish NHPD) and unidimensionality (Spanish NHPD). Here the NHPD shows excellent internal consistency, correlations supporting its validity, and ability to differentiate among PD severity subgroups These data add to past experiences and support that the NHPD is a useful measure in PD. Additional studies addressing, e.g., test-retest reliability, dimensionality, and equivalence among language versions are, however, warranted.

\section{7/ TREATMENT AND HEALTHCARE SATISFACTION AMONG} PEOPLE WITH MIGRAINE HEADACHES

Joan A. Mackell, Pfizer Inc., New York, NY; Diana D. McDonnell, Consumer Health Sciences, Princeton, NJ

Satisfaction with healthcare has been linked to positive health-related behaviors and improved efficacy of care. Among people with migraine headaches, there is unmet need for appropriate diagnosis, medications, and effective care. This analysis will therefore explore whether treatment of migraines with prescription medications improves healthcare satisfaction. Data were from a mailed survey fielded in Spring 2000 . A total of 21,986 questionnaires were returned $(56 \%)$ from an initial mailing to 39,491 US adults whose names were purchased from a large market research company. The 12-page survey covered a range of health topics including opinions, health history, and treatments. Respondents were asked how satisfied they were with their healthcare on a 6-point scale from "strongly disagree" (1) to "strongly agree" (6). Migraine status was assessed by asking whethe respondents had migraines (yes/no), if these were diagnosed by a doctor (yes/no), and if they used a prescription drug (Rx) to treat them (yes/no). Severity was self-reported as mild, moderate, or severe. A total of 4,069 people (19\%) reported having migraines. Of those, $65 \%$ $(n=2,605)$ were diagnosed and $42 \%(n=1,090)$ of those reported currently using an $\mathrm{Rx}$. Compared to those without migraines, people with migraines were less likely to be satisfied with their healthcare $(59 \% \mathrm{v}$. $51 \%, p<.001)$. However, those treating their migraines with a prescription medication were more likely to be satisfied than those who were not taking an $\operatorname{Rx}(59 \%$ v. $50 \%$ p<.001). These bivariate associations remained after controlling for potential confounders (healthcare resource use, overall physical and mental health), when comparing migraineurs to nonmigraineurs $(B=-.07, p=.026)$ as well as diagnosed $v$. undiagnosed $(B=.25, p<.001)$ and people with at least moderately severe conditions $(B=.26, p<.001)$. People who are successfully treated have higher healthcare satisfaction. Physicians' should therefore work with patients to find a medication that is effective for the spectrum of migraine symptoms.
178/ TREATMENT AND QUALITY OF LIFE AMONG PEOPLE WITH MIGRAINES

Joan A. Mackell, Pfizer, Inc., New York, NY; Diana D. McDonnell, Consumer Health Sciences, Princeton, NJ

Migraines affect an estimated 11 million people in the US. Using selfreports, the effects on quality of life are comparable to, if not worse than, angina, arthritis, or depression. This analysis explores whether treatment with prescription medication improves migraineurs' quality of life. Data were from a survey mailed in Spring 2000. A total of 21,986 questionnaires were returned $(56 \%)$ from an initial mailing to 39,491 US adults whose names were purchased from a large market research company. The 12-pages covered a range of health topics including opinions, health history, and treatments. Quality of life was evaluated using the mental score of the SF12, a generic measure of health status (Ware, et al 1996). The mean for the adult US population is 50; higher scores indicate better well being. Migraine status was assessed by asking whether respondents had migraines (yes/ no), if these were diagnosed by a doctor (yes/no), and if they used a prescription drug (Rx) to treat them (yes/no). Severity was self-reported as mild, moderate, or severe. A total of 4,069 people $(19 \%)$ reported having migraines. Of those, $65 \%(n=2,605)$ were diagnosed and $42 \%(n=1,090)$ of those currently used an Rx. In bivariate analyses, those taking an Rx had slightly higher quality of life than those not taking a drug (46.6 v. $45.9, p=.164)$, even though $R x$ users had more severe migraines and severe migraineurs tended to have lower life quality. Since the relationship between quality of life and Rx use was confounded by disease severity, a linear regression model was run, controlling for this and other potential confounders (age, comorbidities, migraine frequency). In this model, those taking an Rx for their migraines had higher quality of life than those not taking a drug $(B=1.08, p=.020)$. Migraineurs who are successfully treated with prescription medications have higher quality of life than those not taking medication. Physicians should work with patients to find a medication that targets their migraine-specific symptoms.

179/ QUALITY OF LIFE IN GERMAN PATIENTS WITH MYASTHENIA GRAVIS

Jörg Klewer, Linda Wondzinski, Annette Friedrichs, Public Health, Dresden Medical School, Dresden, Germany; Dieter Pöhlau, Department of Neurology, Hachen Hospital, Sundern, Germany; Joachim Kugler, Public Health, Dresden Medical School, Dresden, Germany

Myasthenia gravis (M.g.) is a common neuromuscular disease with prevalences of 10 patients per 100000 residents. Progress in medical therapy has continuously increased life expectancy in M.g. patients. In this study we focused on the determinants of quality of life in M.g. patients. In collaboration with the German Myasthenia Association, the self-help organization for patients, 2150 patients were asked to work on a mailed questionnaire on health care situation. The questions were related to demographical data, impairments, therapeutical course, use of complementary therapies, illness related costs and quality of life (SF-36). 1525 patients (average age: 57 years; m/f: $40 /$ 60 ) participated resulting in a response rate of $71 \%$. More than $70 \%$ of the patients had problems in using their arms and moving around, about $40 \%$ reported difficulties in swallowing and ptosis. Nearly all had received acetylcholinesterase inhibitors and about 50\% experienced thymectomy. Approximately $18 \%$ were treated by neurologists and $11 \%$ received care primarily from general practitioners. Despite coverage of neurological therapy by state health insurance, over $50 \%$ spent 25 to 500 USD monthly to ease illness related problems, mainly help in housekeeping and transportation. Quality of life was reduced in all dimensions of the SF-36, especially in vitality and experienced health. Decreased quality of life correlated significantly with increased impairments and the amount of money spent. The results indicate, that despite prolonged life-expectancy, patients quality of life is still reduced. Health care system should provide more measures for improving living situation of M.g. patients. 
180/ QUALITY OF LIFE IN PATIENTS WITH MULTIPLE SCLEROSIS LIVING AS SINGLE

Joachim Kugler, Public Health, Dresden Medical School, Dresden, Germany; Dieter Pöhlau, Department of Neurology, Hachen Hospital, Sundern, Germany; Jörg Klewer, Public Health, Dresden Medical School, Dresden, Germany

Multiple sclerosis (MS) is the most common non-traumatic neurological disease of the central nervous system in younger adults. In this survey, we compared the impact of disease and social problems in patients with MS either living as a single or with a partner. The members of the „Berlin Section" of the "German Multiple Sclerosis Association" were evaluated by using a standardized questionnaire (IRES) including questions on social situation, daily problems, daily health impairment, social support and MS-specific questions about course of disease and therapy. 645 patients (489 female, 156 male) volunteered in the study, which resulted in a response rate of $62 \%$. One third $(n=210)$ of the sample lived as single (166 female, 44 male). Therapeutic regimen and frequency of general medical complaints were not significantly different between both groups. But, singles rated higher on EDSS, reported reduced mobility and more communication disabilities (e.g. hearing, reading). Furthermore, single living MS patients experienced significantly lower quality of life. Moreover, single living patients with MS (especially female patients) were significantly older and suffered from a longer course of MS than patients with a partner. Regression analysis showed that reduced quality of life is significantly determined by living as a single, higher age and female gender. Living conditions are important for the quality of life of patients with MS. The results support the notion that single-living patients have more problems to cope with the disease. That is why these patients require more attention from the health care system to improve their mental and physical health.

181/ ASSESSMENT OF HEALTH STATUS IN PATIENTS WITH CEREBRAL PALSY: WHAT IS THE ROLE OF QUALITY OF LIFE MEASURES?

Michael G. Vitale, Pediatric Orthopaedics, Children's Hospital of New York, New York, NY; Julie C. Choe, Pediatric Orthopaedics, Annetine C. Gelijns, Alan J. Moskowitz, Surgery, InCHOIR, New York, NY; Joshua E. Hyman, Francis Y. Lee, David P. Roye, Pediatric Orthopaedics, Children's Hospital of New York, New York, NY

A prospective study of 60 children with cerebral palsy (CP) seeking orthopaedic treatment was conducted between 1998 and 2000. The purpose of this study was to explore their quality of life (QOL), using two recently published outcome measures. Nearly half of these children were diagnosed with quadriplegia (45\%) and the rest with diplegia $(38 \%)$ or hemiplegia $(13 \%)$; only about half of the patients were able to ambulate $(52 \%)$. Many of the children met developmental milestones $(43 \%)$, but the rest were either delayed $(30 \%)$ or completely involved $(28 \%)$. Since many procedures performed on children with $\mathrm{CP}$ aim to improve QOL, we administered QOL questionnaires (American Academy of Orthopaedic Surgeons' Pediatric Outcomes Instrument and the Child Health Questionnaire) to the parents to identify those measures that are sensitive to this unique population. Scores for pre-operative patients showed floor effects $(33 \%$ of patients scoring near 0 ) for most physical function domains and ceiling effects (33\%-62\% scoring near 100) for most psychosocial domains. Compared to "normal" children, our patients scored significantly lower on all physical function domains (t-test, $p<0.05$ ), but not on any psychosocial domains. Children with quadriplegia scored much lower in physical function scores, but not in psychosocial scores. Similarly, those who were able to ambulate had significantly higher physical function scores than non-ambulators, but psychosocial scores were not affected by ambulatory status. Both questionnaires exhibited appropriate psychometric properties in this population. Our data suggests that rigorous evaluation of specific patients subgroups (eg. quadriplegia) will demand disease-specific measures. These surveys are a significant improvement in assessment methodology for some of our patients and will serve a valuable role in the assessment and comparison of outcomes for these patient groups.
182/ A COMPREHENESIVE REVIEW OF CAREGIVER BURDEN MEASURES IN ALZHEIMER'S DISEASE

Jeff Borenstein, Cedars-Sinai Health System, Zynx Health Inc., Beverly Hills, CA; Sobin Chang, Outcomes Research, Novartis Pharmaceuticals Corporation, East Hanover, NJ; Eileen Reyes, Simcha M. Russak, Research, Cedars-Sinai Health System, Zynx Health Inc., Beverly Hills, CA; Aaryn S. Cohen, Outcomes Research, Novartis Pharamaceuticals Corporation, East Hanover, NJ; Chiun-Fang Chiou, Research, Cedars-Sinai Health System, Zynx Health Inc., Beverly Hills, CA

The purpose of this research was to review and assess current caregiver burden measures in Alzheimer's Disease (AD). A systematic search of MEDLINE, HEALTHSTAR, and PUBMED databases was conducted to identify all disease-specific and general caregiver burden measures used within the AD population. The accuracy and ultimate value of a measure was assessed through 3 general psychometric indices of performance: reliability, validity, and responsiveness. The evaluation of caregiver burden instruments along these psychometric properties were identified and compared across measures. A total of 62 articles were found to contain background information or application of $A D$ caregiver burden measures. Of these articles, 10 measures were identified. Four were AD-specific (Screen for Caregiver Burden, Caregiving Hassles Scale, Caregiver Burden Inventory, and Caregiver Burden Scale) and 6 were generic measures (Burden Interview, Memory and Behavior Problems Checklist, 3 Caregiver Burden Scales, and Rabins, Mace and Lucas' Structured Interview) used within an AD population. The available evidence of each measure's reliability, validity and responsiveness varied. While a majority of the measures ( 9 out of 10 ) reported reliability, few contained content validity and responsiveness data. The Screen for Caregiver Burden was the only measure that reported reliability, construct and content validity, and responsiveness and performed well. The lack of psychometric properties reported in the majority of measures may limit the ability to accurately assess caregiver burden and to evaluate changes in response to interventions intended to lessen this burden. Future validation studies of $A D$ caregiver burden measures will benefit by addressing these issues.

183/ THE INFLUENCE OF GENDER ON HEALTH-RELATED QUALITY OF LIFE (HRQL) OF FAMILY CAREGIVERS OF STROKE SURVIVORS

Carole L. White, Sharon Wood-Dauphinee, Nancy E. Mayo, James Hanley, Epidemiology \& Biostatistics, McGill University, Montreal, QC, Canada

Most stroke survivors return to live in the community despite many suffering some degree of permanent disability. This is achieved largely through the assistance provided by informal care providers, most of who are women. There is limited evidence concerning the impact of caregiving on $\mathrm{HRQL}$ and whether this effect is modified by gender. As part of a longitudinal study of stroke, 97 family caregivers were assessed every 6 months for two years with a battery of measures including the Burden Interview, SF-36 and a visual analogue scale measuring quality of life (QoL). Caregivers were $74 \%$ female, an overall mean age of 57 years and were most likely to be spouses. A comparison of scores on the SF-36 at baseline with Canadian age-matched norms showed that women scored between 17 and $24 \%$ below the norms on the mental sub-scales while men scored 9 to $13 \%$ lower. Although scores for men improved over time, scores changed little for women, thus widening the differences between genders. Caregiver scores on the physical sub-scales were similar or even higher than the age, sex-matched norms and remained stable over time. Although increased burden was initially associated with decreased scores on the Mental Component Summary score (MCS) of the SF-36, over time this association was modified by gender. While increasing burden did not impact on MCS for males, for females, increased burden was associated with decreased MCS scores. Burden was not associated with the physical sub-scales. There was a positive relationship between the MCS and QoL and this was significantly stronger in women than in men. These results suggest that the caregiving role impacts on both mental and social health of family caregivers and this effect is different in women and men. The high scores on physical health may be suggestive of a "healthy caregiver effect". Understanding the mechanisms of gender differences in caregiving and $\mathrm{HRQL}$ will assist in designing interventions for family caregivers. 
184/ QUALITY OF LIFE OF ENROLLEES AND NON-ENROLLEES IN FORMAL WEIGHT-LOSS PROGRAMS

Donald L. Patrick, Health Services, University of Washington, Seattle, WA; Donald M. Bushnell, Health Research Associates, Seattle, WA; Laura A. Glauda, Dennis D. Gagnon, Margaret Rothman, The R.W. Johnson Pharmaceutical Research Institute, Raritan, NJ

To compare the quality of life of persons enrolled and not enrolled in formal weight-loss programs over 12 weeks and to examine important quality-of-life correlates of weight change, including demographic characteristics, bothersomeness of weight-related symptoms, and depressive symptomatology. Two groups of overweight persons were compared: 1) 137 enrollees in diet, exercise, and health programs for losing weight, and 2) 154 non-enrollees recruited from the general public. Subjects had a Body Mass Index of 27 through 50 and were between the ages of 18-75. Respondents completed the Weight-Related Symptom Measure (WRSM), Obesity and Weight-Loss Quality of Life Instrument (OWLQOL), Centers for Epidemiological StudiesDepression Scale (CES-D), Perceived Quality of Life (QCOL) Scale and demographics. QOL improvements at end of 12 weeks were observed for both enrollees and non-enrollees, but greater improvements were reported by enrollees in the OWLQOL and QCOL. (Table) Sixty-six \% of enrollees lost weight (mean $=2.9 \mathrm{~kg} ; \mathrm{SD}=2.6$ ), and $60 \%$ of non-enrollees lost weight (mean=2.4 kg;SD=2.1). Losing weight and being depressed at baseline increased QoL differences between enrollees and non-enrollees in a similar pattern. Being enrolled in a formal weight loss program, losing weight, and having depressive symptomatology were associated with improvements in obesity-specific and generic quality of life.

\begin{tabular}{|l|l|l|l|l|}
\hline Study Group & WRSM Change (SD) & OWLQOL Change (SD) & QCOL Change (SD) & CES-D Change (SD) \\
\hline New Enrollees (N=137) & $-8.4(14.0)$ & $10.7(12.3)$ & $0.4(0.9)$ & $-2.2(7.4)$ \\
\hline Non-enrollees (N=154) & $-5.9(14.0)$ & $4.6(12.4)$ & $0.0(1.1)$ & $-1.3(8.2)$ \\
\hline F stat (Sig.) & 2.3 (ns) & $17.4(0.001)$ & $9.2(0.01)$ & $0.8(\mathrm{~ns})$ \\
\hline
\end{tabular}

185/ A PHILOSOPHICAL ANALYSIS OF THE MEANING OF SUFFERING

Laura M. DeHelian, Nursing, Cleveland State University, Cleveland, $\mathrm{OH}$

It is well accepted that the suffering of persons experiencing illness or injury, particularly chronic or terminal illness and function altering injury, directly affects those individuals' evaluation of the current and future quality of their lives. The purpose of a philosophical analysis of suffering is to formulate a common understanding of what it means to suffer. A review of the literature clearly indicates that none currently exists. Such an understanding can serve as a theoretical framework in the development of a mechanism for accurately assessing the nature of the suffering of particular individuals. This theoretical framework will also aid in the development and employment of strategies to adequately address such suffering, thus positively affecting the quality of life for both the sufferer and those affected by him. Not only does the theoretical framework presented address the concerns of assessment and intervention, but also serves to argue for the position that once adequately understood, the concept of suffering offered lends great weight to the ethical imperative to appropriately address it. The method employed was a wide review and philosophical analysis of expositions on the concept of suffering, which suggests that the commonality of the experience of suffering can be characterized as a serious disruption in the meaning structure in which one's identity is imbedded. The illness or injury an individual undergoes invalidates his previous meaning structure. Suffering is experienced in the interval before a new meaning structure, one in which the changes brought about by the illness or injury are successfully incorporated, is formed. Although the experience of being caught in the gap between meaning structures is a common one, there remains great variation in the purpose that various individuals believe such suffering serves in their lives. This variation is responsible for the complexity of the suffering experience, making accurate assessment and appropriate intervention so crucial.
186/ RELATING CONCEPTS OF DISEASE \& ILLNESS IN HRQL RESEARCH

Michael A. Hagan, Researcher, Health Systems Strategies, Columbia University, Mailman School of Public Health, Denville, NJ; Steven M. Albert, Assistant Professor of Neuropsychology and Public Health, Gertrude H. Sergievsky Center, Columbia University, Denville, NJ

Anthropological concepts of disease and illness as coined by Kleinman have not been related to HRQoL. In our research we used hemoglobin $(\mathrm{Hb})$ as a biomedical indicator of disease (i.e. anemia) and Pain Score, recorded on a linear analog scale assessment (LASA), as an indicator of patient-perceived disability associated with hypercalcemia. These are secondary analyses on a dataset $(n=200)$ from a clinical trial of cancer patients with metastases to the bone. A series of least square regression models were used; from linear through quadratic and cubic models. The coefficient of determination increased from 0.301 to 0.305 and 0.353 for the linear quadratic and cubic models respectively. On the average, the Pain Scores improved by 10 $\mathrm{mm}$ for each $1 \mathrm{~g} / \mathrm{dl}$ increase in $\mathrm{Hb}$. The inflection points were calculated to be at 8.92 and $15.15 \mathrm{~g} / \mathrm{dl}$. The releveance of the increase in explanation of the variability in the model was tested through a stepwise regression model to test the hypothesis $\mathrm{H} 0$ : The higher order terms do not make a difference. It can be rejected at the 0.01 significance level. Although the disease indicator $(\mathrm{Hb})$ in continuous, patients perceive their health in terms of thresholds. Below a Hb level of $10 \mathrm{~g} / \mathrm{dl}$, further decrements in health are not detected well. Similarly, improvements in health for $\mathrm{Hb}$ higher than $15 \mathrm{~g} / \mathrm{dl}$ are not detected well. Therefore, in this population, while the biomedical measure of disease $(\mathrm{Hb})$ is continuous, the HRQoL (Pain Measure) of illness is discrete and based on perceptual factors.

\section{7/ ASSESSING THE SUBJECTIVE IMPACT ON INFORMAL CAREGIVERS OF CARING FOR CANCER PATIENTS: A REVIEW OF CONCEPTS AND MEASURES}

Claire F. Snyder, Division of Cancer Control and Population Sciences, National Cancer Institute, Bethesda, MD

Subjective caregiver impact is defined here as the effect of the patient's disease and its treatment on the informal caregiver's everyday life and overall well-being. The impact on informal caregivers is an increasingly important consideration as patient care is shifted from the inpatient to the outpatient setting and informal (unpaid) caregivers take on significantly more responsibility for patient care. As a result of the physical and emotional demands of caring for a cancer patient, informal caregivers may experience effects on their physical, emotional, social, and role functioning. These subjective impacts are distinct from objective impacts (e.g., \# of hours spent caregiving). Instruments have been developed to assess the subjective impact on informal caregivers specific to cancer and generically. This review will focus on the areas of the informal caregiver's life affected by caring for a patient with cancer and the measures that have been developed to assess this impact. A conceptual model of subjective caregiver impact will be discussed. The model outlines the relationship among the patient's disease and its treatment, the negative impacts on the caregiver (e.g., fatigue, anxiety), and the positive impacts on the caregiver (e.g., satisfaction, feelings of usefulness) as they relate to overall subjective caregiver impact. Measures developed to assess subjective caregiver impact will be presented with regards to the following: content, psychometric evidence (validity, reliability, responsiveness, interpretability), and practical considerations. The relative strengths and weaknesses of the measures will be compared. Measures developed specifically for informal caregivers of cancer patients (e.g., Caregiver Quality of Life Index-Cancer) and measures that are not specific to cancer caregivers (e.g., Burden Interview) will be considered. Potential applications for subjective caregiver impact research and future needs to develop the science will be discussed. 
188/ FACTORS THAT PREDICT HRQOL IN PATIENTS WITH RELAPSING REMITTING MS: RESULTS OF AN 8-YEAR STUDY Deborah M. Miller, Richard Rudick, Mellen Center, Neurology, Cleveland Clinic Foundation, Cleveland, OH; Monika Baier, Garry Cutter, Center for Research Methodology and Biometrics, AMC Cancer Center, Lakewood, CO; David Daughtery, Neurology, Walter Reed Army Medical Center, Washington, DC; Bianca Weinstock-Guttman, Neurology, Buffalo General Hospital, Buffalo, NY; Michele Mass, Neurology, Oregon Health Sciences Center, Portland, OR; Elizabeth Fisher, Biomedical Engineering, Cleveland Clinic Foundation, Cleveland, $\mathrm{OH}$; Nancy Simonian, Medical Research, Biogen, Inc., Cambridge, MA

The purpose of this study was to determine the impact of objective disease measures and $\mathrm{HRQOL}$ in predicting final $\mathrm{HRQOL}$ in patients with relapsing remitting multiple sclerosis (RR-MS) over 8 years. Much remains to be learned about HRQoL changes in patients with progressive disease. We hypothesized an association between changes in objective disability measures and the physical aspects of future $\mathrm{HRQOL}$ while future psychosocial $\mathrm{HRQ}$ oL would be more closely associated with previous measures of the same dimension. We conducted a secondary analysis of data from a follow up study of RR-MS patients who participated in a phase III study of interferon Beta-1a (Avonex) to determine the predictive value of previous subjective and objective disease measures on future HRQoL. Measures of Sickness Impact Profile, Expanded Disability Status Scores (EDSS), MRI exams and Multiple Sclerois Composite Scores (MSFC) were obtained at baseline, year-2 and year-8. One-hundred fifty-three subjects participated in this secondary analysis. All indicators of disease severity worsened from baseline to year-8 follow-up. Worsening of the Physical Dimension of the SIP was statistically significant between baseline and follow-up. There was a trend towards worsening Psychosocial Dimension that was not statistically significant. Regression analysis indicated that the follow-up SIP Physical was most strongly associated with the change in the MSCF from year-2 to follow-up, overall $\mathrm{R} 2=88.06$. Follow-up SIP Psychosocial was most strongly associated with the baseline SIP Psychosocial, overall R2=52.8. Regression results indicate that in this follow-up the final Physical Dimension is largely explained by objective changes in physical status and the final regression model accounts for a large proportion of the final score. Much remains unexplained about the final Psychosocial Dimension.

\section{9/ WHAT DO WE MEASURE: HRQL OR HRQL DETERMI- NANTS?}

Marie-Claude Simeoni, Christophe Sapin, Audrey Clement, Stéphanie Antoniotti, Pascal Auquier, Public Health Department, Medical School, Marseille, France

One major issue in $\mathrm{HRQL}$ measurement is dealing with the definition of the concept which should be assessed. Part of this debate consists in differentiating HRQL from its determinants. In the literature some determinants of $\mathrm{HRQL}$ are already identified. We studied the impact of various determinants on HRQL in adolescents (11-17 years). The variables that we selected to be studied as determinants were: socio-demographic features including gender, age, social class, residence, familial structure - family resources - health status: disabilities, illnesses - health behaviours: risk behaviours, protective factors - health services utilisation HRQL was assessed by the VSP-As, a validated HRQL instrument specifically designed for adolescents and developed from their exclusive viewpoint. This tool provides an outcome measurement in the way that it measures the impact of health status on everyday life, including functioning but also feelings and emotional aspects. The questionnaire, comprising VSP-As and items related to determinants, was self-administered and completed in two random samples of school adolescents $(n 1=2,941$, mean age $=14.2 \pm 2.0$ years; $n 2=1,371$, mean age $=15.0 \pm 0.9$ years . Differences were found according to most of the criteria that were studied, notably concerning gender, age group, social class, health status, health behaviours (smoking, practice of sports...), but the importance of their impact varied a lot from one to another. In conclusion, we stressed that some HRQL instruments developed for adolescents and children include the assessment of HRQL determinants. We should then be careful when computing HRQL scores so that determinants are not taken into account both as determinants of HRQL and as outcome measurement of the impact of these determinants. In a pragmatic perspective, in our attempts at improving the HRQL of the adolescents, we should focus on those determinants for which changes are achievable.
190/ A NEW CONCEPTUAL MODEL FOR TREATMENT SATISFACTION: WHAT IS IT? WHY MEASURE IT? AND, HOW BEST TO USE IT

Meryl Brod, Vice President, Scientific and Research Affairs, Susan Mathias, Vice President, Health Outcomes Research, The Lewin Group, San Francisco, CA; Erin Sullivan, Research Scientist, The Lewin Group, Boston, MA; Shoshana Colman, Senior Research Manager, The Lewin Group, San Francisco, CA

The inclusion of treatment satisfaction as an important endpoint for clinical and late phase trials is receiving increasing attention from the scientific, pharmaceutical and regulatory communities. While there is some consensus on the broad concepts of satisfaction, there is less agreement on the key factors necessary for appropriately developing and validating satisfaction measures. These factors include adequate definition of terms, identification of key domains, and a commonly accepted conceptual model. Additionally, development and validation of satisfaction measures is challenging for multiple reasons, ranging from choosing the appropriate research design to analytic issues. Issues which must be addressed include the selection of generic versus disease or treatment specific items. Additionally, how best to use treatment satisfaction data is poorly understood. Understanding these issues and evaluating the strengths and weaknesses of treatment satisfaction measurement and validation techniques is crucial to the development of the field if these measures are to be included in clinical and late phase trials and considered as an outcome worthy of labeling claims or regulatory review. We have developed a new conceptual framework and model for Treatment Satisfaction derived from interviews with clinicians, patients and providers, as well as literature review and qualitative experience. The five key domains which have emerged are satisfaction with: efficacy, side effects, symptom relief, convenience and willingness to use. This paper will present this new framework and the full conceptual model of domains, mediators and endpoints. A state-of-the-art review of the field of Treatment Satisfaction will be provided. Additionally, tailoring the model to specific teatments, and conceptual, instrument development and validation issues will be addressed. Reasons for assessing treatment satisfaction from the industry, provider and patient perspective will be highlighted. The challenges to adequately measuring Treatment Satisfaction as well as best practices for using the data will be outlined.

191/ TIME PRESSURE AND EMOTIONAL WELL-BEING IN THE LIVES OF CANADIAN ADOLESCENTS AGED 12 TO 18 (1986 TO 1998)

Jiri Zuzanek, Roger Mannell, Recreation and Leisure Studies, University of Waterloo, Waterloo, ON, Canada

The paper is based on the analyses of Canadian time use and population health data, collected by Statistics Canada during the period from 1986 to 1998. In particular, the data from the following surveys will be examined: the 1986, 1992 and 1998/99 General Social Surveys (time use, time pressure, life satisfaction) and the 1994, 1996, and 1998 National Population Health Surveys (stress, mental health, emotional well-being). An attempt will be made to compare Canadian trends with trends observed in other developed industrial countries. The paper will address the following questions: How have Canadian adolescents' self-assessed health, life satisfaction and emotional wellbeing changed over the past 15 years? Are the adolescents' emotional well-being and health affected by time use and subjective sense of time pressure? Specifically, how does participation in physically active and social leisure affect adolescents' physical and mental health and their emotional well being? How do Canadian adolescents' selfassessed health and subjective sense of well-being compare to that of youth in other highly developed industrial countries? 
192/ DETERMINANTS FOR ADOLESCENT QUALITY OF LIFE: A LONGITUDINAL STUDY

Lynn H. Meuleners, Andy H. Lee, Colin W. Binns, School of Public Health, Curtin University of Technology, Perth, Western Australia, Australia; Anthony G. Lower, General Practice, Combined Universities Centre for Rural Health, Geraldton, Western Australia, Australia

There is currently little research available that documents the dynamic progression for adolescent quality of life (QOL) over time. The purpose of this study is to prospectively investigate the stability of determinants for adolescent quality of life (QOL) over a six months period. The generic Quality of Life Profile-Adolescent Version questionnaire was administered to 363 adolescents on two occasions. Subjects were aged 10 to 19 years and were currently enrolled in 20 high schools within the Perth metropolitan area. Five latent constructs (physical health, social, psychological, opportunities and environment) constituting adolescent QOL were modelled by means of multiple indicators based on a second-order confirmatory factor analysis. In addition, four single indicator background variables were hypothesized as predictors for adolescent QOL based on the results of a longitudinal multilevel model. The stability of the determinants over six months was tested using a structural equation modelling approach (SEM). A satisfactory fit was obtained for the SEM model. The amount of control perceived by the adolescent and their perceptions of health were found to be significantly associated with QOL. The findings support the application of a SEM approach in assessing determinants for adolescent QOL over time.

\section{3/ OVERALL QUALITY OF LIFE IN THE GENERAL NORWE-} GIAN POPULATION MEASURED BY THE QOLS-N

Astrid K. Wahl, Faculty of Health, Buskerud College, Drammen, Norway; Berit R. Hanestad, Department of Public Health and Primary Health Care, University of Bergen, Bergen, Norway; Tone Rustoen, Faculty of Nursing, Oslo University College, Oslo, Norway; Anners Lerdal, Øistein Knudsen Jr., Faculty of Health, Buskerud College, Drammen, Norway

The purpose of this study was to validate the Norwegian version of the Quality of Life Scale (QOLS-N) in a random sample of the general population. The QOLS was developed by Flanagan and further adapted by Burckhardt et al., and intends to measure overall quality of life. The questionnaire contains 16 items with regard to satisfaction with physical and material well - being, personal development, relationships with others, participation in social, community and civic activities, and recreation. The possible scale score range from 16 to 112. Higher scores indicate better quality of life. Four thousand randomly assigned people were sent the questionnaire by mail. Eighteen hundred and sixty seven responded after one reminder. Fiftytwo percent of the sample was men and the mean age was 45 years ( SD 15.9). Sixty-two percent had one or two years at upper secondary school or less. Twenty $\%$ had polytechnic or university less than 4 years, and $17 \%$ reported more than four years in polytechnic or university. Seventeen percent of the sample lived alone, and $28 \%$ reported to suffer from chronic diseases. Results showed an overall quality of life mean score of 84.2 (SD 12.2). Men scored significantly lower than women $(p=0.002)$. Higher age and higher levels of education were positively associated with better quality of life. People living alone reported significantly worse quality of life $(p=0.000)$ than those living together with someone. In addition, people with chronic diseases had worse overall quality of life than those who reported themselves as healthy $(p=0.000)$. The Cronbach's alpha of the QOLS-N was estimated to 0.88 . The correlation coefficient between the QOLS score and the global question of quality of life (question 1) in the WHOQOL-BREF was $0.63(p=0.000)$. In conclusion the questionnaire seems to be a reliable and valid instrument for measuring overall quality of life in the general Norwegian population, discriminating between different subgroups as expected.
194/ QUALITY OF LIFE AND SELF-RATED HEALTH

Dagmar Dzurova, Charles University, Prague, Czech Republic; Eva Dragomirecka, Prague Psychiatric Center, Prague, Czech Republic

In the Czech Republic, the overall quality of life as well as several health status indicators have improved remarkably in the post-1989 period (for instance, life expectancy increased by 6 years between 1989 and 2000). However, these improvements have not been uniformly distributed throughout the population; in fact, different population subgroups and regional units have undergone very different changes. The aim of this survey is to analyze information about the subjective views of quality of life, including respondents' self-rated satisfaction with health and changes over time in their attitudes. In 1996-97 and 2000-01, two samples representative of the Czech population aged $15-79$ years (488 and 893 persons, respectively) completed the SQUALA questionnaire. The SQUALA is designed to assess 23 areas of life (value preferences); to measure satisfaction with these areas; and to derive an overall score termed 'the score of the quality of life' (QOL). Self-rated health (SRH - one of the 23 areas of life) was measured on a five-point scale. Associations between SRH and $\mathrm{QOL}$ were estimated using the logistic regression. Associations between poor $\mathrm{SRH}$, poor QOL and other factors were expressed in terms of odds ratios. Results are presented for three models: (a) adjusted for age and sex; (b) adjusted for age, sex, level of education, marital status, material well-being and size of place of residence; and (c) with all variables included. Analyses were performed using the statistical software 'SPSS for Windows'. Material deprivation is strongly associated with both $\mathrm{SRH}$ and QOL. This finding confirms the hypothesis that economic well-being is an important factor of the quality of life, especially when it declines below a certain level. The association is a reflection of the current social reality in which negative consequences of the post-communist transformation disproportionately affect the economically less successful segments of the population. This research was supported by the grant No. VZ MSM 113100007 and CEZ MZ 00000023752.

195/ HEALTH RELATED TRADITIONAL BEHAVIORS CAN ALSO BE DISCRIMINATED BY HRQOL INSTRUMENTS: WHOQOL-BREF IN A POPULATION AND HEALTH SURVEY

Erhan Eser, Public Health (Halk S.), Gonul Dinç, Cemil Ozcan, Ümit C. Atman, Ahmet M. Oral, Public Health, Celal Bayar University Medical Faculty, Department of Public Health, Manisa, Turkey

Quality of Life Instruments can be used in demonstrating socioeconomic inequalities as well. The aims of this study were to define the population standards of the WHOQOL for the reproductive age women and to compare the migrated and native women's physical, psychological, social and environmental quality of life scores. This study is a cross sectional study which is a part of the Manisa Demographic and Health Survey finalized in February 2000. Manisa is a 200000 censused city in the western part of Turkey. Data were collected from 1641 married women of reproductive (15-49 years) age. The World Health Organization quality of life instrument WHOQOL-BREF which includes physical, psychological, social and environment domains were used as a tool of qol measure. The mean scores were 15.1+2.3 for physical; $13.7+-2.5$ for psychological; $14.8+-2.5$ for social and 13.5+- 2.2 for environment domains. The women living in urban areas have given statistically higher $(p<0.0001)$ qol scores than the women living in the sub-urban settlements. Similarly, the higher the years of education, the greater the qol scores were held $(p<0.0001)$. The low socio-economical class (unskilled workers plus blue collar industry workers plus unemployed) has given lower scores than the high socioeconomic class (the rest of them) in every domain scores. Migration has a negative effect on the qol scores for all of the domains except social relations domain. The women who have migrated from the eastern part (i.e. most deprived part ) of the country have shown lower scores $(p<0.0001)$ compared either with the immigrants from the other regions or native inhabitants. Cultural and ethnic solidarity among the eastern immigrants could possibly have neutralized the expected difference in the social domain score. WHOQOL-BREF can be accepted as a very appropriate instrument in demonstrating the socio-cultural inequalities. 
196/ EFFECTS OF ALTERNATIVE MEDICINE ON THE QUALITY OF LIFE OF RESIDENTS IN A JAPANESE LARGE POPULATION SURVEY

Kauzo Uebaba, International Research Center for Traditional Medicine, Toyama City, Toyama Prefecture, Japan; Feng-Hao Xu, Akira Nagata, International Research Center for Traditional Medicine Toyama City, Toyama Prefecture, Japan; Hideki Origasa, Toshihisa Morioka, Biostatistics, Toyama Medical \& Pharmaceutical University, Toyama City, Toyama Prefecture, Japan

There has been an increasing interest in complimentary and alternative medicine(CAM) in general population. It is also expected that CAM may enhance the QOL of people. We have already known the result of WHOQOL for Japanese population as Nakane\&Tazaki's report (Soc Med.1999; 9:123-130). Our region is well known to have a high prevalence of CAM users. The purpose of this study was to clarify the effects of CAM by comparing the QOL in our region with the Nakane \& Tazaki's report that would be considered as the Japanese standard of WHOQOL data. We mailed a WHOQOL26 questionnaire with supplemental sheets about utilization of CAM, to all of the residents of 60 and 61 years old $(\mathrm{N}=1568)$ in our region from the Basic resident registers. We compared QOL of our survey with Nakane \& Tazaki's report by the age-adjusted method. We also surveyed the same content to current CAM users $(\mathrm{N}=248)$ of the same age $(58$ to 62 years old) who are attending our centers for Traditional Medicine. The QOL of these CAM users was compared with the data of the residents of the same age as above. A total of 928 residents (response rate $59.3 \% ; 428$ male, 500 female) responded to our WHOQOL questionnaire. We obtained 129 answers from the current CAM users (response rate $52.0 \% ; 29$ male, 100 female). Mean of the QOL score in 928 residents was 3.38 (male; 3.39 , female; 3.37 ), and in CAM users was 3.49 (29 male; $3.55,100$ female; 3.47 ), which values were higher than the previous report in Japanese population of 580 people of 50 to 79 years old (267 male; $3.30,313$ female; 3.36 ). There was only 3 to $4 \%$ difference in each of the four domains between a general population and CAM users; however, the QOL of CAM users was consistently higher than residents. We found that Quality of life could be enhanced by CAM in older people.

\section{7/ MEASURING FATIGUE IN THE GENERAL POPULATION}

Anners Lerdal, Faculty of Health, Buskerud College, Drammen, Norway; Torbjørn Moum, Faculty of Medicine, University of Oslo, Oslo, Norway; Astrid K. Wahl, Faculty of Health, Buskerud College, Drammen, Norway; Tone Rustøen, Faculty of Nursing, Oslo University College, Oslo, Norway; Øistein Knudsen Jr., Faculty of Health, Buskerud College, Drammen, Norway; Berit R. Hanestad, Department of Public Health and Primary Health Care, University of Bergen, Bergen, Norway

Fatigue, or the feeling of lack of energy, is reported to be a common complaint in the general population. When people become acutely ill or experience chronic illness, fatigue is often one of their symptoms. There are several instruments designed to measure fatigue. One of the instruments of self-reported fatigue witch has been used in different patient populations in different countries, are the 9 item Fatigue Severity Scale (FSS) developed by Krupp et al. In addition to the FSS, three specific items measuring sleep disturbance were used. These items had been developed separately and used earlier in a population study of individuals with Multiple Sclerosis. To interpret responses from this study, data from the general population was needed for comparison. A study of fatigue in the Norwegian population was undertaken using the FSS. Four thousand randomly assigned people were sent the questionnaire by mail. Eighteen hundred and sixty seven responded after one reminder. Fiftytwo \% of the sample was men and the mean age was 45 years (SD 15.9). Sixtytwo \% had one or two years at upper secondary school or less. Twenty $\%$ had polytechnic or university less than 4 years, and $17 \%$ reported more than 4 years in polytechnic or university. Seventeen $\%$ of the sample lived alone, and $28 \%$ reported to suffer from chronic diseases. The study was undertaken the autumn of 2000 . Fatigue correlated with sleep disturbance $(r=0.40, p<0.001)$, self reported chronic illness ( $r$ $=-0.35, p<0.001)$ and level of education $(r=-0.20, p<0.001)$. Fatigue showed a slight correlation to gender and age.
198/ THE HEALTH STATUS OF MIGRANT FARM WORKERS IN GEORGIA - AN INVISIBLE BUT INTEGRAL SEGMENT OF OUR POPULATION

Tom Himelick, Emory Physician Assistant Program, Alison Lauber, Department of Family and Preventive Medicine, Emory University, Atlanta, GA; Teresa Zyczynski, Health Economics and Outcomes Research, AstraZeneca LP, Wayne, PA; Cheryl Silberman, Elizabeth Adams, Health Economics and Outcomes Research, AstraZeneca LP, Wilmington, DE

Migrant farm workers and their families represent one of the most vulnerable, yet economically essential, populations in the United States. Their frequent relocation across state lines, arduous manual labor, cultural barriers, unstable political and economic status, and limited access to health care predispose this population to a greater risk for work-related injuries and of developing chronic and communicable diseases. A number of national and local programs have been implemented to assist communities in providing care to this underserved population. Because of the unpredictability of both the crop season and the migrant farm worker pool, communities have difficulty in planning for the impact these individuals have on local resources. Thus, this study will collect health care utilization, HRQL, health status and other lifestyle factors, which will assist in short and long term program implementation. A medical and health status assessment of approximately 500 migrant farm workers working in southern Georgia will be implemented in June 2001. Volunteers from the Emory Physician Assistant Program, Emory School of Medicine Department of Family and Preventive Medicine, Centers for Disease Control and Prevention, and AstraZeneca will be working collaboratively with community volunteers (local growers, churches, Spanish interpreters, local hospital and health department) at a farm clinic providing care and assessing the health status of the migrant farm workers. The SF-12 will be administered, by a Spanish translator as necessary, to the population and linked to other health data collected on site, through a program developed by Assist Technologies. Recommendations from the assessment will be used in further planning to assure adequate care in the short and long term for this vulnerable patient population.

199/ WELLNESS BEHAVIORS AS AN INDICATOR OF HEALTHRELATED QUALITY OF LIFE

Michelle L. Melia-Gordon, Fuschia M. Sirois, Timothy A. Pychyl, Psychology, Carleton University, Ottawa, ON, Canada

Wellness behaviors reflect action taken to maintain and enhance one's health and well-being. However, research suggests that these behaviors decrease when quality of life is compromised. This study explored the utility of wellness behaviors as a marker for health-related quality of life in first-year university students. A sample of 248 students (169 females, 79 males; age 19.6, +/- 1.4, range $17-25$ ) were selected from a larger sample $(n=415)$ and screened for absence of chronic health conditions. They completed self-report questionnaires on health-related quality of life (HRQOL) dimensions - financial stress (FINS), general social stress (GSS), interpersonal stress (IPS), satisfaction with academic performance (ACSAT), vitality, and common health problems. Students also completed the Wellness Behaviors Checklist (WBC), a 10-item inventory previously developed and validated to assess the frequency of preventive health behaviors (e.g. sleep, diet and exercise habits). Linear regression analyses explored associations between HRQOL dimensions and the practice of wellness behaviors. All variables were forced into the model equation in the first analysis. Although the overall model was significant $(\mathrm{R}$-square $=$ $.16, p<.000)$, only vitality (Beta $=.19)$, common health problems (Beta $=-.24)$, and ACSAT (Beta $=.12$ ) were significant individual predictors of WBC scores. FINS and IPS approached significance. A stepwise multiple regression assessed the relative strengths of the WBC predictors. Only common health problems (R-square $=.08$ ), and then vitality $(\mathrm{R}$-square $=12)$ entered as significant predictors $(\mathrm{p}$ $<.001)$. The relationships found in the current study may be generalisable to other QOL relevant populations, as students often experience significant stress. Results support the utility of wellness behaviors as an overall marker for components of HRQOL, particularly the health-related dimensions. Suggestions for the use of this behavioral index to identify and track important changes in HRQOL are discussed. 
200/ WORKERS' QUALITY OF LIFE: TOWARDS A NEW APPROACH OF INDUSTRIAL MEDICINE?

Jean-Louis San Marco, Public Health Department, Medical School, Marseille, France, Véronique Pellissier, Christian Mechetti, Jacques Viala-Artigues, Christophe Sapin, Pascal Auquier, Public Health Department, Marseille, France

It is now well known that work conditions can have several positive and negative repercussions on workers' physical and psychological well-being, despite the great efforts of industrial medicine to limit risky behaviours. The Marseilles city transport authority (RTM) is the third employer of our south-eastern city of France. The RTM management is aware of the impact of work conditions on workers' health status especially in a urban transport company where risky behaviours can not be admitted. They organise every four years an exhaustive survey about work conditions and health status. An exhaustive survey was conducted in 2000 . Each consenting employee had to fulfil a questionnaire dealing with demographic variables, work conditions in RTM, work status, tobacco and alcohol consumption, and two HRQL measurement: the SF-36 and the Duke Health Profile (DHP). Three subgroups were compared, defining by their status in the company: the managerial staff, the technicians and the train crews. The comparison of these populations was performed using Chi-square tests and ANOVA. 2,064 employees fulfilled the questionnaires (participation rate $>99 \%$ ). The distribution between the 3 subgroups was: $\mathrm{MS}=15 \% ; \mathrm{Te}=30 \% ; \mathrm{Dr}=55 \%$. The drivers were younger than the 2 other groups, but their drug or alcohol consumption did not significantly differ from those of the other groups. Their HRQL, assessed by DHP, did not significantly differ from MS or Te's one, while SF-36 scores were more impaired in the Role-Emotional ( $80 \pm 32$ versus $85 \pm 27$ for both MS and Te; $p<0.01)$ and Role-Physical $(80 \pm 29$ versus $87 \pm 22$ for both MS and Te; $p<0.001$ ) dimensions. The results of this observational study point out an impairment of workers' HRQL assessed by SF-36, while DHP dimensions were not able to discriminate these three populations. If these results were confirmed in others surveys, the HRQL assessment could be integrated in the industrial medicine to predict such impairment.

201/ HEALTH-RELATED QUALITY OF LIFE AND OCCUPATION Trond Riise, Bente E. Moen, Monica W. Nortvedt, Department of Public Health, University of Bergen, Bergen, Norway

The impact of occupational factors on health and a sense of wellbeing are the central issues in occupational health science. As traditional physical and chemical exposures have been reduced among workers in the industrial countries the past years, an increased focus has been put on various psychosocial stressors that leads to a number of health problems such as musculoskeletal symptoms and mental health problems. Quality of life questionnaires enable measurements of the consequences of such objectively less perceptible health problems for the workers' daily living. We used the SF-12 Health Survey in a general population of 23,312 individuals with age 40-47 to estimate the level of physical and mental health in occupational groups. The scores of the physical and mental component summary scales were adjusted for the effect of sex, level of education, smoking, alcohol consumption, body mass index and physical activity using a 6 way analysis of covariance. Administrative workers scored highest on both the physical and the mental component. Workers in transport and communication scored lowest on the physical scale probably reflecting known physical stressors for this type of work. Workers in primary occupations scored lowest on the mental health component corresponding to other studies reporting of various mental health problems including a higher suicide rate among these workers. Although selection bias related to the choice of occupation cannot be ruled out the differences found in the SF-12 scales most likely reflect the effect of various occupational health stressors. The SF-12 seems to be a responsive and an adequate measure of the accumulation of the health effects caused by such stressors.
202/ THE INFLUENCE OF LIFE-STYLE FACTORS ON HEALTHRELATED QUALITY OF LIFE

Monica W. Nortvedt, Trond Riise, Department of Public Health and Primary Health Care, University of Bergen, Bergen, Norway

Smoking, excessive alcohol consumption and overweight are related to a number of adverse health effects, including cardiovascular diseases. Health-related quality of life questionnaires give an overall measure of the consequences of varying health on the daily living. We estimated the relation between life style factors and health related quality of life in a general population of 23,312 individuals with age 40-47. Effects of smoking, alcohol consumption, body mass index (BMI) and physical activity on the physical and mental component scales of the SF-12 Health Survey were estimated using a 3way analyses of covariance. Marked and significant relations were found for all variables. Smoking was related to reduced physical and mental health with clear dose-response relations reaching mean scores of more than $0.8 \mathrm{SD}$ below the general mean for both scales for the individuals smoking more than 30 cigarettes per day. Ex-smokers had almost the same mean levels as non-smokers. Inverse Ushape relationships were found for alcohol consumption reaching a maximum mean physical score for the category of 10-20 units per week and 3-4 units for mental health. These results support the hypothesis of the positive relation between moderate alcohol consumption and health, although the maximum mental health was reached at a relatively low level of intake. Inverse U-shape relationships were found also for the BMI. Maximum mean score for the physical component was found at $22 \mathrm{~kg}$ per $\mathrm{m}^{2}$, while the maximum for the mental health component was found for the individuals scoring as high as 30 $\mathrm{kg}$ per $\mathrm{m}^{2}$. This latter score is outside the recommended range according to evidence-based guidelines $(18.5-24.9)$ and suggests marked differences in the mechanisms between overweight and physical and mental health. Physical training was strongly related to increased physical and mental health. To reduce the effect related to cardiovascular diseases in the estimates the analyses were repeated excluding all individuals having had cardiac infarction, angina pectoris or stroke $(n=233)$. Almost identical results were found including those for smoking and BMI. Causal interpretation of these results needs to be done with caution due to the cross-sectional design, but they nevertheless suggest marked influence of life-style factors on health-related quality of life.

203/ HOW MIGRATION AFFECTS THE HEALTH RELATED QUALITY OF LIFE?: THE COMPARISON OF THE HRQOL OF THE NATIVE AND IMMIGRANT REPRODUCTIVE AGED WOMEN

Erhan Eser, Gonul Dinc, Cemil Ozcan, Public Health (Halk S.), CBU Medical Faculty, Manisa, Turkey

Quality of Life Instruments can be used in demonstrating socioeconomic inequalities as well. The aims of this study were to define the population standards of the WHOQOL for the reproductive age women and to compare the migrated and native women's physical, psychological, social and environmental quality of life scores. This study is a cross sectional study which is a part of the Manisa Demographic and Health Survey finalized in February 2000. Manisa is a 200000 censused city in the western part of Turkey. Data were collected from 1641 married women of reproductive (15-49 years) age. The World Health Organization quality of life instrument WHOQOL-BREF which includes physical, psychological, social and environment domains were used as a tool of qol measure. The mean scores were $15.1 \& \# 61617 ; 2.3$ for physical; $13.7 \& \# 61617 ; 2.5$ for psychological; $14.8 \& \# 61617 ; 2.5$ for social and $13.5 \& \# 61617 ; 2.2$ for environment domains. The women living in urban areas have given statistically higher $(p<0.0001)$ qol scores than the women living in the sub-urban settlements. Similarly, the higher the years of education , the greater the qol scores were held $(p<0.0001)$. The low socio-economical class (unskilled workers plus blue collar industry workers plus unemployed) has given lower scores than the high socioeconomic class (the rest of them) in every domain scores. Migration has a negative effect on the qol scores for all of the domains except social relations domain. The women who have migrated from the eastern part (i.e. most deprived part ) of the country have shown lower scores $(p<0.0001)$ compared either with the immigrants from the other regions or native inhabitants. Cultural and ethnic solidarity among the eastern immigrants could possibly have neutralized the expected difference in the social domain score. WHOQOL-BREF can be accepted as a very appropriate instrument in demonstrating the socio-cultural inequalities. 
204/ IMPACT OF COMMUNITY MENTAL HEALTH SERVICES ON USERS' QUALITY OF LIFE: A COMPARISON OF THREE SYSTEMS

Leo R. Poirier, Malijai Caulet, Louise Fournier, Public Health, Montreal Health and Social Services Board, Montreal, QC, Canada; Celine Mercier, Div. de Recherche Psychosociale, Centre de Recherche de I'hopital Douglas, Verdun, QC, Canada; Alain D. Lesage, Centre de Recherche Fernand-Seguin, Hopital L. H. Lafontaine, Montreal, QC Canada; Deena White, GRASP, Universite de Montreal, Montreal, QC, Canada

It is generally assumed that integrated community-based resources can provide the full range of health and social services needed by people with psychotic disorders in a way that ensures continuity of care. However very few work has been done on the relationship between level of integration and outcome measures such as quality of life. The purpose of our study is to investigate this relationship. Since mental health services are sectorised in Montreal, we compared the level of integration in three sectors, using concepts and tools borrowed from social network analysis. Then, in a prospective cohort study, outcome data (quality of life, satisfaction of needs and symptomatology) were obtained in a representative sample of severely mentally ill adults living in these sectors. They were interviewed both as they leave a crisis center, an emergency room or a short-term hospital ward (Time 1) and one year later (Time 2). Quality of life was measured using the Wisconsin Quality of Life Index (W-QLI), in a Canadian version. It is a self-administered instrument that allows the respondents to determine the weight to be given to each of the eight dimensions of the scale.These dimensions were identified as important for quality of life and they had been shown to be good predictors of outcome in schizophrenia. The three resources network can be roughly characterized by their degree of density and of centrality. Density describes the general level of linkage among resources while centrality concerns prominence within the whole network. The network featuring the highest level of density is the one where the final score on the W-QLI shows the most significant increase between Time 1 and Time 2. Dimensions contributing to this result include psychological well-being, social relations/support and activities of daily living. Possible explanations, based on a finer description of the networks, and more detailed results, will be presented.

205/ USING A HEALTH STATUS INDICATOR TO INFORM PROGRAM DECISION-MAKING IN TWO OUTPATIENT MENTAL HEALTH SERVICES

David L. Lewis, Father Sean O'Sullivan Research Centre, St Joseph's Healthcare Hamilton, Hamilton, ON, Canada; Gail C. Coulas, Sociology, McMaster University, Hamilton, ON, Canada

Generic health status indexes provide measurable health outcomes When applied before and after an intervention, they can be used to establish the degree to which that intervention affects health. When the index has been normed against a population, it is also a usefu method to facilitate decision-making in health services. It can be applied across diverse clinical populations and treatment modalities thereby showing (a) whether a particular program or treatment method is successful, (b) the relative impact of one program compared to another on the health of populations, and (c) specific strengths and weaknesses of one program or intervention. We report on data collected by two outpatient mental health services in Hamilton, Canada: Community Psychiatric Services (CPS) and East Region Mental Health Services (ERMHS). As part of ongoing data collection, enrollment and post-discharge was obtained for consecutive patients de-enrolled in 2000 (CPS; $n=108$ ) and 2000-2001 (ERMHS; n=452). Information collected was socio-demographic, DSM-IV diagnosis (Axes I and II), Global Assessment of Functioning (DSM-IV Axis V), intervention type and length of time in program, service utilization (using a validated self-report instrument), satisfaction, and health status using the SF12. Results indicate, among other things, that the overall improvement in "mental component score" was 8.9 at ERMHS $(p<.01)$ and 3.9 at CPS $(p<.01)$; that the impact was greatest on adjustment disorders $(11.4 ; p<.01)$, and so on. By relating the results to clinician, audits are also possible. This information is, in turn, used by the Services involved to determine funding priorities, areas for quality improvement, and the like. In this paper, we will relate the process by which this technique was chosen and implemented, the results obtained, and how they have been employed in each Service.
206/ QUALITY OF LIFE AND PRECARIOUSNESS: COMPARISON OF OBJECTIVE AND SUBJECTIVE HEALTH ASSESSMENT Stéphanie Antoniotti, Public Health Department, Medical School, Marseille, France; Rémi Didelot, Doria Health Centre, Marseille, France; Christophe Sapin, Marie-Claude Simeoni, Public Health Department, Medical School, Marseille, France; Pascal Auquier, Public Health Department, Medical School, Marseille, France

In 1998, the French Public Health High Committee revealed in its report the transitional time of precariousness. Classical health indicators are currently unable to detect such population, because they evolve slowly. So we decided to suggest an alternative approach of health assessment, based on Health-Related Quality of Life (HRQL), and to compare the results of these two approaches in the pointing of precarious people. This transversal survey was conducted during June, 1999 in a Health Centre where the poorest can get free of charge health care. Collected were socio-demographic data, clinical data, a Clinical Global Impression (CGI) rated by the physician, an anxiety scale (STAI) and a HRQL measure (SF-36). The comparison of these populations was performed using Chi-square tests and ANOVA. 977 people were recruited, 388 precarious and 589 not precarious. Precarious people were younger $(41 \pm 12$ years versus $53 \pm 13$ years, $p<0.001)$. The rates of healthy people $(C G I)$ were not different between the two populations ( $35 \%$ versus $36 \%$, NS), while the precarious' anxiety level was significantly greater $(42 \pm 11$ versus $37 \pm 10$, $p<0.001)$, and their HRQL was more impaired in the Social Functioning $(71 \pm 26$ versus $75 \pm 25, p<0.05)$, Role Emotional $(68 \pm 37$ versus $75 \pm 36, p<0.05)$, Mental Health $(60 \pm 20$ versus $65 \pm 19, p<0.001)$ and Vitality $(55 \pm 19$ versus $58 \pm 19, p<0.05)$. The results of this observational study point out an impairment of HRQL of precarious people, while the objective assessment of health does not allow to discriminate between these two groups. If these results were confirmed in others surveys, the HRQL assessment could be integrated in the medical decision process to study the impact of such impairment on the becoming of precarious people.

207/ DOES HEALTH-RELATED QUALITY OF LIFE ASSESSMENT IN ROUTINE PRACTICE IMPROVE PATIENT OUTCOMES?

P. L. Lua, M. S. Salek, Centre for Socioeconomic Research, Cardiff University, Cardiff, UK.; N. Pratheepawanit, Faculty of Pharmaceutical Sciences, Khon Kaen University, Khon Kaen, Thailand; I. G. Finlay, Velindre NHS Trust \& University of Wales, College of Medicine, Cardiff, UK

Evidence is limited on the impact of HRQOL assessment in routine practice. The study was aimed at determining the impact of routine use of QOL information by physicians on QOL outcomes for in-patients with advanced cancer. This was a UK single centre, randomisedcontrolled-parallel study recruiting in-patients with advanced cancer who gave verbal consent and met the inclusion/exclusion criteria. $\mathrm{HRQOL}$ was assessed using the revised McGill QOL Questionnaire (MQOL), administered on admission day and a week later. At baseline, patients completed forms and were randomised (blocks of 10) into either study or control group. Physicians completed the physicianversion MQOL to assess patients' QOL and the ECOG Score within $24 \mathrm{hrs}$ of completion by patient. Only study patients' QOL information was then shown to the physicians. The same procedure was repeated at follow-up (a week later or on discharge day if less than a week). Statistical tests were performed to examine any significant difference in QOL between the 2 groups with ' $p$ ' set at $5 \%$ level. A total of 334 patients were admitted into the wards over the 13-week study period. Of these, $110(32.9 \%)$ patients participated in the study (mean age $=62.4$, median $=63.5$, age range $=24-89$ years, male $=47, \mathrm{fe}-$ male $=63), 48$ patients $(14.4 \%)$ refused (main reason: being tired) and the rest were not approached (too ill). From a total of 315 forms completed, only 42 (study=24, control=18) were evaluable. Baseline demographic characteristics and QOL scores for the two groups were not significantly different except that control patients had poorer ECOG scores $(p=0.04)$. At follow-up, the study patients had significant improvement in their existential $(p=0.034)$ and support domains $(p=0.038)$ whereas the control patients deteriorated in these domains. Generally, physicians over-estimated the physical symptoms domain scores for the study group but under-estimated for the control patients at baseline and during follow-up $(p<0.05)$. Limitations of study include small sample size and inability to account for differences in treatment types. Nevertheless results demonstrate the usefulness of HRQOL information in routine practice to improve the outcomes of in-patients with advanced cancer. 
208/ USING HEALTH STATUS QUESTIONNAIRES IN CLINICAL PRACTICE: A SURVEY OF HEALTHCARE PROFESSIONALS' VIEWS

Marissa Vicari, Dorothy L. Keininger, Mapi Research Institute, Lyon, France

While there is much discussion regarding the potential use of health status measures as an aid in clinical decision making, evidence suggests that they are not being integrated into routine clinical practice. Information available on the behaviours and attitudes of Healthcare Professionals' (HCP) regarding the use of health status measures in clinical practice is often limited to select populations of HCP specialising in chronic conditions. To better understand what HCP consider to be the benefits and barriers to the use of health status measures in clinical practice, and what resources would facilitate their use, a survey was developed with the purpose of assessing the views of a general population of HCP of varied specialisation, in two areas the state of Massachusetts, USA and the Rhone-Alps region of France. The survey, written in English and translated into French for the Rhone-Alps study, was comprised of 33 attitudinal and behavioural statements and was accompanied by a cover letter, a question and answer sheet and a copy of the SF-36 Health Survey. The survey was piloted in both areas on a group of $50 \mathrm{HCP}$; and 4 phone interviews were carried out in each language. In Massachusetts, the sample frame included Medical Doctors and Nurses listed on state Registers, provided by the Massachusetts Boards of Registration in Nursing and Medicine. In the Rhone-Alps, the sample frame included a list of officially registered Medical Doctors from France Telecom and the complete register of Nurses from the DRASS (Direction Régiona des Affaires Sanitaires et Sociales de Rhone-Alpes). The survey sample was determined by stratifying the registers by postal code and performing systemic sampling. The survey was sent to 2654 Nurses and Medical Doctors in Massachusetts and 2470 Nurses and Medical Doctors in the Rhone-Alps Region in France. Results to be announced.

209/ FUNCTION AND QUALITY OF LIFE MORBIDITY IN THE TREATMENT OF EXTREMITY SOFT TISSUE SARCOMA: THE NEED FOR SHARED DECISION-MAKING

Aileen M. Davis, Research, Toronto Rehabilitation Institute, Toronto ON, Canada; Brian O'Sullivan, Charles N. Catton, Radiation Oncology, Princess Margaret Hospital, Toronto, ON, Canada; Robert Turcotte, Orthopaedics, L'Hopital Maisonneuve-Rosemont, Montreal QC, Canada; Robert S. Bell, Jay S. Wunder, Surgery, Mount Sinai Hospital, Toronto, ON, Canada; Anna Sadura, Andrew Day, Joe Pater Benny Zee, Clinical Trials Group, National Cancer Institute, Queen's University, Kingston, ON, Canada

Wound complications may translate into disability and quality of life disadvantages for patients treated with pre-operative radiotherapy (RT) for soft tissue sarcoma (STS) of the extremity. Alternatively, late effects of fibrosis, oedema and joint stiffness more common with postoperative radiotherapy (due to the higher dose and large area of treatment) may also prove detrimental to patient function. The results of this randomized trial comparing pre vs. post-operative RT in extremity STS evaluate the effect of early vs late treatment morbidity on patient function and quality of life. Eligibility included surgery and RT for local management. The primary trial end-point was the incidence of wound complications, with function, quality of life, and late RT morbidity secondary end-points. The Musculoskeletal Tumor Society Rating Scale (MSTS), the Toronto Extremity Salvage Score (TESS) and the Shortform-36 (SF-36), were collected at randomization, 6 weeks, 3, 6 and 12 months post surgery. Thirty-one of 88 eligible and evaluable subjects $(35 \%)$ had wound complications with preoperative RT compared to 16 of $92(17 \%)$ treated postoperatively $(p=0.01)$. The patients treated with post-operative RT had higher function and health status scores, MSTS $(p<0.01)$, TESS $(p=0.01)$ and SF-36 bodily pain $(p=0.03)$, only at 6 weeks post-surgery. Wound complication, large, lower extremity tumour, and prior incomplete excision were adverse predictors of function. Late radiation tissue morbidity, grade 2 or greater subcutaneous fibrosis $(p=0.003)$ and oedema $(p=0.014)$ was more common in the post-operative RT arm. Dmax dose radiotherapy dose and TESS score were associated with subcutaneous fibrosis, bone and joint toxicity $(p<0.05)$. Wound complications more common in preoperative RT have a significant detrimental effect on patient function. However, postoperative RT results in greater subcutaneous tissue fibrous, oedema and joint stiffness. There needs to be shared decision-making between the patient and clinician in determining acceptable risks for impaired function and quality of life based on the known risk of wound complications with preoperative RT versus the late radiation morbidity of postoperative RT.

210/ ANGIOPLASTY PATIENT 36 MONTH SURVIVAL: THE ROLE OF QUALITY OF LIFE IN CLINICAL PRACTICE DECISIONS

Colleen M. Renier, Division of Education and Research, Carl E. Heltne Regional Heart Center, Jeanette A. Palcher, Division of Education and Research, St. Mary's/Duluth Clinic Health System, Duluth, MN

As Quality of Life (QoL) assessment becomes more common clinical practice, two endpoints emerge as those of greatest importance to clinicians: 1) Will patient QoL be significantly impacted by treatment, and the more traditional clinical question 2) Can baseline QoL assessment assist in predicting patient mortality. To assess the role of QoL assessment in prediction of mortality for patients receiving Percutaneous Transluminal Coronary Angioplasty (PTCA), the SF-36, was given to patients scheduled to have their first PTCA. Z-scores (US population) were calculated for each of the SF-36 subscales and then recoded to Below normal (z-score $<-1.0)$ vs Normal (z-score $\geq-$ 1.0). Medical records were abstracted to collect patient specific data available to the physician when making treatment decisions: Age, gender, ejection fraction, and pre-existing health conditions. Mortality was tracked through clinic and hospital records, and regional obituaries. Survival analysis, using conditional stepwise Cox Regression, was then performed to determine what role SF-36 QoL assessment could play in improved prediction of 36 month survival status. On the univariate level several variables were found to be significant predictors $(p<.05)$ of 36 month mortality: Age, history of cancer, history of other circulatory problems, history of pulmonary disease, history of traumatic injury, and two SF-36 subscales, general health and social functioning scale. Multivariate analysis yielded a model containing: History of cancer, history of other circulatory problems, history of pulmonary disease, history of traumatic injury and the SF-36 social functioning scale. It is too simplistic to view coronary patient survival in terms of both current and prior disease history. It is also important to know how the patient interacts with other people closest to them. This added information can be effectively provided by the SF-36 social functioning scale, so that more accurate mortality risk assessment can be made.

211/ INTRODUCING HRQL INTO DAILY ROUTINE PRACTICE: HOW DO THE PHYSICIANS RESPOND?

Preben Bendtsen, Socialmedicine, Matti Leijon, Margareta Kristenson, Public Health Science, Health and Environment, Linköping, Sweden

Title: Introducing HRQL into daily clinical practice: how do the physicians respond? Authors: Preben Bendtsen, Matti Leijon, Margareta Kristenson Main Authors Institution: Social Medicine and Public Health Science, Institution of Health and Environment, University Hospital, S- 58185 Linköping, Sweden. Chronic obstructive pulmonary disease (COPD) is a major health problem. It is an important cause of death in older adults but also a source of significant disability leading to decreased QOL. During the recent years it has become more and more obvious that there is only a weak correlation between traditional physiological parameters of lung function and the patients' perception of treatment outcome. However, HRQL measurements are not very widely accepted for use in routine clinical practice both various logistics reasons and probably also a disbelief in patient oriented evaluation methods. HRQL measurement was decided to be part of the daily routine practice in an outpatient department in a Chest Disease department in a University hospital in Sweden. In order to evaluate the physicians understanding and use of the HRQL measurement a focus group interview was performed before and 6 month after the introduction of HRQL measurements. The interviews revealed a general positive attitude towards using $\mathrm{HRQL}$ as an evaluation of treatment in COPD. Although the physicians did not believe that the measurements gave more information than they usually had access to, the HRQL helped them focus upon the patients' perspective rather than the traditional physiological parameters. Introducing HRQL into daily routine practice has helped the physicians to apply a more holistic perspective upon the patients with COPD. 
212/ MEASUREMENT OF CONTINUITY AND CO-ORDINATION OF PATIENT CARE IN A CANCER CENTRE

Laura Booth, Peter Selby, Pamela Lynch, Cancer Medicine Research Unit, Imperial Cancer Research Fund, Leeds, UK; Julia Brown, Northern and Yorkshire Clinical Trials and Research, University of Leeds, Leeds, UK; Galina Velikova, Cancer Medicine Research Unit, Imperial Cancer Research Fund, Leeds, UK

Modern cancer treatment is mainly administered in large cancer centres as the concentration of expertise allows safe delivery of complex treatments. The patients are looked after by teams of specialists and do not necessarily see the same doctor during the course of treatment. The aim of this study was twofold: 1 ) to validate an instrument measuring the continuity and co-ordination of medical care and 2) to describe cancer patients' views on the continuity of their care in a tertiary cancer centre. A 21 item instrument was adapted from a validated questionnaire "Components of Primary Care Index (Flocke SA, 1997).It was given to 285 patients attending the outpatient oncology clinics (during the clinic visit-130 patients or by post-155).One hundred and ninety seven patients returned the questionnaire $(70 \%), 159$ female and 38 male patients with a mean age 56.12 (sd 12.7).Descriptive statistics and factor analysis were used.Based on pre-set criteria for frequency of endorsement 6 items were eliminated.A 3 factor structure best fitted the data: patient preferences for continuity (4 items), co-ordination of care (6 items) and interpersonal skills (5 items). The internal consistency of these sub-scales was $0.82,0.74$ and 0.77 respectively. Eighty percent of the patients stated that they rarely see the same doctor in clinic and $68 \%$ felt it was important to see a regular doctor.More than $70 \%$ of the patients felt that the doctors knew about their previous problems and results of tests, but still $53 \%$ said that they often have to repeat their problems to different doctors and 30\% felt that doctors don't always know enough about their care. In conclusion, it appears possible to measure patient views on the continuity of their care and the instrument is currently used in a prospective study. The clinicians found the descriptive results of the study very informative and useful and as a result a new appointments system was implemented aiming to improve the continuity and co-ordination of care in the Unit.

213/ QUALITY OF LIFE IN ELDERLY PERSONS SUPPORTED BY MANDATORY HEALTH CARE SYSTEM

Michiko Kobayashi, Ken Matsuoka, Tomoko Namba, Sachiyo Okabayashi, Shizuko Sugimoto, Michiko Ogushi, Akikazu Fukuoka, Sumie Masuyama, Kouji Miura, Tsukasa Kawanishi, Naoko Yui, Atsuko Suzuki, Haruyasu Fujita, Soichiro Maruyama, Kozaburo Adachi, Kanehisa Morimoto, Takashi Mandai, Japanese Society of Quality of Life Research, Kobe, Hyogo, Japan

The purpose of this study was to evaluate the quality of life(QOL) in elderly persons supported by the mandatory long-term care insurance for the elderly. Sixty-nine elderly persons supported by the insurence participated in this study. Both personal interviews and our self-administered questionnaire including 40 questions divided into 15 categories with the Life Satisfaction Index were used. Pearson's correlation coefficients between our questionnaire and the Life Satisfaction Index was $r=0.82(P<0.01)$. Cronbach's alpha coefficients of our questionnaire were high enough to accept for clinical use; 0.91 in environmental problems, 0.90 in well-being and dietary problems, 0.86 in economic problems, and 0.80 in medical service etc., respectively. Our questionnaire contained 11 main factors and cumulative contribution was 0.85 . After nine months' supporting care service, some persons indicated improved total QOLs, others showed deteriorated total QOLs against our expectations. In the improved QOL persons, significant improvements of QOL were demonstrated in the strata of well-being, physical function, mental function, cognitive function, social participation, and environmental problems $(P<0.05)$, but there were significant deteriorations of QOL in well-being, dietary problems, sleep disturbance, physical function, mental function, medical service, social participation, work performance, environmental problems, and economic problems $(\mathrm{P}<0.05)$ in the deteriorated persons, too These findings indicate that our questionnaire has high enough reliability and potency of validity to use for elderly persons supported by the mandatory long-term care insurance for the elderly. Although health care system for the elderly is one of the most important world wide problem in the 21 st century, we must face and conquer many kinds of difficult problems, i.e. decreasing the proportion of younger generation, financial problems, and sociomedical problems etc. We should pay more attention for the utility of QOL evaluation technology on the mandatory health care system for the elderly in all over the world.
214/ HOW DO WE AGE? AN ANALYSIS OF SYMPTOMS AND FUNCTION IN A COMMUNITY COHORT

T. G. Ganiats, Family and Preventive Medicine, K. M. David, R. M. Kaplan, E. Barrett-Connor, UC San Diego, La Jolla, CA

How does the pattern of morbidity change as a population ages? Subjects in a population-based cohort in Rancho Bernardo, California participate in a longitudinal study of chronic disease. From 199295,1335 members of this cohort age 35 to 95 years completed the Quality of Well-being (QWB) scale. Total and subscale scores and individual responses were analyzed by age group $(35-54, \mathrm{n}=79 ; 55$ $64, n=192 ; 65-74, n=259$, and $>74$ years, $n=402)$ and gender $(61 \%$ female). Mean QWB scores showed a systematic decline with age $(35-54=0.75,55-64=0.73,65-74=0.71$, and $>74$ years $=0.67, p<$ $0.001)$ with men having higher scores $(0.71)$ than women $(0.69)(p<$ 0.02 ). Function accounted for half the 0.08 QWB difference between the youngest and oldest participants. Most $(77 \%)$ of the change in function came in the Physical and Social Activity scales, while the rest came in the Mobility scale. The older age groups reported more symptoms (a mean of 5.5 symptom clusters in the oldest group vs. 3.6 in the youngest group, $p<0.001$ ), but the distribution of symptoms was very similar for all age groups. The 5 most common QWB symptom complexes (CPX-20: wearing contacts/glasses, CPX-19: taking medication, CPX-18: pain or other symptom in head, CPX-17: weight or skin problem, and CPX-7: joint, stomach, or chest pain) were in the top 4 for each age group. The rank order lists of the 4 top symptom complexes for each age group were: ages 35-54 and 5564: CPX-20, CPX-19, CPX-17, CPX-18; age 65-74: CPX-20, CPX19, CPX-18, CPX-7; and age >74: CPX-20, CPX-19, CPX-7, CPX18. The proportion of patients in each CPX varied from $78 \%$ to $37 \%$ in the youngest to $92 \%$ to $43 \%$ in the oldest. There were minimal differences between the genders. While an age-related decrement in quality of life is expected, the finding that the pattern of symptoms does not vary significantly with age is surprising. If confirmed in other populations, this finding lends support to the practice of using only the most troublesome symptom in the generation of the QWB score.

215/ CAN PHARMACEUTICAL CARE IMPROVE THE HEALTH-RELATED QUALITY OF LIFE FOR PATIENTS WITH REACTIVE AIRWAYS DISEASE? A RANDOMIZED TRIAL

Morris Weinberger, Michael D. Murray, William M. Tierney, Medicine, Regenstrief Institute, Indianapolis, IN; David G. Marrero, Michael G. Lykens, Lisa E. Harris, Roopa Seshadri, Helena Caffrey, Medicine, Indiana University, Indianapolis, IN; Nancy Brewer, Medicine, Regenstrief Institute, Indianapolis, IN; Jeffrey A. Newell, Community Care, CVS/pharmacy, Woonsocket, RI; Joyce Collins, CVS/pharmacy, Indianapolis, IN

Pharmacists may improve patients' health-related quality of life (HRQOL) by engaging in pharmaceutical care (PC) activities (e.g., monitoring symptoms, counseling about medications, helping resolve drug-related problems). We conducted a randomized trial in $36 \mathrm{com}-$ munity drug stores to evaluate the effectiveness of PC for 1,113 enrolled patients with asthma or chronic obstructive pulmonary disease. Drug stores were divided into 12 matched clusters of 3 stores. Within each cluster, stores were randomized into one of three groups: PC with monitoring peak flow rates (PFR), PFR monitoring only control, usual care control. Pharmacists in the $P C$ group were provided with: recent patient-specific data (PFR, medication compliance rates, acute exacerbations resulting in emergency department or hospital visit); materials to facilitate implementation of $\mathrm{PC}$; and training on the program. Outcomes (PFR, HRQOL, acute exacerbations, medication compliance) were assessed at baseline, 6 and 12 months. While HRQOL and medication compliance improved for patients in the PC program, there was no significant difference across the three study groups. PFR was higher in the PC group compared to the usual care group, but not the group that received PFR monitoring only. Surprisingly, asthma patients in the PC stores were more significantly $(p<0.05)$ more likely to have an acute exacerbation compared to those receiving usual care (Odds Ratio=2.16). We conclude that, our PC program to enhance pharmacists' involvement in the care of patients with respiratory disease did not improve HRQOL. This may be attributed to the unexpected substantial improvement in HRQOL within the two control groups. Investigators who assess HRQOL as an outcome in randomized controlled trials should be aware that simply monitoring patients may result in improved HRQOL. However, had we not included a control group, we would have concluded that pharmaceutical care was effective in improving HRQOL. 
216/ LONG-TERM QUALITY OF LIFE IMPROVEMENTS IN ASTH MATIC ADULTS UNDERGOING EVIDENCE-BASED ASTHMA DISEASE MANAGEMENT PROGRAM

Ágota Szende, Health Economics, Astra Zeneca, Torokbalint, Hungary; Gabriella Jona, Zsolt Mogyorosy, Melinda Szaraz, Gizella Berki, Agnes Kuntar, Medical, Misszio Egeszsegugyi Kozpont, Veresegyhaz Hungary, Eva Farkas, Respiratory Outpatient Clinic, Godollo, Hungary, Gyula Berta, Pulmonology, Hospital for Chest Diseases, Mosdos, Hungary

The purpose of this study was to detect changes in health-related quality of life in asthmatic patients undergoing an experimental evidence-based asthma disease management program. Fifty-nine asthmatic adult patients entered the study in one of the experimental integrated health care provider settings in Hungary. All patients had been treated with asthma in ordinary care before entering the study. The experimental asthma disease management program consisted of the development and use of an evidence-based local clinical protocol, patient pathway protocol, drug use protocol, extensive patient education and asthma nurse consultation program, and a self-management program. One of the indicators used to measure effectiveness of the ADM program was health-related quality of life. QoL was measured with the utility-based generic EQ-5D questionnaire. A before-and-after study design was applied. Patients filled in the questionnaire at the time of entering the study and then around 6 months later. T-tests were used to test statistical significance. Quality of life has improved in participating patients. There was a mean increase of 0.094 ( $p=$ 0.0062 ) from 0.79 to 0.89 in EQ-5Dindex values and a mean increase of 9.7 ( $p=0.0033$ ) from 68 to 77 in EQ-5Dvas values. According to $E Q-5 D$ results from previous asthma study, these changes correspond to a difference of one severity group in the GINA classification system. It is concluded that a well-designed ADM program can substantially improve the QoL of already treated asthmatic patients.

\section{7/ QUALITY OF LIFE IN MYOPIC PATIENTS TREATED WITH DAY SURGERY \\ Takashi Mandai, Kozaburo Adachi, Kanehisa Morimoto, Shunji Tsuboi,} Haruyasu Fujita, Soichiro Maruyama, Fumio Nomura, Atsuko Suzuki, Michiko Kobayashi, Yoshiko Naka, Rika Hayashida, Sachiyo Okabayashi, Tomoko Namba, Ken Matsuoka, Japanese Society of Quality of Life Research, Kobe, Hyogo, Japan

The purpose of this study was to evaluate the quality of life(QOL) in myopic patients treated with Laser Insitu Keratomileusis(LASIK) operation. Eighty myopic patients treated with LASIK operation participated in this study. Both personal interviews and our self-administered questionnaire including 40 questions divided into 15 categories with the Life Satisfaction Index were used. Pearson's correlation coefficients between our questionnaire and the Life Satisfaction Index was $r=0.79(P<0.05)$. Cronbach's alpha coefficients of our questionnaire were high enough to accept for clinical use; 0.94 in mental function, 0.85 in dietary problems, 0.84 in medical service, 0.83 in environmental problems, 0.80 in social pariticipation etc., respectively. Our questionnaire contained 11 main factors and cumulative contribution was 0.84 . After LASIK operation, one half patients indicated the improved total QOLs but another one showed the deteriorated total QOLs. In the improved QOL patients, significant improvements of QOL were demonstrated in the strata of well-being, medical service, social participation, physical function, mental function, enviromental problems, and economic problems $(P<0.05)$, but there were significant deteriorations of QOL in mental function, physical function, and cognitive function $(P<0.05)$ in the deteriorated patients, too. These findings indicate that our questionnaire has high enough reliability and potency of validity to use for myopic patients. Although eyesight test indicates that LASIK operation for myopic patient plays very valuable role in quantitative improvement on eyesight in our study, qualitative deteriorations on eyesight i.e. cloudy vision etc after LASIK operation might deteriorate patients' total QOLs against our expectations. We are easy to understand the merit of day surgery for myopic patient by quantitative evaluation, but we should pay more attention for the qualitative postoperative eyesight care and counseling in the complicated cases.
218/ CONTRIBUTION OF DIFFERENT CLINICAL VISUAL OUTCOMES TO THE IMPROVEMENT IN PERCEIVED VISUAL FUNCTION AFTER CATARACT SURGERY

Xavier Castells, Department of Studies, Hospital del Mar, Health Services Research Unit, IMIM-IMAS, Barcelona, Spain; Mercè Comas, Department of Studies, Hospital del Mar, Health Services Research Unit, IMIM, Barcelona, Spain; Ana Martínez-Palmer, Miguel Castilla, Department of Ophthalmology, Hospital del Mar, Barcelona, Spain; Josep Garcia-Arumí, Vicente Martinez, Department of Ophthalmology, Hospital de la Vall d'Hebron, Barcelona, Spain; Jordi Alonso, Health Services Research Unit, Institut Municipal d'Investigació Mèdica, IMIM-IMAS, Barcelona, Spain

Some studies have shown that clinical measures, like visual acuity, have a small but relevant correlation with perceived visual function in cataract patients. The purpose of this study was to assess the contribution of clinical measures of visual function (visual acuity, contrast sensitivity and stereopsis) to perceived visual function as measured by the VF14 index. Patients from the ophthalmology departments of two teaching hospitals were assessed preoperatively and two months after first eye cataract surgery. Patients had bilateral cataracts with visual acuity in either eye not higher than 20/40. Visual function was measured with the VF-14 index, and visual acuity, contrast sensitivity and stereopsis with standardized clinical tests. Analysis of correlations and multiple linear regression were performed to assess the relative contribution (standardized regression coefficient) of each clinical variable to the VF-14 scores. Data was collected from 300 patients. Correlations of VF-14 scores with logMAR visual acuity, log contrast sensitivity and stereopsis were, respectively: $-0.36,0.39$ and -0.25 preoperatively; $-0.48,0.51$ and -0.38 postoperatively; and -0.34 , 0.39 and -0.07 for change. The standardized coefficients for preoperative VF-14 were: -0.17 for visual acuity, 0.23 for contrast sensitivity and -0.13 for stereopsis; for postoperative VF-14 were $-0.24,0.32$ and -0.12 and for change were $-0.18,0.29$ and -0.08 . Contrast sensitivity, a test that is not routinely performed, has a more important association with perceived visual function than other clinical indicators (i.e., visual acuity and stereopsis) in bilateral cataract patients. The inclusion of the measurement of contrast sensitivity can improve the clinical assessment of these patients.

\section{9/ EVALUATION OF A GENERAL AND DISEASE-SPECIFIC} QUALITY OF LIFE INSTRUMENT FOR CYTOMEGALOVIRUS RETINITIS

Barbara K. Martin, Adele M. Kaplan Gilpin, Epidemiology, Douglas A. Jabs, Ophthalmology, Albert W. Wu, Health Policy and Management, Johns Hopkins University School of Public Health, Baltimore, $\mathrm{MD}$

The objective of the study was to assess the reliability, validity and responsiveness of the general and disease-specific components of a health-related quality of life questionnaire developed for use in cytomegalovirus (CMV) retinitis research. Data were obtained from the Cytomegalovirus Retinitis Retreatment Trial, conducted by the Studies of Ocular Complications of AIDS Research Group(SOCA), for the 279 people enrolled. General scales included measures of general health perceptions, quality of life, physical function, energy, pain mental health, cognitive function, social function, and role function. Disease-specific scales included measures of visual function, visual symptoms, and treatment impact. Baseline data were used to assess internal consistency (Cronbach's alpha) and discriminant construct validity (multitrait analysis). Pearson correlations and t-tests were used to examine the association of scales with clinical measures at baseline and generalized estimating equations regression was used to assess responsiveness over time. Cronbach's alpha ranged from .68 to .88. Most scales adequately discriminated from other scales, although the energy scale appeared to overlap with the general health perceptions scale. Moderate correlations were obtained between baseline general scales and Karnofsky score (.14 to .49) and between baseline vision scales and visual acuity (.22 to .55$)$, visual field (.30 to .55), and extent of CMV retinitis (-.27 to -.41). Patients with a 10-point or greater decline in Karnofsky score over 6 months showed declines in all general scales except cognitive function $(p<.01$ to .37$)$. Changes in the visual function scale were strongly associated with changes clinical measures of vision and the extent of retinitis in patients' better eye $(p<.000001)$. These results provide evidence for the reliability, validity, and responsiveness of the tested measures in patients with CMV retinitis and AIDS. 
220/ PAIN SCALE RATINGS AND SATISFACTION WITH PAIN CONTROL IN THE HOSPICE SETTING: RESPONSES TO CHANGES IN THE PATIENT'S LEVEL OF PAIN

Stephan F. Gohmann, Economics, University of Louisville, Louisville, $\mathrm{KY}$

Hospice patient satisfaction with palliative care depends upon the responsiveness of patients to the analgesics used and their tolerance for pain. Hospices often use a pain scale to measure a patient's level of pain. The scale ranges from zero (no pain) to ten (the worst pain imaginable). During each visit to a patient, the nurse notes the patient's level of pain, the analgesic administered, and the patient's satisfaction with the level of pain control. If the hospice is responsive to a patient's pain, then as the pain level increases, a more potent analgesic should administered. This paper examines the relationship between drug treatment regimens, changes in pain, and satisfaction with the level of pain control for quarterly data for 2,690 hospice patients in 23 hospices over a two year period. For a given pain level, the drug treatment is examined to determine if patients are receiving adequate pain relief medication as suggested by the WHO analgesic stepladder and to determine to what extent these patients are satisfied with their level of pain control given their drug treatment. Since patients are observed over multiple visits, we can determine a patient's satisfaction with the pain control as the level of pain changes from mild to moderate to severe and as the analgesics change. Furthermore, by examining a patient's highest pain level ever, which is reported upon entry into the hospice, we can determine differences in pain tolerance between patients who report a high value for the highest level of pain relative to those reporting a low level. The results show that in most cases patients with severe pain do receive the opioids suggested by the WHO stepladder, but are often unsatisfied with the level of pain control. Further, patients who report a high value for their worst pain ever are less likely to be satisfied with the pain control relative to those reporting a low level for the highest pain ever.

\section{1/ DISCRIMINATIVE ASPECTS OF QUALITY OF LIFE IN PA-} TIENTS WITH VULVAL DISEASES

Silke Schmidt, Department of Medical Psychology, University Hospital of Hamburg, Hamburg, Germany; Claudia Hartmann, University Hospital of Jena, Jena, Germany; Bernhard Strauss, Department of Medical Psychology, University Hospital of Jena, Jena, Germany

The relationship between quality of life (QoL) determinants and its outcomes in chronic health conditions is a very complex issue. Two studies focussed on the interaction of QoL and determinants of QoL in patients with vulval diseases with the aim of a better understanding on the psychosocial antecedents, concomitants and consequences of QoL in affected patients. Different subgroups of vulval diseases were identified according to the recent standards of the International Society of Classification of Vulval Diseases (ISCVD). The first study comprised 53 patients with vulval diseases, mostly of dysaesthetic vulvodynia, vestibulitis syndrome and vulval dermatoses from an interdisciplinary care unit. Patients completed questionnaires on psychological complaints (BSI) and QoL (Skindex) as well as some supplemental scales at two points of measurement (beginning and end of treatment). In the second study, a sample of 80 patients with vulval diseases were assessed with the BSI and Skindex again, but additionally coping self and observer-rating scales as well as social support and relationship questionnaires over the course of treatment. The partners were investigated as well, however with a questionnaire response rate of only $45 \%$. Findings revealed significant differences in psychosocial and QoL profiles in different subgroups of vulval disease patients. Patients with dysaesthetic vulvodynia and vulval dermatoses showed very high levels of impairment, however patients with vulval dermatoses showed greater and significant improvement in the course of treatment. In contrast, patients with dysaesthetic vulvodynia hardly improved while revealing the highest levels of negative emotional coping. Patients with vulval vestibulitis syndrome did not show any deviations from the normal population reference values except for high elevations in shame and intimacy related issues. These findings indicate that the relationship between quality of life and its determinants follows differential pathways in subsets of vulval disease patients and highlight the necessity to develop different psychosocial treatment approaches as well as evaluative instruments for these patients.
222/ EXPLORATIVE STUDY ON COMPLIANCE AND ACCEP. TANCE OF CHRONIC PAIN PATIENTS TREATED WITH BUPRENORPHIN TRANSDERMAL SYSTEM (BTDS)

Astrid Anderson-Hillemacher, Medical Affairs + Health Economy, Grünenthal $\mathrm{GmbH}$, Aachen, Germany; Alarcos Cieza, Heribert Limm, Institute for Medical Psychology, University, Munich, Germany

BTDS is a proven effective opioid for pain applied as a patch (wearing period $72 \mathrm{~h}$ ). During its clinical development program a remarkable acceptance among patients (pts) was observed. Thus, the aim of this study was to identify factors which contribute to this acceptance and to assess the difference in satisfaction and compliance/ adherence among the treatment with BTDS, tablets and other analgesic forms as evaluated by the pts and their physicians (phys). The study was conducted as a multicenter $(\mathrm{N}=11)$ cross-sectional study with 32 pain pts (mean pain duration 7.7 years, mean 56.8 years old). Two questionnaires were developed: for pts self-assessment and for phys to evaluate pts-satisfaction and compliance with BTDS, including a comparison with former pain treatments. SF-36 was used to assess the QoL of the pts. All results were analysed descriptively. Moderate or high satisfaction was rated by $78 \%$ for efficacy and by $90 \%$ for the handling of BTDS. Since $78 \%$ evaluated the duration of the analgesic effect as important or very important, this seems to be an essential factor for that positive resonance. BTDS appears to contribute to an increased feeling of self efficacy: $78 \%$ feel less helpless and $72 \%$ experience to have more control over the pain. For $84 \%$ the use of BTDS was more or much more pleasant and for $66 \%$ the analgesic effect better or much better than the use of tablets or drops. Only $6 \%$ have been highly satisfied with their previous analgesics, but $59 \%$ of the pts expressed a high satisfaction with BTDS. QoL was poor in all SF-36 subscales, specially within the physical dimensions: physical functioning (mean $=38.4$ ), body pain (mean $=32.5$ ), even if BTDS was perceived by pts and phys as a potent treatment which contributes to the general well-being and specially to the physical well-being. This study has confirmed the observed acceptance of BTDS during its development phase and represents a basis for further studies.

223/ EVALUATION OF A NOVEL PAIN MANAGEMENT PROGRAMME AND ITS IMPACT ON QUALITY OF LIFE

N. Pratheepawanit, Faculty of Pharmaceutical Sciences, Khon Kaen University, Khon Kaen, Thailand; M. S. Salek, Centre for Socioeconomic Research, Cardiff University, Cardiff, UK; I. G. Finlay, Velindre NHS Trust, University of Wales College of Medicine, Cardiff, UK

Chronic non-malignant pain is one of the most difficult problems demanding effective therapy. Therefore, there is a growing need for evaluating pain control programmes using relevant outcomes. The aim of the study was to compare the impact of the traditional and a novel system of chronic pain management on patients' quality of life (QoL). Patients in both groups were assessed, using the Revised McGill Quality of Life Questionnaire (MQOL), at baseline (first visit) and at their follow-up visit to the weekly pain clinic and a 3-month Chronic Pain Management Group (CPMG) of the Velindre hospital, Cardiff, UK. The MQOL consists of 16 items covering 5 domains $(0-$ 10 scale with anchor ends). Over a two-year period $(1998-2000)$, 242 new patients attended the outpatient pain clinic; 121 (50\%) patients completed the Revised MQOL at their first and follow-up visits (female $=79$, mean age 48). The mean time period from first to follow-up visit was 5 weeks. However, for the CPMG, 20 patients completed the Revised MQOL before and 6 weeks after the programme. There was no difference between the two groups with respect to demographic characteristics and baseline QoL scores, except for physical symptoms which were more impaired in CPMG $(p=0.03)$. In the follow-up visits, the QoL scores for pain clinic patients were changed slightly or unchanged compared to the baseline values $(p>0.05)$. In contrast, there was improvement in CPMG patients in particular in existential domain $(p=0.04)$. In addition, the mean differences in QoL scores showed significant difference in existential domain $(p=$ 0.03 ). Limitations of the study were small sample size and potentially uncontrolled variables in both groups. Nevertheless, the findings suggest a positive impact of the CPMG upon patients' QoL as a novel approach in pain management. 
224/ MEASUREMENT OF CHANGES IN DISEASE-SPECIFIC AND GENERIC QUALITY OF LIFE (QOL) OF END-STAGE RENAL DISEASE PATIENTS AFTER KIDNEY TRANSPLANTATION

Gabriele H. Franke, Psychology of Rehabilitation, University of Applied Sciences, Stendal, Germany; Saxonia-Anhaltina, Jens Reimer, Thomas Philipp, Uwe Heemann, University of Essen, Department of Nephrology and Hypertension, Essen, Germany, Northrhine-Westfalia

The End-Stage Renal Disease Symptom Checklist - Transplantation Module (ESRD-SCL-TM) measures disease-specific QoL of renal transplant recipients: (1) Limited Physical Capacity (10 items, internal consistency: Cronbach's alpha=0.85), (2) Limited Cognitive Capacity (8, alpha $=0.82)$, (3) Cardiac and Renal Dysfunction (7, alpha=0.76), (4) Side Effects of Corticosteroids (5, alpha=0.77), (5) Increased Growth of Gum and Hair (5, alpha=0.78), and Transplantation Associated Psychological distress (8, alpha=0.80). Scales (1) (6) contribute to the scoring of the "Global Severity Index". All questions are scored on a five-point Likert scale. The ESRD-SCL-TM proved to have adequate reliability and validity (Franke et al 1999). Investigation of changes in disease-specific QoL in a sample of 180 patients within one year (critical difference $=\left(1,96 *{ }^{*} D^{*} S Q R(2 *(1-\right.$ alpha)))) demonstrated a statistically significant improvement (worsening) of distress (ESRD-SCL-TM-GSI) in 19, reduced distress in 20 and a stable situation in 141 patients. The three groups of patients did not differ regarding demographic (gender, age), and clinical variables (duration of graft function, creatinine level), but global QoL (SF36) (especially Vitality, and Social Functioning) changed in the same direction. Measurement of changes in QoL of end-stage renal disease patients after kidney transplantation should incorporate diseasespecific and global measures.

225/ LONG-TERM QUALITY OF LIFE AND COMORBID CONDITIONS IN THE DUTCH LUNG TRANSPLANT PROGRAM: A LONGITUDINAL STUDY

Karin M. Vermeulen, Jan-Paul Ouwens, Office for MTA, Wim van der Bij, Department of Pulmonary Diseases, Wim J. de Boer, Department of Thoracic Surgery, Gerard H. Koëter, Department of Pulmonary Diseases, Elisabeth M. TenVergert, Office for MTA, University Hospital Groningen, Groningen, The Netherlands

The purpose of the present study was to investigate if Health Related Quality of Life (HRQL) after lung transplantation (LgTX) is influenced by changes in the number of comorbid conditions caused by immunosuppression. Twenty-eight surviving $\mathrm{LgTX}$ patients completed selfadministered questionnaires before $\operatorname{LgTX}$ and at 4,7,19,31, 43 and 55 months after LgTX. HRQL measures were: the Nottingham Health Profile (NHP), the State-Trait Anxiety Inventory (STAI), Self-Rating Depression Scale (ZUNG) and the Index of Well-Being (IWB). Furthermore, a number of questions was composed to measure independence while performing activities of daily life (ADL). The number of comorbid conditions was collected from the patients records. Before LgTX, patients reported major restrictions on the NHP-dimensions mobility, energy and sleep, suffering from dyspnea, depressive symptoms and a lower level of experienced well-being. Most patients also experienced difficulties in performing activities of daily life. Four months after LgTX, most HRQL measures improved statistically significant and were within or close to the reference values. From 4 months after LgTX until 43 months after LgTX, scores on most dimensions remained stable. After 43 months scores on several dimensions of the NHP, grades of dyspnea that patients experience during making haste, STAI- ZUNG- and IWB-scores declined. The number of comorbid conditions appeared to increase i.e the number of patients suffering from Bronchiolitis Obliterans Syndrome, diabetes mellitus, and renal dysfunction increased. It may be concluded that overall HRQL improved and remained stable until 43 months after LgTX. Scores on several dimensions declined after 43 months, which may be explained by an increase of comorbidity.
226/ EVALUATION OF THE SF-36 AND EQ-5D FOR USE IN RENAL TRANSPLANTATION CLINICAL TRIALS

Diane Whalley, Stephen McKenna, Galen Research, Manchester, UK; Zoltan Kalo, Novartis Pharma AG, Basel, Switzerland

Renal transplantation is an indication for which generic measures of health status are often employed in an attempt to assess the impact of intervention from the patient's perspective. The aim of the present study was to evaluate the performance of two such instruments (SF36 and $E Q-5 D)$ in the context of a post-renal transplantation clinical trial. Secondary analyses were conducted on data collected in a double-blind, randomized, international renal transplantation clinical trial. Data were available for 407 post-transplant patients on the SF36 and 379 patients on the EQ-5D at baseline ( 7 days post-transplant) and at 3,6,9 and 12 months. Instruments were examined in terms of end effects and inter-scale correlations. Evaluation of internal validity (factor analysis and item response (Rasch) scaling) focused on the SF-36. Substantial end effects $(>20 \%)$ were found with the SF-36 role physical (RP), bodily pain (BP), social functioning (SF) and role emotional (RE) scales and with the EQ-5D tariff scores. Most inter-scale correlations were moderate $(0.4-0.6)$. The only low correlations (average 0.32 ) were between the physical functioning (PF) and mental health $(\mathrm{MH})$ scales. EQ-5D tariff scores and VAS were both moderately associated with all SF-36 scales (average $0.40-0.67$ ). Factor analysis confirmed the dimensional structure of the SF-36 at baseline but not at subsequent time points; eg the RE and RP scales tended to form a single factor and the VT scale separated into two factors. Rasch analysis showed problems of unidimensionality with the PF scale. Both instruments produced substantial end effects, reducing their responsiveness. Problems were evident with the internal structure of the SF-36 and the distinction between mental and physical health was far from clear. Given the focus of both measures on physical health, it is likely that they will miss much of the emotional and psychological impact of renal transplantation. It cannot be concluded that either the SF-36 or EQ-5D is appropriate for use in renal transplantation clinical trials.

\section{7/ PREVALENCE OF ERECTILE DYSFUNCTION OF MALE RE-} NAL TRANSPLANT PATIENTS AND ITS RELATION WITH HEALTH RELATED QUALITY OF LIFE

Pablo Rebollo, Nefrología-I, H. Central Asturias. Institute Reina Sofia of Nephro, Oviedo, Asturias, Spain; Francisco Fernandez-Vega, Nefrología-I, H.Central Asturias, Oviedo, Asturias, Spain; Francisco Ortega, Jose M. Baltar, Servicio de Nefrología-I, H.Central Asturias, Institute Reina Sofia, Oviedo, Asturias, Spain; Jaime Alvarez-Grande, Servicio de Nefrología-I, H. Central Asturias, Oviedo, Asturias, Spain

Erectile Dysfunction (ED) has been studied in end-stage renal disease patients, as a frequent problem associated to renal replacement therapy. But the relationship between ED and Health Related Quality of Life (HRQOL) in these patients has not been studied. We studied the prevalence of ED (using the IIEF-5 items), the HRQOL (using the SF-36 Health Survey) and the satisfaction with life (using the Fulg-Meyer Life Satisfaction Check List) in all renal transplant patients of our region ( $\mathrm{N}=243), 44$ of them aged 65 or over (elderly pts). We also collected socio-demographic and clinical data. Included pts. $=200(82.3 \%)$. Excluded pts $=35$ pts. no collaboration; 4 pts. with no sexual activity; 2 pts. with cognitive problems; 2 pts. returned to dialysis. Prevalence of ED (IIEF-5 score $=<21$ points)in the whole sample was $55.3 \%$. Prevalence of ED in elderly was $94.4 \%$ and in younger pts. was $46,6 \%$. Only 5 elderly with ED (13.9\%) were satisfied with their sexual life, however SF-36 standardized scores showed that their HRQOL was similar to that of the general population of the same age and gender. 89 younger pts. with ED (56.3\%) were satisfied with their sexual life and their HRQOL was also similar to that of the general population norm. We have not found any statistically significant correlation between IIEF-5 score and SF-36 standardized scores. Patients without ED showed slightly higher score on Physical Component Summary (PCS) but lower score on the Mental Component Summary (MCS). Elderly pts.: PCS score $=$ pts. with ED 50.8 \pm 7,9 vs $56 \pm 0.6$ pts. without ED; MCS score= pts. with ED $51.8 \pm 9,5$ vs $43.4 \pm 21.6$ pts. without ED. Younger pts.: PCS score= pts. with ED $47.6 \pm 8.3$ vs $49.8 \pm 6.3$ pts. without ED; MCS score= pts. with ED $50.9 \pm$ 9.6 vs $47.9 \pm 14.9$ pts. without ED. Elderly patients with ED showed higher satisfaction with their partnership relation $(4.96 \pm 0.89$ vs $4.22 \pm$ $1.05)$, and higher scores in Physical Function $(0.32 \pm 0.74$ vs $0.03 \pm 0.69)$, General Health $(0.14 \pm 1.16$ vs $-0.37 \pm 1)$, and Social Function $(0.24 \pm 0.76$ vs $-0.47 \pm 1.49)$ of the SF-36 than younger patients with ED. Prevalence of ED in kidney transplant patients of our region is high, mainly in elderly, but it has not influence on patient's HRQOL. 
228/ QUALITY ASSURANCE IN KIDNEY TRANSPLANTATION THE APPROACH IN ESSEN, GERMANY

Jens Reimer, Department of Neurology, Ruhr University Bochum Bochum, Germany; Gabriele H. Franke, Institute of Medical Psychology, Thomas Philipp, Uwe Heemann, Department of Nephrology, University of Essen, Essen, Germany

The Essen approach for quality assurance in kidney transplantation (KTX) comprises besides careful medical diagnosis and intervention a comprehensive psychodiagnostic program. Generic QOL (SF-36, MLDL [German]), disease-specific QOL (ESRD-SCLTM), psychological distress (BSI) and social relations (K-22 [German]) are assessed in yearly intervals. ESRD-patients after KTX and ESRD-patients on the waiting list for KTX and healthy controls were explored. Each group consisted of 149 persons, matched for age and gender. QOL of KTXpatients and healthy controls was - with exception of social relations - comparable and statistically significant higher than QOL of dialysis patients. As QOL-issues of KTX-patients are not sufficiently covered by generic QOL-instruments, a specific instrument was developed. After analysis of data of more than 450 patients, the ESRD-Symptom Checklist Transplantation Module with its six subscales was constructed (Nephron 1999). KTX-results are restricted partly due to immunosuppressive therapy. Therefore, the impact of different immunosuppressive agents on generic (SF-36) and disease-specific QOL (ESRD-SCLTM) was evaluated. KTX-patients, either treated by a cyclosporine- or tacrolimus-based regimen, were matched as to sociodemografic and medical parameters (each group $n=63$ ). Patients with the tacrolimus-based regimen reported statistically significant better generic ("Physical Component Summary") and disease-specific QOL. Overall, KTX improves QOL in ESRD-patients. Transplantation brings along new problems and challenges to QOL-research: physical QOL is affected by side effects of immunosuppressive agents, fear of rejection touches on psychological issues and social support decreases. The Essen approach for quality assurance in kidney transplantation led to identification and understanding of vital QOL-issues for KTX-patients. Implementation and validation of specific intervention strategies are future tasks.

\section{9/ A PROSPECTIVE, CONTROLLED STUDY ON THE EFFECT OF DIALYSIS TO THE QUALITY OF LIFE OF RENAL FAILURE PATIENTS IN CHINA}

Li Lingjiang, Mental Health Institute, Hunan Medical University, Changsha, Hunan, P.R.China

To evaluate the quality of life of end-stage renal failure patients and the effect of dialysis therapy. The quality of life of 99 end stage renal failure patients (32 hemodialysis, 32 peritoneal dialysis and 35 renal therapy) was measured before and 1 month after started dialysis and was compared with that of 99 healthy controls. Every dimension of quality of life of the renal failure patients was significantly lower than the healthy controls. The psychological functioning, social functioning and living conditions of the dialysis patients were much better than the conservative therapy patients while there were no significant differences in the physical functioning. The dialysis had different influences on different dimensions of quality of life of the patients, but the overall quality of life was not significantly changed before and after dialysis. The quality of life of hemodialysis and peritoneal dialysis patients was also different in some dimensions while the overall quality of life was almost the same. We recommended peritoneal dialysis as the first choice of therapy of end stage renal failure in China. Key words: End stage renal failure peritoneal dialysis hemodialysis quality of life.
230/ USE OF KARNOFSKY PERFORMANCE SCALE FOR EVALUATION QUALITY OF LIFE IN CLINICAL GRADING CAPD PATIENTS IN NORTH-EAST OF THAILAND

Supaphan Sunpithayakul, Srinagarind Hospital, Faculty of Medicine, Chonlatip Pongsakol, Jirayut Junma, Medicine Department, Faculty of Medicine, Khon Kaen University, Khon Kaen, Thailand

End Stage Renal Disease (ESRD) patients require long -long support treatment .Continuous Ambulatory Peritoneal Dialysis (CAPD) is a therapeutic modeling to give them a better quality of life (QOL).Our center use clinical grading index (i.e. 'A' for 'Excellent', B for 'Good', ' $C$ ' for 'Fair' and ' $D$ ' for 'Bad') based on blood pressure, hematocrit, serum albumin and potassium, infection rate, psychological status and patient degree of satisfaction, as an assessment index.There was no report study QOL related a clinical grading in CAPD Patients. Using Karnofsky performance scales is an instrument to evaluation the factors associated with health related quality of life (HRQOL) in CAPD patients. QOL in 107 CAPD patients. They come to follow-up at out patient department every 4-6 weeks, were attended by using clinical grading index.Of those were male 61 cases and female 46 cases, age $<60$ years $(54 \%)$ and $>60$ years $(49 \%)$.The major underlying diseases were hypertension(32\%), diabetes mellitus(25\%), stone(16\%) and gout(13\%). Of those who had grade $A$ $14 \%$, grade B $51 \%$, grade C $30 \%$ and grade D $5 \%$, and analyzed on associated with Karnofsky Activity Scales by 1) normal physical activity $18 \%, 2$ ) normal physical activity part of time $69 \%, 3)$ selfcare only $8 \%$ and 4 ) require assistance or some special care $7 \%$ Both of grade $A$ and $B$ had normal physical activity and normal physical activity part of time.Grade D was only able to carry out physical activities involving self care and required some assistance,may require special care from caregiver in their family. Using Karnofsky index would be acceptable level of measure reliability and evaluation the quality of life in the grading CAPD patients. This study will help the health personal to develop the grading index to be acceptable to measure the quality of life, especially in CAPD patients in each cultural and region.

\section{1/ QUALITY OF LIFE DETERMINANTS IN A DIALYSIS POPU-} LATION

Laura Schwartzmann, Department of Medical Psychology, School of Medicine, University of Uruguay, Montevideo, Uruguay; Sonia Dergazarian, Inés Olaizola, Aida Guerra, Servicio de Asistencia Renal Integral, Montevideo, Uruguay; Teresa Ceretti, Graciela Porley, Department of Medical Psychology, School of Medicine, University of Uruguay, Montevideo, Uruguay

The purpose of the study was to analyze a hemodialysis and a healthy population sample, matched by age, gender and education to assess differences between groups. The reliability and validity of SF-36 to detect those differences was also assessed.Predictive models for health-related quality of life (HRQL) in hemodialysis patients were developed. Patients 18 years of age and older undergoing dialysis for at least 3 months in 5 institutions in Montevideo, Uruguay, were asked to complete the SF-36, the effect of Kidney Disease domain of the KDQOL and additional socio-economic information. A sample of healthy individuals attending preventive medicine clinics, within an age range similar to the dialysis population completed the SF36.Chi square test and Student $t$ were used to assess differences.Least square linear regression models were developed for each SF-36 domain to determine the combination of factors influencing each outcome. Patient population included 300 patients; $60 \%$ males; age: 60 \pm 16 ; mean time in HD: $60 \pm 46$ months; $65 \%$ Grammar School only. Healthy population comprised 313 subjects; mean age $54 ; 53 \%$ males, $50 \%$ Grammar School only. Patients differed significantly from healthy population in QL physical components: Physical Function, Physical Role, Pain, Vitality and General Health. However differences were not significant in Mental Health and Social Functioning.SF-36 showed a good capacity for discriminating between these populations. The most important predicting factors for variance in SF 36 scales were: age, sex, number of hospitalizations, income perception, dietary restrictions, impact of the disease on sexual function and ability to continue working. In spite of important limitations posed by the disease,the social and psychological well being of patients is not significantly different from a healthy population of almost similar age. The variations of standards and expectations after the disease may account for this result. The impact of patient characteristics as predicting factors of HRQL should be considered in treatment strategies. 
232/ QUALITY OF LIFE AND THE DURATION OF TREATMENT WITH ORAL ANTICOAGULANTS IN PATIENTS WITH VENOUS THROMBOSIS

Mirjam Locadia, Mirjam Sprangers, Department of Medical Psychology, Martin Prins, Department of Clinical Epidemiology and Biostatistics, Academic Medical Centre, Amsterdam, The Netherlands

The first objective of this study was to explore the course of quality of life $(\mathrm{QoL})$ in patients with venous thrombosis treated for 3 months with oral anticoagulants (OAC), compared to patients treated longer than 3 months. The second objective was to investigate which demographic, clinical and QoL factors predict the duration of treatment with OAC. Patients with venous thrombosis completed the MOS-SF-20 and the Rotterdam Symptom Checklist adapted to thrombosis, before treatment, after 2 weeks, after 3 months and after 6 months. Differences in the course of QoL were assessed by a two factor (group $x$ time) repeated measures analyses of variance. Demographic, clinical and QoL factors that differed between the two patient groups were included in a stepwise multivariate logistic regression analysis $(p<0.10)$. The final multivariate logistic regression model was constructed using both forward and backward selection $(p<0.05)$. Of 360 eligible patients, 216 were treated for 3 months and 144 patients were treated for more than 3 months. No significant differences in QoL were found between the two patient groups. In both groups QoL improved significantly over time $(p<0.001)$. An interaction effect between group and time was found for physical functioning $(p=0.028)$. Regression analyses indicated that treatment centre, the presence of one or more permanent risk factors, duration of hospitalisation and mobility prior to deep-vein thrombosis were independent predictors of duration of treatment. In our study QoL did not differ between patients treated for 3 months and patients treated longer than 3 months. Qol improved over time, irrespective of duration of treatment with OAC. Interestingly, QoL was not a predictive factor of duration of treatment The most important predictor was treatment centre, indicating that practice variation has a great influence on decisions regarding duration of treatment with OAC.

\section{3/ ASSESSMENT OF HEALTH-RELATED QUALITY OF LIFE IN} HYPERTENSION: DO WE NEED MORE QUESTIONNAIRES?

Kasem S. Akhras, Global Outcomes Research, Pharmacia Corporation, Skokie, IL; Kunle Adesina, Linda N. Abetz, Mapi Values, Bollington, Macclesfield, UK

Health-related quality of life (HRQL) in hypertensive patients is becoming increasingly important in the selection of antihypertensive therapy among the nine classes of drugs currently in use. We sought to evaluate which HRQL questionnaires were used in the last decade to measure HRQL in hypertensive patients, and which domains are captured. MedLine search of the English language using the terms: "quality of life" and "hypertension" in randomized, controlled trials published between January 1990 and September 2000. In addition MAPI Research Institute databases were reviewed to include any potentially relevant studies that may have been missed by the MedLine search. A total of 75 studies met the inclusion criteria. Questionnaires used to assess HRQL in the different studies included the following: eight hypertension-specific, multi-dimensional questionnaires; ten generic, multi-dimensional questionnaires; ten single-dimensional, hypertension-specific scales; and 12 single-dimensional, generic scales. A breakdown of the domains measured by types of scales is presented in the table below. Most trials used a 'battery approach.' Between 20 and 30 instruments were used to capture physical, psychological, and social domains, indicating significant variations in assessment across trials. Given that hypertension is a 'silent disease' resulting in measurement that often focuses on the impact of adverse events, it is noteworthy that $10-12$ instruments were used to capture recognized adverse events (symptoms, sexual and cognitive functioning). Results were not consistent across trials and seemed to vary, depending on treatments, study design and on the questionnaires chosen. Significant variations exist in measuring HRQL in hypertension. Multiple measures are used for assessing the same domains, leading to conflicting results across a variety of trials. This may contribute to confusion and lack of acceptance of $\mathrm{HRQL}$ as a valid measure in clinical decision making in hypertension. Further evaluation and analysis of these measures is needed before an agreement can be reached on what domains are most important in hypertension and which measures are most sensitive.

See table — next column of page

\begin{tabular}{|l|l|l|l|l|l|l|l|}
\hline & Physical & Psychological & Social & General Healt & Symptoms & Sexual & Cognitive \\
\hline & MD/SD & MD/SD & MD/SD & MD/SD & MD/SD & MD/SD & MD/SD \\
\hline HTN-Specific & $7 / 6$ & $7 / 3$ & $5 / 6$ & $4 / 1$ & $5 / 1$ & $5 / 2$ & $4 / 4$ \\
\hline Generic & $11 / 6$ & $10 / 9$ & $8 / 2$ & $6 / 1$ & $1 / 3$ & $2 / 1$ & $3 / 1$ \\
\hline Total & 30 & 29 & 21 & 12 & 10 & 10 & 12 \\
\hline
\end{tabular}

234/ DEVELOPING AN INTERVENTION TO ENHANCE QUALITY OF LIFE AFTER MYOCARDIAL INFARCTION: WHAT ARE THE PATIENTS‘ NEEDS?

Tove A. Hanssen, Jan E. Nordrehaug, Kjellaug N. Shandiz, Department of Heart Disease, Haukeland University Hospital, Bergen, Norway; Berit R. Hanestad, Department of Public Health and Primary Health Care, University of Bergen, Bergen, Norway

Shorter hospital stays imply less time to give patients necessary information on how to comply with medication and to support them coping with the stress of the disease. The aim of this study was to increase the knowledge about patients' information needs and followup preferences, post-discharge an acute myocardial infarction (MI). Patients, 75 years or younger, discharged from Haukeland University Hospital within the last 3 months, with main diagnosis MI, were invited to participate in a focus group session. Of the 46 invited, 19 returned the informed consent-form and 14 participated in 1 of the 3 focus-group interviews conducted. The main questions were what information, support and follow-up they received and what they wanted, per- and post- hospitalisation. The sample comprised 2 females and 12 males. The age ranged from 42-69 years. Data was digital recorded and transcribed to facilitate content analysis. Most of the informants were satisfied with the information they received at the hospital in relation to acute care and treatment. They reported that the staff was very skilled and talked to them in an empathic way. Few got any follow-up contact except from one compulsory visit at the outpatient clinic. Only a few joined any rehabilitation programme. Areas in which the participants would have liked to have more information concerned how to cope after discharge, in relation to such as physical activity. When asked what kind of support or follow up they wanted to have but did not receive, most of them thought a contact telephone and telephone follow-up would have been useful ways of receiving counselling, information and support post discharge a MI. Based on a literature review and the results from these focus-group interviews, it will at Haukeland University Hospital, be developed and implemented an intervention, using open telephone line and telephone follow-up, to increase coping and quality of life after MI.

235/ ASSESSING QUALITY OF LIFE IN HYPERTENTION PATIENTS WITH THE WHOQOL-100 QUESTIONNAIRE

Popi Kounti, Maria Coccossis, Gina Triantafillou, Department of Psychiatry, loannis Tountas, Department of Hygiene and Epidemiology, Pavlos Sakkas, George N. Christodoulou, Department of Psychiatry, Medical School, University of Athens, Athens, Greece

Hypertention is a major risk factor for the development of cardiovascular disorders. The influence of treatment in the patients quality of life is an area that needs to be given emphasis and to be investigated since securing or improving a patient's quality of life is as important as it is to add years of life. The WHOQOL -100 questionnaire was administered in 160 hypertention patients who were given treatment in the context of a special programme for hypertention in the General Hospital of Ag. Olga in Athens. A percentage of $87,5 \%$ agreed to participate in this study. In terms of gender, $54 \%$ were female, while in terms of family status $71 \%$ were married. $62 \%$ of these patients were diagnosed by their physician as suffering from a type II medium level of hypertention. Concerning the WHOQOL- 100 domains, the patients' total score referring to the somatic domain is $57(S D+18)$, the psychological domain is $63(S D \pm 14)$, the domain referring to the level of independence is 64 (SD \pm 17 ), domain of social relations is 62 (SD \pm 16$)$, the domain of environment is $58(S D \pm 14)$, and the domain of spirituality and religion is $67(S D \pm 12)$. Concerning the sociodemographic characteristics, sex differences statistially significant were found referring specifically to the higher scores of men in the somatic domain and the corresponding facets at a level of $p<0,05$ Also, statistically significant differences at a level of $p<0,05$ were found in all the WHOQOL -100 domains in reference to the level of severity hypertention, that is type I patients (low level of hypertention) presented significantly higher scores than the type III patients (high level of hypertention). Differences were also found in terms of work status, that is employed patients showing significantly higher levels of overall quality of life than those who were retired $(p<0,05)$. 
236/IS SELF PERCEPTION OF HEALTH THE MOST IMPORTANT FACTOR CONTRIBUTING TO IMPROVEMENT OF QUALITY OF LIFE IN HIGH RISK CORONARY PATIENTS AFTER THE OPERATION?

Bogoljub B. Mihajlovic, Djordje Jakovljevic, Zoran R. Potic, Marijela M. Potic, Ninoslav Radovanovic, Cardiovascular surgery, Institute of CVD, Sremska Kamenica, Vojvodina, Yugoslavia

Quality of life (QOL), before and six months after myocardial revascularisation, was analyzed in 360 patients, which were categorized into two groups regarding expected operative risk according to V. Parsonnet risk stratification system 1996: Group I (312 pts) with low risk (0-6\%) and Group II (48 pts) with high operative risk (>6\%). For assessment of QOL we used our own, disease specific and health related QOL index, as an integral numerical value (QOLi-NS), ranged from $0-100$. After summarizing the theoretically maximal number of points for each question from specific domain, we calculated the maximal contribution of each of the four domains to overall index: physical status (PS) - $25.5 \%$; mental status (MS) - $36.2 \%$; social status (SS) - $8.2 \%$; self perception of health (SPOH) $-30.0 \%$. The average improvement of each domain was the difference of its mean value before and six months after the operation. Analyzing the improvement of each domains of QOLi-NS, self perception of health improved significantly and seems to be the most important factor contributing to improvement of $\mathrm{QOL}$ in high risk coronary patients, six months after myocardial revascularisation.

\begin{tabular}{|l|l|l|l|l|}
\hline Domain & Total & Low Risk & High Risk & Difference \\
\hline PS & 5.6 & 5.5 & 6.6 & $p=0.23$ \\
\hline MS & 25.7 & 25.2 & 28.6 & $p=0.10$ \\
\hline SS & 0.68 & 0.74 & 0.28 & $p=0.17$ \\
\hline SPOH & 11.9 & 11.4 & 15.6 & $p<0.001$ \\
\hline
\end{tabular}

237/ RESPONSIVE HEALTH-RELATED QUALITY OF LIFE DOMAINS IN THE TREATMENT OF HYPERTENSION: A SYSTEMATIC REVIEW OF PUBLISHED STUDIES AND MEASURES

Kunle B. Adesina, Linda N. Abetz, Health Outcomes, Mapi Values, Macclesfield, Cheshire, UK; Kasem S. Akhras, Global Outcomes Research, Pharmacia, Skokie, IL; Marie-Pierre Emery, Health Outcomes, Mapi Research Institute, Lyon, France

The objective of this study was to indentify domains of health-related quality of life (HRQL) that are most responsive to clinically important changes observed in hypertension trials. A systematic review was conducted of published trials (1990-2000) of anti-hypertensive agents and HRQL, using a Medline, Excerpta Medica, and in-house resources of The Mapi Research Institute. Only studies with named, referenced HRQL instruments used with hypertensive populations were selected. A total of 91 original studies including 75 trials of anti-hypertensive treatments were reviewed. We identified 22 generic and 20 hypertension-specific measures assessing domains (or dimensions) of physical, psychological and social functioning as well as general health perceptions, symptoms-specific, side-effects of treatment, sexual and cognitive functioning. Hypertension-specific instruments were found to have the most domains responsive to clinical changes. The most frequently responsive domain was physical functioning, including sleep problems and fatigue, and followed very closely by symptoms-specific/side-effects, psychological functioning and sexual functioning. The least frequently responsive domain was social functioning. The instruments that were found to be most consistently responsive across trials were: The Psychological General Well-Being Index, the Bulpitt \& Fletcher Quality of Life Questionnaire, Croog's Physical Symptoms Distress Index, Testa's Side-effects and Symptoms Distress Index. Our findings suggest the need for careful consideration of domains included in $H R Q L$ measures intended for use in clinical trials of antihypertensive agents. Careful selection of HRQL measures should improve the design of such trials as well as minimize respondent burden by targeting HRQL assessment at those domains that are most likely to reveal the impact of treatments on patient quality of life.
238/ RELATIONSHIP BETWEEN SYMPTOMS AND SF-36 PCS AND MCS SCORES IN TREATED HYPERTENSIVE PATIENTS Steven R. Erickson, College of Pharmacy, Brent C. Williams, Larry D. Gruppen, School of Medicine, University of Michigan, Ann Arbor, MI

The purpose of this study was to describe the relationship between symptoms and the SF-36 PCS and MCS scores in treated hypertensive patients. This cross-sectional study included all hypertensive patients seen during a 6-month period in a general medicine or a hypertension clinic. Patients were age 30 years or older and prescribed 1 or more antihypertensive. Data were from a mailed questionnaire and medical records and included the SF-36 PCS and MCS, demographic (age, gender, race, education, income), disease (blood pressure, years with hypertension), and medication (number of antihypertensives and duration of regimen). The Symptom Distress Checklist (SDC) provided a symptom count (number of symptoms reported) and symptom distress (sum of distress reported per symptom). Linear regression determined the relationship of symptom data to PCS and MCS while accounting for the contribution of patient, disease, and treatment characteristics. Stepwise elimination of variables with $p<0.05$ led to the final models. Separate models were run for each SDC and SF-36 variable. 125 of 225 patients responded. The mean age was $59.0(11.2)$ years; $47.2 \%$ were male; $75.2 \%$ were Caucasian; and averaged 16 years with hypertension. The mean blood pressure was $139.6(20.7) / 85.5(11.8) \mathrm{mmHg}$. The mean number of medications was 1.5(0.7) and were taken an average of 3.8(4.6) years. 122 reported one or more symptom. The mean symptom count was 8.8(7.8); mean total symptom distress was 31.6(46.2). The mean PCS was 48.7(9.3); MCS 51.6(10.1). The final PCS model had an adjusted $r$-squared of 0.31 and contained the variables (with standardized coefficient): age (-0.28), duration of drug therapy $(-0.21)$, and symptom count $(-0.51)$. The final MCS model had an adjusted $r$-squared of 0.41 and contained gender(-0.15) and symptom count(-0.62). Results for symptom distress models were similar. Symptoms have a greater impact on PCS and MCS scores than patient characteristics, blood pressure, and medication-related factors.

239/ DIFFERENTIAL WEIGHTINGS OF THE CLINICAL IMPORTANCE OF QUALITY OF LIFE STATEMENTS BY PROFESSIONALS IN TWO COUNTRIES: EXPERIENCE WITH THE EPILEPSY PSYCHO-SOCIAL EFFECTS SCALE

John E. Chaplin, Department of Paediatrics, Lund University, LUND, Sweden; Kristina Malmgren, Institute of Clinical Neuroscience, Sahlgrenska University Hospital, Gothenburg, Sweden; Pamela Thompson, Institute of Neurology, Sahlgrenska University Hospital, London, UK

The study identified differences in the weightings assigned to quality of life items by professionals in two countries. The Epilepsy PsychoSocial Effects Scale (EPSES) contains 42 statements derived from in-depth interviews with people with epilepsy. The statements are grouped into fourteen domains of psycho-social affect. When completing the questionnaire, the person with epilepsy responds to each statement by indicating their level of agreement on a five-point scale. The weightings for each statement are based on the judgements of professionals in terms of their clinical significance. In this study, the weightings of professionals in England and Sweden were compared. The procedure used a paired comparison method using the judgements of medical staff including doctors, nurses, psychologists and social workers. Each statement was weighted for clinical importance against the other statements in its' domain. A comparison between the weightings derived in Sweden and the UK revealed a general agreement in the clinical estimation of the statements' importance but there were some significant differences. Explanations for the findings can be sought in the differences in health care, medical training and cultural background of the two countries. Professional differences were also identified. The implications for cross-cultural research are discussed in relation to the use of quality of life as a medical concept. 
240/ IS THE EFFECT REAL OR AN ARTIFACT?

Michael A. Hagan, Health Systems Strategies, Columbia University, Mailman School of Public Health, New York, NY; Zhiliang Ying, Department of Statistics, Columbia University, New York, NY

The analytic tool of choice for HRQoL research is often regression analysis. Data captured through the use of Linear Analog Scale Assessment (LASA) may exhibit floor or ceiling effects that would manifest in a flattening of the regression curve. In regressing Pain Score (dependent variable) on hemoglobin $(\mathrm{Hb})$ (independent variable) we found that the best fit to the data was a cubic model. The question we propose to answer here is: Is this effect real or an artifact of the bounded condition of the LASA scale?

We proposed the following regression model:

$E(H R Q o L \mid H b)=K_{1}+\left\{K_{2}{ }^{*} e^{\alpha+\beta^{*}(H b)}\right\} /\left\{1+e^{\alpha+\beta^{*}(H b)}\right\}$

Where $\alpha$ and $\beta$ are the usual logistic regression parameters and $K_{1}+$ $\mathrm{K}_{2}$ are additional parameters used to evaluate to possibility of an artifact. All four parameters were estimated by non-linear least squares. Specifically, the estimators are defined as the minimizers of the equation:

$\sum\left\{\mathrm{Y}_{\mathrm{i}}-\left[\mathrm{K}_{1}+\mathrm{K}_{2}{ }^{*} \mathrm{e}^{\alpha+\beta^{\star}(\mathrm{Hb})}\right] /\left[\left(1+\mathrm{e}^{\alpha+\beta^{\star}(H b)}\right)\right]\right\}^{2}$

$=1$

If $\mathrm{K}_{1}$ converges to 0 (i.e. the highest possible LASA) then a flooring effect exists. If $\mathrm{K}+\mathrm{K}_{2}$ converge to 100 (i.e. the lowest possible LASA) then a ceiling effect exists. Since $K_{1}$ converges to 28 and $K_{1}+K$ converges to 40 neither a floor nor a ceiling effect exist. Hence the functional form of the relationship between disease indicator $(\mathrm{Hb})$ and patient-reported illness (Pain) is cubic, involving thresholds of perceived deficits.

241/ PHYSICAL PERFORMANCE, TOXICITY, AND QUALITY OF LIFE AS ASSESSED BY THE PHYSICIAN AND THE PATIENT

Minna-Liisa Luoma, Department of Psychology, Liisa HakamiesBlomqvist, Swedish School of Social Science, University of Helsinki, Helsinki, Finland; Johanna Sjöström, Department of Oncology Helsinki University Central Hospital, Helsinki, Finland; Carl Blomqvist Department of Oncology, Uppsala University Hospital, Uppsala, Sweden

The aim of the present study was to study the relationship between physician assessed Quality of Life (QoL) parameters, i.e., toxicity and physical performance, and patients' self reports of their QoL. In a multicentre trial 283 patients, with advanced breast cancer, were randomized to receive either docetaxel $(T)$ or sequential methotrexate5-fluorouracil (MF). QoL data were available for 245 patients. QoL was assessed at baseline and before each treatment using the EORTC QLQ-C30. The WHO performance score (PS) and toxicity were assessed before each treatment in physical interviews. The correlations between the WHO PS and the QLQ-C30 functioning scale scores varied from weak to moderate, depending on the scale. The strongest correlations between QLQ-C30 scales and WHO PS were found in physical functioning (varying from -0.60 to -0.42 ), in social functioning (varying from -0.48 to -0.31 ), in global QoL (varying from 0.47 to -0.29 ), and in role functioning (varying from -0.43 to -0.21 ) Fairly low correlations were found between emotional functioning and the WHO PS (varying from -0.34 to -0.14 ) and also between cognitive functioning and the WHO PS (varying from -0.27 to -0.14 ). The QLQ-C30 nausea/vomiting and diarrhoea scales correlated moderately ( ie. $>.40$ ) to corresponding WHO scores. Multiple linear regression analysis was used to analyze the contribution of toxicity variables to the global QoL. The best model (having more severe nausea, infection, fatigue, and oedema) predicted worse global QoL. However, these four variables only explain $7 \%$ of the variance of the global QoL. The present findings highlight the importance of independent QoL assessments focused on those aspects of QoL not captured in clinical interviews with the physician.
242/ RESULTS FROM THREE PHYSICIAN CONSENSUS PANELS DETERMINING CLINICALLY SIGNIFICANT CHANGES IN HEALTHRELATED QUALITY OF LIFE AMONG CHRONIC DISEASE OUTPATIENTS

K. W. Wyrwich, Division of Health Services Research, F. D. Wolinsky, School of Public Health, Saint Louis University, St. Louis, MO; W. M. Tierney, Division of General Internal Medicine and Geriatrics, K. Kroenke, Regenstrief Institute for Healthcare, Indiana University, Indianapolis, IN, A. N. Babu, Department of Medicine, Saint Louis University, St. Louis, MO

Standards for establishing the amount of change over time needed in a health-related quality of life (HRQoL) measure in order for that change to be considered important or relevant can be determined empirically by between-persons and within-person change studies. However, these anchor-based methods incorporate only the patients' perspectives of important change, and do not reflect an informed clinical evaluation of HRQoL improvement or decline. Without clinical input on what constitutes a significant change, these measures and resulting change standards are less likely to be adopted by clinicians for use in their daily practices. As a first step to developing clinically important difference standards for the SF-36 (version 2.0) and three disease-specific HRQoL measures: Asthma Quality of Life Questionnaire-Juniper (AQLQ), Chronic Heart Failure Questionnaire (CHQ) and Chronic Respiratory Questionnaire (CRQ), we assemble a nineperson expert panel of North American physicians for each of the three following chronic diseases: asthma, heart disease, and chronic obstructive pulmonary disease. All panelists were familiar with the use of at least one of the HRQoL measures among patients with the panel-specific disease. Using two rounds of the Delphi process, one in-person meeting of each panel, and an iterative improvement process for circulating and correcting the final report of the panel meeting, these three panels each set clinically important change thresholds for all eight scales of the SF-36 among patients with the panelspecific disease, as well as the domains of their respective diseasespecific HRQoL measure (AQLQ, CHQ, or CRQ).

243/ AGREEMENT BETWEEN SELF AND PROXY ASSESSMENT IN ELDERLY PATIENTS WITH HIP FRACTURES

C. Allyson Jones, Faculty of Pharmacy \& Pharmaceutical Sciences, University of Alberta, Edmonton, AB, Canada; David H. Feeny, Institute of Health Economics, Edmonton, AB, Canada

Confusion in patients with hip fracture is well recognized and reduces the number of patients who are able to respond to health status questionnaires. Are family members a reasonable alternative to self assessment? The objective of this study is to examine interrater reliability for pain, disability and health status by examining agreement between patients and proxy respondents in a patient cohort that has recently fractured their hips. Agreement will be assessed for a) an 11-point pain rating scale, b) a performance-based disability measure, Functional Independence Measure (FIM), and c) the Health Utilities Index Mark 2 and 3 (HUI2/3). 105 patients with Mini-Mental State Examination scores of $>17 / 30$ were seen within 3 to 5 days after surgery to stabilize a fractured hip. Questionnaires were interview-administered. Family caregivers were recruited as proxy respondents. Agreement was evaluated using intraclass correlation (ICC). The mean age of patients was 80 years (sd 8). The patient cohort was comprised of mostly women $(74 \%)$ with a mean number of 5.4 (sd 3.1) comorbidities. The majority of the proxies (78\%) were either a son/daughter or spouse who felt they knew the patient's health well. For pain there was moderate agreement between patient and proxies responses $(I C C=0.48)$. Strong agreement was seen with the FIM $($ ICC >0.50). Moderate to strong agreement was seen for overall and single attribute utility scores of the HUI2/3 except for HUI2 mobility $(I C C=0.12)$. In general, there was reasonable agreement between self and proxy assessments in patients with recent hip fractures with respect to pain, physical function and overall health status. Further study during less acute phases of recovery will be required but it may be reasonable to enlist family proxy respondents for hip fracture patients who are unable to respond on their behalf. 
244/ RESPONSE SHIFT OF A HEALTH-RELATED QUALITY OF LIFE ASSESSMENT AS A PREDICTOR OF SUSTAINED WEIGHT LOSS IN OBESE PATEINTS

Ivan Barofsky, Department of Psychiatry and Behavioral Sciences, Johns Hopkins University School of Medicine, Baltimore, MD; Kevin R. Fontaine, Department of Medicine, University of Maryland, Baltimore, MD; Susan J. Bartlett, Shawn C. Franckowiak, Ross E. Andersen, Department of Medicine, Johns Hopkins University Schoolf of Medicine, Baltimore, MD

To determine 1)if a response shift in a Health-Related Quality of Life (HRQL) assessment occurred following participation in a 13-week weight loss program, and if so 2) whether the response shift predicted the maintenance of weight loss one year later. 38 mild to moderately obese persons completed a weight loss program, and were administered the Medical Outcomes Study Short-Form Health Survey (SF-36) once at baseline, and twice at the completion of the intervention. Participants were asked to complete the second post-test administration of the SF-36, as they perceived themselves to have been at baseline (the "thentest"). 32 participants completed the study and returned one year later for a follow-up evaluation. Evidence of a response shift $(P<0.009)$ was found for some (e.g., physical functioning, general health, social functioning, and mental health) but not all SF-36 domains. In all SF-36 domains, except role-physical, respondents reported a lower level of functioning during the "thentest" than reported at baseline. The relationship between the response shift and weight status at one year follow-up was also examined. Respondents who regained $(\mathrm{N}=21)$ or maintained $(\mathrm{N}=11)$ their weight loss were classified in terms of whether they were above or below the median response shift for each domain. Finally, the pre-post change in each SF-36 domain was also calculated. For the physical functioning domain respondents who regained weight reported minimal pre-post change independent of whether they reported a low or high response shift. In contrast, respondents who maintained their weight loss, reported a statistically significant difference in their physical functioning on the basis of whether they reported a low or high response shift low shift respondents reported greater pre-post change in physical functioning. When these analyses were repeated for other SF-36 domains only general health approached statistical significance. In conclusion, response shift may be a useful predictor of whether respondents maintain or regain body weight. The current data, if generalized, suggest that those who report significantly higher change in physical functioning also report less response shift. This implies that persons who fail to maintain their weight loss alter their perception of their baseline physical functioning.

245/ EFFECT OF MODE OF ADMINISTRATION ON THE WALKING IMPAIRMENT QUESTIONNAIRE IN PERIPHERAL ARTERIAL DISEASE PATIENTS

Karin S. Coyne, Mary Kay Margolis, MEDTAP International, Bethesda, MD; Judy Regensteiner, University of Colorado Health Sciences Center, Denver, CO; Susan Grandy, Kim A. Gilchrist, DuPont Pharmaceuticals Company, Wilmington, DE; Dennis A. Revicki, MEDTAP International, Inc., Bethesda, MD

Peripheral arterial disease (PAD) can have a significant impact on patients' walking function and health-related quality of life (HRQL). Recent studies have examined factors contributing to variations in patient reported outcome (PRO) measures and the impact of using self-administered questionnaires or in-person interviews. Studies in other patient populations suggest that administering PRO assessments via telephone may decrease costs, patient burden, and frequency of missing data. The purpose of this study was to examine the effect of mode of administration (MOA) on patient responses to the Walking Impairment Questionnaire (WIQ). The WIQ, consisting of 4 subscales (pain, distance, speed, stairs), has been an intervieweradministered questionnaire; however for large clinical trials, such administration is excessively burdensome. The WIQ was modified to be self- (SA) or telephone-administered (TA). At two clinic sites, patients were recruited and randomized into two groups: Group 1 received SA then TA; Group 2 received TA then SA. The two administrations took place 4-7 days apart. Additional questionnaires (SF-36 $E Q-5 D$ ) were also included to further assess the psychometric validity of the WIQ. Telephone interviews were conducted by trained interviewers using standardized scripts. Test-retest reliability was assessed at 2 weeks. Sixty patients were recruited ( $n=30$ per group); $78 \%$ were men; mean age $=67.1$ years; $83 \%$ were caucasian. Mean duration of PAD symptoms was 6.8 years. There were no significant differences between the SA and TA subscale scores of the WIQ. No interaction effects between order and MOA were noted. Cronbach alphas for each subscale ranged from 0.82-0.91. Subscale intra-class correlations between MOA ranged from $0.55-0.83$. The modified WIQ demonstrated good reliability and validity; self and telephone administration of the WIQ can be used reliably and efficiently in the clinical trial setting.

246/ OBTAINING SELF-REPORTS ABOUT HRQL FROM COGNITIVELY IMPAIRED RESPONDENTS

Sarah C. Smith, Donna L. Lamping, Health Services Research Unit, London School of Hygiene \& Tropical Medicine, London, UK; Beth Foley, Joanna Murray, Sube S. Banerjee, Institute of Psychiatry, King's College, London, UK

For respondents with cognitive impairment (eg dementia, stroke, aphasia), the measurement of health-related quality of life (HRQL) presents a particular challenge. Our recent experience in developing a self-report measure of HRQL for people with dementia (DEMQOL) highlights a number of conceptual and methodological issues that must be resolved in order to balance psychometric rigour with the needs of a cognitively impaired population. Conceptually the challenge is linked to how the subjective perceptions that are fundamental to HRQL can be reliably elicited from cognitively impaired respondents. The standard way of avoiding this problem through the use of proxy reports is methodologically limited (Sprangers \& Aaronson 1992), and does not capture the patient's subjective perceptions. Methodologically there are also several problems in meeting the standard psychometric criteria for good questionnaire design. It is generally recommended that a mix of positive and negative questions is used to minimise acquiescence bias and that time frames are clearly specified. For cognitively impaired respondents, a switch from negative to positive questions can be confusing and time frames may be meaningless to people with memory difficulties. The choice of response scale presents other challenges. A limited number of response options can help respondents with severe impairment, but frustrating to those with mild impairment. Standard verbal response options may be too semantically complex for cognitively impaired respondents. Although pictorial response options may be a helpful alternative for those with a specific language deficit, they may create extra cognitive demand for people with dementia. We discuss several of these challenges and proposed solutions in the context of designing an instrument that balances psychometric and methodological rigour with the practical constraints of evaluating HRQL by self-report in respondents with cognitive impairment.

247/ IMPACT OF A CHANGE IN MODE OF ADMINISTRATION ON QUESTIONNAIRE RETURN DURING A STUDY OF THE OUTCOME OF HIP AND KNEE REPLACEMENT

Francois A. Bethoux, Deborah M. Miller, The Mellen Center / U10, George M. Muschler, Department of Orthopaedic Surgery / A41, The Cleveland Clinic Foundation, Cleveland, $\mathrm{OH}$

The mode of administration of questionnaires can have a significant impact on the quantity and quality of outcomes data collected. We had the opportunity to observe the results of a change from on-site data collection to mailing during an outcomes study conducted in a large orthopaedic surgery department. Consecutive patients with a diagnosis of osteoarthritis $(\mathrm{OA})$, and scheduled for primary unilateral total hip or knee replacement (THR or TKR), were asked to fill out the American Academy of Orthopaedic Surgeons MODEMS questionnaire during their presurgical assessment visit. The questionnaire is composed of 57 items, and includes the SF-36, as well as additional disease-specific items. Recruitment took place between September 1998 and August 2000. From September 1998 to September 1999, patients were asked to fill out the baseline questionnaire in the clinic. After this date, the questionnaires were mailed to the patients' home with a pre-paid return envelope one month before surgery. Potential candidates were identified from a computerized operating room scheduling system, using diagnosis and CPT codes as filters. Three-hundred seventy-six patients were enrolled during the study period, out of 798 potential candidates identified (overall enrollment rate: $47 \%$ ). There was a significant increase in enrollment rate after switching to mailing (from $27 \%$ to $71 \%$ ). The average completion rate by item among the questionnaires returned did not change significantly. Although collection of data at the time of an outpatient clinic appointment was thought to yield a higher return rate than mailing, our experience showed the opposite. Lack of time, both for the clinicians who were supposed to identify and give the questionnaires to potential candidates, and for the patients who were hurrying between appointments, was identified as the main limiting factor. 
248/ COMPARISON OF PHYSICIAN AND PATIENT RATINGS OF QUALITY OF LIFE IN URUGUAYAN CANCER PATIENTS

Juan J. Dapueto, Departmento de Psicología Médica, Facultad de Medicina, Universidad de la República, Montevideo, Uruguay; Carla Francolino, Departmento de Psicología Médica, Liliana Servente, Irene Gotta, Departmento de Biofísica, Facultad de Medicina, Montevideo, Uruguay

The purpose of this study was to determine the agreement between patient and physician assessments of the patient's quality of life (QOL). Ratings of the Spitzer's Quality of Life Index doctor (SQLI-d) and patient version (SQLI-p) were compared in 91 cases. In reliability testing, correlations between SQLI-d and SQLI-p items and the tota scores of the scales ranged from $r=0.35$ to $r=0.79$ with the exception of the SQLI-p Support item ( $r=0.09)$. Cronbach's alpha for SQLI-d scale was 0.63 and lower for SQLI-p (0.49). Cronbach's alpha for SQLI-d and SQLI-p scales increased when the Support item was deleted from the scale ( 0.68 and 0.62 , respectively). Significant correlations were found between doctor and patient SQLI total scores $(r=.63, p<.001 ; \mathrm{ICC}=.63)$, Activity $(r=.71, \mathrm{p}<.001 ; \mathrm{ICC}=.70)$, Daily Living $(r=63, p<.001$; ICC $=.62)$ but were lower for Health $(r=.32, p<.001$; $I C C=.32)$, Support $(r=.30, p<.001 ; I C C=.30)$ and Outlook $(r=.20, p<.05$ $I C C=.19)$ items. Mean difference between total scale scores, Activity and Outlook items were significant $(p<.001)$ with a small but systematic bias $(d=0.30-0.41)$. These findings suggest a good agreement between physician and patient assessments in the overall scale and physical impairment items. Agreement was poor for QOL items related to emotional and social aspects. Although small, a systematic bias was observed with doctors reporting better patient QOL, activity and outlook levels. Support items must be revised in order to improve scale reliability in future studies.

249/ RESPONSIVENESS OF THE ACNE-SPECIFIC QUALITY OF LIFE QUESTIONNAIRE (ACNE-QOL)

Sheri Fehnel, RTI Health Solutions, Lori McLeod, Statistics Research Division, Research Triangle Institute, RTP, NC; Jane Brandman, Outcomes Research, Pfizer Pharmaceutical Group, New York, NY; Connie McLaughlin-Miley, Outcomes Research, Pfizer Pharmaceutical Group, Ann Arbor, MI

The Acne-QoL was developed by Girman et al. (1996) to measure psychosocial impacts of acne such as self-consciousness and social withdrawal. This self-administered questionnaire contains four subscales: Self-Perception, Role-Emotional, Role-Social, and Acne Symptoms. The objectives of the current study were to evaluate the responsiveness of the Acne-QoL and to extend the developer's psychometric validation of the instrument. The Acne-QoL was utilized in two identically designed, randomized, double-blind, placebo-controlled trials conducted to assess the efficacy of Estrostep (a low-dose oral contraceptive containing 20,30, and $35 \mathrm{mcg}$ ethinyl estradiol and 1 $\mathrm{mg}$ norethindrone acetate) in the treatment of moderate facial acne in females. Combining the data from both trials, a total of 593 patients received either Estrostep or placebo. The Acne-QoL was completed at baseline, cycle 3, and study exit during the six 28-day treatment cycles. The responsiveness of the Acne-QoL was demonstrated through its ability to detect both small (baseline to cycle 3) and moderate (baseline to study end) treatment advantages for subjects who received Estrostep. In addition, confirmatory factor analysis strongly supported the current subscale structure: all items loaded highly on their respective factors and fit indices were excellent (e.g., GFI=.98) All four subscales were also internally consistent with Cronbach's alpha coefficients ranging from .86 to .96 at the final visit. Convergent and discriminant validity were further supported by comparing AcneQoL subscale scores with clinical measures and obtaining correlations in both the direction and relative magnitude hypothesized. Finally, the results of item response theory (IRT) analyses confirmed that each item is highly related to its subscale's latent construct, and that each subscale is sensitive across a broad range of the underlying continuum. The results of this evaluation confirm that the AcneQoL is responsive, internally consistent, and valid.
250/ PSYCHOMETRIC PROPERTIES OF THE NEI-VFQ - A PSYCHODIAGNOSTIC INVENTORY MEASURING DISEASE-SPECIFIC QUALITY OF LIFE OF VISUALLY IMPAIRED PEOPLE

Gabriele H. Franke, Psychology of Rehabilitation, University of Applied Sciences, Stendal, Germany; Saxonia-Anhaltina, Jens Reimer, Ruhr University Bochum, Department of Neurology, St. Josef Hospital, Bochum, Germany; Northrhine-Westfalia, Joachim Esser, University of Essen, Center for Ophthalmology, Essen, Germany, NorthrhineWestfalia

The NEI-VFQ (Mangione, Lee \& Hays, 1996) measures disease-specific quality of life (QoL) of visually impaired patients: (1) General Health, (2) General Vision, (3) Ocular Pain, (4) Near Activities, (5) Distance Activities, (6) Social Functioning, (7) Mental Health, (8) Role Difficulties, (9) Dependency, (10) Driving, (11) Color Vision, and (12) Peripheral Vision. 424 visually impaired patients and 360 healthy controls were investigated. Analysis of reliability (internal consistencies) demonstrated acceptable results: controls: alpha(min $)=0.64$ scale (3), alpha $(\max )=0.95$ scale $(10)$; patients: alpha $(\min )=0.53$ scale (3), alpha $(\max )=0.92$ scale (4). The NEI-VFQ was able to differentiate between patients and controls - even after controlling sociodemographic variables (age, gender). Investigation of the effects of cataract surgery in a subsample of 102 patients before and after operation (critical difference $=\left(1,96 * \mathrm{SD}^{\star} \mathrm{SQR}\left(2^{*}(1\right.\right.$-alpha $\left.\left.)\right)\right)$ ) demonstrated a statistically significant improvement of vision-specific QoL in 4 patients (scale (1)) to at least 34 patients (scale (4)) and a decrease in zero patients (scale (3)) to at least 13 patients (scale (8)). The scale structure of the NEI-VFQ should be revised: (1) Visionspecific Dependency (15 items), (2) Near Activities (13 items), (3) Ability to Work (2 items), (4) General Health (2 items), (5) Worry about Visual Function (2 items), (6) Ocular Pain (2 items).

\section{1/ THE STROKE SURVIVOR FUNCTIONING AND WELL-BEING} SCALE

Patrick J. Doyle, Geriatric Research Education and Clinical Center, VA Pittsburgh Healthcare System, Pittsburgh, PA; Malcolm R. McNeil, Department of Communication Science and Disorders, University of Pittsburgh, Pittsburgh, PA; Joseph M. Mikolic, Geriatric Research Education and Clinical Center, VA Pittsburgh Healthcare System, Pittsburgh, PA; Luis Prieto, FPCE Blanquerna, Universitat Ramon Llull, Barcelona, Spain

Stroke frequently results in depression and activity limitations across multiple domains of functioning. However, most instruments used to describe health outcomes in stroke survivors are narrowly focused on physical aspects of functioning and rarely assess other functional domains or emotional well-being. As such, the Stroke Survivor Functioning and Well Being Scale (SSFWB) was developed to provide a comprehensive assessment of health outcomes in stroke survivors. The SSFWB is a patient reported health status instrument whose conceptual structure is grounded in the ICIDH-2 model of functioning and disability, and whose item content was derived from cross cultural qualitative research employing stroke survivors, family caregivers, and healthcare providers. The SSFWB is unique relative to instruments commonly used to assess health outcomes in stroke survivors in that it is conceptually grounded in the ICIDH-2 model of disablement and functioning, quantifies activity limitations across the full range of functional domains commonly impacted by stroke, and quantifies the effects of domain specific activity limitations on survivors' appraisals of their emotional states, life satisfaction, and participation in society. The pilot version of the instrument consists of 75 items comprising 7 facets of functioning index by ICIDH-2 and endorsed by relevant stakeholders as negatively impacting quality of life in stroke survivors (mobility, self-care, communication, cognition, vision/proprioception, social relationships, and energy \& sleep). An additional 37 items assess the impact of domain specific activity limitations on positive and negative mood states, life satisfaction, and participation in society. The instrument is currently being validated in a prospective sample of 250 stroke survivors and 250 gender and age matched healthy controls from which the internal consistency, test-retest reliability and factor structure of the instrument will be reported. 
252/ PSYCHOMETRIC VALIDATION OF THE ITALIAN PAVK-86 QUESTIONNAIRE

Inge Kirchberger, QUALI-team, Research \& Consulting in Health and Soci., Augsburg, Germany; Edmundo E. Dagrosa, Jürgen Müller, Medical Department/Clinical Development, Aventis Pharma Germany, Bad Soden, Germany

The PAVK-86 questionnaire is a German instrument to assess QoL of patients with peripheral arterial occlusive disease. To enable its use in international clinical trials the German questionnaire was translated into Italian using forward-backward translation and pilot patient testing of the Italian version. To evaluate the psychometric properties the Italian PAVK-86 was included in a clinical trial to assess the effect of pentoxifylline on the QoL of patients with intermittent claudication. Data of 215 Italian patients were analysed. Psychometric analysis included feasibility (Number of missing data), reliability (Internal consistency), construct validity (Item internal consistency, item discriminant validity), concurrent validity and discriminant (known-groups) validity. The Italian PAVK- 86 showed an acceptable feasibility. About $20 \%$ of the patients completed the questionnaire without any missing answers. Some of the items were missed frequently, probably due to lacking relevance for the sample, patients with Fontaine stage II. Four subscales showed excellent reliability with internal consistency coefficients ranging from 0.90 to 0.94 . The subscales "Pain" and "Social Life" (Alpha=0.65) almost reached the minimum requirement for internal consistency of 0.70 . Only few items did not correlate highly with their own subscale (Item internal consistency) and most items correlated higher with their hypothesized subscale than with other subscales (Item discriminant validity), reflecting good construct validity. Scaling success, a measure for item discriminant validity, proved to be perfect for almost all subscales. Concurrent validity was supported by substantial correlations of the PAVK-86 subscales with comparable dimensions of the SF-36. Moreover, three of the subscales proved to be able to discriminate patients according to walking distance. These results showed that the Italian version of the PAVK-86 is a suitable instrument with satisfactory reliability and excellent validity.

253/ CONCEPTUALIZATION AND DESIGN OF AN INDIVIDUALIZED MEASURE OF BPH IMPACT

Asha V. Hareendran, Outcomes Research, Pfizer Ltd., Sandwich, UK Mona L. Martin, Donald M. Bushnell, Jane E. Goodman, Health Research Associates, Inc., Seattle, WA

Patient's report of the impact of Benign Prostatic Hyperplasia(BPH)symptoms on their lives are important for treatment decisions making and evaluating outcomes of new treatments. The relevance and importance of the impact of $\mathrm{BPH}$ on different aspects of patients'lives are diffuse and patterns of occurrence among the targeted patient group are unpredictable. Eliciting this information from patients using standardised response options tends to statistically "normalise"them,leaving no room for individuality. Hence we developed a measure of BPH-specific patient impact that would benefit from both the statistical strength of a standardised measure as well as from the adaptability and high content relevance of an individualised measure. Qualitative interviews and literature review provided an initial list of 71 different topic areas that were grouped into 9 separate domains. The item generation process yielded an initial long-list of three types of questions:Functional impact items ( $n=34)$;BPH symptom items $(n=21)$; and Psychological impact items $(n=13)$. An initial measure was drafted which incorporated these items into a standardised format with a five point Likert-type response scale. The content was duplicated in a prompter list followed by an individualised section for the top five individualised patient choices. The two versions of the measure were pilot tested on 49 patients. Final designation of items to the standardised versus individualised versions was determined through a combination of qualitative and quantitative methods. This resulted in the development of the BPH Patient Impact Measure with 15 standardised items (highest frequency of reporting by the pilot group).Those items representing a lower report-frequency (yet still presented important and relevant data to a portion of the patient group) were assembled into a prompter list for the individualised portion of the measure.
254/ INITIAL PSYCHOMETRIC PERFORMANCE OF AN INDIVIDUALIZED MEASURE OF BPH PATIENT IMPACT (BPH-PIM)

Mona L. Martin, Health Research Associates, Inc, Seattle, WA; Asha V. Hareendran, Outcomes Research, Pfizer Ltd., Sandwich, UK; Donald M. Bushnell, Jane E. Goodman, Health Research Associates, Inc., Seattle, WA

In order to increase representativeness of the content and still focus on key impact areas of importance to patients, the BPH-PIM (Benign Prostatic Hyperplasia-Patient Impact Measure)was developed. This measure addresses Physical Impacts (10 standardized items), Psychological Impact (5 standardized items) and provides a 5-item individualized section preceded by a prompter list to facilitate patient responses. This research aims to evaluate the initial psychometric performance of the BPH-PIM, for both the summary score and the two subscales. Initial psychometric data was collected on a sample of 48 individuals with BPH. Internal consistency reliability was evaluated using Cronbach's alpha. Test/retest reliability was assessed using the intraclass correlation coefficient (ICC). Convergent validity was assessed by comparing the BPH-PIM standardized and individualized scales the SF-36. Known groups of $\mathrm{BPH}$ severity were used to determine the ability of the measure to discriminate. The correlation between the standardized and individualized measure scores was (0.70). The BPH-PIM demonstrates good internal consistency (alpha $=0.93$ ) and the test-retest reliability (at 6 days) is 0.91 . Acceptable convergent validity was demonstrated with relevant subscales of the SF-36 (ranging between 0.40 and 0.56 ; significant at the 0.05 level). As expected, lesser associations were seen with the bodily pain and physical subscales $(0.29$ and 0.13$)$. The BPH-PIM demonstrated the ability to discriminate between symptom severity levels and the AUA problem index scores. $(0.40$ and $0.68 / \mathrm{p}<0.05)$. In summary, this new BPH Patient Impact Measure is short in length, low in patient burden, high in content validity, and demonstrates acceptable psychometric performance.

255/ QUALITY OF LIFE AND DEMOGRAPHICS IN PRIMARY CARE AND RHEUMATOLOGY SAMPLES

Erik J. Groessl, R. M. Kaplan, T. G. Ganiats, K. M. David, W. J. Sieber, University of California San Diego, La Jolla, CA

The self-administered version of the Quality of Well-Being Scale (QWB-SA) is a generic measure of health-related quality of life (HRQOL). The purpose of the present study was to examine the validity of the QWB-SA for measuring HRQOL in primary care and rheumatology clinic groups of varying demographic backgrounds. Hypotheses based on prior research are that a) rheumatology patients will have lower QOL than primary care patients; b) males will have higher QWB-SA scores than females; and that c) QWB-SA scores will increase as the patient's level of education increases. The participants were 896 people (562 primary care, 334 rheumatology patients) who received medical care in University of California San Diego clinics. They were $63 \%$ female, and 50 years of age on average. The QWB$\mathrm{SA}$ was administered along with a demographic questionnaire and a measure of self-rated health status. Independent-sample t-tests showed that rheumatology patients had significantly lower mean QWBSA scores $(.508)$ than primary care patients $(.651)(p<.001)$. Overall, females $(.571)$ had lower scores than males $(.651)(p<.001)$, but a significant 2-way interaction between clinic group and gender showed that this difference was significant only in the rheumatology group. Significant increases in QOL were found as education increased, although this trend $(p=.059)$ was much more pronounced for the primary care patients. Finally, Asian-Americans were found to have higher QOL than non-Asian Americans among both primary care and rheumatology patients. The results support the validity of the QWBSA for measuring HRQOL in primary care and rheumatology clinic samples. The measure detected differences that have been previously associated with demographic or pre-existing differences such as gender and education level. An interesting finding was that AsianAmericans had higher QOL scores, even among rheumatology patients. Possible explanations include that they have higher HRQOL and/or respond to the QWB-SA questions differently than non-Asians. 
256/ THE VALIDITY AND RELIABILITY OF THE TAAQOL: A HEALTH-RELATED QUALITY OF LIFE INSTRUMENT COMPRISING HEALTH STATUS WEIGHTED BY THE IMPACT OF PROBLEMS ON WELL BEING

Jeanet Bruil, Minne Fekkes, Ton Vogels, Erik Verrips, Child Health Quality of life, TNO Prevention and Health, Leiden, The Netherlands

There is a growing awareness that health status and health-related quality of life (hrqol) are distinct concepts and that hrqol should include impact of health status problems. In this study, hrqol is defined as a person's health status, weighted by the impact of health status problems on well being. The TNO-AZL Questionnaire for Adult's Health-Related Quality of Life (TAAQOL) for people of 16 years and older was constructed to enable a systematic, valid and reliable description of hrqol according to the definition described above. Aim of the study was to evaluate the validity and reliability of the TAAQOL. The survey instrument included the TAAQOL and the SF-36 as well as information on demographic variables, chronic illness and use of medical care. A total number of 8,800 households, drawn at random from the national telephone registry, were included in the study, of which $4,427(50 \%)$ returned a completed questionnaire (1882 men $(45 \%)$ and 2271 women $(54 \%))$. The age range was $16-97$ years. The TAAQOL includes 12 scales. Factor analysis using varimax rotation reflects the supposed scale structure very well. The scales have good reliabilities ranging from 72 to 90 . Scales of the TAAQOL and the corresponding SF-36 scales measuring the same concept have a correlation coefficient of .50 to .70 , indicating both a shared similarity as well as a clear distinction between the two concepts (health status versus hrqol). The validity of the distinction between health status and hrqol is supported by the finding that, depending on the scale $37 \%$ (social functioning) to $74 \%$ (gross motor functioning) of the health status problems reported were associated with negative emotional reactions. To assess whether the TAAQOL is able to detect differences in hrqol scores between adults with a chronic illness $(n=2604$ $(59 \%))$ and healthy adults, multivariate variance analyses (MANOVA) were performed using illness and gender as factor and age as covariate. Adults with a chronic illness scored significantly lower on all TAAQOL scales than healthy adults $(p<.001)$. Clearly, the TAAQOL allows for a reliable and valid measurement of hrqol at group level, intrinsically subjective as the concept of hrqol may be.

\section{7/ THE SCOPA-PS: A PSYCHOSOCIAL QUESTIONNAIRE FOR} PATIENTS WITH PARKINSON'S DISEASE

Johan Marinus, Martine Visser, Bob van Hilten, Neurology, Anne M. Stiggelbout, Medical Decision Making, Leiden University Medical Center, Leiden, The Netherlands

To construct and validate a short psychosocial questionnaire for patients with Parkinson's Disease (PD). The 12 initial items of the SCOPA-PS were largely derived from existing generic and diseasespecific quality-of-life (QoL) instruments and together covered the psychosocial domain in PD. The scale was tested in a survey that included several other health measurement instruments. This survey was sent to 205 Dutch patients with idiopathic PD, visiting an outpatient neurology clinic of a university hospital. One-hundred-seventyseven usable questionnaires were returned, constituting a response rate of $86 \%$. Seventy patients were classified as mildly affected, while 64 patients were moderately, and 32 patients severely affected. One item was removed because of floor effects. Deletion of this item did not affect the reliability and content validity. The internal consistency of the remaining 11 items, measured as a Cronbach's a, was 0.84 . Test-retest reliability over a two-week interval, calculated as an intraclass correlation coefficient, was 0.85 . Retest reliability for individual items, calculated by means of a weighted kappa, ranged from $0.50-0.83$. The construct validity, established by computing the correlation between the SCOPA-PS on the one hand, and related scales on the other hand, was 0.80 for the Parkinson's Disease Questionnaire (PDQ-39), 0.72 for the Hospital Anxiety and Depression Scale (HADS), and -0.65 for a visual analogue scale that assessed QoL. A summary index, calculated over the sum of the items revealed a significant trend in the expected direction between patients grouped on the basis of disease severity. The SCOPA-PS is a new, short psychosocial questionnaire for patients with $P D$ and has very good clinimetric properties. Because of the latter, the SCOPA-PS may be preferred over other short QoL scales in PD, such as the Parkinson's Impact Scale (PIMS) and the Parkinson's Disease Questionnaire - 8 item version (PDQ-8)
258/ QOL-OLD INSTRUMENT TO ASSESS QUALITY OF LIFE IN THE ELDERLY

Erzebet AC-Nikolic, Olja Niciforovic Surkovic, Svetlana Kvrgic, Institute for Public Health, Novi Sad, Vojvodina, Yugoslavia; Zoran Potic, Institute for Cardiovasculari Diseases, Novi Sad, Vojvodina, Yugoslavia

Demographically old population of Vojvodina (Eastern Europe) from one side and importance of quality of life measurement in assessing outcomes of medical and social interventions on the other side, are pointing out the need of creating an instrument for the assessment of quality of life in the elderly. The random sample of 373 elderly people, who were generally speaking healthy, fulfilled the questionnaire, which was compiled from several other instruments. In order to reduce the number of items and obtain appropriate psychometric characteristics, reability and factor analyses were performed. The final questionnaire consists 36 items grouped in six domains (physical function, self-care, interpersonal relations, social usefulness, social functioning and self assessment scale). Reliability was determined by using Cronbach Alpha coefficient which showed high values for all six domains respectively (values between 0,70 and 0,87 ). Validity was determined by using known age groups $(p<0,01)$. The QOL_OLD index is overall numerical value composed by values of all six domains weighted with regression coefficients obtained principal component analyses. This value is transformed on scale $0-100$, and for our group the average value is about 70 . The created instrument showed high reliability and validity and can be used in assessment of quality of life in elderly in population as well as for individuals who are generally speaking healthy for their age.

259/ THE PSYCHOMETRICS OF THE HOSPITAL ANXIETY \& DEPRESSION SCALE: FACTOR AND ITEM-RESPONSE BASED ANALYSES

Adam B. Smith, Galina Velikova, Peter J. Selby, ICRF Cancer Medicine Research Unit, St. James's University Hospital, Leeds, West Yorkshire, UK

The Hospital Anxiety and Depression (HADS) has been used as a common screening tool for psychological distress in general hospital wards and clinics (Zigmond \& Snaith, 1983). In this study we explored the factor structure and Item-Response Theory (IRT) based analyses of the HADS in a large $(n=1479)$ heterogeneous population taken from oncology wards and clinics. The factor analysis (FA) of the entire dataset revealed a two-factor structure corresponding to the two subscales, Anxiety (accounting for $38 \%$ of variance) and Depression $(12 \%$ variance). This factor structure remained when the data were divided into male/female and split-half reliability subsets; and different age groups. Internal reliability analyses demonstrated Cronbach's alpha of 0.85 for Anxiety and 0.70 for Depression. Finally, only one question (item 4) from the Anxiety subscale loaded onto the Depression subscale. The dataset was then analysed using the Graded Response Model (Muraki, 1990), which has been designed for ratingscale questionnaires with the same number of response categories. The analysis demonstrated high item discrimination (slope) parameters for four of the questions from the Anxiety subscale, indicating that these item discriminate well between individuals with different levels of anxiety. An item information curve indicated that these items also provided the greatest amount of information across the estimated latent trait. In line with the FA results the slope parameters for item 4 were particularly low. The Depression subscale revealed only 2 items (questions 3 and 6 ) with high slope parameters. Although the traditional FA method demonstrated a stable two-factor structure of the HADS, the IRT analysis extended the analysis and highlighted the different discrimination properties of the questions and the information they provided about the subscales. 
260/ RELIABILITY AND VALIDITY OF 'NEWSQOL': THE NEWCASTLE STROKE-SPECIFIC QUALITY OF LIFE MEASURE Deborah Buck, Ann Jacoby, Primary Care, University of Liverpool Liverpool, UK; Gary A. Ford, Wolfson Unit of Clinical Pharmacology, University of Newcastle, Newcastle upon Tyne, UK

The purpose of this study was to develop and validate a new strokespecific quality of life instrument - the Newcastle Stroke-specific Quality of Life measure (NEWSQOL). This presentation will focus on Phase II of the study, where the main objective was to test NEWSQOL for construct validity and stability. During Phase I, qualitative work with 28 stroke patients, a focus group with informal carers, and a pretesting phase with 30 patients identified key QOL areas for inclusion in the measure. An item reduction phase with 100 patients identified poorly performing items, and factor analysis identified likely domains. The final version of NEWSQOL contains 56 items across 11 domains: Feelings, ADL/Self-care, Cognition, Mobility, Emotion, Sleep, Interpersonal Relationships, Communication, Pain/Sensation, Vision, and Fatigue. During Phase II, NEWSQOL, the Nottingham Health Profile (NHP), the Barthel Index (BI), and the National Institutes of Health Stroke Scale (NIHSS) were administered to 106 patients to test for construct validity and test-retest reliability. Internal consistency was re-examined. Cronbach's alpha was high for NEWSQOL domains $(0.71-0.90)$. Item-total correlations ranged from moderate to high in all except the Pain/Sensation domain. Test-retest reliability was high ( $r$ range $0.78-0.92$ ). All items correlated more highly with their own than with other domains, providing evidence of item-discriminant validity. Domain scores were moderately to highly significantly correlated $(0.45-0.76)$ with relevant NHP and selected NIHSS items or B scores (except for the Cognition domain). The present study provided useful evidence about the psychometric properties of NEWSQOL. Ongoing studies are needed to provide additional information, in particular the evaluation of its responsiveness to changes in quality of life over time, and the validity of the Cognition domain.

261/ FEASIBILITY OF THE SF-12 HEALTH SURVEY IN A REGIONAL POPULATION SURVEY IN AUSTRIA

Inge Kirchberger, QUALI-team, Research \& Consulting in Health and Soci., Augsburg, Germany; Franz Piribauer, Public Health Directorate, State Government, Graz, Styria, Austria; Elisabeth Santigli, University Clinic for Dental Medicine, University of Graz, Graz, Styria, Austria

Up to now few international experience about feasibility and utility of the SF-12 in population surveys is published. The German version of the SF-12 is increasingly used in Austria. However, the question whether the German translation of the questionnaire is also adequate for its use in Austria has not yet been examined. Our paper reports about experiences with the German SF-12 derived from a survey on an Austrian population sample. In Styria, one of the nine states of Austria, the Microcensus on health was supplemented by the SF-12. In 2640 Styrian households 5881 persons were asked the SF-12 items in face-to-face interviews. If household members were not present proxy interviews took place. $5222(88.8 \%)$ of the 5881 subjects visited agreed to answer the SF-12. Subjects who refused did not differ significantly from the responders. For 1654 subjects $(31.7 \%)$ a member of the same household answered the questions on behalf of them. 5124 subjects $(98.1 \%)$ answered the SF-12 completely, whereas $1.9 \%$ did not answer one or more questions. Significant differences in age, gender and general health status between subjects with complete versus incomplete questionaires could not be detected. The two items belonging to the subscale "Role-emotional" (Item 6,7$)$ and the question on pain (Item 8 ) were most frequently not answered by the interviewees $(n=13,0.25 \%) .317$ subjects $(6.1 \%)$ asked the interviewer for an explanation of particular questions. Compared with those subjects who did not demand an explanation, this group was 13 years older in average and judged their own health condition significantly worse. The items which needed an explanation most frequently were both questions on emotional role-functioning (Item 6,7) and the question on disturbance of social activities (Item 12). SF-12 scores were found to be affected by age and gender wheras the interviewer did not affect the results. Differences between self- and proxy ratings were found depending on the age-group studied.
262/ ATTITUDES TOWARDS QUALITY OF LIFE AND SURVIVAL IN METASTATIC BREAST CANCER: A COMPARISON OF INSTRUMENTS

Anne M. Stiggelbout, Sylvia J. Jansen, Department of Medical Decision Making, Marianne A. Nooy, Department of Clinical Oncology, Job Kievit, Department of Medical Decision Making, Leiden University Medical Center, Leiden, The Netherlands

Attitudes towards palliative chemotherapy relate to tradeoffs between dimensions of quality of life, and between quality of life and uncertain survival benefits. We assessed such attitudes with a probability tradeoff instrument (PT) and the Quality-Quantity-Questionnaire (QQQ). Aim was to study the relationship between the instruments, and to assess the attitudes over time (before, during, and after treatment), in breast cancer patients treated with palliative chemotherapy or hormonal therapy. The PT described two scenarios: wait\&see and chemotherapy. The patient was asked for the response rate of chemotherapy at which she would prefer chemotherapy to wait\&see. The QQQ consists of 8 items assessing patients' attitudes towards tradeoffs between quality and length of life (1). It forms two scales, a Q- and an Lscale, stressing preferences for Quality of life and Length of life, respectively. Fifty-one patients with metastatic breast cancer were interviewed: 36 receiving chemotherapy, 15 receiving hormonal therapy only. Reliability of the $Q Q Q$ was adequate (alpha=0.73). The PT correlated positively with the $\mathrm{Q}$-scale, negatively with the L-scale (e.g. during treatment $r$ was .56 for the $Q$ - and -.56 for the $L$-scale). Over time, the correlations were most consistent for the controls. As expected, the control group required a significantly larger response from treatment than the chemotherapy group. The required benefit increased over time in both groups, though not significantly so (medians increased from $1 \%$ to $7.5 \%$ in the chemotherapy group, from $20 \%$ to $30 \%$ in the control group). The two instruments are useful complements in assessing attitudes towards palliative chemotherapy. The direction of the correlations between the PT and the QQQ seems to imply that although symptom relief was stressed in the PT, patients associated palliative chemotherapy mostly with survival benefits. 1 Stiggelbout et al. Med Decis Making 1996;16:184-92.

263/ A HEALTH RELATED QUALITY OF LIFE MEASURE FOR USE IN PATIENTS WITH ERECTILE DYSFUNCTION: A VALIDATION STUDY

Deborah P. Lubeck, David Latinin, Hilary Colwell, Urology, University of California, San Francisco, San Francisco, CA; David Penson, Urology, University of Washington, Seattle, WA; Shilpa Mehta, Outcomes Research, TAP Pharmaceutical Products Inc., Lake Forest, IL

Male erectile dysfunction has substantial impact on health-related quality of life (HRQoL). The purpose of this paper was to examine the psychometric properties of the disease-specific HRQoL scales in the ExCEED Baseline Quality of Life questionnaire. The ExCEED (Exploratory and Comprehensive Evaluation of Erectile Dysfunction) Baseline Quality of Life questionnaire was administered to patients enrolled in a long-term observational study of men with erectile dysfunction (ED). The questionnaire incorporated a number of standard scales of psychosocial characteristics, as well as two experimental sets of questions developed from comments made during a focus group of men with ED. Patients enrolled in the study complete the questionnaire at enrollment, three-month, six-month, and twelvemonth follow-ups. Responses to existing scales were scored according to the standard scoring instructions for those scales. Factor analysis was used to evaluate patterns of responses to the two sets of experimental questions. The questionnaire was completed by 111 men at baseline and three-month follow-up. The factor analysis of the psychological aspects of ED questions resulted in two new scales. The internal consistency reliability of all scales was moderate to good (Cronbach's alpha $=0.66-0.95)$. Test-retest reliability was moderate to good for all scales (intra-class correlation $=0.48-0.77$ ). The disease-specific HRQOL measures significantly differentiated between men with varying degrees of ED. Conclusions: evaluation of the ExCEED Baseline questionnaire indicated that the existing scales showed adequate psychometric properties in this sample. Two new scales were developed to measure the psychological impact of ED. The disease-specific HRQL measures were able to discriminate between men reporting different levels of ED 
264/ PSYCHOMETRIC VALIDATION OF CONTRACEPTIVE RING ACCEPTABILITY QUESTIONNAIRE

Annoesjka Novak, Health Economics Department, NV Organon, Oss, The Netherlands; Linda Abetz, MAPI Values Ltd., Cheshire, UK; Christine de la Loge, MAPI Values, Lyon, France

A self-completion acceptability questionnaire was developed to assess womens valuation of a novel vaginal hormonal contraceptive NuvaRing $\AA$ in a large clinical trial. The 21 -item questionnaire was completed by 2145 women at regular intervals during the 12-month study period and covered: ease of use (ring/package), clarity of instructions, sexual comfort, menstruation, compliance and satisfaction. The robustness and predictive value of the questionnaire were assessed using cycle 3 data $(n=1950)$. The quality of completed questionnaires, item content analysis, construct validity, internal consistency reliability, known-groups validity, and predictive value were evaluated. Excluding non-ordinal items, on average $0.6 \%$ of data were missing. Principle component analysis of 15 ordinal items indicated that linked domains gave consistent results. Hence, 'ease of package use' and 'clarity of instructions' were combined. Item convergent validity (the degree of correlation between an item and its own scale) was excellent for 'ease of use' and 'satisfaction', moderate for 'clarity of instructions' and 'sexual comfort' and poor for 'menstruation'. Item discriminant validity (that an item correlates higher with its own scale than with any other) was excellent for all domains. Women without adverse events, those choosing NuvaRing as the best method of contraception and completers responded most positively to the questionnaire, indicating known groups validity. The level of satisfaction and compliance captured by the questionnaire at cycle 3 were the best predictors of early discontinuation after cycle 3 , indicating the predictive validity of the questionnaire. It was concluded that the acceptability questionnaire has good psychometric properties and can predict early discontinuation of the method. This questionnaire could be used in general practice to monitor user compliance.

\section{5/ QUALITY OF LIFE IN DERMATOLOGY: DEVELOPMENT OF VQ-DERMATO'S SINGLE INDEX}

Christophe Sapin, Public Health Department, Medical School, Marseille, France; Jean-Jacques Grob, Dermatology Department, St. Marguerite Hospital, Marseille, France; Stéphane Robitail, MarieClaude Simeoni, Pascal Auquier, Public Health Department, Medical School, Marseille, France

VQ-Dermato is the only French multidimensional self-administered Health-Related Quality of Life (HRQL) instrument, based on patients' viewpoint, which assessed the impact of chronic skin diseases in patients' everyday life. It comprises 28 items, allowing the calculation of 7 dimensions. One of the major problem for the dermatologists' community is the lack of a single index, so that the HRQL evaluation could be used in the medical decision process. So we decided to compare several weighting methods to develop a single index for VQ-Dermato. A literature review led us to select 6 weighting processes among all the methods initially developed in the Marketing and Operational Research frameworks. These methods were already used in the Outcome Measurement field : Diakoulaki, Entropy, simple cardinal evaluation (SCE), analytic hierarchical process (AHP), partial-least-square regression (PLS) and conjoint analysis (CA). Due to the problem of transposability of regressive methods (PLS and CA) in the Quality of Life field, we decided to compare the composite weighted global indexes derived from Diakoulaki, Entropy, SCE and AHP methods in terms of validity, fidelity, acceptability and responsiveness. The data used were those of the validation step, comprising 300 patients presenting 12 different skin disorders. All the 4 indexes, except AHP, showed a rather good external validity: high levels of correlation with the skin disorder severity and the percentage of skin surface involved. They also were reliable (test-retest correlations always greater than $0.90)$ and sensitive to change. The acceptability was good, with less than $5 \%$ of missing data. The validation of such a single score needs further investigations. However, it seems relevant to think that a global index for VQ-Dermato can help the dermatologists' community to adapt the therapeutic strategies by using the HRQL evaluation in the medical decision.
266/ VALIDATION OF A FRENCH INSTRUMENT MEASURING HOSPITALISED PATIENT SATISFACTION: QSH

Stéphanie Antoniotti, Public Health Department, Medical School, Marseille, France; José Labarere, Public Health Department, Grenoble, France; Laurent Gerbaud, Public Health Department, Clermont-Ferrand, France; Dominique Bertrand, Public Health Department, Annecy, France, Christophe Sapin, Audrey Clement, Public Health Department, Marseille, France; Patrice Francois, Public Health Department, Grenoble, France; Pascal Auquier, Public Health Department, Marseille, France

A literature review found three hospitalised patient satisfaction measurements with available validation results. None were widely validated and developed from the exclusive patients' viewpoint. We report the development and the validation of such a measurement in French: the QSH questionnaire. 95 interviews were conducted with patients of different subgroups. Content analysis of these interviews led to 124 candidate items. Item reduction step was planned in 75 services of publicly-owned hospitals (medical wards: 38; surgical: 35; obstetrical: 2). 1,200 subjects were included the last day of their hospitalisation and 69 items were selected (classical methods and Rasch analyses). The validation study was conducted on a random sample of 3,000 patients of 10 hospitals in France. Demographic variables, time length of hospitalisation, load of nurses care, clinical diagnosis and case mix adjustment were also collected. The final questionnaire -QSH- included less than 50 items, isolating different domains covering the 3 main fields: hospitalisation process (admittance, cares, discharge), co-ordination and organisation (waiting time), environmental factors (food, living arrangements). Known group comparisons were studied according to: socio-demographic variables, load of nurses care... The criterion validity was shown (clinical related group). Reproducibility was assessed on a randomised sample (1/ 10) 48 hours after hospital discharge. The evolution of patient satisfaction over time was explored on a sample of 800 subjects at day $15,21,30,45$ and 60 after hospital discharge. The percentage of missing responses was low, completion time was always under 20 minutes, therefore it seems possible to assess satisfaction with this only self-administered questionnaire constructed on the basis of patient's viewpoint: QSH.

267/ PSYCHOMETRIC PROPERTIES OF IBS-QOL QUESTIONNAIRE AND ITS RELATIONSHIP WITH SF-36

Fabio Arpinelli, Giovanni Visona, Luca Cavazzuti, Medical Department, GlaxoWellcome S.p.A., Verona, Italy; Mauro Niero, Nadia C. Oprandi, University of Verona, Verona, Italy

We compared the Italian version of the Irritable Bowel Syndrome Quality of Life Questionnaire (IBS-QoL) with SF-36, in order to study pros and cons of both instruments. From January to July 2000, questionnaires were administered to 326 people suffering from IBS according to the GPs judgement. During the analysis, $43.3 \%$ of patients resulted IBS positive according Manning and Rome 2 criteria, 47.8\% were IBS negative and $8.9 \%$ showed discrepancies between Manning and Rome 2 criteria. These groups were compared during the analysis. SF-36 showed better scaling properties and a smaller ceiling and floor effect than the IBS-QoL. The IBS-QoL was more specific in detecting IBS impact on patients' lives. Such specific characteristics were tested on the basis of a construct validation model. Hypoteses included in the model were met by the IBS-QoL many more times than the SF-36. In particular, IBS-QoL sub-scale variance resulted to be explained by IBS symptoms (pain, bloating, distension). Moreover, the sub-scale variance was explained also by acid regurgitation, vomit, nausea and heartburn, though they are not specific IBS symptoms. Sensitivity to change of the IBS-QoL questionnaire was higher than SF-36. A moderate convergence was shown between the psychological scales of both questionnaires. Despite its positive aspects, the IBS-QoL shows technical shortcomings which should be improved: 1) a number of its sub-scales show very high scores on a 0-100 scale, where 100 means the best QoL; 2) subscales physical and sex have a great number of missing values; 3 ) sub-scales emotional and mental are similar: this can create redundancies; 4) sub-scales variance is not related to bowel movements 5) several non IBS-related symptoms affect IBS-QoL variance; 6) the food domain is not explained by any of the symptoms. This means that appropriate predictive variables are missing, or that the problem concerns all patients suffering from gastrointestinal symptoms. 
268/ VALIDATION OF THE COMMUNITY DEMENTIA QUALITY OF LIFE PROFILE (CDQLP): COMPARISON WITH THE EUROQOL5D (EQ-5D)

M. D. Walker, M. S. Salek, Centre for Socioeconomic Research, Cardiff University, Cardiff, UK; A. J. Bayer, Department of Geriatric Medicine, University of Wales College of Medicine, Cardiff, UK

The EQ-5D is a generic instrument that has been used to assess Quality of Life (QOL) of patients with dementia. The CDQLP is a disease specific instrument specifically developed to assess the QOL of such patients as well as that of their carers. Comparison of these two instruments was carried out with a view to providing evidence in support of the construct validity of the CDQLP. Five a priori predictions were made about how items on the CDQLP would correlate with items on the EQ-5D. A cross-sectional comparative study in 66 patients with dementia was carried out and Spearman's rank correlation coefficients between items assessing similar domains on both instruments were calculated. The predictions were confirmed or refuted on the basis of these coefficients. The CDQLP and EQ-5D were completed by 66 carers (Age: mean $=63$, range $=34-88$ - Sex: male $=27$; female $=39$ ) acting as proxies for 66 patients living in the community (Age: mean $=80.4$, range $=61-94$ - Sex: male $=22$; female $=44$ - Duration of disease (months): Mean $=54$, range=9-132). Examining associations between the CDQLP and EQ-5D produced 20 correlation coefficients of which 17 demonstrated significance at the $1 \%$ level and 3 at the $5 \%$ level. Areas of association included mobility, selfcare, usual activities, anxiety \& depression and overall patient health. All five a priori predictions were confirmed by the analysis. The EQ$5 \mathrm{D}$ measures general QOL using single items for each domain. The CDQLP measures a larger number of domains including all but one of those in the EQ-5D. The CDQLP has several items for each domain and assesses QOL more extensively for patients with dementia. There are no items on the CDQLP that correspond to pain/discomfort and therefore no predictions were made for this domain. Areas commonly assessed by the CDQLP and the EQ-5D were highly correlated as predicted. These findings further support the validity of the CDQLP.

269/ COMPARISON OF TWO DISEASE-SPECIFIC QUALITY OF LIFE (QOL) MEASURES: VALIDATION OF THE COMMUNITY DEMENTIA QUALITY OF LIFE PROFILE (CDQLP)

M. D. Walker, M. S. Salek, Centre for Socioeconomic Research, Cardiff University, Cardiff, UK; A. J. Bayer, Department of Geriatric Medicine, University of Wales College of Medicine, Cardiff, UK

The CDQLP is a disease-specific measure specifically developed to assess the QOL of patients with dementia as well as that of their carers. The Progressive Deterioration Scale (PDS) is a well-established measure that has also been used to assess QOL in patients with dementia. In order to provide further evidence supporting the validity of the CDQLP, areas of $Q O L$ assessed by both instruments were compared and the association among items was determined. A cross-sectional comparative study was carried out in 66 dementia patients. A total of 9 a priori predictions were made about how items on the CDQLP would correlate with items on the PDS. These were then confirmed or refuted by calculating Spearman's rank correlation coefficients. The CDQLP and PDS were completed by 66 carers (Age: mean $=63$, range $=34-88$ - Sex: male $=27$; female $=39$ ) acting as proxies for 66 patients living in the community (Age: mean=80.4, range $=61$ 94 - Sex: male=22; female=44 - Duration of disease: Mean=54 months, range=9-132). Examining associations between the CDQLP and PDS produced 31 correlation coefficients of which 27 demonstrated significance at the $5 \%$ level. Areas of association included handling finance, conversational ability, household maintenance, mobility, use of utensils, recreation \& pastime, dressing and social activities. Only items relating to confusion were not significantly correlated. Areas commonly assessed by both the CDQLP and the PDS were found to be highly correlated and represent QOL domains that are of great importance to patients with dementia. The PDS distinguishes between stages of dementia measured by the Global Deterioration Scale. The CDQLP assesses carer QOL as well as that of the patient and can also distinguish between patient populations. Measurement of $\mathrm{QOL}$ outcomes using the CDQLP could be carried out on a routine basis with a view to assessing the impact of disease as well as drug treatments.
270/ THE QUALITY OF LIFE INSTRUMENTS DATABASE (QOLID)

Marie-Pierre Emery, Mapi Research Institute, Lyon, France; Marcello Tamburini, Istituto Nazionale Tumori, Milano, Italy; Elodie Pasquier, Mapi Research Institute, Lyon, France

The Information Resources Centre of Mapi Research Institute, France, in collaboration with Pr Marcello Tamburini, Istituto Nazionale Tumori, Italy, is currently developing the Quality of Life Instruments Database (QOLID), a comprehensive and unique source of information on $\mathrm{Pa}$ tient-Reported Outcomes (PROs) measures available on the Internet. Over the last 20 years, a large number of PROs instruments have been developed and are increasingly used in multinational research and clinical practice. The success of a study involving PROs measurement depends on the choice of the most appropriate instrumentation. The aim of the QOLID database is to facilitate the collection of relevant information through a structured presentation of synthesized, reliable and updated data on PROs instruments. Using and restructuring information contained in the existing QLMed.org website and completed by the extensive information collected over the years by the Information Resources Centre of Mapi Research Institute, QOLID.org will unite detailed and structured information on over 400 PROs measures. 2 distinct forms of access will be proposed: the first level will provide free access to a description of each instrument including the developer's name, the measure's objectives, pathology and population studied and the available languages. The second level based on individual subscriptions will include references, copyright, detailed contact information as well as copies of the questionnaires themselves and their user manuals. Any information included in the database will be approved by and updated in collaboration with the developers of each instrument. Its facilitated and unique access will make QOLID the first source of accurate, reliable and regularly updated information providing an overview of existing instrumentation on the Internet.

271/ RELIABILITY AND VALIDITY OF A NEWLY DEVELOPED QUALITY-OF-LIFE QUESTIONNAIRE SPECIFIC TO MENOPAUSE York F. Zoellner, Social Pharmacy, Humboldt University, Berlin, Germany; Phillip Oliver, General Practice \& Primary Care, John E. Brazier, Scharr, University of Sheffield, Sheffield, UK; Jeannette Alt, Women's Health, Solvay Pharmaceuticals, Hannover, Germany; Marion Schaefer, Social Pharmacy, Humboldt University, Berlin, Germany

The purpose of this study was to develop and validate a comprehensive climacteric quality-of-life questionnaire including both menopausal symptoms and potential adverse effects (AEs) of hormone replacement therapy (HRT). Development stages: (1) Prelim.: psychometric analysis, from clinical trial data on file, of an exhaustive 45-item questionnaire $(n=367)$. (2) Pilot: face and content validity check $(n=20)$. (3) Fielding phase A: test-retest reliability $(n=107), 28$ days interval. (4) Fielding phase B: mail-out of new tool and SF-36 to 1080 patients from 6 GP practices to determine internal consistency, construct-, concurrent and known-groups validity ( $n=785,32 \%$ on HRT). Preliminary: Reduction from 45 to 39 items. Face/content: items understandable, comprehensive; some changes in wording; scaling reduced from 5 to 4 levels. Test-retest: Items generally reproducible (Pearson's $r>0.60$ ); those 7 testing $<0.60$ earmarked for possible deletion. Construct: Factor analysis yielded 6 robust factors. Eventually, 23 items could be meaningfully grouped into the following 6 domains: psychological, sexual, somatic, menstrual, vasomotor and androgenic, explaining $62.5 \%$ of the variance and all meeting the criteria for item convergent and item divergent validity. Consistency: Sub-scales showed high (sexual, psychological, vasomotor) or reasonable (somatic, menstrual) internal consistency ( $>0.80$ and $>.50$, respectively), with only the androgenic factor revealing some heterogeneity (Crohnb.'s alpha $=0.40)$. Known groups: All but 3 of the symptomitems could distinguish between 'symptomatic' and 'non-symptomatic' women; AE-items did not appear to differentiate between users and non-users of HRT. Concurrent: The 'generic' (psychological and somatic) domains showed strong and significant correlations with the relevant SF-36 sub-scales. The instrument has performed well in all reliability and validity assessments. Forthcoming research will document its usefulness in clinical practice. 
272/ A SPECIFIC QUALITY OF LIFE SCALE IN UPPER LIMB LYMPHQEDEMA: THE ULL-27 QUESTIONNAIRE

Robert Launois, Alexandre Coffi Megnigbeto, Sante Publique, Universite Paris XIII, Bobigny Cedex, France

Lymphœdema of the arms is a serious complication of breast cancer The aim of the study, sponsored by the French society of lymphology, was to construct a specific quality of life self-completed questionnaire. The questionnaire was developed in three stages: a qualitative survey was conducted to identify patients'complaints. This questionnaire was administered to 154 patients. Principal component analysis was used to identify the dimensions of impairment. A validation study was carried in 304 patients. Six instruments have been used in the case report form on D0 and D28: the ULL-27 and SF-36 scales have been completed by patients. A composite symptom scale was completed by clinicians from patient interviews. Finally, doctors and patients gave their overall opinion. Differences in volume between the healthy and the affected arms were calculated. 304 patients were included in the study. Factorial analysis isolated 3 dimensions: The Spearman correlation between individual item score and the subscale score were greater than 0.40 . The Cronbach coefficients were greater than 0,80 . In stable patients, correlation coefficients between D0 and D28, were all greater than 0.84 and statistically significant. Correlations were significantly positive between the ULL-27 sub-scales and the SF-36 homologous dimensions. The population was stratified according to the volume of œdema, physical and social dimensions of ULL-27 scale were significantly correlated with the severity of illness. It was not the case for the psychological dimension. The sensitivity analysis between D0 and D28 in patients with active disease, demonstrated significant differences between mean scores for all ULL-27 dimensions. Volume of œdema poorly reflects the impact of the illness upon the patient. The ULL- 27 scale seems to be a consistent instrument to measure outcomes lymphœdema.

273/ PRELIMINARY FINDINGS IN THE DEVELOPMENT OF THE SPIRITUALITY MODULE FOR THE WHOQOL IN URUGUAY Betina Caporale, Adriana Bagnulo, Medical Psychology, Medical College, State University, Montevideo, Montevideo, Uruguay

The importance of spiritual aspects in people's health is being studied more deeply nowadays. This study is framed in the World Health Organization (WHO) Project which proposes incorporation of a Spiritual, Religiousness and Personal Beliefs (SRPB) module to the existing WHOQOL instrument. Uruguay is one of the 13 centers included in this project. Ten focus groups, with an average of 80 participants were run to discuss the questions and facets included in the original questionnaire proposed by WHO. After integrating the modifications suggested by the different centers, a new questionnaire was created to carry out the pilot study in these countries. The study comprised the administration of the SPRB module which has (105 items, comprising 15 facets, each one including 7 items; 21 importance questions and the WHOQOL-100.In our country, the questionnaires, previously translated to our language according to the WHO methodology, were administered at a General Hospital and to general population. Trained interviewers administered the questionnaires in those cases when the respondents showed any difficulties in answering. The sample was composed by 217 individuals, with a mean age of 44,18 (range $18-84$ ); $57,6 \%$ women, $42,4 \%$ men, $44,2 \%$ healthy and $55,8 \%$ ill. The purpose of the study was to describe the distribution of the answers to the facets of the SRPB module in our sample, comparing the different groups by religion, health status and educational level. The frequency of the answers were analyzed calculating and comparing means of different groups through a Student-t. Significant differences between groups were found in 36 of the 105 questions Two of the 15 facets(Conectedness to a Superior Force and Faith) showed the highest discriminatory power. By opposition, Death and Spiritual Strength were the only facets where no significant differences between groups could be detected. As this last finding is not concordant with the previous hypothesis that these facets would allow to detect differences between ill and healthy people, the reformulation of these questions will be reviewed as a first step. Further analysis on the complexities of developing questionnaires on such mobilizing topics should follow.
274/ VALIDATION AND RESCORING OF THE MINNESOTA LIVING WITH HEART FAILURE QUESTIONNAIRE

Stacie A. Hudgens, Elizabeth A. Hahn, Center on Outcomes, Research and Education (CORE), Randall E. Williams, Karen Spronk, Michael Memmini, Cardiology, Evanston Northwestern Healthcare (ENH), Evanston, IL; Thomas S. Rector, Medicine/Cardiovascular Division, University of Minnesota, Minneapolis, MN

The Minnesota Living with Heart Failure questionnaire (LHFQ) is a 21 -item scale that measures general effects of congestive heart failure (CHF) on patients' lives including physical (PC) and emotional (EC) components. In an ongoing randomized trial of $\mathrm{CHF}$ telemanagment, a baseline LHFQ was completed by interviewing 157 $\mathrm{CHF}$ patients during initial hospitalization. We used these data to evaluate the measurement properties of the LHFQ. An extension of the one-parameter Rasch measurement model for rating scale data was used to determine whether the LHFQ defines a unidimensional measure of impairments due to CHF. Raw scores and Rasch person measures were compared across severity groups, as defined by physician based New York Heart Association (NYHA) classifications (Class $\mid=11 \%$, Class $\mid I=57 \%$, Class $|I|=28 \%$, and Class IV $=5 \%$ ). Mean age of the respondents was 74 (range 40-98). Evaluation of the 6 point scale $(0=$ no, $1=$ very little, $2-4=$ undefined, $5=$ very much) suggested that the middle categories were less orderly and had less distinct step calibrations. A collapsed 3-point scale (0 vs. 1-3 vs. 4-5) resulted in more orderly steps and balanced distribution of the response categories. The weighted mean-square fit statistics were acceptable for most items (range 0.67 to 1.62 ) indicating that items worked well together to define the construct. One item ("working to earn a living") had a higher proportion of misfit, likely due to lower relevance in an elderly sample. The Rasch model reliability using the 3 -point scale was 0.89 versus 0.86 for the 6 -point scale. Overall, both raw scores and Rasch person measures on the total scale, PC and EC were significantly different $(p<0.05)$ across NYHA classes. These results suggest that the LHFQ is a reliable measure of CHF-related impairments and discriminates differences in disease severity.

275/ THE RELIABILITY AND VALIDITY OF A NEW GENERIC MEASURE OF HEALTH-RELATED QUALITY OF LIFE FOR USE AMONGST THE KAMBA OF KENYA

Annabel Bowden, Julia A. Fox-Rushby, Public Health and Policy, London School of Hygiene and Tropical Medicine, London, UK; Lilian Nyandieka, John W. Kangeri, Centre for Public Health Research, Kenya Medical Research Institute, Nairobi, Kenya

Following a lengthy period of participative qualitative research accessing local notions of 'health, a new measure of health-related quality of life (HRQL) has been developed for use amongst Kamba people aged over 15 in Makueni district, Kenya. It is designed to capture the public face of personal perceptions of HRQL in terms of 'contentment', 'corporeal capability', 'cleanliness', 'co-operation' and 'completeness'. This paper assesses the construct validity and test/re-test reliability of the KENQOL survey questionnaire. A two-stage randomised survey $(n=550$ individuals) was conducted in Makueni district during 1999. All HRQL questions were answerable as yes, no, or don't know (and note was taken of any refusals to answer). In addition to the data on $\mathrm{HRQL}$, a range of information was also asked, and observed, about socio-economic and demographic status. 23.6\% were re-interviewed within a two-week period. Descriptive analyses and hypotheses testing is currently being undertaken using chisquared tests and Mann-Whitney $U$ tests for independent samples. The test/re-test analysis uses the Wilcoxon matched-pairs signed rank test. The overall response rate was $81.3 \%$ for the first questionnaire and $68.5 \%$ for the retest questionnaire. The mean item response rate was $98.1 \%(\mathrm{SD}=3.6)$. Results will examine the relationship between responses to the KENQOL questionnaire with socio-economic (e.g. acres of land owned, education, occupation) and demographic (e.g. number children alive/dead, age, gender) characteristics. Discussion will focus on how sensitive the instrument is likely to be, and in relation to which types of interventions. 
276/ TESTING THE PSYCHOMETRIC PROPERTIES OF THE PSORIQOL: A PSORIASIS-SPECIFIC QUALITY OF LIFE INSTRUMENT

Stephen P. McKenna, Sharon A. Cook, Diane Whalley, Lynda C. Doward, Galen Research, Manchester, UK; Daniel van Assche, Novartis Pharma AG, Basel, Switzerland

Psoriasis is a common chronic disfiguring skin disorder that has a major impact on quality of life (QoL). A 56 item version of the PSORIQoL was derived from content analysis of qualitative interviews with 67 psoriatics. This paper describes studies undertaken to identify the final version of the UK questionnaire and to assess its reliability and face, content and construct validity. Field-test interviews were conducted with 21 patients to assess acceptability and comprehension and the questionnaire was then administered in a test-retest postal survey, with two weeks between administrations. Interviewees found the content of the PSORIQoL relevant and comprehensive and that the measure was easy to understand and complete. Test-retest questionnaires were completed by 78 patients ( $44 \%$ male; mean (SD) age $=46$ (15) years). As a result of the postal survey, a version of the measure was identified that formed an unidimensional scale with adequate item fit. Internal consistency was 0.93 and test-retest reliability 0.94 . Scores on the PSORIQoL were unrelated to age, sex or marital status but were associated with perceived severity and presence of lesions on the hands $(p<0.001)$. The PSORIQoL is well-accepted by respondents who consider its content comprehensive and highly relevant. It also has excellent psychometric properties suggesting that it will be a valuable tool for clinical trials. It remains necessary to establish the instrument's validity further and its responsiveness to change in disease severity.

\section{7/ DEVELOPMENT AND VALIDATION OF DEEP VEIN THROM-} BOSIS QOL SCALE

Stacie A. Hudgens, Center on Outcomes, Research and Education (CORE), Joseph A. Caprini, Surgery, Carol A. Caprini, David Cella, CORE, Lakshman R. Sehgal, Surgery, Evanston Northwestern Healthcare, Evanston, IL

Deep Vein Thrombosis (DVT) is a serious problem that annually affects 900,000 people. Symptoms of venous insufficiency are not always associated with objective signs such as veriscosities, dilated veins, ulcers or other lesions. Most cases of DVT following surgery are asymptomatic and thought not to be clinically significant. Untreated asymptomatic clots may later leg symptoms and decrease their QOL. The objective of this project was to document symptom severity and validate a questionnaire to measure those symptoms in patients with DVT during the first year following an acute episode. Eighty-eight patients hospitalized with an acute DVT and treated with a standard anticoagulation pathway protocol were interviewed by telephone at 3-7 days, 30-40 days, and 12-months following discharge. Using a 14-item questionnaire, patients were queried regarding leg pain, swelling, skin discoloration, cosmetic appearance, activity tolerance, emotional distress, and leg-related sleep problems. These seven areas were rated for each leg on a 5 -point Likert scale $(0=$ 'no problem at all',5='severe problem'). Internal consistency reliability at 3-7 days was high for the 7-item left leg rating (LLR) scale (alpha=0.87), 7item right leg rating $(R L R)$ scale $(a l p h a=0.82)$, and the 14-item total (alpha=0.81). Coefficients were lower at 30-40 day follow up $(L L R=0.73, R L R=0.73$, total=0.72), but remained acceptable for group comparisons. At 12-month follow up, coefficients were high for each scale $(L L R=0.82, R L R=0.87$, total $=0.86$ ). Patients were able to reliably diffentiate and localize symptom reports based on leg involvement. For patients affected with a right DVT, RLR scores were significantly higher $(p<0.05)$ than LRL scores at baseline and 30-40 days. After removing two patients with a history of DVT/PE and diabetes, 12-month differences were also detected. For patients affected with a left leg DVT, LLR scores were significantly higher $(p<0.05)$ than RLR scores at all three time points. These findings suggest that this symptom scale can be used to evaluate the natural history of legassociated symptoms following an acute episode of DVT. This tool is highly reliable and could be utilized to investigate DVT-related leg symptoms-QOL.
278/ MEASURING SOCIAL PARTICIPATION: DEVELOPING A SHORT FORM OF THE GLASGOW HEALTH STATUS INVENTORY: THE PARTICIPATION SCALE

Graeme Hawthorne, Centre for Health Program Evaluation, The University of Melbourne, West Heidelberg, Victoria, Australia; Anthony Hogan, Simpson Centre for Health Innovation, University of New South Wales Clinical School, Liverpool Health Service, Sydney, NSW, Australia

As health care resources are limited, evidence of program effectiveness is necessary. To demonstrate this, measurement should be conducted at the specific illness level, the generic health status level and the utility level. To avoid cognitive overload, response burden or refusal to participate instruments need to be parsimonious. Critical analysis of the Glasgow Hearing Status Inventory (GHSI) suggested that several items were redundant and the instrument could be shortened. We administered the GHSI and Assessment of Quality of Life (AQoL) instruments to 148 deafened adults with cochlear implants $(\mathrm{Cl})$ and to 54 without Cls, as part of a cross-sectional study. We used standard psychometric procedures to examine the GHSI's structure, resulting in the removal of half the items. The short version of the GHSI we labeled the Participation Scale (PS) to avoid confusion with the GHSI. The PS is an 11-item instrument measuring self-esteem, social handicap and hearing handicap. Factor analysis suggested that each sub-scale was unidimensional. All items loaded on the principal component. Correlation with the GHSI was 0.95 , suggesting the two instruments could be used interchangeably. Both the PS and GHSI provided evidence of monotonicity when used to predict AQoL scores. They were equally sensitive at differentiating between implantees and non-implantees. Although these findings need to be confirmed, the PS is ready to be used in studies of interventions for deafness. At a time when evaluators are being asked for evidence of program effect, the parsimonious PS achieves similar results to but uses half the items in the GHSI.

279/ THE RELATIVE RESPONSIVENESS OF GENERIC AND DISEASE-SPECIFIC MEASURES OF HRQOL IN PATIENTS UNDERGOING CORONARY REVASCULARISATION

Sara Schroter, Donna L. Lamping, Health Services Research Unit, London School of Hygiene \& Tropical Medicine, London, UK

The Coronary Revascularisation Outcomes Questionnaire (CROQ; Schroter \& Lamping, 2000) is a reliable, valid and responsive patientbased measure of outcome for patients undergoing coronary artery bypass graft surgery (CABG) and percutaneous transluminal coronary angioplasty (PTCA). We compared the relative responsiveness of the CROQ with the SF-36 and the Seattle Angina Questionnaire (SAQ) at 3 months after revascularisation. A total of 199 patients $(127$ CABG, 72 PTCA) completed the CROQ; $72 / 55$ CABG and $38 / 34$ PTCA patients also completed the SF-36 or SAQ, respectively. Compared with SF-36 PCS/MCS scores, effect sizes for CROQ Physical/ Psychosocial Functioning scores were larger in both CABG (1.19/ 1.52 CROQ vs. 0.85/0.67 SF-36) and PTCA (0.73/0.64 CROQ vs. 0.56/0.07 SF-36) samples. Compared with SAQ Exertional Capacity/ Anginal Frequency scores, effect sizes for CROQ Physical Functioning/Symptoms scores were similar in both CABG (1.74/2.74 CROQ vs. $1.56 / 2.59 \mathrm{SAQ})$ and PTCA (0.67/1.09 CROQ vs. $0.84 / 0.80 \mathrm{SAQ})$ samples. In both CABG and PTCA patients, the disease-specific CROQ was more responsive than the generic SF-36 and similar in responsiveness to the disease-specific $S A Q$. One advantage of the CROQ over the SAQ is that it is more conceptually appropriate for measuring outcomes after coronary revascularisation as it includes items to evaluate cognitive functioning, complications and satisfaction with outcome. 
280/ FINAL PSYCHOMETRIC EVALUATION OF THE CORONARY REVASCULARISATION OUTCOME QUESTIONNAIRE (CROQCABG/PTCA)

Sara Schroter, Donna L. Lamping, Health Services Research Unit, London School of Hygiene \& Tropical Medicine, London, UK

Our preliminary psychometric evaluation of the Coronary Revascularisation Outcome Questionnaire (CROQ-CABG/PTCA; Schroter \& Lamping, 2000) in 569 (289 CABG, 280 PTCA) patients produced an item-reduced version and provided preliminary evidence of its reliability, validity and responsiveness as a measure of symptoms, physical, psychosocial and cognitive functioning, complications and satisfaction with treatment in coronary revascularisation. The itemreduced version of the $C R O Q$ produced in the preliminary field test then underwent final field testing by postal survey at 3/9 months after revascularisation in independent samples of 415/100 CABG and 345/ 80 PTCA patients, respectively. Results confirmed that the final (itemreduced) version of the $C R O Q$ was highly acceptable to patients (low missing data, good response rates), satisfied scaling assumptions (good item convergent and discriminant validity), and showed good internal consistency (Cronbach's alphas $>0.80$ ) and reproducibility (test-retest reliability $>0.80$ ). Construct validity was demonstrated by: high correlations between subscales and total scores; moderate correlations between subscales; convergent and discriminant correlations with the SF-36 and three other cardiac-specific measures; support for hypothesised group differences in CROQ scores between patients with different levels of clinically-defined (CCS, NYHA) angina and dyspnoea severity and of global improvement. Responsiveness was demonstrated by the ability to detect significant improvements 3 and 9 months after revascularisation. The CROQ is a psychometrically robust, patient-based measure that is also the most conceptually appropriate instrument for evaluating outcomes after CABG and PTCA.

\section{1/ DEVELOPMENT AND VALIDATION OF DISEASE-SPECIFIC MEASURES FOR CHOROIDAL MELANOMA - A CANCER OF THE EYE}

Michele Melia, Department of Ophthalmology, Johns Hopkins University, Baltimore, MD; Claudia S. Moy, Neuroscience, NINDS, Bethesda, MD; Sandra M. Reynolds, Department of Ophthalmology, Johns Hopkins University, Baltimore, MD; Carol M. Mangione, Division of General Internal Medicine, UCLA School of Medicine, Los Angeles, CA; David Cella, Center on Outcomes Research and Education, Evanston Northwestern Healthcare, Evanston, IL

To develop and validate scales measuring satisfaction with appearance, fear of cancer recurrence, and difficulty with vision-dependent activities requiring stereopsis/binocularity. 842 patients with choroidal melanoma (CM) who were enrolled in the Collaborative Ocular Melanoma Study (COMS) randomized trial for medium-sized tumors. Patients were randomized to radiation therapy (eye retained) or enucleation (eye removed). Patients are interviewed annually using standard HRQOL instruments (SF-36, NEI-VFQ 25, HADS). However, these instruments did not address 2 additional major concerns of CM patients, namely effect of treatment on appearance, and fear of cancer recurrence. Also, although the NEI-VFQ measures difficulty with a wide range of vision-dependent activities, the feasibility of using these items for constructing a stereopsis/binocularity scale had not been investigated. All 3 of these measures are hypothesized to differ by treatment. Items addressing these concerns were developed and added to the end of the COMS HRQOL interview. Item analysis, multitrait scaling analysis, and principal components analysis were performed to investigate the newly-compiled scales. On a scale from 0 to 100 , mean recurrence score was $74.8+/-19.0$, with range $0-100,17 \%$ of patients at the ceiling and $1 \%$ at the floor. Mean appearance score was $71.0+/-15.4$ with range $10-100,2 \%$ at ceiling, and $0 \%$ at floor. Mean stereopsis score was $81.2+/-15.9$ with range $19-100,11 \%$ at ceiling, and $0 \%$ at floor. All items correlated well with their hypothesized scales $(r=0.67-0.85)$ and less well with other scales. Cronbach's alpha for a 3 -item recurrence scale was 0.78 , a 5 item appearance scale was 0.82 , and an 8 -item stereopsis scale was 0.88 . Principal components analysis supports that appearance and recurrence items form 2 distinct scales, while the stereopsis scale is not independent of other visual-functioning scales.
282/ EXPLORATORY FACTOR ANALYSIS (EFA) OF THE QUALITY OF LIFE RADIATION THERAPY INSTRUMENT \& HEAD AND NECK MODULE (QOL-RTI/HN) USING MULTI-NATIONAL SAMPLES

Clement K. Gwede, Darlene Johnson, Bonnie Sauder, Andy Trotti, Radiation Oncology, Moffitt Cancer Center, Tampa, FL

The QOL-RTI/HN, developed in English for use in patients undergoing radiation therapy, has been translated and validated in German and Japanese and is being validated in Chinese, Italian, Spanish, and Greek in different countries. We report the multidimensionality of the general tool (QOL-RTI) and Head \& Neck (HN) module to assess construct validity. Pretreatment data from 3 countries (United States $\mathrm{N}=48$, Austria $\mathrm{N}=92$, and Japan $\mathrm{N}=51$ ) were pooled together for analysis. The patients were undergoing radiation therapy for advanced stage head and neck cancer of the oral cavity, pharynx and larynx. The two instruments were assessed independently using EFA and correlation of the resulting subscales was evaluated. Four meaningful factors were identified for the 24-item QOL-RTI with Chronbach's alphas ranging from .57 to .70 . For the HN module two subscales emerged: a functional scale associated with patient's perceived level of functional impairment and a discomfort index related to perceived level of discomfort or bothersomeness of symptoms. These findings suggest that head and neck specific concerns represent two important concepts that make up a common construct. Coefficient alphas were 69 for the functional subscale, .79 for the discomfort subscale and .81 for the entire 14-item HN module. The two subscales were strongly but divergently correlated with the total $\mathrm{HN}$ score (functional, $r=.84$, discomfort, $r=-.70$ ) as expected and moderately correlated with each other $(r=-.40), p=<.0001$. Mean scores for the QOL-RTI domains were poorly to moderately correlated with each other, and with the QOL-RTI total and the two HN subscales. The results support the multidimensionality of both the QOL-RTI and HN module. The data for the HN module provide a practical and clinically meaningful perspective for understanding head and neck-specific QOL and function.

283/ INTERPRETING THE MOS SF-36: HOW MANY SUM SCORES ARE APPROPRIATE?

Corina Guethlin, Harald Walach, Institute of Environmental Medicine \& Hospital Epidemiology, University Hospital, Freiburg, Germany

The MOS SF-36 is a widely used instrument to assess generic health status or illness related quality of life. It is translated in several languages and well validated in German as well as in English. Nevertheless the discussion about the factor structure (1 mental and 1 physical factor or one general factor) is still ongoing. We report psychometric analyses of the German version of the SF-36 health survey. Our analysis is based on questionnaires of patients suffering from a variety of diseases, mainly pain syndromes, who sought acupuncture treatment $(n=2000)$. The questionnaires are given before and after treatment and once every year for 4 more years. The purported factorial structure of the questionnaire can be approximately reproduced across items and scales. A confirmatory factor analysis confirms either two intercorrelated factors "mental health" and "physical health" or one general factor. The two dimensions are correlated (phi.51), the residuals are high and intercorrelated. We discuss advantages and disadvantages concerning the interpretation of the results and the properties of the questionnaire as one factor or as two factors, and we report scaling algorithms for one general factor. In our opinion it is more appropriate and closer to the data to use one single factor which is easier to analyse and interpret. 
284/ METHODOLOGY FOR CHOICE OF ITEMS : DESIGNING A QUALITY OF LIFE INSTRUMENTS FOR HIP AND KNEE OSTEOARTHRITIS

Francis Guillemin, Epidemiologie et Evaluation Cliniques, Hopital Marin, Nancy, France; Joel Coste, Departement Biostatistique et Informatique Medicale, Hopital Cochin, Paris, France; Jacques Pouchot, Service de Médecine Interne, Hopital Louis Mourier, Paris, France; Nathalie Retel-Rude, Laboratoire de Biostatistique, Faculté de Médecine, Besançon, France; Elisabeth Spitz, Departement de Psychologie, Université de Metz, Metz, France; Michèle Baumann, Ecole de Sante Publique Upres, Faculte de Medecine, Vandoeuvre les Nancy, France; Cyrille Tarquinio, Departement de Psychologie, Université de Metz, Metz, France

In the construction of composite health status measurement scales, item generation targets the content of the scale. It proceeds usually in three stages : item elicitation to obtain a coverage of content in all potential affected areas, testing instrument scaling properties, and item selection to ensure feasibility and acceptability of the scale. The objective of this study was to investigate the relative contribution of several methods of item development and choice, with an application to designing a lower limbs osteoarthritis (OA) QoL scale. This study was conducted with the working hypothesis that various item generation methods may each contribute the addition of relevant statements and words. The methods tested included patients cognitive interview, patient individual or focus group interview, health professional individual or focus group interview aimed to generate wordings, speech and verbatim, followed by various methods of content analysis. A total of 128 OA patients and 32 health professionals was interviewed A frame for content analysis was developed by a sociologist team. Two other sociologist teams operated independently a semantic theme content analysis of tape recorded and transcribed interviews, generated a list of words, speeches and verbatim, completed by hierarchical cluster analysis. This allowed to identify 140 items pertaining to QoL dimensions. The separate contribution of each generation method confirmed the working hypothesis. The primary stages of scale construction include concepts specification and content descriptions. The comparison of methods helps contribute to future methodological guidelines to generate quality of life questionnaires.

\section{5/ HOW MUCH IS "VERY MUCH"? DEVELOPING A RATING SCALE FOR PORTUGUESE SPEAKING COUNTRIES}

Benjamin J. Arnold, S. Eremenco, C. H. Chang, D. Cella, CORE, ENH, Evanston, IL; J. L. P. Ribeiro, Faculdade de Psicologia, Universidade do Porto, Porto, Portugal; M. P. Doro, Bone Marrow Transplant Unit, University Hospital, Curitiba, Parana, Brazil; R. Harris, CORE, ENH, Evanston, IL

Developing linguistically appropriate and psychometrically sound response scales for use in questionnaires is critical to ensure scores obtained from the summation of responses are meaningful. Translating these answer categories while retaining their linguistic and measurement equivalence poses great challenges. In this study, we examined the translated 5-point Likert-type rating scale used in the Portuguese Functional Assessment of Cancer Therapy-General(FACTG)27-item questionnaire. We applied Andrich's rating scale model(RSM)to explore the answer category structure, identify possible problematic answer categories, investigate alternatives, and develop one set of equivalent translations to be used in Brazil(BR)and Portugal(POR). A Portuguese version was translated and tested in both $\operatorname{BR}(n=81)$ and $\operatorname{POR}(n=81)$. Through $R S M$ analyses, we found the translated answer categories(nem um pouco, um pouco, mais ou menos, bastante, muito) did not represent equal intervals on the two highest categories. We then carried out cognitive debriefing to pinpoint the problem and find better answer categories. A survey with 15 possible alternatives to cover the answer category continuum was compiled. Using a Q-sort method, 62 subjects (32 BR; 30 POR) arranged the alternatives in order of increasing intensity and were encouraged to comment. Data were tabulated using median ranking for each answer category. Results showed that high-end (bastante and muito) answer categories were perceived as having the same intensity for the subjects as a whole independent of literacy, health status, and country. The answer category set(nem um pouco, um pouco, mais ou menos, muito, muitíssimo)was then finalized based on the median ratings and comments of patients. This method produced a Portuguese language rating scale with categories judged to be of equal interval across both countries.
286/ SHOULD SYMPTOMS BE SCALED FOR INTENSITY, FREQUENCY, OR BOTH?

Chih-Hung Chang, Center on Outcomes, Research and Education, David Cella, Susan Clarke, Evanston Northwestern Healthcare, Evanston, IL; Jamie H. von Roenn, Northwestern University Medical School, Chicago, IL; Allen W. Heinemann, Rehabilitation Institute of Chicago, Chicago, IL

The purpose of the study was to evaluate the comparability between two 5-point Likert-type rating scales commonly used in symptom measurement. One measured intensity (category ranging from "not at all" to "very much") and one measured frequency ("none of the time" to "all of the time"). Data were collected as part of a larger study to develop a fatigue computerized adaptive testing platform. Thirteen questions comprising the Functional Assessment of Chronic Illness Therapy (FACIT)- Fatigue were asked using both response formats and separated by "interference" questions. Patients $(N=161)$ were from three disease groups: Cancer $(n=60)$, stroke $(n=51)$ and HIV $(n=50)$. These two rating scales were calibrated separately using Andrich's rating scale model to distance and compare the order of steps from one response category to its higher (adjacent) category. Each item was evaluated for its position on the fatigue measurement continuum relative to the other items. The comparability of measured fatigue from each rating scale was also evaluated. The order of the two sets of step measures was in the expected direction and was comparable across both rating scales. There was a high correlation $(r=.86, p<$ .001) between two sets of person fatigue measures measured by intensity and frequency, also supporting their measure equivalence regardless of rating scale being used. There was not a major distinction between frequency versus intensity measures in assessing fatigue in chronically ill patients, suggesting results from one or the other approach are roughly comparable.

287/ TEST-RETEST RELIABILITY AND INTERPRETATION OF UTILITY SCORES: EVIDENCE FROM ELECTIVE TOTAL HIP ARTHROPLASTY

David H. Feeny, Chris Blanchard, Institute of Health Economics, Edmonton, AB, Canada; Jeffrey Mahon, Department of Epidemiology and Biostatistics, Robert Bourne, Cecil Rorabeck, Department of Surgery, Larry Stitt, Susan Webster-Bogaert, Department of Epidemiology and Biostatistics, University of Western Ontario, London, ON, Canada

The first purpose is to assess test-retest reliability of standard gamble(SG)utility scores for repeated assessments in a prospective study. The second purpose is to provide evidence on the markerstate approach in interpreting utility scores. SG scores for three hypothetical marker states (mild, moderate, and severe osteoarthritis(OA))and the patient's current state were obtained at multiple points in time in a longitudinal study of elective total hip arthroplasty (THA). Patients were assessed repeatedly throughout the process of waiting to see a surgeon, waiting for surgery, and recovery after THA. Test-retest reliability was assessed using the intraclass correlation coefficient (ICC). Analysis of covariance (ANCOVA) was used to assess the effects of time on SG scores. The mean (SD) time from first assessment (A1) to A2 was 5.2 months (2.0); the mean from $A 2$ to $A 3$ was 5.5 (1.8); from $A 3$ to $A 45.0$ (2.3). ICCs varied from 0.65 to 0.83 . Time was insignificant $(p<0.05)$ in 8 of 9 ANCOVAs. Mean (SD) baseline SG scores for mild, moderate, and severe marker states were $0.71(0.29), 0.61(0.32)$, and $0.39(0.33)$. Mean SG scores for current health improved from $0.62(0.31)$ at baseline to $0.76(0.23)$ at follow-up after THA. Test-retest reliability of SG scores over an extended period of time is acceptable at the group level. The marker states assist in interpreting results. On average, THA converted moderate OA to better than mild. 
288/ UTILITY ASSESSMENT AMONG PATIENTS WITH DRY EYE DISEASE

John G. Walt, Global Pharmaoceconomic Strategy \& Research; Rhett Schiffman, Ophthalmology Clinical Research, Allergan, Irvine, CA; Stacy Evans, Gary Lebovics, Research, Analytica, New York, NY

To determine utilities for mild, moderate and severe forms of dry eye disease. Patients previously diagnosed with dry eye were invited to undergo utility assessment by three standard methods of health utility assessment, including Standard Gamble (SG), Time-Trade-Off (TTO), and Rating Scale (RS), using a computerized utility assessment tool called U-titer. These utilities were computed with and without adjustment for medical comorbidities. After completing the utility assessment, patients underwent a clinical examination to assess the severity of their dry eye disease. Fifty-six patients completed the utility assessment with internally consistent results. Reported unadjusted utilities by SG ranged from 0.97 for mild disease to 0.80 for the most severe forms of dry eye requiring tarsorrhaphy for management. For TTO, utilities from 0.90 to 0.65 and by RS, utilities ranged from 0.90 to 0.57 . Utilities for the more severe forms of dry eye are in the range of other conditions more recognized for lowering health utilities such as home dialysis $(0.64)$ and moderate angina $(0.70)$. These results underscore how patients value dry eye compared with other conditions, and these utilities can now be used for cost-utility analyses.

289/ HOW TO COMBINE PREFERENCE-BASED QOL SCORES OF PATIENTS COLLECTED IN LONGITUDINAL CLINICAL TRIALS WITH CLINICAL DATA TO ARRIVE AT ONE OVERALL OUTCOME MEASURE?

Paul Krabbe, Margriet Hartman, Medical Technology Assessment, Roland Laan, Rheumatology, University Medical Centre Nijmegen, Nijmegen, The Netherlands

One way of examining trade-offs between quantity and quality of life (QOL) is to combine them into a single measure as quality-adjusted life year (QALY). For the computation of QALYs individual preference scores to express QOL are used, preferably repeatedly assessed during follow-up. Life years are simply determined as the study period or extrapolated by imputing the life expectancy of patients. An alternative approach is the Q-TWIST method. Here, a limited number of distinct clinical health states are distinguished and each of them is assigned with a preference score, whereas the estimation of the proportion of patients in each health state at a specific time period is based on survival curves. We investigated an extension of these two approaches to arrive at a method that is appropriate to deal with longitudinal multi-state QOL-preference data collected in clinical studies. To demonstrate the features of our methodological approach we used data from a clinical study focussing on the effect of methotrexate (MTX) in patients with rheumatoid arthritis. The basic assumption was that toxicities due to methotrexate can be reduced with co-administration of folic and folinic acid, without loss of anti-inflammatory efficacy. The study was a double blind, placebo controlled trial with three parallel groups: MTX + placebo, MTX + folic acid, MTX + folinic acid. Patients (145 in each group) were followed for 48 weeks. Success of the therapy was determined at intervals of 3 weeks. QOLpreferences were individually assessed 5 times at intervals of 12 weeks by applying the EQ-5D. Our results show that the approach allows handling of several characteristics inherent to clinical trials: 1) repeated (unscheduled) assessments, 2) dropout during follow-up, 3) confounding factors. Moreover, various graphical representations support the expression of differences between treatment groups by integrating QOL and clinical data.
290/ MODELING PREFERENCES FOR COMPLEX HEALTH STATES

Jacek A. Kopec, Health Care and Epidemiology, University of British Columbia, Vancouver, BC, Canada; Rollin Brant, Community Health Sciences, University of Calgary, Calgary, AB, Canada

The objective of this study was to derive and compare alternative statistical models for estimating rating scale preferences for complex (multi-deficit) health states. The health state descriptions were based on the Health Utilities Index (HUI3), but only 6 of the 8 health attributes were included (vision, ambulation, dexterity, emotion, cognition and pain). Of the 32,400 possible states, we selected 227 states to be rated. Preferences were measured with a feeling thermometer in a self-administered format. Seven versions of the questionnaire were developed, with 32 version-specific states and 3 standard states common to all versions. Each version was mailed to a stratified random sample of 300 households selected from a database of telephone numbers in Ontario, Canada. Of those, 601 persons returned usable questionnaires. Mean preference values for the health states ranged from 0.11 to 0.91 . Our analysis began by modeling health preference scores following a mixed effects linear regression approach incorporating random intercepts and attribute-specific gradients for each subject. Overall, the coefficients associated with levels within the individual attributes followed the anticipated decreasing pattern. The steepest gradients were associated with emotion and cognition, with the weakest being dexterity. There was evidence of super-additivity of effects for the higher levels of health and sub-additivity for the lower levels. We also modeled a derived utility score, following a multiplicative multiattribute utility theory model, incorporating random effects as above. Findings were similar with regard to the contributions of individual attributes. Again, there were indications of departures from multiplicity over the spectrum of health. We believe this is the first study to demonstrate significant departures from both the additive and multiplicative preference models in a large sample of complex health states.

\section{1/ COST-UTILITY ANALYSIS OF UNIVERSAL AND INDICATED PREVENTION PROGRAMS}

E. Michael Foster Ph.D., Eunjeong Kang MPH, Health Policy and Administration, The Pennsylvania State University, State College, PA

This paper accomplishes three aims. First, it examines the cost-utility of indicated vs universal interventions targeted to young children with behavior problems. This analysis addresses an important policy question-should resources be focused on children showing severe problems early in life or spread less intensively across more children? Second, the paper examines the validity of the Health Utilities Index Mark II for use in prevention research. Of particular interest is the degree to which the measure's emotion attribute correlates with standard assessments of a child's emotional health. Third, we consider the validity of parental reports of quality of life by considering whether those reports correlate with parental characteristics such as marital stress and caregiver depression. The data are obtained from a multicomponent intervention targeted to young children with emergent behavior problems. The intervention comprises three components. All children in study classrooms receive teacher-implemented comprehensive children's social skills program; teachers in those classrooms receive a second component, training in classroom behavior management. To parents of children showing early behavior problems, a third component is added-a 12-week parenting program is provided. The evaluation is being conducted in 54 first-grade classrooms in Oregon. The study is tracking children receiving the indicated $(n=162)$ and universal components $(n=648)$ of the study over time. Children are randomly assigned within participating schools to treatment or control classrooms. Parents of participating children complete a wide range of survey instrument including the Child and Adolescent Disruptive Behavior Inventory, a well validated measure of children's mental health. The instrument also includes the HUI Mark II which is the basis for our cost-utility analysis. Information on the child's behavior is also provided by his or her teacher. Finally, the evaluation also includes a comprehensive assessment of the program's costs. 
292/ CAN WE AND HOW TO WEIGHT THE DIMENSIONS OF QUALITY OF LIFE MEASURES

Kaiping G. Yao, Chui De Chiu, Psychology, National Taiwan University, Taipei, Taiwan, R.O.C.

In the past, quality of life $\left.{ }_{j}\right] \mathrm{QOL}_{i}{ }^{\wedge}$ researchers usually sum the scores from several dimensions/items with equal weights to obtain individual's overall QOL score. However, this approach has been inquired. One of the arguments is that QOL dimensions/items may have different meanings to individuals in terms of importance. Equal weighting approach may underestimate the QOL dimensions/items with more importance and overestimate the QOL dimensions/items with less importance to individuals. As a result, individual's true QOL level cannot be estimated appropriately. To examine this issue in a more clear way, several questions should be raised. One is "Can we sum scores from different dimensions/items?" The answer may be yes and may be not because this depends on how people define the "overall QOL score". Under certain conditions, we may sum scores from different dimensions/items to form an overall QOL score. Consequently, we would further ask "How to sum the scores?" The purpose of this exploratory study is to find the appropriate ways to weigh the dimensions of QOL measures so that the assessment of QOL can describe subject' true QOL level better. As a result, the overall QOL score is much more meaningful. Two main questions will be discussed in this study. First, WHO should give the weights? Should it be the health service users, the health service providers, the health policy makers, or the public? Second, HOW to weight the dimensions? Should we use regression approach, factor analysis approach, multi-attribute utility approach, or direct rating? Is there any optimal method? In this study, we will explore these questions. Three types of subjects participate in this study. They are patients, health-care providers, and college students. Besides answering the WHOQOL questionnaire, they give both importance ranking and rating scores on the $6 \mathrm{QOL}$ domains (physical, psychological, social, independence, environment, and spirituality/personal belief/religion) and $41 \mathrm{QOL}$ facets originally designed from the WHOQOL research project. Several weighted and non-weighted indices are considered for comparisons. The quality of these weights are examined according to their applications to the QOL measures in terms of reliability and validity.

293/ A FRAMEWORK FOR CALCULATING CANADIAN DISABILITY WEIGHTS

Karla M. Nobrega, Julie Bernier, Jean-Marie Berthelot, Health Analysis and Modeling Group, Statistics Canada, Ottawa, ON, Canada; Robert A. Spasoff, Epidemiology, University of Ottawa, Ottawa, ON, Canada

In this presentation we will provide a detailed overview of the disability weights framework for the Canadian Burden of Disease, Injury and Risk Factor study. Disability weights quantify the consequences of a disease by attaching a value from zero to one to a particular health state. These weights will be calculated using a modification of the person-trade off method used by the World Health Organization (WHO) in their Global Burden of Disease study. Since weighting preferences can change based on who elicits them, the approach will involve lay people, health care professionals and health care policy makers in a delphi group setting. In addition to the modified WHO time-trade-off approach, we will perform a population-level standard gamble to assess the differences between aggregate individual utilities and population utilities. The disability weights obtained for Canada will be compared to the weights derived by the WHO and the Netherlands to assess valuation differences due to social context (global vs. the Netherlands vs. Canadian). In addition, the population norms of the Health Utilities Index (HUI), calculated using Canada's National Population Health Survey (NPHS) for specific levels of disability, will be compared to the complement of the disability weights in order to study the potential use of population health surveys to estimate disability weights. Work will also be done in the evaluation of disability weights in the presence of co-morbidities. Overall, this work will add to the understanding of the differences in the valuation of health states.
294/ USING THE WHOQOL-BREF INSTRUMENT AND TIMETRADE-OFF UTILITY FOR INSTITUTIONALIZED ELDERLY PEOPLE IN TAIWAN

Kuan-Lang Lai, Institute of Life Sciences, National Defense Medical Center, Taipei, Taiwan; Rong-Jye Tzeng, Department of Education and Training, Tri-Service General Hospital, Taipei, Taiwan; Bing-Long Wang, Department of Research, Senyeong Kao, School of Public Health, National Defense Medical Center, Taipei, Taiwan

The purpose of this study is to explore the health-related quality of life and health utility among institutionalized elderly people in Taiwan. 428 elderly people lived in 63 long-term-care institutions located at 12 counties in Taiwan were investigated. Taiwan's abbreviated version of the World Health Organization Quality of Life (WHOQOLBREF) and Time-Trade-Off (TTO) utility questionnaires were administered. The average age of the participants is 77.9 years and the average length of stay in institutions is 20.2 months. Among the 428 persons who completed the WHOQOL-BREF measurement, 298 persons $(70 \%)$ also completed the TTO measurement. Other 81 persons $(19 \%)$ did not understand the meaning of TTO and the other 49 persons $(11 \%)$ refused to answer. The WHOQOL-BREF shows acceptable internal consistency (alpha range: $0.85-0.88$ across domains). Good validity is demonstrated by its correlation with the selfrated health status, and its ability to discriminate between the diverse samples in this study. Activity of daily living has strong impact on the Physical domain $\left(R^{2}=0.40\right)$ and moderate impact on the Social Relationship domain $\left(R^{2}=0.11\right)$. The mean score of self-rated health status (total 100) is 61.3 [16.2; 10-100][SD; range]. Most of the elderly people $(88 \%)$ were unwilling to trade time for excellent health. The mean utility among the 298 persons is 0.92 [0.22; 0.05-1]. There is no statistical relationship between TTO utility with the domain scores or facet scores of WHOQOL-BREF. In conclusion, the results indicate that WHOQOL-BREF (Taiwan version) is suitable for evaluating the health-related quality of life of institutionalized elderly people. Longitudinal study is needed to explore the relationships between health-related quality of life and health utility for institutionalized elderly people. (Key words: Institutionalized, Elderly, Health-Related Quality of Life, Utility, WHOQOL-BREF, Time-Trade-Off, Taiwan.)

295/ SOCIAL COMPETENCE, EMOTIONAL/BEHAVIORAL PROBLEMS AND GLOBAL SELF WORTH IN A SWEDISH SAMPLE OF 7-9-YEAR-OLD CHILDREN WITH ASTHMA

Kjell L. Reichenberg, Nordic School of Public Health, Göteborg, Sweden; Anders G. Broberg, Department of Psychology, Göteborg University, Göteborg, Sweden

This study is a comparison of psychological adjustment in healthy children and children with asthma. The importance of asthma severity for psychological adjustment was studied. Parents of 61 Swedish 7-9-year-olds with asthma scored the Child Behavior Checklist (CBCL), and the children the I Think I am questionnaire, a measure of global self worthChildren with asthma had more emotional/behavioral problems than healthy children, but no less total social competence or global self worth. In a bivariate analysis, scores were related to urinary eosinophil protein X (U-EPX), the child's own and parents' grading of asthma symptoms, and also to sex, presence of eczema, family socio-economic status and cohabitation status of parents. In a multivariate analysis, all these factors except U-EPX and sex were independent predictors of at least one of the measures of psychological adjustment. We conclude that preadolescent children with asthma are at risk for acquiring emo-tional/behavioral problems. Adjustment is related to both disease-specific and social factors. 
296/ FAMILY CLOSENESS, PARENTAL LOCUS OF CONTROL AND PARENTING EFFICACY IN CHILDHOOD ASTHMA

Kjell Reichenberg, Nordic School of Public Health, Göteborg, Sweden; Anders G. Broberg, Department of Psychology, Göteborg University, Sweden

In developing outcome measures of family counselling in childhood asthma, we studied if family closeness, the parents' locus of control (PLOC) and their sense of efficacy as parents were related to social factors, the presence of allergy-related disor-ders, asthma severity and children's and parents' psychological adjustment to the child's asthma. 61 7-9-year-old children with mild $(n=11)$, moderate $(n=40)$ or severe $(n=10)$ asthma participated. Parents $(52$ mothers and 9 fathers) scored the self-rated ques-tionnaires Family Climate, a shortened PLOC-questionnaire, a Parenting Efficacy Scale, the Child Behaviour Checklist (CBCL), children's asthma symptoms and the Paediatric Asthma Caregiver's Quality of Life Questionnaire (PACQLQ). Children scored the Paediatric Asthma Quality of Life Questionnaire (PAQLQ) and a measure of global self worth. In bivariate analyses, Family Closeness, PLOC and Parenting Efficacy were related to each other. All measures of adjustment were related to at least one of the family/parent scales. Adjusting for background social background, allergy-related conditions and asthma severity, PLOC was that of the 3 measures accounting for the major part of the explained variance of adjustment. We conlude that Family Closeness and PLOC should be tested in trials of interventions attempting to improve parents' ability to cope with the asthma and improve psychological adjustment. Parenting Efficacy should be further validated in cross-sectional studies.

\section{7/ QUALITY OF LIFE IN CHILDREN AND ADOLESCENTS WITH DISABILITIES AND THEIR FAMILIES INTRODUCING THE EURO- PEAN DISABKIDS PROJECT}

Monika Bullinger, Department of Medical Psychology Universitätskrankenhaus Eppendorf, Hamburg, Germany; Ute Thyen, Department of Paediatrics Medical University Luebeck, Lübeck, Germany; Henrik Koopman, Department of Paediatrics Leiden University Medical, Leiden, The Netherlands; Peter Hoare, Department of Psychiatry, University of Edinburgh, Edinburgh, Scotland; Marie Claude Simeoni, Department of Public Health, University of Marseille, Marseille, France; Anathanasios Vidalis, Department of Paediatric Respiratory Unit, Hippocratio Hospital, Thessaloniki, Greece; John Eric Chaplin, Univesity of Lund, Lund, Sweden; Michael Quittan, Department of Physical Medicine and Rehabilitation, University of Vienna Vienna, Austria; DISABKIDS Group, Department of Medical Psychology, Universitätskrankenhaus Eppendorf, Hamburg, Germany

The European Commissions' 5th Framework Programme on "Quality of Life and Management of Living Resources" solicited grant proposals within the area of "Disabilities". This opportunity was taken up by a group of physicians, social /behavioural scientists and statisticians from 6 European countries by submitting the DISABKIDS Project which is funded for 3 years starting February 1st, 2001. The aim of the project is to enhance the quality of life of children and adolescents with disabilities and their families by developing, testing and implementing in 7 countries disease specific QOL self-report instruments, for children and adolescents aged 4-7, 8-12,13-16 years with Asthma, Epilepsy, Diabetes, Rheumatoid Arthritis, Cerebral Palsy and Cystic Fibrosis. Using literature searches, expert delphi rounds and focus groups with the children/ adolescents and families, items are developed and translated into the respective project languages. After pilot testing and analysis, a field study will be conducted with a minimum of 60 families per at least 3 conditions per country (total $n=1280$ ). Item response theory and structural equation modelling are used to examine the psychometric characteristics of the questionnaires. The new tools will be available in a paper - pencil and in a computerised version for easy application in the clinical context. Implementation of the new tools in the clinical settings will be planned, conducted and evaluated. The final analysis will involve national and cross - cultura analysis of the data set. The project tasks of development, testing, application of the new instruments are described in eleven work packages, also identifying participants responsible. News, materials and project reports will be available on the DISABKIDS homepage. Project tasks and methods will be presented. The DISABKIDS project, as a European project, will help to assess challenges of and solutions to European cooperation in the paediatric QOL field. The DISABKIDS project cooperates closely with the partner project KIDSCREEN coordinated by U. Ravens-Sieberer, Robert Koch Institut, Berlin, Germany.
298/ THE HEALTH OF CHILDREN IN EUROPE-A QUALITY OF LIFE PERSPECTIVE

Monika Bullinger, Department of Medical Psychology, Universitätskrankenhaus Eppendorf, Hamburg, Germany; Ulrike Ravens-Sieberer, KIDSCREEN group, Department of Epidemiology and Health Reporting, Robert Koch Institute, Berlin, Germany, DISABKIDS Group, Department of Medical Psychology, Universitätskrankenhaus Eppendorf, Hamburg, Germany

The health related quality of life (QOL) of children and adolescents is considered a relevant topic for research. Generic and disease specific instruments to assess QOL in children and adolescents are developed and applied in epidemiological surveys, clinical studies, quality assurance and health economics projects. Cross-cultural research on QOL in children is rare International data such as the Global Burden of Disease Report do not include self or parent-reports of physical, emotional and social wellbeing or functioning in children, nor do they address the family perspective on health care needs and services. Also the relationship of QOL in children to determinants such as health behaviours, coping processes, social support systems, family climate and socio-economic conditions need to be studied. From a public health perspective only few countries have engaged a comprehensive surveys on child health, which differ widely in the methods applied. Clinical studies with exception of the few multinational clinical trials involving children with a chronic health conditions, suffer from comparable problems. Based on a comprehensive literature search on international QOL research in children and adolescents in Medline over 18000 abstracts were identified and evaluated by an expert group on child health and quality of life using a rating procedure. Results showed that descriptive cross-sectional studies were in the foreground clinically and that representative health surveys were rare. From the literature search conceptual, methodological and practical problems of available studies can be identified, which have to be addressed to guide further research into child and adolescent health in Europe. In order to scientifically guide health planning instruments for self-report and parents report of QOL in children/adolescents should be developed and applied.

299/ THE CORE VSP-A AND ITS SPECIFIC MODULE: A GENERIC HRQL INSTRUMENT FOR ILL AND HEALTHY ADOLESCENTS

Marie-Claude Simeoni, Christophe Sapin, Audrey Clement, Mohammed El Khammar, Pascal Auquier, Public Health Department, Medical School, Marseille, France

The development of the VSP-A, a generic HRQL instrument designed for adolescents was based on the exclusive adolescents' viewpoint. Two parallel approaches were conducted independently in school adolescents and ill adolescents following the same item generation procedures, using both individual interviews and focus groups. We aimed at studying the differences in the perceptions of HRQL between ill and healthy adolescents. We analysed the feasibility of producing a single HRQL core questionnaire relevant in both ill and healthy adolescents. Our approach revealed no major difference between these 2 groups of adolescents in their perceptions of HRQL. The VSP-As developed in school-based samples led to a 42-item version validated with 2,941 adolescents. The VSP-Am, developed and validated with 683 ill adolescents with various types of diseases (in medical, surgical and psychiatric wards) and levels of severity, comprised 39 items describing 8 domains. The similarity of the wording and content of the questionnaires (except for a dimension linked to the relationships with the medical staff) led us to propose a core questionnaire, called VSP-A, for use with ill and healthy adolescents. $A$ validation survey was set up to assess reliability, validity and acceptability of the VSP-A involving both school adolescents and inand out-patients. The final core questionnaire comprises less than 40 item, covering psychological, physical well-being, and social relationships. An additional module specific to ill adolescents is dealing with relationships with medical staff. The acceptability of the questionnaire is high. Good levels of reliability (Cronbach's alpha > 0.7), item discriminant validity and factorial validity were found. Known group comparisons according to socio-demographic and clinical criteria showed expected differences. The assessment of its reproducibility and sensitivity to changes is on-going in specific longitudinal surveys. 
300/ VALIDATION IN FRENCH OF THE CHILD HEALTH QUESTIONNAIRE PARENT FORM (CHQ-PF50) IN CHILDREN AGED 919 YEARS SUFFERING FROM CANCER

Chantal Rodary, Biostatistics, Nicolas Bellon, Institut Gustave Roussy, Villejuif, France; Jeanne Landgraf, Health Act, Boston, MA; Chantal Kalifa, Institut Gustave Roussy, Villejuif, France; Pascal Chastagner, CHRU Hopital d'enfants, Vandoeuvre les Nancy, France; Jean-Pierre Vannier, Hôpital Charles Nicolle, Rouen, France

To validate the French version of the CHQ-PF50 in order to supplement the child assessment. This generic instrument was initially developed by J. Landgraf in 1993 for children aged 5-18 years. The CHQ-PF50 is a comprehensive, multidimensional quality of life (QoL) assessment tool comprising 50 questions grouped in 11 dimensions - Physical Functioning (PF), Role/Social Physical (RP), Bodily Pain (BP), General Health (GH), General Behaviour (BE), Role/Socialemotional/Behavioral (REB), Self Esteem (SE), Mental Health (MH), Family Activities (FA), Parental Impact Time (PIT), Parental Impact Emotional (PIE), resulting in 11 scores ranging from 0 (worst QoL) to 100 (best QoL). 282 parents of children suffering from 8 types of cancer treated in ten centres completed the CHQ-PF50. Children were free of disease and off treatment for at least 6 months. Psychometric computations were performed using the Revised Multitrait-Analysis Program. Compliance was good (98\%) as well as the acceptability (median duration of completion $15 \mathrm{mn}$ ). Floor effect was negligible, but ceiling effects $>40 \%$ were observed for PF, BP, FA, PIT, REB and RP. Reliability of the scale, estimated using Cronbach's alpha coefficient was satisfactory $(>0.70)$ for seven dimensions. For three dimensions (GH, BE, and PIE) it was between .65 and .70. Ninety percent of the items achieved acceptable internal consistency. The tests for convergent and discriminant validity were successful in $88 \%$ of the time. Mean scores ranged from $63(\mathrm{GH})$ to 90 (PF, RP). The use of the $\mathrm{CHQ}-\mathrm{PF} 50$ allowed us to detect differences in profiles of scores between the 4 cancers presenting more than 30 patients (total 161 pts). The results obtained in children having suffered from cancer are satisfying for the psychometric properties. It would be necessary to test the instrument's sensibility over time. We also need to compare the profiles obtained by parent reports and self-reports.

\section{1/ DEVELOPMENT, VALIDATION OF HRQL MEASUREMENT} IN CHILDREN: VSP-AE

Pascal Auquier, Public Health Department, Medical School, Marseille, France; Audrey Clement, Christophe Sapin, Mohammed El Khammar Jean-Louis San Marco, Public Health Department, Medical School, Marseille, France; Marie-Claude Simeoni, Public Health Department, Medical School, Marseille, France

In France only one HRQL instrument is widely used in school adolescents (10-17 yr.), the VSP-As, based on adolescents' viewpoint. It was proposed in follow-up surveys and as a part of the medical school check-up to point out the most vulnerable pupils. The implementation of programs relying on this approach is already very useful, but it would be even more relevant to act earlier. Therefore we develop a instrument designed for children of 8-10 yr. (VSP-Ae) following the same methodology than the one used for VSP-As. 96 school children -aged $8-11 \mathrm{yr}$., $54 \%$ girls, with a large range of socioeconomic family status levels- were involved in audiotaped interviews: 46 individual interviews and 12 focus groups (4-5 subjects). Content analysis of interviews isolated 53 candidate items. The acceptability of questionnaire was explored ( 90 children) including cognitive debriefing Item reduction step was led with 251 patients in 3 different areas of France: Paris, Grenoble, Marseille and their region. Non participation rate was $6 \%$ only due to lack of parental consent. Validation study was conducted on a random sample of 1,000 pupils (stratification: urban-rural, socioeconomically disadvantaged area or not, age group). The final questionnaire included less than 45 items: VSP-Ae. The major dimensions were psychological and physical well-being, social relationships with friends, family, leisure, self-esteem... Cronbach's alphas were high (> 0.7). Known group comparisons were studied according to sociodemographic criteria, health status. Our approach revealed a major overlap between the adolescents and the children in the identified domains of HRQL. However specificity of the wording was found in this age group, due to lower cognitive and abstraction skills. Reproducibility and sensitivity to change will be assessed in a survey planned with 3,000 pupils. The VSP-A questionnaire and the new validated VSP-Ae will allow to follow up children and adolescents from 8 to 17 .
302/ QUALITY OF LIFE IN CARERS OF SEVERELY DISABLED CHILDREN WITH SEVERE FEEDING PROBLEMS

Santanu Maity, Adrian G. Thomas, Department Of Paediatric Gastroenterology, Booth Hall Childrens Hospital, Manchester, UK; Alison Bachlet, P. B. Sullivan, John Radcliffe Infirmary, Oxford, UK; M. Eltumi, Watford General Infirmary, Watford, UK; Bridget Lambert, John Radcliffe Infirmary, Oxford, UK

Feeding problems are common in disabled children and may result in an enjoyable social activity becoming distressing for the child \&/or carer. They may also cause malnutrition and result in prolonged feeding times. We have previously developed an instrument to assess QoL in the carers of these children and are currently using it to determine the effect of gastrostomy feeding in the child on the carers QoL. To establish the baseline QoL and the most important concerns of the carers of severely disabled children with feeding problems before gastrostomy placement. The carers of 42 severely disabled children completed the QoL questionnaire before gastrostomy placement in their child. Major concerns were divided into 4 areas: the child in general, feeding difficulties, practical difficulties for the carer, psychologi$\mathrm{cal} /$ emotional difficulties for the carer. The main concerns, as recorded by answers 'definitely and mostly true' to the questions asked were; worried about the future for my child $(95 \%)$, concerned that my child needs help with feeding $(91 \%)$, having a disabled child can be stressful $(91 \%)$, concerned that no one else can look after my child $(91 \%)$, worried that my child can't eat lumpy food (83\%). The top ranking questions that the carers didn't agree with were; other people understand my child's problems $(24 \%)$, other people understand my problems with regard to the care and needs of my child $(24 \%)$, there are always adequate changing facilities when I need them for my child $(36 \%)$. The preliminary results of the questionnaire give us an understanding of the main concerns of carers with disabled children who are about to have a gastrostomy placed. The next stage of the study is to determine whether gastrostomy feeding improves the carers' QOL, and to see whether there is any change in their concerns.

303/ RELIABILITY AND DISCRIMINANT VALIDITY OF THE CHILD HEALTH QUESTIONNAIRE 28-ITEM PARENT FORM (CHQ-PF28) AS PEDIATRIC GENERIC HEALTH STATUS MEASURE

Hein Raat, Marie-Louise Essink-Bot, Department of Public Health, Erasmus University, Rotterdam, ZH, The Netherlands; Jeanne M. Landgraf, Health Act, Boston, MA

The 50-item version of the CHQ-PF is a much-used generic health status measure in pediatrics. Cross-culturally validated $\mathrm{CHQ}$-translations are available in 21 languages (32 countries). As in adult instruments (e.g. SF36, SF12) there is a demand for the shortest possible, yet reliable and valid version of this instrument. A 28-item CHQ-PF is available, but sparsely used until now, as few data are available on its reliability and validity. The purpose of this study is to assess the internal reliability and discriminant validity of the CHQ-PF28. A random sample of 2040 parents of elementary school children in The Netherlands received in March 2001 a mail questionnaire with the CHQ-PF28 (age 5-13; response 70\%). Data of the first 787 respondents were already processed and eligible for analyses. The parents also completed questions on chronic diseases, medical consumption and a 0-100 Visual Analogue Scale-rating (VAS) of the child's health. The Cronbach alphas of 8 CHQ-PF28-scales were on average .60 (range .36-.78; 4 were >.70). The General health perceptions scale, with an alpha of .49 , is however one of the best discriminating $\mathrm{CHQ}$ domains in measuring differences between groups with low vs. high medical consumption, zero vs. $>=2$ chronic diseases, overall child health via VAS-rating $>=90$ vs. $<90$. The CHQ-PF28-Physical summary measure also has good discriminating power with regard to these health indicators. E.g.: ICC of General health perceptions and Physical summary with VAS-rating $.37(p<.01)$, respectively .43 $(p<.01)$. In conclusion, internal reliabilities of the PF28-scales are variable, while discriminant validity equals that of the longer PF50. The $\mathrm{CHQ}$ PF28 may be applied if there is not sufficient place for the often-used CHQ-PF50, in order to augment outcome assessment, in addition to disease-specific and clinical measures. 
304/ SCREENING FOR AND PROMOTION OF HEALTH RELATED QUALITY OF LIFE IN CHILDREN AND ADOLESCENTS - A EUROPEAN PUBLIC HEALTH PERSPECTIVE

Ulrike Ravens-Sieberer, Child and Adolescent Health, Robert Koch Institute, Berlin, Germany; Thomas Abel, Social and Preventive Medicine, University of Bern, Bern, Switzerland; Pascal Auquier, Department of Public Health, University of Marseille, Marseille, France; Bärbel-Maria Bellach, Epidemiology and Health Reporting, Robert Koch Institute, Berlin, Germany; Jeanet Bruil, Prevention and Health, TNO, Leiden, The Netherlands; Wolfgang Duer, Ludwig Bolzmann Institute for Sociology of Health an, Vienna, Austria; Mick Power, Department of Psychiatry, University of Edinburgh, Edinburgh, Great Scotland, Luis Rajimil, Catalan Agency for Health Technology, Assessment, Barcelona, Spain; the European KIDSCREEN Group

Within the 5th Framework-Programme Quality of Life Management of Living Resources by the European Commission the KIDSCREEN project was favorably reviewed within the sub-programme Activities of a Generic Nature, Area Public Health. KIDSCREEN is funded for three years, started February 1st, 2001, involving seven European countries: D, E, UK, F, A, NL, CH. The project aims at a co-operative cross-cultural European development of a standardized screening measure for children's quality of life which will be used in representative national and European health surveys. It also aims at identifying children at risk at terms of their subjective health to suggest early intervention possibilities by including the measure in health services research and health reporting. Instrument development pertained to construct a psychometrically sound HrQol measure taking into account the existing state of the art by using literature searches, expert consulting and delphi method. Items were developed by conducting focus groups with children (8-17 years) to identify relevant QoL dimensions and acceptable wording. According to international guidelines items were translated into languages of the participating countries and a pilot instrument was obtained which will be tested in 300 children in each country. Item response theory and structural equation modeling are used to determine the optimal item and scale characteristics of the questionnaire in addition to common psychometric analysis. The final instrument will be used in representative mail and telephone surveys on HrQoL in 1800 children and parents per country $(\mathrm{n}$ total $=12.600)$ and norm data will be provided. The potential implementation of the measure in European health services and health reporting will be planned, tested and evaluated. The final analysis will involve national and European cross cultural comparison of the instrument. News, continuing documentation and publications of work from the project will be available, on the KIDSCREEN homepage. The KIDSCREEN project can be helpful in discussing challenges and possible solutions of European cooperation within the quality of life field.

\section{5/ A LONGITUDINAL STUDY ON HEALTH RELATED QUALITY} OF LIFE IN PATIENTS WITH HIV-INFECTION

Claes G. Cederfjäll, Department of Nursing, Karolinska Institutet, Stockholm, Sweden; Ann Langius-Eklöf, Department of Nursing, Knut Lidman, Department of Medicine, Karolinska Institutet, Stockholm Sweden; Regina Wredling, Department of Nursing, Karolinska Institutet, Stockholm, Sweden

The purpose of this study was to analyse health related quality of life in relation to biomedical variables and antiretroviral treatment during a period of 2 years. One hundred and four patients $(71 \% \mathrm{men})$ attending an outpatient clinic for HIV-infected patients in a major hospital in Stockholm were enrolled in the study. The mean age was 42 with a mean duration of 8.6 years since HIV diagnosis. The patients were asked to fill in questionnaires at 3 times with 12 months interval (1997-1998-1999). At the same time demographics and biomedical variables were collected from the patients medical records. Questionnaires used was; Health Index measuring general health (9 items), HIV symptom scale (9 items), The Well-being Scale (depression 6 items, anxiety 6 items, energy 4 items, positive well-being 6 items), social support (10 items) and the Sense of Coherence scale (29 items). Biomedical variables included disease stage, the amount of antiretroviral treatment, CD4 cell count/mm3 and HIV/RNA copies/ $\mathrm{ml}$. The results showd that the amount of antiretroviral treatment increased $(p=0.0001)$ and the CD4 cell count increased from 331 to 535 over two years $(p=<0.0001)$. In the health index scale the patients experienced more problems with bowel function $(p=0.0004)$ and a decrease in mobility $(p=0.008)$. The patients reported more weightloss over the two years $(p=0.026)$ and more symptoms from the mucous membranes of the mouth $(p=0.0005)$ in the HIV symp- tom scale. All other scales did not show any significant changes with the three measurements. Since there were an increase in antiretroviral treatment and a higher amount of CD4 cells but more symptoms reported one might argue that those symptoms stems from side effects of the treatment. The conclusion is that this group of patients got healthier regarding biomedical variables but experienced more symptoms and their quality of life did not increase.

\section{6/ HEALTH-RELATED QUALITY OF LIFE IN HIV+ PATIENTS TAKING HAART}

Rita Murri, Cosmo Del Borgo, Prospero Limongelli, Infectious Diseases, Catholic University of Rome, Rome, Italy

The purpose of this study was to assess role of protease inhibitors (PIs) in change of HRQoL in HIV+ patients (pts). Prospective multicenter, cohort study on Italian HIV+ pts at any stage of disease taking PIs. From 10/97 to 5/98, consecutive patients were enrolled in 7 Italian Centers. The MOS-HIV was used as tool of the study and administered every 6 months. Two summary scores for physical health (PHS) and mental health (MHS) were obtained. Outcomes of the study were considered a decrease of at least 5 points in the PHS or MHS scales at 3rd follow-up. 446 patients were enrolled into the study. Mean age: 36 years, females: $29 \%$, intravenous drug users: $47 \%$, CDC's group C: $46 \%$. Median CD4+ cells count: $203 / \mathrm{ml}$; undetectable HIV RNA: $40 \%, 54 \%$ of pts were taking indinavir (IDV), $15 \%$ ritonavir (RTV), 26\% saquinavir (SAQ), 1\% nelfinavir and $4 \%$ both RTVISAQ . At 3rd follow-up visit, $24.1 \%$ and $29.4 \%$ had respectively at least a 5-point decrease of PHS and MHS. At bivariable analysis, taking IDV or SAQ, being aware of HIV infection status since more than 80 weeks, CD4 cell count $<200 / \mathrm{ml}$ at enrollment, detectable HIV RNA at enrollment, changing HAART during follow-up, and PHS.

\section{7/ SATISFACTION WITH SEXUAL LIFE IN HIV+ PATIENTS}

Rita Murri, Massimo Fantoni, Cosmo Del Borgo, Prospero Limongelli, Infectious Diseases, Catholic University of Rome, Rome, Italy

The purpose of this study was to assess prevalence and determinants of poor satisfaction with sexual life (SatSexL) in HIV+ pts in the new antiretrovirals (HAART) era. Prospective, multicenter, cohort study on Italian HIV+ pts at any stage of disease. Consecutive patients were enrolled in 7 Italian Centers from 10/97 to 05/98. The MOS-HIV, of which the Italian version has been previously validated, was used as tool of the study. It evaluates $11 \mathrm{HRQ}$ oL domains: pain, physical, role, social, and cognitive functioning, mental health, vitality, health distress, general health perception, health transition, overall QoL. Two summary scores for physical (PHS) and mental health (MHS) were obtained. A symptom list as well as a single item on SatSexL were added to the original version. Outcome of the study was considered poor SatSexL ("not at all" or "a little" to the question: "During the last 4 weeks, have you been satisfied with your SexL"?) 514 patients were enrolled into the study. $>35$ years: $44 \%$, females: $30 \%$, intravenous drug users: $47 \%$, CDC's group C: $28 \%$. Median CD4+ cells count 400/ml; undetectable HIV RNA: $37 \% .471$ pts (83.5\%) were taking HAART $(67 \%$ with a protease inhibitors-containing regimen, $20 \%$ with a NNRTI and $13 \%$ with both); $49.7 \%$ and $50.1 \%$ had, respectively, a PHS and MHS below the median. $309(60 \%)$ reported poor SatSexL. Factors indipendently associated with poor Sat SexL were being $>35$ years-old (OR 1.67; 95\% Cl 1.13-2.49), IDU (OR 0.56; 95\% Cl 0.380.83), reporting a poor PHS (OR 3.36; 95\% Cl 1.94-5.82), and having a high symptom score (OR 2.26; 95\% Cl 1.49-3.44). At chi-square test, taking ritonavir was associated with poorer SatSexL $(p=0.03)$ while taking nelfinavir was associated with better Sat SexL $(p=0.03)$. Older people and people with poorest physical health have poorest satisfaction with sexual life; presence of symptoms negatively influences satisfaction with sexual life. 
308/ IMPLEMENTING QUALITY OF LIFE IN EORTC CLINICAL

TRIALS

Andrew Bottomley Ph.D., Quality of Life Unit, EORTC Data Center, Brussels, Belgium; Dr. Henning Flechtner, Klinik fuer Psychiatrie und Psychotherapie des Kinde, Universitaet zu Koeln, Koeln, Germany, on behalf of EORTC Quality of Life Group

For cancer patients, Quality of Life (QL) is now becoming an increasingly accepted endpoint in cancer clinical trials. However, reports in published literature suggest that fewer than $10 \%$ of all clinical trials have QL assessment, although it is believed such reports may be biased by time lag factors in the new and emerging field of $Q \mathrm{~L}$. This paper therefore examines the extent of QL studies that are conducted within one of the largest cancer clinical trial organization in Europe the EORTC, investigating both completed and ongoing clinical trials. An examination of all clinical trials conducted by the EORTC was undertaken from January 1990 to January 2000 by reviewing data from various databases, publications and records. Trials were systematically selected if they involved any aspect of $\mathrm{QL}$, as clearly specified in the protocol. In total, some 112 EORTC clinical trials were identified as having a QL component. Over 10,000 patients had been entered into these trials. All of the trials involved multinational patient recruitment, with the highest recruitment from The Netherlands, France and Germany and the lowest from Malta, Estonia and Slovakia. Approximately 14 disease groups have been actively recruiting patients over the last decade, with the major number of patients being from disease groups of Genito-urinary, Breast and Lung cancers. A clear linear trend was noted, with increasing numbers of clinical trials involving QL components over this ten-year period. Of all the trials, 74 studies were phase III design, 15 were phase II design, and the remainders were feasibility studies. Presently, 45 trials are ongoing, and open to patient entry, 19 are nearly mature for data analysis, 15 have now being published and 10 are now being analyzed. In the last year, 30 new studies have been submitted for research involving $Q L$, suggesting that quality of life is a highly important endpoint in present day trials across the European setting. While QL was not a major component of EORTC clinical trials in the early 1990's, it is now highly integrated into EORTC cancer clinical trials, and almost a standard secondary endpoint. This suggests that clinicians and researchers alike in the European context are increasingly seeing the importance of patient based outcome assessment methods for assessing the value of cancer therapeutic modalities.

309/ A DECISIVE STEP TOWARDS THE RECOGNITION OF PATIENT REPORTED OUTCOMES IN CLINICAL TRIALS: REPORT OF A MEETING AT THE FDA ON FEBRUARY 16, 2001 Catherine Acquadro, ERIQA Group, Mapi Research Institute, Lyon, France

At the initiative of Mapi Research Institute, two Harmonization meetings brought together members of ERIQA, ISOQOL, ISPOR, PhRMA HOC, and FDA observers on March 31st, 2000 and on September 14 th, 2000, in Rockville, MD, to focus on issues related to Heathrelated Quality of Life (HRQL) outcomes. One of the conclusions was to continue discussions under the coordination of a Committee of representatives from each organization and the FDA. The purpose of the February Meeting was to continue to clarify aspects and components of Patient Reported Outcomes (PROs) evaluation, specifically to discuss its added value, and to suggest use of this information within labeling and promotional claims. Four key issues were addressed. 1.Conceptual and Definitional Issues; 2. The Value of PROs; 3. Methodological considerations in obtaining PROs in clinical trials; 4. Interest in and Demand for PROs. The meeting gathered representatives of the 4 organizations and of the FDA (30). The audience did not question the definitions of PROs and HRQL, and expressed a great interest in the presentation of PROs added value. The group recognized that reliable and valid instruments are available to measure PROs, but deplored the lack of quality of some studies. The interest of including PROs in clinical trials was recognized as adding value for decision-making. Concerns about PROs were also expressed. There is a need to clarify a) the uniqueness of PROs methodology; b) the meaning of clinical significance, c) when are the data meaningful, d) the methods to handle missing data; e) the level of evidence needed to support a claim, f) how should results be presented in the label. Although additional research is needed, this meeting could be considered as a crucial step in the future recognition of PROs as relevant evaluation criteria of drugs in clinical trials. The meeting was a seen by many participants as a turning point and an encouraging sign for the future of the field.
310/ USEFULNESS OF QUALITY OF LIFE (QOL) INFORMATION IN A TRIAL OF A MATRIX METALLOPROTEINASE INHIBITOR (MMPI) MARIMASTAT

Andrea Bezjak, DRO, Frances A. Shepherd, Department of Medical Oncology, Princess Margaret Hospital, Toronto, ON, Canada; Lesley Seymour, A. Sadura, Dongsheng Tu, Joseph Pater, Central Office, NCIC Clinical Trials Group, Kingston, ON, Canada; G. Giaconne, C. Debruyne, S. Ahmedzai, EORTC, EORTC Central Office, Brussels, Belgium

QOL is a frequently employed secondary endpoint in cancer clinical trials. When agents with a favorable toxicity profile are studied, there may be a desire to omit QOL assessment since it is felt to be of limited clinical usefulness. In this study of an MMPI that had been reported to have generally mild toxicity limited to joint pain, inclusion of $\mathrm{QOL}$ as an endpoint proved to be highly useful. A Phase III placebocontrolled trial of Marimastat was conducted jointly by NCIC CTG and EORTC centers. 555 patients (pts) with limited or extensive stage small cell lung cancer and response to induction chemotherapy +/radiotherapy were randomized to Marimastat $10 \mathrm{mg}$ bid or placebo for 2 yrs. The primary endpoint was overall survival. Secondary endpoints included QOL, assessed using EORTC QLQ-C30 and the lung specific module at baseline, 3, 6, 12, 18 and 24 months, and then yearly. There were no significant differences in progression-free or overall survival (median survival $9.6 \mathrm{mo}$ ), in the entire cohort nor in subgroup of pts with limited disease. Toxicity, as reported on casereport forms, was similar in both groups except for increased musculoskeletal symptoms (63\% vs $38 \%$ ) and lethargy (33\% vs $25 \%)$ in the Marimastat arm. QOL data at 3 months revealed significant differences with more pain in arm/shoulder in the Marimastat arm (mean change score 33.3 vs 8.3 in the placebo arm, $p=0.0001$ ), greater overall pain $(21.3$ vs $8.9, p=0.0002)$, worse social functioning $(-4.59$ vs $1.52, p=0.03)$ and emotional functioning $(-6.07 v s-1.18, p=0.03)$. Similar significant differences in pain and global QOL were observed at 6 months. This was reflected in decreased compliance with Marimastat in comparison to the placebo arm. In this study, QOL information highlighted the clinical implications of toxicity and prompted discontinuation of study therapy shortly after the final analysis so that patients could be spared further adverse effects on QOL.

\section{1/ PSYCHOMETRIC PROPERTIES OF CYCLOSPORINE CAP-} SULE SATISFACTION SURVEY (CCSS)

Amitabh Singh, Talat Ashraf, Azmi Nabulsi, Rebecca Hoffman, Global Epidemiology and Outcomes Research, Abbott Laboratories, Abbott Park, IL

CCSS assesses the impact of cyclosporine's smell, taste and size on patient satisfaction with cyclosporine capsule. Prior research has indicated patients' dissatisfaction with smell, odor, taste and size of cyclosporine. The purpose of this research was to establish the psychometric properties of CCSS. A six-month, open-label study was conducted in which de novo renal transplant $(N=100)$ recipients were randomized (1:1) to receive BID dosing of either Gengraf (Abbott cyclosporine) or Neoralâ followed by an open-label extension period with all subjects receiving Gengraf. CCSS was self-administered at week 4 , month 3 , and month 6 . The raw scale scores were transformed to a 0-100 scale. CCSS's psychometric properties were evaluated at week 4 . Factor analysis was performed to assess the underlying factors that are responsible for the correlation in the item scores. The minimum eigenvalue for a retained factor was one. Factor loadings were computed using varimax rotation. Both item and scale-level reliability were assessed. Item-level discriminant validity analyses were conducted to estimate scaling success. Cronbach's alpha, item-homogeneity, and average inter-item correlation were computed to estimate internal consistency of items within a scale. Factor analysis confirmed presence of 3 underlying factors - smell, taste and sizewith eigenvalue greater than one. The uniqueness estimates illustrate correlation in item scores are explained well by the three retained factors. Although, floor effects in item-scores were detected, the item-scale Pearson correlations were consistently statistically significant and exceeded 0.40 . Item-scale discriminant validity analysis with multitrait/multi-item analysis showed that $83.3,100$, and 75 scaling success for smell, size, and taste scales respectively. Scales were highly reliable with Cronbach's Alpha 10 estimates of $0.87,0.94$ and 0.82 for smell, size, and taste scales respectively. CCSS is a reliable and valid method to assess subject's preference for alternative cyclosporine formulations. 
312/ QUALITY OF LIFE OF INFLAMMATORY BOWEL DISEASE (IBD) PATIENTS WHO HAVE EXPERIENCED FAECAL INCONTINENCE (FI): A PATIENT CENTRED APPROACH

C. Speed, On behalf of the QoLiFI Research Team, University of Newcastle upon Tyne, Newcastle, UK

We report on the first stage of a study to develop an instrument to measure the impact of FI in people with IBD. We present the findings of a series of in-depth interviews with patients who have experienced $\mathrm{FI}$. FI is the involuntary or inappropriate passage of faeces, liquid matter or gas. It is a significant feature of IBD yet under-reported by patients and rarely asked about by clinicians. Any attempt to measure QOL in FI needs an informed understanding of what FI means to those experiencing incontinence. Using a patient-centred approach we aim to better understand the physical, social and psychological impact of FI. The interviews will form the basis for the QOL instrument which will be revised in line with factor analyses and assessments of psychometric properties. To develop the instrument, a sample of $\mathrm{FI}$ patients was selected on the basis of frequency and severity of symptoms. Patients were interviewed in-depth about their experiences of $\mathrm{FI}$ and the impact it had upon their lives. These interviews presented the opportunity to cover any issues surrounding FI that were pertinent to the patients. Using a matrix based approach, interview transcripts were analysed and salient themes extracted. Several common themes emerged from the analyses of the transcripts. These included: planning, isolation (physical and psychological), embarrassment (discussing $\mathrm{FI}$ and also the consequences of $\mathrm{FI}$ ), and control The lack of public awareness and understanding of FI was also raised Humour was a common coping strategy used but the ability to share this with someone else was important. Whilst there are many differences in these personal accounts there are commonalities. Many of them were negatively biased, as would be expected in any chronic condition that hinders daily life. The most striking similarity between patients who maintained a positive frame of mind was a good relationship with partners and family and a high level of social support.

\section{3/ SYMPTOMS OF GASTRO-OESOPHAGEAL REFLUX DIS-} EASE HAVE A NEGATIVE IMPACT ON HEALTH-RELATED QUALITY OF LIFE

Nicholas J. Talley, Professor of Medicine, Division of Medicine, University of Sydney, Penrith, Australia; Ola Junghard, Biostatistics Clinical Science; Ingela K. Wiklund, Outcomes Research, AstraZeneca R\&D Mölndal, Mölndal, Sweden

The aim of this study was to investigate how symptoms of gastrooesophageal reflux disease (GORD) affect peoples' lives. 984 patients (18-80 years) with endoscopy-negative GORD received esomeprazole $20 \mathrm{mg}$ or $40 \mathrm{mg}$ once daily for four weeks as part of two separate clinical trials. Data derived from the esomeprazole treatment arms of both clinical trials were pooled. The validated diseasespecific questionnaires, Quality-of-Life in Reflux and Dyspepsia (QOLRAD) and Gastrointestinal Symptom Rating Scale (GSRS), were completed at baseline and after 2 and 4 weeks of treatment with esomeprazole. QOLRAD questionnaire data showed the proportion of patients affected by heartburn or acid regurgitation 'at least some of the time' during the preceding week. The GSRS was used to evaluate symptom relief over the study period. Change-score correlations were calculated linking symptom relief with improvement in HealthRelated Quality of Life (HRQL). Before esomeprazole treatment, the most pronounced dysfunctions related to eating/drinking problems $(45-81 \%)$, sleep problems $(39-49 \%)$, lack of vitality $(41-58 \%)$ and emotional well-being (40-55\%). Most daily activities were affected in approximately $20 \%$ of patients, although as many as $44 \%$ of the total population avoided bending over because of heartburn. After treatment, all complaints were reduced to between $7 \%$ and $19 \%$ at 2 weeks and between $6 \%$ and $16 \%$ at 4 weeks. At the start of the trial, heartburn and acid regurgitation caused at least 'moderate discomfort' in $77.3 \%$ and $51.8 \%$ of patients, respectively. This was reduced to $13.3 \%$ and $9.7 \%$, respectively, after 2 weeks and to $7.2 \%$ and $5.7 \%$, respectively, after 4 weeks. Symptom relief at 2 weeks correlated with improvement in all of the most pronounced dysfunctions $(p<0.0001)$. To conclude, effective acid suppression with esomeprazole reduces symptoms and rapidly improves HRQL in patients with GORD.
314/IMPACT OF GASTROINTESTINAL SYMPTOMS AND AFFECTIVE DISORDERS ON HEALTH-RELATED QUALITY OF LIFE (HRQL) IN US PATIENTS WITH IRRITABLE BOWEL SYNDROME (IBS)

Ingela Wiklund, Outcomes Research, AstraZeneca, Molndal, Sweden; George Longstreth, Kaiser Permanente, San Diego, CA; Emeran Mayer, UCLA, Los Angeles, CA; Jorgen Naesdal, Ingalill Wilson, AstraZeneca, Molndal, Sweden

The irritable bowel syndrome (IBS) is a common clinical problem, which disrupts health-related quality of life (HRQL). The aim of this study was to investigate how IBS symptoms and emotions affect HRQL. A total of 124 patients (female $77 \%$; mean age 46 ) with moderate to severe IBS symptoms according to the Rome I criteria were included in a "mock trial" for 12 weeks. Treatment was provided at the discretion of the investigator. The patients completed an IBS symptom questionnaire (GSRS-IBS; best score 1, worst score 7) and the Hospital Anxiety and Depression (HAD) scale. General health status was evaluated by the Short Form 36 (SF-36) and HRQL by the Irritable Bowel Syndrome Quality of Life instrument (IBS-QOL; best score 1 , worst score 5) and the Disease Specific Questionnaire - Irritable Bowel Syndrome (DSQ-IBS; best score 100, worst score 0). According to the HAD scale, $32 \%$ had definite anxiety, $19 \%$ had definite depression, and $10 \%$ had both. The most bothersome symptoms were pain, bloating and diarrhoea (scores 4.1,3.9 and 3.8, respectively). The presence of both anxiety and depression significantly increased these symptoms with depression having a larger impact than anxiety on pain and bloating. In the IBS-QOL, symptoms resulted in dysphoria, interference with daily activities and food avoidance (scores 2.7, 2.8 and 3.1 , respectively), while DSQ-IBS indicated impairment in emotions, energy and activity (scores 48.1,52.0 and 45.8, respectively). The SF- 36 had low scores in all dimensions compared to reference values, with particularly emotional and role functioning being worsened by anxiety and depression. The predominant IBS symptoms correlated with impaired HRQL. In summary, pain, diarrhoea and bloating impair HRQL. The presence of affective disorders significantly worsens $\mathrm{HRQL}$ and symptoms of IBS, with depression having a greater effect than anxiety in this study.

315/ EVALUATION OF A COMPUTERISED TOUCH-SCREEN QUESTIONNAIRE TO MEASURE QUALITY OF LIFE IN CHILDREN WITH INFLAMMATORY BOWEL DISEASE

Santanu Maity, Adrian G. Thomas, Department Of Paediatric Gastroenterology, Booth Hall Childrens Hospital, Blackley, Manchester, UK

Inflammatory bowel disease (IBD) has an enormous impact on the Quality of Life (QoL) of affected children but QoL is often ignored because of difficulty in measurement. A questionnaire to measure QoL in children with IBD has been developed in Canada and modified for use in the Netherlands and UK (IMPACT-II). The questions are answered on a Visual Analogue Scale which is time consuming and difficult to analyse, limiting its clinical application. The aims of this study were: 1) to develop a touch-screen computerised version of the IMPACT-II questionnaire and assess its use in the outpatient clinic. 2) to compare patient preference and time taken to complete the paper \& computerised versions. The IMPACT-II questionnaire was adapted onto a Fujitsu Siemens B Series Lifebook by Streets Havers Healthcare Computing. 19 children with IBD attending the outpatient clinic completed (random order) the paper \& computerised versions. Time to complete the questionnaire, patient preference and reasons were recorded. Seventeen children preferred the computerised version because it was more fun, easier or because they liked computers. Two children preferred the paper version because they didn't like computers. The mean time to complete the computerised version was $5.1 \mathrm{~min} v 5.3 \mathrm{~min}$ for the paper version $(p=n s)$. The adaptation of the IMPACT II questionnaire onto a touch-screen portable computer was very acceptable to children with IBD attending the outpatient clinic and is a major step towards providing rapid information about QoL in the clinical setting. Further studies are required to determine validity, reliability and sensitivity and most importantly, to see whether the information obtained can be used to improve the children's QoL. 
316/ DETERMINANTS OF QUALITY OF LIFE IN PEOPLE WITH DIABETES MELLITUS

Pedro L. Ferreira, Conceicao Neves, Centre for Health Studies and Research, Manuela Carvalheiro, Faculty of Medicine, University of Coimbra, Coimbra, Portugal

The main objective of this research was to assess the health related quality of life in people with diabetes mellitus according to the various types of treatment, as well as to identify some variables that determine the perception people have about their own health. A cross sectional sample of 423 individuals with diabetes mellitus was selected in the external consultations of five hospitals in the central zone of the country. From the people studied, the majority had more than 50 years old $(53.7 \%)$, was married $(69.5 \%)$ and had a low instruction leve $(55.3 \%)$. In what concerns the clinical characteristics the majority was diabetic type $2(62.6 \%)$, had diabetes since less than 10 years $(55.3 \%)$ with various chronic complications, such as retinopathy $(37.8 \%)$, nephropathy $(27.4)$, neuropathy $(39.5 \%)$, hypertension $(42.6 \%)$, limb injuries $(13.2 \%)$ and vascular accident $(11.3 \%)$. It was also noticed some acute complications, namely severe hypoglicemy $(16.6 \%)$ and severe hyperglicemy $(57.2 \%)$. The majority did glicemic autocontro $(89.4 \%)$ and showed $\mathrm{HbA} 1 \mathrm{c}$ scores higher than $7.5 \%$ (56\%). People with diabetes type 1 under intensive treatment showed higher quality of life than those under conventional treatment. Significant differences were found in the SF-36's dimensions 'physical health' and 'vitality'. People with diabetes type 2 under treatment with oral antidiabetics only showed higher quality of life than those under treatment with insulin only. Significant differences were found in all the dimensions of both instruments (SF-36 and DHP). The results indicate a negative impact of some clinical characteristics, especially acute and chronic complications, as well as different depressive frames, in the perception of the health related quality of life of individuals with diabetes mellitus.

317/ SYMPTOMS OF DIABETIC RETINOPATHY AND THEIR IMPACT ON HEALTH-RELATED QUALITY OF LIFE

Mary Kay Margolis, Karin Coyne, MEDTAP International, Inc. Bethesda, MD; Gwendolyn Kiebert, MEDTAP International, Inc., London, UK; Tessa Kennedy-Martin, Lilly Research Centre, Windlesham, Surrey, UK; Timothy Baker, MEDTAP International, Inc., Bethesda, MD; Ronald Klein, Ophthalmology and Visual Sciences, University of Wisconsin, Madison, WI; Helen Simpson, Guys, Kings, and St. Thomas' School of Medicine, London, UK, Dennis Revicki, MEDTAP International, Inc., Bethesda, MD

Diabetic retinopathy (DR) affects $50-85 \%$ of people with diabetes and may result in visual impairment that has a substantial impact on $\mathrm{HRQL}$. The impact of DR on HRQL must be better quantified as there are few data available regarding the sensitivity of existing vision-specific HRQL instruments in people with DR. We conducted 6 focus groups $(F G), 4$ US $(n=15)$ and 2 UK $(n=23)$, to explore symptoms experienced by people with DR and their impact on HRQL. Patients (pts) > 18 yrs with Type 1 or Type 2 diabetes and DR were recruited from 3 physician offices. Physicians rated DR severity as mild $(n=15)$, moderate $(n=14)$ or severe $(n=9)$. 19 pts had undergone laser photocoagulation. Issues discussed during the FG included symptoms experienced, HRQL impact, coping behaviors, and feedback on 2 visionspecific questionnaires, VFQ-25 and VF-14. Content analysis and descriptive statistics were used to analyze the data. Pts described a range of symptoms from transient distorted vision, flashes of light, floaters, blurry, cloudy and wavy vision, and peripheral vision loss. Difficulty driving, especially at night, and trouble reading were noted at each severity level. Severe DR pts described giving up important aspects of their lives such as work, driving, reading, and sports. Many pts described difficulty performing diabetes care activities (e.g., exercising, reading nutritional labels, preparing insulin injections, and blood testing). Loss of independence, especially mobility and increased fear of accidents, had a profound impact on social activities. For some, asking for transportation assistance was demoralizing and caused activity avoidance. Pts felt the VFQ-25 questionnaire was more comprehensive and reflective of their experiences than the VF-14. Pts at all levels of DR severity experienced varying symptoms. DR had a significant impact on HRQL in the FG pts, particularly in the areas of independence, mobility, leisure, and self-care activities.
318/ GENDER DIFFERENCES IN QUALITY OF LIFE OF PATIENTS WITH DIABETES MELLITUS

Corinna Petersen, Matthias Morfeld, Monika Bullinger, Department of Medical Psychology, University Hospital Hamburg- Eppendorf, Hamburg, Hamburg, Germany

In the past years quality of life has been increasingly considered as a relevant outcome criterion in epidemiological, clinical and health economic studies. While the assessment methodology has advanced in terms of developing psychometrically tested instruments and applying generic as well as condition-specific measures, the question whether these instruments are sensitive to gender related differences has not been appropriately considered. Until recent years, the conception of women's health has been limited to attempts to protect and promote physical health, women's QoL has been relatively neglected. The current study analysed QoL data to investigate gender differences. In a cross sectional study, patients with diabetes mellitus associated in patient organisations were contacted by a telephone interview to assess their perception of their health condition and its impact on their daily life. 206 of 1000 diabetes mellitus patients participated in the study. The mean age was 56.9 years and $54.1 \%$ were female. Health- related quality of life was assessed with the SF12 Health Survey and the Nottingham Health Profile (NHP). Additionally, several standard questionnaires were used to assess psychosocial determinants of quality of life. Statistical analysis included correlation, variance and regression analysis. In the SF-12 physical scale, impairments for female as compared to male diabetes mellitus patients were found. Stronger impairments in the NHP Subscales Energy, Pain, Sleep and Mobility were noted for women. The regression analysis showed that similar variables for women and men explain the sum scores of the SF-12, but the number and the weight of the variables differ. Determinants of quality of life for both genders were comorbidity, age, stress and loneliness. The results suggest that women and men differ in their health-related quality of life and its determinants, and that gender sensitive instruments are necessary to detect those differences.

\section{9/ COPING AND DISTRESS BEFORE AND AFTER OBESITY TREATMENT}

Anna Ryden, Jan Karlsson, Lars-Olof Persson, Charles Taft, Health Care Research Unit, Jarl S. Torgerson, SOS Secretariat, Internal Medicine, Marianne Sullivan, Health Care Research Unit, Göteborg University, Göteborg, Sweden

The purpose of this study was to explore effect of weight change on disease-specific coping and distress in severely obese persons. The Swedish Obese Subjects (SOS) study is a clinical trial of surgical vs. conventional weight reduction treatment. The Obesity Coping and the Obesity Distress Scales were administered pre- and 2 years posttreatment in 1192 surgical candidates and their conventionally treated counterparts. Structural Equation Modeling (SEM) was used to elucidate the relation between coping and distress. SEM makes simultaneous analysis of several proposed relations between multiple variables possible. The model showed that problem-focused strategies Social Trust and Fighting Spirit were adaptive, i.e. was negatively associated with distress. Emotion-focused Wishful Thinking proved maladaptive, i.e. was positively associated with distress. Fighting Spirit was most strongly related to distress factor Helplessness, Wishful Thinking was most strongly associated with distress factor Intrusion. At follow-up the surgically treated had lost weight, decreased in maladaptive coping and distress. A weight reduction of $>20 \mathrm{~kg}$ also increased adaptive coping and was related to even greater improvement in distress. Half of the conventionally treated gained weight, decreased in adaptive but increased in maladaptive coping leaving distress unchanged. Those losing $<20 \mathrm{~kg}$ decreased in maladaptive coping and thus improved on distress. A small number of the conventionally treated lost $>20 \mathrm{~kg}$ and reported more adaptive and less maladaptive coping, resulting in even greater reduction of distress. In conclusion, increase in weight abated whereas reduction in weight augmented adaptive coping. The greater the changes in weight the greater the change in coping and distress. This lends support to the belief that psychological dysfunction is a result, not a cause, of obesity. Maladaptive coping decreased in all groups suggesting a general intervention effect of receiving professional aid and support. 
320/ HEALTH-RELATED QUALITY OF LIFE IN OBESE SEEKING TREATMENT COMPARED TO NON-OBESE REFERENCE SUBJECTS

Jan Karlsson, Health Care Research Unit, Lars Sjöström, Marianne Sullivan, Health Care Research Unit, Institute of Internal Medicine, Göteborg, Sweden

Obesity is a chronic disease with multiple adverse effects on healthrelated quality of life (HRQL). Although much has been learned in recent years, it is still unclear and debated how obesity affects specific HRQL areas. The aim of the study was to compare obese subjects seeking treatment with non-obese reference subjects and to evaluate the impact of obesity on different HRQL dimensions. Data on 6863 obese and 1017 non-obese, middle-aged subjects included in the Swedish Obese Subjects (SOS) Registry and Reference studies were utilized. According to WHO classification, $10.4 \%$ were obese, $43.2 \%$ were severely obese and $46.2 \%$ were massively obese. Only $0.5 \%$ of the reference subjects were underweight, while $57.9 \%$ were of normal weight and $41.6 \%$ were overweight. A battery of generic and study-specific quality-of-life instruments (SOS-QoL Survey) was used to assess HRQL. Fisher's nonparametric test was used for testing of group differences and effect sizes were calculated to estimate the importance of significant results. Mental well-being (overall mood anxiety, depression, self-esteem) was significantly worse in the obese. The impact was small to moderate in men and moderate to large in women. General health perceptions were less positive in the obese and group differences were large in both genders. Functional health limitations were more common among the obese. The impact was moderate to large and women were more affected than men. Also, psychosocial dysfunction was markedly more common in obese men and women. Eating behavior disturbances caused by intense hunger feelings and uncontrolled eating patterns were frequent in the obese and group differences were large. Overall quality of life ratings were more negative in obese subjects and the affect was moderate in men and large in women. In summary, the impact of obesity on HRQL is substantial in both men and women and concerns physical, psychosocial as well as mental health aspects.

\section{1/ DO MEN AND WOMEN RESPOND DIFFERENTLY TO} WEIGHT CHANGE?

J. Michael Woolley, Jinhai Shi, M. Haim Erder, Amgen Inc., Thousand Oaks, CA

The purpose of this research was to determine whether men and women had similar health-related quality of life (HRQOL) responses to weight change. HRQOL data were collected as part of a clinical trial in obesity where all subjects had a body mass index (BMI, calculated as the weight in kilograms divided by the height in meters squared) between 27.5 and 38 . 141 subjects completed both the SF36 and the Obesity Quality of Life (OBQOL) instrument at baseline and after six months. The OBQOL consists of 77 items measuring 16 HRQOL domains ranging from physical functioning, work, obesity discomfort, and energy, to sexuality, body image, anxiety, and self-esteem. The analysis focused on the effect of gender on perceived changes in HRQOL that resulted from weight change. Specifically, the change in each $H R Q O L$ scale was regressed on the change in $\mathrm{BMI}$, with a gender-change in BMI interaction included. The results indicate that men and women were similar in their responses to weight loss. Of the $16 \mathrm{OBQOL}$ scales analyzed, only one scale showed a statistically significant $(p<0.05)$ difference between men and women and the magnitudes of the differences were small. For the SF-36, there was some evidence of a pattern in responsiveness, as men experienced bigger improvements in the 5 more physical scales, and smaller improvements in the 3 more mental scales. However, none of the gender interactions were statistically significant. Furthermore the magnitude of the coefficients indicated that for a similar one unit change in BMI (about 3 to $4 \mathrm{~kg}$ ), the change in HRQOL for men and women were within 3 points of each other. In sum, the evidence for differential response was weak. It appears that there is not a great difference between men and women in their HRQOL response to weight change.
322/ QUALITY OF LIFE \& PSYCHOLOGICAL DISTRESS AMONG CAREGIVERS OF DEMENTIA PATIENTS IN INDIA

Santosh K. Chaturvedi, Psychiatry, National Institute of Menatl Health

\& Neurosciences, Bangalore, India

Quality of life of caregivers of dementia patients was studied as an International multicentric study including centers from Japan, Russia, Spain and India. The observations made in the study center in India, of 54 dementia patients, are presented here. Quality of life of caregivers of dementia patients in India was assessed using the WHOQOL-Bref scale, and psychological distress assessed using the GHQ-12 scale. Caregivers situation and behaviour problems in the dementia patients was also recorded systematically using a questionnaire. The correlation between QOL scores and psychololgical distress and demographic variables were computed. The subjective perception of QOL on WHOQOL had a mean score of 3.22 (SD 0.42). QOL scores were higher for the male caregivers (3.32), and for caregivers above 60 years (3.60) as compared to the younger ones. The QOL scores didnot vary significantly with gender, age, educational status, their marital status, health, relationship with the dementia patients, the type of residence, family type or whether they received help. $37 \%$ of the caregivers had psychological distress as measured by GHQ-12. The correlation between WHOQOL total score and $G H Q$ was found to be $-0.39(P<0.003)$ confirming poor quality of life in relation to increasing psychological distress. The findings emphasize the importance of detecting and reducing psychological distress among the caregivers in order to improve their quality of life. Other efforts should also be made to improve their quality of life in order to enable them to care for their dementia patients better.

323/ ASSOCIATIONS BETWEEN FIVE-FACTOR MODEL OF PERSONALITY AND QUALITY OF LIFE IN INDONESIAN WOMEN WITH BREAST CANCER

Magdalena S. Halim, Psychology, AtmaJaya Indonesia Catholic University, Jakarta Selatan, Indonesia; Jan J. L. Derksen, Cees P. F. van der Staak, Clinical Psychology and Personality, University of Nijmegen, Nijmegen, The Netherlands

The aim of the present study is to examine the associations between five-factor model of personality (i.e.: Neuroticism, Extraversion, Openness to experience, Agreeableness, and Conscientiousness) and quality of life in Indonesian breast cancer patients. The Revised NEO Personality Inventory (NEO PI-R) together with the Rotterdam Symptom Cheklist (RSCL) were administered to 106 breast cancer patients approximately one month after their surgery in Jakarta. Three months later, 95 of the 106 breast cancer patients completed the second measurement of RSCL. The results showed that Neuroticism (N) was associated with physical distress, psychological distress, and overall quality of life at two different times of measurement $(.05<p<.001)$. Neurotic breast cancer patients, who were particularly high on anxiety, angry hostility, depression, and vulnerability scores, were found to have many physical disturbances, many psychological problems, and a worse overall quality of life. Extraversion (E) and Agreeableness (A) were only associated to QOL at the second time of measurement $(.05<p<.01)$. Those patients high on $E$ and in particular on warmth, assertiveness, and activity facets tended to have a better overall QOL at three months follow-up. Agreeable patients, who were high on trust, compliance, and modesty facets reported less physical and psychological distress. The other two factors of the five-factor model of personality (i.e.: Openness to experience and Conscientiousness) appeared to have no association with QOL either at the first measurement or at three-month follow-up. As a conclusion, the results showed five-factor model of personality to associate significantly - with the exception of Openness and Conscientiousness - with QOL in Indonesian breast cancer patients. Furthermore, it might also indicate a possibility that $\mathrm{N}, \mathrm{E}$, and A could be considered as predictors of QOL. 
324/ QUALITY OF LIFE AND MENTAL HEALTH IN YOUNG IRANIAN POPULATION

Ali Montazeri, Kazem Hosseini, Jila Sadighi, Mohammad Shariati, Farzaneh Maftoon, Mohammad Heydari, Iranian Institute of Health Sciences Research, Tehran, Iran

It is well known that Iran has a very young population. To assess global quality of life and mental health in this population and to examine the relationship between global quality of life and mental health a cross sectional study was carried out in Tehran, Iran. Using a single measure of global quality of life ( ranging from extremely poor to excellent) and the General Health Questionnaire (GHQ-12), a sample of 748 young people aged 18 to 25 years old were entered into the study. The mean age of the respondents was $21.1(\mathrm{sd}=2.1)$ and most were female $(76 \%)$, single $(84 \%)$ and college/university student $(51 \%)$. While $10 \%$ of the respondents reported an extremely poor, poor and rather poor global quality of life, $28 \%$ indicated neither good nor bad, and $31 \%$ moderately good global quality of life. On the other hand, the GHQ mean score was found to be $3.7(s d=3.5)$. Considering the cut-off threshold (people scoring above the mean), the result indicated that about $44 \%$ of the respondents scored high on the GHQ and showed an indication of a mental health disorder. However, there were significant differences between the respondentsâ $€^{\mathrm{TM}} \mathrm{GHQ}$ score and their marital, educational, and employment status $(P=0.005, P$ $<0.00001, \mathrm{P}=0.0003$ respectively). In addition, a multiple logistic regression analysis showed that global quality of life was the most significant predictor of mental health $(P<0.00001)$. The study findings suggest that mental health in young people is poor and it is strongly associated with their quality of life. Since poor mental health in young people could be regarded as risk factor for psychological disorders such as antisocial behaviour, criminal activity, suicidal behaviour, substance abuse, depression, and eating disorders, the importance of improving quality of life in this age group becomes selfevident.

\section{5/ ISSUES INVOLVED IN ASSESSING QUALITY OF LIFE IN BI-} POLAR DISORDER

Stephen P. McKenna, PhD., David M. Meads, BS.c., Sharon A. Cook, BS.c., Galen Research, Manchester Science Park, Manchester, UK; Mairead M. Kearney, MD, Global Health Economics and Disease Management, Novartis Pharma AG, Basel, Switzerland

Bipolar disorder (BPD) is a severe chronic mental illness affecting between 1 and $2 \%$ of the population. BPD presents a particular challenge to researchers wishing to assess quality of life (QoL) as the disease is characterised by two quite different health states; mania and depression. A literature search failed to identify any QoL instruments specific to BPD, with reliance in clinical studies being placed on generic measures of health status or mental health. Several authors question the validity of information given by respondents who are in a manic state and difficulty can also be found in gaining responses from individuals who are severely depressed. The assessment of QoL in such extreme states could be considered less important than clinical judgement of symptom severity. However, QoL assessment is essential in longer term prophylactic treatment studies where the aim is to prevent recurrence of (hypo)manic/depressive symptoms. Studies also indicate that there is psychosocial impairment during the inter-episode intervals. One reason for the absence of a QoL measure specific to BPD could be the difficulty of producing an instrument that works well in both manic and depressed states. A potentially useful approach would be to develop a measure that adopts the needs-based model of QoL. Qualitative unstructured interviews with individuals in both manic and depressed stages of the illness would be conducted to identify needs that were affected by both mania and depression. This would ensure that the instrument's content was relevant to all potential respondents at all stages of their illness.
326/ COGNITIVE FACTORS IN SYMPTOM ASSESSMENT: ARE WE ASKING TOO MUCH?

Deborah L. Jonas, Nancy Kline Leidy, MEDTAP International, Inc., Bethesda, MD; Cheryl C. Silberman, AstraZeneca, Wilmington, DE; Mary Kay Margolis, MEDTAP International, Inc., Bethesda, MD; Anne Heyes, AstraZeneca, Cheshire, UK

Measures of symptom distress ask people to attribute their functioning and well being to a particular symptom with the goal of understanding how symptoms impact patients' lives. However, limits in cognitive functioning can lead to inaccurate and incomplete reporting, and problems with interpretation. For example, patients may easily attribute their decreased ability to walk to increased breathlessness. However, patients may not attribute other outcomes, such as decreased social activities, to breathlessness, even when there is a causal relationship. Measures of symptom distress, which force subjects to consider relationships that may or may not be real, are unlikely to capture the comprehensive impact of symptoms on patients' lives. When participants experience multiple symptoms, accurate attributions may be particularly difficult. Cancer patients are often asked "how much trouble have you had in the last 2 weeks as a result of being nauseated?" (Breast Chemotherapy Questionnaire). If individuals also experience pain, vomiting, or other symptoms, their responses may over- or underestimate the impact of nausea on outcomes. Interpreting change in health status based on measures of symptom distress can also be problematic. These measures simultaneously ask people to determine 1) the degree of symptom experience and 2) how much the experience troubles them. To respond, individuals are required to think about the relationship between two concepts. If responses change following an intervention, there are at least two possible causes. One is that the symptom subsided, the other is that study participants accommodated to their situation and no longer perceived their symptoms as troublesome. Measures of symptom distress make it impossible to distinguish between these two outcomes. This presentation will further explicate the cognitive implications of symptom attributions on health outcome measures and recommend ways to avoid the cognitive pitfalls of asking too much.

327/ WAS IT A GOOD MOVE? IMPROVEMENT IN QUALITY OF LIFE AMONG CHRONIC MENTAL PATIENTS MOVING FROM A HOSPITAL TO A HOSTEL

Roberto Umansky, Division of Psychiatry, Marianne Amir, Dafna Chen, Department of Behavioral Sciences, Boris Nemetz, Monika Friedman, Etty Zidon, Division of Psychiatry, Ben-Gurion University of the Negev, Beer-Sheva, Israel

This study examined whether transition from mental hospital to rehabilitation hostel improved chronic mental patients' quality of life. This longitudinal, prospective study compared the quality of life of 16 patients who moved from the mental hospital to a hostel with that of 20 matched patients who remained hospitalized. We hypothesized that the patients moving to a hostel would exhibit improved quality of life and that the quality of life of patients remaining hospitalized would not improve. The participants were administered a generic quality of life instrument, the WHOQOL-Bref and a mental health specific questionnaire, Lehman's TL 30S questionnaire, before the move and again six months later. The hospitalized patients were administered the questionnaires at the same time points. We also assessed the similarity of the psychiatric patients' self-reports with the evaluation of the medical staff. The findings supported the hypotheses: No changes were reported in the quality of life of hospitalized patients, while those who moved to the hostel exhibited significant improvement in all aspects of life, as reflected by both patient and staff report on the WHOQOL-Bref and subjective indicators in the TL $30 \mathrm{~S}$ questionnaire. Patients' self-reports reflected a higher quality of life improvement than staff reports, but both displayed similar trends regarding changes in quality of life. 
328/ IMPACT OF DEPRESSIVE SYMPTOMS IN SOCIAL FUNCTIONING AND QUALITY OF LIFE IN A BRAZILIAN PRIMARY CARE SERVICE USERS

Marcelo P. Fleck, Ana Flávia B. Lima, Sérgio N. Louzada, Gustavo Schestatsky, Alexandre Henriques, Vivian R. Borges, Suzi Camey, LIDO Group, Psychiatry, Universidade Federal do Rio Grande do Sul, Porto Alegre, RS, Brazil

Depressive disorders constitute a major public health problem due to their high prevalence and psycho-social impact. Depressed patients are assiduous users of primary care services, even though they are often not diagnosed as such. The objective of this study is to describe and to evaluate depressive symptoms in individuals that seek primary care services in Porto Alegre, south Brazil, and to characterize those patients with respect to impacts quality of life and social functioning. 2201 primary care users were evaluated in the city of Porto Alegre, south Brazil in relation to their physical and emotional health using the following instruments: 2 questions from the World Health Organization Quality of Life instrument (WHOQOL-Bref); Medical Outcomes Study Short-Form 12 (SF-12) and MHI 5 (MHI-5); Centers for Epidemiologic Studies - Depression (CES-D), plus additional questions about work loss days and health care utilization. $79.5 \%$ of the individuals were female, avering 40 years of age. The intensity of depressive symptoms (measured by CES-D) was 20.2 for women and 16.2 for men. All the appraised parameters had an inverse relationship with the intensity of depressive symptoms. Patients with worse perceptions of their health, worse life quality, worse social functioning, more days as inpatients, larger number of medical consultations and more missing working days presented larger intensity of depressive symptoms. The data reinforce findings in other countries that depressive symptoms have a strong impact in social functioning, quality of life and health resource utilization in primary care patients. Keywords LIDO Study - Depression - Primary Care - Quality of life Social Functioning - Evaluation

\section{9/ QUALITY OF LIFE FOR POOR AFRICAN-AMERICAN WOMEN WITH DEPRESSION}

Lori Frank, Dennis Revicki, MEDTAP International, Inc., Bethesda, MD; Kelly Grotzinger, Global Health Outcomes, GlaxoSmithKline, Collegeville, PA

Little is known about the mental health needs and associated quality of life of poor African-American (AA) women in the US. The WE Care study is designed to describe and address the mental health needs of poor, depressed AA, Latina, and White women. Subjects were screened for depression while obtaining health care or other social services. Prior to treatment, subjects were administered the Hamilton Depression and Anxiety Rating Scales (HAM-D, HAM-A) and the SF36. A total of $114 \mathrm{AA}$ women met criteria for depression (HAM-D>16), mean age 28.8( \pm 8.4$)$. Compared with age-adjusted US norms for females, SF-36 scores for the depressed AA women were lower on all 8 SF-36 subscales, MCS, and PCS $(p<0.05)$; results were the same when comparison was made to non-depressed $A A$ women from the study $(\mathrm{N}=30)$. Relative to White and Latina depressed women in the sample $(\mathrm{N}=100) \mathrm{SF}-36$ scores for the AA women were lower on 5 subscales (role-physical, general health, vitality, social functioning, role-emotional) and the MCS $(p<0.05)$. Because depression with comorbid anxiety may be associated with poorer quality of life than depression alone, SF-36 scores for AA women with comorbid anxiety (HAM-A>12) were compared to scores for those with depression alone. Scores were lower for all but the vitality subscale $(p<0.05)$. All subscale and component summary scores were significantly and negatively correlated with HAM-A scores ( $r=-0.22$ to -0.43$)$; only 2 subscales were not correlated with HAM-D score $(p<0.05)$. Age was negatively correlated with physical functioning, bodily pain, general health, PCS and MCS scores ( $r=-0.21$ to -0.36$)$. All but 9 depressed subjects had at least 1 child (range 0 to 6 ); number of children was negatively correlated with physical functioning, role-physical, bodily pain, general health, and PCS scores (range $r=-0.22$ to -0.36 ; $p<0.05$ ). Results indicate impaired quality of life for poor African-American women with depression relative to depressed White and Latina women. Presence of comorbid anxiety is associated with lower quality of life than depression alone.
330/ SOCIAL SUPPORT AND QUALITY OF LIFE IN CHRONIC MENTAL DISEASE PATIENTS SUBMITTED TO DIFFERENT HOUSING TREATMENT PROGRAMS

Jose L. Pais-Ribeiro, Faculdade de Psicologia, Universidade do Porto, Porto, Portugal; Clara Guterres, ISPA, ISPA, Lisboa, Portugal

The aims of the present study is to inspect the impact of Social Support and Housing on the Quality of Life (QOL) of long term controlled mental health patients submitted to two different housing program treatment Participants where 123 long-term mental health patients $(63.4 \%$ males) living in two conditions: Living with Family with outpatient day care support (56.9\%)(A group) and; Community House treatment program (43.1\%). Material used includes the "Escala de Suporte Social Percebido", a Portuguese Social Support Scale, which evaluates Perceived Social Support, with five dimensions, Satisfaction with the Friendship, Satisfaction with Intimate Friend Relationships, Satisfaction with Family, Satisfaction with Social Activities, and Satisfaction with Neighbours, and the Portuguese adaptation of the WHOQOL Bref. The WHOQOL Group developed a quality of life questionnaire with 100 items. Recently produced an abbreviated form with 26 items covering four domains: Physical Capacity, Psychological, Social Relationships, and Environment. Results show no differences in QOL and Perceived Social Support between the two housing treatment groups. Correlation's between dimensions of Social Support and dimensions of QOL vary between the two treatment groups: Correlation's between QOL domains and Satisfaction with Intimate Friend Relationships, Satisfaction with the Family, and Satisfaction with Neighbours are positive and statistically significant for the Community House treatment Group and not for the Living With The Family Group: Correlation's between Social Activities and QOL domains are negative and statistically significant for the Living With The Family treatment group and not for the Community House Group. Results suggest that the two kind of treatment can induce satisfaction with different aspects or domains of Social Support, resulting in similar impact on QOL.

\section{1/ DOES CHANGE IN DEPRESSION STATUS PREDICT} CHANGE IN HRQOL?

Marianne Amir, Behavioral Sciences, Ben-Gurion University of the Negev, Beer-Sheva, Israel; Stephen McKenna, Galen Research, Manchester, UK; Mona L. Martin, Donald M. Bushnell, Health Research Associates, Inc., Seattle, WA; Diane Whalley, Galen Research, Manchester, UK; Donald L. Patrick, Department of Health Services, University of Washington, Seattle, WA

A strong association between depressive symptoms and quality of life (QOL) has been established throughout the literature. The objective of the present report was to investigate the changes in QOL as a result of changes in depression or the correlates of depression in the LIDO Study. The relationship between treatment and change in QOL was also examined. The sample consists of all enrolled patients $(\mathrm{N}=$ 1117) across six sites (Israel, Spain, Australia, Brazil, Russia, and the United States). Using the baseline and 9-month assessment of the quality of life measures (WHOQOL-Bref, QLDS, and SF-12), and the measures of depression status at 9 months (CIDI and CES-D scores), we examined the associations between changes in QOL scores and depression status, hypothesizing that patients who have less depressive symptoms, or who were no longer clinically depressed, should show improvements in QOL. To assess the relationship between QOL and treatment, we compared the enrolled patients having any mental health treatment with those receiving no treatment. We expected participants having undergone treatment to improve more than others with regard to QOL measures, even when controlling for initial depressive symptoms. Results showed that changes in depression symptom scores (by CES-D) were significantly associated with changes in QOL scores (WHOQOL-Bref Psychological 0.69, QLDS 0.67, and SF-12 MCS 0.67). In addition, treatment significantly improved quality of life scores. Those with anti-depressant treatment improved by 2.0 points on the WHOQOL-Bref Psychological scale compared to 1.1 points for those not receiving medication $(F=15.8$, $\mathrm{p}<0.001$ ); and 5.0 points on the QLDS for treatment versus 3.1 points for no treatment $(F=8.4, p<0.01)$. Therefore, this study demonstrates a significant association between depressive symptoms and multiple HrQOL indicators. 
332/ HEALTHCARE RESOURCE USE AMONG CAREGIVERS OF PEOPLE WITH SCHIZOPHRENIA

Richard E. White, Audra N. Boscoe, Health Economics and Outcomes Research, John Travers, Senior Medical Director, AstraZeneca, Wilmington, DE; Diana D. McDonnell, Consumer Health Sciences, Princeton, NJ

With increasing medical costs, the health of schizophrenia caregivers cannot be ignored. This research evaluated healthcare resource use among caregivers of people with varying severities of schizophrenia. In June 2000, 376 schizophrenia caregivers from national support groups completed self-administered questionnaires. They reported whether they were hospitalized $(17 \%)$, visited the ER $(19 \%)$, or had physicians' appointments (48\%) in the past 6 months. Caregivers rated schizophrenia severity by reporting how often (on a scale from 0 to 4 ) their patient experienced 23 problems. Scores were then computed as the overall mean. Logistic regression was used to control for other variables that could confound resource use - caregivers' demographics as well as overall mental and physical health, and patients' demographics, time with schizophrenia, and antipsychotic use. Controlling for these confounders, caregivers of people with severe schizophrenia were about 3 times more likely to have been hospitalized $(O R=2.8$ $p<.001)$ or to have visited an ER $(O R=3.1, p<.001)$ in the previous 6 months. The effect of severity on the number of physician visits was not significant $(p=0.798)$. Medications and other strategies that help control patients' severity - or help maintain people at lower severity may reduce caregivers' use of hospitals and emergency rooms and ultimately lower the overall costs associated with treating schizophrenia.

333/ MENTAL WELL BEING AMONG CAREGIVERS OF PEOPLE WITH SCHIZOPHRENIA

John Travers, Senior Medical Director, Richard E. White, Audra N Boscoe, Health Economics and Outcomes Research, AstraZeneca, Wilmington, DE; Diana D. McDonnell, Consumer Health Sciences, Princeton, NJ

Caregivers' mental health, and the effect of schizophrenia severity, may be critical to patients' quality of care. This research therefore evaluates how the symptoms of schizophrenia affect the mental well being of those providing informal patient care. In June 2000,376 schizophrenia caregivers from national support groups completed selfadministered questionnaires. Mental well being was measured using the SF-12 (Ware, et al 1996). Schizophrenia symptoms were evaluated as a 4-level variable: high negative/high positive (35\%), high negative/low positive (17\%), low negative/high positive $(10 \%)$, and low negative/low positive (38\%). To control for confounders to caregivers' well being - caregivers' demographics and involvement, and patients' demographics and time with schizophrenia - a linear regression model was used. The mean SF-12 score was 48.7 ( $S D \pm 10.6)$. In bivariate, chi-squared analysis, caregivers' mental well being decreased as schizophrenia symptoms increased $(p<.001)$. Controlling for confounders, symptom severity remained significant. Caregivers of people with low positive and negative symptoms had average SF-12 scores 6 points higher than those caring for people with high symptoms $(p<.001)$. Even caregivers of people with only high positive symptoms scored about 5 points higher $(p=.015)$. Caregivers of people with high negative symptoms did not differ from those with both high negative and positive symptoms $(p=.616)$. Medications and strategies that help control patients' symptoms, especially negative symptoms, can also help caregivers experience more positive well being.
334/ FACTOR STRUCTURE OF THE QUALITY OF LIFE IN REFLUX AND DYSPEPSIA (QOLRAD) QUESTIONNAIRE EVALUATED IN PATIENTS WITH HEARTBURN PREDOMINANT REFLUX DISEASE

Károly R. Kulich, Ingela Wiklund, Outcomes Research, Ola Junghard, Statistician, AstraZeneca R\&D Mölndal, Mölndal, Sweden

The aim of this study was to verify and confirm the original factor structure of the QOLRAD in two different populations: a) patients with Nordic languages and b) English-speaking patients. Clinical trial patients with heartburn without esophagitis completed the Nordic versions $(\mathrm{N}=634)$ and the English version $(\mathrm{N}=1185)$. The factor structure was examined using models generated by exploratory and confirmatory factor analysis. The exploratory factor analysis confirmed the original five-factor structure in both populations. The Nordic versions explained $67 \%$ and the English version $72 \%$ of the variance. The factor loading of twenty-three out of twenty-five items was > .55. A confirmatory factor analysis indicated an acceptable goodness of fit of the five-factor solution to the data with a goodness-of-fit index of 0.88 in population a) and 0.91 in population $b$ ). The internal consistency reliability ranged from 0.70 to 0.94 (in the Nordic versions) and from $0.85-0.92$ (in the English version) supporting the homogeneity of the items within the factors and thus their construct validity. The QOLRAD was sensitive to differences in severity and frequency of heartburn. The factor structure and dimensionality of the original QOLRAD was confirmed by both the exploratory and confirmatory factor analysis. The QOLRAD is a valid instrument for use in clinical trials. Keywords: Health-related quality of life, Dyspepsia, Gastroesophageal reflux disease, Factor analysis, Psychometric documentation.

335/ USING THE MANN-WHITNEY STATISTIC TO IMPROVE THE INTERPRETATION OF ORDINAL SCALES: AN APPLICATION TO THE PATIENT SATISFACTION WITH INSULIN THERAPY QUESTIONNAIRE

Joseph C. Cappelleri, Robert A. Gerber, Global Research \& Development, Pfizer Inc., Groton, CT; Avanish K. Mishra, Pfizer Pharmaceuticals Group, Pfizer Inc., New York, NY

Our objective is to show and illustrate how the $U$ statistic from the Mann-Whitney test, a powerful nonparametric test to compare two groups, can improve the interpretation of ordinal scales. Subjects with diabetes, who were interested in participating in an inhaled insulin (INH) study, from two Phase 2 trials were pooled and randomized to either a regimen including INH $(n=63)$ or only subcutaneous insulin $(n=65)$ for 3 months (study end). The Mann-Whitney $U$ statistic on the change score, from baseline to 3 months, was derived for each of 15 items on the Patient Satisfaction with Insulin Therapy Questionnaire (Diabetes Care 23:1799-1803, 2000). Subjects indicated, at baseline and study end, their level of agreement with each satisfaction item using a 5-point Likert scale (strongly disagree to strongly agree). In addition, the 5-point Likert scale item "I would like to continue to take insulin the way I took it during the study" was asked at study end. The Mann-Whitney $U$ statistic resulted in a probability that a randomly chosen subject given the INH regimen will have more satisfaction, from baseline to study end, than a randomly chosen subject given subcutaneous (SC) insulin. This probability was higher, favoring the $\mathrm{INH}$ regimen, on 14 of the 16 items (the two items that favored SC insulin were not significant, $p>0.05$ ) and statistically so on 7 items $(p<0.05)$. For example, there was a $73.2 \%$ chance $(p=0.0001)$ that a subject given INH therapy would have more convenience than a subject given SC therapy, and there was a $74.6 \%$ chance $(p=0.0001)$ that a subject given INH therapy would want to continue with that therapy than a subject given SC therapy would want to continue with SC therapy. Converting the Mann-Whitney U statistic into a meaningful and useful probability can enhance the interpretation of ordinal scales for the comparison of two groups. 
336/ VALID RESULTS DESPITE MISSING DATA?

Corina Guethlin, Harald Walach, Institute of Environmental Medicine \& Hospital Epidemiology, University Hospital, Freiburg, Germany

Measuring quality of life is one of the central issues nowadays. Longitudinal designs provide the most valid quality-of-life-data, but collecting data over a long period of time implies an increase of missing data, too. Depending on the pattern of missings, one can distinguish between "missing completely at random" (MCAR) and "missing at random" (MAR) or "not missing at random" (NMAR). There is no consensus in recommendations about statistical procedures in imputing missing data. We collected quality-of-life-data in a longitudinal observational study about the effects of acupuncture and homoepathy in 5000 patients over 4 years, using the MOS SF-36. The patients had to fill in questionnaires pre and post treatment and 1 time per year. Other outcome criteria were the doctors' and the patients rating of the improvement. Depending on the imputing routine we analysed the pre-posttreatment change of 500 or 350 patients. The pattern of missing data is MAR. Our results show an increase in quality of life between $6 \%$ and $19 \%$ (pre/posttreatment) depending on the scale, using only the complete cases. The effect size for physical functioning is $d=.44$ (Power=1.0). Imputing missing data with the mean plus 2 standard deviations the increase is between $18 \%$ and $46 \%$, the effect size is $d=.75$ (Power=1.0). Imputing with the mean minus 2 standard devices there is a decrease between $2 \%$ and $14 \%$ (results are negative), the effect size is $d=.16$ (Power= .61). Imputation routines like regression analyses or propensity score show nearly the same curve over the first year after treatment as the curve without any imputation do. The effect size using complete cases is medium sized while the non-conservative estimation suggest a large effect. The conservative way of imputation provide the impression of no effect. Astonishingly we found no difference between the imputation routines nor between the data with and without imputation (regression, propensity score).

\section{7/ THE DEVELOPMENT AND UTILISATION OF THE EORTC QUALITY OF LIFE ITEM BANK}

Stephanie Vachalec, Andrew Bottomley, Quality of Life Unit, EORTC Data Center, Brussels, Belgium; Jane Blazeby, Department of Surgery, Bristol Royal Infirmary, Bristol, UK; Pascal Ruyskart, IT Unit, EORTC Data Center, Brussels, Belgium; Henning Flechtner, Klinik fuer Psychiatrie und Psychotherapie des Kinde, EORTC Data Center, Koeln, Germany; Kristin Bjordal, Radiotherapy \& Oncology, Norwegian Radium Hospital, Montebello, Norway

The EORTC Quality of Life Group has developed a modular approach to assessing patients' Quality of Life (QL) in cancer clinical trials. Based on this approach, a number of modules specific to tumor sites, treatment modalities or QL dimensions not included in the EORTC QLQ$\mathrm{C} 30$ have been developed to be administered with the EORTC QLQ$\mathrm{C} 30$. The number of EORTC modules has rapidly increased, resulting in a degree of overlap between Items and their numerous translations. In addition, we have seen an increasing demand for trial specific Items, a demand, which could not be met with the present module development process. A computerized database of all existing EORTC Items and translations was developed. This aimed to improve the quality and the speed of development as well as translations of new modules, in addition to providing a database of valid items for trial specific questions. Over two years of development have been necessary to finalize the development of the database and to store and classify over 400 Items from all current EORTC modules and more than 5400 translations Initial results of this Internet based instrument are positive. It has been used by EORTC members during the development process of new EORTC QL modules for High Dose Chemotherapy, Ophthalmic and Chronic Lymphocytic Leukemia. Initial feedback from module developers has been positive, and quality and speed have increased in the module development process. In addition, it has been used by EORTC members for selection of additional validated QL items to be used in EORTC Phase III clinical trials involving Gynecological and Genito-urinary patients. Our initial results suggest that the approach on computerizing items from EORTC $\mathrm{QL}$ measures is successful, and has led to both improved use of QL items in our clinical trials, speeded up the process of selecting items for such trials and also speeded up the module development process. We plan to systematically evaluate the tools' use after one year of use within the EORTC. Presently, we are considering the possibility of linking real time data to QL items with a possibility of making the Item Bank a more functional research based tool.
338/ CLINICAL POTENTIAL OF LONGITUDINAL QOL PROFILES Barbara A. Elliott, Department of Family Medicine, UMD-School of Medicine, Duluth, MN; Ronald R. Regal, Department of Mathematics and Statistics, UMD, Duluth, MN; Colleen M. Renier, Thomas E. Elliott, Division of Education and Research, SMDC, Duluth, MN

The purpose of this study was to describe the longitudinal QoL scores among people with stages $0,1,2$, and 3 breast cancer or colo-rectal cancer. In a study designed to enhance rural practitioners' cancer care, patient outcomes including QoL were measured. A population of 237 recently diagnosed breast and 103 colo-rectal cancer patients in 13 rural Midwestern (USA) communities were interviewed and invited to complete QoL (FLIC) forms nine times over 2 years: at baseline (within one month of diagnosis) and at 2, 4, 6, 9, 12, 15, 18, and 24 months. The resulting profiles were analyzed with mixed models repeated measures techniques. Statistically significant effects on overall QoL profiles for these patients included the length of time since diagnosis $(p=0.0001)$, type of treatment being experienced (chemo vs. no chemo: $p<0.05$ ), and the patients' scores on the Sense of Coherence (SOC) scale $(p=0.0001)$. Statistically significant effects on subscale profiles included time $(p=0.0001)$ and age $(p<0.05)$ on the physical, psychological, and social scores; and time $(p=0.0001)$, treatment $(p<0.0005)$ and cancer type $(p<0.01)$ on nausea and hardships scores. There were also statistically significant interactions between time, treatment age, and cancer type for total as well as subscale QoL scores. The subscale and total profiles can be used clinically to compare patients' profiles to standard profiles, given the patients' background, including age and SOC. Discussion of the findings and clinical implications will be included in the presentation.

\section{9/ METHODS FOR INTEGRATING THE SF-12 INTO A ROUTINE} CENSUS BASED HOUSEHOLD SURVEY

Franz Piribauer, Public Health Directorate, State-Government, Graz, Styria, Austria; Inge Kirchberger, Quali-Team Augsburg, Quality of Life Consulting Company, Augsburg, Bavaria, Germany; Elisabeth Santigli, University Clinic for Dental Medicine, University of Graz, Graz, Styria, Austria; Jeannette Klimont, Health and Population Statistics, Statistics Austria Vienna, Vienna, Austria

Measuring the health status of a nation may be costly. Methods to reduce costs will help to increase the regular and representative monitoring of HRQOL in large populations. In September 1999 we could modify the delivery of one routine household interview survey in Austria. Such "Microcensus" surveys are applied in Austria and Germany by the federal statistical offices regularly. On average in Austria ( 8 Mio. inhabitants) in $70 \%$ of the 30.800 finally selected housing units 60.000 persons can be addressed by the interviewers four times a year since 1967. Most interviewers have long - time contracts and are picked from the local communes. The 131 interviewers in the Styrian region (1,2 Mio inhabitants) were instructed to perform the additional part; including the SF-12 and 4 four short questions on blood pressure measurement and cholesterol screening at the end of the interview. The machine-readable interview form requested from the interviewer him/herself to answer finally, if SF-12 items, and if yes what items, had to be explained. Incentives to raise response rates were created with additional fees for the interviewers and family weekend of choice in an upper class skiing resort or in thermal spa hotel for the households. 3 EUROs were spent per returned interview form, and 4 luxury weekends for the households. In 2.640 Styrian households data on 5.881 persons were collected, as a second round of telephone contacts to 92 interviewers could reduce the number of machine-unreadable forms from 503 to 17 . As our regional sample data can be linked via an identifier to the Microcensus data, conclusions from Styria to Austrian population can be drawn, and advantage can be taken of the extensive time-series which are available on a score of variables. Reducing costs of surveying by our "Microcensus" method may have as a shortcoming a social response bias due to the familiarity of the interviewers with their communes. 
340/ CHANGE IN QUALITY OF LIFE IN RELATION TO HEALTHRELATED BEHAVIOUR

Wolfgang Weidenhammer, Centre for Complementary Medicine Research, Department of Internal Medicine II, Technical University Munich, München, Germany; Stefanie Wunderlich, Sanitas Dr.KöhlerParkkliniken, Hospital for Complementary Medicine, Bad Elster, Germany; Dieter Melchart, Centre for Complementary Medicine Research, Department of Internal Medicine II, Technical University Munich, München, Germany

A major intention of complementary medicine is change in healthrelated behaviour in order to prevent morbidity or to improve coping with chronic diseases. This approach is triggered by improving quality of life (QoL). Which relations exist between $\mathrm{QoL}$ and behaviour and its treatment induced changes? A sample of 240 in-patients treated with complementary medicine was investigated within a prospective cohort study. Two questionnaires (SF-36 and FEG, an assessment instrument for health-related behaviour) were handed to the patients twice, before treatment and 6 months after. Main diagnostic groups were neurasthenia, back pain syndromes and headache with a median duration of disease of 8 years. Mean age of the patients was $52.6+/-13.7$ years, $78.8 \%$ of the patients were female. The results of the SF-36 are presented in terms of the standard values of the component scores PCS (Physical component) and MCS (Mental component). For the FEG sex and age adjusted standard scores were used. Before treatment $Q$ oL in average was reduced in patients under investigation (mean PCS 39.9 resp. MCS 41.0). The increase in QoL and the improvement in behavioural aspects covering feeding and physical activity were statistically significant comparing pre and post measurements. Effect sizes were higher in QoL (PCS 0.475 resp. MCS 0.426 ) than in behaviour (range from 0.162 to 0.421 ). Regarding the pre measurements the only significant correlations could be found between the mental health component of QoL and behavioural aspects. The pre-post-differences correlated weakly. The most prominent coefficient (Spearman's rho $=0.225$ ) was found for change in physical health and change in satisfaction with physical activity. The results suggest no clear coincidence of improvements in QoL and changes in health-related behaviour. Closer relationship was found when cognitive evaluation of behavioural aspects is considered.

341/ MULTIPLE FACTOR ANALYSIS: APPLICATION IN THE VALIDATION OF MULTI-ITEM SCALES

Benoit Arnould, Patrick Marquis, Mapi Values, Lyon, France; Jérôme Pagès, ENSAR-INSFA, Rennes, France

The discussion of the metrologic properties of scores calculated from multi-item scales is essential in Health Related Quality of Life (HRQL) assessment. Therefore, we need tools and criteria to assess: 1/ Structural stability for the validation of score comparison in populations changing over time and between cross-cultural versions; 2/ Fitness to the conceptual model for the development of short forms; 3/ Clinical validity; 4/ Conceptual equivalence and overlapping, for concurrent validity and battery assessment; 5 / Overall concordance for inter-rater reliability and comparison of patient's and physician's perspectives. For all these issues, it is necessary to analyse different datasets together. Our solution is to build multiple tables in which a same population of patients is described by several sets of variables. Each set of variables induces a specific configuration of patients population. The comparison of these configurations is a classical methodological issue in statistical science. Among the available methods, Multiple Factor Analysis (MFA) has many interesting properties with direct practical application: the different sets of variables are first weighted in order to balance their importance in the consecutive global analysis; the factors common to the different sets of variables are highlighted; several correlation coefficients are provided to drive the interpretation. Detailed listings support accurate analysis of the significance of the results, while graphical displays support a synthetic view over the data. We describe several applications of MFA to various instruments used in therapeutic areas such as schizophrenia, alzheimer disease, veinous insufficiency, erectile dysfunction, upper gastro-intestinal disorders, and urinary incontinence. Reference values for the correlations levels are proposed for the interpretation of results in the different applications.
342/ COHEN CONFIRMED? EMPIRICAL EFFECT SIZES FOR THE QLQ-C30

Madeleine T. King, Centre for Health Economics Research \& Evaluation, University of Sydney, Sydney, NSW, Australia

Effect sizes (ES) are useful for interpreting health-related quality of life (QOL) outcomes. The guidelines Cohen proposed for "small", "medium" and "large" ES are widely used. Cohen described these as "arbitrary conventions ... recommended for use only when no better basis for estimating the effect size is available". Available evidence for QLQ-C30 was collated to confirm or replace Cohen's guidelines with empirically based ones. Groups of patients defined by clinical criteria were used to determine the relative size of differences in mean QOL scores, and ES was calculated with the SD of QOL at baseline. Changes observed during familiar treatments were used to determine the relative size of changes in QOL, and ES was calculated with the SD of change in QOL. A literature search identified 55 studies that reported QLQ-C30 scores. Valid estimates of change in QOL were provided by 26 studies. For each scale, the median sample SD was used to calculate ES. For all but three scales (role, emotional, cognitive function), a difference of up to 2 points was unlikely to have clinical relevance ("trivial"), a difference of about 5 points was relatively small but may have been clinically important ("small"), a difference of about 10 points was likely to have clinical significance ("moderate"), and a difference of 20 or more was relatively large and had clear clinical relevance ("large"). These generalities held whether differences were measured cross-sectionally or longitudinally, and for all scales except the role function scale on which larger differences and changes were recorded. There was insufficient evidence for the emotional and cognitive function scales to judge the relative size of effects. The sampling distribution of standard deviations varied somewhat among scales, and the SD of change tended to be a little smaller than the SD at baseline. Nowithstanding such variation, the empirical ES were generally similar to Cohen's guidelines for small $(0.2)$, medium (0.5) and large (0.8).

\section{3/ TESTING RASCH ITEM ANALYSIS}

Jeremy C. Hobart, Neurological Outcome Measures Unit, Institute of Neurology, London, UK; Benjamin D. Wright, MESA Psychometric Laboratory, University of Chicago, Chicago, IL; Afsane Riazi, Alan J. Thompson, Neurological Outcome Measures Unit, Institute of Neurology, London, UK

Summed rating scales have important limitations. Total scores (indicators of patients' health) are dependent on the rating scale used, and item descriptive statistics (indicators of item difficulty) are sample dependent. Thus, accurate comparisons of studies that use different rating scales are difficult. Rasch analysis claims to overcome this problem by estimating patient measures that are "scale-free" and item calibrations that are "sample-free". We tested these assumptions by comparing estimates of patients' physical health generated by four subsets of items chosen from the 20-item physical component of the Multiple Sclerosis Impact Scale (MSIS-29). And, we examined the stability of item calibrations across multiple subsamples. Data from 713 MS patients were examined. Agreement between estimates generated by the four subsets were determined using product-moment correlations, intraclass correlations, and scatterplots. Agreement was high $(r>0.94$, ICC $=0.95)$. Scatterplots confirmed that all four subsets generated health estimates within the confidence intervals of estimates generated by each other, and by all 20 -items. Item calibrations were stable across multiple subsamples defined by gender, age, and social class, but differed with disability level. Nevertheless, scatterplots confirmed the similarity of patient measures generated using these different item calibrations. These findings support claims that Rasch analysis can generate scale-free health estimates from ordinal-level rating scale data. Although item calibrations showed irregularities, these did not significantly affect estimates of patients' measures. These findings lay the foundation for instrument equating and computer adative measurement. 
344/ UNDERSTANDING CHANGE OVER TIME IN THE SELFRATED HEALTH OF SURVIVING OLDER ADULTS

Kathleen W. Wyrwich, Division of Health Services Research, Saint Louis University, St. Louis, MO; Robert B. Wallace, College of Public Health, University of lowa, lowa City, IA; Fredric D. Wolinsky, School of Public Health, Saint Louis University, St. Louis, MO

Self-rated or self-reported health (SRH) is gaining increased attention as an important indicator of health-related quality of life, yet very little is know about how this measure changes over time in older adults who continue to survive. This study explores changes in SRH and the effect of baseline characteristics on these SRH changes among surviving participants of the lowa Established Populations for the Epidemiologic Studies of the Elderly (EPESE) cohort (1982-1992). Using hierarchical linear modeling techniques, we developed separate models for estimating the intercept and slope parameters of SRH measurements over time among healthy (excellent or good $\mathrm{SRH}$ at baseline) and unhealthy (fair, poor or very poor SRH at baseline) women and men. Each model incorporated the effects of baseline characteristics (demographics, health status, cognitive impairment depression, and functional limitations) on the predicted intercepts and slopes. Generally, SRH slowly decreased among healthy surviving participants, while it noticeably increased among the surviving unhealthy baseline participants. Rosow-Breslau limitations had significant effects on the predicted SRH trajectories, but very little of the variation in individuals' slopes could be explained by baseline characteristics. From these results we can conclude that among white healthy older adults, a decrease by more that one response level in less than a decade should be seen as an important individual decline. White unhealthy older adults' SRH should be expected to improve, and proceeding to a lower SRH state without first improving should be seen as an important decline that is not indicative of a long-term survivor

345/ COULD LINEAR REGRESSION BE REPLACED BY ITEM RESPONSE THEORY ANALYSIS WHEN ADDRESSING CRITERIONREFERENCE INTERPRETATION OF TEST SCORES? A DISCUSSION EXAMPLE

Luis Prieto, FPCE Blanquerna, Universitat Ramon Llull, Barcelona, Spain; Jordi Alonso, Jose M. Valderas, Health Services Researh Unit Institut Municipal d'Investigació Mèdica (IMIM), Barcelona, Spain

In order to attach meaning to a test score, some external point of reference is needed. With a criterion-referenced approach, scores are interpreted relative to a well-defined variable that is assumed to measure the same trait. Although linear regression analysis is usually applied to establish criterion-reference, its basic assumptions are often ignored (e.g. linearity and additivity, normalidity, homocedasticity) The present study inspects the claim that Item Response Theory (IRT) analysis could yield an alternative option to linear regression for this particular aim. By means of a sample of 198 subjects recruited in a multicenter prospective study of patients referred for first cataract surgery, Rasch analysis was applied to estimate the precise relationship between the scores of a questionnaire measuring functional capacity related to vision (VF14) and the ability to ditinguish details and shapes of objects as measured by the Snellen Visual Acuity Test (VA). The VF14 contained 14 items in a rating scale response format describing visual difficulties to perform daily life activities affected by cataracts. The Snellen test assigned patients into one of eleven categories based on decimal VA in the better eye. The measurementbased "regression" analysis was done in two steps: first, the 14 items of the questionnaire were independently calibrated using Winsteps computer program; after anchoring to these calibrations, the VA categories were inserted in the analysis to show how well its categories were predicted from the overall variable implied by the items of the VF14. Category probability curvers finally provided estimates of the most likely VA in the better eye category for a given VF14 score. Personian chi-square fit statistics were satisfactory, suggesting the adequacy of the results found. Based on these facts, IRT seems to provide an useful alternative to linear regression when criterion interpretations of test scores are assessed.
346/ APPLYING ITEM RESPONSE THEORY (IRT) ON ITEMS DEVELOPED WITHIN THE FRAMEWORK OF CLASSICAL TEST THEORY (CTT)

Elof Dimenas, Ingela Wiklund, Outcomes Research, Jonas Carlsson, Biostatistics, AstraZeneca R\&D, Molndal, Sweden

Most health status measures are based on CTT and thus primarily relevant for evaluation of populations rather than specific individuals. With the increased focus on individual patient combined with increased interaction via the Internet the need of having measures for individual evaluation and monitoring of QoL becomes critical. How can the traditionally developed measures be used in developing IRT models? The objective of this study was to evaluate the item-characteristic curves for items included in some previously validated questionnaires. The analysis is based on a randomly selected normal population $(n=2162)$ using four different questionnaires, PGWB (Psychology General Well-Being), GSRS (Gastrointestinal Symptom Rating Scale), SSA-P (Subjective Symptom Evaluation Profile), and the MSE-P (Minor Symptom Evaluation Profile). An explanatory factor analysis varimax rotation including all items gave 20 factors with the two first explaining a significant portion of the variance. Most of the items from PGWB and some of the items from SSA-P and MSE-P were included in the first factor (Well-being). The second factor (contentment) consists of items primarily from the MSE-P. Applying a Rasch analysis on two first factors showed that the items were distributed according to severity with acceptable infit and outfit values for most items. Item equivalence for the well-being items was observed for gender and for the majority of items across age. The study indicate that it is possible to characterise items from previously validated instruments and that it is likely that they could be adjusted to be used in Computerised Adaptive Testing-models. Furthermore, using IRT models will make it more relevant to compare results across patientgroups and over time.

\section{7/ MODELING LONGITUDINAL QOL DATA USING REGRES-} SION SPLINES

Carl A. de Moor, Departments of Behavioral Science and Biostatistics, University of Texas MD Anderson Cancer Center, Houston, TX

Randomized trials of QOL often involve multiple post-intervention assessments. The commonly used statistical tests of treatment group differences may include any one of several approaches: multiple pointwise comparisons at the individual follow-up time points, comparisons on data summarized across all follow-up points, or comparisons using repeated measures analysis with time by group interaction terms. However, these procedures may suffer from inflated Type I error rates or lack of power, or they may overly simplify or obscure the specific pattern of differences across time. A seldom used but powerful alternative involves using regression splines to explicitly model the pattern of changes in QOL. Regression splines are particularly useful when the patterns of QOL scores contain important nonlinearities over time that are difficult to fit using simple linear or polynomial regression. In this presentation, we will describe the regression spline methodology in the context of randomized trials with multiple follow-ups. We show that it can be used to model complex patterns of change over time and produce a powerful overall test of treatment differences, as well as point-wise comparisons between treatment groups. We also present a simple numerical method for constructing confidence bands for the time dependent difference in QOL between the two treatment groups. We illustrate the method using data from a randomized trial for smoking cessation including graphical presentation of the fitted spline functions, time specific differences in QOL, and confidence bands associated with those differences. The power of this approach will be compared to more standard approaches. We demonstrate that regression spline analysis is relatively straightforward to implement and can be conducted with any standard regression software package. 
348/ SOME LOGICAL PROBLEMS WITH TESTS FOR CLINICALLY IMPORTANT CHANGES IN HRQOL

Dennis D. Gagnon, RWJ Pharmaceutical Research Institute, Raritan, NJ

The objective of this presentation is to logically evaluate several proposed methods for ascertaining the clinical importance of observed health-related quality-of-life (HrQoL) changes in clinical trials. The five proposed methods are: effect sizes, changes of 0.5 per item in multi-item measures, anchor-based, numbers needed to treat, and inspection of cumulative distribution curves. Each of these methods are separately evaluated in terms of being sufficient criteria for conferring clinical importance. Upon a conceptual analysis it is found that effect sizes can be manipulated by inclusion/exclusion criteria. Thus they measure efficiency of study design rather than clinical importance. Tests based upon changes of 0.5 per item are specific to measures where each item offers responses on a seven-point scale. Many common measures include items that do not offer responses on sevenpoint scales. Additionally, this test reduces to an anchor-based test. Anchor-based tests are sensitive to the chosen anchor and therefore can give different indications of clinical importance for the very same change in HrQoL depending upon the specific anchor. The numbersneeded-to-treat test assumes a threshold of clinical importance. Thus this test does not answer whether an observed change is clinically important. While inspection of cumulative distribution curves can determine dominance of one treatment over another, it likewise does not answer the question of whether the observed changes are clinically important. Based upon an extension of the work of the 20th century philosopher Ludwig Wittgenstein to the area of clinical interpretation, it appears that clinical importance of HrQoL results is not a matter of the application of a mechanical test upon the data. Rather, an evaluation of clinical importance is a function of the researcher's understanding of the study population, design, and results; including a thorough familiarity with the HrQoL measures used in that study.

349/ RELIABILITY AND VALIDITY OF MODIFIED LUNG CANCER SYMPTOM SCALE (LCSS) IN MULTINATIONAL SAMPLE OF PATIENTS WITH PLEURAL MESOTHELIOMA

Astra M. Liepa, Global Economic Affairs, Eli Lilly and Co., Indianapolis, IN; Patricia J. Hollen, School of Nursing, Northeastern University Boston, MA; Richard J. Gralla, Division of Medical Oncology, Columbia University, New York, NY; James J. Rusthoven, Medical Group, Alimta Team, Eli Lilly and Co., Indianapolis, IN

No health-related quality of life (HRQoL) instrument had been validated for patients (pts) with malignant pleural mesothelioma (MPM). The LCSS, developed and validated for non-small and small cell lung cancers, was identified as a potential instrument that could apply to MPM. The LCSS patient scale was modified slightly (ie, "lung illness" substituted for "lung cancer") since MPM is discussed differently with pts. The LCSS was incorporated into 2 multinational trials of pemetrexed disodium (ALIMTA, MTA) in chemonaive pts with unresectable MPM. Translations were developed using standard backward/forward process and pilot-testing. Preliminary baseline characteristics $(\mathrm{N}=466)$ are: median age $=62 ; 81 \%$ male; $83 \%$ Karnofsky performance status (KPS) $>70 ; 80 \%$ stage III-IV. Despite good performance status, pts reported the following baseline LCSS median scores (based on 0-100 mm VAS where 100=worst possible): anorexia $=20$, fatigue $=36$, cough $=6$, dyspnea $=31$, hemoptysis $=0$, pain $=19$ symptom distress $=33$, activity level $=45$, global $Q \circ L=44$. Results from this sample demonstrate acceptable reliability for the modified LCSS with Cronbach's alpha coefficient $=0.85$. Cronbach's alphas for each trial are $0.84(\mathrm{~N}=381)$ and $0.88(\mathrm{~N}=54)$. Data from participating countries with at least 10 pts (ie, Argentina, Australia, Belgium, Finland, France, Germany, India, Italy, Mexico, Poland, Spain, Turkey, UK USA) demonstrate acceptable reliability for translations with Cronbach's alphas $=0.78-0.88$. Test-retest reliability using Pearson correlation with 2 baseline assessments completed 3-5 days apart is 0.86 . Construct validity is supported by relationship of median total LCSS scores with known groups (ie, lower HRQoL scores in higher KPS groups): KPS $=70$, LCSS $=42 ; \mathrm{KPS}=80$, LCSS $=33 ; \mathrm{KPS}=90$ LCSS $=18$; KPS $=100$, LCSS $=12$. The modified LCSS appears to be reliable and valid for assessing HRQoL in pts with MPM. These data can also serve as a normative data set for further studies in MPM.
350/ AN INDONESIAN VERSION OF THE ROTTERDAM SYMPTOM CHECKLIST: A PSYCHOMETRIC EVALUATION

Magdalena S. Halim, Psychology, Atmajaya Indonesia Catholic University, Jakarta Selatan, Indonesia; Jan J. L.Derksen, Cees P. F. van der Staak, Clinical Psychology \& Personality, University of Nijmegen, Nijmegen, The Netherlands

The purpose of this study is to assess reliability and balidity of the Rotterdam Symptom Checklist (RSCL) in Indonesian breast cancer patients. We prospectively analysed 106 women with breast cancer from July 1998 to August 1999. All patients completed the RSCL and the Revised NEO Personality Inventory (NEO PI-R)approximately one month after surgery as the first time measurement. Three months later, 95 of 106 breast cancer patients completed the RSCL for the second time. Internal consistency reliability of RSCL were assessed by Cronbach's alpha. Relationship between Neuroticism (N) factor of the NEO PI-R, including Anxiety and Depression facets, and the RSCL were evaluated for vvalidity assessment. The results showed that physical, psychological, and functional activity subscales of RSCL had a good internal reliability (Cronbach's alpha .86; .90;.85). At three months follow-up, test-retest reliability ranged from .83 (functional activity) to .88 (physical and psychological subscales). A Pearson correlation coefficient ranged from .43 to.54 ( $p<.01)$ was found between Neuroticism (including Anxiety and Depression scale) and the psychological subscale of RSCL. The structure of Indonesian version of RSCL was also analysed by factor analysis reproducing results of the English version. In conclusions, we found that the psychometric quality of the Indonesian version of RSCL was satisfactory. The Indonesian version of RSCL can therefore be considered as conceptually equivalent to the original version.

351/ RELIABILITY AND VALIDITY OF THE FUNCTIONAL ASSESSMENT OF CANCER THERAPY-BREAST (FACT-B): WOMEN'S HEALTH OUTCOME STUDY-01 (WHOS-01)

Kojiro Shimozuma, Japan Medical Association Research Institute (JMARI), Tokyo, Japan; Yasuo Ohashi, School of Health Science and Nursing, University of Tokyo, Tokyo, Japan; Kimio Yoshimura, National Cancer Center Research Institute, Tokyo, Japan; Shigemichi Iha, Kurashiki Medical Center, Kurashiki, Okayama, Japan; Hideyuki Saeki, Shozo Ohsumi, National Shikoku Cancer Center, Matsuyama, Ehime, Japan; Katsumasa Kuroi, Toyosu Hospital, Showa University School of Medicine, Tokyo, Japan; Noriyuki Katsumata, National Cancer Center Hospital, Tokyo, Japan; Takahiro Okamoto, Tokyo Women's Medical University, Tokyo, Japan; Katsuhiro Tanaka, Kawasaki Medical School, Kurashiki, Okayama, Japan

The objective of this study was to verify the reliability and validity of the Japanese version of the Functional Assessment of Cancer Therapy-Breast (FACT-B: General scale [FACT-G] plus Breast Cancer Subscale [BCS]) for Japanese breast cancer (BC) patients. We developed the Japanese version of the FACT-B in collaboration with Evanston Northwestern Healthcare. Between December 1998 and March 2001, 128 post-surgical BC patients were enrolled in a study investigating rehabilitation needs and predictive factors of quality-oflife (QOL)(Women's Health Outcome Study [WHOS]-01). We serially assessed QOL using the FACT-B (Version 4) in conjunction with the Hospital Anxiety and Depression (HADS) scale at 1, 6, and 12 mos. after surgery. The response rate for the FACT-B was $93.5 \%$ even at $12 \mathrm{mos}$. and the compliance to complete each item of the instrument ranged from 89.7 to $100 \%$ except for one item inquiring about sexual satisfaction (40.3-50.5\%). As for the internal consistency, Cronbach coefficients alpha ranged from 0.73 to 0.88 for the FACT-G subscales, from 0.48 to 0.67 for the BCS, from 0.84 to 0.91 for the FACT-G, from 0.84 to 0.92 for the FACT-B, and from 0.86 to 0.88 for the Trial Outcome Index (TOI). High correlation coefficients $(r=0.51-0.79)$ were observed between the Emotional Well-Being (EWB) subscale and the HADS subscales. Multitrait scaling analysis confirmed the hypothesized structure of the FACT-B except for the Physical Well-Being (PWB) subscale. Sensitivity to change over time (from 1 to 6 mos.) was statistically significant for the Social Well-Being (SWB) subscale, the Functional Well-Being (FWB) subscales, and the TOI. In conclusion, the results of this study demonstrate an ease of administration, reliability and validity of the FACT-B for Japanese BC patients. 
352/ VALIDITY OF THE SPANISH CHILD HEALTH AND ILLNESS PROFILE, ADOLESCENT EDITION (CHIP-AE)

V. Serra, Agència d'Avaluació Tecnologia i Recerca Mèdiques, Barcelona, Spain; L. Rajmil, AATM, Barcelona, Spain, J. Alonso, Institut Municipal d'Investigació Mèdica, Barcelona, Spain; B. Starfield, A. Riley, Johns Hopkins University, Baltimore, MD

The Spanish version of the CHIP-AE is the first generic health status measure for adolescents available in Spanish. The CHIP-AE allows to create health profiles to identify systematic differences in adolescent populations. The aim of this study was to assess convergent and structural validity of the Spanish version of the CHIP-AE. A representative sample of 902 adolescents aged 12-18 from public and private schools and different socioeconomic areas of Barcelona city, and a rural sample of 551 were included in the analyses. The CHIP $A E$, Child Depression Inventory (CDI) and State-Trait Anxiety Inventory for Children (STAIC) were administered in a sub-sample of adolescents to assess convergent and discriminant validity. Subdomain and domain scores of the CHIP-AE were standardized to a mean of 20 and standard deviation of 5 . The scores of the CDI and STAIC were correlated with the CHIP-AE subdomains. Intercorrelations of domains and subdomains were also computed. Exploratory and confirmatory factor analyses were carried out, expecting similar patterns with the original U.S. profile. Girls reported lower satisfaction and selfesteem and more Discomfort. Boys scored more physical activity and risky behaviours. CHIP-AE emotional discomfort and self-esteem scores showed higher correlations with the STAIC and the CDI than with other subdomains (range: $0.48-0.80$ ). Adolescents reporting more Satisfaction also reported less Discomfort. Disorders tends to correlate positively with Discomfort $(p<0.01)$. The factors useful to create the profile-types for the assessment of health needs in Spain, were clearly defined: Satisfaction, Discomfort, Resilience and Risks. The results are similar to the US version. These results and previuos psychometric testing confirm that the Spanish CHIP-AE has acceptable validity to describe the health of adolescents in Spain and to compare it internationally.

353/ QUALITY OF LIFE IN THE AUSTRIAN GENERAL POPULATION: "NORMATIVE DATA" FOR THE FACT-G

Bernhard Holzner, Department of Biological Psychiatry, Georg Kemmler, Department of General Psychiatry, Christina de Paoli, Department of Biological Psychiatry, Martin Kopp, Department of General Psychiatry, Richard Greil, Department of Internal Medicine Wolfgang Fleischhacker, Barbara Sperner-Unterweger, Department of Biological Psychiatry, University Hospital Innsbruck, Innsbruck, Austria

The aim of this study was to derive population-based norms for the Functional Assessment of Cancer Therapy Scale - General (FACT$\mathrm{G}$ ) and to compare these with FACT-G data of a sample of patients with chronic lymphatic leucaemia (CLL). A randomly selected sample of 3000 people of the Austrian population was included in this postal study. The FACT-G as well as some questions concerning demographic data and health status were sent by mail. Additionally a sample of patients with CLL (presently receiving no cancer treatment) was asked to fill in the FACT-G. These patients were compared with an age- and sex-matched subsample of the reference group. Concerning the population-based study, 667 questionnaires were returned giving an overall response rate of $23.2 \%$ (female $50.5 \%$, age $49.6 \& \# 61617 ; 16.8)$. When analysing the impact of sociodemographic variables on the FACT-G subscales the following results were found people with higher education report higher Qol-values in all FACT-G subscales; divorced and widowed persons have significantly lower Qol scores. Concerning physical, emotional and social well-being women report lower Qol-values than men. Higher age is accompanied by lower Qol-scores in the physical and functional subscale as well as in the total score. CLL-patients $(n=81,40.7 \%$ female, age $67.1 \& \# 61617 ; 10.3$, time since initial diagnosis $4.8 \& \# 61617 ; 3.2$ ) report significantly lower Qol-scores in the subscales 'physical well-being' (effect size: 0.57), 'emotional well-being' (effect size: 0.24) and in the total score (effect size: 0.31 ) than age and sex-matched population based controls. Sociodemographic variables (e.g. age, sex, marital status, education) should always be taken into consideration when interpreting QoL scores both in the general population and in cancer patients. CLL-patients without current cancer treatment often experience reduced Qol and report more problems than the general population
354/ PRELIMINARY ANALYSIS OF THE CULTURAL VALIDATION OF THE WHOQOL-100 IN URUGUAY

Adriana Bagnulo, Laura Schwartzmann, Medical Psychology, Medical College, State University Montevideo, Montevideo, Uruguay

The World Health Organization Quality of Life assessment instrument (WHOQOL-100) is a cross cultural, generic quality of life measure that has been developed in over 40 countries worldwide. This paper reports a preliminary analysis of the cultural adaptation of the WHOQOL-100 in Uruguay. Previous Spanish versions ( Argentina, Spain) were revised. Focus groups were run to discuss the importance of the dimensions included and the formulation of the questions. An additional new facet related to the impact of dieting on QL was proposed. The application was performed by medical students as part of their curricular Medical Psychology Module. The instrument was piloted on 342 persons with a mean age of 40.19 (S.D. $18,80) ; 54 \%$ in-patients from a General Hospital and $46 \%$ healthy subjects. The analysis included descriptive statistics, score distribution, internal consistency, content validity and discriminant validity. Internal consistency was satisfactory for all domains (alpha $>.70$ ) with the exception of the social relationship domain (alpha= .67). Ability to discriminate between healthy and ill subjects was good (MannWhitney $U>.05$ ) for all domains with the exception of spirituality domain. Content validity is still being processed. Preliminary results suggest that the Uruguayan version of WHOQOL-100 demonstrates good psychometric properties.

355/ SEQUENTIAL VS. PARALLEL APPROACH TO MULTICULTURAL SCALE DEVELOPMENT: CASES FROM THE FACIT MEASUREMENT SYSTEM

Sonya L. Eremenco, Chih-Hung Chang, David Cella, CORE, ENH, Evanston, IL

As quality of life (QOL) assessment in international clinical trials becomes increasingly prominent, more attention has been paid to how QOL scales are developed, translated and adapted for use crossculturally. This study compared two approaches (sequential and parallel) to scale development used in the FACIT (Functional Assessment of Chronic IIIness Therapy) Measurement System. In the sequential approach, a scale is developed in one culture and later adapted to other cultures through translation. In the parallel approach, a scale is developed in multiple cultures with the goal of deriving a common set of items across countries. The Functional Assessment of Cancer Therapy - Breast (FACT-B) subscale followed the more common sequential approach in which the items were developed in the US and later translated to over 20 languages. Through linguistic validation with 240 patients, we found 2 items which were less relevant in other cultures and 1 item which was difficult to translate. These issues were due in part to the cultural specificity of the original wording. In order to address this potential problem, the parallel approach can be implemented in new scale development. One of the newer FACIT measures, the FACT-Gastric subscale has undergone parallel development in the US and Japan. Item generation was conducted via expert and patient interviews in both countries simultaneously. As a result, items which were culturally irrelevant or otherwise problematic for translation were detected prior to linguistic validation and finalization of the subscale. An advantage of the parallel approach is that by taking into account cultural differences during development, the resulting questionnaire may be more equivalent at the conceptual level. In comparing the advantages and disadvantages of these approaches to scale development, the sequential approach may be more efficient and feasible, while the parallel approach may yield better conceptual comparability of the resulting scales across cultures and languages. 
356/ THE LOGISTICS OF HEALTH-RELATED QUALITY OF LIFE (HRQL) RESEARCH AMONG ETHNIC MINORITY CANCER POPULATIONS

Rianne Hoopman, Psychosocial Research and Epidemiology, The Netherlands Cancer Institute, Amsterdam, The Netherlands: Sahsenem Celik, Fadoua El Haddouchi, Neil K. Aaronson, Psychosocial Research and Epidemiology, The Netherlands Cancer Institute, Amsterdam, The Netherlands

In the Netherlands there are two main ethnic minority groups, the Turkish and Moroccan population. The applicability of HRQL instruments within these groups is largely unknown. Most 1st generation Turks and Moroccans lack proficiency in Dutch, thus inhibiting their participation in clinical and health services research in which HRQL is assessed. Additionally, identification and recruitment of individuals from these minority ethnic groups can be quite complex. In this paper we will present our experiences with the logistics of carrying out a validation study of 4 widely used HRQL questionnaires (the SF-36, the EORTC QLQ-C30, the RSCL, and the COOP/WONCA charts) among approximately 100 cancer patients (and their significant others) from each of these two ethnic minority groups. Specifically, we will address: (1) use of phonetic script for translating and administering translated versions of HRQL questionnaires when there is no written language (e.g., Rif-Berbers, Moroccan-Arabic); (2) identifying eligible patients when nationality and ethnicity are not standardly recorded in hospital administrative databases and medical records; (3) recruiting patients who often are illiterate and unfamiliar with surveys (4) recruiting patients from minority communities where privacy (sometimes in connection with legal status) is a salient issue; and (5) recruiting patients and significant others from minority communities where cancer is considered taboo and often is not discussed within the family.

357/ DYSPEPSIA-RELATED QUALITY OF LIFE: TRANS-CULTURAL ADAPTATION AND PSYCHOMETRIC VALIDATION INTO SPANISH OF THE DISPEPSIA-RELATED HEALTH SCALES Miguel Ruiz, Faculty of Psychology, Autonoma University Madrid, Madrid, Spain; Rejas Javier, Health Outcomes Research, Medical Division, Pfizer Spain, Alcobendas, Madrid, Spain; Soto Javier, Health Outcomes Research, Medical Division, Pharmacia Spain Madrid Madrid, Spain

Dyspepsia is a prevalent disorder, affecting $25 \%$ of population and impacting negatively on Quality of Life (QoL). New instruments have been developed to assess QoL in such patients. The purpose was to culturally adapt and psychometrically validates into Spanish the new instrument DRHS. A longitudinal-prospective study was designed to perform adaptation. 234 out-patients (60,7\% females), 18 - 93 years (mean age + SD: 49,1+18,7) were enrolled; 163 with active dyspepsia, and 71 matched-healthy volunteers. An expert panel coordinated the entire validation process. Original scales were forward and backward translated in duplicate and a cognitive debriefing and a pilot testing in 16 subjects were conducted to produce a final version. The instrument was administered three times separately 1 to 4 days to assess test-retest reliability, and 1 to 3 weeks to evaluate sensitivity to change after dyspepsia treatment. Also, internal consistency, construct validity, convergent validity with Euro-QoL and discriminant capacity of the questionnaire was evaluated. Inter-rater Hambleton \& Rovinelli index was below 0,41 . Factor analysis showed that scales could be considered as one-dimension for both patients and healthy subjects. Spanish DRHS was internally consistent (overall Cronbach alpha $=0,92$ ) and stable (intraclass coefficient $=0,95$ ), and demonstrated moderate, even satisfactory, capacity to measure $Q o L(r=0,54$ $p<0,001)$. Predictive validity was fair $(r=0,47, p<0,001)$ and also was able to separate patients and healthy subjects $(p<0,001)$ and different dyspepsia-related health status after therapy $(p<0,001)$. Spanish version of DRHS entitled QoL-PEI showed good psychometric properties and it was able to predict dyspepsia evolution. This new too may become of help for clinicians and investigators to both in their daily activity and as an investigational instrument.
358/ PROSPECTIVE VALIDATION STUDY OF SPANISH VERSION OF THE KIDNEY TRANSPLANT QUESTIONNAIRE (KTQ)

Pablo Rebollo, Servicio de Nefrología-I, H.Central de Asturias, Institute Reina Sofia of Neph, Oviedo, Asturias, Spain; Francisco Ortega, Servicio de Nefrología-I, H.Central de Asturias, Institute Reina Sofia, Oviedo, Asturias, Spain; Monica Garcia-Mendoza, Jose M. Baltar, Jaime Alvarez-Grande, Servicio de Nefrología-I, H.Central de Asturias, Oviedo, Asturias, Spain

The interest of evaluate the Health Related Quality of Life (HRQOL) of patients on Renal Replacement Therapy is growing. In Spain there are two specific HRQOL assessment instruments validated for patients on dialysis, but there is not any specific questionnaire for kidney transplant patients. Here is presented the Spanish validation of the first specific HRQOL assessment instrument for transplant patients: the kidney transplant questionnaire (KTQ). Prospective study of 40 patients on transplant waiting list who received their first kidney transplant in our region. Patients were evaluated before transplant and after 1, 3, 6 and 12 months, using the KTQ and the SF-36 Health Survey. Feasibility ( $\%$ who complete questionnaire and time), validity (correlation coefficients among KTQ dimensions, and between the KTQ and the SF-36), reliability (Cronbach's alpha and Intraclass correlation coefficients), and responsiveness (correlation coefficients with clinical variables collected) were evaluated. We collected data of 67 patients on RRT entering transplant waiting list: 55 of them received their first kidney transplant (15 patients excluded because missing follow-up). Mean time of administration of the KTQ was 12 minutes, except for the first administration $(20 \mathrm{~m}$.). All correlation coefficients among KTQ dimensions were moderate-high ( $r>0.4)$. Correlation coefficients of KTQ dimensions with SF-36 PCS were low $(r<0.4)$, and with SF-36 MCS were moderate-high ( $r>0.4)$ except for Physical Symptom dimension $(r=0.303)$. Cronbach's Alpha was satisfactory for all KTQ dimensions $(>0.7)$ : Physical Symptoms $=0.80 ;$ Fatigue $=0.93$; Uncertainty/Fear $=0.79$; Appearance $=0.72$; Emotional $=0.91$. Intraclass correlation coefficients were similar to that of the original KTQ version: ranged between 0.49 for Physical Symptoms and 0.86 for Uncertainty/Fear. All patients showed significant improving in KTQ scores after receive the transplant. We confirmed a transitory decrease of $\mathrm{KTQ}$ scores between $3 \mathrm{rd}$ and $6^{\circ}$ month, which we have observed in a previous prospective study. Results of validation study show that feasibility, validity, reliability and responsiveness of the Spanish version of the KTQ are similar to that of original KTQ version.

359/ LINGUISTIC VALIDATION OF THE KING'S HEALTH QUESTIONNAIRE (KHQ) IN 34 LANGUAGES

Katrin Conway, Laurence Chevallet, Mapi Research Institute, Lyon, France; Tara Symonds, Pfizer Ltd, Sandwich, UK

The King's Health Questionnaire (KHQ) developed in UK English was originally designed for use in women suffering from urinary incontinence, but has since been validated for use in men as well. The domains assessed include daily activities, physical \& social limitations, sexuality, emotional consequence, strategies for coping with urinary incontinence and overall quality of life. Prior to use in an international trial involving both men and women, the original questionnaire underwent linguistic validation in 34 languages. The process involved the recruitment of a QoL specialist in each target country as supervisor and followed a rigorous methodology which consisted of: 1) two separate forward translations produced by professional translators, native speakers of the target language and bilingual in English or updating of existing translations; 2) comparison and reconciliation of the translations by QoL specialist and translators; 3 ) a backward translation by a native English speaker; 4) comparison of source with backward version; 5) comprehension test in a sample target population (i.e. both men and women); and 6) international harmonisation. Through the translation process a number of conceptual and linguistic issues arose. The item "household tasks" followed by a parenthesis referring to "cleaning, shopping," had to have the addition of "DIY" (or equivalent) in the translations to include the male population. To cover both short and long distances contained in the item "travel", the literal translation had to be completed by reference to "travel by car, bus, train or plane" in the translations. In both the Japanese and Hungarian versions, the response option "not applicable" had to be adapted to each item to be clear for patients. A rigorous translation methodology was performed to ensure conceptual equivalence and cross-cultural acceptability of the KHQ translated versions. Data generated by a clinical trial in each country should ideally be compared to ensure response similarity across countries. 
360/VALIDITY AND RELIABILITY OF EQ-5D DOMAINS IN ASIANS IN SINGAPORE WITH RHEUMATIC DISEASES

Nan Luo, Department of Pharmacy, National University of Singapore Singapore; Ling-Huo Chew, School of Health Sciences, Nanyang Potytechnic Singapore, Singapore; Kok-Yong Fong, Dow-Rhoon Koh Swee-Cheng Ng, Kam-Hon Yoon, Sheila Vasoo, Department of Medicine, Shu-Chuen Li, Department of Pharmacy, Julian Thumboo, Department of Medicine, National University of Singapore, Singapore

The purpose of this study was to evaluate the validity and reliability of the EQ-5D in Singaporeans with rheumatic diseases. Consecutive English-speaking patients attending rheumatology outpatient clinics at a tertiary referral hospital were interviewed twice within two weeks using a standardised questionnaire containing the EQ-5D, Short-Form 36 Health Survey (SF-36) and other scales. To assess construct validity of the EQ-5D, 13 a-priori hypotheses relating EQ-5D domains or the EQ-5D visual analogue scale (EQ-5D VAS) to SF-36 scores and other variables were examined using the Mann-Whitney $U$ test t-test or Spearman's correlation. Test-retest reliability was assessed using Cohen's kappa. Sixty-six patients (osteoarthritis: 9, rheumatoid arthritis: 26 , systemic lupus erythematosus: 23 , spondyloarthropathy: 8; Female: $72.7 \%$; mean age: $\mathbf{4 4 . 3}$ years) completed baseline questionnaires. Ten, 53 and 3 patients respectively reported no, moderate or severe health problems as defined by the EQ-5D. Ten of 13 apriori hypotheses relating EQ-5D domains or VAS scores to external variables were fulfilled, supporting construct validity. For example patients reporting problems in any EQ-5D domain had lower SF-36 scores than patients not reporting problems; patients reporting depression/anxiety had poorer mental health $(p<0.001)$ but similar physical functioning $(p=0.44)$ versus subjects without depression/anxiety and the EQ-5D VAS correlated negatively with age $(-0.39, p<0.01)$ and patient's global assessment of pain $(-0.36, p<0.01)$. Cohen's kappa for test-retest reliability $(n=52)$ ranged from 0.29 to 0.58 . EQ$5 \mathrm{D}$ domains and the EQ-5D VAS appear to be valid in measuring health status in Singaporeans with rheumatic diseases; however their reliability warrants further investigation. These data provide a basis for further studies evaluating validity of the EQ-5D in Singapore.

361/ CROSS-CULTURAL ADAPTATION OF THE SF-36 AND SF12 PHYSICAL AND MENTAL SUMMARY SCALES ON A THE CHINESE POPULATION

Cindy L. Lam, Family Medicine Unit, Department of Medicine, The University of Hong Kong, Hong Kong SAR, China; Barbara Gandek Health Assessment Lab, Boston, MA

The two principal factor structure of the SF-36 was found in the Chinese population in Hong Kong (HK) in previous studies, suggesting that the SF-36 and SF-12 physical and mental summary scales (PCS/ MCS) may be applicable to our population. The aim of this study was to find out if the US standard weights and item selection for the SF-36 and SF-12 PCS/MCS were applicable to the Chinese population in HK. 2410 Chinese adults randomly selected from the general population in HK completed the Chinese (HK) SF-36 and a questionnaire on demographic and morbidity data. The standard SF-36 and SF-12 PCS/MCS scores were calculated by the standard US algorithm. The HK specific SF-36 PCS/MCS were calculated from the population specific mean, SD and factor coefficients. Multiple regression of the SF-36 PCS/MCS scores on the SF-36 items was used to select 12 items that could explain most of the variance in the SF-36 PCS/MCS. The standard and HK specific PCS/MCS scores were compared between disease groups to assess their sensitivities. Six of the HK specific SF-12 items were different from those of the standard SF-12. The HK SF-12 PCS /MCS explained $88 \%$ and $90 \%$ of the variance in the SF-36 PCS/MCS scores, respectively; the standard SF-12 PCS/ MCS explained $82 \%$ and $89 \%$ of the variance of the SF-36 PCS/ MCS, respectively. The differences in the HK specific SF-12 PCS between disease groups had significantly bigger effect sizes than those of the standard SF-12 PCS. There was little difference between the sensitivities of the HK specific and standard SF-36 PCS/MCS or SF12 MCS. The US standard SF-36 PCS/MCS scoring algorithm was applicable to the Chinese population in Hong Kong, but population specific items and weights may be more appropriate for the SF-12 PCS/ MCS.
362/ SWEDISH SF-36 VERSION 2.0 - CONSTRUCT AND CRITERION-RELATED VALIDITY

Charles Taft, Jan Karlsson, Marianne Sullivan, Health Care Research Unit, Sahlgrenska University Hospital, Göteborg, Sweden

The changes incorporated in SF-36 version 2.0 imply potential improvement in the instrument's data quality, reliability, score precision and responsiveness. Initial studies from the US, UK and Sweden have documented gains in internal consistency reliability and reductions in floor/ ceiling effects, particularly in relation to the role functioning scales. Evidence replicating v1.0 findings of the hypothesized associations between the 8 SF-36 scales and the two major theoretical constructs of health, physical and mental, has been reported for the US and UK versions. The UK version has also been shown to adequately discriminate between groups known to differ on key demographic variables. The aim was to evaluate the construct and criterion-related validity of the Swedish SF-36 v2. The Swedish SF-36 v2 normative sample comprised 2,237 subjects aged 18-98 randomly selected from the Swedish population. Sociodemographic distributions of the sample were comparable with v1.0. Construct and criterion-related validity were evaluated following procedures widely used in v1.0 validation studies. Assessed were 1) the relative validity of the SF-36 scales as measures of the physical and mental health constructs, and 2) the efficacy of the scales to discriminate between groups known to differ in physical and mental health. Overall, results were generally identical with those obtained for v1.0. Slightly more of the total variance was explained by the mental and physical health constructs $(73 \%$ vs $70 \%)$ and associations between scales and constructs were more polarized. Relative validity estimates were unchanged, except for the two role scales where slight increases were noted. In known group analyses v2 performed as well or better than v1.0 in differentiating groups differing on key clinical and sociodemographic variables. The modifications in the Swedish SF-36 v2 appear to have improved the reliability and validity of the instrument without compromising its underlying structure. The changes will likely further enhance its sensitivity to change.

363/ EVALUATING THE PERFORMANCE OF A NEW SLOVAK VERSION OF THE INCONTINENCE-RELATED QUALITY OF LIFE MEASURE (I-QOL)

Donald M. Bushnell, Mona L. Martin, HRA, Inc, Seattle, WA; Jan Svihra, Department of Urology, Jessenius School of Medicine, Comenius University, Martin, Slovak Republic

International clinical trials of treatment for urinary incontinence (UI) have been increasing in both frequency and global scope. This has increased the demand for incontinence-specific quality of life measures for application in regions not previously active in clinical trials, including Eastern Europe. Standard translation criteria were followed to develop a Slovak language version of the I-QOL. This new translation was then administered to 100 women reporting symptoms of incontinence as a part of a local epidemiologic study. The mean age of the sample was 59 years, with $29 \%$ reporting moderate to severe symptoms, and $50 \%$ reporting symptoms of both stress and urge incontinence. Psychometric evaluation of the I-QOL in this population showed good internal consistency (alpha $=0.97$ ), and the ability of the $\mathrm{I}-\mathrm{QOL}$ to discriminate between degrees of self-reported severity, length of time with UI, and clinical indicators such as number of pads used and amounts of urine leakage. Mean I-QOL scores [Avoidance \& Limiting Behavior (ALB), Social Embarrassment (SE), Psychosocial Impacts $(\mathrm{PI})$ ] were compared to those in previous validation studies in other European countries, see table. We conclude the new Slovak version of the $\mathrm{I}-\mathrm{QOL}$ demonstrates performance characteristics and mean scores in line with those found in other European countries. As clinical trials for UI expand into Eastern Europe, this version of the IQOL will be both useful and appropriate.

\begin{tabular}{|l|l|l|l|l|l|}
\hline I-QOL mean (SD) & France $\mathrm{n}=62$ & Spain $\mathrm{n}=65$ & Sweden $\mathrm{n}=64$ & Germany $\mathrm{n}=68$ & Slovakia $\mathrm{n=100}$ \\
\hline Total Score & $62(22)$ & $42(21)$ & $66(22)$ & $51(24)$ & $64(25)$ \\
\hline ALB & $59(23)$ & $42(22)$ & $63(23)$ & $44(23)$ & $64(24)$ \\
\hline SE & $54(26)$ & $25(22)$ & $56(27)$ & $41(27)$ & $58(27)$ \\
\hline PI & $70(23)$ & $51(26)$ & $75(23)$ & $62(27)$ & $68(26)$ \\
\hline
\end{tabular}


364/ KOREAN VALIDATION OF THE KIDNEY DISEASE QUALITY OF LIFE (KDQOL) INSTRUMENT: A PILOT STUDY

Sehyun Kim, Preventive Medicine, Hye J. Park, Nursing, College of Medicine, Pochon CHA University, Sungnam, Kyonggi-do, Korea; Jin S. Yong, Sung S. Han, Nursing, Catholic University, Seoul, Korea; Kyeong S. Kim, Dong H. Yang, Nephrology, College of Medicine, Pochon CHA University, Sungnam, Kyonggi-do, Korea

Assessment of quality of life of patients with chronic kidney disease will provide information to evaluate the care delivered. The objective of this study was to validate a Korean translation of the Kidney Disease Quality of Life (KDQOL-SF 1.3) instrument. We have been following rigorous translation steps guided by the KDQOL Working Group. Two translators who are native Korean speakers and fluent in English translated KDQOL instructions, items, and response choices, and rated the difficulty of translating each item and response scale. After completing a forward translation, we have conducted a pilot study for cognitive testing. The translated KDQOL instrument was administered to 15 dialysis outpatients. Both translators have agreed on the difficulty of translation (agreement rate $=92 \%$ ). In particular, both had difficulty translating the following scale, 'Most of the time,' 'A good bit of the time,' because these represent similar quantities in Korean and the item, 'Walking one block,' due to a 'block' is not an usual measure of distance in Korea. Completion rates were excellent so that the calculated KDQOL scale scores were based on answers by all respondents. Mean (+/- sd) scale scores ranged from $31.67(+/$ $22.09)$ for the burden of kidney disease to $81.11(+/-18.76)$ for the cognitive function scale. Additionally, mean ( $+/-\mathrm{sd})$ overall health and patient satisfaction score was $46.00(+/-17.65)$ and $57.78(+/-18.76)$ respectively. There were high internal consistency reliabilities (Chronbach's Alpha $>=0.74$ for 7 out of 10 domains). The results of the cognitive testing support for the reliability of the Korean-translated KDQOL in its present form. However, further refinement for rewording of the response choice and for providing conceptually equivalent meaning of 'Walking one block' is needed. We are currently planning to convene a panel in order to finalize forward translation prior to submit to the KDQOL Working Group for approval.

365/ USING THE DELPHI METHOD TO DETERMINE EXPERT CONSENSUS IN THE CONSTRUCTION OF A NEW EUROPEAN HEALTH-RELATED QUALITY OF LIFE QUESTIONNAIRE (HRQOL) FOR CHILDREN AND ADOLESCENTS

Michael Herdman, Luis Rajmil, and the KIDSCREEN and DISABKIDS groups, Catalan Agency for Health Technology Assessment, Barcelona, Spain

The KIDSCREEN project is a large European project to develop a generic HRQOL measure for use with children and adolescents in public health surveys. For the first stage of the project, a Delphi method was used to determine the degree of consensus among experts regarding the conceptual basis, format and content of the new measure. Three questionnaire rounds were administered to a multidisciplinary group of 24 experts from 7 European countries. The questionnaire contained 3 sections dealing with conceptualisation and operationalistion of $\mathrm{HRQOL}$, questionnaire construction, and content. The first Delphi round consisted largely of open questions, and answers to this round provided the basis for later versions. Consensus was considered to be reached when at least $90 \%$ of experts either agreed or disagreed with a given item, or where median scores were over 6 on a scale of 1-10, and score dispersion was within predefined limits. Responses were received from 20 respondents in the 3 rounds. There was agreement that the new instrument should be a multidimensional, profile measure with $30-49$ items covering 5 to 8 dimensions, taking 10-15 minutes to complete. Agreement was also reached on 8 specific dimensions to include in the questionnaire (psychological well-being, self-esteem, body image, cognitive functioning, mobility, energy/vitality, social relations, and family/home function). The consensus was generally against having an individualised questionnaire, or one which measured both functioning and satisfaction with functioning. Focus groups with children, parents, and workers in the field, and literature and instrument reviews were thought to be the most appropriate sources of content. This is only the first stage of the KIDSCREEN project, and it will be interesting to compare expert opinion on questionnaire content with the opinions of children and parents interviewed in the next phase.
366/ COMPARATIVE PERFORMANCE OF THE SF-36 BY ETHNICITY

Denise R. Globe, Joanne Wu, Pharmaceutical Economics and Policy, University of Southern California, Los Angeles, CA

The purpose of this analysis was a comparative assessment of selfreported health status across diverse ethnic groups receiving care from a large health maintenance organization. The data set consisted of sociodemographic, pharmacy, and survey data collected from 13,448 members. Quality of Life (QOL) was assessed using the English version of the SF-36. The number and type of comorbidities were assessed from automated pharmacy data. Mean subgroup scores by ethnicity were compared using one way ANOVA to determine if there was a systematic difference in responses to the instrument by race. Separate regression analyses were completed to determine the association of ethnicity on the QOL scores, controlling for covariates age, gender, income, smoking, employment status, and the presence of chronic disease. The majority of the cohort was Caucasian $(55 \%)$ with a representative sample of African Americans $(22 \%)$, Latinos (14\%), and Asians (7.2\%). Depression, pain and inflammation, diabetes and rheumatologic conditions were associated with health status scores among Caucasian, African American and Latino subgroups. Unadjusted mean SF-36 subscale scores were significantly different, with Caucasians receiving the lowest scores and Asians the highest. In the regression analysis, combined demographic and clinical covariates explained $25 \%$ of the variation in the physical health subscale for African American, $28 \%$ for Caucasian, and $17 \%$ for the Asian and Latino subgroups $(P=0.0001)$. In general, after adjusting for covariates, Asians scored significantly higher on all subscales compared to the other three ethnic subgroups. This is important for those utilizing $\mathrm{QOL}$ instruments in diverse populations. Even after controlling for sociodemographic and clinical conditions some differences in the levels of reported health status between ethnic groups remained. Additionally, the similarity in health status scores across different ethnic groups may be reflective of the study's sampling strategy including only English speaking and high risk subjects.

367/ TESTING FOR VALIDITY OF A NEWLY DEVELOPED JAPANESE VERSION OF THE MOS SOCIAL SUPPORT SURVEY

Hideki Origasa, Biostatistics, Toyama Medical and Pharmaceutical University Toyama, Toyama, Japan; Hideki Nomura, General Medicine, Kanazawa University, Kanazawa, Ishikawa, Japan; Masami Matsumura, Nephrology, Ishikawa Prefectural Hospital, Kanazawa, Ishikawa, Japan; Toshihisa Morioka, Tomoharu Matsukura, Biostatistics, Toyama Medical and Pharmaceutical University Toyama, Toyama, Japan

Significant impact of perceived social support (SS) on quality of life of patients with chronic conditions has been emphasized. However, a reliable tool to evaluate the SS status for trans-cultural comparison has been unavailable in Japan. To develop and validate a Japanese version of the MOS SS Survey questionnaire that comprises of 18 questions with 4 domains; emotional/informational support, tangible support, affectionate support, and positive social interaction. The MOS SS Survey questionnaire was translated into Japanese by two independent investigators and discrepancies were discussed to reach a consensus. This Japanese version, as well as SF-36 Japanese version, was then applied to 153 patients with chronic renal failure undergoing hemodialysis, after obtaining an informed consent. Construct validity was evaluated by factor analysis and item response theory. Convergent/divergent validity was confirmed by factor analysis and correlation with SF-36. Internal consistency was confirmed by standardized alpha coefficients among domains. Sensitivity was explored by subgroup analysis based on the value of SF-36. The translation of the phrase "someone who hugs youth" was changed to "someone who take your hands or put hands on your shoulder," following Japanese cultural custom. A ceiling (indicating the best support) effect was found in a range of $20 \%$ to $30 \%$. Construction of the four domains was almost validated except that there was an ambiguity between tangible and affectionate supports. This measure clearly discriminated from physical and mental domains of the SF-36, whereas the 4 domains of this measure were similar. Internal consistency revealed a satisfactory level $(>0.8)$ for all the domains. A group of high SF-36 (>70) was approximately 10\% higher than a low SF-36 $(<70)$ group, in each of the four domains. A new Japanese version of the MOS Social Support Survey showed a good property of the validity using a sample of Japanese HD patients. 
368/ VALIDITY OF A NEWLY DEVELOPED JAPANESE SS-QOL (STROKE-SPECIFIC QUALITY OF LIFE) MEASURE

Hideki Origasa, Biostatistics, Toyama Medical and Pharmaceutical University Toyama, Toyama, Japan; Takeshi Nakano, Neurology, Toyoshina Red-Cross Hospital, Matsumoto, Nagano, Japan; Toshihisa Morioka, Biostatistics, Toyama Medical and Pharmaceutical University Toyama, Toyama, Japan; Mufumi Ohwa, Cardiology, Shinshu University School of Medicine, Matsumoto, Nagano, Japan

The incidence of stroke is relatively high in Japan. Although there has been an increasing interest in quality of life after stroke, there is no reliable questionnaire available in Japan to conduct cross-cultural studies. Therefore, we developed a Japanese version of the 2 nd version of SS-QOL (Stroke 1999; 30:1362-9) and have already shown the result of reliability at the Pan-pacific QOL conference in Tokyo. To confirm the validity of a Japanese version of the SS-QOL that comprises of 49 questions with 12 domains. A Japanese version of the SS-QOL, together with SF-36 and EQ-5D questionnaires, was applied to 120 stroke patients attending at a local hospital neurology department. Scaling validity was explored by obtaining both floor and ceiling effects. Construct validity was confirmed by item-response theory in addition to factor analysis. Item response theory was also used for solving a problem of item redundancy. Convergent/divergent validity was confirmed by a multitrait-multimethod analysis. Internal consistency was evaluated by standardized alpha coefficients among domains. Sensitivity was explored by comparing a group of worse after stroke with that of unchanged. A ceiling effect indicating the best QOL was found in a range of 10 to $60 \%$. Each of the 12 domains was independently constructed and there appeared to be converged into two groups, i.e., physical dysfunction and mental/social aspects. Two questions ("I didn't join in activities just for fun with my family; " $X$ and I had sex less often than I would like") were picked up as showing invalid responses, due to the Japanese cultural difference of family relationships. The domain of physical dysfunction was remarkably convergent among different scales, whereas emotional domain did not show a good convergence. A group of worse after stroke was $17 \%$ lower than a group of unchanged after stroke. Internal consistency revealed a satisfactory level for all the domains. A newly developed Stroke Specific QOL Japanese version showed a good property of validity.

369/ AN INNOVATIVE APPROACH TO THE DEVELOPMENT OF A SINGLE SPANISH TRANSLATION FOR USE IN MORE THAN ONE COUNTRY

Alyson M. Grove, Erika D. Ammerman, Oxford Outcomes, Oxford, UK; Silvia C. Bonicatto, QoL and Pharmacoeconomics, Fundonar Foundation, La Plata, Argentina; Eduardo Medina Cárdenas, University of Chile, Santiago, Chile; Carlos Kiyan, University of Peru, Lima, Peru; George R. Parkerson, Department of Community and Family Medicine, Duke University Medical Center, Durham, NC; Sandra L. Ford, Global Outcomes Research, Pharmacia Corporation, Kalamazoo, MI

With pharmaceutical companies increasingly looking to recruit patients for trials in multiple countries speaking the same language, there is often pressure on agencies providing cultural adaptations to compromise the recommended methodologies in order to limit the cost of producing useable translations. This presentation describes an innovative use of recommended methodology in order to develop a single Spanish translation of the 17-item Duke Health Profile (DUKE) for use in 3 South American countries - Argentina, Chile and Peru. With agreement from the developer, the Castillian Spanish DUKE questionnaire was reviewed concurrently by quality of life experts in each of the three target countries. The results of these three reviews were discussed and a single set of changes to the Castillian Spanish agreed. These were made to the questionnaire and this draft Spanish translation was then pilot-tested (debriefed) concurrently in the three target countries and again the results pooled. The final set of changes was reviewed by the developer to ensure the conceptual coherence of the original Castillian Spanish translation had not been compromised, and that conceptual equivalence with the original English had been maintained. His approval resulted in a single Spanish translation for use in Argentina, Chile and Peru. In an attempt to make a distinction between mere 'translations' and instruments which have been 'translated and culturally validated', sometimes agencies involved in the development and international use of outcomes research measures are afraid that any deviation from the basic recommended methodology is necessarily a compromise. This study shows that, in certain specific circumstances, innovative approaches based on the principles of accepted methodological standards can result in culturally valid translations which are not restricted for use in a single country.

370/ CULTURAL ADAPTATION OF QUALITY OF LIFE INSTRUMENTS FROM THE WEST TO THE EAST: WHAT IS DIFFERENT? Eva Dragomirecka, Psychiatric Demography Unit, Pavla Selepova, Prague Psychiatric Center, Prague, Czech Republic

The main purpose of this presentation is to highlight the items that cannot be mechanically taken over in the process of adaptation of questionnaires to the Czech language since their relation to the quality of life is different in the Anglo-American context to the East-European (specifically Czech) reality. These items are usually related to the lifestyle and can be divided into three categories: 1) description of aspects/activities that are far less represented in the Czech culture, and the absence of them does not necessarily mean that the quality of life is limited or that the respondent is disabled; 2) description of aspects/activities that have a high value for the quality of life of the Czech respondent but since they represent a different lifestyle they are often not involved in the QOL instruments and 3) new aspects/ activities that are only accepted by a certain part of the Czech population (usually young people living in larger cities) and therefore these variables depend strongly on the age and location of respondents. The presentation is supplemented with examples of QOL items, confronted with the corresponding data about the lifestyle in the Czech Republic. This work was supported by CEZ MZ 00000023752

371/ THE SPANISH NIH-CHRONIC PROSTATITIS SYMPTOM INDEX: TRANSLATION AND LINGUISTIC VALIDATION

Mark S. Litwin, Departments of Urology and Health Services, UCLA Los Angeles, CA; Michael P. O'Leary, Alex Adler, Department of Surgery, Harvard Medical School, Division of Urologic Surgery, Boston, MA; Elizabeth A. Calhoun, Institute for Health Services and Health Policy Studies, Northwestern University, Chicago, IL; Michel A. Pontari, Department of Urology, School of Medicine, Philadelphia, PA; Sonya L. Eremenco, Chih-Hung Chang, Linda Odom, CORE, ENH, Evanston, IL; Mary McNaughton Collins, General Medicine Division, Massachusetts General Hospital, Boston, MA

Due to the increasing prominence of health-related quality of life (HRQOL) endpoints in international clinical research, standardized measures are necessary to allow for cross-cultural comparisons. The $\mathrm{NIH}-$ Chronic Prostatitis Symptom Index (NIH-CPSI) assesses symptoms and $\mathrm{HRQOL}$ in men with chronic prostatitis. To expand its use to Spanish-speaking patients, we conducted a translation and linguistic validation. The NIH-CPSI was translated into Spanish according to a standard translation methodology consisting of 2 forward translations, 1 reconciled version, a back-translation of the reconciled version, and 3 independent reviews by bilingual experts. The goal of the translation process was to obtain a single universal Spanish version that would be acceptable to native Spanish speakers inside and outside the United States (US). After completing the translation process, the Spanish version was pre-tested in Argentina, Mexico, Spain and the US. The internal consistency of the CPSI was evaluated using Cronbach's alpha. Patient responses were analyzed and comments compiled to identify necessary modifications. Data were collected from chronic prostatitis patients in Argentina $(\mathrm{N}=15)$, Mexico $(\mathrm{N}=15)$, the US $(\mathrm{N}=4)$, and Spain $(\mathrm{N}=3)$. The translation had high reliability overall and in all subscales (Cronbach's alphas .87 to .94). In pre-testing, patients' only difficulty was in distinguishing the response categories "a menudo" ("often") from "normalmente" ("usually"). We revised "a menudo" to "muchas veces" and "normalment" to "casi siempre" to improve the scale. The Spanish NIH-CPSI has excellent reliability and face validity in Spanish-speaking men from a variety of countries. The Spanish NIH-CPSI will permit cross-cultural comparisons of men with chronic prostatitis. 
372/ TRANSLATION, RELIABILITY, AND VALIDITY OF THE 12 ITEM GENERAL HEALTH QUESTIONNAIRE AMONG YOUNG PEOPLE IN IRAN

Ali Montazeri, Faranak Farzadi, Azita Goshtasebi, Mariam Vahdani, Gholamreza Garmaroudi, Abolfazl Fateh, Iranian Institute of Health Sciences Research, Tehran, Iran

The objective this study was to translate and to test reliability and validity of the 12-item General Health Questionnaire (GHQ-12) in Iran. Using standard â€ backward-forwardâ€ ${ }^{\mathrm{TM}}$ translation procedure, the English-language version of the questionnaire was translated into Persian (Iranian language). Then, a sample of 748 young people aged 18 to 25 years old completed the questionnaire. In addition, a short questionnaire containing demographic questions and a single measure of global quality of life (ranging from extremely poor to excellent) was administered. The mean age of respondents was $21.1(\mathrm{sd}=2.1)$ years and most were female $(76 \%)$, single $(84 \%)$, and college/university student $(51 \%)$. Employing the recommended method of scoring (ranging from 0 to 12), the mean GHQ score was found to be 3.7 $(s d=3.5)$. However, the single measure of global quality of life indicated that many $(28 \%)$ experiencing neither good nor bad and $31 \%$ moderately good quality of life. To test reliability, the internal consistency of the questionnaire was assessed by Cronbachâ€ $€^{\mathrm{TM}} \mathrm{S}$ alpha coefficient and it was found to be 0.87 . Validity was performed using known groupsâ€TM comparison analysis. The results of the ANOVA of GHQ score with global quality of life status (as the grouping variable) indicated that there were significant differences between groups in the expected direction. Those with a better global quality of life scored lower on the $\mathrm{GHQ}$ and those with an extremely poor or poor globa quality of life reported more psychological problems $(F=58.6, P$ $<00001$ ). Finally, to suggest a cut-off score for 'caseness', it was found that the mean GHQ score for the whole population of respondents provided a rough guide to the best threshold. The findings of this study indicated that the Iranian version of the GHQ-12 is an acceptable, reliable and valid instrument of measuring psychological wellbeing in young peopel in Iran, where more than $50 \%$ of populations are under 30 years old in age.

373/ CHALLENGES AND SOLUTIONS TO DESIGNING AND CONDUCTING GLOBAL RESEARCH

Catherine Marle, The Lewin Group, Levallois-Perret, Cedex, France; Meryl Brod, The Lewin Group, San Francisco, CA; Pascale Peeters, The Lewin Group, Levallois-Perret, Cedex, France; Erin Sullivan, The Lewin Group, Boston, MA

Post approval global studies incorporating patient-reported health outcomes and pharmacoeconomic data are becoming more common. Data from these studies are increasingly used by pharmaceutical companies to demonstrate treatment benefits of novel therapies to regulatory agencies and formulary committees. However, designing these global studies and measuring health and pharmacoeconomic outcomes cross culturally pose unique methodological and logistical challenges to researchers. Definitions of research designs and the use of health outcomes data vary widely not only between the US and Europe but within Europe. Designing a study that meets the regulatory and marketing needs of each participating country is an enormous challenge that requires scientific expertise as well as diplomacy. Standardization of designs and data collection processes are made difficult due to differences in technologies, treatment norms, available resources, cultural expectations, and characteristics in the health care system across countries and continents. Additionally, potential cultural variation in perception of disease and treatment related side effects, statistical issues regarding pooling of data and interpretation of outcomes must all be addressed. Successful implementation of a global study requires input from all involved countries on design, measurement and logistical decisions. In addition, cross culturally valid methodologies and measurement tools must be incorporated, and guidelines for data collection may need to be uniquely tailored to each country. This presentation will offer a review of the state of the art for conducting global research and outline design, measurement, anaIytic, and interpretation issues which should be considered. The challenges associated with conducting global research will be highlighted along with differences in potential applications of health outcomes data in Europe and the US. Case examples from large multinational trials will be presented to illustrate these challenges and solutions.
374/ THE VALIDATION STUDY OF RUSSIAN VERSION GASTROINTESTINAL SYMPTOM RATING SCALE (GSRS) IN PEPTIC ULCER PATIENTS

I. Wiklund, AstraZeneca R\&D Molndal, Molndal, Sweden, A Novik Department of Hematology, Military Medical Academy, St. Petersburg, Russia; T. Ionova, QOL Group, N. L. Denisov, A. V. Kishtovich, V. A. Shuvaev, Multinational Center of Quality of Life Research, St. Petersburg, Russia

The aim of this study was to translate in Russian and perform crosscultural adaptation of the disease-specific questionnaire GSRS. A total of 176 patients were enrolled in this study according to next criteria: males and females over 18 years of age, no previous gastric surgery and previous history of peptic ulcer disease documented by endoscopy. All patients completed russian versions SF-36 and GSRS and undergo upper endoscopy and ultrasound examination of abdomen. The design of validation was consisted from double translation and investigation of reliability, validity and responsiveness derived Russian version of GSRS. As result of factor analysis structure of dimensions were regrouped in abdominal pain syndrome (where nausea was excluded) and reflux syndrome(where nausea was included). For this modified structure reliability were shown good: Cronbach a ranged from 0,75 to 0,87 and reproducibility (Spearmann $r$ ) ranged from 0,53 to 0,77 . The content validity was approved by group of expert gastroenterologists. Construct validity was evaluated in terms of correlation with dimensions of SF-36 (most highly was - 0,53 for abdominal pain syndrome GSRS and bodily pain of SF-36), knowngroups validity (for patient with or without relapse of ulcer mean of effect size was ranged from 0,50 to 1,07 for different dimensions of GSRS).Responsiveness was assessed by evaluation quality of life before and after ulcer healing. In this way Gyatt index was ranged from 0,36 to 1,22 ,indicating the dimension of abdominal pain syndrome as most responsive. Thus, russian version of GSRS is reliable, valid and responsive for peptic ulcer disease and may use for quality of life research of this group patients.

375/ A COMPARATIVE STUDY OF PHYSICAL AND MENTAL HEALTH IN RUSSIA AND THE UNITED STATES

Tatyana I. Ionova, Multinational Center of Quality of Life Research, Military Medical Academy, St. Petersburg, Russia; Barbara Gandek, Health Assessment Lab, Boston, MA; Andrei A. Novik, Anna A. Tsepkova, Anton V. Kishtovich, Yuriy A. Sukhonos, Multinational Center of Quality of Life Research, Military Medical Academy, St. Petersburg, Russia

Tatyana lonova, Barbara Gandek, Andrei Novik, Anna Tsepkova, Anton Kishtovich, Yuriy Sukhonos. Multinational Center of Quality of Life Research, St. Petersburg, Russia The health of the Russian population has declined during the past 10 years, as measured by indicators such as declining life expectancy and increasing complicated birth rates. This research adds to these indicators by comparing the self-reported physical and mental health of inhabitants of Russia and the US, using survey data collected in both countries in 1998. St. Petersburg data was from a representative sample of 2,207 adult inhabitants of St. Petersburg (age range 16-85, 40.5\% male). US data was from a nationally representative sample of 1,982 adults (age range $18-96,40.4 \%$ male). Both surveys included the SF-36 Health Survey, which has 8 scales measuring functioning and well-being and two summary measures of physical and mental health. To facilitate comparisons, all SF-36 scales and summary measures were scored such that a value of 50 is the mean and 10 is the standard deviation in the 1998 US general population. Across all age groups, SF-36 scores were lower in Russia than the US, with differences ranging from 0.3 (Vitality) and 1.2 (PF-Physical Functioning) to 9.8 points (MH-Mental Health) $(p<0.05$ for all scales and summary measures except Vitality). However, differences varied by age group. For example, among adults age 18-44, PF scores were not significantly different (Russia $=52.5$, US $=53.0$ ), while $\mathrm{MH}$ scores were 7 points lower in Russia. For adults age 65 and older, PF scores were 3 points and $\mathrm{MH}$ scores were 11 points lower in Russia. Overall, differences between the two countries were much greater for mental health than for physical health, and the differences were greater for older age groups. These findings may partly reflect the psychological pressures caused by social changes in Russia during the past 10 years, particularly for older Russians who have primarily lived under a different political and economic system. 
376/ PROPOSED GUIDELINES FOR THE 1ST STEP OF CROSS CULTURAL ADAPTATION OF PATIENT REPORTED OUTCOMES (PROS) MEASURES

Katrin Conway, Isabelle Mear, Catherine Acquadro, ERIQA Group, Mapi Research Institute, Lyon, France

With increased international collaboration and the growing use of Patient Reported Outcomes (PROs) evaluation in clinical research, the need for international measures has grown. As most are developed in English, they need to be culturally adapted, i.e. linguistically and psychometrically validated, to obtain equivalence between the source and target measures. In response to European regulators' concern about the methodology employed to achieve this, the ERIQA Group and Mapi Research Institute are investigating current guidelines for cross-cultural adaptation of PROs questionnaires focusing on the linguistic validation of quality of life questionnaires (QoL). The objective of the research is to identify current guidelines for crosscultural adaptation of PROs measures, and to propose minimal requirements for their linguistic validation. Relevant papers were identified from Medline, Embase, and Mapi Research Institute's database. The first two databases were explored with: "quality of life," "questionnaires," "health status indicators" matched with "translating" and "cross-cultural comparison." 173 references were identified. The search on the Institute's database used "translation issues," "crosscultural comparison," "cross-cultural research" as keywords and identified 234 references. Titles and abstracts of the 407 references were reviewed for relevance. Papers were included if they proposed recommendations or reviewed methods to cross-culturally adapt PROs measures. 33 papers met the inclusion criteria. First results identified 11 sets of guidelines developed either by identified Groups, Institutions or individuals, i.e. the EORTC Group, EUROQOL Group, FACIT Group, Guillemin et al, Herdman et al, IQOLA Group, Mapi Research Institute, MOT, NHP group, Patrick et al, and the WHO. Each methodology will be outlined and minimal requirements for the Linguistic Validation of PROs measures will be developed. Further research to explore empirical evidence of the effectiveness of methods will be suggested.

377/ RELIABILITY AND VALIDITY OF A SWEDISH VERSION OF CHILD HEALTH QUESTIONNAIRE (CHQ)

Ulla Norrby, Department of Pediatrics, Sahlgrenska University Hospital/Ostra, Goteborg, Sweden; Lena A. Nordholm, Department of Occupational Therapy and Physiotherapy, Goteborg University, Goteborg, Sweden

Child Health Questionnaire (CHQ) is an American self-administered instrument to assess the physical and psychosocial health status for children (CHQ-CF) and parents (CHQ-PF). It has been found valid and reliable in the USA and several other countries, but as yet reliability and validity have not been determined for the Swedish version. Therefore, the objectives of this study was to determine the psychometric characteristics of CHQ in a sample of 200 Swedish children (aged 9-15) and their parents. The children were registered as outpatients at the Queen Silvia's Children's Hospital, Sahlgrenska University Hospital, Göteborg, Sweden. Diagnoses of the children included: asthma, diabetes, juvenile chronic arthritis and short stature. As of date all data have been collected and preliminary analyses on some of the groups have been carried out. Cronbach's alpha was used to calculate the internal consistency of the CHQ-scales and was found to range between 0.76 and 0.98 for children with juvenile chronic arthritis, which compares well with previous studies. Validity will be assessed by correlating $\mathrm{CHQ}$ with three different instruments as appropriate to each group of children: Child Health Assessment Questionnaire, Paediatric Asthma Quality of Life Questionnaire and a Visual Analogue Scale. The sensitivity will be evaluated by correlating $\mathrm{CHQ}$ with objective disease-specific findings. These results will be reported at the conference. 
378/ PSYCHOLOGICAL ADJUSTMENT THAN CLINICAL SEVERITY IS MORE STRONGLY ASSOCIATED WITH HQOL OF PATIENTS WITH PARKINSON'S DISEASE

Yoshimi Suzukamo, Epidemiology and Health Care Research, Kyoto University, Kyoto, Japan; Sadayoshi Ohbu, Neurology, Yokohama Municipal Citizen's Hospital, Yokohama, Japan; Tomoyoshi Kondo, Junko Kohmoto, Neurology, Wakayama Medical University, Wakayama, Japan; Masanori Nagaoka, Neurology, National Rehabilitation Center for the Disabled, Tokorozawa, Saitama, Japan; Shunichi Fukuhara, Epidemiology and Health Care Research, Kyoto University, Kyoto, Japan

The objective of this study was to assess how health-related quality of life (HQOL) of patients with Parkinson's disease(PK) is associated with psychological adjustment to their disease. Cross-sectional data from 218 patients with PK (88 men with a mean age of 65 years) were analyzed. Validated Japanese versions of SF-36 and PDQ39 were used to measure global and disease-specific HQOL respectively. The Nottingham adjustment scale (NAS) was used to measure psychological adjustment. We focused on the "acceptance of disability(AD)" and "self efficacy(SE)" subscales of NAS. Primary physicians provided clinical data, such as the Hohn and Yahr disease severity indices(HY). We ran general linear models, including HQOL as an outcome variable, and age, gender and comorbid conditions as independent variables. We compared the increase in $\mathrm{R}<$ sup $>2<1$ sup $>$ from the basic model between the HY model adding clinical severity and the NAS model adding psychological adjustment, respectively, to the basic model. We also used covariance structure analysis to compare intervariable association with these two models. The $\mathrm{R}<$ sup $>2</$ sup $>$ of the basic model ranged from 0.02 to 0.15 . In analysis using SF-36 results as outcomes, in 7 out of 8 subscales the increases in $R<$ sup $>2</$ sup $>$ were significantly larger when "AD" of NAS was used than in the HY model. The differences were greater in the subscales "social functioning," "role emotional," "mental health," and "vitality." "SE" of NAS also increased R<sup $>2<$ /sup $>$ for "general health perception" of SF-36. The "mobility" and "ADL" subscales of $P D Q 39$ had larger increases in $\mathrm{R}<$ sup $>2</$ sup $>$ with the HY model than with the NAS model. However, for other subscales, the NAS model using "AD" showed larger $R<$ sup $>2</$ sup $>$ increases than did the HY model. On covariance structural analysis, psychological adjustment also influenced HQOL more strongly than did clinical severity. Psychological adjustment, such as "AD" and "SE," was much more strongly associated with the HQOL of patients with PK than was disease severity itself. This finding suggests that psychosocial intervention may help improve the HQOL of patients with PK.

\section{9/ QUALITY OF LIFE IMPAIRMENT IN MULTIPLE SCLEROSIS PATIENTS}

Petr I. Denisov, Andrei A. Novik, Tatyana I. Ionova, Elena M. Vereschagina, Anton V. Kishtovich, Multinational Quality of Life Research Center, Military Medical Academy, St. Petersburg, Russia

It is well known that multiple sclerosis (MS) considerably detriments patients quality of life (QoL). The aim of the study was to assess QoL of MS patients and to provide the comparison with the sample of St.Petersburg population. Twenty-nine patients with MS from St.-Petersburg Association of MS patients agreed to participate in the study. The characteristics of the sample was: $19 / 10$ females/males; mean age $41.0+/-6,5$; the duration of the disease -12.4 years (from 6 to 28 ); EDSS $7.2+/-1.8$ (from 4 to 9 ). The comparison group was formed from the representative sample of St. Petersburg population with the same demographic properties (age and sex). SF-36 questionnaire (1.0 Russian version) was used for $\mathrm{QoL}$ assessment. The patients got the questionnaire by mail (with phone doubling). Statistical analysis was provided using Shapiro-Wilkoxon and Mann-Whitney tests. QoL parameters were dramatically lower in MS patients as compared with the control group. The greatest, more than 10-fold difference was observed for "physical functioning" scale (7.4 vs. 75.3 ). All other scales values (except bodily pain) were much worse in MS patients as well. There was 4 -fold decrease of role physical functioning (16.4 vs. 62.0$), 1.5$-fold of general health ( 28.4 vs. 45.4$), 1.5$-fold of vitality ( 35.7 vs. 51.2 ), 2 -fold - of social functioning scale ( 28.4 vs. 65.9 ), 4 fold of role emotional functioning (17.2 vs. 65.5) and 1.3-fold of mental health (43.2 vs. 55.2 ). The difference in bodily pain scale parameters was not significant between MS patients and control group (48.1 vs. 64.4). The data obtained confirm the results for MS patients made in such countries as Great Britain, France and Norway. Thus, MS has dramatic detriment to QoL of MS patients. All QoL parameters of FS36 are considerably lower in MS patients as compared with the con- trol group. Russian version of SF-36 questionnaire is a reliable and valid instrument for QoL assessment of MS patients.

380/ ADVANTAGES OF JOINT USE OF EORTC QLQ-C30 AND FACT-G IN MALIGNANT LYMPHOMA PATIENTS

Andrei A. Novik, Tatyana I. Ionova, Anton S. Povzun, Anton V. Kishtovich, Multinational Center of Quality of Life Research, Military Medical Academy, St. Petersburg, Russia

EORTC QLQ-C30 and FACT-G are the most frequently used questionnaires in quality of life (QoL) assessment of cancer patients. The purpose of the study was to compare these questionnaires in case of malignant lymphoma and to reveal a correlation between their scales. One-hundred sixty-one malignant lymphoma patients (non-Hodgkin's lymphoma and Hodgkin's disease) were studied with two questionnaires in different time points during and after chemotherapy. Threehundred sixty-five pairs of questionnaires were compared. Statistical analysis was provided by Spearmen method ( $p$ less then 0,01 ). Physical well-being (PWB) scale of FACT-G questionnaire had significant correlation with physical functioning scale $(r=0,55)$ of EORTC QLQC30. There wasn't any correlation between social/family well-being scale of FACT-G and EORTC QLQ-C30 scales. FACT-G emotional well-being (EWB) scale correlated with emotional functioning scale $(r=0,53)$ of EORTC QLQ-C30. Functioning well-being scale (FWB) had correlation with 4 of 5 EORTC QLQ-C30 functional scales with average correlation coefficients. FACT-G total score correlated with general health status scale $(r=0,51)$ of EORTC QLQ-C30 questionnaire. Thus, three of four FACT-G scales had a quantity correlation with similar EORTC QLQ-C30 scales. Information on social/family well-being of a patients can be gained only from FACT-G. On the contrary, the presence of symptoms scales of EORTC QLQ-C30 gives more insight physical aspects of QoL and offers the advantage of identifying somatic symptoms related with the disease and its treatment In conclusion, EORTC QLQ-C30 and FACT-G provide similar information on many aspects of QoL of malignant lymphoma patients. However a number of limitations of both questionnaires make it reasonable their joint use in comprehensive QoL assessment.

\section{1/ QUALITY OF LIFE AND AGE RELATIONSHIP IN RHEUMA-} TOID ARTHRITIS PATIENTS

A. Tsepkova, Multinational Center of Quality of Life Research, St. Petersburg, Russia; T. Shemerovskaya, Rheumatological Center, St. Petersburg, Russia; A. Kishtovich, T. Ionova, Multinational Center of Quality of Life Research, St. Petersburg, Russia

It is widely known that age is a most important factor influencing quality of life (QoL). The aim of the study was to identify the relationship between age and QoL of rheumatoid arthritis (RA) patients and to assess the influence of RA in different age groups. We included 126 patients with definite RA, with age from 26 to 76 years (mean age $54,5 ; \mathrm{SD}=11,1)$. The majority of patients were women $(87 \%)$, with disease duration of 3-10 years, II degree of disease activity, (+) rheumatoid factor, III radiology stage, and II functional class. The patients were divided in 3 age groups: younger than 50 years $(n=46)$, from 50 to 60 years $(n=38)$, and older than 60 years $(n=42)$. As a control three groups from general St-Petersburg population with comparable age/ gender structure were chosen. SF-36 questionnaire was administered to RA patients at acute disease phase. As a result, values of all SF36 scales were significantly lower in three RA patients groups as compared with the controls of the same age $(p<0,001)$. In RA group younger than 50 years $P F, R P, R E$ and BP parameters were the most deteriorated as compared with controls. Their values were from 2 to 5 -fold worse than in control group $(39,5 \mathrm{vs} 76,4 ; 11,4 \mathrm{vs} 58,8$; $21,7 v s 67,6$; and $32,0 v s 63,6$ ). RA group aged $50-60$ showed more pronounced, from 3 to 10 -fold impairment of the above scales. Moreover, VT, MH and SF scale values were extremely low $(24,4 \mathrm{vs} 50,2$; $37,7 \mathrm{vs} 52,0 ; 36,5 \mathrm{vs} 61,8)$. RA patients after age 60 also showed significant QoL impairment, but there was improvement of all QoL parameters, especially of VT and SF $(p<0,05)$ as compared with the group of $50-60$ years. In conclusion, RA extremely deteriorates patients QoL. Age elder than 50 years results in more pronounced detriment of QoL parameters than younger age. The worst QoL parameters were revealed in the patients group of $50-60$ years. 
382/ ARE QUALITY OF LIFE PARAMETERS OF PROGNOSTIC VALUE FOR THE PRESENCE OF DUODENAL ULCER RELAPSE? Vassily Shuvaev, Multinational Center of Quality of Life Research, St. Petersburg, Russia

The aim of the research was to study prognostic value of the quality of life (QoL) parameters for prediction of duodenal ulcer relapse. A total of 115 patients were enrolled in this study according to next criteria: males and females over 18 years of age, symptoms of ulcer relapse, previous history of duodenal ulcer disease documented by endoscopy, no previous gastric surgery. Russian versions SF-36 and Gastrointestinal Symptom Rating Scale (GSRS) were administered to the patients. Discriminant analysis was provided. As a result of endoscopy all patients were divided two groups: 1) with the presence of duodenal ulcer relapse ( 90 cases); 2 ) with the absence of duodenal ulcer ( 25 cases). The following scales were significantly important for model construction: abdominal pain syndrome (APS), indigestion syndrome (IS), diarrhea syndrome (DS) of GSRS and general health $(\mathrm{GH})$, physical functioning (PF) of SF-36. The classification function for the presence of ulcer relapse was as follows: $19,08+2,20^{\star} \mathrm{APS}+0,94^{\star} \mathrm{IS}+0,79^{\star} \mathrm{DS}+0,08^{*} \mathrm{GH}+0,25^{\star} \mathrm{PF}$; the function for the absence of ulcer: $-18,92+1,54^{\star} A P S+1,47^{*} I S+1,28^{*} D S+0$ $12^{*} \mathrm{GH}+0,23^{*} \mathrm{PF}$. Classification matrix demonstrated $64 \%$ sensitivity and specificity for the presence of ulcer relapse. Thus, strong relationship between QoL parameters and morphological changes was shown. The following QoL parameters - abdominal pain syndrome, indigestion syndrome, diarrhea syndrome, general health and physical functioning might be the candidates as prognostic factors for the prediction of ulcer relapse. Further research is necessary to confirm this statement.

\section{3/ PERSONALITY AND QUALITY OF LIFE}

Guus Van Heck, Jolanda De Vries, Clinical Health Psychology, Tilburg University, Tilburg, The Netherlands

Quality of life (QOL) reflects the possession of resources necessary to the satisfaction of individual needs and desires. It concerns the perceived position in life in relation to individual goals, expectations, standards, and concerns. This definition suggests a link between personality and QOL. First, we review the literature on individual differences in QOL. Then, we present data of our own. In a first study, using a combined patient and non-patient group, all dimensions of the Big Five model of personality were related to $\mathrm{QOL}$ as measured by the WHOQOL-100. E.g., extraversion and neuroticism correlated 0.50 and -0.45 , respectively, with overall QOL and perceived genera health. Substantial correlations were also obtained with QOL domains (e.g., neuroticism correlated -0.54 with Psychological Health). In a second study, using the WHOQOL-Bref in a representative sample of the Dutch working population, the Big Five factors as well as Pavlovian temperament variables were examined. Again, substantive links were found. E.g., extraversion, emotional stability and autonomy/ openness to experience correlated significantly with all four QOL domains (correlations ranging from 0.21 to 0.65 ). Lower correlations were found with respect to agreeableness and conscientiousness. Also the Pavlovian variables, strength of excitation, strength of inhibition and mobility of nervous processes, reflecting individual differences in the behavioral expression of nervous system properties, were strongly related to QOL. Finally, this paper reports on the relationships between (i) the temperament dimensions of Cloninger's psychobiological model, novelty seeking, harm avoidance, reward dependence and persistence, (ii) the dimensions of character distinguished in this model, self-directedness, cooperativeness and self-trancendence, and (iii) QOL as assessed by the WHOQOL-Bref. In conclusion, it is stated that examination of the relationships between personality and temperament, on the one hand, and QOL, on the other hand, might aid in understanding and refining QOL research.
384/ DEVELOPMENT OF RUSSIAN VERSION OF THE PEDSQL GENERIC CORE SCALES

Andrei Novik, Tatyana lonova, Tatyana Nikitina, Anton Kishtovich, Multinational Center of Quality of Life Research, Military Medical Academy, St. Petersburg, Russia

The Pediatric Quality of Life Inventory (PedsQL, Varni et al) is a comprehensive modular instrument for measuring health-related quality of life in children ages 2-18. The aim of this study was to develop Russian version of the PedsQL 4.0 Generic Core Scales for children ages 5-7 (form 1) and 8-12 (form 2) as well as for their parents (forms $3,4)$. The Phase I of the study was the translation according to standard PedsQL translation methodology. It consisted of forward and back translations. After the review of the first version of PedsQL-Rus by the author Prof. J.W. Varni retranslating procedure was provided and Russian version of the PedsQL Generic Core Scales was created. The Phase II included cultural adaptation and pilot testing of Russian version of PedsQL (forms 1,2,3 and 4). For this goal PedsQLRus was administered to children recruited from a primary school of one of St-Petersburg's gymnasia. There were two age groups of children: $5-7$ years, boys/girls $=34 / 22 ; 8-12$ years, boys/girls $=35 / 23$ and their parents as proxy $(n=99)$. The mean Total Score for the group of 5-7 years was 70,3 ; for the group of $8-12$ years $-79,1$; for the parents of 5-7 years children - 72,2; for the parents of 8-12 years children 74,0 (maximum score is 100). Differences between child/parent concordances were significant for scales of Physical, Emotional, and School Functioning $(p<0,001)$ for $5-7$ years children and for scales of Physical, Emotional, Social, Psychosocial Functioning and Total Scale $(p<0,05)$ for 8-12 years children. Thus, after measuring of psychometric properties of Russian version of PedsQL Generic Core Scales it might be used in the pediatric quality of life research in Russia. 

Quality of Life Research 10: 291-298, 2001.

\section{Author Index}

\begin{tabular}{|c|c|}
\hline Author & Page(s) \\
\hline AC-Nikolic, Erzebet & 257 \\
\hline Antoniotti, Stéphanie & $240,244,259$ \\
\hline Atman, Umit & 241 \\
\hline Auquier, Pascal & $\begin{array}{c}210,212,240,243,244,259,259 \\
268,268\end{array}$ \\
\hline Azorin, Jean-Michel & 212 \\
\hline Aaronson, Neil & $194,210,225,225,226,282$ \\
\hline Abel, Thomas & 269 \\
\hline Abetz, Linda & $251,252,259$ \\
\hline Acquadro, Catherine & 270,287 \\
\hline Adachi, Kozaburo & 246,247 \\
\hline Adams, Elizabeth & 242 \\
\hline Adesina, Kunle & 251,252 \\
\hline Adler, Alex & 286 \\
\hline Agins, Bruce & 204 \\
\hline Ahmed, Sara & 204 \\
\hline Ahmedzai, S & 270 \\
\hline Akhras, Kasem & 251,252 \\
\hline Albert, Steven & 239 \\
\hline Albertsen, Peter & 211 \\
\hline Albright, Carol & 202 \\
\hline Alderson, D & 227 \\
\hline Allen, Mark & 198 \\
\hline Alonso, J & 220,281 \\
\hline Alonso, Jordi & 247,279 \\
\hline Alt, Jeannette & 261 \\
\hline Alvarez-Grande, Jaime & 250,282 \\
\hline Amir, Marianne & $236,275,276$ \\
\hline Ammerman, Erika & 223,285 \\
\hline Andersen, Ross & 254 \\
\hline Anderson, Donna & 219 \\
\hline Anderson-Hillemacher, Ast & 248 \\
\hline Andreas, Lori & 232 \\
\hline Andresen, E & 199 \\
\hline André, Baruchel & 216 \\
\hline Antonicelli, Leonardo & 201 \\
\hline Antoniu, Sabina & 220,220 \\
\hline Antó, J & 220 \\
\hline Aptel, Isabelle & 231 \\
\hline Arnold, Benjamin & 264 \\
\hline Arnould, Benoit & 213,278 \\
\hline Arpinelli, Fabio & 260 \\
\hline Ashraf, Talat & 271 \\
\hline Au, Heather-Jane & 228 \\
\hline Auquier, Pascal & 269 \\
\hline Axdorph, Ulla & 224 \\
\hline Bansal, Manishi & 198 \\
\hline Berki, Gizella & 247 \\
\hline Berta, Gyula & 247 \\
\hline Bertrand, Dominique & 259 \\
\hline Bey, Pierre & 227 \\
\hline Bredart, Anne & 227 \\
\hline Briancon, Serge & 195,227 \\
\hline Babu, A. & 253 \\
\hline Bachlet, Alison & 268 \\
\hline Badger, Shirlene & 231 \\
\hline Bagnulo, Adriana & 261,281 \\
\hline Baier, Monika & 236,240 \\
\hline Baker, Timothy & 272 \\
\hline Baltar, Jose & 250,282 \\
\hline Banerjee, Sube & 254 \\
\hline Banik, D. & 212 \\
\hline Barham, C & 227 \\
\hline Barofsky, Ivan & 197,254 \\
\hline Barr, Judith & 213 \\
\hline Barr, Ronald & 197 \\
\hline Barrett-Connor, E & 246 \\
\hline Bartlett, Susan & 254 \\
\hline Basen-Engquist, Karen & 229 \\
\hline Basque, Noreen & 204 \\
\hline Baumann, Michèle & 264 \\
\hline Bayer, A & 260,260 \\
\hline Beaton, Dorcas & 221 \\
\hline Becker, Gideon & 236 \\
\hline
\end{tabular}

Becker, Marion 215

Begerow, Bettina 214

Beinfeld, Molly 193

Bell, Robert 245

Bellach, Bärbel-Maria

Belloso, Waldo 207

Bendtsen, Preben 245

Benzer, Werner 206

Berend, M. 225

Bergman, Bengt 230

Bernardo, Arthur

Bernier, Julie 197, 266

Berrisford, R 227

Berthelot, Jean-Marie 197, 266

218, 255

Bezjak, Andrea $194,211,270$

Binns, Colin 241

Birbeck, Gretchen 221

Bjordal, Kristin 277

Bjorner, Jakob 195, 196, 203, 216

Björkholm, Magnus 224

Blanchard, Chris 208, 265

Blazeby, J 227

Blazeby, Jane 277

Bleiker, Eveline 225, 225, 226

Blomqvist, Carl 253

Blood, Paul 211

Bloom, Joan 195

Bode, Rita 203

Bodner, T. 230

Bodurka-Bevers, Diane 229

Boehmer, Sonja 196

Bonicatto, Silvia 285

Booth, Laura 246

Borenstein, Jeff 238

Borges, Vivian 275

Bosch, Johanna 193

Boscoe, Audra 276, 276

Boshuizen, Hendriek 217

Bottomley, Andrew 277

Bottomley Phd, Andrew 270

Bourne, Robert 208, 265

Bowden, Annabel 262

Bowling, Ann 224

Boyle, Deneane 200

Brady, Marianne 197

Brandman, Jane 255

Brandt, Dieter 206

Brant, R 196

Brant, Rollin 265

Brazier, John 222, 261

Brewer, Nancy 247

Broberg, Anders 267, 267

$\begin{array}{ll}\text { Brod, Meryl } & 240,286\end{array}$

Brown, Julia 246

Bruil, Jeanet 257, 269

Brundage, Michael 194, 211

Brülde, Bengt 214

Buck, Deborah 258

Buerk, , Guido 200

Bullinger, Monika $\quad 199,267,267,272$

Burnett, Paul 231

Bury, Caron 202

Bushnell, Donald 200, 215, 239, 256, 256, 276, 284

Buskila, Dan 236

Byng, Sally 233

Chandra, Prabha 273

Chaturvedi, Santosh 273

Clement, Audrey $240,259,268,268$

Cady, Paul 236

Caffrey, Helena 247

Calhoun, Elizabeth 208, 286

Camey, Suzi 275

Caporale, Betina 261

Cappelleri, Joseph 277

Caprini, Carol 262

Caprini, Joseph 262

Carlsson, Jonas 279 


\begin{tabular}{|c|c|}
\hline Carmack Taylor, Cindy & 229 \\
\hline Carone, Mauro & 220,220 \\
\hline Carriere, Kc & 210 \\
\hline Carroll, Peter & 226 \\
\hline Carvalheiro, Manuela & 272 \\
\hline Castells, Xavier & 247 \\
\hline Castilla, Miguel & 247 \\
\hline Catlin, $\mathrm{T}$ & 199 \\
\hline Catton, Charles & 245 \\
\hline Caulet, Malijai & 244 \\
\hline Cavazzuti, Luca & 260 \\
\hline Cederfjäll, Claes & 269 \\
\hline Celik, Sahsenem & 282 \\
\hline Cella, David & $\begin{array}{c}197,203,203,218,222,262,263, \\
264,282,216,212,234, \\
264\end{array}$ \\
\hline Ceretti, Teresa & 251 \\
\hline Chan, C & 219,229 \\
\hline Chan, Kitty & 214 \\
\hline Chan, Wing & 235 \\
\hline Chang, Chih-Hung & $203,203,264,282,286,264,212$ \\
\hline Chang, Sobin & 238 \\
\hline Chantal, Kalifa & 216,268 \\
\hline Chantal, Rodary & 216,268 \\
\hline Chaplin, John & 253,267 \\
\hline Charbonneau, Cecile & 231 \\
\hline Chavez, Shannon & 213 \\
\hline Chen, Dafna & 275 \\
\hline Chevallet, Laurence & 283 \\
\hline Chew, Ling-Huo & 283 \\
\hline Chhabra, Rosy & 204 \\
\hline Chiou, Chiun-Fang & 238 \\
\hline Chiu, Chui De & 266 \\
\hline Choe, Julie & $201,218,238$ \\
\hline Chou, Ann & 195 \\
\hline Christodoulou, , George & 252 \\
\hline Cieza, Alarcos & 248 \\
\hline Clarke, Ann & 209 \\
\hline Clarke, Susan & 264 \\
\hline Cleeland, Charles & $221,230,230$ \\
\hline Clegg, Limin & 211 \\
\hline Coccossis, Maria & 252 \\
\hline Cohen, Aaryn & 238 \\
\hline Cohen, S Robin & 231 \\
\hline Collins, Joyce & 247 \\
\hline Colman, Shoshana & 240 \\
\hline Colowick, A & 229 \\
\hline Colwell, Hilary & 259 \\
\hline Comas, Mercè & 247 \\
\hline Conroy, Thierry & 227 \\
\hline Consiglio, Ezequiel & 207 \\
\hline Conway, Katrin & 283,287 \\
\hline Cook, Sharon & 262,274 \\
\hline Correia, Edgar & 202 \\
\hline Coste, Joel & 264 \\
\hline Cottrell, Catherine & 236 \\
\hline Coulas, Gail & 244 \\
\hline Covinsky, Kenneth & 214 \\
\hline Coyle, Nessa & 205 \\
\hline Coyne, Karin & $199,200,254,272$ \\
\hline Crosby, Ross & 221 \\
\hline Culbertson, Vaughn & 236 \\
\hline Cutter, Garry & 240 \\
\hline Dallery, Jean & 212 \\
\hline Desandes, Emmanuel & 195,227 \\
\hline Didelot, Rémi & 244 \\
\hline Dinc, Gonul & 241,243 \\
\hline Dagrosa, Edmundo & 256 \\
\hline Dapueto, Juan & 255 \\
\hline Daughtery, David & 240 \\
\hline David, $\mathrm{K}$ & 215,246 \\
\hline David, KM & 257 \\
\hline Davis, Aileen & 245 \\
\hline Day, Andrew & 245 \\
\hline De Boer, Wim & 249 \\
\hline De Haan, Rob & $195,196,203$ \\
\hline De Koning, Harry & 207 \\
\hline De Moor, Carl & 280 \\
\hline De Paoli, Christina & 281 \\
\hline
\end{tabular}

\begin{tabular}{ll} 
De Witte, Luc & 219 \\
De La Loge, Christine & 259 \\
De Vries, Jolanda & 289 \\
Dehelian, Laura & 239 \\
Debruyne, C & 270 \\
Degner, Lesley & 194 \\
Del Borgo, Cosmo & 269,270 \\
Denisov, N. & 286 \\
Denisov, Petr & 288 \\
Dergazarian, Sonia & 251 \\
Derksen, Jan J. & 274 \\
Derkx, Bert & 200,217 \\
Deschamps, Claude & 198 \\
Determann, Mechthild & 227 \\
Detmar, Symone & 194 \\
Dewar, Abigail & 235 \\
Dewey, Jim & 203 \\
Dharma-Wardene, Melina & 228 \\
Diamond, Ronald & 215 \\
Dijkers, Marcel & 223 \\
Dimenas, Elof & 279 \\
Dinant, Huibert & 217 \\
Dixon, Peter & 211 \\
Dobrez, Deborah & 208 \\
Doll, Helen & 223 \\
Domingo-Salvany, A & 220 \\
Donner, Claudio & 220,220 \\
Doro, M. & 264 \\
Doward, Lynda & 235,262 \\
Doyle, Patrick & 256 \\
Dragomirecka, Eva & 241,285 \\
Duchesne, Inge & 213 \\
Duer, Wolfgang & 269 \\
Dupere, David & 228 \\
Duprat-Lomon, Isabelle & 223 \\
Durnin, Colin & 230 \\
Dzurova, Dagmar & 241 \\
& \\
\hline &
\end{tabular}

El Khammar, Mohammed $\quad 210,268,268$

Empereur, Fabienne 195, 227

Eremenco, Sonya 282

Eser, Erhan 241, 243

Easley, Mary 230

El Haddouchi, Fadoua $\quad 282$

Elfström, Magnus 234

Ellert, Ute 203

Elliott, Barbara $\quad 277$

Elliott, Thomas $\quad 219,277$

Elsner, Peter 246

Eltumi, M 268

Emery, Marie-Pierre $\quad 252,260$

Erder, H 229, 273

Eremenco, S. 264, 286

Erica, Quintana 216

Erickson, Pennifer 197, 208

Erickson, Steven 202, 252

Eriksen, John 199

Esser, Joachim 255

Essink-Bot, Marie-Louise 207, 269

$\begin{array}{ll}\text { Eton, David 222, } 230 & \end{array}$

Evans, Stacy 265

Exbrayat, Catherine 231

Farkas, Eva $\quad 247$

Francois, Patrice 259

Fairclough, Diane 211

Faloon, T. 233

Fantoni, Massimo 270

Faris, P 196

Farzadi, Faranak $\quad 286$

Fateh, Abolfazl 286

Feeny, David $\quad 208,210,254,265$

Fehnel, Sheri 255

Fekkes, Minne $\quad 257$

Feldman, Ira 204

Feldman-Stewart, Deb 194

Fernandez-Vega, Francisco 250

Ferrazzi, S. 233

Ferreira, Pedro 229, 272 


\begin{tabular}{|c|c|}
\hline Ferrer, M & 220 \\
\hline Fielding, R & 219,229 \\
\hline Fink, Nancy & 198 \\
\hline Finlay, I & $212,244,249$ \\
\hline Fischer, Tobias & 246 \\
\hline Fisher, Elizabeth & 240 \\
\hline Fitzpatrick, Ray & 216 \\
\hline Flechtner, Henning & 270,277 \\
\hline Fleck, Marcelo & 275 \\
\hline Fleischhacker, Wolfgang & 281 \\
\hline Fleiss, Anja & 228 \\
\hline Foley, Beth & 254 \\
\hline Fong, Kok-Yong & 283 \\
\hline Fontaine, Kevin & 254 \\
\hline Ford, Gary & 258 \\
\hline Ford, Helen & 209 \\
\hline Ford, Sandra & 285 \\
\hline Foster, E. Michael & 266 \\
\hline Fournier, Louise & 244 \\
\hline Fox-Rushby, Julia & $207,208,262$ \\
\hline Franckowiak, Shawn & 254 \\
\hline Francolino, Carla & 255 \\
\hline Frank, Lori & 275 \\
\hline Franke, Gabriele & $228,249,250,255$ \\
\hline Friedman, Monika & 275 \\
\hline Friedrichs, Annette & 237 \\
\hline Fujita, Haruyasu & 246,247 \\
\hline Fukuhara, Shunichi & 288 \\
\hline Fukuoka, Akikazu & 246 \\
\hline Fukuroku, Keiko & 235 \\
\hline Fukuuchi, Atsushi & 194 \\
\hline Furlong, William & 197 \\
\hline Félez, M & 220 \\
\hline Gagnon, D & 216 \\
\hline Gagnon, D. & 234 \\
\hline Gagnon, Dennis & $200,239,280$ \\
\hline Galbraith, D & 196 \\
\hline Gandek, Barbara & $222,283,287$ \\
\hline Ganiats, T & 215,246 \\
\hline Ganiats, Tg & 257 \\
\hline Garcia-Arumí, Josep & 247 \\
\hline Garcia-Aymerich, J & 220 \\
\hline Garcia-Mendoza, Monica & 282 \\
\hline Garmaroudi, Gholamreza & 286 \\
\hline Garnerin, Philippe & 214 \\
\hline Gazelle, Scott & 193 \\
\hline Gelijns, Annetine & 201,238 \\
\hline Georgiou, Amanda & 209 \\
\hline Gerbaud, Laurent & 259 \\
\hline Gerber, Robert & 277 \\
\hline Gerritsma, Miranda & 225,226 \\
\hline Ghali, W & 196 \\
\hline Giaconne, G & 270 \\
\hline Gilchrist, Kim & 254 \\
\hline Gilliland, Frank & 211 \\
\hline Gilsbach, Joachim-Michael & 209 \\
\hline Glauda, Laura & 200,239 \\
\hline Globe, Denise & $193,193,284$ \\
\hline Glueer, Maren Gesina & 214 \\
\hline Gohmann, Stephan & 248 \\
\hline Gómez, Juan & 217 \\
\hline Gonzalves, Sheila & 200 \\
\hline Goodman, Jane & 256,256 \\
\hline Goshtasebi, Azita & 286 \\
\hline Gotay, Carolyn & $224,232,233$ \\
\hline Gotta, Irene & 255 \\
\hline Goudeman, Michel & 212 \\
\hline Grabowski, Martin & 237 \\
\hline Graf V.D. Schulenburg, Ma & 202 \\
\hline Grafton, Clive & 211 \\
\hline Gralla, Richard & 228,280 \\
\hline Grandy, Susan & 254 \\
\hline Greenhalgh, Joanne & 209 \\
\hline Greil, Richard & 281 \\
\hline Greimel, Karoline & 219 \\
\hline Greiner, Wolfgang & 202 \\
\hline Grob, Jean-Jacques & 259 \\
\hline Groenvold, Mogens & $196,212,232$ \\
\hline Groessl, E & 215 \\
\hline
\end{tabular}

\begin{tabular}{|c|c|}
\hline Groessl, Erik & $\begin{array}{l}257 \\
213\end{array}$ \\
\hline $\begin{array}{l}\text { Grogg, Amy } \\
\text { Grootenhuis, Martha }\end{array}$ & $\begin{array}{l}213 \\
200,201\end{array}$ \\
\hline Grosclaude, Pascale & 231 \\
\hline Gross, Cynthia & 194, 202, 234 \\
\hline Grossfeld, Gary & 226 \\
\hline Grotzinger, Kelly & 275 \\
\hline Grove, Alyson & 285 \\
\hline Gruppen, Larry & 252 \\
\hline Guelfi, Julien-Daniel & 212 \\
\hline Guerra, Aida & 251 \\
\hline Guethlin, Corina & 264,277 \\
\hline Guillemin, Francis & $195,227,264$ \\
\hline Guillemin, Francois & 227 \\
\hline Guterres, Clara & 275 \\
\hline Gwede, Clement & 263 \\
\hline Hagan, Michael & 239,253 \\
\hline Hagell, Peter & 237 \\
\hline Hahn, Elizabeth & 218,261 \\
\hline Hak, Tony & 215 \\
\hline Hakamies-Blomqvist, Liisa & 253 \\
\hline Halim, Magdalena & 274,280 \\
\hline Halpern, Elkan & 193 \\
\hline Hammerlid, Eva & 225 \\
\hline Han, Sung & 284 \\
\hline Hanestad, Berit & $206,241,242,251$ \\
\hline Hanley, James & 238,204 \\
\hline Hanson, John & 228 \\
\hline Hanssen, Tove & 251 \\
\hline Harada, Takao & 235 \\
\hline Hareendran, Asha & 256,256 \\
\hline Harris, Lisa & 247 \\
\hline Harris, R. & 264 \\
\hline Hartley, Guilford & 221 \\
\hline Hartman, Margriet & 265 \\
\hline Hartmann, Claudia & 248 \\
\hline Hawthorne, Graeme & 207,262 \\
\hline Hayashi, Kazuo & 194 \\
\hline Hayashida, Rika & 247 \\
\hline Hays, Ron & 206,221 \\
\hline Heemann, Uwe & 249,250 \\
\hline Heffernan, $\mathrm{N}$. & 212 \\
\hline Heinemann, Allen & 203,264 \\
\hline Helgeson, Vicki & 230 \\
\hline Heltne, Carl & 245 \\
\hline Henne-Bruns, Doris & 227 \\
\hline Henning, James & 226 \\
\hline Henriques, Alexandre & 275 \\
\hline Herdman, Michael & 284 \\
\hline Hertz, Marshall & 234 \\
\hline Heydari, Mohammad & 274 \\
\hline Heyes, Anne & 213,274 \\
\hline Hilari, Katerina & 233 \\
\hline Himelick, Tom & 242 \\
\hline Himmelberger, David & 201 \\
\hline Hoare, Peter & 267 \\
\hline Hobart, Jeremy & 216,279 \\
\hline Hoffman, Rebecca & 271 \\
\hline Hoffman, Richard & 211 \\
\hline Hogan, Anthony & 262 \\
\hline Hogg-Johnson, Sheilah & 221 \\
\hline Hollen, Patricia & 228,280 \\
\hline Hollenstein, Judith & 225 \\
\hline Holman, Rebecca & $195,196,203$ \\
\hline Holzner, B. & 230,281 \\
\hline Hoopman, Rianne & 282 \\
\hline Horsman, John & 197 \\
\hline Hosseini, Kazem & 274 \\
\hline Hsu, David & 231 \\
\hline Hudgens, Stacie & 261,262 \\
\hline Hurley, Laurie & 221 \\
\hline Hurst, Keith & 209 \\
\hline Hyman, Joshua & 201,238 \\
\hline Höfer, Stefan & 206 \\
\hline Hütter, Bernd-Otto & 209 \\
\hline
\end{tabular}

Groessl, E

215 
Iha, Shigemichi

Im, Soo

Imai, Hirohisa

Ingham, Mike

lonova, $\mathrm{T}$.

lonova, Tatyana

Irvine, E

Isitt, J

Jona, Gabriella

Jabs, Douglas

Jackson-Thompson, J

Jacobi, Catharina

Jacoby, Ann

Jakovljevic, Djordje

Jane, Landgraf

Janjan, Nora

Jansen, Sylvia

Javier, Rejas

Javier, Soto

Jean-Pierre, Vannier

Jeanne, Landgraf

Jeffrey, Janet

Jensen, Pernille

Johnson, Darlene

Johnson, Jeffrey

Johnson, Laura

Jonas, Deborah

Jones, C. Allyson

Jones, Paul

Junghard, Ola

Junma, Jirayut

Klein, Sylvie

Kono, Suminori

Kuntar, Agnes

Kallich, J

Kalo, Zoltan

Kalthoff, Holger

Kangeri, John

Kao, Senyeong

Kaplan, R

Kaplan, Rm

Kaplan Gilpin, Adele

Karafa, M.

Karlsson, Jan

Katsumata, Noriyuki

Kavadas, V

Kawanishi, Tsukasa

Kearney, Mairead

Keininger, Dorothy

Kelly, Brian

Kemmler, Georg

Kennedy, Carol

Kennedy-Martin, Tessa

Khalaf, A

Kiebert, Gwendolyn

Kievit, Job

Kim, Kyeong

Kim, Sehyun

Kinchen, Kraig

King, Madeleine

Kirchberger, Inge

Kirkels, Wim

Kirking, Duane

Kishtovich, A.

Kishtovich, Anton

Kiyan, Carlos

Klee, Marianne

Klein, Ronald

Klewer, Jörg

Klimont, Jeannette

Kline Leidy, Nancy

Klinger, Regine

Kluijt, Irma

Knopp, Werner

Knudsen, Øistein

Knudsen Jr, Øistein
281
218
226
213
288
$286,288,288,289$
233

247

248

199

217

209, 258

205, 220, 252

268

230

258

282

282

268

216

223

232

263

210

211

213, 274

254

220, 220

271,276

250

195

218

247

229

249

227

262

266

215, 246

257

248

205

$273,273,283$

281

227

246

274

245

231

230, 281

221

272

220

272

258

284

221, 284

198

278

$256,258,278$

207

202

286, 288

288, 288, 289

285

232

272

$234,237,238$

278

202, 213, 274

219

225,226

234

206

241, 242
Knudtson, M

Kobayashi, Michiko

Koh, Dow-Rhoon

Kohlboeck, Gabriele

Kohlmann, Thomas

Kohmoto, Junko

Kollenbaum, Volker

Kolotkin, Ronette

Komaki, Ritsuko

Kondo, Tomoyoshi

Koopman, Henrik

Kopec, Jacek

Kopp, Martin

Korfage, Ida

Kosinski, Mark

Kounti, Popi

Koëter, Gerard

Krabbe, Paul

Kreitzer, Mary Jo

Kremer, Bernd

Kreuter, Margareta

Kristenson, Margareta

Kroenke, K

Kugler, Joachim

Kuhl, David

Kulich, Károly

Kunitoh, Hideo

Kuroi, Katsumasa

Kvrgic, Svetlana

Köhler, F.

Köhler, K.

L.Derksen, Jan

Labarere, José 259

Lancon, Christophe 212

Lash, Ayhan 217

Launois, Robert 261

Leonard, Isabelle $\quad 195,227$

Leplege, Alain 212

Llorca, Pierre-Michel $\quad 212$

Laan, Roland 265

Laforêt, Céline 213

Lai, Jin-Shei $\quad 222,203$

Lai, Kuan-Lang 266

Laimer, Herbert 206

Laliberte, Kimberly $\quad 216$

Lam, Cindy 283

Lamarca, R 220

Lambert, Bridget $\quad 268$

Lamping, Donna 216

Langendijk, Johannes $\quad 210$

Langius-Eklöf, Ann $\quad 224,269$

Last, Bob

200,201

259

242

214

236

265

241

201, 238

213

219

245

194,231

213

222

211

230

206, 241, 242

244
232

201

244

283

228

269

197

228, 280 


\begin{tabular}{|c|c|}
\hline Lima, Ana Flávia & 275 \\
\hline Limm, Heribert & 248 \\
\hline Limongelli, Prospero & 269,270 \\
\hline Lindeboom, Robert & $195,196,203$ \\
\hline Lindvall, Olle & 237 \\
\hline Lingjiang, Li & 250 \\
\hline Lipscomb, Joseph & 233 \\
\hline Litwin, Mark & 207,286 \\
\hline Lograsso, Mary & 218 \\
\hline Lobo, Francis & 194 \\
\hline Locadia, Mirjam & 251 \\
\hline Long, Andrew & 209 \\
\hline Longstreth, George & 271 \\
\hline Loonen, Hester & 200,217 \\
\hline Louzada, Sérgio & 275 \\
\hline Lower, Anthony & 241 \\
\hline Lua, P & 212,244 \\
\hline Lubeck, Deborah & 226,259 \\
\hline Luo, Nan & 283 \\
\hline Luoma, Minna-Liisa & 253 \\
\hline Lykens, Michael & 247 \\
\hline Lynch, Pamela & 246 \\
\hline Mechetti, Christian & 243 \\
\hline Megnigbeto, Alexandre Cof & 261 \\
\hline Mogyorosy, Zsolt & 247 \\
\hline Mohanti, Bk & 198 \\
\hline Morita, Takako & 218 \\
\hline Ma, Yungsheng & 204 \\
\hline Machin, David & 232 \\
\hline Mackell, Joan & 237,237 \\
\hline Madalinska, Joanna & 207 \\
\hline Maftoon, Farzaneh & 274 \\
\hline Mahon, Jeffrey & 208,265 \\
\hline Maity, Santanu & 268,272 \\
\hline Malmgren, Helge & 214 \\
\hline Malmgren, Kristina & 253 \\
\hline Mandai, Takashi & 246,247 \\
\hline Mangione, Carol & 263 \\
\hline Mannell, Roger & 240 \\
\hline Manno, Michael & 221 \\
\hline Marden, Susan & 205 \\
\hline Margolis, Mary Kay & $213,254,272,274$ \\
\hline Marinelli, William & 202 \\
\hline Marinus, Johan & 257 \\
\hline Marle, Catherine & 286 \\
\hline Marquis, Patrick & 278 \\
\hline Marrades, $\mathrm{R}$ & 220 \\
\hline Marrero, David & 247 \\
\hline Marsh, Jane & 198 \\
\hline Martin, Barbara & 248 \\
\hline Martin, Mona & $215,256,256,276,284$ \\
\hline Martinez, Vicente & 247 \\
\hline Martínez-Palmer, Ana & 247 \\
\hline Maruyama, Soichiro & 246,247 \\
\hline Mason, Emanual & 213 \\
\hline Mass, Michele & 240 \\
\hline Masuyama, Sumie & 246 \\
\hline Mata, Pedro & 202 \\
\hline Mathees, Barbara & 194 \\
\hline Mathias, Susan & 240 \\
\hline Matsuda, Tomohiro & 231 \\
\hline Matsukura, Tomoharu & 285 \\
\hline Matsumoto, Taketoshi & 226 \\
\hline Matsumura, Masami & 285 \\
\hline Matsuoka, Ken & 246,247 \\
\hline Matthees, Barbara & 234 \\
\hline Mayer, Emeran & 271 \\
\hline Mayo, Nancy & $204,209,209,210,238$ \\
\hline Mcdonnell, Diana & $237,237,276,276$ \\
\hline Mckenna, Stephen & $235,249,262,274,276$ \\
\hline Mckenzie, Deirdre & 221 \\
\hline Mclaughlin-Miley, Connie & 255 \\
\hline Mcleod, Lori & 255 \\
\hline Mcnaughton Collins, Mary & 286 \\
\hline Mcneil, Carmelita & 231 \\
\hline Mcneil, Malcolm & 256 \\
\hline Meads, David & 274 \\
\hline Mear Isabelle & 287 \\
\hline
\end{tabular}

\begin{tabular}{|c|c|}
\hline Medina Cárdenas, Eduardo & 285 \\
\hline Mehta, Shilpa & 259 \\
\hline Melchart, Dieter & 278 \\
\hline Melia, Michele & 263 \\
\hline Melia-Gordon, Michelle & 233,242 \\
\hline Memmini, Michael & 261 \\
\hline Mendoza, Tito & $221,230,230$ \\
\hline Menko, Fred & 225,226 \\
\hline Meraner, V. & 230 \\
\hline Mercier, Celine & 244 \\
\hline Meuleners, Lynn & 241 \\
\hline Michaels, J. & 205 \\
\hline Mihajlovic, Bogoljub & 205,252 \\
\hline Mikolic, Joseph & 256 \\
\hline Miller, Daniel & 198 \\
\hline Miller, Deborah & $205,218,236,240,255$ \\
\hline Minne, Helmut & 214 \\
\hline Mishra, Avanish & 277 \\
\hline Miura, Kouji & 246 \\
\hline Mobley, Gary & 230 \\
\hline Moen, Bente & 243 \\
\hline Moenkemoeller, Kirsten & 200 \\
\hline Monsó, E & 220 \\
\hline Montazeri, Ali & 274,286 \\
\hline Morales, Leo & 206 \\
\hline Morfeld, Matthias & 272 \\
\hline Morimoto, Kanehisa & 246,247 \\
\hline Morioka, Toshihisa & $242,285,285$ \\
\hline Moskowitz, Alan & 201,238 \\
\hline Moum, Torbjørn & 242 \\
\hline Moy, Claudia & 263 \\
\hline Muia, Patrick & 207 \\
\hline Muller, Martin & 210 \\
\hline Mulroy, Liam & 211 \\
\hline Muraoka, Miles & 224 \\
\hline Murray, Joanna & 254 \\
\hline Murray, Michael & 247 \\
\hline Murri, Rita & 269,270 \\
\hline Muschler, George & 218,255 \\
\hline Müller, Jürgen & 256 \\
\hline Nagano, Jun & 218 \\
\hline Nagaoka, Masanori & 288 \\
\hline Nishibayashi, Yasurou & 218 \\
\hline Nabulsi, Azmi & 271 \\
\hline Nadesan, Bala & 236 \\
\hline Naesdal, Jorgen & 271 \\
\hline Naess, Siri & 199 \\
\hline Nagano, Takuzou & 235 \\
\hline Nagata, Akira & 242 \\
\hline Naka, Yoshiko & 247 \\
\hline Nakano, Takeshi & 285 \\
\hline Namba, Tomoko & 246,247 \\
\hline Narkiss, Tal & 236 \\
\hline Naujoks, Christel & 235 \\
\hline Nayer, Marla & 221 \\
\hline Nelson, D. & 205 \\
\hline Nemetz, Boris & 275 \\
\hline Neuparth, Nuno & 202 \\
\hline Neves, Conceicao & 272 \\
\hline Newell, Jeffrey & 247 \\
\hline $\mathrm{Ng}$, Swee-Cheng & 283 \\
\hline Nichol, Michael & 193 \\
\hline Nichols, Francis & 198 \\
\hline Niciforovic Surkovic, Olj & 257 \\
\hline Nicolas, Bellon & 216,268 \\
\hline Niero, Mauro & 260 \\
\hline Nieuwkerk, Pythia & 204 \\
\hline Nikitina, Tatyana & 289 \\
\hline Nishi, Tsunehiro & 194 \\
\hline Nobrega, Karla & 266 \\
\hline Noda, Kazumasa & 226 \\
\hline Nomura, Fumio & 247 \\
\hline Nomura, Hideki & 285 \\
\hline Nooy, Marianne & 258 \\
\hline Nordholm, Lena & 287 \\
\hline Nordrehaug, Jan & 251 \\
\hline Norrby, Ulla & 287 \\
\hline Norris, Colleen & 196 \\
\hline
\end{tabular}


Nortvedt, Monica

Novak, Annoesjka

Novik, Andrei

Nyandieka, Lilian

Nzioka, Charles

O'Leary, Michael

O'Sullivan, Brian

Oral, Ahmet

Ozcan, Cemil

Odom, L.

Odom, Linda

Oga, Katsuhiko

Ogino, Satoshi

Ogushi, Michiko

Ohashi, Yasuo

Ohbu, Sadayoshi

Ohman, Susan

Ohsumi, Shozo

Ohwa, Mufumi

Okabayashi, Sachiyo

Okamoto, Takahiro

Olaizola, Inés

Oliver, Phillip

Olsen, Alf

Oort, Frans

Oprandi, Nadia

Origasa, Hideki

Ortega, Francisco

Osoba, David

Ouwens, Jan-Paul

Pellissier, Véronique

Poirier, Marie-France

Pagès, Jérôme

Pai, Mohan

Pairolero, Peter

Pais-Ribeiro, Jose

Palcher, Jeanette

Palmer, Martin

Palmer, Michael

Parasuraman, Bhash

Pare, $\mathrm{P}$.

Park, Hye

Parkerson, George

Parsons, Susan

Pascal, Chastagner

Pasquier, Elodie

Pater, Joseph

Patrick, Donald

Paul, D.

Payne, Richard

Pedersen, Lise

Peeters, Pascale

Pelusi, Dan

Penson, David

Perneger, Thomas

Persson, Lars-Olof

Peterman, Amy

Petersen, Corinna

Petersen, Morten

Petruson, Karin

Pfeifer, Michael

Philipp, Thomas

Pickard, Simon

Piotrowski, Wolfgang

Piribauer, Franz

Poirier, Leo

Poissant, Lise

Pongsakol, Chonlatip

Pontari, Michel

Popov, Miroslava

Porley, Graciela

Porterfield, Patricia

Post, Marcel

Post, Wendy

Potic, Marijela

Potic, Zoran
243, 243

259

$286,288,288,289$

262

207

286

245

241

241, 243

212

286

226

235

246

226, 281

288

213

281

285

246, 247

281

251

261

212

204

260

$242,285,285$

250,282

197

249

243

212

278

197

198

202, 227, 275

219,245

228

211

215

233

284

285

201

268

260

194, 211, 270, 245

200, 239, 276

212

205

212

286

231

259

214

234,273

197, 222

272

196

225

214

249, 250

210

219

258, 278

244

209

250

286

220

251

231

219

232

205, 220, 252

$205,220,252,257$
Potosky, Arnold

Pouchot, Jacques

Poulsen, E

Povzun, Anton 288

Powe, Neil

Power, Mick

Pratheepawanit, N

Prieto, Luis

Prins, Martin

Pychyl, Timothy

Pöhlau, Dieter

Quittan, Michael

Reine, Gilles

Robitail, Stéphane

259

259

205, 220, 252

Radovanovic, Ninoslav

269

Rajimil, Luis

Rajmil, Luis

281,284

Rance, $L$.

Rapaport, Mark

Rapkin, Bruce

Rapp, Stephen

Raspe, Heiner

Ravens-Sieberer, Ulrike

Rebollo, Pablo

Recklitis, Christopher

Rector, Thomas

Reed, George

Reeve, Bryce

Regal, Ronald

Regensteiner, Judy

Reichenberg, Kjell

Reimer, Jan

Reimer, Jens

Reise, Steven

Renier, Colleen

Rentz, Anne

Retel-Rude, Nathalie

Revicki, Dennis

Reyes, Eileen

Reyes-Gibby, Cielito

Reynolds, Sandra

Riazi, Afsane

Ribeiro, Helena

Ribeiro, J.L.P.

Richardson, Jeffrey

Riise, Trond

Riley, A

Ritvo, Paul

Robertson, Marguerite

Rodrigues, Vitor

Rorabeck, Cecil

Rose, M.

Rossi, G

Rothman, Margaret

Roye, David

Rudick, Richard

Ruiz, Miguel

Rupnow, Marcia

Rupp, Ines

Russak, Simcha

Rusthoven, James

Rustoen, Tone

Ruyskart, Pascal

Ryden, Anna

Samuelian, Jean-Claude

San Marco, Jean-Louis

Sapin, Christophe

Simeoni, Marie-Claude

Szaraz, Melinda

Szende, Ágota

Sa, Eunice

213

204

236

222

250,282

201

261

204

211

277

254

267, 267

237

206

$219,245,277$

199

264

238

221

263

216,279

229

264

207

243,243

281

194, 231

231

229

208, 265

206

200, 239

201, 218, 238

240

282

213

217

228,280

206, 241, 242

277

273, 234

212

243, 268 268

247

247

227

$199,203,267,269,200$

$228,249,250,255$

$199,254,272,275$

210, 240, 243, 244, 259, 259, 268,

$212,240,244,259,268,268$ 


\begin{tabular}{|c|c|}
\hline Sacristán, José & 217 \\
\hline Sadighi, Jila & 274 \\
\hline Sadura, Anna & 245,270 \\
\hline Saeki, Hideyuki & 281 \\
\hline Sagnier, Pierre-Philippe & 223 \\
\hline Sakkas, , Pavlos & 252 \\
\hline Saleh, Mahassen & 197 \\
\hline Salek, M & $198,212,244,249,260,260$ \\
\hline Sandlund, Birgitta & 237 \\
\hline Santigli, Elisabeth & 258,278 \\
\hline Sauder, Bonnie & 263 \\
\hline Saunders, D & 196 \\
\hline Savik, Kay & 234 \\
\hline Schaefer, Marion & 261 \\
\hline Scherbring, Mary & 231 \\
\hline Schestatsky, Gustavo & 275 \\
\hline Schiffman, Rhett & 265 \\
\hline Schmand, B. & 195 \\
\hline Schmidt, Peter & 206 \\
\hline Schmidt, Silke & 246,248 \\
\hline Schroter, Sara & 263,263 \\
\hline Schumacher, Gerald & 213 \\
\hline Schwartz, Carolyn & 204 \\
\hline Schwartzmann, Laura & 251,281 \\
\hline Sehgal, Lakshman & 262 \\
\hline Sek, Julia & 197 \\
\hline Selby, Peter & 246,258 \\
\hline Selepova, Pavla & 285 \\
\hline Serra, V & 281 \\
\hline Serra-Batlle, J & 220 \\
\hline Servente, Liliana & 255 \\
\hline Seshadri, Roopa & 247 \\
\hline Seymour, Lesley & 270 \\
\hline Sham, J & 219,229 \\
\hline Shandiz, Kjellaug & 251 \\
\hline Shariati, Mohammad & 274 \\
\hline Sharp, Mark & 204 \\
\hline Shepherd, Frances & 270 \\
\hline Shemerovskaya, $\mathrm{T}$. & 288 \\
\hline Sherbourne, Cathy & 193 \\
\hline Shi, Jinhai & 273 \\
\hline Shilling, B & 216,234 \\
\hline Shimozuma, Kojiro & 281 \\
\hline Shuaib, Ashfaq & 210 \\
\hline Shuvaev, V. & 286 \\
\hline Shuvaev, Vassily & 289 \\
\hline Sieber, W & 215,257 \\
\hline Siebmann, J.-U. & 228 \\
\hline Silander, Ewa & 225 \\
\hline Silberman, Cheryl & $213,242,274$ \\
\hline Simeoni, Marie Claude & 267 \\
\hline Simonian, Nancy & 240 \\
\hline Simpson, Helen & 272 \\
\hline Sinclair, Samuel & 222 \\
\hline Singh, Amitabh & 271 \\
\hline Sirois, Fuschia & 233,242 \\
\hline Sjogren, Per & 212 \\
\hline Sjöström, Johanna & 253 \\
\hline Sjöström, Lars & 273 \\
\hline Skyrnarz-Murphy, Kerry & 200 \\
\hline Smith, Adam & 258 \\
\hline Smith, Sarah & 254 \\
\hline Snyder, Claire & 233, 239 \\
\hline Spasoff, Robert & 266 \\
\hline Speed, C & 271 \\
\hline Spellmann, Mark & 218 \\
\hline Sperner-Unterweger, B. & 230,281 \\
\hline Spies, James & 200 \\
\hline Spitz, Elisabeth & 264 \\
\hline Sprangers, Mirjam & 204,251 \\
\hline Springer, Carolyn & 204 \\
\hline Spronk, Karen & 261 \\
\hline Stanford, Janet & 211 \\
\hline Starfield, B & 281 \\
\hline Steinbock, Clemens & 204 \\
\hline Stephenson, Robert & 211 \\
\hline Stiggelbout, Anne & $232,257,258$ \\
\hline Stitt, Larry & 208,265 \\
\hline Strauss, Bernhard & 246,248 \\
\hline Stromgren, Annette & 212 \\
\hline
\end{tabular}

Sturly, Ann

Sugimoto, Shizuko

Sukhonos, Yuriy

Sullivan, Erin

Sullivan, Marianne

Sullivan, PB

Sullivan, Patrick

Sunpithayakul, Supaphan

Suzukamo, Yoshimi

Suzuki, Atsuko

Svihra, Jan

Symonds, Tara

Thibault, Jean-Luc

Taal, Babs

Taft, Charles

Takayama, Tomoko

Talley, Nicholas

Tamburini, Marcello

Tanaka, Katsuhiro

Tarquinio, Cyrille

Tenvergert, Elisabeth

Teng, Josephine

Testa, Marcia

Thomas, Adrian

Thomas, Christiane

Thompson, Alan

Thompson, Pamela

Thompson, W

Thornton, David

Thumboo, Julian

Thyen, Ute

Tierney, William

Toi, Masakazu

Torgerson, Jarl

Tountas, , loannis

Trastek, Victor

Travers, John

Triantafillou, , Gina

Trotti, Andy

Tsepkova, A.

Tsepkova, Anna

Tsevat, Joel

Tsuboi, Shunji

Tu, Dongsheng

Turcotte, Robert

Turner, Ralph

Tzeng, Rong-Jye

Uebaba, Kauzo

Umansky, Roberto

Verdoux, Hélène

Viala-Artigues, Jacques

Vachalec, Stephanie

Vahdani, Mariam

Valderas, Jose

Valdimarsdottir, Heiddis

Van Gool, R.

Van Assche, Daniel

Van Beurden, Marc

Van Heck, Guus

Van Hilten, Bob

Van Laerhoven, Henriette

Van Den Bos, Geertrudis

Van Der Bij, Wim

Van Der Staak, Cees P.

Vandoros, Christina

Vardy, Daniel

Varghese, Frank

Vasoo, Sheila

Velikova, Galina

Velji, Karima

Vereschagina

Vermeulen, Karin

Vermeulen, $\mathrm{M}$.

Verrips, Erik
213

246

287

240, 286

$234,273,273,283$

268

193

250

288

246, 247

284

283

210

225, 226

273,283

194

271

260

281

264

249

209

222

268, 272

203

216, 279

253

233

215

283

267

247, 253

194

273

252

198

276, 276

252

263

288

286

214

247

194, 211, 270

245

222

266 


\begin{tabular}{|c|c|}
\hline Vicari, Marissa & 245 \\
\hline Vickery, C & 227 \\
\hline Vickrey, Barbara & 221 \\
\hline Vidalis, Anathanasios & 267 \\
\hline Vilagut, G & 220 \\
\hline Visona, Giovanni & 260 \\
\hline Visser, Martine & 257 \\
\hline Vitale, Michael & $201,218,238$ \\
\hline Vogels, Ton & 257 \\
\hline Von Roenn, Jamie & 264 \\
\hline Wahl, Astrid & $206,241,242$ \\
\hline Walach, Harald & 264,277 \\
\hline Walker, Irwin & 197 \\
\hline Walker, M & 260,260 \\
\hline Wallace, Robert & 279 \\
\hline Walt, John & 265 \\
\hline Wang, Bing-Long & 266 \\
\hline Wang, Shelley & 221 \\
\hline Wang, Xin Shelley & 230 \\
\hline Ward-Smith, Peggy & 224 \\
\hline Ware Jr, John & $203,216,222$ \\
\hline Watanabe, Koshiro & 226 \\
\hline Webster, K. & 212 \\
\hline Webster-Bogaert, Susan & 208,265 \\
\hline Weeks, J. & 212 \\
\hline Weidenhammer, Wolfgang & 278 \\
\hline Weinberger, Morris & 247 \\
\hline Weinert, Craig & 202 \\
\hline Weinstock-Guttman, Bianca & 240 \\
\hline Westerman, Marjan & 215 \\
\hline Wettergren, Lena & 224 \\
\hline Wever, Lidwina & 225,226 \\
\hline Whalley, Diane & $249,262,276$ \\
\hline Wheeler, H. Brownell & 204 \\
\hline White, Carole & 238 \\
\hline White, Deena & 244 \\
\hline White, Richard & 276,276 \\
\hline Wiklund, Ingela & $271,271,276,279,286$ \\
\hline Williams, Brent & 252 \\
\hline Williams, G. & 221 \\
\hline Williams, Randall & 261 \\
\hline Wilson, Ingalill & 271 \\
\hline Winn, R. & 212 \\
\hline Wolinsky, Fredric & 279,253 \\
\hline Wondzinski, Linda & 237 \\
\hline Wood-Dauphinee, Sharon & $204,209,209,210,238$ \\
\hline Woolley, J. Michael & 235,273 \\
\hline Wrede, Anne & 200 \\
\hline Wredling, Regina & 269 \\
\hline Wright, Benjamin & 279 \\
\hline Wright, Jim & 211 \\
\hline Wu, Albert & $198,199,214,248$ \\
\hline Wu, Joanne & 193,284 \\
\hline Wunder, Jay & 245 \\
\hline Wunderlich, Stefanie & 278 \\
\hline Wyrwich, Kathleen & $279,199,253$ \\
\hline $\mathrm{Xu}$, Feng-Hao & 242 \\
\hline Yamazaki, Yoshihiko & 194 \\
\hline Yang, Dong & 284 \\
\hline Yao, Kaiping & 266 \\
\hline Ying, Zhiliang & 253 \\
\hline Yong, Jin & 284 \\
\hline Yoon, Kam-Hon & 283 \\
\hline Yoshimura, Kimio & 281 \\
\hline Young, Carolyn & 209 \\
\hline Young, J. & 205 \\
\hline Yount, S. & 212 \\
\hline Younts, Joanna & 218 \\
\hline Yu, Clara & 219,229 \\
\hline Yui, Naoko & 246 \\
\hline Ziegler, Marc & 210 \\
\hline Zagari, M & 216,234 \\
\hline Zavazava, Nicholas & 227 \\
\hline Zee, Benny & 245 \\
\hline Zeidler, Henning & 222 \\
\hline Zeimet, G. & 230 \\
\hline
\end{tabular}


TOPIC INDEX

ANALYTIC ISSUES (LONGITUDINAL STUDIES/ MISSING DATA/ MULTIPLICITY OF ENDPOINTS)

\# PAGE TITLE

13196 COMPARISON OF THREE DIFFERENT STATI

15196 THE NOTTINGHAM HEALTH PROFILE IN PO

17197 A COMPARISON OF ANALYSIS METHODS FO

23198 ORODENTAL HEALTH, ACUTE AND LATE MO

37202 THE EFFECT OF ASTHMA SEVERITY ON HE

70210 DEFINING SYMPTOM AND FUNCTIONAL HEA

71210 AGREEMENT BETWEEN PROXY ASSESSMENT

72210 THE IMPORTANCE OF CONSIDERING MISSI

73211 INCORPORATING DEATH INTO THE STATIS

74211 IMPACT OF MISSING HRQOL DATA IS IT

75211 HRQOL OUTCOMES AMONG MEN MANAGED WI

81213 THE PATIENT'S PERSPECTIVE IN SCHIZO

109220 ASSOCIATION OF GENERIC AND SPECIFIC

110220 PREDICTORS OF MORTALITY IN PATIENTS

111220 IS IT POSSIBLE TO PREDICT PATIENT'S

112220 RELATIONSHIP BETWEEN COMORBIDITIES

114221 DEFINING RECOVERY: THE APPLICATION

115221 ROC ANALYSIS OF WEIGHT LOSS TO ACHI

120222 USING STRUCTURAL EQUATION MODELS (S

136227 A PROSPECTIVE RANDOMIZED STUDY ON T

157232 QUALITY OF LIFE OF BREAST CANCER SU

169235 THE PRACTICALITIES OF QUALITY OF LI

191241 DETERMINANTS FOR ADOLESCENT QUALITY

193241 QUALITY OF LIFE AND SELF-RATED HEAL

215247 CAN PHARMACEUTICAL CARE IMPROVE THE

224249 MEASUREMENT OF CHANGES IN DISEASE-S

230250 USE OF KARNOFSKY PERFORMANCE SCALE

250255 PSYCHOMETRIC PROPERTIES OF THE NEI-

270260 THE QUALITY OF LIFE INSTRUMENTS DAT

289265 HOW TO COMBINE PREFERENCE-BASED QOL

292266 CAN WE AND HOW TO WEIGHT THE DIMENS

306269 HEALTH-RELATED QUALITY OF LIFE IN H

307270 SATISFACTION WITH SEXUAL LIFE IN HI

336277 VALID RESULTS DESPITE MISSING DATA?

338277 CLINICAL POTENTIAL OF LONGITUDINAL

344279 UNDERSTANDING CHANGE OVER TIME IN T

345279 COULD LINEAR REGRESSION BE REPLACED

347280 MODELING LONGITUDINAL QOL DATA USIN

373286 CHALLENGES AND SOLUTIONS TO DESIGNI

383289 PERSONALITY AND QUALITY OF LIFE

\section{CARDIOVASCULAR DISEASE}

\# PAGE TITLE

4 193 CONVERGENT VALIDITY OF SF-36 UTILIT

13196 COMPARISON OF THREE DIFFERENT STATI

50205 SYMPTOM EXPERIENCE AND HEALTH RELAT

51205 PREDICTING UTILIZATION AND COSTS IN

52205 LONG TERM QUALITY OF LIFE AFTER OPE

53206 DETERMINANTS OF THE QUALITY OF LIFE

54206 PSYCHOMETRIC PROPERTIES OF THE MACN

111220 IS IT POSSIBLE TO PREDICT PATIENT'S

173236 AN ASSESSMENT OF HEALTH-RELATED QUA

209245 ANGIOPLASTY PATIENT 36 MONTH SURVIV

232251 QUALITY OF LIFE AND THE DURATION OF

233251 ASSESSMENT OF HEALTH-RELATED QUALIT

234251 DEVELOPING AN INTERVENTION TO ENHAN

236252 IS SELF PERCEPTION OF HEALTH THE MO

237252 RESPONSIVE HEALTH-RELATED QUALITY O

238252 RELATIONSHIP BETWEEN SYMPTOMS AND S

245254 EFFECT OF MODE OF ADMINISTRATION ON

252256 PSYCHOMETRIC VALIDATION OF THE ITAL

274261 VALIDATION AND RESCORING OF THE MIN

279263 THE RELATIVE RESPONSIVENESS OF GENE

280263 FINAL PSYCHOMETRIC EVALUATION OF TH

\section{CHILDREN}

\# PAGE TITLE

26199 THE REVISED KINDL-R: FINAL RESULTS

31200 COPING STYLES PREDICT QUALITY OF LI

32200 "I THINK MY LIFE IS BETTER THAN MOS PEDIATRIC SPINE DEFORMITY: ASSESSIN HEALTH RELATED QUALITY OF LIFE OF C USING ITEM RESPONSE THEORY TO IMPRO THE CHILD HEALTH QUESTIONNAIRE(CHQ) A COMPARISON OF LIKERT SCALE AND VI PERSPECTIVES ON PEDIATRIC OUTCOMES: ASSESSMENT OF HEALTH STATUS IN PATI WHAT DO WE MEASURE: HRQL OR HRQL DE TIME PRESSURE AND EMOTIONAL WELL-BE DETERMINANTS FOR ADOLESCENT QUALITY COST-UTILITY ANALYSIS OF UNIVERSAL SOCIAL COMPETENCE, EMOTIONAL/BEHAVI QUALITY OF LIFE IN CHILDREN AND ADO THE CORE VSP-A AND ITS SPECIFIC MOD VALIDATION IN FRENCH OF THE CHILD H DEVELOPMENT, VALIDATION OF HRQL MEA QUALITY OF LIFE IN CARERS OF SEVERE RELIABILITY AND DISCRIMINANT VALIDI SCREENING FOR AND PROMOTION OF HEAL EVALUATION OF A COMPUTERISED TOUCHUSING THE DELPHI METHOD TO DETERMIN RELIABILITY AND VALIDITY OF A SWEDI

\section{CHRONIC DISEASE}

\# PAgE TITLE

1193 COMPARING FIVE METHODS OF UTILITY A

20197 HEMOPHILIA: CONSTRUCT VALIDITY OF T

25199 DIABETES AND CHANGE IN PSYCHOSOCIAL

32200 "I THINK MY LIFE IS BETTER THAN MOS

35201 HEALTH RELATED QUALITY OF LIFE OF C

40202 COPING AND QUALITY OF LIFE IN ADULT

50205 SYMPTOM EXPERIENCE AND HEALTH RELAT

92215 DIFFERENCES IN PERCEPTION OF QUALIT

95216 PSYCHOMETRIC PROPERTIES OF FACT-AN

107219 MAJOR DEPRESSIVE DISORDER (MDD) IN

109220 ASSOCIATION OF GENERIC AND SPECIFIC

123223 ASSESSMENT OF QUALITY OF LIFE IN CH

162233 QUALITY OF LIFE IN UNIVERSITY STUDE

164234 DETERMINANTS OF QUALITY OF LIFE IN

165234 FACT-AN SHOWS SENSITIVITY TO CHRON

184239 A PHILOSOPHICAL ANALYSIS OF THE MEA

222248 EXPLORATIVE STUDY ON COMPLIANCE AND

229250 A PROSPECTIVE, CONTROLLED STUDY ON

230250 USE OF KARNOFSKY PERFORMANCE SCALE

235252 ASSESSING QUALITY OF LIFE IN HYPERT

239253 DIFFERENTIAL WEIGHTINGS OF THE CLIN

242253 RESULTS FROM THREE PHYSICIAN CONSEN

250255 PSYCHOMETRIC PROPERTIES OF THE NEI-

286264 SHOULD SYMPTOMS BE SCALED FOR INTEN

312271 QUALITY OF LIFE OF INFLAMMATORY BOW

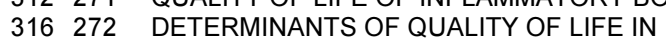

317272 SYMPTOMS OF DIABETIC RETINOPATHY AN

318272 GENDER DIFFERENCES IN QUALITY OF LI

320273 HEALTH-RELATED QUALITY OF LIFE IN O

321273 DO MEN AND WOMEN RESPOND DIFFERENTL

322273 QUALITY OF LIFE \&AMP; PSYCHOLOGICAL

335277 USING THE MANN-WHITNEY STATISTIC TO

364284 KOREAN VALIDATION OF THE KIDNEY DIS

378288 PSYCHOLOGICAL ADJUSTMENT THAN CLINI

383289 PERSONALITY AND QUALITY OF LIFE

\section{CLINICAL TRIALS}

\# PAge tittLe

$6 \quad 194$ CANCER PATIENTS' PREFERENCES FOR CO

17197 A COMPARISON OF ANALYSIS METHODS FO

52205 LONG TERM QUALITY OF LIFE AFTER OPE

70210 DEFINING SYMPTOM AND FUNCTIONAL HEA

74211 IMPACT OF MISSING HRQOL DATA. IS IT

76211 IS MORE BETTER? QUALITY OF LIFE (QO

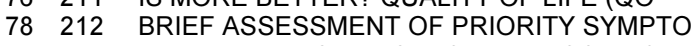

81213 THE PATIENT'S PERSPECTIVE IN SCHIZO

84213 WHAT ROLE DO SYMPTOMS PLAY IN HEALT

94216 ITEM REDUCTION: DO DIFFERENT METHO

102218 THE RELIABILITY AND VALIDITY OF THE

116221 RESPONSIVENESS OF THE QUALITY OF LI 
132226 QUALITY OF LIFE ASSESSMENT IN RANDO

136227 A PROSPECTIVE RANDOMIZED STUDY ON T

142228 TESTING THE LUNG CANCER SYMPTOM SCA

145229 IMPROVEMENT IN HEMOGLOBIN LEVELS RE

156232 SEXUAL FUNCTION IN WOMEN WITH A HIS

167235 ASSESSING QUALITY OF LIFE IN OSTEOP

168235 EVIDENCE SUPPORTING THE VALIDITY OF

169235 THE PRACTICALITIES OF QUALITY OF LI

189240 A NEW CONCEPTUAL MODEL FOR TREATMEN

208245 FUNCTION AND QUALITY OF LIFE MORBID

215247 CAN PHARMACEUTICAL CARE IMPROVE THE

217247 QUALITY OF LIFE IN MYOPIC PATIENTS

219248 EVALUATION OF A GENERAL AND DISEASE

226249 EVALUATION OF THE SF-36 AND EQ-5D F

229250 A PROSPECTIVE, CONTROLLED STUDY ON

233251 ASSESSMENT OF HEALTH-RELATED QUALIT

237252 RESPONSIVE HEALTH-RELATED QUALITY O

241253 PHYSICAL PERFORMANCE, TOXICITY, AN

264259 PSYCHOMETRIC VALIDATION OF CONTRACE

281263 DEVELOPMENT AND VALIDATION OF DISEA

289265 HOW TO COMBINE PREFERENCE-BASED QOL

308270 IMPLEMENTING QUALITY OF LIFE IN EOR

309270 A DECISIVE STEP TOWARDS THE RECOGNI

310270 USEFULNESS OF QUALITY OF LIFE (QOL)

313271 SYMPTOMS OF GASTRO-OESOPHAGEAL REFL

319273 COPING AND DISTRESS BEFORE AND AFTE

325274 ISSUES INVOLVED IN ASSESSING QUALIT

334276 FACTOR STRUCTURE OF THE QUALITY OF

335277 USING THE MANN-WHITNEY STATISTIC TO

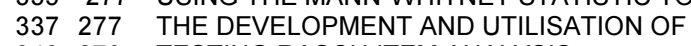

343279 TESTING RASCH ITEM ANALYSIS

347280 MODELING LONGITUDINAL QOL DATA USIN

348280 SOME LOGICAL PROBLEMS WITH TESTS FO

349280 RELIABILITY AND VALIDITY OF MODIFIE

351281 RELIABILITY AND VALIDITY OF THE FUN

373286 CHALLENGES AND SOLUTIONS TO DESIGNI

$\begin{array}{ll}376 & 287 \\ \text { PROPOSED GUIDELINES FOR THE 1ST STE }\end{array}$

\section{DERMATOLOGY}

\# PAGE TITLE

170235 QUALITY OF LIFE IN PATIENTS WITH AT

171236 HELIOTHERAPY AT THE DEAD SEA IMPROV

172236 A METHOD FOR ESTIMATING THE CONTRIB

211246 QUALITY OF LIFE AND COPING STRATEGI

221248 DISCRIMINATIVE ASPECTS OF QUALITY O

249255 RESPONSIVENESS OF THE ACNE-SPECIFIC

265259 QUALITY OF LIFE IN DERMATOLOGY: DEV

276262 TESTING THE PSYCHOMETRIC PROPERTIES

\section{DISABILITYIINJURYIREHABILITATION}

\# PAGE TITLE

38202 QUALITY OF LIFE AFTER INTENSIVE CAR

65209 THE COMPLEX RELATIONSHIP BETWEEN CO

68209 THE MS SYMPTOM AND IMPACT DIARY: A

72210 THE IMPORTANCE OF CONSIDERING MISS I

105219 RELATIONSHIPS BETWEEN SATISFACTION

121223 VALUE SHIFT AFTER DISABILITY ONSET:

122223 COMPARISON OF UNWEIGHTED SATISFACTI

160233 HRQOL IN PEOPLE WITH APHASIA: PRELI

166234 EFFECTS OF COPING ON MENTAL OUTCOME

224249 MEASUREMENT OF CHANGES IN DISEASE-S

250255 PSYCHOMETRIC PROPERTIES OF THE NEI-

251256 THE STROKE SURVIVOR FUNCTIONING AND

278262 MEASURING SOCIAL PARTICIPATION: DEV

297267 QUALITY OF LIFE IN CHILDREN AND ADO

302268 QUALITY OF LIFE IN CARERS OF SEVERE

327275 WAS IT A GOOD MOVE? IMPROVEMENT IN

330275 SOCIAL SUPPORT AND QUALITY OF LIFE

340278 CHANGE IN QUALITY OF LIFE IN RELATI

\section{DISEASE PREVENTION / PUBLIC HEALTH}

\# PAGE TITLE

39202 EFFECTS OF DISEASE MANAGEMENT ON QU

41203 USING ITEM RESPONSE THEORY TO IMPRO
MEASURING THE QUALITY OF LIFE AND Q COLORECTAL CANCER SCREENING: COMPLI QUALITY OF LIFE IN PATIENTS WITH AT WHAT DO WE MEASURE: HRQL OR HRQL DE DETERMINANTS FOR ADOLESCENT QUALITY WORKERS' QUALITY OF LIFE: TOWARDS A THE INFLUENCE OF LIFE-STYLE FACTORS QUALITY OF LIFE AND PRECARIOUSNESS: LONG-TERM QUALITY OF LIFE IMPROVEME DEVELOPMENT AND VALIDATION OF DEEP USING THE WHOQOL-BREF INSTRUMENT AN DEVELOPMENT, VALIDATION OF HRQL MEA SCREENING FOR AND PROMOTION OF HEAL QUALITY OF LIFE AND MENTAL HEALTH I METHODS FOR INTEGRATING THE SF-12 I CHANGE IN QUALITY OF LIFE IN RELATI

\section{ELDERLY}

\# PAGE TITLE

46204 RESPONSE SHIFT EFFECTS OF AN ADVANC

140228 THE NEED FOR PSYCHOSOCIAL SUPPORT I

154231 DECISIVE FACTORS OF QUALITY OF LIFE

195242 EFFECTS OF ALTERNATIVE MEDICINE ON

213246 QUALITY OF LIFE IN ELDERLY PERSONS

243254 AGREEMENT BETWEEN SELF AND PROXY AS

258257 QOL-OLD INSTRUMENT TO ASSESS QUALIT

268260 VALIDATION OF THE COMMUNITY DEMENTI

269260 COMPARISON OF TWO DISEASE-SPECIFIC

288265 UTILITY ASSESSMENT AMONG PATIENTS W

294266 USING THE WHOQOL-BREF INSTRUMENT AN

344279 UNDERSTANDING CHANGE OVER TIME IN T

\section{END OF LIFE CARE}

\# PAGE TITLE

16196 USING ITEM RESPONSE THEORY TO SHORT

49205 RESPONSE SHIFT IN PATIENTS' LIVING

73211 INCORPORATING DEATH INTO THE STATIS

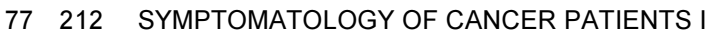

88214 QUALITY OF LIFE IN SERIOUSLY ILL AD

152231 THE PATIENT AND THEIR DOCTOR: A CON

153231 QOLLTI-F: A MEASURE OF THE QOL OF F

184239 A PHILOSOPHICAL ANALYSIS OF THE MEA

220248 PAIN SCALE RATINGS AND SATISFACTION

\section{EPIDEMIOLOGYIPOPULATION BASED STUDIES}

\# PAGE TITLE

13196 COMPARISON OF THREE DIFFERENT STATI

15196 THE NOTTINGHAM HEALTH PROFILE IN PO

19197 COMPARISON OF THE HEALTH UTILITY IN

20197 HEMOPHILIA: CONSTRUCT VALIDITY OF T

25199 DIABETES AND CHANGE IN PSYCHOSOCIAL

26199 THE REVISED KINDL-R: FINAL RESULTS

27199 RETEST RELIABILITY AND VALIDITY OF

36201 THE RELATIONSHIP BETWEEN RESOURCE C

37202 THE EFFECT OF ASTHMA SEVERITY ON HE

56206 VALIDATION OF THE WHOQOL-BREF IN A

61208 MEASURING THE QUALITY OF LIFE AND Q

62208 WHOSE VALUES COUNT? A CRITIQUE OF D

86214 HOW IMPORTANT IS HEALTH FOR OVERALL

91215 TOWARDS BRIEFER QOL INSTRUMENTS: A

113221 SURVIVAL, QUALITY OF LIFE AND THE S

123223 ASSESSMENT OF QUALITY OF LIFE IN CH

126224 QUALITY OF LIFE OF MALIGNANT BRAIN

150230 THE RELATIONSHIP BETWEEN PAIN SEVER

161233 CONSTIPATION AND QUALITY OF LIFE IN

172236 A METHOD FOR ESTIMATING THE CONTRIB

173236 AN ASSESSMENT OF HEALTH-RELATED QUA

$\begin{array}{lll}176 & 237 & \text { TREATMENT AND HEALTHCARE SATISFACTI }\end{array}$

190240 TIME PRESSURE AND EMOTIONAL WELL-BE

192241 OVERALL QUALITY OF LIFE IN THE GENE

193241 QUALITY OF LIFE AND SELF-RATED HEAL

194241 HEALTH RELATED TRADITIONAL BEHAVIOR

195242 EFFECTS OF ALTERNATIVE MEDICINE ON

196242 MEASURING FATIGUE IN THE GENERAL PO 
199243 WORKERS' QUALITY OF LIFE: TOWARDS A

200243 HEALTH-RELATED QUALITY OF LIFE AND

201243 THE INFLUENCE OF LIFE-STYLE FACTORS

202243 HOW MIGRATION AFFECTS THE HEALTH R

214246 HOW DO WE AGE? AN ANALYSIS OF SYMPT

227250 PREVALENCE OF ERECTILE DYSFUNCTION

243254 AGREEMENT BETWEEN SELF AND PROXY AS

261258 FEASIBILITY OF THE SF-12 HEALTH SUR

273261 PRELIMINARY FINDINGS IN THE DEVELOP

288265 UTILITY ASSESSMENT AMONG PATIENTS W

293266 A FRAMEWORK FOR CALCULATING CANADIA

299268 THE CORE VSP-A AND ITS SPECIFIC MOD

303269 RELIABILITY AND DISCRIMINANT VALIDI

324274 QUALITY OF LIFE AND MENTAL HEALTH I

338277 CLINICAL POTENTIAL OF LONGITUDINAL

339278 METHODS FOR INTEGRATING THE SF-12 I

346279 APPLYING ITEM RESPONSE THEORY (IRT)

353281 QUALITY OF LIFE IN THE AUSTRIAN GEN

354281 PRELIMINARY ANALYSIS OF THE CULTURA

361283 CROSS-CULTURAL ADAPTATION OF THE SF

365284 USING THE DELPHI METHOD TO DETERMIN

372286 TRANSLATION, RELIABILITY, AND VALID

375287 A COMPARATIVE STUDY OF PHYSICAL AND

\section{FAMILYICAREGIVERS}

\# PAGE TITLE

151231 EFFECT OF CAREGIVER PERCEPTION OF P

153231 QOLLTI-F: A MEASURE OF THE QOL OF F

181238 A COMPREHENESIVE REVIEW OF CAREGIVE

182238 THE INFLUENCE OF GENDER ON HEALTH-R

186239 ASSESSING THE SUBJECTIVE IMPACT ON

296267 FAMILY CLOSENESS, PARENTAL LOCUS OF

322273 QUALITY OF LIFE \&AMP; PSYCHOLOGICAL

332276 HEALTHCARE RESOURCE USE AMONG CAREG

333276 MENTAL WELL BEING AMONG CAREGIVERS

\section{GASTROINTESTINAL DISEASE}

\# PAGE TITLE

24198 QUALITY OF LIFE AFTER ESOPHAGEAL RE

31200 COPING STYLES PREDICT QUALITY OF LI

130225 COLORECTAL CANCER SCREENING: COMPLI

131226 GENETIC COUNSELING FOR COLORECTAL C

135227 A PROSPECTIVE COMPARISON OF QUALITY

161233 CONSTIPATION AND QUALITY OF LIFE IN

244254 RESPONSE SHIFT OF A HEALTH-RELATED

267260 PSYCHOMETRIC PROPERTIES OF IBS-QOL

312271 QUALITY OF LIFE OF INFLAMMATORY BOW

313271 SYMPTOMS OF GASTRO-OESOPHAGEAL REFL

314271 IMPACT OF GASTROINTESTINAL SYMPTOMS

315272 EVALUATION OF A COMPUTERISED TOUCH-

320273 HEALTH-RELATED QUALITY OF LIFE IN O

334276 FACTOR STRUCTURE OF THE QUALITY OF

357282 DYSPEPSIA-RELATED QUALITY OF LIFE:

382289 ARE QUALITY OF LIFE PARAMETERS OF P

\section{GYNECOLOGYIOBSTETRICS/ENDOCRINE}

\# PAGE TITLE

28199 THE SEXUAL RELATIONS SCALE: PSYCHOM

29200 VALIDATION OF THE UFS-QOL, A DISEAS

30200 PSYCHOMETRIC EVALUATION OF THE OBES

120222 USING STRUCTURAL EQUATION MODELS (S

156232 SEXUAL FUNCTION IN WOMEN WITH A HIS

183239 QUALITY OF LIFE OF ENROLLEES AND NO

221248 DISCRIMINATIVE ASPECTS OF QUALITY O

271261 RELIABILITY AND VALIDITY OF A NEWLY

\section{HEALTH SERVICES RESEARCH/ TECHNOLOGY}

\section{ASSESSMENT}

\# PAGE TITLE

$4 \quad 193$ CONVERGENT VALIDITY OF SF-36 UTILIT

42203 ITEM BANKING TO IMPROVE, SHORTEN AN

55206 THE FACTOR STRUCTURE OF THE SF-36 P
60207 NEGATIVE UTILITY SCORES: THEORETICA

63208 COMPARING COMMUNITY-PREFERENCE BASE

67209 CONTRIBUTIONS OF HEALTH-RELATED QUA

83213 ETHNIC DIFFERENCES IN VALUES FOR HE

91215 TOWARDS BRIEFER QOL INSTRUMENTS: A

140228 THE NEED FOR PSYCHOSOCIAL SUPPORT I

185239 RELATING CONCEPTS OF DISEASE \&AMP:

203244 IMPACT OF COMMUNITY MENTAL HEALTH S

204244 USING A HEALTH STATUS INDICATOR TO

210245 INTRODUCING HRQL INTO DAILY ROUTINE

213246 QUALITY OF LIFE IN ELDERLY PERSONS

214246 HOW DO WE AGE? AN ANALYSIS OF SYMPT

223249 EVALUATION OF A NOVEL PAIN MANAGEME

228250 QUALITY ASSURANCE IN KIDNEY TRANSPL

240253 IS THE EFFECT REAL OR AN ARTIFACT?

266259 VALIDATION OF A FRENCH INSTRUMENT M

270260 THE QUALITY OF LIFE INSTRUMENTS DAT

287265 TEST-RETEST RELIABILITY AND INTERPR

316272 DETERMINANTS OF QUALITY OF LIFE IN

366284 COMPARATIVE PERFORMANCE OF THE SF-3

373286 CHALLENGES AND SOLUTIONS TO DESIGNI

\section{INDIVIDUALIZED MEASURES}

\# PAGE TITLE

18197 THE GOAL INTERFERENCE SCALE: EVALUA

36201 THE RELATIONSHIP BETWEEN RESOURCE C

115221 ROC ANALYSIS OF WEIGHT LOSS TO ACHI

121223 VALUE SHIFT AFTER DISABILITY ONSET:

122223 COMPARISON OF UNWEIGHTED SATISFACTI

124224 AN INDIVIDUALIZED APPROACH IN THE M

161233 CONSTIPATION AND QUALITY OF LIFE IN

181238 A COMPREHENESIVE REVIEW OF CAREGIVE

213246 QUALITY OF LIFE IN ELDERLY PERSONS

217247 QUALITY OF LIFE IN MYOPIC PATIENTS

222248 EXPLORATIVE STUDY ON COMPLIANCE AND

251256 THE STROKE SURVIVOR FUNCTIONING AND

253256 CONCEPTUALIZATION AND DESIGN OF AN

254256 INITIAL PSYCHOMETRIC PERFORMANCE OF

263259 A HEALTH RELATED QUALITY OF LIFE ME

302268 QUALITY OF LIFE IN CARERS OF SEVERE

346279 APPLYING ITEM RESPONSE THEORY (IRT)

358282 PROSPECTIVE VALIDATION STUDY OF SPA

366284 COMPARATIVE PERFORMANCE OF THE SF-3

383289 PERSONALITY AND QUALITY OF LIFE

\section{INFECTIOUS DISEASE/ HIVIAIDS}

\# PAGE TITLE

28199 THE SEXUAL RELATIONS SCALE: PSYCHOM

43203 ONE- VERSUS TWO-PARAMETER ITEM RESP

45204 FACTORS ASSOCIATED WITH STABILITY I

47204 USING ITEM RESPONSE THEORY (IRT) AN

59207 QUALITY OF LIFE ASSESSMENT IN HIV

219248 EVALUATION OF A GENERAL AND DISEASE

305269 A LONGITUDINAL STUDY ON HEALTH RELA

306269 HEALTH-RELATED QUALITY OF LIFE IN H

307270 SATISFACTION WITH SEXUAL LIFE IN HI

\section{INSTRUMENT CALIBRATION/ EQUIVALENCY AMONG SCALES}

\# PAGE TITLE

5194 ESTIMATION AND VALIDATION OF DERIVE

16196 USING ITEM RESPONSE THEORY TO SHORT

19197 COMPARISON OF THE HEALTH UTILITY IN

51205 PREDICTING UTILIZATION AND COSTS IN

60207 NEGATIVE UTILITY SCORES: THEORETICA

135227 A PROSPECTIVE COMPARISON OF QUALITY

143229 RELATIONSHIP BETWEEN CANCER LOCUS O

199243 WORKERS' QUALITY OF LIFE: TOWARDS A

256257 THE VALIDITY AND RELIABILITY OF THE

262258 ATTITUDES TOWARDS QUALITY OF LIFE A

278262 MEASURING SOCIAL PARTICIPATION: DEV

315272 EVALUATION OF A COMPUTERISED TOUCH-

380288 ADVANTAGES OF JOINT USE OF EORTC QL 


\section{INTERPRETATION OF HRQOL DATA}

\# PAGE TITLE

6194 CANCER PATIENTS' PREFERENCES FOR CO

32200 "I THINK MY LIFE IS BETTER THAN MOS

34201 PEDIATRIC SPINE DEFORMITY: ASSESSIN

35201 HEALTH RELATED QUALITY OF LIFE OF C

45204 FACTORS ASSOCIATED WITH STABILITY

50205 SYMPTOM EXPERIENCE AND HEALTH RELAT

53206 DETERMINANTS OF THE QUALITY OF LIFE

67209 CONTRIBUTIONS OF HEALTH-RELATED QUA

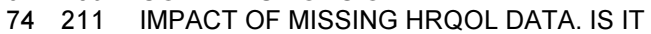

$76 \quad 211$ IS MORE BETTER? QUALITY OF LIFE (QO

85214 SUBJECTIVE VERSUS OBJECTIVE QUALITY

95216 PSYCHOMETRIC PROPERTIES OF FACT-AN

99217 FATIGUE AND QUALITY OF LIFE IN PATI

103218 PERSPECTIVES ON PEDIATRIC OUTCOMES

104218 SATISFACTION AND DISSATISFACTION: A

105219 RELATIONSHIPS BETWEEN SATISFACTION

110220 PREDICTORS OF MORTALITY IN PATIENTS

112220 RELATIONSHIP BETWEEN COMORBIDITIES

115221 ROC ANALYSIS OF WEIGHT LOSS TO ACHI

125224 QUALITY OF LIFE IN LONG-TERM PROSTA

138227 IMPACT OF COPING, FUNCTIONALITY, AN

141228 DETERMINING CLINICALLY SIGNIFICANT

143229 RELATIONSHIP BETWEEN CANCER LOCUS O

145229 IMPROVEMENT IN HEMOGLOBIN LEVELS RE

147230 SYMPTOMS PREDICT REDUCED HRQOL DURI

148230 EARLY QUALITY OF LIFE IN LOCALIZED

149230 MENTAL ADJUSTMENT TO CANCER AND SOC

150230

151231

155232

$165 \quad 234$

$170 \quad 235$

174236

$\begin{array}{ll}176 & 237\end{array}$

$\begin{array}{ll}177 & 237\end{array}$

180238

182238

185239

188240

189240

198242

200243

203244

204244

$205 \quad 244$

208245

214246

218247

$227 \quad 250$

231251

$233 \quad 251$

236252

237252

238252

239253

242253

256257

310270

316272

318272

322273

$329 \quad 275$

$330 \quad 275$

331276

332276

333276

335277

$340 \quad 278$

$342 \quad 278$

344279

$345 \quad 279$

348280

353281

$375 \quad 287$

378288

381288

382289
THE RELATIONSHIP BETWEEN PAIN SEVER EFFECT OF CAREGIVER PERCEPTION OF P MPACT OF A SECOND PRIMARY CANCER O QUALITY OF LIFE IN PATIENTS WITH AT HOW CONSISTENT IS SELF-REPORTED HEA TREATMENT AND HEALTHCARE SATISFACTI TREATMENT AND QUALITY OF LIFE AMONG ASSESSMENT OF HEALTH STATUS IN PATI THE INFLUENCE OF GENDER ON HEALTH-R RELATING CONCEPTS OF DISEASE \&AMP;

WHAT DO WE MEASURE: HRQL OR HRQL DE A NEW CONCEPTUAL MODEL FOR TREATMEN WELLNESS BEHAVIORS AS AN INDICATOR HEALTH-RELATED QUALITY OF LIFE AND IMPACT OF COMMUNITY MENTAL HEALTH S USING A HEALTH STATUS INDICATOR TO QUALITY OF LIFE AND PRECARIOUSNESS: FUNCTION AND QUALITY OF LIFE MORBID HOW DO WE AGE? AN ANALYSIS OF SYMPT TITLE: CONTRIBUTION OF DIFFERENT CL PREVALENCE OF ERECTILE DYSFUNCTION QUALITY OF LIFE DETERMINANTS IN A D ASSESSMENT OF HEALTH-RELATED QUALIT IS SELF PERCEPTION OF HEALTH THE MO RESPONSIVE HEALTH-RELATED QUALITY O RELATIONSHIP BETWEEN SYMPTOMS AND S DIFFERENTIAL WEIGHTINGS OF THE CLIN RESULTS FROM THREE PHYSICIAN CONSEN THE VALIDITY AND RELIABILITY OF THE USEFULNESS OF QUALITY OF LIFE (QOL) DETERMINANTS OF QUALITY OF LIFE IN GENDER DIFFERENCES IN QUALITY OF LI QUALITY OF LIFE \&AMP; PSYCHOLOGICAL QUALITY OF LIFE FOR POOR AFRICAN-AM SOCIAL SUPPORT AND QUALITY OF LIFE DOES CHANGE IN DEPRESSION STATUS PR HEALTHCARE RESOURCE USE AMONG CAREG MENTAL WELL BEING AMONG CAREGIVERS USING THE MANN-WHITNEY STATISTIC TO CHANGE IN QUALITY OF LIFE IN RELATI COHEN CONFIRMED? EMPIRICAL EFFECT S UNDERSTANDING CHANGE OVER TIME IN T COULD LINEAR REGRESSION BE REPLACED SOME LOGICAL PROBLEMS WITH TESTS FO QUALITY OF LIFE IN THE AUSTRIAN GEN A COMPARATIVE STUDY OF PHYSICAL AND PSYCHOLOGICAL ADJUSTMENT THAN CLIN QUALITY OF LIFE AND AGE RELATIONSHI ARE QUALITY OF LIFE PARAMETERS OF P FACT-AN SHOWS SENSITIVITY TO CHRONI
ITEM RESPONSE THEORY (IRT)

\# PAGE TITLE

11195 EVALUATING THE LATENT DISTRIBUTION

41203 USING ITEM RESPONSE THEORY TO IMPRO

42203 ITEM BANKING TO IMPROVE, SHORTEN AN

43203 ONE- VERSUS TWO-PARAMETER ITEM RESP

158232 HOW TO MEASURE THE ATTITUDE OF WOME

343279 TESTING RASCH ITEM ANALYSIS

346279 APPLYING ITEM RESPONSE THEORY (IRT)

\section{MEDICAL PRACTICE/QUALITY OF CARE}

\# PAGE TITLE

$7 \quad 194$ DOCTOR-PATIENT COMMUNICATION ABOUT

8194 PHYSICIAN FS COMMUNICATION STYLE, C

9195 MEASURING QUALITY OF LIFE IN CLINIC

10195 THE IMPACT OF SHARED TREATMENT DECI

21198 ONE YEAR OUTCOMES OF HEMODIALYSIS A

23198 ORODENTAL HEALTH, ACUTE AND LATE MO

46204 RESPONSE SHIFT EFFECTS OF AN ADVANC

51205 PREDICTING UTILIZATION AND COSTS IN

75211 HRQOL OUTCOMES AMONG MEN MANAGED WI

79212 DEVELOPMENT OF THE SHORT FORM MCGIL

80212 DEVELOPMENT AND VALIDATION OF QUALI

82213 SOAP-51: A SELF-ADMINISTERED, HEALT

101218 THE OUTCOME OF HIP AND KNEE REPLACE

104218 SATISFACTION AND DISSATISFACTION: A

106219 QUALITY OF LIFE OF CHINESE CANCER P

107219 MAJOR DEPRESSIVE DISORDER (MDD) IN

108219 PREDICTING PSYCHOSOCIAL OUTCOME OF

131226 GENETIC COUNSELING FOR COLORECTAL C

133226 HOW POTENT IS POTENT? EVALUATION OF

137227 QUALITY OF LIFE IMPACT ON SATISFACT

138227 IMPACT OF COPING, FUNCTIONALITY, AN

144229 THE RELATIONSHIP BETWEEN INFORMATIO

146229 PSYCHOLOGICAL DISTRESS AND SEXUAL F

159233 THE CANCER OUTCOMES MEASUREMENT WOR

177237 TREATMENT AND QUALITY OF LIFE AMONG

207245 USING HEALTH STATUS QUESTIONNAIRES

209245 ANGIOPLASTY PATIENT 36 MONTH SURVIV

210245 INTRODUCING HRQL INTO DAILY ROUTINE

212246 MEASUREMENT OF CONTINUITY AND CO-OR

231251 QUALITY OF LIFE DETERMINANTS IN A D

232251 QUALITY OF LIFE AND THE DURATION OF

234251 DEVELOPING AN INTERVENTION TO ENHAN

238252 RELATIONSHIP BETWEEN SYMPTOMS AND S

239253 DIFFERENTIAL WEIGHTINGS OF THE CLIN

242253 RESULTS FROM THREE PHYSICIAN CONSEN

247255 IMPACT OF A CHANGE IN MODE OF ADMIN

248255 COMPARISON OF PHYSICIAN AND PATIENT

263259 A HEALTH RELATED QUALITY OF LIFE ME

266259 VALIDATION OF A FRENCH INSTRUMENT M

272261 A SPECIFIC QUALITY OF LIFE SCALE IN

357282 DYSPEPSIA-RELATED QUALITY OF LIFE:

367285 TESTING FOR VALIDITY OF A NEWLY DEV

\section{MINORITY POPULATIONS}

\# PAGE TITLE

2193 COMPARISON OF THREE MEASURES OF UTI

55206 THE FACTOR STRUCTURE OF THE SF-36 P

59207 QUALITY OF LIFE ASSESSMENT IN HIV:

197242 THE HEALTH STATUS OF MIGRANT FARM W

205244 QUALITY OF LIFE AND PRECARIOUSNESS:

323274 ASSOCIATIONS BETWEEN FIVE-FACTOR MO

329275 QUALITY OF LIFE FOR POOR AFRICAN-AM

356282 THE LOGISTICS OF HEALTH-RELATED QUA

360283 VALIDITY AND RELIABILITY OF EQ-5D D

364284 KOREAN VALIDATION OF THE KIDNEY DIS

\section{MODES OF ADMINISTRATION}

\# PAGE TITLE

89215 ELECTRONIC VERSUS PAPER QUESTIONNAI

90215 ADMINISTRATION OF SEIQOL-DW: THREAT

91215 TOWARDS BRIEFER QOL INSTRUMENTS: A 
160233 HRQOL IN PEOPLE WITH APHASIA: PRELI 245254 EFFECT OF MODE OF ADMINISTRATION ON 246254 OBTAINING SELF-REPORTS ABOUT HRQL F 247255 IMPACT OF A CHANGE IN MODE OF ADMIN 339278 METHODS FOR INTEGRATING THE SF-12।

\section{MUSCULOSKETAL/RHEUMATOLOGY}

\# PAGE TITLE

34201 PEDIATRIC SPINE DEFORMITY: ASSESSIN

63208 COMPARING COMMUNITY-PREFERENCE BASE

85214 SUBJECTIVE VERSUS OBJECTIVE QUALITY

99217 FATIGUE AND QUALITY OF LIFE IN PATI

100217 QUALITY OF LIFE IN SYSTEMIC LUPUS E

101218 THE OUTCOME OF HIP AND KNEE REPLACE

102218 THE RELIABILITY AND VALIDITY OF THE

103218 PERSPECTIVES ON PEDIATRIC OUTCOMES:

114221 DEFINING RECOVERY: THE APPLICATION

118222 MULTISAMPLE MEAN AND COVARIANCE STR

163234 EVALUATING QUALITY OF LIFE IN PATIE

167235 ASSESSING QUALITY OF LIFE IN OSTEOP

168235 EVIDENCE SUPPORTING THE VALIDITY OF

169235 THE PRACTICALITIES OF QUALITY OF LI

180238 ASSESSMENT OF HEALTH STATUS IN PATI

223249 EVALUATION OF A NOVEL PAIN MANAGEME

247255 IMPACT OF A CHANGE IN MODE OF ADMIN

255257 QUALITY OF LIFE AND DEMOGRAPHICS IN

284264 METHODOLOGY FOR CHOICE OF ITEMS : D

287265 TEST-RETEST RELIABILITY AND INTERPR

289265 HOW TO COMBINE PREFERENCE-BASED QOL

360283 VALIDITY AND RELIABILITY OF EQ-5D D

381288 QUALITY OF LIFE AND AGE RELATIONSHI

\section{NEUROLOGY}

\# PAGE TITLE

48204 RESPONSE SHIFT IN THE ASSESSMENT OF

65209 THE COMPLEX RELATIONSHIP BETWEEN CO

66209 VALIDATION OF THE EUROQOL STROKE UT

67209 CONTRIBUTIONS OF HEALTH-RELATED QUA

68209 THE MS SYMPTOM AND IMPACT DIARY: A

69210 PDQ-39SI, PDQ-8SI: TOWARDS A FRENCH

71210 AGREEMENT BETWEEN PROXY ASSESSMENT

72210 THE IMPORTANCE OF CONSIDERING MISSI

87214 WHAT IS SUBJECTIVE QUALITY OF LIFE?

94216 ITEM REDUCTION: DO DIFFERENT METHO

116221 RESPONSIVENESS OF THE QUALITY OF LI

121223 VALUE SHIFT AFTER DISABILITY ONSET:

160233 HRQOL IN PEOPLE WITH APHASIA: PRELI

166234 EFFECTS OF COPING ON MENTAL OUTCOME

174236 HOW CONSISTENT IS SELF-REPORTED HEA

175237 THE NHP INDEX OF DISTRESS IN PARKIN

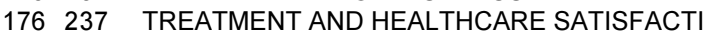

177237 TREATMENT AND QUALITY OF LIFE AMONG

178237 QUALITY OF LIFE IN GERMAN PATIENTS

179238 QUALITY OF LIFE IN PATIENTS WITH MU

181238 A COMPREHENESIVE REVIEW OF CAREGIVE

182238 THE INFLUENCE OF GENDER ON HEALTH-R

187240 FACTORS THAT PREDICT HRQOL IN PATIE

246254 OBTAINING SELF-REPORTS ABOUT HRQL F

251256 THE STROKE SURVIVOR FUNCTIONING AND

257257 THE SCOPA-PS: A PSYCHOSOCIAL QUESTI

343279 TESTING RASCH ITEM ANALYSIS

368285 VALIDITY OF A NEWLY DEVELOPED JAPAN

378288 PSYCHOLOGICAL ADJUSTMENT THAN CLINI

379288 QUALITY OF LIFE IMPAIRMENT IN MULTI

\section{ONCOLOGY}

\# PAgE TITLE

6194 CANCER PATIENTS' PREFERENCES FOR CO

7194 DOCTOR-PATIENT COMMUNICATION ABOUT

8194 PHYSICIAN FS COMMUNICATION STYLE, C

10195 THE IMPACT OF SHARED TREATMENT DECI

17197 A COMPARISON OF ANALYSIS METHODS FO

18197 THE GOAL INTERFERENCE SCALE: EVALUA

23198 ORODENTAL HEALTH, ACUTE AND LATE MO

42203 ITEM BANKING TO IMPROVE, SHORTEN AN
ONE- VERSUS TWO-PARAMETER ITEM RESP TRANSLATION AND DEVELOPMENT OF A DU PATIENT PREFERENCE AND COMPREHENSIO DEFINING SYMPTOM AND FUNCTIONAL HEA HRQOL OUTCOMES AMONG MEN MANAGED WI IS MORE BETTER? QUALITY OF LIFE (QO SYMPTOMATOLOGY OF CANCER PATIENTS I BRIEF ASSESSMENT OF PRIORITY SYMPTO DEVELOPMENT OF THE SHORT FORM MCGIL THE CHILD HEALTH QUESTIONNAIRE(CHQ) QUALITY OF LIFE OF CHINESE CANCER P SURVIVAL, QUALITY OF LIFE AND THE S WHAT IS A CLINICALLY MEANINGFUL CHA QUALITY OF LIFE IN LONG-TERM PROSTA QUALITY OF LIFE OF MALIGNANT BRAIN EFFECTS OF PSYCHOSOCIAL INTERVENTIO PSYCHOSOCIAL CONSEQUENCES OF PREVEN "GENDER DIFFERENCES IN FACTORS PRED COLORECTAL CANCER SCREENING: COMPLI GENETIC COUNSELING FOR COLORECTAL C QUALITY OF LIFE IMPACT ON SATISFACT IMPACT OF COPING, FUNCTIONALITY, AN "AN EMPIRICAL META-ANALYSIS OF 5 DI DETERMINING CLINICALLY SIGNIFICANT TESTING THE LUNG CANCER SYMPTOM SCA RELATIONSHIP BETWEEN CANCER LOCUS O THE RELATIONSHIP BETWEEN INFORMATIO IMPROVEMENT IN HEMOGLOBIN LEVELS RE PSYCHOLOGICAL DISTRESS AND SEXUAL F SYMPTOMS PREDICT REDUCED HRQOL DURI EARLY QUALITY OF LIFE IN LOCALIZED MENTAL ADJUSTMENT TO CANCER AND SOC EFFECT OF CAREGIVER PERCEPTION OF P DECISIVE FACTORS OF QUALITY OF LIFE IMPACT OF A SECOND PRIMARY CANCER O QUALITY OF LIFE OF BREAST CANCER SU THE CANCER OUTCOMES MEASUREMENT WOR RELATING CONCEPTS OF DISEASE \&AMP; ASSESSING THE SUBJECTIVE IMPACT ON DOES HEALTH-RELATED QUALITY OF LIFE FUNCTION AND QUALITY OF LIFE MORBID MEASUREMENT OF CONTINUITY AND CO-OR IS THE EFFECT REAL OR AN ARTIFACT? PHYSICAL PERFORMANCE, TOXICITY, AN COMPARISON OF PHYSICIAN AND PATIENT THE PSYCHOMETRICS OF THE HOSPITAL A ATTITUDES TOWARDS QUALITY OF LIFE A A SPECIFIC QUALITY OF LIFE SCALE IN DEVELOPMENT AND VALIDATION OF DISEA HOW MUCH IS "VERY MUCH"? DEVELOPING VALIDATION IN FRENCH OF THE CHILD H IMPLEMENTING QUALITY OF LIFE IN EOR USEFULNESS OF QUALITY OF LIFE (QOL) ASSOCIATIONS BETWEEN FIVE-FACTOR MO COGNITIVE FACTORS IN SYMPTOM ASSESS THE DEVELOPMENT AND UTILISATION OF CLINICAL POTENTIAL OF LONGITUDINAL RELIABILITY AND VALIDITY OF MODIFIE AN INDONESIAN VERSION OF THE ROTTER RELIABILITY AND VALIDITY OF THE FUN QUALITY OF LIFE IN THE AUSTRIAN GEN SEQUENTIAL VS. PARALLEL APPROACH TO THE LOGISTICS OF HEALTH-RELATED QUA

\section{PROXY MEASURES}

\# PAge titLe

33201 QOL AFTER PEDIATRIC STEM CELL TRANS

71210 AGREEMENT BETWEEN PROXY ASSESSMENT

93216 THE CHILD HEALTH QUESTIONNAIRE(CHQ)

218247 TITLE: CONTRIBUTION OF DIFFERENT CL

220248 PAIN SCALE RATINGS AND SATISFACTION 243254 AGREEMENT BETWEEN SELF AND PROXY AS 248255 COMPARISON OF PHYSICIAN AND PATIENT 


\section{PSYCHIATRIC DISORDERSISUBSTANCE ABUSE}

\# PAge titLe

3193 CAN UTILITY-WEIGHTED HRQOL ESTIMATE

80212 DEVELOPMENT AND VALIDATION OF QUALI

81213 THE PATIENT'S PERSPECTIVE IN SCHIZO

82213 SOAP-51: A SELF-ADMINISTERED, HEALT

83213 ETHNIC DIFFERENCES IN VALUES FOR HE

92215 DIFFERENCES IN PERCEPTION OF QUALIT

97217 PSYCHOMETRIC PROPERTIES OF THE GLOB

107219 MAJOR DEPRESSIVE DISORDER (MDD) IN

203244 IMPACT OF COMMUNITY MENTAL HEALTH S

204244 USING A HEALTH STATUS INDICATOR TO

259258 THE PSYCHOMETRICS OF THE HOSPITAL A

291266 COST-UTILITY ANALYSIS OF UNIVERSAL

324274 QUALITY OF LIFE AND MENTAL HEALTH I

325274 ISSUES INVOLVED IN ASSESSING QUALIT

327275 WAS IT A GOOD MOVE? IMPROVEMENT IN

328275 IMPACT OF DEPRESSIVE SYMPTOMS IN SO

329275 QUALITY OF LIFE FOR POOR AFRICAN-AM

330275 SOCIAL SUPPORT AND QUALITY OF LIFE

331276 DOES CHANGE IN DEPRESSION STATUS PR

332276 HEALTHCARE RESOURCE USE AMONG CAREG

333276 MENTAL WELL BEING AMONG CAREGIVERS

372286

\section{PSYCHOMETRIC METHODS/ SCALING}

\# PAGE TITLE

27199 RETEST RELIABILITY AND VALIDITY OF

40202 COPING AND QUALITY OF LIFE IN ADULT

47204 USING ITEM RESPONSE THEORY (IRT) AN

54206 PSYCHOMETRIC PROPERTIES OF THE MACN

69210 PDQ-39SI, PDQ-8SI: TOWARDS A FRENCH

94216 ITEM REDUCTION: DO DIFFERENT METHO

95216 PSYCHOMETRIC PROPERTIES OF FACT-AN

96216 PRECISION AND BREVITY: VITALITY ASS

97217 PSYCHOMETRIC PROPERTIES OF THE GLOB

98217 A COMPARISON OF LIKERT SCALE AND VI

108219 PREDICTING PSYCHOSOCIAL OUTCOME OF

118222 MULTISAMPLE MEAN AND COVARIANCE STR

172236 A METHOD FOR ESTIMATING THE CONTRIB

246254 OBTAINING SELF-REPORTS ABOUT HRQL F

254256 INITIAL PSYCHOMETRIC PERFORMANCE OF

264259 PSYCHOMETRIC VALIDATION OF CONTRACE

265259 QUALITY OF LIFE IN DERMATOLOGY: DEV

267260 PSYCHOMETRIC PROPERTIES OF IBS-QOL

274261 VALIDATION AND RESCORING OF THE MIN

311271 PSYCHOMETRIC PROPERTIES OF CYCLOSP

326274 COGNITIVE FACTORS IN SYMPTOM ASSESS

334276 FACTOR STRUCTURE OF THE QUALITY OF

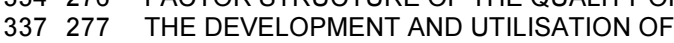

341278 MULTIPLE FACTOR ANALYSIS: APPLICATI

350280 AN INDONESIAN VERSION OF THE ROTTER

352281 VALIDITY OF THE SPANISH CHILD HEALT

363284 EVALUATING THE PERFORMANCE OF A NEW

\section{QUALITATIVE METHODS}

\# PAGE TITLE

$7 \quad 194$ DOCTOR-PATIENT COMMUNICATION ABOUT

49205 RESPONSE SHIFT IN PATIENTS' LIVING

59207 QUALITY OF LIFE ASSESSMENT IN HIV:

90215 ADMINISTRATION OF SEIQOL-DW: THREAT

126224 QUALITY OF LIFE OF MALIGNANT BRAIN

152231 THE PATIENT AND THEIR DOCTOR: A CON

173236 AN ASSESSMENT OF HEALTH-RELATED QUA

187240 FACTORS THAT PREDICT HRQOL IN PATIE

234251 DEVELOPING AN INTERVENTION TO ENHAN

284264 METHODOLOGY FOR CHOICE OF ITEMS : D

312271 QUALITY OF LIFE OF INFLAMMATORY BOW

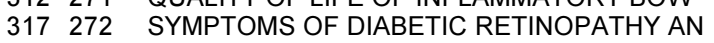

\section{RESPIRATORY DISEASE}

\# PAge titLe

36201 THE RELATIONSHIP BETWEEN RESOURCE C

37202 THE EFFECT OF ASTHMA SEVERITY ON HE

38202 QUALITY OF LIFE AFTER INTENSIVE CAR

39202 EFFECTS OF DISEASE MANAGEMENT ON QU

40202 COPING AND QUALITY OF LIFE IN ADULT

84213 WHAT ROLE DO SYMPTOMS PLAY IN HEALT

89215 ELECTRONIC VERSUS PAPER QUESTIONNAI

110220 PREDICTORS OF MORTALITY IN PATIENTS

112220 RELATIONSHIP BETWEEN COMORBIDITIES

210245 INTRODUCING HRQL INTO DAILY ROUTINE

215247 CAN PHARMACEUTICAL CARE IMPROVE THE

216247 LONG-TERM QUALITY OF LIFE IMPROVEME

295267 SOCIAL COMPETENCE, EMOTIONAL/BEHAVI

296267 FAMILY CLOSENESS, PARENTAL LOCUS OF

\section{RESPONSE SHIFT}

\# PAGE TITLE

8194 PHYSICIAN FS COMMUNICATION STYLE, C

33201 QOL AFTER PEDIATRIC STEM CELL TRANS

45204 FACTORS ASSOCIATED WITH STABILITY I

46204 RESPONSE SHIFT EFFECTS OF AN ADVANC

47204 USING ITEM RESPONSE THEORY (IRT) AN

48204 RESPONSE SHIFT IN THE ASSESSMENT OF

49205 RESPONSE SHIFT IN PATIENTS' LIVING

90215 ADMINISTRATION OF SEIQOL-DW: THREAT

244254 RESPONSE SHIFT OF A HEALTH-RELATED

\section{RESPONSIVENESS/ INTERPRETATION}

\# PAGE TITLE

22198 USE OF ERYTHROPOIETIN IN EARLY ANAE

113221 SURVIVAL, QUALITY OF LIFE AND THE S

114221 DEFINING RECOVERY: THE APPLICATION

116221 RESPONSIVENESS OF THE QUALITY OF LI

117222 WHAT IS A CLINICALLY MEANINGFUL CHA

119222 A COMPARISON OF THE DISCRIMINATIVE

120222 USING STRUCTURAL EQUATION MODELS (S

123223 ASSESSMENT OF QUALITY OF LIFE IN CH

141228 DETERMINING CLINICALLY SIGNIFICANT

146229 PSYCHOLOGICAL DISTRESS AND SEXUAL F

150230 THE RELATIONSHIP BETWEEN PAIN SEVER

167235 ASSESSING QUALITY OF LIFE IN OSTEOP

174236 HOW CONSISTENT IS SELF-REPORTED HEA

200243 HEALTH-RELATED QUALITY OF LIFE AND

223249 EVALUATION OF A NOVEL PAIN MANAGEME

240253 IS THE EFFECT REAL OR AN ARTIFACT?

241253 PHYSICAL PERFORMANCE, TOXICITY, AN

249255 RESPONSIVENESS OF THE ACNE-SPECIFIC

279263 THE RELATIVE RESPONSIVENESS OF GENE

321273 DO MEN AND WOMEN RESPOND DIFFERENTL

327275 WAS IT A GOOD MOVE? IMPROVEMENT IN

361283 CROSS-CULTURAL ADAPTATION OF THE SF

\section{SCALE DEVELOPMENT/ VALIDATION}

\# PAGE TITLE

24198 QUALITY OF LIFE AFTER ESOPHAGEAL RE

26199 THE REVISED KINDL-R: FINAL RESULTS

27199 RETEST RELIABILITY AND VALIDITY OF

28199 THE SEXUAL RELATIONS SCALE: PSYCHOM

29200 VALIDATION OF THE UFS-QOL, A DISEAS

30200 PSYCHOMETRIC EVALUATION OF THE OBES

56206 VALIDATION OF THE WHOQOL-BREF IN A

64208 PATIENT PREFERENCE AND COMPREHENSIO

66209 VALIDATION OF THE EUROQOL STROKE UT

68209 THE MS SYMPTOM AND IMPACT DIARY: A

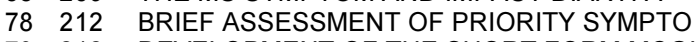

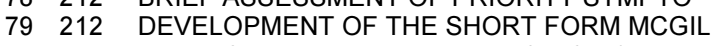

80212 DEVELOPMENT AND VALIDATION OF QUALI

82213 SOAP-51: A SELF-ADMINISTERED, HEALT

96216 PRECISION AND BREVITY: VITALITY ASS

98217 A COMPARISON OF LIKERT SCALE AND V

102218 THE RELIABILITY AND VALIDITY OF THE 
139228 "AN EMPIRICAL META-ANALYSIS OF 5 DI

142228 TESTING THE LUNG CANCER SYMPTOM SCA

153231 QOLLTI-F: A MEASURE OF THE QOL OF F

154231 DECISIVE FACTORS OF QUALITY OF LIFE

166234 EFFECTS OF COPING ON MENTAL OUTCOME

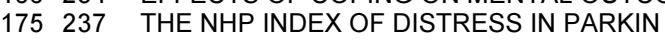

186239 ASSESSING THE SUBJECTIVE IMPACT ON

187240 FACTORS THAT PREDICT HRQOL IN PATIE

189240 A NEW CONCEPTUAL MODEL FOR TREATMEN

192241 OVERALL QUALITY OF LIFE IN THE GENE

194241 HEALTH RELATED TRADITIONAL BEHAVIOR

196242 MEASURING FATIGUE IN THE GENERAL PO

202243 HOW MIGRATION AFFECTS THE HEALTH R

212246 MEASUREMENT OF CONTINUITY AND CO-OR

219248 EVALUATION OF A GENERAL AND DISEASE

226249 EVALUATION OF THE SF-36 AND EQ-5D F

249255 RESPONSIVENESS OF THE ACNE-SPECIFIC

252256 PSYCHOMETRIC VALIDATION OF THE ITAL

255257 QUALITY OF LIFE AND DEMOGRAPHICS IN

256257 THE VALIDITY AND RELIABILITY OF THE

257257 THE SCOPA-PS: A PSYCHOSOCIAL QUESTI

260258

264259

265259

266259

268260

269260

271261

272261

273261

275262

276262

277262

278262

280263

281263

282263

283264

286264

291266

296267

299268

300268

301268

303269

308270

311271

$325 \quad 274$

$326 \quad 274$

349280

352281

354281

355282

358282

360283

362283

363284

365284

367285

368285

RELIABILITY AND VALIDITY OF 'NEWSQO

PSYCHOMETRIC VALIDATION OF CONTRACE

QUALITY OF LIFE IN DERMATOLOGY: DEV

VALIDATION OF A FRENCH INSTRUMENT M

VALIDATION OF THE COMMUNITY DEMENTI

COMPARISON OF TWO DISEASE-SPECIFIC

RELIABILITY AND VALIDITY OF A NEWLY

A SPECIFIC QUALITY OF LIFE SCALE IN

PRELIMINARY FINDINGS IN THE DEVELOP

THE RELIABILITY AND VALIDITY OF A N

TESTING THE PSYCHOMETRIC PROPERTIES

DEVELOPMENT AND VALIDATION OF DEEP

MEASURING SOCIAL PARTICIPATION: DEV

FINAL PSYCHOMETRIC EVALUATION OF TH

DEVELOPMENT AND VALIDATION OF DISEA

EXPLORATORY FACTOR ANALYSIS (EFA) O

INTERPRETING THE MOS SF_36: HOW MAN

SHOULD SYMPTOMS BE SCALED FOR INTEN

COST-UTILITY ANALYSIS OF UNIVERSAL

FAMILY CLOSENESS, PARENTAL LOCUS OF

THE CORE VSP-A AND ITS SPECIFIC MOD

VALIDATION IN FRENCH OF THE CHILD H

DEVELOPMENT, VALIDATION OF HRQL MEA

RELIABILITY AND DISCRIMINANT VALIDI

IMPLEMENTING QUALITY OF LIFE IN EOR

PSYCHOMETRIC PROPERTIES OF CYCLOSP

ISSUES INVOLVED IN ASSESSING QUALIT

COGNITIVE FACTORS IN SYMPTOM ASSESS

RELIABILITY AND VALIDITY OF MODIFIE

VALIDITY OF THE SPANISH CHILD HEALT

PRELIMINARY ANALYSIS OF THE CULTURA

SEQUENTIAL VS. PARALLEL APPROACH TO

PROSPECTIVE VALIDATION STUDY OF SPA

VALIDITY AND RELIABILITY OF EQ-5D D

SWEDISH SF-36 VERSION 2.0 - CONSTR

EVALUATING THE PERFORMANCE OF A NEW

USING THE DELPHI METHOD TO DETERMIN

TESTING FOR VALIDITY OF A NEWLY DEV

VALIDITY OF A NEWLY DEVELOPED JAPAN

\section{SCALE TRANSLATION/CULTURAL ADAPTATION}

\# PAgE TITLE

2193 COMPARISON OF THREE MEASURES OF UTI

55206 THE FACTOR STRUCTURE OF THE SF-36 P

56206 VALIDATION OF THE WHOQOL-BREF IN A

57207 HOW WELL DO HEALTH STATE VALUATION

58207 TRANSLATION AND DEVELOPMENT OF A DU

69210 PDQ-39SI, PDQ-8SI: TOWARDS A FRENCH

192241 OVERALL QUALITY OF LIFE IN THE GENE

196242 MEASURING FATIGUE IN THE GENERAL PO

252256 PSYCHOMETRIC VALIDATION OF THE ITAL

273261 PRELIMINARY FINDINGS IN THE DEVELOP

285264 HOW MUCH IS "VERY MUCH"? DEVELOPING

304269 SCREENING FOR AND PROMOTION OF HEAL

350280 AN INDONESIAN VERSION OF THE ROTTER

351281 RELIABILITY AND VALIDITY OF THE FUN

354281 PRELIMINARY ANALYSIS OF THE CULTURA

355282 SEQUENTIAL VS. PARALLEL APPROACH TO

357282 DYSPEPSIA-RELATED QUALITY OF LIFE:

359283 LINGUISTIC VALIDATION OF THE KING'S

361283 CROSS-CULTURAL ADAPTATION OF THE SF

364284 KOREAN VALIDATION OF THE KIDNEY DIS

366284 COMPARATIVE PERFORMANCE OF THE SF-3

367285 TESTING FOR VALIDITY OF A NEWLY DEV

368285 VALIDITY OF A NEWLY DEVELOPED JAPAN

369285 AN INNOVATIVE APPROACH TO THE DEVEL

370285 CULTURAL ADAPTATION OF QUALITY OF L

371286 THE SPANISH NIH-CHRONIC PROSTATITIS

372286 TRANSLATION, RELIABILITY, AND VALID

$\begin{array}{lll}376 & 287 & \text { PROPOSED GUIDELINES FOR THE 1ST STE }\end{array}$

377287 RELIABILITY AND VALIDITY OF A SWEDI

379288 QUALITY OF LIFE IMPAIRMENT IN MULTI

384289 DEVELOPMENT OF RUSSIAN VERSION OF T

\section{SURGERYITRANSPLANTATION}

\# PAGE TITLE

5194 ESTIMATION AND VALIDATION OF DERIVE

24198 QUALITY OF LIFE AFTER ESOPHAGEAL RE

52205 LONG TERM QUALITY OF LIFE AFTER OPE

108219 PREDICTING PSYCHOSOCIAL OUTCOME OF

111220 IS IT POSSIBLE TO PREDICT PATIENT'S

134226 EVALUATION OF QUALITY OF LIFE AFTER

164234 DETERMINANTS OF QUALITY OF LIFE IN

217247 QUALITY OF LIFE IN MYOPIC PATIENTS

218247 TITLE: CONTRIBUTION OF DIFFERENT CL

224249 MEASUREMENT OF CHANGES IN DISEASE-S

225249 LONG-TERM QUALITY OF LIFE AND COMOR

226249 EVALUATION OF THE SF-36 AND EQ-5D F

236252 IS SELF PERCEPTION OF HEALTH THE MO

311271 PSYCHOMETRIC PROPERTIES OF CYCLOSP

319273 COPING AND DISTRESS BEFORE AND AFTE

\section{THEORIES OF QUALITY OF LIFE}

\# PAGE TITLE

9195 MEASURING QUALITY OF LIFE IN CLINIC

10195 THE IMPACT OF SHARED TREATMENT DEC

57207 HOW WELL DO HEALTH STATE VALUATION

62208 WHOSE VALUES COUNT? A CRITIQUE OF D

84213 WHAT ROLE DO SYMPTOMS PLAY IN HEALT

85214 SUBJECTIVE VERSUS OBJECTIVE QUALITY

86214 HOW IMPORTANT IS HEALTH FOR OVERALL

87214 WHAT IS SUBJECTIVE QUALITY OF LIFE?

92215 DIFFERENCES IN PERCEPTION OF QUALIT

105219 RELATIONSHIPS BETWEEN SATISFACTION

106219 QUALITY OF LIFE OF CHINESE CANCER P

137227 QUALITY OF LIFE IMPACT ON SATISFACT

144229 THE RELATIONSHIP BETWEEN INFORMATIO

159233 THE CANCER OUTCOMES MEASUREMENT WOR

162233 QUALITY OF LIFE IN UNIVERSITY STUDE

164234 DETERMINANTS OF QUALITY OF LIFE IN

184239 A PHILOSOPHICAL ANALYSIS OF THE MEA

190240 TIME PRESSURE AND EMOTIONAL WELL-BE

207245 USING HEALTH STATUS QUESTIONNAIRES

293266 A FRAMEWORK FOR CALCULATING CANADIA

295267 SOCIAL COMPETENCE, EMOTIONAL/BEHAVI

323274 ASSOCIATIONS BETWEEN FIVE-FACTOR MO

\section{UROLOGYINEPHROLOGY}

\# PAGE TITLE

21198 ONE YEAR OUTCOMES OF HEMODIALYSIS A

22198 USE OF ERYTHROPOIETIN IN EARLY ANAE

133226 HOW POTENT IS POTENT? EVALUATION OF

139228 "AN EMPIRICAL META-ANALYSIS OF 5 DI

148230 EARLY QUALITY OF LIFE IN LOCALIZED

227250 PREVALENCE OF ERECTILE DYSFUNCTION

228250 QUALITY ASSURANCE IN KIDNEY TRANSPL

231251 QUALITY OF LIFE DETERMINANTS IN A D

253256 CONCEPTUALIZATION AND DESIGN OF AN

254256 INITIAL PSYCHOMETRIC PERFORMANCE OF 
263259 A HEALTH RELATED QUALITY OF LIFE ME

358282 PROSPECTIVE VALIDATION STUDY OF SPA

363284 EVALUATING THE PERFORMANCE OF A NEW

371286 THE SPANISH NIH-CHRONIC PROSTATITIS

\section{UTILITIES}
\# PAGE TITLE
1193 COMPARING FIVE METHODS OF UTILITY A
2193 COMPARISON OF THREE MEASURES OF UTI
3193 CAN UTILITY-WEIGHTED HRQOL ESTIMATE
4193 CONVERGENT VALIDITY OF SF-36 UTILIT
5194 ESTIMATION AND VALIDATION OF DERIVE
20197 HEMOPHILIA: CONSTRUCT VALIDITY OF T
39202 EFFECTS OF DISEASE MANAGEMENT ON QU
57207 HOW WELL DO HEALTH STATE VALUATION
60207 NEGATIVE UTILITY SCORES: THEORETICA
61208 MEASURING THE QUALITY OF LIFE AND Q
62208 WHOSE VALUES COUNT? A CRITIQUE OF D
63208 COMPARING COMMUNITY-PREFERENCE BASE
64208 PATIENT PREFERENCE AND COMPREHENSIO
66209 VALIDATION OF THE EUROQOL STROKE UT
83213 ETHNIC DIFFERENCES IN VALUES FOR HE
119222 A COMPARISON OF THE DISCRIMINATIVE
140228 THE NEED FOR PSYCHOSOCIAL SUPPORT I
195242 EFFECTS OF ALTERNATIVE MEDICINE ON
198242 WELLNESS BEHAVIORS AS AN INDICATOR
209245 ANGIOPLASTY PATIENT 36 MONTH SURVIV
216247 LONG-TERM QUALITY OF LIFE IMPROVEME
228250 QUALITY ASSURANCE IN KIDNEY TRANSPL
277262 DEVELOPMENT AND VALIDATION OF DEEP
287265 TEST-RETEST RELIABILITY AND INTERPR
288265 UTILITY ASSESSMENT AMONG PATIENTS W
290265 MODELING PREFERENCES FOR COMPLEX HE
293266 A FRAMEWORK FOR CALCULATING CANADIA
294266 USING THE WHOQOL-BREF INSTRUMENT AN
356282 THE LOGISTICS OF HEALTH-RELATED QUA 


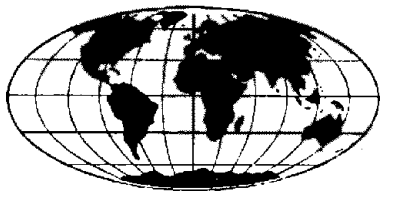

Neil Aaronson, Co-Chair (The Netherlands)

Mirjam Sprangers, Co-Chair (The Netherlands)

Kasem Akhras (USA)

Jordi Alonso (Spain)

Marianne Amir (Israel)

Xavier Badia (Spain)

Judith Barr (USA)

Rick Berzon (USA)

Francois Bethoux (USA)

Andrea Bezjak (Canada)

Jane Blazeby (UK)

John Brazier (UK)

Monika Bullinger (Germany)

Joseph C. Cappelleri (USA)

David Cella (USA)

Chih-Hung Chang (USA)

Desmond Curran (Ireland)

Aileen Davis (Canada)

David Feeny (Canada)

Dennis Gagnon (USA)

Barbara Gandek (USA)

Carolyn Gotay (USA)

Cynthia Gross (USA)

Asha Hareendran (UK)

Tatyana lonova (Russia)

Paul Kind (UK)
ISOQOL would like to acknowledge the 2001 Annual Meeting Abstract Reviewers

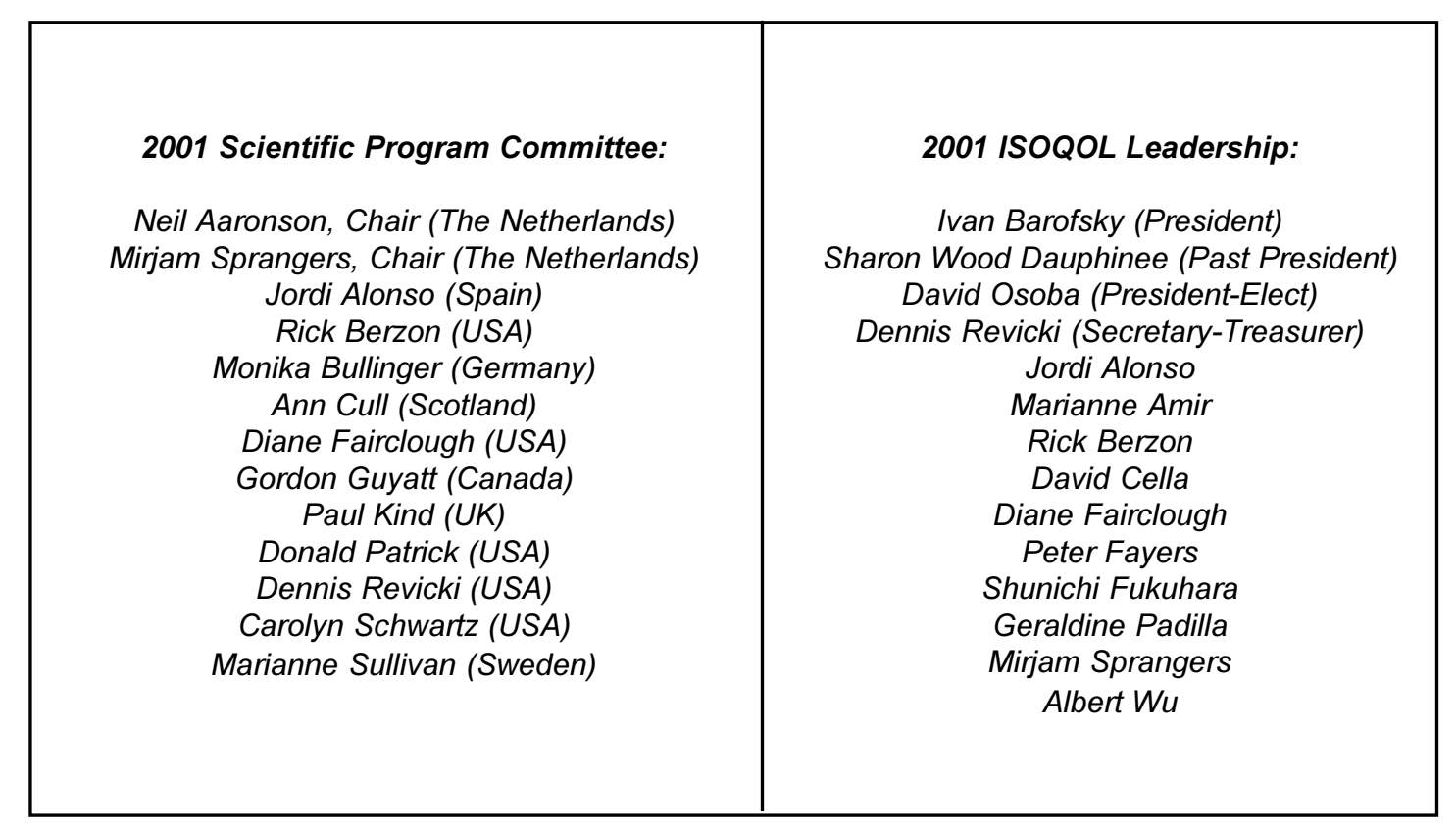

Donna Lamping (UK)

Christopher Lee (Canada)

Deborah P. Lubeck (USA)

Yasmin Maor (Israel)

Mona Martin (USA)

Susan Mathias (USA)

Elaine McColl (UK)

Stephen McKenna (UK)

Alex C. Michalos (Canada)

Ali Montazeri (Iran)

Richard Osborne (Australia)

David Osoba (Canada)

Joseph Pellizzari (Canada)

Amy Peterman (USA)

Arnold Potosky (USA)

Luis Prieto (Spain)

Marlene Reimer (Canada)

Dennis Revicki (USA)

Gerald Schumacher (USA)

Carolyn Schwartz (USA)

Kevin Smith (USA)

Marianne Sullivan (Sweden)

Tara Symonds (UK)

Alan Tennant (UK)

Claudette Varricchio (USA)

Galina Velikova (UK) 


\section{See you in 2002...}

Mark your calendars...

...for the ISOQOL 2002 meeting!

\section{October 30 - November 2 in Orlando, FL (USA)}

\section{The Caribe Royale Resort Suites \& Villas}

The Caribe Royale is located in the Lake Buena Vista part of Orlando and offers complimentary transportation to Walt Disney World.

All rooms are suites - a beautiful property.

The meeting space will fit our program very nicely.

Watch the ISOQOL website, www.isoqol.org, for the Call for Abstracts in January!

Again, be sure to mark your calendars for October 30 - November 2, 2002! 